\title{
MORPHOLOGICAL STUDIES OF POLYPHOSPHAZENES AND THEIR NANOCOMPOSITES USING SOLID-STATE NUCLEAR MAGNETIC RESONANCE SPECTROSCOPY
}

\author{
CHUCHU SUN \\ B.Sc., University of Chao Hu, China, 2011
}

\author{
A Thesis \\ Submitted to the School of Graduate Studies \\ of the University of Lethbridge \\ in Partial Fulfilment of the \\ Requirement for the Degree

\section{MASTERS OF SCIENCE} \\ Department of Chemistry and Biochemistry \\ University of Lethbridge \\ LETHBRIDGE, ALBERTA, CANADA
}

(C) Chuchu Sun 2014 
To my family 


\section{ABSTRACT}

Solution state NMR of ${ }^{1} \mathrm{H},{ }^{19} \mathrm{~F}$ and ${ }^{31} \mathrm{P}$ of poly[bis(trifluoroethoxy)] (PBFP) in THFd8 were acquired to study the polymer morphology in the solution state. Solid state high resolution ${ }^{1} \mathrm{H},{ }^{19} \mathrm{~F}$ and ${ }^{31} \mathrm{P}$ MAS DP NMR spectra were studied using a deconvolution method to understand how each component proportion varies with synthetic approach and processing methods, such as solvent casting, heat cycling, and inclusion of inorganic nanoparticles.

The ${ }^{19} \mathrm{~F}$ and ${ }^{31} \mathrm{P}$ NMR relaxation time data were acquired at an MAS spinning rate of $10 \mathrm{kHz}$, over a temperature range from 20 to $80^{\circ} \mathrm{C}$ for samples PBFP, annealed PBFP, NC $5 \%$ and annealed NC $5 \%$. The ${ }^{19} \mathrm{~F}$ NMR spectra were deconvolved using a threecomponent model for samples PBFP and NC 5\% and a four-component model for samples annealed PBFP and annealed NC 5\% from 20 to $40^{\circ} \mathrm{C}$. A four-component model was used for all four samples at high temperature range, from $50-80^{\circ} \mathrm{C}$. The ${ }^{31} \mathrm{P}$ NMR spectra were deconvolved using a four-component model for all four samples from 20 to $40^{\circ} \mathrm{C}$. A fivecomponent model was applied to all four samples over 50 to $80^{\circ} \mathrm{C}$. The models were established and adjusted to be consistent for all the relaxation arrays over their corresponding temperature ranges.

The relaxation time constants of each component were analyzed and compared between samples and temperatures. All ${ }^{31} \mathrm{P} \mathrm{T}_{1}$ constants decrease with increasing temperature and their $T_{2}$ values increase, indicating the polymer backbone is in the slow motion regime. For any particular component, the annealed PBFP always has the largest $\mathrm{T}_{1}$, the annealed NC $5 \%$ has the second longest $\mathrm{T}_{1}$, followed by the PBFP and NC $5 \%$ has the shortest $\mathrm{T}_{1}$, suggesting that annealing increases the crystallite size and thus slows the 
motion of the backbone; the filling of nano- $\mathrm{TiO}_{2}$ suppresses the growth of the crystallites and enhances the motion of the polymer backbone. The ${ }^{19} \mathrm{~F} T_{1}$ values increase with temperature and their $T_{2}$ also increase with temperature, suggesting the polymer sidechain is in the slow motion regime. For any particular component, the $\mathrm{NC} 5 \%$ always has the largest $T_{1}$ and the PBFP has the second largest $T_{1}$, followed by the annealed NC $5 \%$ and annealed PBFP has the shortest $\mathrm{T}_{1}$; indicating that annealing increases the crystallite size and thus slows the motion of the polymer sidechains; the nano- $\mathrm{TiO}_{2}$ suppresses the growth of the crystallite and enhances the motion of the polymer sidechain, similar effects as what are observed for polymer backbone.

The ${ }^{1} \mathrm{H}$ to ${ }^{31} \mathrm{P}$ and ${ }^{19} \mathrm{~F}$ to ${ }^{31} \mathrm{P}$ NMR cross polarization results at $20^{\circ} \mathrm{C}$ for all four samples, indicated that there are two $\mathrm{CP}$ curves for ${ }^{1} \mathrm{H}$ to ${ }^{31} \mathrm{P}$. One has a much faster building up and decay rate than the other. Only one $\mathrm{CP}$ curve for ${ }^{19} \mathrm{~F}$ to ${ }^{31} \mathrm{P} \mathrm{CP}$ was observed. This suggests that the protons from the middle of the polymer sidechains are more sensitive to the motion of the polymer backbone than the fluorines near the terminal of the sidechains. The model for ${ }^{31} \mathrm{P}$ is further refined based on the $\mathrm{CP}$ curves and the relaxation data and could be correlated to different phases of the polymer. 


\section{ACKNOWLEDGEMENTS}

I would like to express my deepest appreciation and sincerest gratitude to my supervisor, Professor Paul Hazendonk for giving the chance to study for my master degree. It has been truly memorable and educative being a member of his research group. His wide knowledge and inspiration have been of great value for me. I am also extremely grateful

for the freedom he gave me to try out new ways, for the incredible amount of patience he had with me, and for the untiring help during my difficult moments.

This work could not have been done without the expert technical support provided by Prof. Paul G. Hayes for his kind assistance in polymer synthesis and helpful discussions and to all members of the Paul Hayes's lab, I want to thank for their assistance. I must give a special thanks to Tony Motina, for offering an excellent instrument supervision and technique help with nuclei magnetic resonance spectrometer. I also have had the pleasure of working with a number of knowledgeable members in our group for their useful discussions and assistance in carrying out research work. I would like to express my warm thanks to Dr. Paul Singh Sidhu for his advices and help. Last but not least, I would like to thank my family and all my friends for their support and patience. 


\section{TABLE OF CONTENTS}

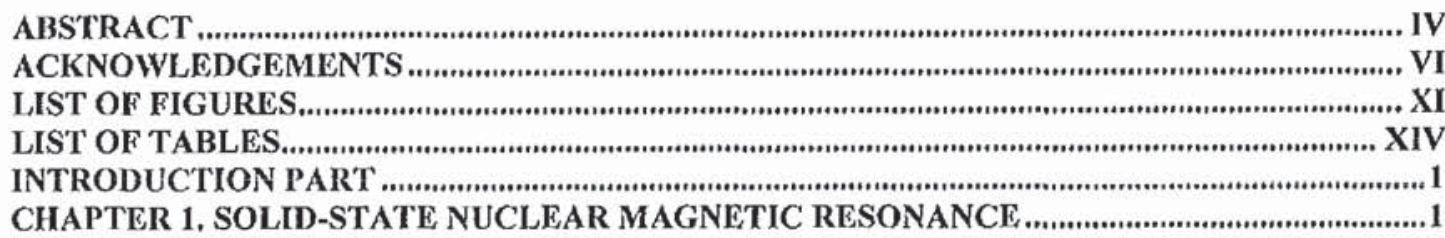

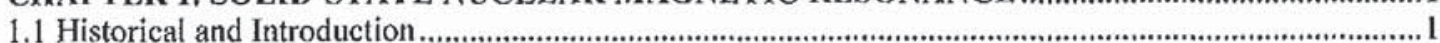

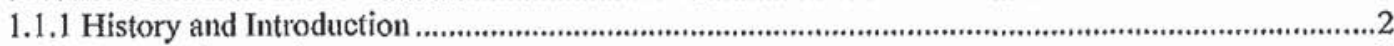

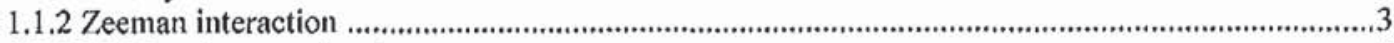

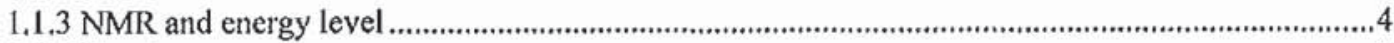

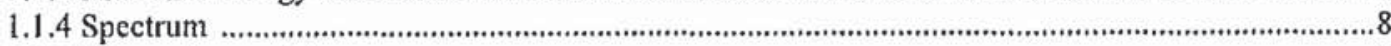

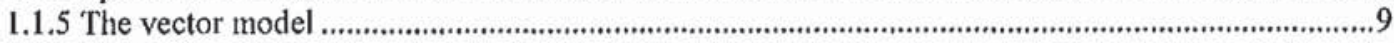

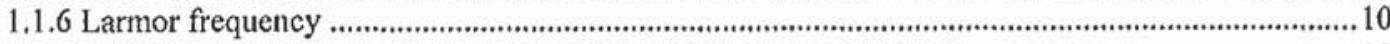

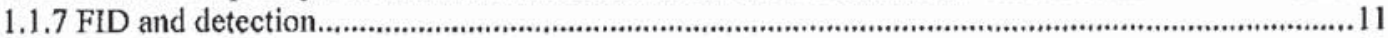

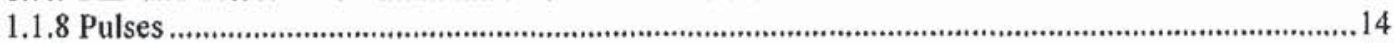

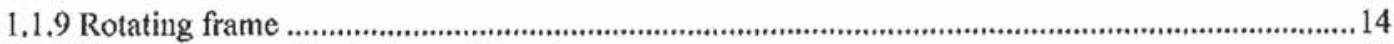

1.1.10 Larmor precession in the rotating frame ..........................................................................14

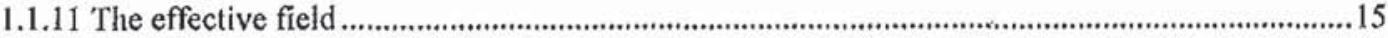

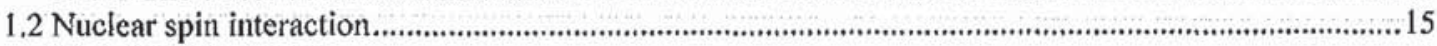

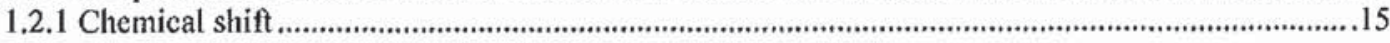

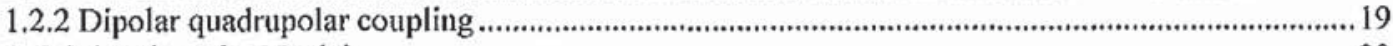

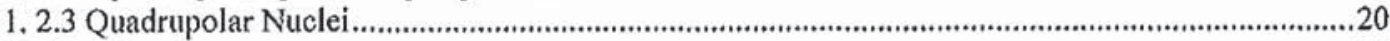

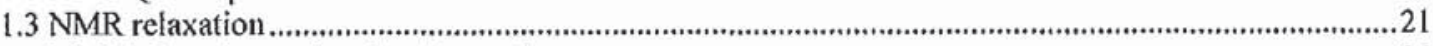

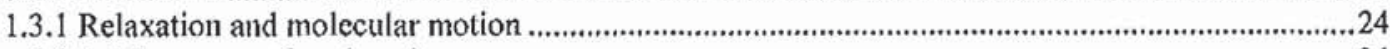

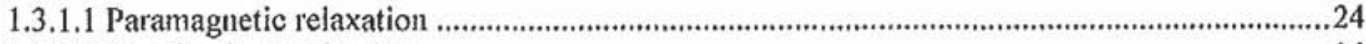

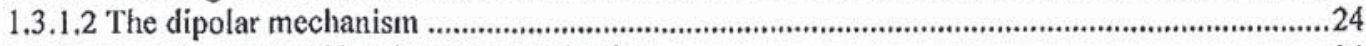

1.3.1.3 The chemical shift anisotropy mechanism...........................................................................24

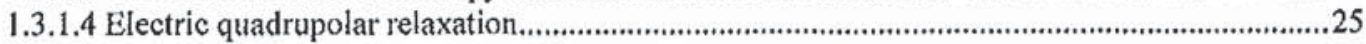

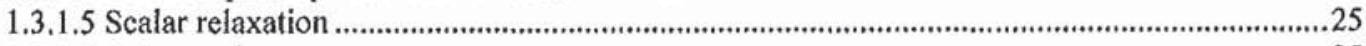

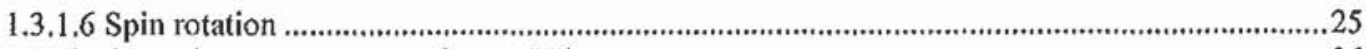

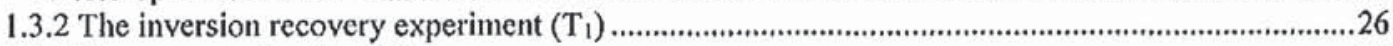

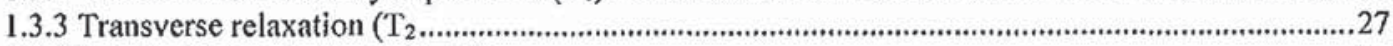

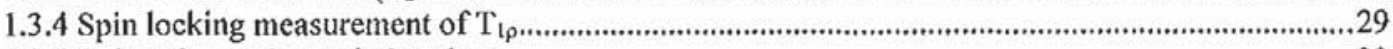

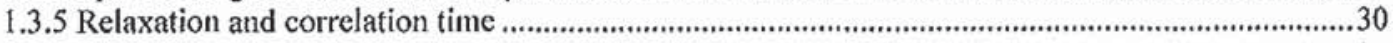

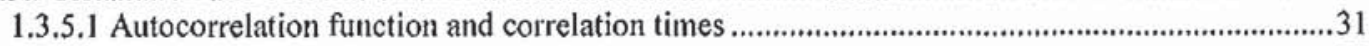

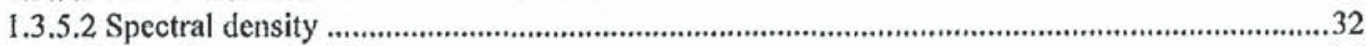

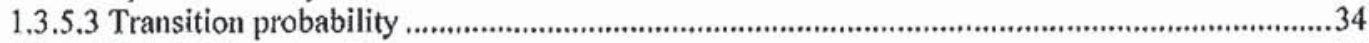

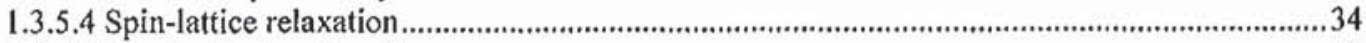

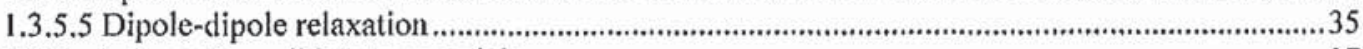

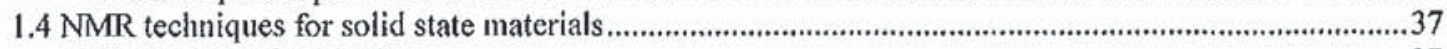

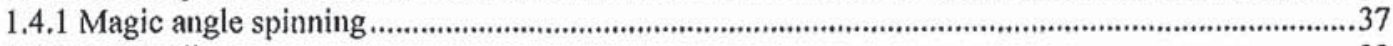

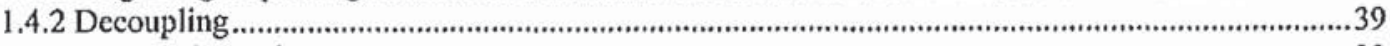

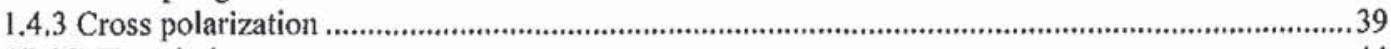

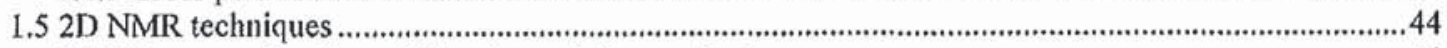

1.5.1 Homonuclear through bond correlation methods .................................................................44

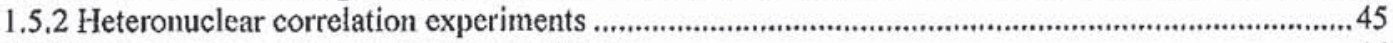

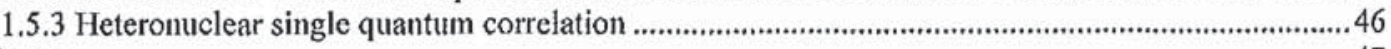

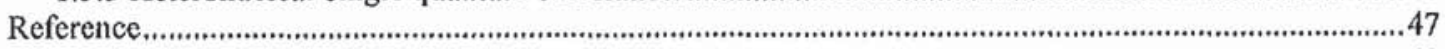

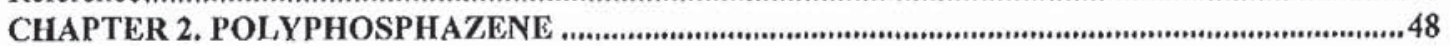

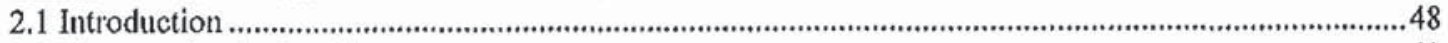

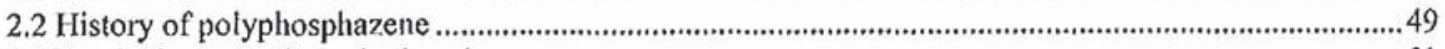

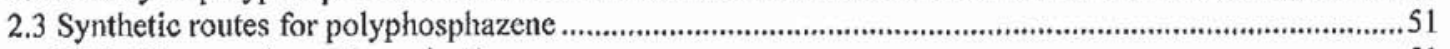

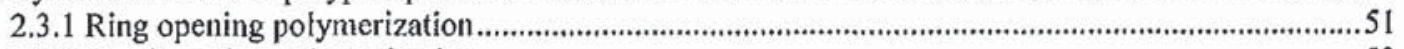

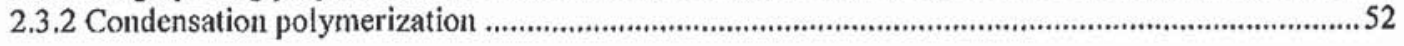


2.3.3 Condensation reaction of $\mathrm{OCl}_{2} \mathrm{PN}=\mathrm{PCl}_{3}$.

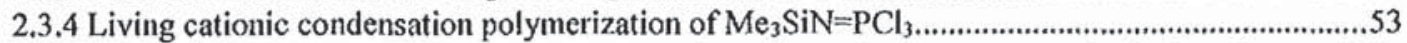

2.3.5 Preparation of polyphosphazene by ring opening polymerization process of substituted or partially substituted cyclophosphazene ......

2.3.6 Condensation polymerization of organic substitution phosphoroanimines ....................................57

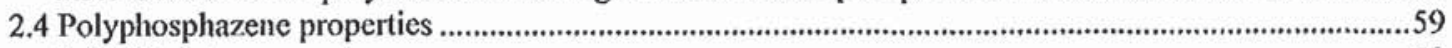

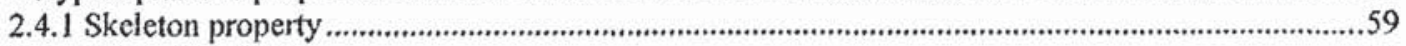

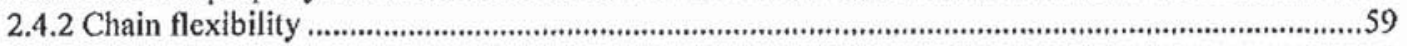

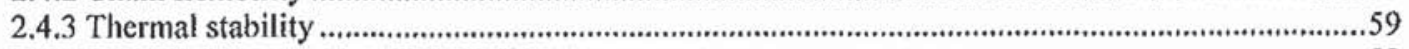

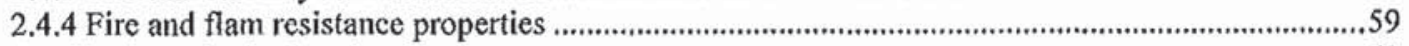

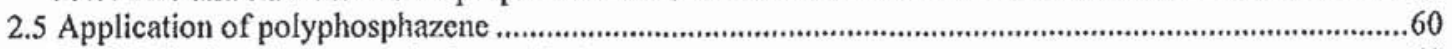

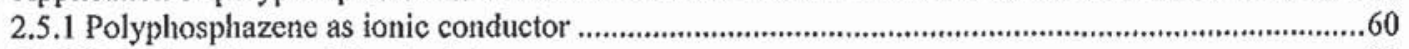

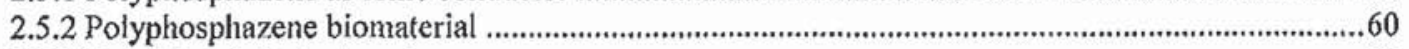

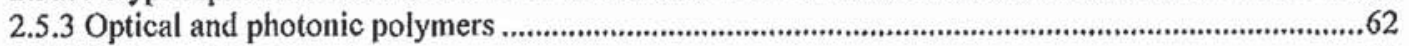

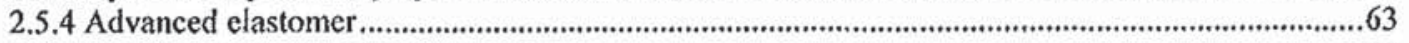

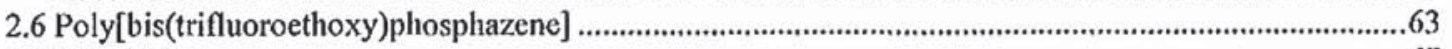

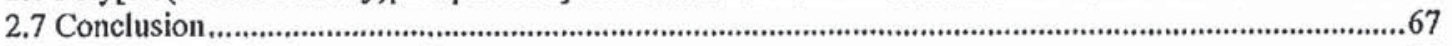

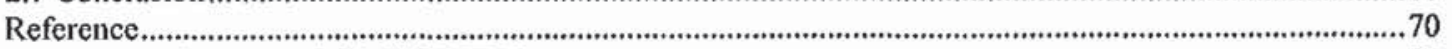

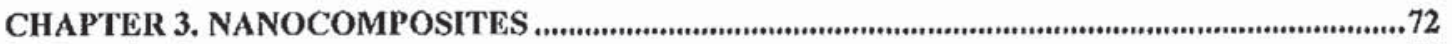

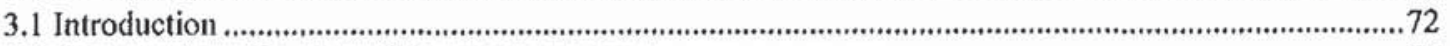

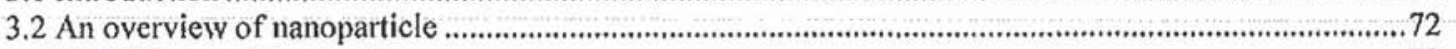

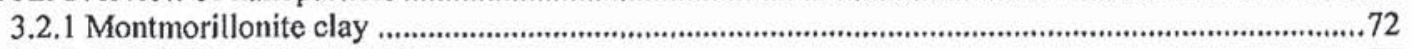

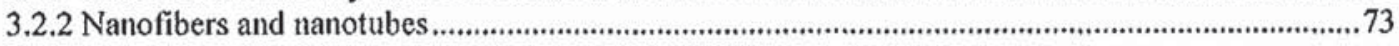

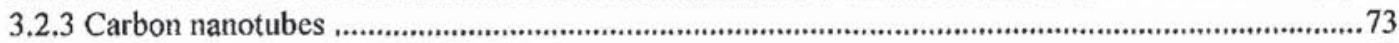

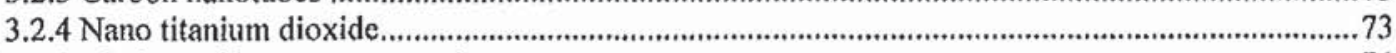

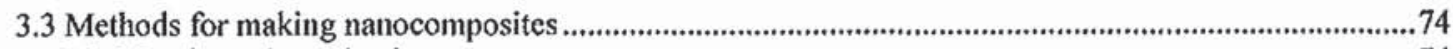

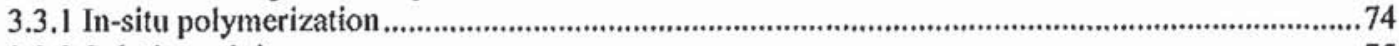

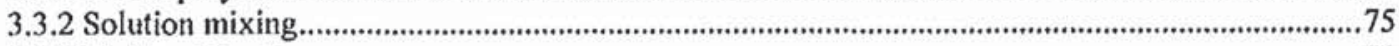

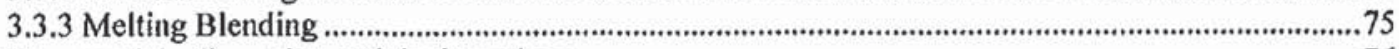

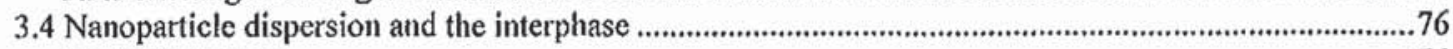

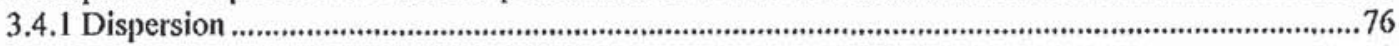

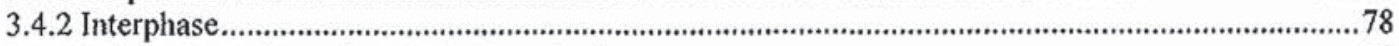

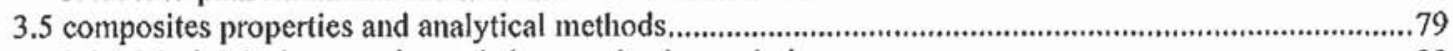

3.5.1 Mechanical properties and characterization techniques ..............................................................8

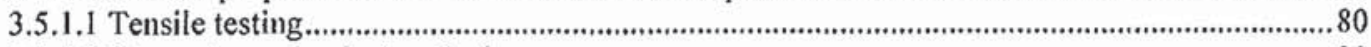

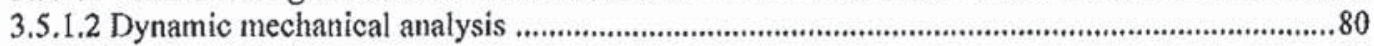

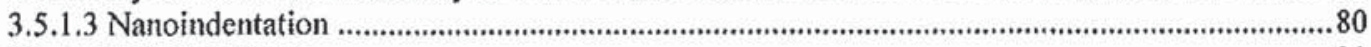

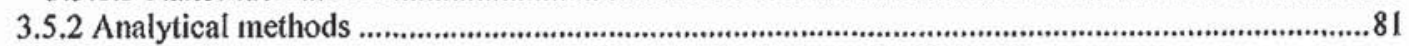

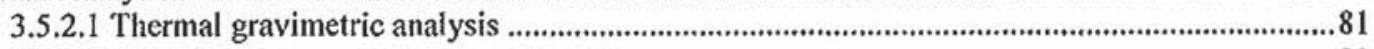

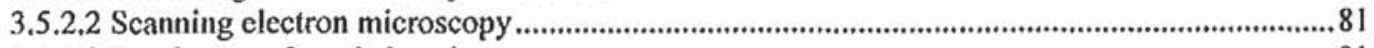

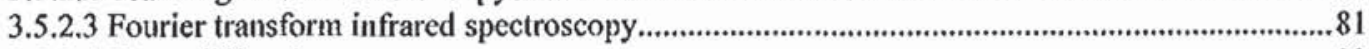

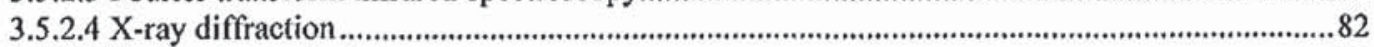

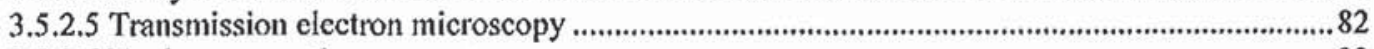

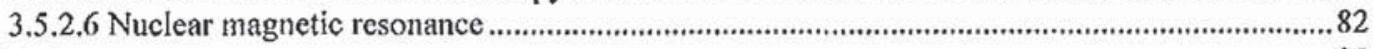

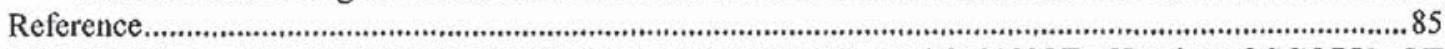

CHAPTER 4 SOLID-STATE NUCLEAR MAGNETIC RESONANCE SPECTROSCOPY OF POLYMER AND NANOCOMPOSITES ..........................................................86

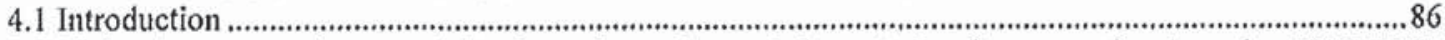

4.2 Molecular structural connectivity dynamics and morphology study of macromolecules using SSNMR 87

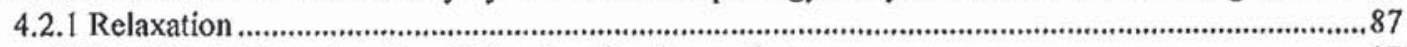

4.2.1.1 Spin-lattice relaxation $\left(\mathrm{T}_{\mathrm{l}}\right)$ and molecular motion..............................................................8

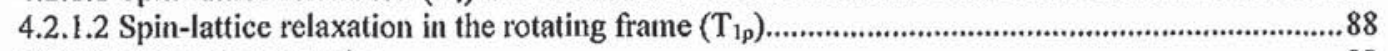

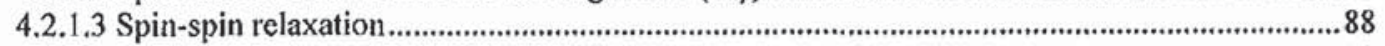

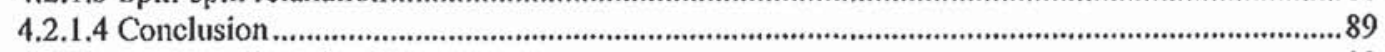

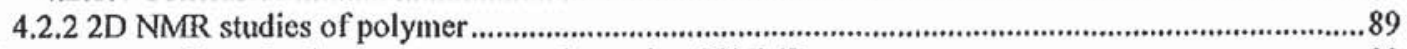

4.3 structure studies of polymer nanocomposites using SSNMR ..........................................................99 


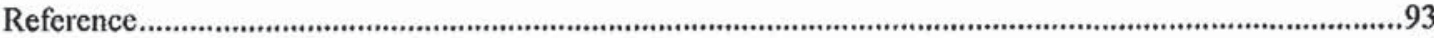

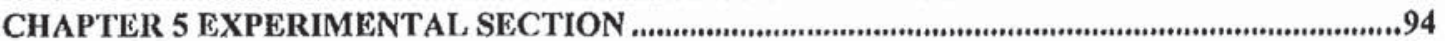

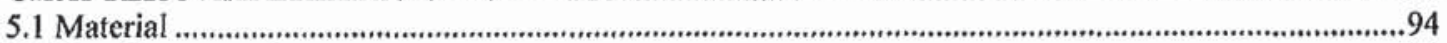

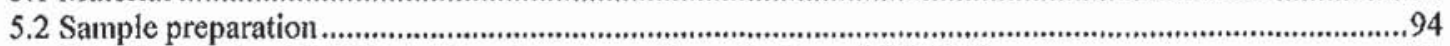

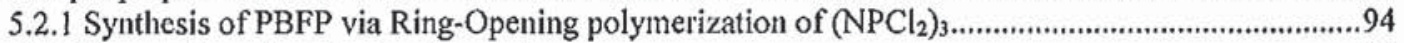

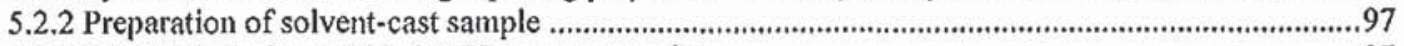

5.2.3 Preparation of nano $\mathrm{TiO}_{2}$ /PBFP nanocomposites ..................................................................97

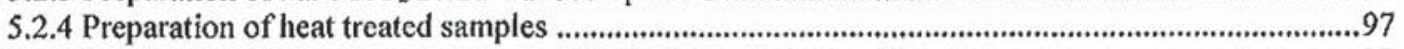

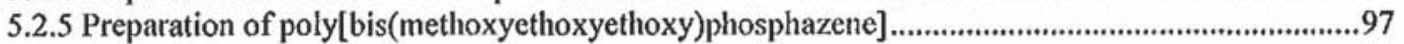

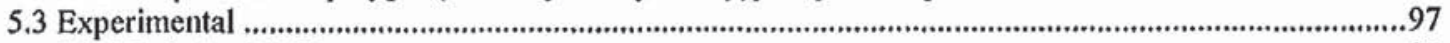

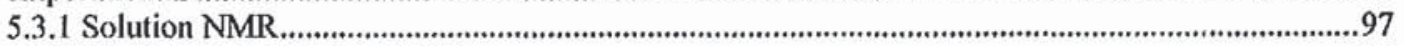

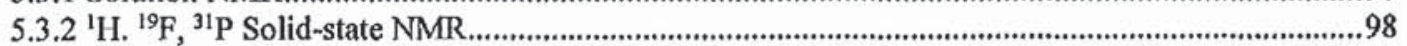

5.3.2.1 ${ }^{1} \mathrm{H}$ DP MAS NMR of PBFP, annealed PBFP, NC 5\% from ring opening polymerization and PBFP from ambient temperature polymerization..................................................................99

5.3.2.2 ${ }^{19} \mathrm{P}$ DP MAS NMR of PBFP, annealed PBFP, NC $5 \%$ from ring opening polymerization and PBFP from ambient temperature polymerization..................................................................99

5.3.2.3 ${ }^{31} \mathrm{P}$ DP MAS NMR of PBFP, annealed PBFP, NC 5\% from ring opening polymerization and PBFP from ambient temperature polymerization....................................................................99

5.3.3 VT solid-state NMR relaxation times of PBFP/annealed PBFP/NC 5\%/annealed NC 5\% ............99

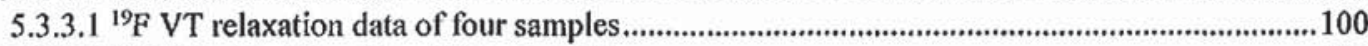

$5.3 .3 .2{ }^{31}$ P VT relaxation data of four samples..................................................................................100

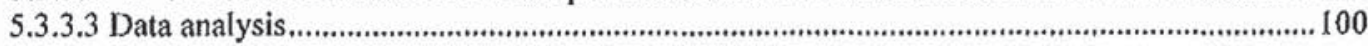

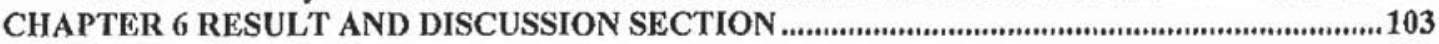

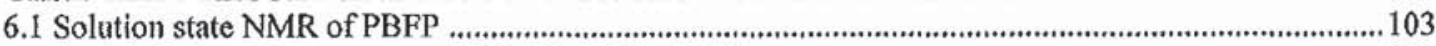

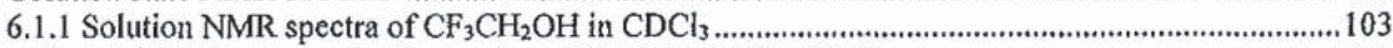

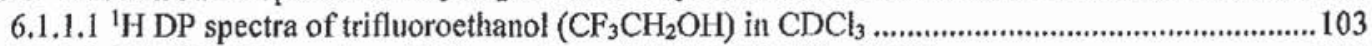

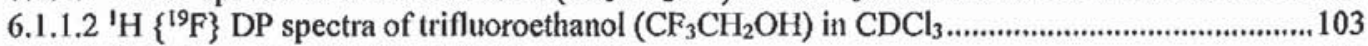

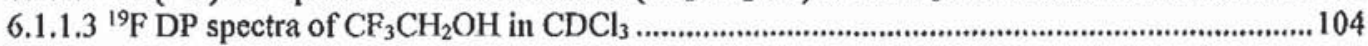

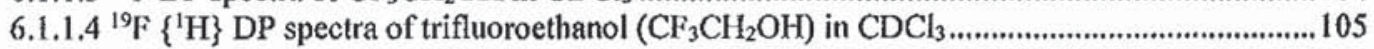

6.1.2 Solution NMR spectra of PBFP in THF_d8 .............................................................................105

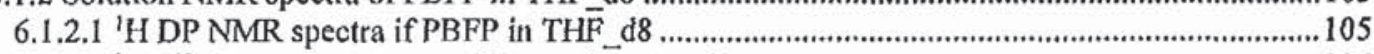

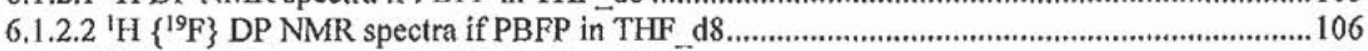

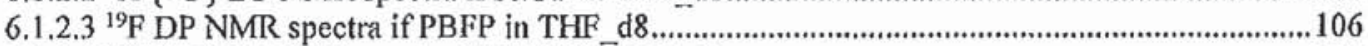

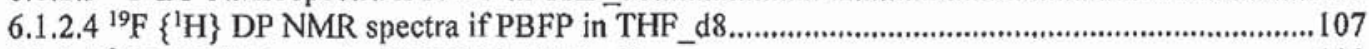

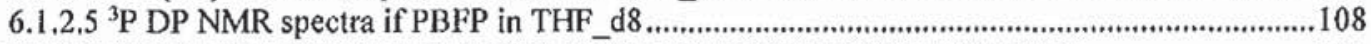

CHATER 7 SOLID-STATE NMR SPECTRA F

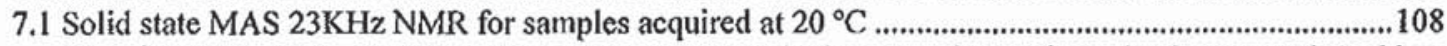

7.1.1 ${ }^{1} \mathrm{H}$ Solid-State MAS $23 \mathrm{kHz}$ NMR Spectra of Ring Opening Polymerization PBFP/ Ambient polymerization PBFP/Heat annealed PBFP/Nano-TiO 2 5\% Nanocomposite.............................109

7.1.2 ${ }^{19} \mathrm{~F}$ Solid-State MAS $23 \mathrm{kHz}$ NMR Spectra of Ring Opening Polymerization PBFP/ Ambient polymerization $\mathrm{PBFP} / \mathrm{Heat}$ annealed $\mathrm{PBFP} / \mathrm{Nano}-\mathrm{TiO}_{2} 5 \%$ Nanocomposite.............................111

7.1.3 ${ }^{31 \mathrm{P}}$ Solid-State MAS $23 \mathrm{kHz}$ NMR Spectra of Ring Opening Polymerization PBPP/ Ambient polymerization $\mathrm{PBFP} / \mathrm{Heat}$ annealed $\mathrm{PBFP} / \mathrm{Nan}-\mathrm{TiO}_{2}, 5 \%$ Nanocomposite............................112

7.2 Morphological Changes of PBFP, annealed PBFP, $5 \%$ Nano-TiO $_{2}$ PBFP nanocomposite (NC 5\%) and Annealed NC 5\% Nanocomposite under Variable Temperature Using Solid State MAS $10 \mathrm{kHz}$ NMR

$7.2 .1{ }^{19} \mathrm{~F}$ DP SSNMR MAS $10 \mathrm{KHz}$ of 4 Samples under Variant Temperature ....................................115

$7.2 .2^{31}$ P DP SSNMR MAS $10 \mathrm{KHz}$ of 4 Samples under Variant Temperature ...................................116

7.2.3 Deconvolution Analysis for ${ }^{19} \mathrm{~F}$ and ${ }^{31} \mathrm{P}$ Using Solid-State NMR MAS $10 \mathrm{kHz}$ at Variant Temperature.

7.2.3.1 ${ }^{31} \mathrm{P}$ DP MAS $10 \mathrm{kHz}$ of four samples deconvolution model over temperature range from 20 to $80^{\circ} \mathrm{C}$

7.2.3.2 ${ }^{31}$ P DP MAS $10 \mathrm{kHz}$ of four samples each deconvolution percentage at temperature range of 20

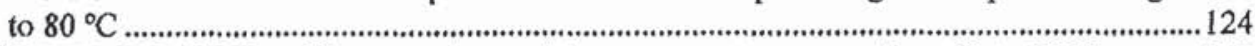

7.2.3.3 ${ }^{19} \mathrm{~F}$ DP MAS $10 \mathrm{kHz}$ of four samples models at temperature range from 20 to $80^{\circ} \mathrm{C} . . . \ldots \ldots \ldots . .128$ 
7.2.3.4 ${ }^{19} \mathrm{~F}$ DP MAS $10 \mathrm{kHz}$ of four samples each deconvolution percentage analysis at temperature

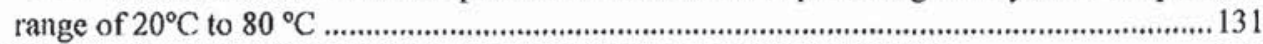

CHAPTER 8 NMR RELAXATION RESULT OF PBRP/NANOCOMPOSITES...............................145

8.1 Relaxation analysis of PBFP and $\mathrm{PBFP}_{\mathrm{TiO}}$ nanocomposites ......................................................... 145

8.2 Solid-state NMR relaxation times of PBFP .................................................................................146

$8.2 .1^{31} \mathrm{P}$ spin-lattice relaxation time of PBFP at an MAS rate of $10 \mathrm{kHz}$............................................146

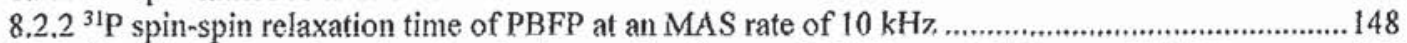

$8.2 .3^{31} \mathrm{P}$ spin-lattice relaxation time in the rotating frame of PBFP at an MAS rate of $10 \mathrm{kHz}$.............149

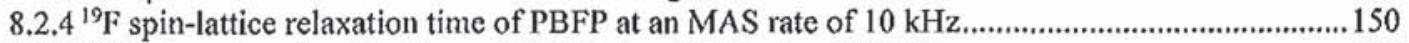

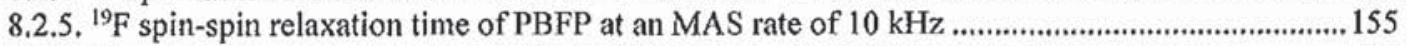

8.2.6 ${ }^{19} \mathrm{~F}$ spin-lattice relaxation time in the rotating frame of PBFP at an MAS rate of $10 \mathrm{kHz} \ldots \ldots \ldots \ldots . . .156$

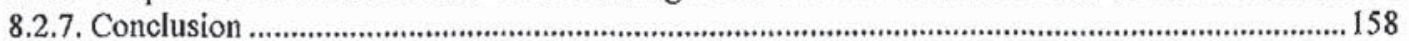

8.3 Solid-state NMR relaxation times of annealed PBFP........................................................................... 159

8.3.1 ${ }^{31} \mathrm{P}$ spin-lattice relaxation time of annealed PBFP at an MAS rate of $10 \mathrm{kHz}$.............................160

8.3.2 ${ }^{31} \mathrm{P}$ spin-spin relaxation time of annealed PBPP at an MAS rate of $10 \mathrm{kHz}$................................162

$8.32 .3^{3 t} \mathrm{P}$ spin-lattice relaxation time in the rotating frame of annealed PBFP at an MAS rate of $10 \mathrm{kHz}$

$8.4^{19} \mathrm{G}$ pin-attion.

8.3.4 F spin-lattice relaxation time of annealed PBFP at an MAS rate of $10 \mathrm{kHz} . . . \ldots \ldots \ldots . . . . . . . . . . . . . . . . . . . .164$

8.3.5. ${ }^{19} \mathrm{~F}$ spin-spin relaxation time of annealed PBFP at an MAS rate of $10 \mathrm{kHz}$.................................

8.3.6 ${ }^{19} \mathrm{~F}$ spin-lattice relaxation time in the rotating frame of annealed PBFP at an MAS rate of $10 \mathrm{kHz}$

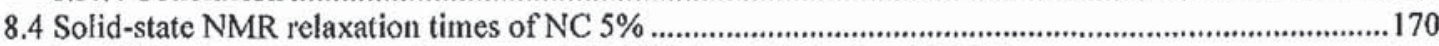

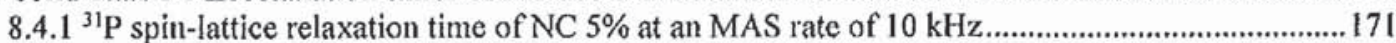

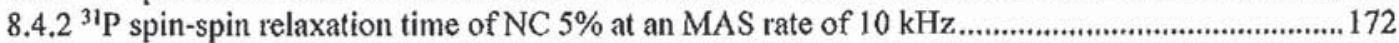

8.4.3 ${ }^{31} \mathrm{P}$ spin-lattice relaxation time in the rotating frame of NC $5 \%$ at an MAS rate of $10 \mathrm{kHz}$......... 173

$8.4 .4^{19} \mathrm{P}$ spin-lattice relaxation time of NC 5\% at an MAS rate of $10 \mathrm{kHz}$........................................174

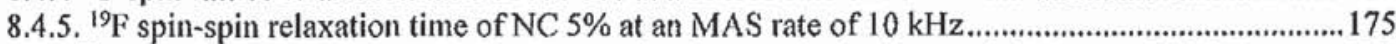

8.4.6 ${ }^{19} \mathrm{~F}$ spin-lattice relaxation time in the rotating frame of $\mathrm{NC} 5 \%$ at an MAS rate of $10 \mathrm{kHz} \ldots \ldots \ldots . .176$

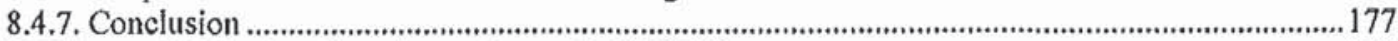

8.5 Solid-state NMR relaxation times of annealed NC 5\% ................................................................... 180

$8.5 \mathrm{I}^{31} \mathrm{P}$ spin-lattice relaxation time of annealed NC $5 \%$ at an MAS rate of $10 \mathrm{kHz}$.............................181

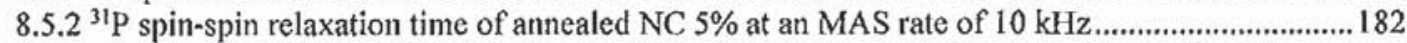

$8.5 .3^{31} \mathrm{P}$ spin-lattice relaxation time in the rotating frame of annealed NC $5 \%$ at an MAS rate of $10 \mathrm{kHz}$

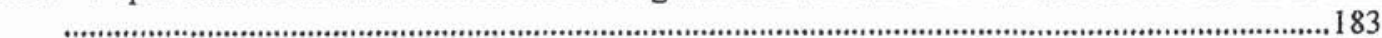

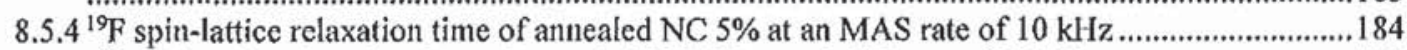

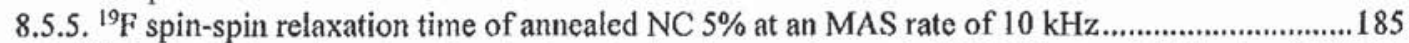

8.5.6 ${ }^{19} \mathrm{~F}$ spin-lattice relaxation time in the rotating frame of annealed NC $5 \%$ at an MAS rate of $10 \mathrm{kHz}$

.

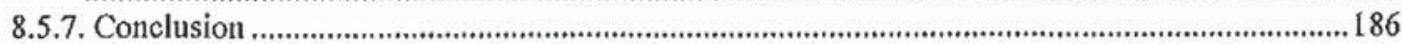

$8.6^{31} \mathrm{P}$ solid-state NMR relaxation times of different preparation methods .................................................187

$8.61{ }^{31} \mathrm{P}$ solid-state $\mathrm{NMR} \mathrm{T}_{1}$ relaxation time of different preparation methods .....................................189

8.5.2 ${ }^{31} \mathrm{P}$ solid-state NMR $\mathrm{T}_{2}$ relaxation time of different preparation methods ...................................192

8.5.3 ${ }^{31} \mathrm{P}$ solid-state NMR $\mathrm{T}_{\mathrm{l} \rho}$ relaxation time of different preparation methods ....................................196

$8.7^{19} \mathrm{~F}$ solid-state NMR relaxation times of different preparation methods..............................................198

$8.61{ }^{19} \mathrm{~F}$ solid-state NMR $\mathrm{T}_{1}$ relaxation time of different preparation methods ....................................199

$8.5 .2^{19} \mathrm{~F}$ solid-state $\mathrm{NMR} \mathrm{T}_{2}$ relaxation time of different preparation methods ...................................200

$8.5 .3^{19} \mathrm{~F}$ solid-state NMR $\mathrm{T}_{1 \mathrm{p}}$ relaxation time of different preparation methods...................................207

CHAPTER 9 Cross Polarization $\left({ }^{19} \mathrm{~F}\right.$ to ${ }^{31} \mathrm{P} \mathrm{CP}$ and ${ }^{1 /} \mathrm{H}$ to $\left.{ }^{31} \mathrm{P} \mathrm{CP}\right)$ for samples PBFP/Annealed PBFP/NC 5\%/Annealed NC 5\% ............................................................................211

$9.1{ }^{19} \mathrm{~F} \rightarrow{ }^{31} \mathrm{P}$ CP NMR for samples PBFP/annealed PBFP and NC 5\%/annealed NC 5\%,.......................211

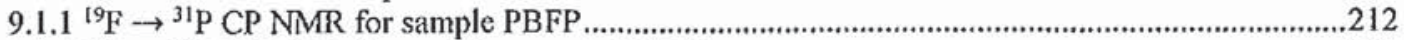

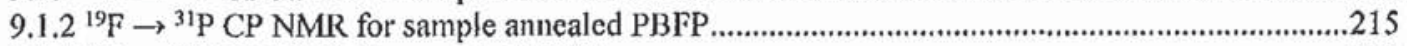

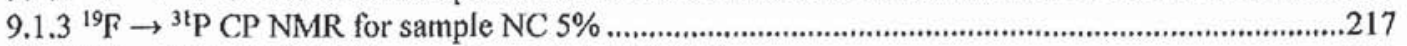

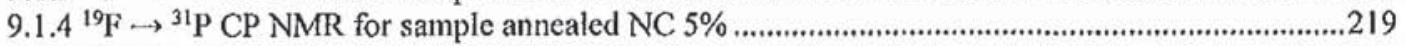

$9.2{ }^{1} \mathrm{H} \rightarrow{ }^{31} \mathrm{P} \mathrm{CP}$ NMR for samples PBFP/annealed PBFP and NC 5\%/annealed NC 5\% ........................221 


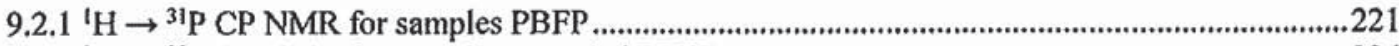

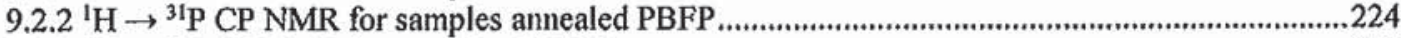

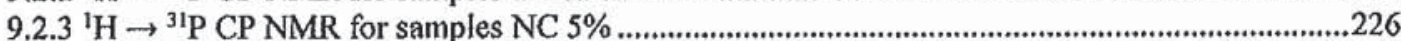

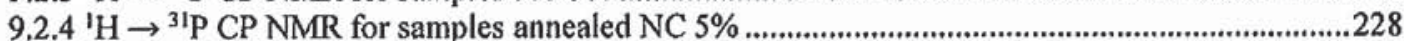

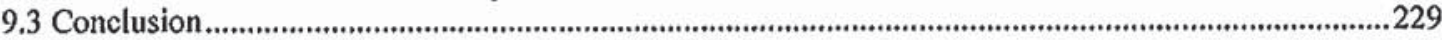

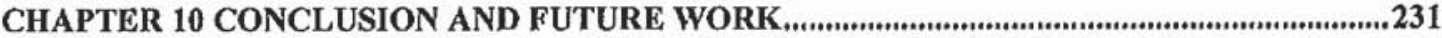

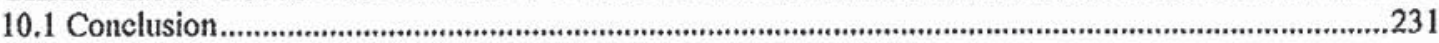




\section{LIST OF FIGURES}

Figure 1.1: Isotropic distribution of the angular momentum in the absence of external fields $\mathrm{B}_{0} \quad 2$ Figure 1.2: The Larmor frequency of nuclei precession about an external field 3

Figure 1.3: Ensemble of spins precessing at $\omega_{0}$ in the presence of the external magnetic field $B_{0}$ along $Z$-axis (left), and the corresponding longitudinal magnetization vector (right)

Figure 1.4: A line in the spectrum is associated with a transition between two energy level 4 $\begin{array}{ll}\text { Figure 1.5: Energy levels for a two spin system } & 7\end{array}$

Figure 1.6: The energy levels of a two-spin system and the corresponding spectrum 9

Figure 1.7: The direction of precession for a nucleus with a positive gyromagnetic ratio and hence a negative Larmor frequency 11

Figure 1.8: Tilting the magnetization vector through an angle $\beta$ gives the $X$-component of $\operatorname{size} M_{0} \sin \beta \quad 12$

Figure 1.9: Illustration of the precession of the magnetization vector in the xy-plane 13

Figure 1.10: Plots of the $\mathrm{x}$ - and $\mathrm{y}$ - components of the magnetization predicted using the approach Fourier transformation of these signals will give rise to the usual spectrum 13

Figure 1.11: The process of signal detection for obtaining the NMR spectrum 14

Figure 1.12: The top rows shows a field rotating at $-\omega_{\mathrm{KF}}$ when viewed in a fixed frame, the same field viewed

in a set of axes rotating at $-\omega_{\mathrm{R}}$ appears to be static $\quad 15$

Figure 1.13: The effective field in the rotating frame 16

Figure 1.14: A "grapefruit" diagram in which the thick line shows the motion of a magnetization vector during an on-resonance pulse $\quad 17$

Figure 1.15: Mechanism of the chemical shift 18

Figure 1.16: When the static magnetic field is applied along a principal axis direction, the induced field is parallel to the static field $\quad 20$

Figure 1.17: Inhomogeneous line broadening mechanisms in NMR spectra caused by CSA 21

Figure 1.18: The direct dipolar coupling between two spins, A and B in the presence of an external magnetic field, $B_{0}$

Figure 1.19: Spin $I=1 / 2$ nuclei have a spherically symmetric nuclear charge distribution (left); quadrupolar nuclei has a non-spherically symmetric charge distribution (right) 24

Figure 1.20: Inversion-recovery pulse sequence $\quad 30$

Figure 1.21: Partial alignment of spins in the transverse plane $\quad 31$

Figure 1.22: Spin echo pulse sequence $\quad 32$

$\begin{array}{ll}\text { Figure 1.23: Full width at half-height } & 33\end{array}$

Figure 1.24: Spin lock pulse sequence $\quad 33$

Figure 1.25: (a) Autocorrelation function of a rapid fluctuating field; (b) Autocorrelation function of a slowly fluctuating field 35

Figure 1.26: Comparison of the fields at short time interval $\quad 35$

Figure 1.27: Comparison of fields at long time interval $\quad 35$

Figure 1.28: Spectral density of a rapid fluctuating field 36

Figure 1.29: Spectral density of a slowly function field $\quad 37$

Figure 1.30: For a fluctuating field, the transition probabilities are proportional to the spectral density at the Larmor frequency $\omega^{0} \quad 38$

Figure 1.31: The spin-lattice relaxation time constant as a function of correlation time 39

Figure 1.32: (a) Single quantum transition probability in a homonuclear AX system; (b) Double and zero quantum transition probability in a homonuclear AX system 39

Figure 1.33: Variation of $T_{1}$ and $T_{2}$ with correlation time, for intramolecular dipole-dipole relaxation 41

Figure 1.34: Geometrical illustration of the magic angle (left); sample rotation at the magic angle in solidstate NMR (right)

Figure 1.35: MAS NMR spectrum with a sideband pattern due to insufficient spinning speed (top); the same spectrum but obtained at higher spinning speed (bottom) 43

Figure 1.36: Cross-polarization pulse sequence $\quad 45$

Figure 1.37: A scheme diagram of $\mathrm{H} \rightarrow \mathrm{X} \mathrm{CP}$ and $\mathrm{CP}$ drain curve 46

Figure 1.38: Structure of two-dimensional NMR experiment 48

Figure 1,39: COSY pulse sequence $\quad 49$

Figure 1.40: Schematic COSY spectrum for two coupled spins, A and X 49

$\begin{array}{ll}\text { Figure 1.41: HSQC pulse sequence } & 51\end{array}$ 
Figure 2.1: Polyphosphazene molecular structure 53

Figure 2.2: Side group substitution of poly(dichlorophosphazene) 55

Figure 2.3: Synthesis of chlorophosphazene $\quad 56$

$\begin{array}{ll}\text { Figure 2.4: Synthesis of Polyphosphazenes } & 57\end{array}$

Figure 2.5: ROP method for synthesis poly(dichlorophosphazene) $\quad 57$

Figure 2.6: Polyphosphazene with two different side groups $\quad 57$

Figure 2.7: Phosphorus pentachloride and ammonia or ammonium chloride react in a stepwise order 58

Figure 2.8: Overall process of Condensation reaction of $\mathrm{OCl}_{2} \mathrm{PN}=\mathrm{PCl}_{3} \quad 59$

Figure 2.9: Methods for preparing $\mathrm{Cl}_{3} \mathrm{P}=\mathrm{NSiMe}_{3}$ using $\mathrm{PCl}_{3}$ and $\mathrm{SO}_{2} \mathrm{Cl}_{2} \quad 60$

Figure 2.10: Different routes for synthesis $\mathrm{Cl}_{3} \mathrm{P}=\mathrm{NSiMe}_{3} \quad 60$

Figure 2.11: Cationic polymerization of trichlorophosphoranimines $\quad 60$

Figure 2.12: Polymerization of variety of organophosphoranimine 64

Figure 2.13: Polymerization of variety of organophosphoranimine $\quad 68$

Figure 2.14: An example of polyphosphazene with controlled refractive index 69

Figure 2.15: Mostly common synthetic route for PBFP

Figure 2.16: Domain structure of semi-crystalline material $\quad 72$

Figure 2.17: Phase transition diagram for solvent-cast PBFP between $T_{g}$ and $T_{m} \quad 73$

Figure 3.1: Illustration of three terms to describe dispersion quality $\quad 85$

Figure 3.2: Schematic of interphase area between filler and the polymer matrix $\quad 87$

Figure 3.3: Schematic representation adsorption characteristics of a metal-polymer nanocomposite $\quad 88$

Figure 5.1: Design of an oven used for the thermal ring-opening polymerization $\quad 106$

Figure 6.1: ${ }^{1} \mathrm{H}$ DP spectrum of $\mathrm{CF}_{3} \mathrm{CH}_{2} \mathrm{OH}$ in $\mathrm{CDCl}_{3} \quad 115$

Figure 6.2: ${ }^{1} \mathrm{H}\left\{{ }^{19} \mathrm{~F}\right\}$ spectrum of $\mathrm{CF}_{3} \mathrm{CH}_{2} \mathrm{OH}$ in $\mathrm{CDCl}_{3} \quad 116$

Figure 6.3: ${ }^{19} \mathrm{~F}$ DP spectrum of $\mathrm{CF}_{3} \mathrm{CH}_{2} \mathrm{OH}$ in $\mathrm{CDCl}_{3} \quad 117$

Figure 6.4: ${ }^{19} \mathrm{~F}\left\{{ }^{1} \mathrm{H}\right\}$ spectrum of $\mathrm{CF}_{3} \mathrm{CH}_{2} \mathrm{OH}$ in $\mathrm{CDCl}_{3} \quad 117$

$\begin{array}{lr}\text { Figure 6.5: ' } \mathrm{H} \text { DP spectrum of PBFP in THF-d8 } & 118\end{array}$

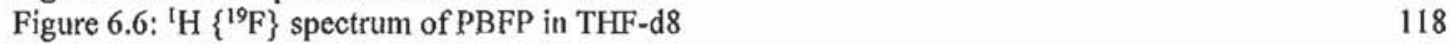

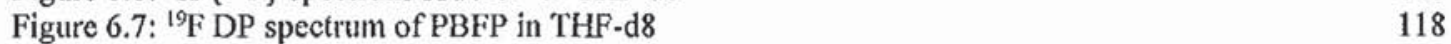

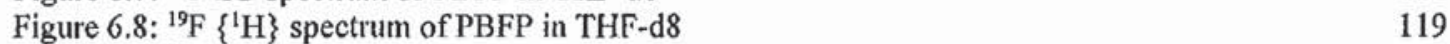

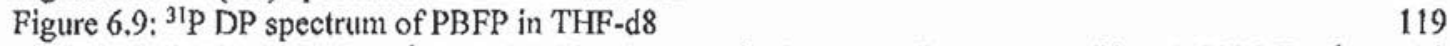

Figure 7.1: (a) SC: $500 \mathrm{MHz}{ }^{1} \mathrm{H}$ MAS NMR spectra of solvent cast ROP PBFP; (b) HT: $500 \mathrm{MHz}{ }^{1} \mathrm{H}$ MAS NMR spectra of heat-treated ROP PBFP; (c) AT: $500 \mathrm{MHz} 1 \mathrm{H}$ MASNMR spectra of AT polymerization PBFP; (d) NC: $500 \mathrm{MHz}{ }^{1} \mathrm{H}$ MAS NMR spectra of ROP PBFP doped with $5 \%$ nc$\mathrm{TiO}_{2}$

Figure 7.2: $500 \mathrm{MHz}{ }^{19} \mathrm{~F}$ DP MAS NMR spectrum of (a) Solvent cast ROP PBFP; (b) Heat-treated ROP PBFP; (c) ALP PBFP; (d) ROP PBFP with $5 \%$ nc-TiO

Figure 7.3: $500 \mathrm{MHz}{ }^{31} \mathrm{P}$ DP MAS NMR spectrum of (a) SC: solvent cast ROP PBFP; (b) NC: ROP PBFP with $5 \%$ nc-TiO ${ }_{2}$; (c) HT: Heat treated ROP PBFP; (d) Heat treated ROP PBFP with $5 \%$ nc-TiO ; (e) ALP: ALP PBFP

Figure 7.4: ${ }^{19} \mathrm{~F}$ SSNMR MAS $10 \mathrm{KHz}$ of 4 samples under variant temperature (a) non annealed PBFP; (b) annealed PBFP; (c) non annealed NC 5\%; (d) annealed NC 5\%

Figure 7.5: ${ }^{31} \mathrm{P}$ SSNMR MAS $10 \mathrm{KHz}$ of 4 samples under variant temperature (a) non annealed PBFP; (b) annealed PBFP; (c) non annealed NC 5\%; (d) annealed NC 5\% 128

Figure 7.6: ${ }^{31} \mathrm{P}$ PBFP virgin $\mathrm{T}_{2}$ relaxation array overlapping at $20^{\circ} \mathrm{C} \quad 128$

Figure 7.7: ${ }^{31} \mathrm{P}$ PBFP virgin $\mathrm{T}_{2}$ relaxation array overlapping at $50^{\circ} \mathrm{C} \quad 128$

Figure 7.8: ${ }^{31} \mathrm{P}$ PBFP virgin $\mathrm{T}_{1}$ relaxation model at $20^{\circ} \mathrm{C}$ for the first spectrum in the relaxation area 130

Figure 7.9: $\mathrm{PBFP}$ virgin ${ }^{31} \mathrm{P} \mathrm{T}_{1}$ relaxation model at $20^{\circ} \mathrm{C}$ for the 8 th spectrum in the relaxation array 130

Figure 7.10: Deconvolution model for ${ }^{31} \mathrm{P}$ of 4 samples at the temperature of $20-40^{\circ} \mathrm{C} \quad 130$

Figure7.11: PBPP virgin $\mathrm{T}_{1}$ relaxation model at $50^{\circ} \mathrm{C}$ for the first spectrum in the relaxation array 131

Figure 7.12: $\mathrm{PBFP}$ virgin $\mathrm{T}_{1}$ relaxation model at $80^{\circ} \mathrm{C}$ for the 8 th spectrum in the relaxation array 131

Figure 7.13: Deconvolution model for ${ }^{31} \mathrm{P}$ of 4 samples at the temperature of $50-80^{\circ} \mathrm{C} \quad 131$

Figure 7.14: ${ }^{31} \mathrm{P}$ deconvolution percentage area changing trends for 4 components over the temperature range of $20-40^{\circ} \mathrm{C}$ for PBFP (left) and annealed PBFP (right) 134

Figure 7.15: ${ }^{31} \mathrm{P}$ deconvolution percentage area changing trends for 4 components over the temperature range of $20-40^{\circ} \mathrm{C}$ for NC $5 \%$ (left) and annealed NC $5 \%$ (right) 
Figure 7.16: ${ }^{31} \mathrm{P}$ deconvolution percentage area changing trends for 5 components over the temperature range of $50-80^{\circ} \mathrm{C}$ for PBFP (left) and annealed PBFP (right)

Figure 7.17: ${ }^{31} \mathrm{P}$ deconvolution percentage area changing trends for 5 components over the temperature range of $50-80^{\circ} \mathrm{C}$ for NC $5 \%$ (left) and annealed NC $5 \%$ (right)

Figure 7.18: Deconvolution model for ${ }^{19} \mathrm{~F}$ of PBFP and NC $5 \%$ samples at the temperature of $20-40{ }^{\circ} \mathrm{C} 138$

Figure 7.19: Deconvolution model for ${ }^{19} \mathrm{~F}$ of annealed PBFP and annealed $\mathrm{NC} 5 \%$ samples at the temperature of $20-40^{\circ} \mathrm{C}$

Figure 7.20: Deconvolution model for ${ }^{19} \mathrm{~F} 4$ samples at the temperature of $50-80^{\circ} \mathrm{C}$

Figure 7.21: ${ }^{19} \mathrm{~F}$ deconvolution percentage area changing trends for different components over the temperature range of $20-40^{\circ} \mathrm{C}$ for PBFP (left) and annealed PBFP (right) 140

Figure 7.22: ${ }^{19} \mathrm{~F}$ deconvolution percentage area changing trends for different components over the temperature range of $20-40^{\circ} \mathrm{C}$ for NC $5 \%$ (left) and annealed NC $5 \%$ (right)

Figure 7.23: ${ }^{19} \mathrm{~F}$ deconvolution percentage area changing trends for 4 components over the temperature range of $50-80^{\circ} \mathrm{C}$ for PBFP (left) and annealed PBFP (right)

Figure 7.24: ${ }^{19} \mathrm{~F}$ deconvolution percentage area changing trends for 4 components over the temperature range of $50-80^{\circ} \mathrm{C}$ for NC $5 \%$ (left) and annealed NC 5\% (right) 143

Figure 8.1: ${ }^{31} \mathrm{P}$ spin-lattice relaxation time $\mathrm{T}_{1}$ for the four components obtained by deconvolution of the ${ }^{31} \mathrm{P}$ solid-state NMR spectra of PBFP, annealed PBFP, PBFP with $5 \%$ of nano-TiO ${ }_{2}$, and annealed sample of PBFP with $5 \%$ of nano- $\mathrm{TiO}_{2}$ over the temperature range of 20 to $40^{\circ} \mathrm{C}$; the biexponential behavior of the two contributions at $-4.55 \mathrm{ppm}$ (bottom)

186

Figure 8.2: ${ }^{31} \mathrm{P}$ spin-lattice relaxation time $\mathrm{T}_{1}$ for the five components obtained by deconvolution of the ${ }^{31} \mathrm{P}$ solid-state NMR spectra of PBFP, annealed PBFP, PBFP with $5 \%$ of nano-TiO 2 , and annealed sample of PBFP with $5 \%$ of nano- $\mathrm{TiO}_{2}$ over the temperature range of 50 to $80^{\circ} \mathrm{C} \quad 188$

Figure 8.3: ${ }^{31} \mathrm{P}$ transverse relaxation time $\mathrm{T}_{2}$ for the four components obtained by deconvolution of the ${ }^{31} \mathrm{P}$ solid-state NMR spectra of PBFP, annealed PBFP, PBFP with $5 \%$ of nano- $\mathrm{TiO}_{2}$, and annealed sample of PBFP with $5 \%$ of nano- $\mathrm{TiO}_{2}$ over the temperature range of 20 to $40^{\circ} \mathrm{C} \quad 190$

Figure 8.4: ${ }^{31} \mathrm{P}$ transverse relaxation time $\mathrm{T}_{2}$ for the four components obtained by deconvolution of the ${ }^{31} \mathrm{P}$ solid-state NMR spectra of PBFP, annealed PBFP, PBFP with $5 \%$ of nano- $\mathrm{TiO}_{2}$, and annealed sample of PBFP with $5 \%$ of nano-TiO $\mathrm{T}_{2}$ over the temperature range of 20 to $40^{\circ} \mathrm{C}$

Figure 8.5: Four examples of ${ }^{31} \mathrm{P} \mathrm{T}_{1} / \mathrm{T}_{2}$ ratio with respect to the changes of temperature for one broad contribution (Left) and one narrow contribution (Right) obtained by deconvolution of the ${ }^{31} \mathrm{P}$ solidstate NMR spectra of PBFP, annealed PBFP, PBFP with $5 \%$ of nano- $\mathrm{TiO}_{2}$, and annealed sample of PBFP with $5 \%$ of nano-TiO 2 over the temperature range of 20 to $40^{\circ} \mathrm{C}$

Figure 8.6: Four examples of ${ }^{31} \mathrm{P} \mathrm{T}_{1} / \mathrm{T}_{2}$ ratio with respect to the changes of temperature for one broad contribution (Left) and one narrow contribution (Right) obtained by deconvolution of the ${ }^{31} \mathrm{P}$ solidstate NMR spectra of PBFP, annealed PBFP, PBFP with $\$ \%$ of nano- $\mathrm{TiO}_{2}$, and annealed sample of PBFP with $5 \%$ of nano- $\mathrm{TiO}_{2}$ over the temperature range of 50 to $80^{\circ} \mathrm{C}$

Figure 8.7: Different components of ${ }^{31} \mathrm{P} \mathrm{T}_{1 \mathrm{p}}$ for 4 sample over the temperature range from $20-40^{\circ} \mathrm{C} \quad 197$

Figure 8.8: Different components of ${ }^{31} \mathrm{P}^{\mathrm{T}} \mathrm{T}_{\mathrm{l \rho}}$ for 4 sample over the temperature range from $50-80^{\circ} \mathrm{C} \quad 200$

Figure 8.9: ${ }^{19} \mathrm{~F}$ spin-lattice relaxation time $\mathrm{T}_{1}$ for the three components obtained by deconvolution of the ${ }^{19} \mathrm{~F}$ solid-state NMR spectra of PBFP, and sample of PBFP with $5 \%$ of nano-TiO ${ }_{2}$ over the temperature range of 20 to $40^{\circ} \mathrm{C}$

Figure $8.10^{19} \mathrm{~F}$ spin-lattice relaxation time $\mathrm{T}_{1}$ for the three components obtained by deconvolution of the ${ }^{19} \mathrm{~F}$ solid-state NMR spectra of amealed PBFP, and sample of annealed PBFP with $5 \%$ of nano- $\mathrm{TiO}_{2}$ over the temperature range of 20 to $40^{\circ} \mathrm{C}$

Figure 8.11: ${ }^{19} \mathrm{~F}$ spin-lattice relaxation time $\mathrm{T}_{1}$ for the three components obtained by deconvolution of the ${ }^{19} \mathrm{~F}$ solid-state NMR spectra of PBFP, annealed PBFP, PBFP with $5 \%$ of nano- $\mathrm{TiO}_{2}$, and annealed sample of $\mathrm{PBFP}$ with $5 \%$ of nano- $\mathrm{TiO}_{2}$ over the temperature range of 50 to $80^{\circ} \mathrm{C}$ 206

Figure 8.12: ${ }^{19} \mathrm{~F}$ transverse relaxation time $\mathrm{T}_{2}$ for the three components obtained by deconvolution of the ${ }^{19} \mathrm{~F}$ solid-state NMR spectra of PBFP, PBFP with $5 \%$ of nano- $\mathrm{TiO}_{2}$ over the temperature range of 20 to $40^{\circ} \mathrm{C}$

Figure $8.13:{ }^{19} \mathrm{~F}$ transverse relaxation time $\mathrm{T}_{2}$ for the three components obtained by deconvolution of the ${ }^{19} \mathrm{~F}$ solid-state NMR spectra of annealed PBFP, annealed PBFP with $5 \%$ of nano- $\mathrm{TiO}_{2}$ over the temperature range of 20 to $40^{\circ} \mathrm{C}$ 
Figure 8.14: ${ }^{19} \mathrm{~F}$ transverse relaxation time $\mathrm{T}_{2}$ for the four components obtained by deconvolution of the ${ }^{19} \mathrm{~F}$ solid-state NMR spectra of PBPF, annealed PBFP, PBFP svith $5 \%$ nano-TiO ${ }_{2}$, and annealed PBFP with $5 \%$ of nano- $\mathrm{TiO}_{2}$ over the temperature range of 50 to $80^{\circ} \mathrm{C}$

211

Figure 8.15: Two examples of ${ }^{19} \mathrm{~F} \mathrm{~T}_{1} / \mathrm{T}_{2}$, ratio with respect to the changes of temperature for one broad contribution (Left) and one narrow contribution (Right) over 20 to $40^{\circ} \mathrm{C}$ for sample PBFP and NC $5 \%$ obtained by deconvolution of the ${ }^{31} \mathrm{P}$ solid-state NMR spectra at an MAS rate of $10 \mathrm{kHz} 213$

Figure 8.16: Two cxamples of ${ }^{19} \mathrm{~F} \mathrm{~T}_{1} / \mathrm{T}_{2}$ ratio with respect to the changes of temperature for one broad contribution (Left), and one narrow contribution (Right) over 20 to $40^{\circ} \mathrm{C}$ for sample annealed PBFP and annealed NC 5\%-TiO $5 \%$ obtained by deconvolution of the ${ }^{31} \mathrm{P}$ solid-state NMR spectra at an MAS rate of $10 \mathrm{kHz}$

Figure 8.17: Two examples of ${ }^{19} \mathrm{~F} \mathrm{~T}_{1} / \mathrm{T}_{2}$ ratio with respect to the changes of temperature for one broad contribution (Left) and one narrow contribution (Right) over 50 to $80^{\circ} \mathrm{C}$ of PBFP, annealed PBFP, PBFP with $5 \%$ of nano0TiO deconvolution of the ${ }^{31} \mathrm{P}$ solid-state NMR spectra at an MAS rate of $10 \mathrm{kHz}$

Figure 8.18: ${ }^{19} \mathrm{~F}$ spin-lattice relaxation time $\mathrm{T}_{\mathrm{to}}$ in the rotating frame for the three components obtained by deconvolution of the ${ }^{31} \mathrm{P}$ solid-state NMR spectra of PBFP, and PBFP with $5 \%$ of nano-TiO ${ }_{2}$ over the temperature range of 20 to $40^{\circ} \mathrm{C}$

Figure 8.19: ${ }^{19} \mathrm{~F}$ spin-lattice relaxation time $\mathrm{T}_{1 p}$ in the rotating frame for the three components obtained by deconvolution of the ${ }^{31} \mathrm{P}$ solid-state NMR spectra of annealed PBFP, and annealed PBFP with $5 \%$ of nano- $\mathrm{TiO}_{2}$ over the temperature range of 20 to $40^{\circ} \mathrm{C}$

Figure 8.20: ${ }^{19} \mathrm{~F}$ spin-lattice relaxation time $\mathrm{T}_{1 \mathrm{p}}$ in the rotating frame for the three components obtained by deconvolution of the ${ }^{31} \mathrm{P}$ solid-state NMR spectra of PBFP, annealed PBFP, PBFP with $5 \%$ nano- $\mathrm{TiO}_{2}$ and $\mathrm{PBFP}$ with $5 \%$ of nano- $\mathrm{TiO}_{2}$ over the temperature range of 50 to $80^{\circ} \mathrm{C}$ 218

Figure 9.1: ${ }^{19} \mathrm{~F}$ to ${ }^{31} \mathrm{P} \mathrm{CP}$ for sample PBFP deconvolution model $\left(\mathrm{a}\right.$, left); ${ }^{19} \mathrm{~F}$ to ${ }^{31} \mathrm{P} \mathrm{CP}$ curve for sample PBFP (b, right)

Figure 9.2: ${ }^{19} \mathrm{~F}$ to ${ }^{31} \mathrm{P} \mathrm{CP}$ for sample annealed PBFP deconvolution model (left); ${ }^{19} \mathrm{~F}$ to ${ }^{31} \mathrm{P} \mathrm{CP}$ curve for sample annealed PBFP (right)

Figure 9.3: ${ }^{19} \mathrm{~F}$ to ${ }^{31} \mathrm{P} \mathrm{CP}$ for sample NC $5 \%$ deconvolution model (left); ${ }^{19} \mathrm{~F}$ to ${ }^{31} \mathrm{P}$ CP curve for sample NC $5 \%$ (right)

Figure 9.4: ${ }^{19} \mathrm{~F}$ to ${ }^{31} \mathrm{P}$ CP for sample annealed NC $5 \%$ (left); ${ }^{19} \mathrm{~F}$ to ${ }^{31} \mathrm{P} \mathrm{CP}$ curve for sample annealed NC $5 \%$ (right)

Figure 9.5: ${ }^{1} \mathrm{H}$ to ${ }^{31} \mathrm{P} \mathrm{CP}$ for sample PBFP deconvolution model (left, a); ' $\mathrm{H}$ to ${ }^{31} \mathrm{P}$ for one component, including the sum of two composition (blue); the faster $\mathrm{CP}$ curve (green); and slow $\mathrm{CP}$ curve (red)(middle, b); ${ }^{1} \mathrm{H}$ to ${ }^{31} \mathrm{P}$ CP curve for sample PBFP (right, c)

Figure 9.6: ${ }^{1} \mathrm{H}$ to ${ }^{3 !} \mathrm{P} \mathrm{CP}$ for sample annealed PBFP deconvolution model (left); ${ }^{1} \mathrm{H}$ to ${ }^{31} \mathrm{P} \mathrm{CP}$ curve for sample annealed PBFP (right)

Figure 9.7: ${ }^{1} \mathrm{H}$ to ${ }^{31} \mathrm{P} \mathrm{CP}$ for sample $\mathrm{NC} 5 \%$ deconvolution model (left); ${ }^{1} \mathrm{H}$ to ${ }^{31} \mathrm{P} \mathrm{CP}$ curve for sample $\mathrm{NC}$ $5 \%$ (right)

Figure 9.8: ${ }^{1} \mathrm{H}$ to ${ }^{31} \mathrm{P} \mathrm{CP}$ for sample annealed $\mathrm{NC} 5 \%$ deconvolution model (left); ${ }^{1} \mathrm{H}$ to ${ }^{31} \mathrm{P} \mathrm{CP}$ curve for sample annealed NC 5\% (right)

Figure 10.1: The transformation scheme of PBFP over temperature 


\section{LIST OF TABLES}

Table 1.1: Four possible combinations of the spin states and the corresponding four energy levels of two spins

Table 1.2: Quantum number $M$ values for two spin system with four levels

Table 1.3: The allowed transitions and the corresponding frequency based on the selection rule for two spin system

Table 1.4: A general idea of spin rotation mechanics and their range of interaction 29

Table 2.1: Examples of some types of polyphosphazene surface modification 68

Table 7.1: Peak parameters of ' ${ }^{\prime} \mathrm{H}$ MAS $23 \mathrm{kHz}$ NMR spectra of SC, HT, ALP and NC samples $\quad 122$

Table 7.2: Peak parameters of ${ }^{19} \mathrm{~F}$ DP MAS $23 \mathrm{kHz}$ NMR spectra of SC, HT, ALP and NC 5\% samples 124

Table 7.3: Peak parameters of ${ }^{31} \mathrm{P}$ NMR spectra of SC, HT, NC 5\%, HT NC 5\%, and ALP samples 125

Table 7.4: PBFP virgin ${ }^{31} \mathrm{P}$ relaxation model parameter 20-40 degree 131

Table 7.5: PBFP virgin ${ }^{31} \mathrm{P}$ relaxation model parameter $50-80^{\circ} \mathrm{C} \quad 132$

Table 7.6: ${ }^{31} \mathrm{P}$ deconvolution percentage area for 4 components over the temperature range of $20-40{ }^{\circ} \mathrm{C}$ for PBFP (left) and annealed PBFP (right)

Table 7.7: ${ }^{31} \mathrm{P}$ deconvolution percentage area for 4 components over the temperature range of $20-40{ }^{\circ} \mathrm{C}$ for samples NC 5\% (left) and annealed NC 5\% (right)

Table 7.8: ${ }^{31} \mathrm{P}$ deconvolution percentage area for 5 components over the temperature range of $50-80{ }^{\circ} \mathrm{C}$ for PBFP (left) and annealed PBFP (right)

Table 7.9: ${ }^{31} \mathrm{P}$ deconvolution percentage area for 5 components over the temperature range of $50-80^{\circ} \mathrm{C}$ for samples NC 5\% (left) and annealed NC 5\% (right) 137

Table 7.10: ${ }^{19} \mathrm{P}$ deconvolution model for PBFP and $\mathrm{NC} 5 \%$ for $20-40^{\circ} \mathrm{C}$

Table 7.11: ${ }^{19} \mathrm{~F}$ deconvolution model for annealed PBFP, and annealed NC $5 \%$ for $20-40^{\circ} \mathrm{C} \quad 138$

Table 7.12: ${ }^{19} \mathrm{~F}$ deconvolution model for 4 samples for $50-80^{\circ} \mathrm{C} \quad 139$

Table 7.13: ${ }^{19} \mathrm{~F}$ deconvolution percentage area for different components over the temperature range of $20-40$

${ }^{\circ} \mathrm{C}$ for PBFP (left) and annealed PBFP (right) 140

Table 7.14: ${ }^{19} \mathrm{~F}$ deconvolution percentage area for different components over the temperature range of $20-40$

${ }^{\circ} \mathrm{C}$ for NC 5\% (left) and annealed NC 5\% (right) 141

Table 7.15: Deconvolution percentage area for 4 components over the temperature range of $50-80{ }^{\circ} \mathrm{C}$ for PBFP (left) and annealed PBFP (right) 142

Table 7.16: ${ }^{19} \mathrm{~F}$ deconvolution percentage area for 4 components over the temperature range of $50-80{ }^{\circ} \mathrm{C}$ for NC $5 \%$ (left) and annealed NC 5\% (right) 142

Table 8.1: ${ }^{31} \mathrm{P} \mathrm{T}_{1}$ times of solid non-annealed PBFP over the temperature range of 20 to $40^{\circ} \mathrm{C}$ obtained with an MAS rate of $10 \mathrm{kHz} \quad 146$

Table 8.2: ${ }^{31} \mathrm{P} \mathrm{T}_{1}$ times of solid non-annealed PBFP over the temperature range of 50 to $80^{\circ} \mathrm{C}$ obtained with an MAS rate of $10 \mathrm{kHz} \quad 147$

Table 8.3: ${ }^{31} \mathrm{P} \mathrm{T}_{2}$ times of solid non-annealed PBFP over the temperature range of 20 to $40^{\circ} \mathrm{C}$ obtained with an MAS rate of $10 \mathrm{kHz} \quad 148$

Table 8.4: ${ }^{31} \mathrm{P} \mathrm{T}_{2}$ times of solid non-annealed PBFP over the temperature range of 50 to $80^{\circ} \mathrm{C}$ obtained with an MAS rate of $10 \mathrm{kHz} \quad 148$

Table 8.5: ${ }^{31} \mathrm{P} \mathrm{T}_{\mathrm{t} \rho}$ times of solid non-annealed PBFP over the temperature range of 20 to $40^{\circ} \mathrm{C}$ obtained with an MAS rate of $10 \mathrm{kHz} \quad 149$

Table 8.6: ${ }^{31} \mathrm{P} \mathrm{T}_{1 \mathrm{p}}$ times of solid non-annealed PBFP over the temperature range of 50 to $80^{\circ} \mathrm{C}$ obtained with an MAS rate of $10 \mathrm{kHz} \quad 151$

Table 8.7: ${ }^{19} \mathrm{~F} \mathrm{~T}_{1}$ times of solid non-annealed PBFP over the temperature range of 20 to $40^{\circ} \mathrm{C}$ obtained with an MAS rate of $10 \mathrm{kHz}$

Table 8.8: ${ }^{19} \mathrm{~F} \mathrm{~T}$ times of solid non-annealed PBFP over the temperature range of 50 to $80^{\circ} \mathrm{C}$ obtained with an MAS rate of $10 \mathrm{kHz}$

Table 8.9: ${ }^{19} \mathrm{~F} \mathrm{~T}_{2}$ times of solid non-annealed PBFP over the temperature range of 20 to $40{ }^{\circ} \mathrm{C}$ obtained with an MAS rate of $10 \mathrm{kHz}$

157

Table 8.10: ${ }^{19} \mathrm{~F} \mathrm{~T}$ times of solid non-annealed PBFP over the temperature range of 50 to $80^{\circ} \mathrm{C}$ obtained with an MAS rate of $10 \mathrm{kHz}$

Table 8.11: ${ }^{19} \mathrm{~F} \mathrm{~T}_{1 \mathrm{p}}$ times of solid non-annealed PBFP over the temperature range of 20 to $40^{\circ} \mathrm{C}$ obtained with an MAS rate of $10 \mathrm{kHz}$ 
Table 8.12: ${ }^{19} \mathrm{~F} \mathrm{~T}_{\mathrm{lp}}$ times of solid non-annealed PBFP over the temperature range of 50 to $80{ }^{\circ} \mathrm{C}$ obtained with an MAS rate of $10 \mathrm{kHz}$

Table 8.13: ${ }^{31} \mathrm{P} \mathrm{T}_{1}$ times of solid annealed PBFP over the temperature range of 20 to $40^{\circ} \mathrm{C}$ obtained with an MAS rate of $10 \mathrm{kHz} \quad 160$

Table $8.14:{ }^{31} \mathrm{P} \mathrm{T}_{1}$ times of solid annealed PBFP over the temperature range of 50 to $80^{\circ} \mathrm{C}$ obtained with an MAS rate of $10 \mathrm{kHz}$

Table 8.15: ${ }^{31} \mathrm{P} \mathrm{T}_{2}$ times of solid annealed PBFP over the temperature range of 20 to $40^{\circ} \mathrm{C}$ obtained with an MAS rate of $10 \mathrm{kHz} \quad 161$

Table 8.16: ${ }^{31} \mathrm{P} \mathrm{T}_{1}$ times of solid annealed PBFP over the temperature range of 50 to $80^{\circ} \mathrm{C}$ obtained with an MAS rate of $10 \mathrm{kHz} \quad 162$

Table 8.17: ${ }^{31} \mathrm{P} \mathrm{T}_{1 \mathrm{p}}$ times of solid annealed PBFP over the temperature range of 20 to $40^{\circ} \mathrm{C}$ obtained with an MAS rate of $10 \mathrm{kHz} \quad 167$

Table 8.18: ${ }^{31} \mathrm{P}_{\mathrm{I} p}$ times of solid annealed PBFP over the temperature range of 20 to $40^{\circ} \mathrm{C}$ obtained with an MAS rate of $10 \mathrm{kHz} \quad 168$

Table 8.19: ${ }^{19} \mathrm{~F} \mathrm{~T}$ times of solid annealed PBFP over the temperature range of 20 to $40^{\circ} \mathrm{C}$ obtained with an MAS rate of $10 \mathrm{kHz}$

Table 8.20: ${ }^{19} \mathrm{~F} \mathrm{~T}_{1}$ times of solid annealed PBFP over the temperature range of 50 to $80^{\circ} \mathrm{C}$ obtained with an MAS rate of $10 \mathrm{kHz}$

Table 8.21: ${ }^{19} \mathrm{~F} \mathrm{~T}_{2}$ times of solid annealed PBFP over the temperature range of 20 to $40^{\circ} \mathrm{C}$ obtained with an MAS rate of $10 \mathrm{kHz}$

Table 8.22: ${ }^{19} \mathrm{~F} \mathrm{~T}_{2}$ times of solid annealed PBFP over the temperature range of 50 to $80^{\circ} \mathrm{C}$ obtained with an MAS rate of $10 \mathrm{kHz}$

Table 8.23: ${ }^{19} \mathrm{~F} \mathrm{~T}_{\mathrm{l} \rho}$ times of solid annealed PBFP over the temperature range of 20 to $40^{\circ} \mathrm{C}$ obtained with an MAS rate of $10 \mathrm{kHz}$

Table 8.24: ${ }^{319} \mathrm{~F} \mathrm{~T}_{10}$ times of solid annealed PBFP over the temperature range of 50 to $80^{\circ} \mathrm{C}$ obtained with an MAS rate of $10 \mathrm{kHz}$

Table 8.25: ${ }^{3} \mathrm{P} \mathrm{T}_{1}$ times of solid NC $5 \%$ over the temperature range of 20 to $40^{\circ} \mathrm{C}$ obtained with an MAS rate of $10 \mathrm{kHz}$

Table $8.26:{ }^{31} \mathrm{P} \mathrm{T}_{1}$ times of solid NC $5 \%$ over the temperature range of 50 to $80^{\circ} \mathrm{C}$ obtained with an MAS rate of $10 \mathrm{kHz}$

Table 8.27: ${ }^{31} \mathrm{P} \mathrm{T}_{2}$ times of solid NC $5 \%$ over the temperature range of 20 to $40^{\circ} \mathrm{C}$ obtained with an MAS rate of $10 \mathrm{kHz}$

Table 8.28: ${ }^{31} \mathrm{P} \mathrm{T}_{2}$ times of solid NC $5 \%$ over the temperature range of 20 to $40{ }^{\circ} \mathrm{C}$ obtained with an MAS rate of $10 \mathrm{kHz}$

Table 8.29: ${ }^{31} \mathrm{P}_{1 \rho}$ times of solid $\mathrm{NC} 5 \%$ over the temperature range of 20 to $40^{\circ} \mathrm{C}$ obtained with an MAS rate of $10 \mathrm{kHz}$

Table 8.30: ${ }^{31} \mathrm{P} \mathrm{T}_{1 \rho}$ times of solid $\mathrm{NC} 5 \%$ over the temperature range of 50 to $80^{\circ} \mathrm{C}$ obtained with an MAS rate of $10 \mathrm{kHz}$

Table $8.31:{ }^{19} \mathrm{~F} \mathrm{~T}$ times of solid NC $5 \%$ over the temperature range of 20 to $40{ }^{\circ} \mathrm{C}$ obtained with an MAS rate of $10 \mathrm{kHz}$

Table 8.32: ${ }^{19} \mathrm{~F} \mathrm{~T}_{1}$ times of solid NC $5 \%$ over the temperature range of 50 to $80^{\circ} \mathrm{C}$ obtained with an MAS rate of $10 \mathrm{kHz}$

Table 8.33: ${ }^{19} \mathrm{~F} \mathrm{~T}_{2}$ times of solid NC $5 \%$ over the temperature range of 20 to $40^{\circ} \mathrm{C}$ obtained with an MAS rate of $10 \mathrm{kHz}$

Table $8.34:{ }^{19} \mathrm{~F} \mathrm{~T}_{2}$ times of solid NC $5 \%$ over the temperature range of 50 to $80^{\circ} \mathrm{C}$ obtained with an MAS rate of $10 \mathrm{kHz}$

Table 8.35: ${ }^{19} \mathrm{~F} \mathrm{~T}_{1 p}$ times of solid NC $5 \%$ over the temperature range of 20 to $40^{\circ} \mathrm{C}$ obtained with an MAS rate of $10 \mathrm{kHz}$

Table 8.36: ${ }^{19} \mathrm{~F} \mathrm{~T}_{1 \rho}$ times of solid $\mathrm{NC} 5 \%$ over the temperature range of 50 to $80^{\circ} \mathrm{C}$ obtained with an MAS rate of $10 \mathrm{kHz}$

Table 8.37: ${ }^{31} \mathrm{P} \mathrm{T}_{1}$ times of solid annealed NC $5 \%$ over the temperature range of 20 to $40^{\circ} \mathrm{C}$ obtained with an MAS rate of $10 \mathrm{kHz}$

Tabie 8.38: ${ }^{31} \mathrm{P} \mathrm{T}_{1}$ times of solid annealed NC $5 \%$ over the temperature range of 50 to $80^{\circ} \mathrm{C}$ obtained with an MAS rate of $10 \mathrm{kHz}$

Table 8.39: ${ }^{31} \mathrm{P} \mathrm{T}_{2}$ times of solid annealed $\mathrm{NC} 5 \%$ over the temperature range of 20 to $40^{\circ} \mathrm{C}$ obtained with an MAS rate of $10 \mathrm{kHz}$ 
Table 8.40: ${ }^{31} \mathrm{P} \mathrm{T}_{2}$ times of solid annealed $\mathrm{NC} 5 \%$ over the temperature range of 50 to $80^{\circ} \mathrm{C}$ obtained with an MAS rate of $10 \mathrm{kHz}$

Table 8.41: ${ }^{31} \mathrm{P} \mathrm{T}_{\mathrm{lp}}$ times of solid annealed NC 5\% over the temperature range of 20 to $40^{\circ} \mathrm{C}$ obtained with an MAS rate of $10 \mathrm{kHz}$

Table 8.42: ${ }^{31} \mathrm{P}_{1 \rho}$ times of solid annealed NC $5 \%$ over the temperature range of 50 to $80^{\circ} \mathrm{C}$ obtained with an MAS rate of $10 \mathrm{kHz}$

Table 8.43: ${ }^{19} \mathrm{~F} \mathrm{~T}_{1}$ times of solid antealed $\mathrm{NC} 5 \%$ over the temperature range of 20 to $40^{\circ} \mathrm{C}$ obtained with an MAS rate of $10 \mathrm{kHz}$

Table 8.44: ${ }^{19} \mathrm{~F} \mathrm{~T}_{1}$ times of solid annealed NC $5 \%$ over the temperature range of 50 to $80{ }^{\circ} \mathrm{C}$ obtained with an MAS rate of $10 \mathrm{kHz} \quad 198$

Table 8.45: ${ }^{19} \mathrm{~F} \mathrm{~T}$ times of solid annealed NC $5 \%$ over the temperature range of 20 to $40{ }^{\circ} \mathrm{C}$ obtained with an MAS rate of $10 \mathrm{kHz}$

Table 8.46: ${ }^{19} \mathrm{~F} \mathrm{~T}$ times of solid annealed $\mathrm{NC} 5 \%$ over the temperature range of 50 to $80^{\circ} \mathrm{C}$ obtained with an MAS rate of $10 \mathrm{kHz}$

Table 8.47: ${ }^{19} \mathrm{~F} \mathrm{~T}_{1 p}$ times of solid annealed NC $5 \%$ over the temperature range of 20 to $40{ }^{\circ} \mathrm{C}$ obtained with an MAS rate of $10 \mathrm{kHz}$

Table 8.48: ${ }^{19} \mathrm{~F} \mathrm{~T}_{1 \mathrm{p}}$ times of solid annealed $\mathrm{NC} 5 \%$ over the temperature range of 50 to $80^{\circ} \mathrm{C}$ obtained with an MAS rate of $10 \mathrm{kHz}$

Table 8.49: ${ }^{31} \mathrm{P} \mathrm{T}_{1} / \mathrm{T}_{2}$ ratio of four samples with the change of temperature for samples over $20-40^{\circ} \mathrm{C}$ obtained with an MAS rate of $10 \mathrm{kHz}$

205

Table 8.50: ${ }^{31} \mathrm{P}^{\prime} \mathrm{T}_{1} / \mathrm{T}_{2}$ ratio of four samples with the change of temperature for samples over $50-80^{\circ} \mathrm{C}$ obtained with an MAS rate of $10 \mathrm{kHz}$

Table 8.51: ${ }^{19} \mathrm{~F} \mathrm{~T}_{1} / \mathrm{T}_{2}$ ratio of PBFP, annealed PBFP, NC $5 \%$ and annealed NC $5 \%$ with the change of temperature over $20-40^{\circ} \mathrm{C}$ obtained with an MAS rate of $10 \mathrm{kHz}$

Table 8.52: ${ }^{19} \mathrm{~F} \mathrm{~T}_{1} / \mathrm{T}_{2}$ ratio of PBFP, annealed PBFP, NC $5 \%$ and annealed $\mathrm{NC} 5 \%$ with the change of temperature over $50-80^{\circ} \mathrm{C}$ obtained with an MAS rate of $10 \mathrm{kHz} \quad 206$

Table 9.1: ${ }^{19} \mathrm{~F}$ to ${ }^{31} \mathrm{P}$ CP curve parameters for sample PBFP 213

Table 9.2: ${ }^{19} \mathrm{~F}$ to ${ }^{31} \mathrm{P}$ CP curve parameters for sample annealed PBFP 216

Table 9.3: ${ }^{19} \mathrm{~F}$ to ${ }^{31} \mathrm{P}$ CP curve parameters for sample NC 5\% 218

Table 9.4: ${ }^{19} \mathrm{~F}$ to ${ }^{31} \mathrm{P}$ CP curve parameters for sample annealed NC 5\% 220

Table 9.5: ${ }^{1} \mathrm{H}$ to ${ }^{31} \mathrm{P}$ CP curve parameters for sample PBFP of part 1 (left) and part 2 (right) and the standard deviation

Table 9.6: ${ }^{1} \mathrm{H}$ to ${ }^{31} \mathrm{P} \mathrm{CP}$ curve parameters for sample annealed PBFP of part 1 (left); part 2 (right) and the deviation error (bottom) $\quad 225$

Table 9.7: ${ }^{1} \mathrm{H}$ to ${ }^{31} \mathrm{P}$ CP curve parameters for sample NC 5\% of part 1 (left) and part 2 (right) 227

Table 9.8: ${ }^{1} \mathrm{H}$ to ${ }^{31} \mathrm{P}$ CP curve parameters for annealed NC 5\% of part one (left) and part two (right) 229 
Abbreviations and Symbols

\begin{tabular}{|c|c|}
\hline$\AA$ & Angstrom \\
\hline ATP & Ambient temperature polymerization \\
\hline $\mathrm{B}_{0}$ & Applied magnetic field \\
\hline$b_{j k}$ & Dipolar-dipolar coupling constant \\
\hline $\mathrm{B}_{\text {loc }}$ & Local magnetic field \\
\hline CNFs & Carbon nanofibers \\
\hline $\cos Y$ & Correlation spectroscopy \\
\hline CODEX & Centerband-only detection of exchange \\
\hline $\mathrm{CP}$ & Cross polarization \\
\hline CSA & Chemical shift anisotropy \\
\hline $\mathrm{CWV}$ & Continuous Wave \\
\hline DSC & Differential scanning spectroscopy \\
\hline DMA & Dynamic mechanical analysis \\
\hline DP & Direct polarization \\
\hline $\mathrm{E}_{\mathrm{a}}$ & Activation energy \\
\hline $\mathrm{E}_{\mathrm{m}}$ & Energy of interaction between $\mu$ and $\mathbf{B}_{0}$ \\
\hline EXSY & Exchange spectroscopy \\
\hline FID & Free induction decay \\
\hline FT & Fourier transformation \\
\hline FT-IR & Fourier transform infrared spectroscopy \\
\hline $\mathrm{G}(\tau)$ & Autocorrelation function \\
\hline GPC & Gel permeation chromatography \\
\hline h & Dirac constant \\
\hline HSQC & Heteronuclear single quantum correlation \\
\hline$I$ & Nuclear spin quantum number \\
\hline$I$ & Nuclear spin angular momentum \\
\hline Iz & z-component of $I$ \\
\hline$J(\omega)$ & Spectral density \\
\hline LOI & Limiting Oxygen Index \\
\hline MAS & Magic angle spinning \\
\hline$m_{1}$ & Spin quantum number \\
\hline $\mathrm{M}_{2}$ & Equilibrium magnetization \\
\hline MMT & Montmorillonite organoclays \\
\hline$N_{\alpha}$ & The number of nuclei in $\alpha$ state \\
\hline$N_{\beta}$ & The number of nuclei in $\beta$ state \\
\hline $\mathrm{NC}$ & Nanocomposite \\
\hline NMR & Nuclear magnetic resonance \\
\hline NOSY & Nuclear Overhauser effect spectroscopy \\
\hline PBFP & Poly[bis(trifluoroethoxy)phosphazene \\
\hline PCL & Polycaprolactone \\
\hline PDCP & Poly(dichlorophosphazene) \\
\hline PEO & Polyethylene oxide \\
\hline PEVA & Polyethylene-co-vinyl acetate \\
\hline PLA & Polylactic acid \\
\hline PLS & Polymer-layered silicate \\
\hline PMMA & Poly(methyl methacrylate) \\
\hline PVA & Polyvinyl alcohol \\
\hline PVP & Poly(Vinylpyrrolidinone) \\
\hline$\gamma$ & Gyromagnetic ratio \\
\hline $\mathrm{R}_{1-}, \mathrm{R}_{2-}$ & Polyphosphazene side groups \\
\hline RF & Radiofrequency \\
\hline ROP & Ring opening polymerization \\
\hline SEM & Scanning electron microscopy \\
\hline $\mathrm{T}_{1}$ & Spin-lattice relaxation constant \\
\hline
\end{tabular}


$\mathrm{T}_{2}$

$\mathrm{T}_{1 p}$

$\mathrm{T}(1)$

TEM

$\mathrm{T}_{8}$

TGA

THF

$T_{m}$

TPPM

V'T

WISE

$\mu$

$\omega_{0}$

$\Omega$

$\delta^{f}$

$\tau_{c}$

$\lambda$
Spin-spin relaxation constant

Spin-lattice relaxation in the rotating frame

Transition temperature for polyphosphazene

Transmission electron microscopy

Glass transition temperature

Thermal gravimetric analysis

Tetrahydrofuran

Melting temperature

Two pulse phase modulated

Variable temperature

Wideline separation

Nuclear magnetic dipole moment

Larmor frequency

Offset

Chemical shift tensor

Correlation time

Coherence decay constant 


\section{CHAP'TER 1}

\section{SOLID-STATE NUCLEAR MAGNETIC RESONANCE}

\subsection{THEORETICAL BACKGROUND}

\subsubsection{History and Introduction}

Nuclear magnetic resonance (NMR) spectroscopy is an experimental technique that explores molecular structure and dynamics through measurement of the interaction between an oscillating radio-frequency electromagnetic field with a collection of nuclei immersed in a strong external magnetic field. It determines the physical and chemical properties of the atoms or molecules to which the nuclei belong and provides detailed information containing the structure, dynamics, reaction state, and chemical environment.

Since discovery of the nuclear magnetic resonance phenomenon by Felix Bloch and Edward Mills Purcell in $1946^{1-2}$, together with advances in superconducting magnet and computing power technology, NMR spectroscopy has become an essential analytical technique to provide insight into the structural and chemical properties of molecules. Solidstate NMR has been an area of intense recent research ${ }^{3-7}$, including studies on proteins ${ }^{8}$, polymers $^{9}$, inorganic materials ${ }^{10}$ and nanocomposites ${ }^{11}$

\subsubsection{Zeeman Interaction}

Many atomic nuclei possess spin, which is an intrinsic quantum mechanical property. The nuclear spin quantum number is conventionally denoted $I . I$ is either a whole or halfpositive integer number, which is determined by vector sum of the intrinsic spin contributions from the protons and neutrons composing the nucleus. The corresponding nuclear spin angular momentum $\mathbf{I}$, is a vector property with magnitude: 


$$
I=h \sqrt{I(I+1)}
$$

The corresponding nuclear magnetic dipole moment, $\mu$, related to the spin angular momentum is as follows:

$$
\boldsymbol{\mu}=\gamma I
$$

where $\gamma$ is known as the gyromagnetic ratio, which is an intrinsic property of the nucleus related to its mass to charge ratio.

Classically, when a nuclear magnetic dipole moment is placed in an external magnetic field, it precesses about an axis defined by the direction of this field. Quantum mechanically speaking, the nuclear spin vector is restricted to only certain fixed orientations determined by its azimuthal spin quantum number, $m_{\mathrm{I}}$. Therefore, the orientation of the spin angular momentum is quantized. The $\mathbf{z}$-component of $\mathbf{I}, \mathbf{I}_{\mathbf{z}}$, has a magnitude given bys:

$$
I_{\mathrm{Z}}=m_{l} \hbar
$$

where $m_{\mathrm{I}}=I,(I-1), \ldots,(I-1),-I$, which represent the sub-levels of a given spin state. The number of these sub-levels is equal to $(2 I+1)$. In the absence of an external magnetic field, these states are degenerates and the direction of the angular momentum vector is completely random, as shown in Figure 1.1.

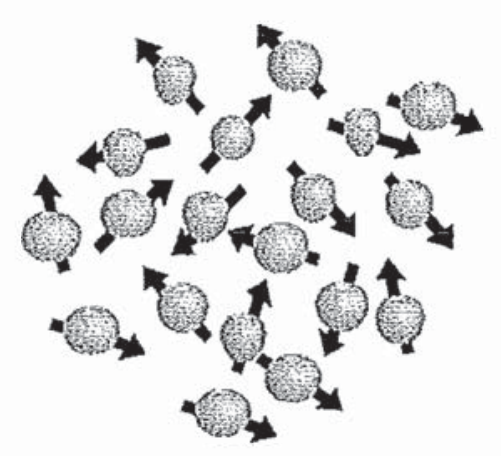

Figure 1.1: Isotropic distribution of the angular momentum in the absence of external fields $\mathrm{B}_{0}{ }^{12}$ 
In the presence of an external magnetic field, B, these sub-states are no longer degenerate; where the angular momentum vectors now have an orientation with respect to the magnetic field direction (commonly assumed to be the $z$-axis, $B=B_{0} k$ ), with different energies which is referred to as Zeeman splitting. The energy of interaction between $\mu$ and B, can be written as:

$$
E_{m}=-\mu \cdot \mathbf{B}=-\mu_{\mathrm{z}} \mathrm{B}_{0}=-\gamma \mathrm{I}_{\mathrm{z}} \mathrm{B}_{0}=-m_{l} \hbar \gamma \mathrm{B}_{0}=m_{l} \hbar \omega_{0}
$$

where

$$
\omega_{0}=-\gamma \mathrm{B}_{0}
$$

s known as the larmor frequency, and is defined as the rate of precession of a nuclear spin vector about the external magnetic field direction.

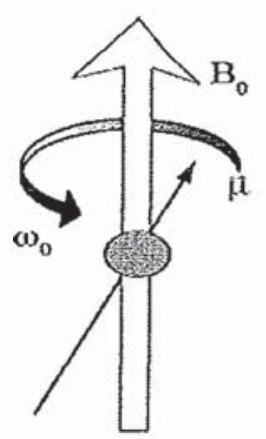

Figure 1.2: The Larmor frequency of nuclei precession about an external field ${ }^{12}$

On the other hand, one can consider a macroscopic sample being composed of an ensemble of randomly oriented spin magnetic vectors. In the presence of an external magnetic field, these vectors will process around the z-axis at their own larmor Frequency. According to the Boltzmann distribution, the spin states are populated according to the Boltzmann distribution for $(I=1 / 2)$ :

$$
\frac{N_{\alpha}}{N_{\beta}}=e^{\frac{-\Delta E}{k^{T}}}
$$


where $N_{\alpha}$ refers to the number of nuclei in the lower energy spin $\left(m_{I}=+1 / 2\right)$ state, $N_{\beta}$ indicates the population of spins in the higher energy spin $\left(m_{I}=-1 / 2\right)$ state, $\Delta \mathrm{E}$ is the energy difference between spin state $\alpha$ and state $\beta$, and $\mathrm{k}$ is a constant. A very modest difference between $N_{\alpha}$, and $N_{\beta}$ results in a slightly higher probability of the magnetic moment being aligned along the external field direction, giving a non-zero longitudinal net magnetization. As depicted in Figure 1.3.

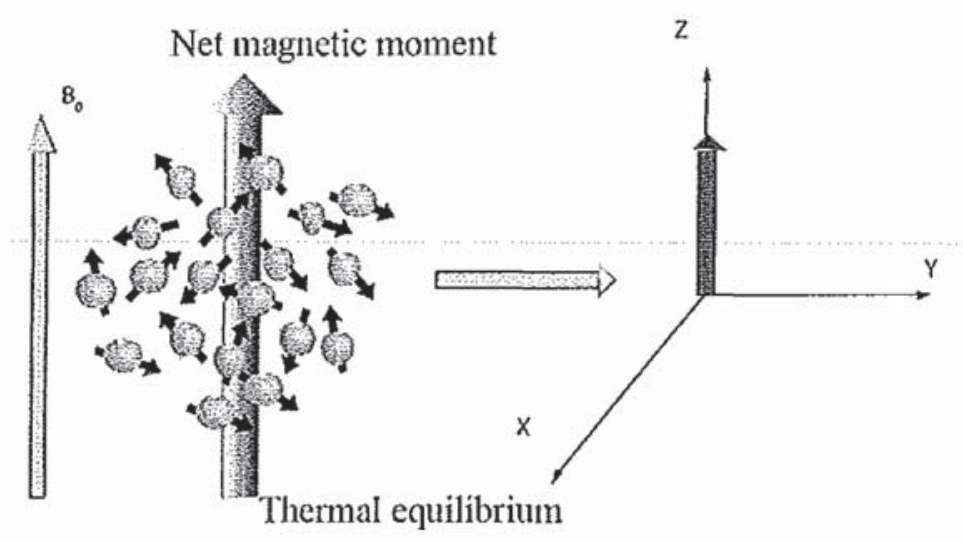

Figure 1.3: Ensemble of spins precessing at $\omega_{0}$ in the presence of the external magnetic field $B_{0}$ along $Z$-axis (left), and the corresponding longitudinal magnetization vector (right) ${ }^{12}$

\subsubsection{NMR and Energy Level}

In general, molecules possess many different energy levels that are determined by the electronic, vibrational rotational and transitional properties of the molecule. Transitions between levels cause the spectra we see in most spectroscopies. The energy of transition is defined as:

$$
\Delta \mathrm{E}=\mathrm{h} v
$$

where $\hbar$ is known as Planck's constant, and $v$ is the frequency of electromagnetic radiation (in $\mathrm{Hz}$ ) that causes the transition. $\Delta \mathrm{E}=\mathrm{E}_{2}-\mathrm{E}_{1}$. As shown in Figure 1.4. 


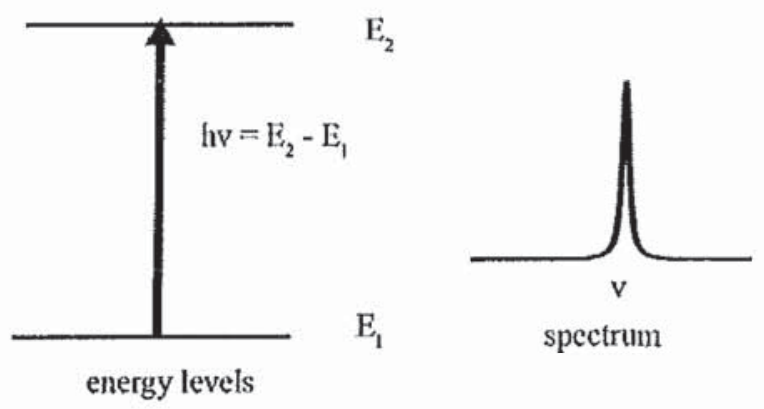

Figure 1.4: A line in the spectrum is associated with a transition between two energy levels ${ }^{13}$ Another way to express frequency in angular units is "radians per second" defined as:

$$
\omega=\frac{2 \pi}{\tau}\left(\frac{\mathrm{rad}}{\mathrm{s}}\right)
$$

$v$ and $\omega$ are related via

$$
v=\frac{\omega}{2 \pi} \text { or } \omega=2 \pi v
$$

The energy of a photon of frequency $v$ is given by

$$
E=\mathrm{h} v=\mathrm{h} \frac{\omega}{2 \pi}=h \omega
$$

The Zeeman energy levels differ in energy by $\Delta E=\hbar \gamma \mathrm{B}_{0}$, and the Hamiltonian describing the interaction of the nucleus with $\mathrm{B}_{0}$ can be written as ${ }^{12}$

$$
\mathbf{H}_{\mathrm{Z}}=-\mathbf{I}_{\mathrm{Z}} \hbar \gamma \mathrm{B}_{0} \quad \text { for } \gamma>0
$$

it is clear from above that, frequency can be expressed as either absolute frequency $\mathrm{Hz}$ or angular frequency rad $\mathrm{s}^{-1}$. It will be more convenient to use frequency units $(\mathrm{Hz})$ throughout.

A $I=1 / 2$ nucleus has two energy levels characterized by the azimuthal spin quantum number $m_{\mathrm{I}}$, this gives us two values for $m_{\mathrm{I}}$ as $-1 / 2$ and $+1 / 2$. In NMR, the energy level which is $m_{\mathrm{I}}=+1 / 2$ is denoted as $\alpha$ and is assigned as the one with lower energy (with 
positive $\gamma$ ). The state with $m_{\mathrm{I}}=-1 / 2$ is denoted as $\beta$, and is assigned as the one with higher energy. Therefore, if we have two spins in our molecule, each spin can be in either the $\alpha$ or $\beta$ state, leading to 4 spin states: $\alpha \alpha, \alpha \beta, \beta \alpha, \beta \beta$, and 3 energy levels as shown in Figure 1.5. For three spins, we get 8 spin states, with 4 energy levels, and so on.

For the one spin system, there are two energy levels labeled $\alpha$ and $\beta$. The energies of these two levels, quantum mechanically speaking, are:

$$
\mathrm{E}_{\alpha}=+\frac{1}{2} \mathrm{v}_{0,1}
$$

and

$$
\mathrm{E}_{\beta}=-\frac{1}{2} \mathrm{v}_{0,1}
$$

where, $v_{0}$ is the Larmor Frequency of the spin.

$$
\mathrm{v}_{0}=-\gamma \hbar \mathrm{B}_{0}
$$

In the case of NMR, the energy transition is controlled by the selection rule, which is related to the quantum number $m$; only transitions in which $m$ changes by one (up or down) are allowed. This can be expressed as

$$
\Delta m=m(\text { initial state })-m(\text { final state })= \pm 1
$$

In the case of single spin-half

$$
\Delta m=+\frac{1}{2}-\left(-\frac{1}{2}\right)=1
$$

so the transition is allowed.

The frequency of the allowed transition can be expressed as:

$$
\begin{gathered}
v_{\alpha, \beta}=E_{\alpha}-E_{\beta} \\
=-1 / 2 v_{0,1}-\left(+1 / 2 v_{0,1}\right)=-v_{0,1}
\end{gathered}
$$

The Larmor frequency of a spin depends on its gyromagnetic ratio, $\gamma$, its chemical shift $\delta$, and the applied magnetic field, $\mathrm{B}_{0}$ : 


$$
\mathrm{v}_{0,1}=-\frac{1}{2 \pi} \gamma_{1}\left(1+\delta_{1}\right) \mathrm{B}_{0}
$$

For two spins system, we will denote the shifts of two spins as "1 and "2, and the corresponding Larmor frequency as $\mathrm{v}_{0,1}$ and $\mathrm{v}_{0,2}$. There are four possible combinations of the spin states of two spins and these combinations correspond to four energy levels, which are given in the following table.

Table 1.1: $\quad$ Four possible combinations of the spin states and the corresponding four energy levels of two spins

\begin{tabular}{c|cc}
\hline Number & Spin states & Energy \\
\hline 1 & $\alpha \alpha$ & $+\frac{1}{2} v_{0,1}+\frac{1}{2} v_{0,2}+\frac{1}{4} J_{1,2}$ \\
2 & $\alpha \beta$ & $+\frac{1}{2} v_{0,1}-\frac{1}{2} v_{0,2}-\frac{1}{4} J_{1,2}$ \\
3 & $\beta \alpha$ & $-\frac{1}{2} v_{0,1}+\frac{1}{2} v_{0,2}-\frac{1}{4} J_{1,2}$ \\
4 & $\beta \beta$ & $-\frac{1}{2} v_{0,1}-\frac{1}{2} v_{0,2}+\frac{1}{4} J_{1,2}$ \\
\hline
\end{tabular}

These energies have the general form:

$$
\mathrm{E}_{\mathrm{m} 1 \mathrm{~m} 2}=m_{1} \mathrm{v}_{0,1}+m_{2} \mathrm{v}_{0,2}+m_{1} m_{2} \mathrm{~J}_{12}
$$

where $m_{1}$ and $m_{2}$ are the $m$ values for spins 1 and 2 , respectively, and $J_{12}$ is the scalar coupling in $\mathrm{Hz}$.

For a homonuclear system, where the two nuclei are of the same type, the energy of the $\alpha \beta$ and $\beta \alpha$ states are rather similar, but very different from the other two states. For a heteronuclear system, where the two nuclei are of different types, the Larmor frequencies differ significantly, the four levels are all of markedly different energies. These points are illustrated in Figure 1.5 


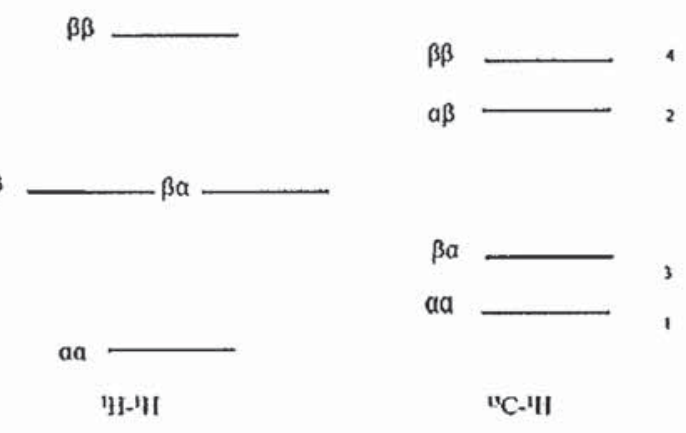

Figure 1.5: Energy levels for two spin system. On the left is a homonuclear system (two protons); where $\alpha \beta$ and $\beta \alpha$ states have the same energy. On the right is the case for a carbon 13 - proton pair. The Larmor frequency of proton is about four times that of carbon -14 , and this clear reflected in the diagram. The $\alpha \beta$ and $\beta \alpha$ states now have substantially different energies. ${ }^{13}$

\subsubsection{Spectrum}

In this case, the quantum number $M$ is found by adding up the in values for each spins:

$$
M=m_{1}+m_{2}
$$

The resulting $M$ values for the four levels are:

Table 1.2: $\quad$ Quantum number $M$ values for two spin system with four leveis ${ }^{13}$

\begin{tabular}{c|cc}
\hline Number & Spin states & $M$ \\
\hline 1 & $\alpha \alpha$ & 1 \\
2 & $\alpha \beta$ & 0 \\
3 & $\beta \alpha$ & 0 \\
4 & $\beta \beta$ & -1 \\
\hline
\end{tabular}

Base on the selection rule that $M= \pm 1$, which means that the allowed transitions are 1 and 2,3 and 4,1 and 3 , and 2 and 4 . The corresponding resulting frequencies can be worked out easily; for example the 1-2 transition:

$$
\mathrm{v}_{1,2}=\mathrm{E}_{2}-\mathrm{E}_{1}
$$

The complete sets of transitions are: 
Table 1.3: The allowed transitions and the corresponding frequency based on the selection rule for two spin system ${ }^{13}$

\begin{tabular}{c|cc}
\hline Transition & Spin states & Frequency \\
\hline 12 & $\alpha \alpha-\alpha \beta$ & $-\mathrm{v}_{0,2}-\frac{1}{2} J_{12}$ \\
34 & $\beta \alpha-\beta \beta$ & $-v_{0,2}+\frac{1}{2} J_{12}$ \\
13 & $\alpha \alpha-\beta \alpha$ & $-v_{0,1}-\frac{1}{2} J_{12}$ \\
24 & $\alpha \beta-\beta \beta$ & $-v_{0,1}+\frac{1}{2} J_{12}$ \\
\hline
\end{tabular}

The energy levels and corresponding schematic spectrum are shown in Figure 1.6. Each allowed transition corresponds to one of the spins flipping from one spin state to the other, which is the active spin, while the spin state of the other spin remains fixed, which is the passive spin. This illustrates that the lines of a multiplet can be associated with different spin states of the coupled (passive) spins.

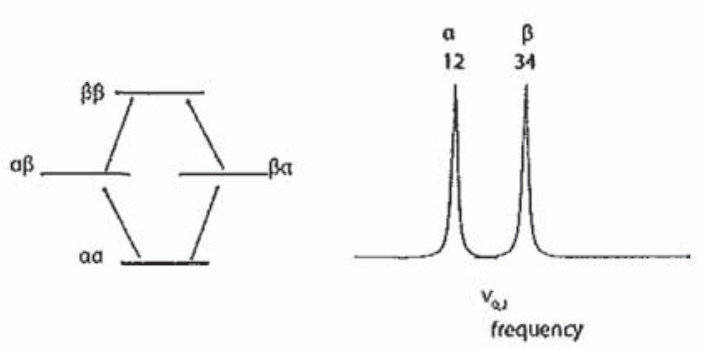

Figure 1.6: On the left, the energy levels of a two-spin system; the arrows show the allowed transitions: solid lines for transitions in which spin 1 flips and dotted for those in which spin 2 flips. On the right, the corresponding spectrum; it is assumed that the Larmor frequency of spin 2 is greater in magnitude than that of spin 1 and that the coupling $J_{12}$ is positive. ${ }^{13}$

There are another two transitions in this two-spin system, which are not allowed by the common selection rule. One is between states 1 and 4 where both spins are active spin, with a $\Delta M$ value as 2 , called as double-quantum transition. Its frequency can be calculated according to the table of energy as $\left(-v_{0,1}-v_{0,2}\right)$. Another transition is between states 2 and 3 , where both spin flip, with a $\Delta \mathrm{M}$ values as 0 , called zero-quantum transition. Its frequency is calculated as $\left(-v_{0,1}+v_{0,2}\right)$. 
The method to calculate energy, to figure the shape of the spectrum, to get the quantum number for two-spin system can also be applied to three-spin system or multispin system.

\subsubsection{The Vector Model}

The vector model of NMR spectroscopy is a simple, very visual way of comprehending the result of radio frequency pulses and delay times in many NMR experiments. It allows one to visualize the motion of the spin systems by seeing the vector rotation. Although the simple vector model fails to predict the behavior of spin systems in which there are interactions, the simplicity of the vector model for describing the motion is unsurpassed.

\subsubsection{Larmor Frequency}

The angular momentum of a rotating object is a vector that may point in any direction in space. It is permissible to think of the nucleus as behaving like a magnetic moment with a magnetic angular momentum that can also be aligned in any direction. The direction of the spin angular momentum is called the spin polarization axis.

In an NMR experiment, what we observe is a large number of vectors instead of just one of them, so we need to think about the net effect of those nuclei, as that is what we observe in the NMR experiment. When a magnetic field (B) is applied, the magnetic moments are no longer aligned randomly but a net magnetic field along the direction of the applied field is obtained at equilibrium. This is called the bulk magnetization of the sample, which can be presented by a vector-magnetization vector-aligned along the direction of the applied field $(\mathbf{k}=\mathrm{z}$-axis $)$ 
The actual response of the spin polarization to an applied field is to move around the field. The magnetic moment of the spin moves on a cone, keeping a constant angle between the spin magnetic moment and the field; see Figure 1.7 This motion is called Larmor precession. The frequency of the Larmor frequency is

$$
\omega_{0}=-\gamma \mathrm{B}_{0}
$$

in unit of $\mathrm{rad} \mathrm{s}^{-1}$, where $\mathrm{B}_{0}$ is the strength of the applied field and $\gamma$ is the gyromagnetic ratio.

Most nuclei have positive $\gamma$, where the Larmor frequency is negative. For nuclei have a negative value of $\gamma$, in which case the Larmor frequency is positive.

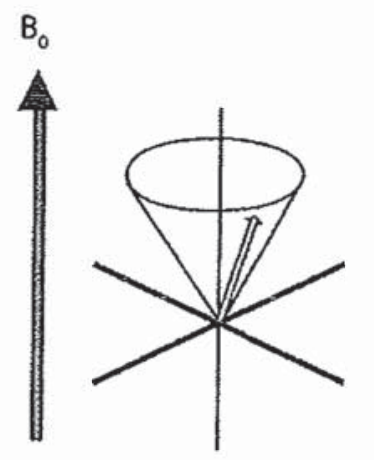

Figure 1.7: If the magnetization vector is titled away from the $\mathrm{z}$ axis it executes a precessional motion in which the vector sweeps out a cone of constant angle to the magnetic field direction. The direction of precession shown is for a nucleus with a positive gyromagnetic ratio and hence a negative Larmor frequency ${ }^{13}$.

There is another way to specify the frequency in units of $\mathrm{Hz}$, or cycles per second. For example, a Larmor frequency of $100 \mathrm{MHz}$ means that the nuclear spin completes 100 million revolutions around its precession cone every second. It is expressed as:

$$
\omega_{0}=-\gamma \mathrm{B}_{0} / 2 \pi
$$


In general, these two frequency units may be converted into each other through the relationships:

Frequency in units if rad $\mathrm{s}^{-1}=2 \pi *$ (frequency in units of $\mathrm{Hz}$ )

Frequency in units of $\mathrm{Hz}=\left(\right.$ frequency in units of $\left.\mathrm{rad} \mathrm{s}^{-1}\right) / 2 \pi$

\subsubsection{FID and Detection}

In an NMR experiment, what we actually detect is the precession of the magnetization vector (the rotating magnetic moment). All we need is a coil with a winding axis along the $x y$-plane. As the magnetization rotates, it "cuts" the coil where a current is induced which we can amplify and then record. This is called free induction signal which is detected in a pulsed NMR experiment. The coil detects the $\mathrm{x}$-component of the magnetization which can be calculated as illustrated in Figure 1.8.

$$
\mathrm{M}_{\mathrm{x}}=\mu_{0} \sin \beta
$$

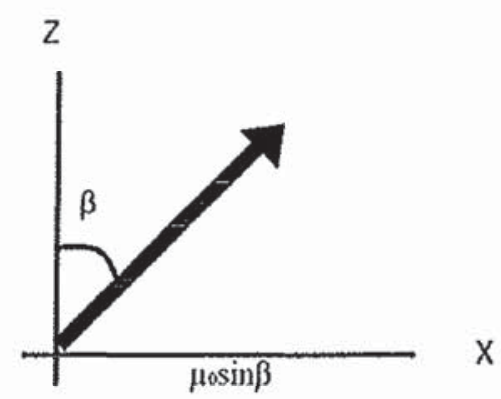

Figure 1.8: Tilting the magnetization through an angle $\theta$ gives an $X$-component of $\operatorname{size} \mu_{0} \sin \beta^{13}$ We can picture what happens to the $x$ - and $y$-component of the magnetization as Figure 1.9 


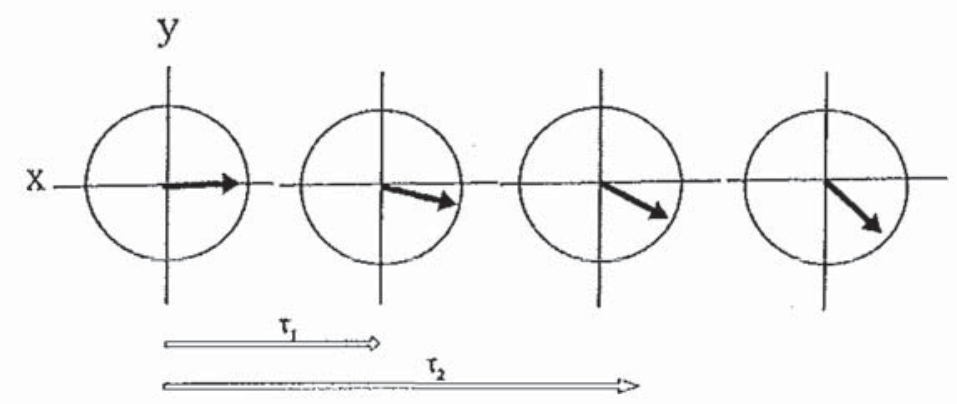

Figure 1.9: $\quad$ Illustration of the precession of the magnetization vector in the xy-plane. The angle through which the vector has precessed is given by $\omega_{0}$. On the right diagram, we see the geometry for working out the $\mathrm{x}$ and $\mathrm{y}$ compounds of the vector

We can deduce that the $x$ - and $y$ - component $M_{x}, M_{y}$ are

$$
\begin{aligned}
& M_{x}=M_{0} \sin \beta \cos \left(\omega_{0} t\right) \\
& M_{y}=M_{0} \sin \beta \sin \left(\omega_{0} t\right)
\end{aligned}
$$

where $\theta=\omega_{0} \mathrm{~T}$, Plots of $\mathrm{M}_{x}$ and $\mathrm{M}_{y}$ are shown in Figure 1.10.

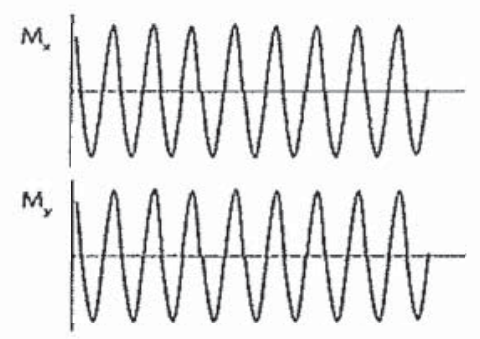

Figure 1.10: Plots of the $\mathrm{x}$ - and $\mathrm{y}$-component of the magnetization predicted using the approach of fig, 9. Fourier transformation of these signals will give rise to the usual spectrum ${ }^{13}$

This week electric signal will decay to zero and this process is called a Free Induction Decay (FID). This time dependent FID signal is amplified and digitized and then transformed from a time domain to a frequency domain via Fourier Transformation (FT). The resulting NMR spectrum, which we usually see in our NMR experiment, is a plot of the signal intensity as a function of frequency, with a peak positioned at the Larmor frequency of the nucleus of interest. This process of signal detection to obtain the NMR spectrum is depicted in Figure 1.11. 

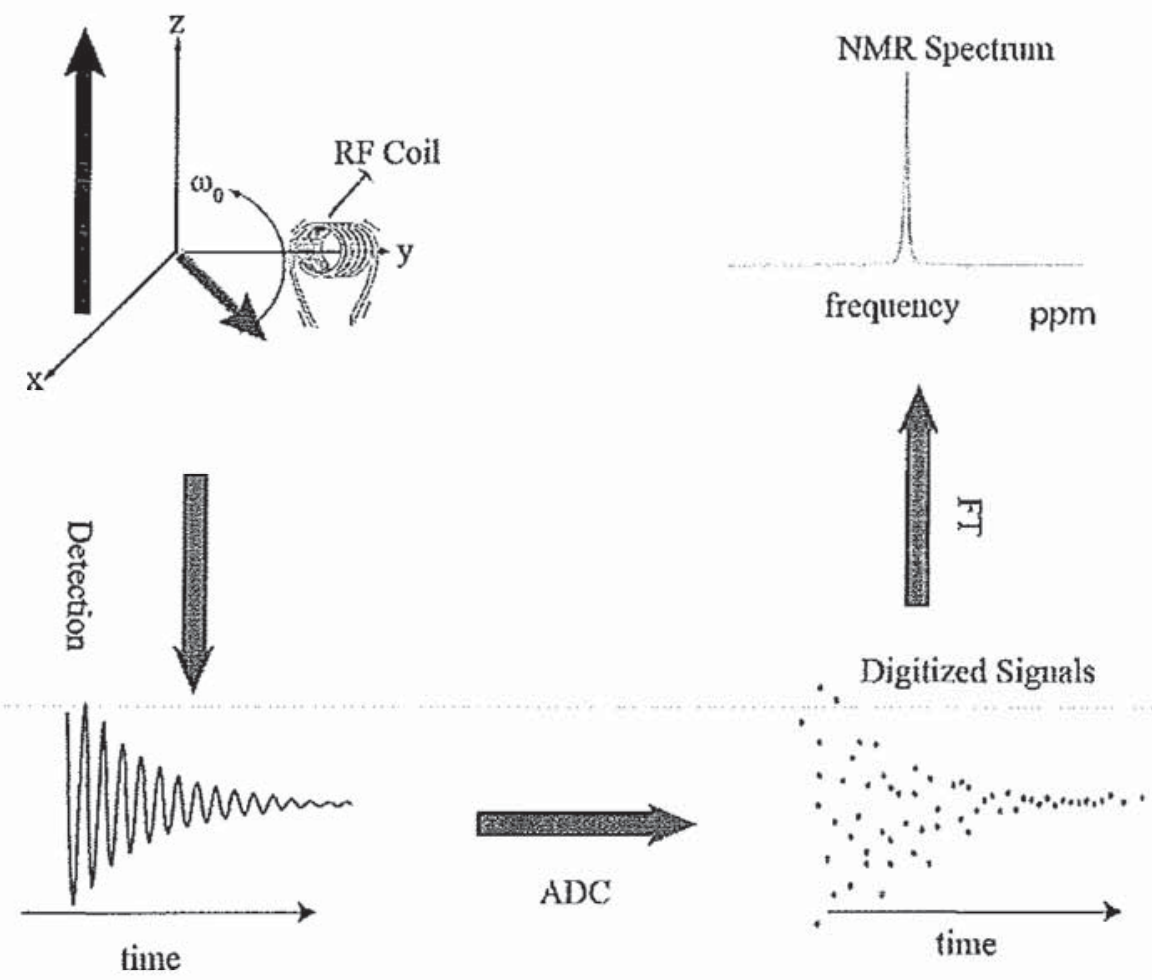

Figure 1.11: The process of signal detection for obtaining the NMR spectrum

\subsubsection{Pulses}

The idea to rotate the Z-magnetization away from it equilibrium is to apply a very small magnetic field along the $\mathrm{x}$-axis but one that is oscillating at or near to the Larmor frequency. When this coil is feed with some radiofrequency (RF) power, an oscillating current is created which in turn creates an oscillating magnetic field along the $\mathrm{x}$ - direction. The RF wave is arranged as its magnetic field oscillates along a direction perpendicular to the $\mathrm{B}_{0}$ field. We can label this component as $2 B_{1}(\mathrm{t})$. A RF transmitter produces this frequency. Such an oscillating field can be thought as the sum of two component rotating about $\mathrm{B}_{0}$ in opposite directions which be written as $+\omega_{\mathrm{rf}}$ and $-\omega_{\mathrm{rf}}$ respectively. This two counter-rotating fields have the same magnitude $B_{1}$. One denoted $B_{1}{ }^{+}$rotates in the positive 
sense, and the other, denoted $B_{1}{ }^{\circ}$, rotates in the negative sense. The component that rotates in the same direction as the precession of the magnetization of $\mathrm{M}_{0}$ has significant effect on Mo. For a positive gyromagnetic ratio nucleus, $B_{1}-$ is in the same direction of rotation that has significant interaction with the magnetization, and the other field, $B_{1}^{+}$will be ignored from now on.

\subsubsection{Rotating Frame}

We can employ a co-ordinate frame that is rotating about the $z$-axis in the same direction and at the same rate as $B_{1}{ }^{\circ}$, called the rotating frame. In this rotating frame, $B_{1}{ }^{-}$ appears to be static and directed along the $\mathrm{x}$-axis of the rotating frame, as shown in Figure 1.12 .
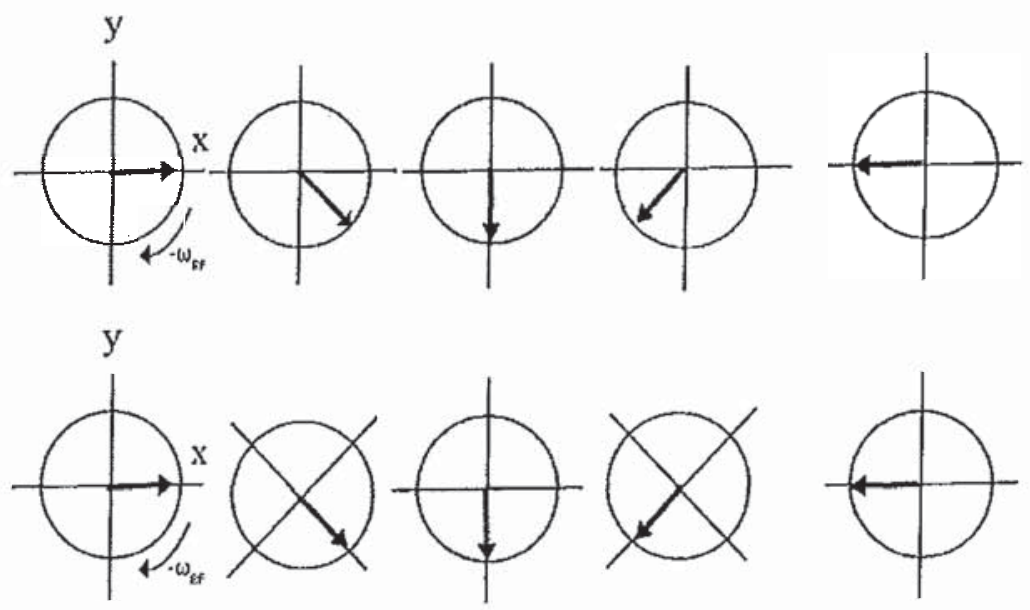

Figure 1.12: The top row shows a field rotating at $-\omega_{\mathrm{RF}}$ when viewed in a fixed frame, the same field viewed in a set of axes rotating at - -()$_{\mathrm{RF}}$ appears to be static ${ }^{13}$

In this way, a difficult time-dependent problem is reduced to a simpler form. From now on we will work in the rotating frame. 


\subsubsection{Larmor Precession in the Rotating Frame}

In the rotating frame the magnetization will appear to be static, the apparent Larmor frequency is zero. In the general case, if the rotating frame is at frequency $\omega_{\mathrm{rf}}$, the Larmor frequency will be at $\left(\omega_{0}-\omega_{\mathrm{rf}}\right)$ this difference is called offset and given the symbol as $\Omega$

$$
\Omega=\omega_{0}-\omega_{\mathrm{rf}}
$$

We can easily calculate the apparent magnetic field B, which is also called reduced field, from the apparent Larmor frequency,

$$
\Omega=-\gamma \Delta \mathrm{B}
$$

Hence

$$
\Delta \mathrm{B}=-\Omega / \gamma
$$

When the rotating frame rotates at the Larmor frequency, the offset would be zero, so will be the apparent field. So the weak RF field can affect the magnetization in the presence of the very strong $\mathrm{B}_{0}$ field. In the rotating frame, $\mathrm{B}_{0}$ shrinks, and under right conditions can become small enough that the RF field is dominate.

\subsubsection{The Effective Field}

In the rotating frame, the apparent field along $\mathrm{z}$-axis and $\mathrm{RF}$ field along $\mathrm{x}$-axis added to give an effective field, $B_{\text {eff }}$ as illustrated in Figure 1.13

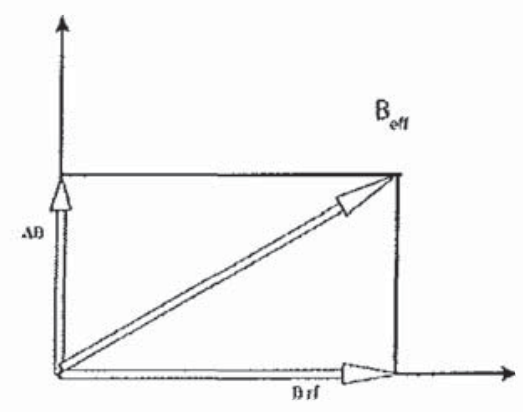

Figure 1.13: The effective field in the rotating frame ${ }^{14}$ 
The effective Larmor frequency about this effective field is given by

$$
\omega_{\mathrm{eff}}=\gamma \mathrm{B}_{\mathrm{eff}}
$$

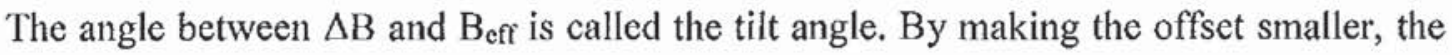
effective field lies more close to the $x y$-plane, when the offset is zero, the magnetization will be rotated from $Z$ to $x y$ plane. When the transmitter frequency is the same as Larmor frequency, the pulse is said to be on resonance. Under this condition, the offset, the reduced field is all zero, and the effective field is equal to $B_{1}$ and lies along the $x$-axis, the tilt angle is 90 .

If the RF would be applied for a time $t_{p}$, the angle $\theta$, through which the magnetization would be rotated, is given by

$$
\Theta=\omega \times \mathrm{t}
$$

$\theta$ is called the flip angle of the pulse. By changing the time the pulse will be applied, we can choose the angle the magnetization is rotated. In most experiment, the commonly used flip angles are $90^{\circ}$ and $180^{\circ}$. The motion of the magnetization vector during an on resonance $90^{\circ}$ and $180^{\circ}$ pulse are shown in Figure 1.14
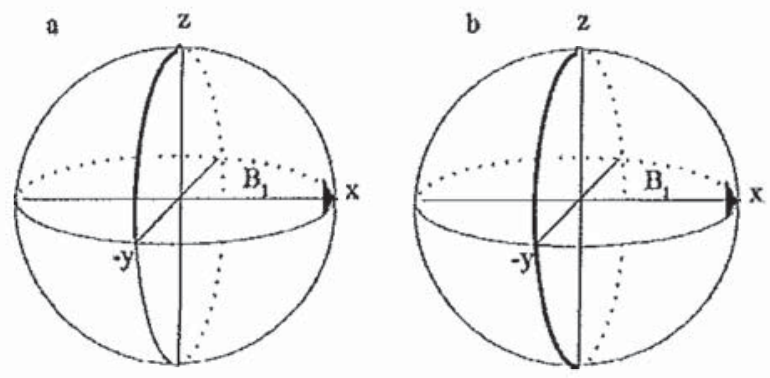

Figure 1.14: A "grapefruit" diagram in which the thick line shows the motion of a magnetization vector during an on-resonance pulse. The magnetization is assumed to start out along $+z$. In (a) the pulse flip angle is $90^{\circ}$. The effective field lies along the $x$-axis and so the magnetization precesses in the yz -plane. The rotation is in the positive sense about $x$ so the magnetization moves toward the $-y$ axis. In (b) the pulse flip angle is $180^{\circ}$ and so the magnetization ends up along $-z .^{13}$ 


\subsection{NUCLEAR SPIN INTERACTION}

\subsubsection{Chemical Shift}

Theoretically, we expect an isolated spin $I=1 / 2$ nucleus should give rise to a single narrow peak that reflects its Larmor frequency in a specific applied field $\mathrm{B}_{0}$. However, experimentally, this is incorrect. For example, protons from $-\mathrm{CH}_{3}$ groups of ethanol molecules show a slightly different Larmor frequency than protons located in the $-\mathrm{CH}_{2}$ groups.

A phenomenon known as shielding (or deshielding) causes the slight shift of the resonance frequency with respect to the Larmor frequency. The shifted signal position is determined by its offset frequency with respect to the Larmor frequency, which is approximated by the frequency of a reference material particular to each nucleus of interest.

For example, for ${ }^{1} \mathrm{H}$ and ${ }^{13} \mathrm{C}$, the primary reference compound is tetramethylsilane (TMS) and the secondary reference is adamantane. This shift is called chemical shift and is expressed in parts per million (ppm) of the reference frequency and the expression is shown in equation.

$$
\delta=\left(\omega^{0} * \omega^{0} \mathrm{TMS}\right) / \omega^{0} \mathrm{TMS}
$$

where $\omega^{0}$ TMS is the Larmor frequency of a spin in the reference compound(TMS) exposed to the same applied field.

$$
\delta_{\mathrm{ref}}=\left(\omega_{\mathrm{ref}}-\omega^{0} \mathrm{TMS}\right) / \omega^{0} \mathrm{TMS}
$$

$\delta_{\text {ref }}$ correspond to the exact center of the NMR spectrum on the ppm scale.

$$
\Omega_{0}=\omega^{0}-\omega_{\text {ref }} \cong\left(\delta-\delta_{\text {ref }}\right) \omega^{0}
$$

Here, we ignore the fine distinction between $\omega_{0}$ and $\omega^{0}$ TMS. 
Shielding arises from the electrons in the atomic shells, and in the chemical bonds that holds the molecules together. The mechanism of chemical shift is (i) the external magnetic field $B_{0}$ induces current in the electron cloud within the molecule. (ii) The circulating currents in turn generate a magnetic field (called the induced field $\mathrm{B}_{j}^{\text {induced }}$ ). These small fields add to or subtract from the external magnetic field.

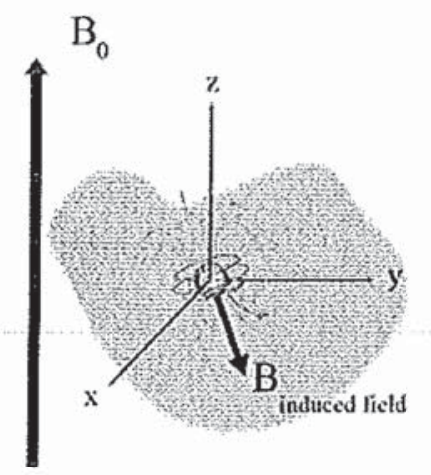

Figure 1.15: Mechanism of the chemical shift ${ }^{15}$

$$
\mathrm{B}_{\mathrm{j}}^{\mathrm{loc}}=\mathrm{B}_{0}+\mathrm{B}_{\mathrm{j}}^{\text {induced }}
$$

The shielding interaction depends on the orientation of the molecular frame with respect to the external field, and is linearly proportional to the applied field, it may be written

$$
B_{j}{ }^{\text {induced }}=\delta^{\mathrm{i}} \cdot \mathrm{B}^{0}
$$

$\delta^{j}$ is called chemical shift tensor, represented by a $3 * 3$ matrix of real numbers

$$
\left(\begin{array}{l}
\mathrm{B}_{\mathrm{j}, \mathrm{x}}^{\text {induced }} \\
\mathrm{B}_{\mathrm{j}, \mathrm{y}}^{\text {induced }} \\
\mathrm{B}_{\mathrm{j}, \mathrm{z}}^{\text {induced }}
\end{array}\right)=\left(\begin{array}{ccc}
\delta_{\mathrm{xx}}^{j} & \delta_{\mathrm{xy}}^{\mathrm{j}} & \delta_{\mathrm{xz}}^{\mathrm{j}} \\
\delta_{\mathrm{yx}}^{j} & \delta_{\mathrm{yy}}^{\mathrm{j}} & \delta_{\mathrm{yz}}^{\mathrm{j}} \\
\delta_{\mathrm{zx}}^{\mathrm{j}} & \delta_{\mathrm{zy}}^{\mathrm{j}} & \delta_{\mathrm{zz}}^{j}
\end{array}\right) \cdot\left(\begin{array}{c}
0 \\
0 \\
\mathrm{~B}^{0}
\end{array}\right)
$$

The tensor is represented in the laboratory frame and is often transformed to Principal Axis System (PAS). For every nucleus, those three directions of the PAS are when the induced field is parallel to the external field. The induced field is parallel to the applied 
field whenever the applied field is in one of those special directions. One of the examples is shown in Figure 1.16.

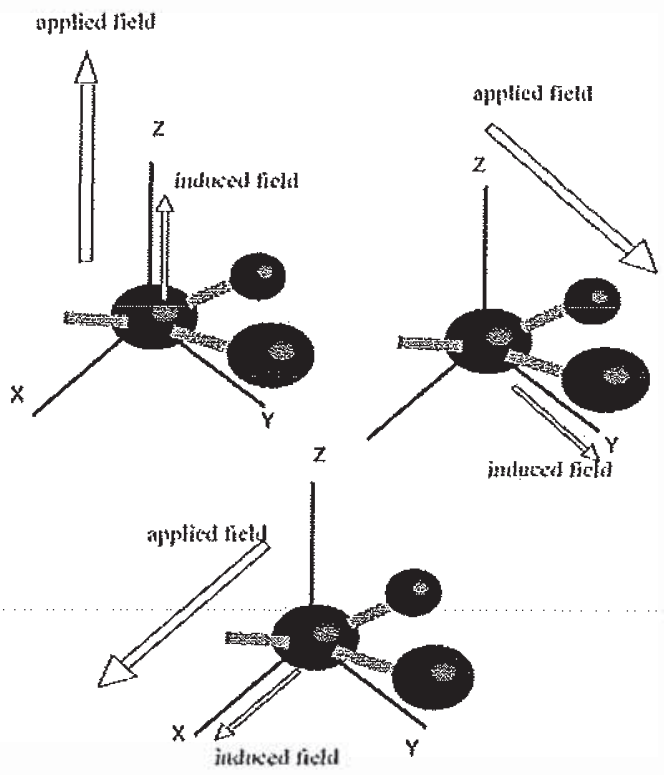

Figure 1.16: If the static magnetic is applied along a principal axis direction, the induced field is parallel to the static field ${ }^{\text {is }}$

The tensor matrix in PAS frame is shown as

$$
\delta_{\mathrm{PAS}}=\left|\begin{array}{ccc}
\delta_{11} & 0 & 0 \\
0 & \delta_{22} & 0 \\
0 & 0 & \delta_{33}
\end{array}\right|
$$

The "off diagonal" shift tensor elements, such as $\delta \mathrm{xz}, \delta \mathrm{yz}$ are zero in the PAS frame system. The average of the tensor is known as a relative isotropic chemical shift as is expressed as

$$
\delta^{i_{i s o}}=1 / 3\left(\delta^{j_{X X}}+\delta_{Y Y}^{j}+\delta_{Z Z} i_{Z}\right)
$$

If all three principal values are equal, then the chemical shift tensor is said to be isotropic, otherwise the chemical shift tensor is said to be anisotropic. The chemical shift anisotropic (CSA) quantifies the deviation from isotropy. Conventionally,

$$
\left|\delta_{Z Z}^{i}-\delta_{j}^{i s o}\right| \geq\left|\delta_{X X}^{j}-\delta_{j}^{i s o}\right| \geq\left|\delta_{Y Y}^{j}-\delta_{f}^{i s o}\right|
$$


The CSA is the largest deviation in chemical shift from the isotropic value (including the sign)

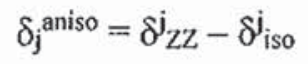

The CSA interaction affects the spectra in liquids and solids quite differently. In solution NMR, The rapid random tumbling averages the CSA NMR interaction and spectra consist of a series of very sharp transitions. This rapid motion cause the CSA term to cause randomly fluctuating fields and thus accelerate the relaxation mechanism. By contrast, in solid state, the samples have different orientation with respect to the applied field, thus the CSA cause inhomogeneous broadening of the signal, which is a result of superimposition of peaks with different chemical shifts due to various orientations, giving rise to a powder pattern.

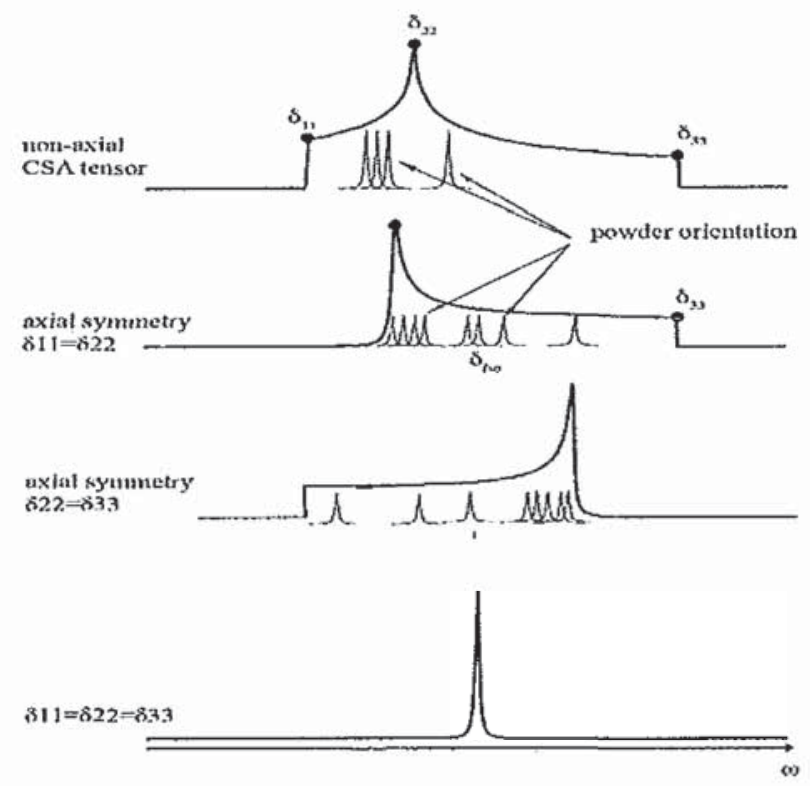

Figure 1.17: Inhomogeneous line broadening mechanism in NMR spectra caused by CSA. Powder pattern characterized by the three different tensor component (top); powder pattern characterized by reduced chemical shift tensor in the case of axial symmetry along bond axis (middle); and single peaks at $\delta_{\text {iso }}$ as a result of fast isotropic motion (bottom)

Tensor components can be scaled down in the molecules that exhibit fast dynamics.

For example, a methyl group that has rapid rotation about the $\mathrm{CH}_{3}-\mathrm{X}$ bond axis leads to 
axial symmetry in the tensor along the bond that results in two principal components becoming equal. (Figure 1.17, middle). Fast isotropic motion cause all three tensor components to become equalized; hence, all orientations have the same shielding, leaving one narrow peak at $\delta_{\text {iso, }}$ as shown in the bottom of Figure 1.17.

The symmetry reflected by the tensor components is the result of a combination of the symmetry in the electron density surrounding the nucleus and the symmetry of any motion that the system undergoes.

\subsubsection{Dipolar Coupling}

Since each nuclear spin is a magnetic, it generates a magnetic field, looping around in its surrounding space, according to the direction of the spin magnetic moment. A second nuclear spin interacts with this magnetic field. The first nuclear spin also experience the field generated by the second nuclear spin, so the interaction is a mutual process. This interaction is called direct dipole-dipole coupling or through space dipole-dipole coupling and can occur between spins of the same or different type of nuclei.

This interaction is proportional to the angle between the coupling vector and the direction of the applied magnetic field, and is inversely proportional to the cube of the internuclear distance. The magnitude of this interaction is given by the dipolar-dipolar coupling constant as presented

$$
b_{j k}=-\frac{\mu_{0}}{4 \pi} \frac{\gamma_{j} \gamma_{k} h}{r_{j k}^{3}}
$$

$\mu_{0}$ is called the permeability of vacuum and is a physical constant at $4 \pi \times 10^{-7} \mathrm{H} \mathrm{m}^{-1}, \gamma_{\mathrm{j}}$ and $\gamma_{\mathrm{k}}$ are the gyromagnetic ratios of the two spins and $r$ is distance between them. 


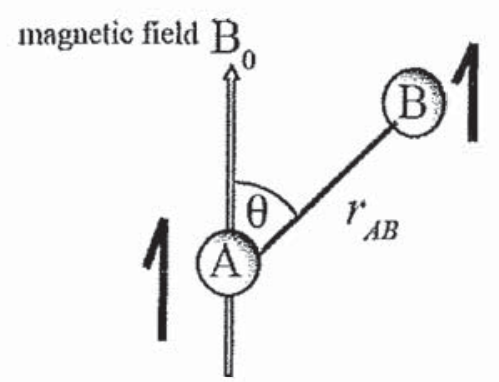

Figure 1.18; The direct dipolar coupling between two spins, $A$ and $B$ in the presence of an external magnetic field, $\mathrm{B}_{0}$

It can be characterized by a symmetric and traceless tensor, meaning that the interaction is symmetric between the two nuclei, and there is no isotropic dipolar coupling.

In solid-state powder sample, every magnetic spin is coupled to every other magnetic spin; dipolar coupling leads to severely broaden NMR spectra. In solution, molecules reorient quickly; nuclear spins feel a time average of the spatial part of the dipolar interaction $<3 \cos ^{2} \theta-1>$ over all orientations.

Strong homonuclear coupling gives rise to homonuclear line broadening, which can limit spectral resolution severely. Heteronuclear interactions do not give rise to homogeneous broadening and can be efficiently surprised using decoupling sequences.

\subsubsection{Quadrupolar Nuclei}

Quadrupolar nuclei have spins $>1 / 2$, and an asymmetric distribution of nucleons giving rise to a non-spherical positive electric charge distribution; this is in contrast to spin $I=1 / 2$ nuclei, which have a spherical distribution of positive electric charge. The difference are shown in Figure 1.19. This interaction is beyond the scope of this work and is the subject of an entire sub-discipline of NMR where unique techniques have been developed to obtain high-resolution spectra. 


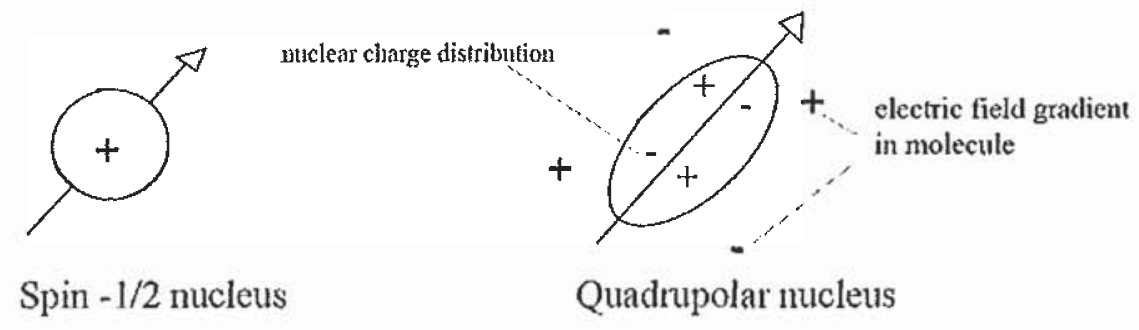

Figure 1.19: $\quad$ spin $I=1 / 2$ nuclei has a spherical positive electric charge (left); quadrupolar nuclei has a non-spherical positive electric charge distribution (right)

\subsection{NMR RELAXATION}

Relaxation is the process where the spins in a sample return to equilibrium, when the population of the energy levels are those predicted by Boltzmann distribution and where no transverse magnetization and hence no coherences present in the system. Experimentally, the rate of spin-lattice relaxation determines how fast an experiment can be repeated. Therefore, it is important to get knowledge how relaxation can be measured and the factor that influence its values. The rate of relaxation is influenced by physical properties, such as elasticity, flow rate, plasticity, etc. of the molecule and the sample, so the study of relaxation can give us the information on these properties.

We know when a NMR sample is placed in a static magnetic field, a net magnetization of the sample along the direction of $\mathrm{B}_{0}$ (traditionally the $\mathrm{z}$-axis) is developed, and magnetization parallel to the applied field is termed longitudinal magnetization. This equilibrium magnetization $\left(\mathrm{M}_{\mathrm{z}}\right)$ arises from the difference in population between the levels of $\alpha$ and $\beta$ spin states.

$$
M_{z} \propto\left(n_{\left.\alpha-n_{\beta}\right)}\right.
$$

where $n_{\alpha}$ and $n_{\beta}$ are the population of $\alpha$ and $\beta$ energy level. 
Suppose at certain time $t, n_{\alpha}$ and $n_{\beta}$ are not at equilibrium values, then for the system to reach equilibrium the population of one level must increase, and the other must decrease. So there must be a transition between these two levels. It is this process which cause relaxation.

We can make an assumption that the transition from $\alpha$ to $\beta$ is proportional to $n_{\alpha}$, and is a first order process with rate $\mathrm{W}$. Base on this assumption, the rate of population loss from $\alpha$ state is $W_{n \alpha}$, and the corresponding loss of population of state $\beta$ is $W_{n \beta}$. Then we can write: the change of population of state $\alpha$ is:

$$
\alpha=-\mathrm{Wn}_{\alpha}+\mathrm{Wn}_{\beta}
$$

because a transition from $\beta$ to $\alpha$ caused the population of level $\alpha$ to decrease, and a transition from $\beta$ to $\alpha$ cause the population of state $\alpha$ to increase. The rate of population change of $\alpha$ and $\beta$ state can be written using the language of calculus as

$$
\begin{aligned}
& \frac{d n_{\alpha}}{d t}=-W n_{\alpha}+W n_{\beta} \\
& \frac{d n_{\beta}}{d t}=-W n_{\beta}+W n_{\alpha}
\end{aligned}
$$

At equilibrium, the population of state $\alpha$ exceeds that of state $\beta$, which is determined by Boltzmann distribution. So we can modify above equation as:

$$
\begin{aligned}
& \frac{d n_{\alpha}}{d t}=W\left(n_{\beta}-n_{\beta}^{0}\right)-W\left(n_{\alpha}-n_{\alpha}^{0}\right) \\
& \frac{d n_{\beta}}{d t}=W\left(n_{\alpha}-n_{\alpha}^{0}\right)-W\left(n_{\beta}-n_{\beta}^{0}\right)
\end{aligned}
$$

where $n_{\alpha}{ }^{0}$ and $n_{\beta}{ }^{0}$ is the population at equilibrium for state $\alpha$ and state $\beta$.

We can work out how the Z-magnetization changes over time:

$$
\begin{aligned}
\frac{d M_{z}}{d t}=\frac{d\left(n_{\alpha}-n_{\beta}\right)}{d t}=\frac{d n_{\alpha}}{d t}-\frac{d n_{\beta}}{d t} & \\
= & W\left(n_{\beta}-n_{\beta}^{0}\right)-W\left(n_{\alpha}-n_{\alpha}^{0}\right)-W\left(n_{\alpha}-n_{\alpha}^{0}\right)+W\left(n_{\beta}-n_{\beta}^{0}\right)
\end{aligned}
$$




$$
=-2 W\left(n_{\alpha}-n_{\beta}\right)+2 W\left(n_{\alpha}^{0}-n_{\beta}^{0}\right)=2 W\left(M_{Z}-M_{Z}^{0}\right)
$$

where $\mathrm{M}_{\mathrm{z}}{ }^{0}=\left(\mathrm{n}_{\alpha}{ }^{0}-\mathrm{n}_{\beta}{ }^{0}\right)$, the equilibrium $\mathrm{z}$-magnetization.

when $\mathrm{M}_{\mathrm{z}}=\mathrm{M}_{\mathrm{z}}{ }^{0}$, the system is at equilibrium, otherwise, there is a rate change of $\mathrm{M}_{\mathrm{z}}$, and this rate is proportional to the difference between $\mathrm{M}_{\mathrm{z}}$ and $\mathrm{M}_{\mathrm{z}}{ }^{0}$.

This equation leads to a differential expression as:

$$
\frac{d M_{Z}(t)}{d t}=-R_{Z}\left(M_{Z}(t)-M_{Z}^{0}\right)
$$

where $R_{z}=2 W$

This equation after integration and rearrangement:

$$
\begin{gathered}
\int \frac{d M_{Z}(t)}{\left(M_{z}(t)-M_{z}^{0}\right)}=\int-R_{z} d t \\
\operatorname{In}\left(M_{z}(t)-M_{Z}^{0}\right)=-R_{z} t+\text { const } \\
\operatorname{In}\left[\frac{M_{z}(t)-M_{z}^{0}}{M_{z}(0)-M_{z}^{0}}\right]=-R_{Z} t \\
M_{z}(t)=\left[M_{z}(0)-M_{z}^{0}\right] \exp \left(-R_{z} t\right)+M_{z}^{0}
\end{gathered}
$$

This equation indicates that the $z$-magnetization returns from $\mathrm{M}_{2}(0)$ to $\mathrm{M}_{\mathrm{z}}{ }^{0}$ following an exponential law. The time constant of the exponential is $1 / R_{z}$ or $1 / 2 \mathrm{~W}$, and this is often called $T_{l}$, which is referred as the longitudinal or spin-lattice relaxation time.

\subsubsection{Relaxation and Molecular Motion}

The relaxation process is induced by field fluctuation due to molecular motion. Nuclei are in constant vibrational and rotational motion within its lattice structure, creating a complex time dependent magnetic field.

A few definitions:

The correlation time- $\tau_{\mathrm{c}}$ (Tau-c): represents the time for a molecular reorient by 1 radian. 
The spectral density $J(\omega)$ describes the ranges of frequency motion that are present. Molecules tumbling at different rate, the range of rates up to the maximum rate of $\left(1 / \tau_{c}\right)$.

The source of magnetic fields which give rise to relaxation and the origin of their time dependence are referred to as relaxation mechanism. There are several relaxation mechanisms, among these only a few are really important for spin $I=1 / 2$ nuclei.

\subsubsection{Paramagnetic relaxation}

Molecular motion modulates the electric field from unpaired electron spin, the large magnetic moment of the unpaired electrons means that paramagnetic species in solution are particularly effective at promoting relaxation, because there is dipole relaxation by the electrons magnetic field and a transfer of unpaired electron density to the relaxing nucleus. Such species include dissolved oxygen and certain transition metal compounds.

\subsubsection{The dipolar mechanism}

We have learned that dipolar interaction is a mutual process and the degree of interaction depends on the inverse cube of the distance between the two nuclei and the direction of the vector that joins the two nuclei relative to the applied field. As the molecule tumbling, the direction of this vector changes and so the corresponding magnetic field changes, changes in the distance between the two nuclei also cause this time dependent magnetic field change.

The pair of mutual interacting nuclei can be in the same or different molecules, resulting intra- and inter- molecular relaxation. Since nuclei in the same molecule can approach much more closely, intra-molecular relaxation is dominant. 
The relaxation induced by dipolar coupling interaction is proportional to the square of the coupling. Thus:

$$
\gamma_{1}^{2} \gamma_{2}^{2} \frac{1}{\mathrm{r}_{12}^{6}}
$$

where $\gamma_{1}$ and $\gamma_{2}$ are the gyromagnetic ratios of the two coupled nuclei. $r_{12}$ is the distance between them.

The equation indicates that the nuclei with high gyromagnetic ratios are most efficient at promoting relaxation. For example, a proton-proton pair will relax 16 times faster than a carbon-proton pair. Dipolar relaxation cannot only distribute energy from one of the spins to the other, but also provides a path by which energy can be transferred between the lattice and the spins.

\subsubsection{The chemical shift anisotropy mechanism}

The magnetic field sensed by the nucleus depends on the chemical shift tensor in the molecule. We know that the chemical shift depends on the CSA, which is responsible for the very wide line width observed in a solid sample. In solution, the CSA is averaged out by molecular tumbling and a sharp isotropic shift is observed, but the time modulation of the shielding can cause relaxation, in the absence of other mechanisms because this mechanism is field dependent.

CSA is an important relaxation mechanism for nuclei with large chemical shift range, for example on ${ }^{31} \mathrm{P}$ and on cadmium-113, but is usually insignificant for protons. 


\subsubsection{Electric Quadrupolar Relaxation}

For nuclei with spin $I>1 / 2$, it is characterized by the non-spherical distribution of electrical charges and possesses an electric magnetic moment. The quadrupolar coupling constant is in the $\mathrm{MHz}$ range. As this relaxation is very large, it dominates over all other mechanisms. This relaxation mainly depends on the quadrupole moment of the nucleus and the electric field gradient, indicating that in a symmetrical environment, the quadrupolar coupling is zero.

\subsubsection{The Inversion Recovery Experiment $\left(\mathrm{T}_{1}\right)$}

Inversion recovery experiment is a method for measuring the longitudinal relaxation time constant of the spin, $\mathrm{T}_{1}$. The value of $\mathrm{T}_{1}$ provides valuable information as to the motion and dynamics of molecules. The pulse sequence is given by:

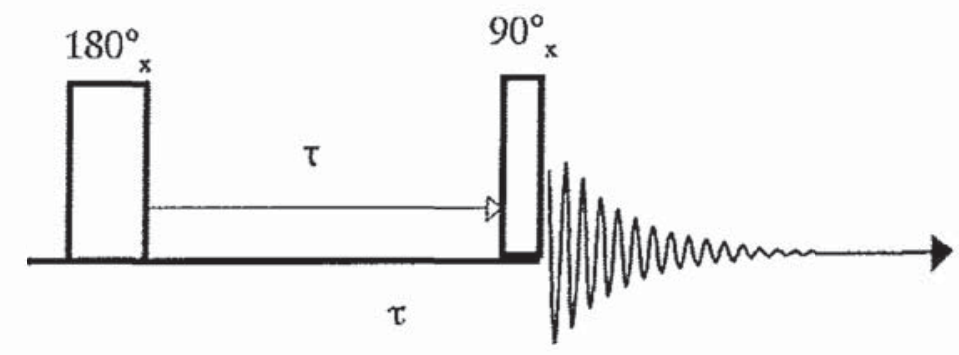

Figure 1.20: Inversion-recovery pulse sequence ${ }^{15}$

The single-headed arrow indicates that the experiment is performed in an arrayed fashion; the pulse is repeated, with different values of intervals $\tau$.

The first $\pi_{x}$ pulse in the sequence generates an inverted population distribution, then the population relax back towards thermal equilibrium during the interval $\tau$, the second $\pi / 2$ pulse converts the population difference into coherence, which induces an NMR signal.

The magnitude of the relaxation time depends highly on the type of nuclei (nuclei with spin $1 / 2$ and low magnetogyric ratio have usually long relaxation time whereas nuclei 
with spin $>1 / 2$ have very short relaxation time), and on other factors, such as the physical state (solid or liquid state), on the viscosity of the solution, the temperature. etc..

$$
M_{\tau}=M_{0}\left(1-2 \exp \left(-\tau / T_{1}\right)\right)
$$

Can be used to calculate the $T_{1}$ after rearrangement

$$
\text { slope }=-1 / \mathrm{T}_{1}
$$

\subsubsection{Transverse relaxation $\left(\mathbf{T}_{2}\right)$}

We have mentioned that relaxation involve two processes: the population returning to equilibrium and the transverse magnetization decaying to zero. We have discussed the

first of these two. The second, where the transverse magnetization decays, is called transverse or spin-spin relaxation.

The transverse contribution behaves in a more complex way as the net transverse magnetization precessing at the Larmor frequency in the transverse plane. We can represent each of the contribution as a vector precessing in the transverse plane. At equilibrium, all the individual contribution will direct in random direction and cancel each other; the net magnetization would be zero in the transverse plane. A RF pulse applied to equilibrium magnetization generates transverse magnetization where all the contribution together has a preference for one direction, which is described as coherence in quantum mechanics. As shown in Figure 1.21. 


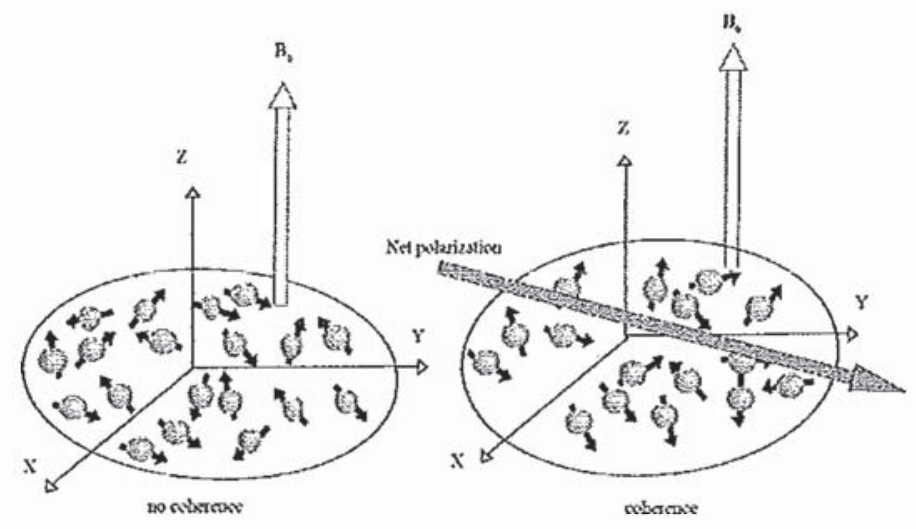

Figure 1.21: Partial alignment of spins in the transverse plane $e^{15}$

Transverse relaxation destroys this coherence by destroying the alignment of the individual contributions. One way to destroy this coherence is to make the vector get out step with each another as a result of precessing at different Larmor frequency; it does not require transitions, just a field to cause a local variation in the magnetic field.

The spin echo pulse sequence is as follows:

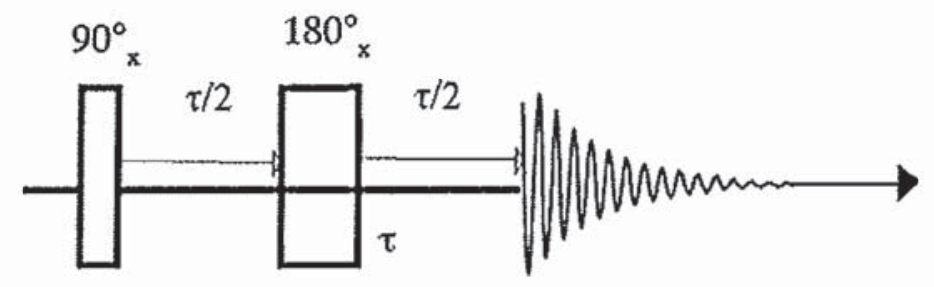

Figure 1.22: spin echo pulse sequence

The Hahn echo is constituted by a 90 degree pulse that flips the magnetization in the $X Y$ plane, during the first $\tau / 2$ delay, the magnetization evolve according to its chemical shift and field inhomogeneity. Then a $180^{\circ}$ pulse is applied, which corresponds to a reversal of the inhomogeneous part of the signal decay. The $180^{\circ}$ pulse causes the magnetization vector to be set up in such a way that the signal grows bigger rather than smaller.

$$
M_{\tau}=M_{0} \exp \left(-\frac{\tau}{T_{2}}\right)
$$

can be used after rearrangement to calculate the $T_{2}$ value in excel with 


$$
\text { Slope }=-\left(1 / \mathrm{T}_{2}\right)
$$

The NMR spectra peak is Lorentzian shape with a finite line width. As shown on the diagram, the peak width at half-height, measure in $\mathrm{rad} / \mathrm{s}$, is equal to $2 / \mathrm{T}_{2}=2 \lambda$, where $\lambda$ is called coherence decay rate constant, and is the inverse of the transverse relaxation time constant.

$$
\lambda=1 / \mathrm{T}_{2}
$$

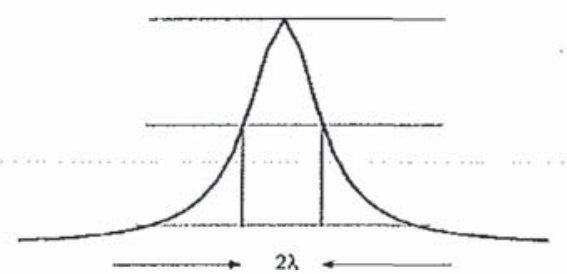

Figure 1.23: Full width at half-height

The peak-width in units of Herts is given by $2 \lambda 2 \pi=\lambda / \pi=1 / \pi T_{2}$

Note that a rapid decay of the transverse magnetization means a small value for $\mathrm{T}_{2}$, and corresponds to a broad spectral peak, while a slow decay of the transverse magnetization corresponds to a narrow spectral peak.

\subsubsection{Spin Locking Measurement of $\mathrm{T}_{1 p}$}

$T_{l \rho}$ is the spin-lattice relaxation in the rotating frame. A resonant $R F$ field is applied to suppress the free evolution of transverse magnetization, locking it to a particular direction in the rotating frame.

A typical spin-locking pulse sequence is as follows: 


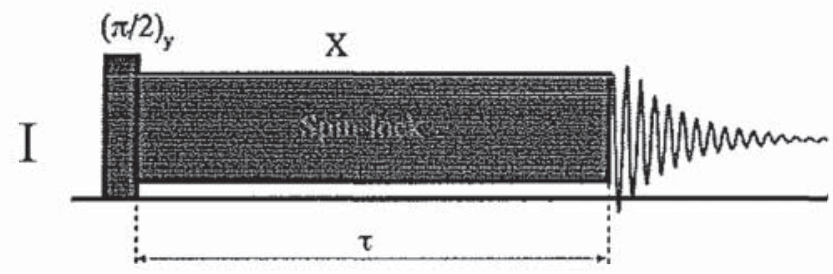

Figure 1.24: Spin lock pulse sequence

The NMR signal intensity is measured as a function of the spin-lock duration $\tau$. The first $\pi / 2$ pulse converts the initial magnetization which is firstly along $\mathrm{z}$-axis into transverse magnetization along the $x$-axis in the rotating frame. The phase of the RF field is switched to $\phi=0$, making the rotating frame $R F$ field is along the $x$-axis. The applied $R F$ is large enough that the transverse magnetization is unable to precess away from the $\mathrm{x}$-axis, which is called spin locked. After time $\tau$, the locking field is turned off, releasing the transverse magnetization and generating a NMR signal.

The equation governing $T_{1}$ relaxation is the exact same as that governing $T_{2}$.

$$
M_{\tau}=M_{0} \exp \left(-\tau / T_{1 \rho}\right)
$$

can be rearranged and used to calculate the $T_{i \rho}$ value with

$$
\text { slope }=1 / T_{1 \rho}
$$

\subsubsection{Relaxation and correlation time}

\subsubsection{Autocorrelation function and correlation times}

Consider a set of isolated spins $I=1 / 2$, when put in the magnetic field, $B_{0}$, they all experience the same static magnetic field along the z-axis, and each spin experiences its own small local magnetic field $\mathrm{B}_{\mathrm{loc}}(\mathrm{t})$ due to its neighboring spins that fluctuates with time. This fluctuating transverse field can be expressed as follows:

The average local field over time is zero 


$$
\left\langle B_{\text {loc }}(t)\right\rangle=0
$$

The mean square local field is not equal to zero

$$
\left\langle B_{\text {loc }}^{2}(t)\right\rangle \neq 0
$$

It has a positive non-zero value.

The autocorrelation function

$$
G(\tau)=\left\langle B_{l o c}(t) B_{l o c}(t+\tau)\right\rangle \neq 0
$$

where $\tau$ is a short time interval. A quickly decaying correlation function with respect to $\tau$ has a rapidly fluctuating local field as presented in Figure 1.25 (a). On the other hand, a slowly decaying autocorrelation function has in its origin a slowly fluctuating local field as shown in Figure 1.25 (b).
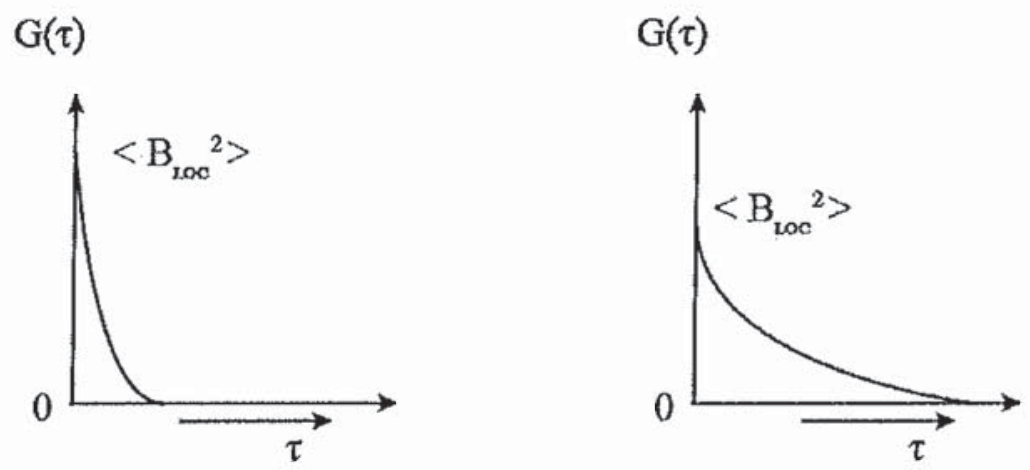

Figure 1.25: (a) Autocorrelation function of a rapidly fluctuating field; (b) Autocorrelation function of a slowly fluctuating field

The decay and form of the autocorrelation function $G(\tau)$ is of great importance for nuclear spin relaxation. The fluctuating field is spin independent, meaning that the autocorrelation function is independent of the time point $t$. This called the stationary assumption.

The general physical meaning of the autocorrelation function is to compare the fluctuating field at any time point $t$ with its value at $t+\tau$. If the interval $\tau$ is small compared 
with $t$, the values of the field at the two points tend to be similar, as illustrated in Figure 1.26. If the interval $\tau$ is long compared with the time scale of the fluctuation $t$, the similarity of the field at these two time points is lost. As illustrated in Figure 1.27.

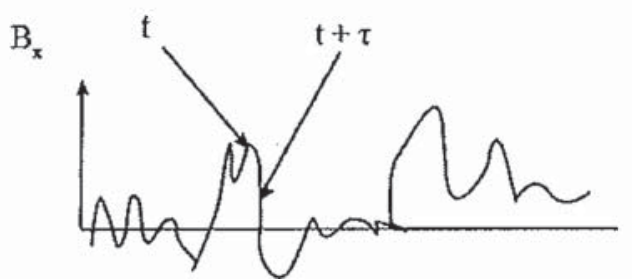

Figure1.26: Comparison of the fields at short time interval

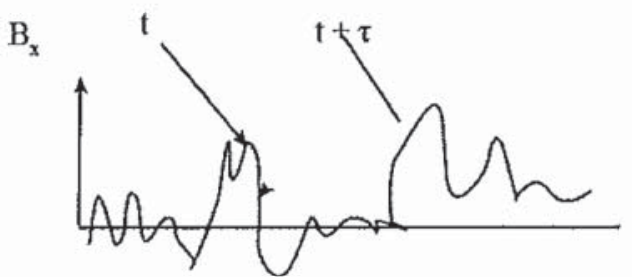

Figure 1.27: Comparison of fields at long time interval

Generally, the autocorrelation function $\mathrm{G}(\tau)$ tends to be large for small value of $\tau$, and tends to zero for large $\tau$. This can be represented in a simple exponential form of the autocorrelation function:

$$
G(\tau)=\left\langle B_{l o c}^{2}\right\rangle e^{-|\tau| / \tau_{c}}
$$

where $\tau_{\mathrm{c}}$ is called the correlation time of the fluctuating field, meaning the time it takes the spin to rotate through one radian. Rapidly moving spins have a rapidly fluctuating field with a very short $\tau_{\mathfrak{c}}$, whereas slowly moving spins have a slowly fluctuating field with a very long $\tau_{c}$.

\subsubsection{Spectral density}

Spectral density $\mathrm{J}(\omega)$ plays an important role in NMR relaxation theory. It is defined as twice the Fourier transform of the autocorrelation function:

$$
J(\omega)=2 \int_{0}^{\infty} G(\tau) \exp \{-i \omega \tau\} d \tau
$$

Explicitly, the spectral density can be written as: 


$$
J(\omega)=2\left\langle B_{l o c}^{2}\right\rangle \frac{\tau_{c}}{1+\omega^{2} \tau_{c}^{2}}
$$

For rapidly fluctuating local fields, the correlation time is short and the spectral density function is broad, as is represented in Figure 1,28.

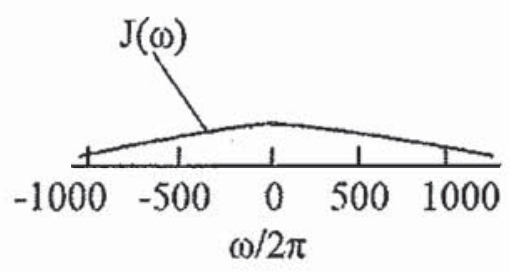

Figure 1.28: Spectra density of a rapid fluctuating field $\left(\tau_{\mathrm{c}}=0.2 \mathrm{~ns}\right)$

For slowly fluctuating local fields, the correlation time is long and the spectral density function is narrow, as shown in Figure 1.29.

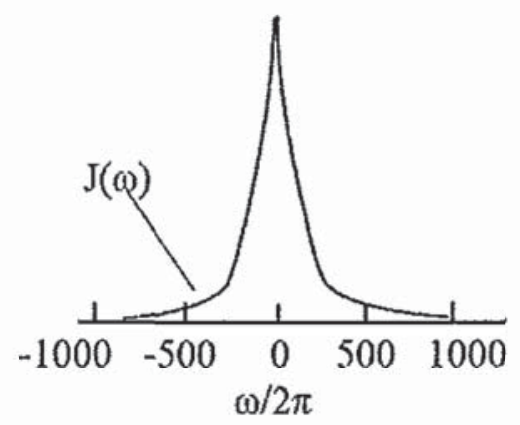

Figure 1.29: Spectral density of a slowly fluctuating field $\left(\tau_{0}=2 \mathrm{~ns}\right)$

Normally, normalized spectral density is denoted as follows:

$$
J(\omega)=A\left(\omega ; \tau_{c}^{-1}\right)=\frac{\tau_{c}}{1+\omega^{2} \tau_{c}^{2}}
$$

\subsubsection{Transition probability}

This fluctuating local field induces transitions between the spin state $\alpha$ and $\beta$. The transition probability per unit time from state $\mid \alpha>$ to state $\mid \beta>$ is defined as

$$
W_{-}=\tau^{-1}\left(\left|\left\langle\beta \mid \alpha^{\prime}\right\rangle\right|^{2}\right)
$$


Similarly, the transition probability per unit time from state $\mid \beta>$ to state $\mid \alpha>$ is defined

as

$$
W_{+}=\tau^{-1} \overline{\left|\left\langle\alpha \mid \beta^{\prime}\right\rangle\right|^{2}}
$$

The subscripts + and - used in the notation for transition probability refer to the change in the angular momentum along the $\mathrm{z}$-axis. The derived transition probability for the random field model is given as:

$$
\mathrm{W}_{-}=\mathrm{W}_{+}=\frac{1}{2} \gamma^{2}\left\langle\mathrm{~B}_{\mathrm{Ioc}}^{2}\right\rangle \mathrm{J}\left(\omega_{0}\right)
$$

The transition probability for the two directions are predicted as equal and are proportional to the spectral density of the random field at the Larmor frequency $\omega_{0}$.

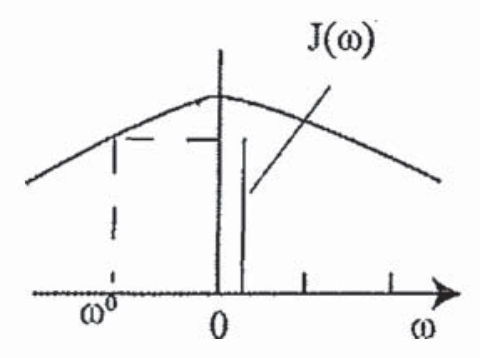

Figure 1.30: For a fluctuating field, the transition probabilities are proportional to the spectral density at the Larmor frequency $\omega_{0}$

\subsubsection{Spin-lattice relaxation and motion}

The kinetic equation for the population in stare $\mid \alpha>$ is defined as:

$$
\frac{\mathrm{d}}{\mathrm{dt}} \rho^{\alpha}=-\mathrm{W}_{-} \rho^{\alpha}+\mathrm{W}_{+} \rho^{\beta}
$$

Similarly, the kinetic equation for the population in state $\mid \beta>$ is defined as:

$$
\frac{d}{d t} \rho^{\beta}=+W_{-} \rho^{\alpha}-W_{+} \rho^{\beta}
$$

The spin magnetization vector along the $\mathrm{z}$-axis is proportional to the difference in the populations of the spin states and is defined as: 


$$
M_{z}=2 B^{-1}\left(\rho^{\alpha}-\rho^{\beta}\right)
$$

After normalization and some rearrangement the z-component of the normalized magnetization vector has the form:

$$
\frac{d}{d t} M_{z}=-2 W\left(M_{z}-1\right)
$$

The equation of motion can be written as:

$$
M_{z}(\tau)=\left(M_{z}(0)-1\right) e^{-2 W t}+1
$$

The spin-lattice relaxation time is equal to:

$$
\mathrm{T}_{1}^{-1}=2 \mathrm{~W}
$$

Meaning that the spin-lattice relaxation rate is equal to twice the mean transition probability per unit time between the states.

The expression for the spin-lattice relaxation rate can be expressed as:

$$
\mathrm{T}_{1}^{-1}=\gamma^{2}\left\langle\mathrm{~B}_{\mathrm{X}}^{2}\right\rangle \frac{\tau_{\mathrm{c}}}{1+\left(\omega_{0} \tau_{c}\right)^{2}}
$$

The relationship between relaxation time constant and correlation time can be expressed as:

$$
\mathrm{T}_{1}=\frac{1}{\mathrm{~B}}\left[\frac{1}{\tau_{\mathrm{c}}}+\omega_{0}{ }^{2} \tau_{\mathrm{c}}\right]
$$

When the correlation time is small; approaching zero, in the fast motion regime, the relaxation time constant is proportional to $1 / \tau_{c}$, as seen in Figure 1.31. Conversely when the correlation time is large approaching infinity, in the slow motion, the relaxation time constant is proportional to $\tau_{\mathrm{c}}$. This curve exhibits a minimum when the correlation time equals $1 / \omega_{0}$. 


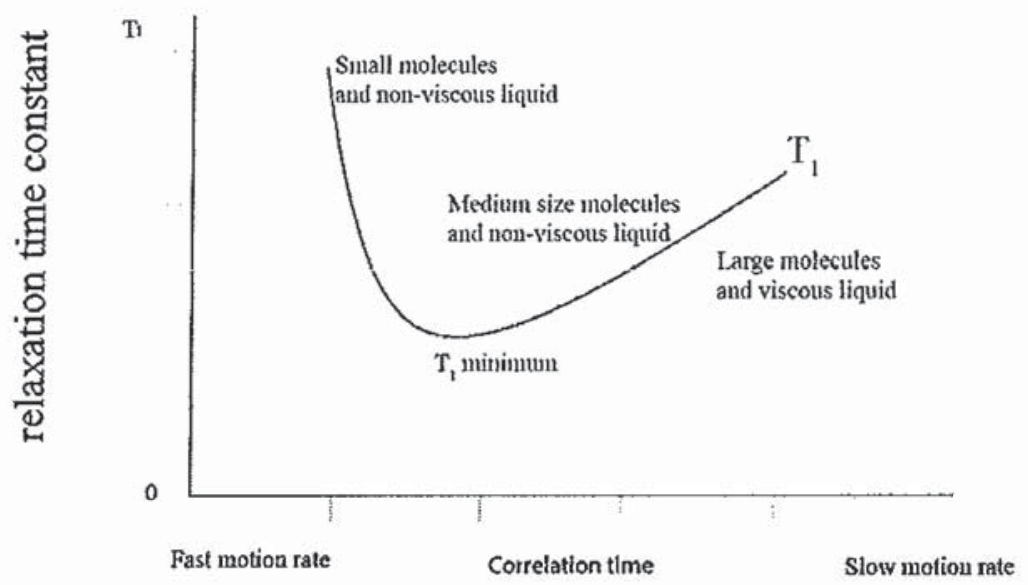

Figure 1.31: The spin-lattice relaxation time constant as a function of correlation time

\subsection{Relaxation time corresponding to temperature and activation energy}

Consider a motion process from $\mathrm{A}$ to $\mathrm{B}$, which is governed by rate $\mathrm{k}$, and $\mathrm{k}$ equals $1 / \tau_{\mathrm{c}}$. As in the equation 1.83

$$
\mathrm{k}=\frac{1}{\tau_{\mathrm{c}}}=\mathrm{Ae}^{-\Delta \mathrm{B}_{\mathrm{a}} / \mathrm{RT}}
$$

After rearrangement

$$
\tau_{c}=A e^{\Delta \mathrm{E} / \mathrm{RT}}
$$

Assuming that $\mathrm{E}_{\mathrm{a}}$ is small and positive, one can say that $\tau_{\mathrm{c}}$ is inversely proportional to temperature to first order.

Now the Equation 1.81 can be expressed as:

$$
T_{1}=\frac{1}{a<B>}\left[T_{\text {temp }}+\omega_{0}^{2} \frac{a^{2}}{T_{\text {temp }}}\right]
$$

The plot of relaxation time with respect to temperature is shown in Figure 1.32. 


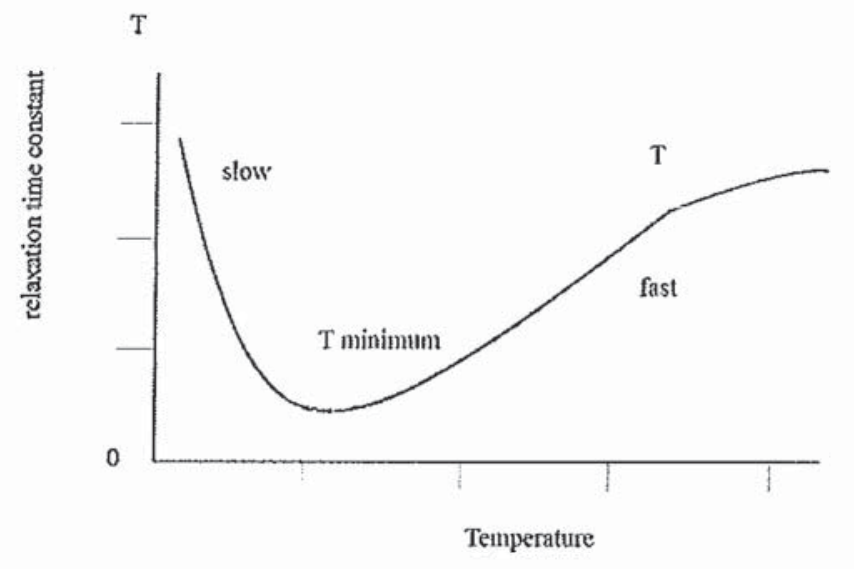

Figure. 1.32: $\quad$ relaxation with respect to temperature

At high temperature, the relaxation time constant is in the fast motion regime where the relaxation time is directly proportion to temperature, meaning that the relaxation time increases with increasing temperature; also the relaxation time constant is directly proportional to the rate of the process rate constant $\mathrm{k}$. Therefore, the relaxation time constant can be used as proxy for the rate constant in the fast motion regime.

At low temperature range, the relaxation time constant is in the slow motion regime where the relaxation time is essentially inversely proportionally to temperature, meaning that the relaxation time decreases with increasing temperature. Furthermore, as the relaxation time constant is inversely proportional to the rate constant. When using the relaxation time constant as a proxy for the rate in the slow motion regime it will lead to negative valued activation energies.

In summary, the fast motion regime is characterized by increasing relaxation time with increasing temperature, and thus gives rise to positive activation energy values. Conversely, the slow motion regime is characterized by decreasing relaxation time with increasing temperature and is typified by negative active energies. The temperature behavior of $T_{1 \rho}$ is the same as $T_{1}$, where the boundary the fast and slow motion regime is 
determined by $\tau_{\mathrm{c}}=1 / \omega_{0}$. While for $T_{2}$, since the relaxation time constant always increases with temperature, the activation energy will always be positive, regardless of the relevant motional regime.

\subsubsection{Dipole-dipole relaxation}

For a weakly coupled AX spin system, there is a spin $I_{1}$ coupled to a spin $I_{2}$, amongst which, four energy eigenstates and 12 transition probabilities exist. There are eight single quantum transitions, four double quantum transitions and four zero quantum transitions, each with different probabilities: as illustrated in Figure 1.33.
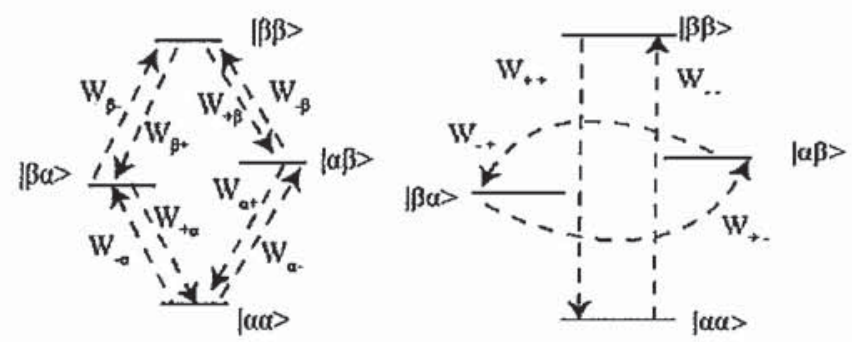

Figure 1.33: (a) Single quantum transition probability in a homonuclear AX system; (b) Double and zero quantum transition probability in a homonuclear AX system

The single-quantum transition probability which is denoted as $\mathrm{W}_{1}$ can be expressed as:

$$
W_{1}=\frac{3}{20} b^{2} J\left(\omega_{0}\right)
$$

where $\mathrm{J}\left(\omega_{0}\right)$ is the spectral density of the dipole-dipole coupling at the Larmor frequency $\omega 0$.

The double quantum transition probability is given by:

$$
W_{2}=\frac{3}{5} b^{2} J\left(2 \omega_{0}\right)
$$

where $J\left(2 \omega_{0}\right)$ is the spectral density at twice the Larmor frequency.

Similarly, the zero quantum transition probability is given as: 


$$
W_{0}=\frac{1}{10} b^{2} J(0)
$$

where $\mathrm{J}(0)$ is the spectral density at zero frequency.

The longitudinal relaxation rate constant can be expressed as:

$$
\mathrm{T}_{1}^{-1}=\frac{3}{10} \mathrm{~b}^{2}\left\{\mathrm{~J}\left(\omega_{0}\right)+4 \mathrm{~J}\left(2 \omega_{0}\right)\right\}
$$

The transverse relaxation rate constant $T_{2}^{-1}$ can be expressed as:

$$
\mathrm{T}_{2}^{-1}=\frac{3}{20} \mathrm{~b}^{2}\left\{3 \mathrm{~J}(0)+5 \mathrm{~J}\left(\omega_{0}\right)+2 \mathrm{~J}\left(2 \omega_{0}\right)\right\}
$$

The relaxation times $T_{1}$ and $T_{2}$ are plotted as a function of correlation time in Figure 1.34 .

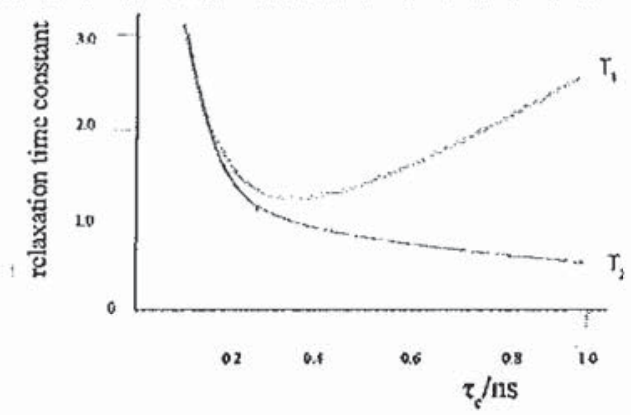

Figure 1.34: variation of $T_{1}$ and $T_{2}$ with correlation time, for intramolecular dipole-dipole relaxation

At very short rotational correlation times, the values of $T_{1}$ and $T_{2}$ are equal. This is called the extreme narrowing limit. As the correlation time increases, $T_{1}$ passes through a minimum and then increases. The transverse relaxation time constant $\mathrm{T}_{2}$, on the other hand, continues to decrease. $T_{2}$ is most efficient when the rate of motion driving the $T_{2}$ relaxation is on the order of $10-1000 \mathrm{~Hz}$, which is similar to the NMR linewidth.

The $T_{1} / T_{2}$ ratio can be used as indication for the rigidity of the nuclear environment. Equation 1.89 and 1.90 are used to give Equation 1.91

$$
\frac{\mathrm{T}_{1}}{\mathrm{~T}_{2}}=\frac{10+37 \omega_{0}^{2} \tau_{\mathrm{C}}^{2}+12 \omega_{0}^{4} \tau_{\mathrm{C}}^{4}}{10+16 \omega_{0}^{2} \tau_{\mathrm{C}}^{2}}
$$


For fast motion, where

$$
\begin{aligned}
& \omega_{0} \tau_{\mathrm{c}}<1 \\
& \mathrm{~T}_{1} / \mathrm{T}_{2} \rightarrow 1
\end{aligned}
$$

For slow motion,

$$
\omega_{0} \tau_{\mathrm{c}} \gg 10
$$

where, for example ${ }^{31} \mathrm{P}$ at $202 \mathrm{MHz}$ or ${ }^{19} \mathrm{~F}$ at $470 \mathrm{MHz}$, the correlation time is larger than 50 or 20 ns, respectively:

$$
\mathrm{T}_{1} / \mathrm{T}_{2} \rightarrow \frac{3}{4} \omega_{0}^{2} \tau_{c}^{2}
$$

The $T_{1} / T_{2}$ with respect to correlation time is given in Figure 1.35.

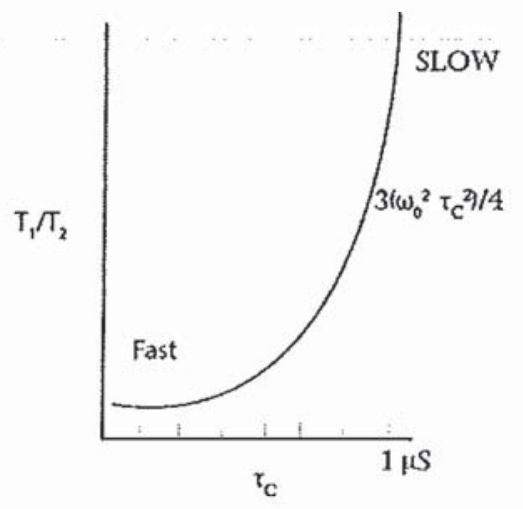

Figure 1.35: $\quad \mathrm{T} 1 / \mathrm{T} 2$ with respect to correlation time

In the limit where the $T_{1} / T_{2}$ approaches $\frac{3}{4} \omega_{0}^{2} \tau_{c}^{2}$, it can be used to the correlation time which in turn indicates mobility in the immediate nuclear environment. Large $T_{1} / T_{2}$ value represents long correlation times and thus rigidity. While small $T_{1} / T_{2}$ values arise from short correlation times and hence indicate mobility. For example, for ${ }^{31} \mathrm{P}$ at $202.34 \mathrm{MHz}$, $T_{1} / T_{2}$ with a value near 100 , has corresponding correlation time around $60 \mathrm{~ns}$, whereas for $\mathrm{T}_{1} / \mathrm{T}_{2}$ value near 30,000 , has corresponding correlation time around $\mathrm{I} \mu \mathrm{s}$.

Spin-lattice relaxation time in the rotating frame $\left(T_{1 \rho}\right)$ is defined as:

$$
\mathrm{T}_{1 \rho}^{-1}=\frac{3}{20} \mathrm{~b}^{2}\left\{3 J\left(\omega_{1}\right)+5 J\left(\omega_{0}\right)+2 J\left(2 \omega_{0}\right)\right.
$$


Analogues to Equation 1.85, which suggests the minimum in $\mathrm{T}_{1}$ is determined by $J(0)$. Equation 1.87 suggests the minimum of the $T_{1 \rho}$ curve is determined by $J\left(\omega_{1}\right)$. This means that at spin locking frequency used experimentally, $10-100 \mathrm{kHz}$, renders $\mathrm{T}_{\mathrm{l} p}$ sensitive to correlation time on the millisecond time scale. In contrast, $T_{1}$ and $T_{2}$ are sensitive to motion at the second or nano-second time scales respectively. Therefore, $T_{1 p}$ gives insight into motion not accessible by $T_{1}$ and $T_{2}$, which are very commonly encountered in the solid-state NMR. In fact, $\mathrm{T}_{1 \rho}$ allows you to select the time scale of motion to which it is sensitive. Unlike $T_{1}$ and $T_{2}$ which are determined by the fixed field strength of the instrument. That is why $T_{1 \rho}$ measurement is commonly used in material science.

\subsection{NMR TECHNIQUES FOR SOLID-STATE MATERIALS}

Over the last few decades, NMR spectroscopy has grown to be an indispensable technique for chemical analysis, structure determination, and the study of dynamics in organic, inorganic, and biological systems. Although most NMR experiments are performed on liquid state samples, solid-state NMR spectroscopy is rapidly emerging as a powerful tool for the study of solid samples and materials. In the following section, some routine NMR techniques or methods relevant to the contents in this thesis will be introduced.

\subsubsection{Magic Angle Spinning (MAS)}

The Hamiltonians for nuclear spin interaction, including chemical shielding interaction, dipolar coupling and quadrupolar interaction are isotropic, meaning that these 
magnetic coupling depends on the relative orientation of the molecule or crystallite with respect to the external applied magnetic field $\mathrm{B}_{0}$. In solution, rapid isotropic tumbling averages this spatial component to zero. For solids materials, however, the orientation depend remain in the Hamiltonians, leading to a broad spectrum for powder samples in NMR experiments. In the late 1950s, Andrew, E. R. Bradbury, A. and Eades, R. G. ${ }^{16}$ and Lowe ${ }^{17}$ independently realized that if the sample rotates around an axis that is tilted at the so-called "magic angle" (at $\left.54.74^{\circ}\right)^{16,18}$, with respect to $B_{0}$, some anisotropic nuclear spin interactions can be removed. Because the time dependent term $3 \cos ^{2} \theta-1=0$ when $\theta=$ 54.74 .The magic angle can be represented as an angle between the Z-axis and the body diagonal in a unit cube. As shown in Figure 1.36.
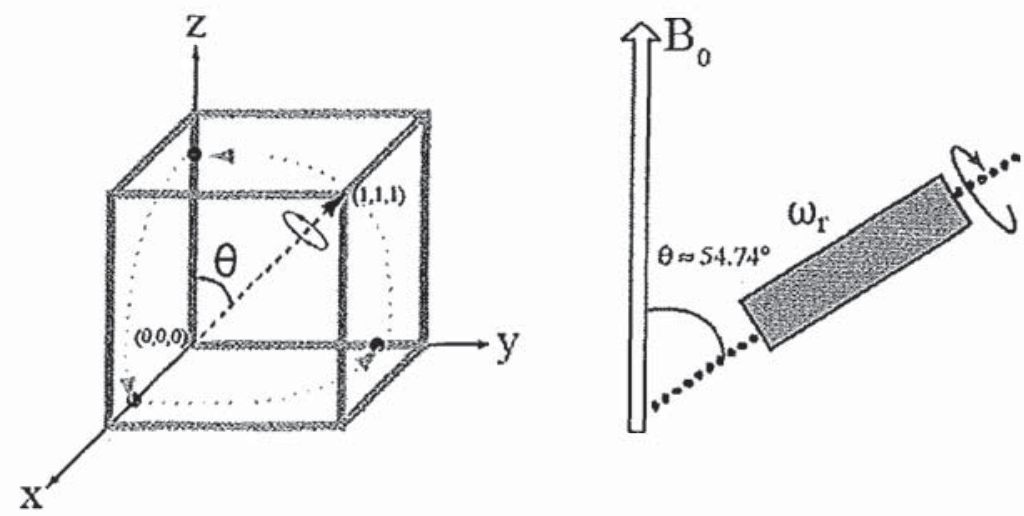

Figure 1.36: Geometrical interpretation of the magic angle (left); sample rotation at the magic angle in solid-state NMR (right).

Strong homonuclear coupling that give rise to extreme homogeneous line broadening can only be removed when the rate of MAS is greater than or equal to the magnitude of the anisotropic interaction. If the sample is spun at a rate less than the magnitude of the anisotropic interaction, a manifold of spinning sidebands will be observed, which are separated by the rate of spinning (in $\mathrm{Hz}$ ). An example is presented in Figure 1.37. 


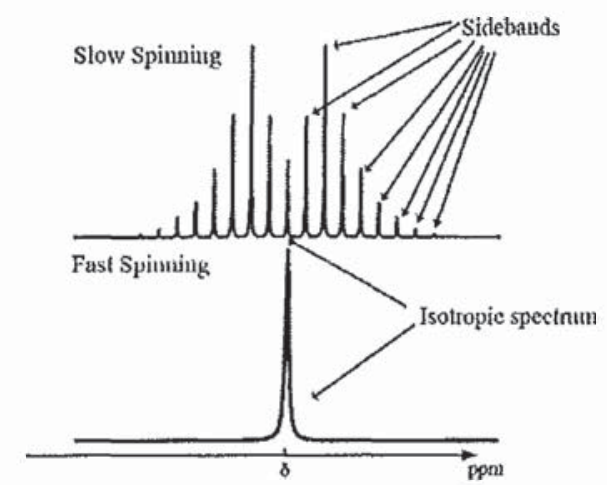

Figure 1.37: MAS NMR spectrum with a sideband pattern due to insufficient spinning speed (top); the same spectrum but obtained at higher spinning speed (bottom)

The isotropic center-band can be identified since it remains in the same position at different spinning rates. As seen in Figure 1.35.

Magic angle spinning is one of the most important NMR techniques for the improvement of the spectral resolution for solids. Since 1958, NMR spectroscopy became a more powerful tool for characterization solid state materials ${ }^{19}$, especially with the development of technology that allows high stable spinning speed, and most of the NMR pulse sequence are designed to be utilized in combination with magic angle spinning.

\subsubsection{Decoupling}

From our discussion above, we know that spins interact with each other in various ways, among which are: scalar coupling and nuclear dipole-dipole interactions, and may result in splitting patterns and inhomogeneous line broadening in NMR spectra. Decoupling consists of irradiating a system of interacting spins continuously and observing the effect on the spectrum. Decoupling is termed as hetero-nuclear decoupling if the observed and irradiated nuclei are different nuclear species or homonuclear if they are the same. 
Since much of the line broadening in ${ }^{13} \mathrm{C}$ NMR spectra of solids is due to dipolar coupling to protons, so we may apply strong continuous RF at proton resonance frequency. Because the interaction maybe of the order of tens of kilohertz, this would totally obscure any chemical shift information if the proton decoupling were not employed. The familiar liquid state $\mathrm{J}$-coupling also plays a role in the solid state. The $\mathrm{J}$-coupling interaction has an anisotropic part that is manifest in solids and cannot be easily distinguished from the through space coupling, because both follow the same tonsorial behavior with respect to rotation.

Standards high power proton decoupling in the solid state involves continuous wave (CW) decoupling. Another technique which provides improvement in both resolution ad sensitivity of the dilute-spin spectrum is known as two pulse phase modulated (TPPM) decoupling. Experimentally, there is an additional pulse length and phase which must be optimized when use TPPM decoupling. Combined MAS and dipolar decoupling will remove both CSA and dipolar broadening. The MAS serve to remove the CSA and any residual dipolar broadening.

\subsubsection{Cross Polarization}

We can enhance the intensity of the NMR signals from low-r nuclei by alternating population distribution. We use spin-spin coupling to transfer the population characterization of high $\gamma$ nuclei to low $\gamma$ nuclei. In the analogous solid-state experiment, the polarizations of low $\gamma$ nuclei are altered by exploiting the dipolar coupling to high $\gamma$ nuclei. This experiment is known as "cross polarization" 
Cross polarization is one of the most important techniques in solid-state NMR. The overall effect is to enhance signal to noise (S/N):

1.Cross polarization enhance signal from dilute spins potentially by a factor of $\gamma_{1} / \gamma_{\text {s }}$, where I is the abundant spin, such as ${ }^{1} \mathrm{H},{ }^{19} \mathrm{~F}$, and $\mathrm{S}$ is the dilute spin, such as ${ }^{13} \mathrm{C},{ }^{15} \mathrm{~N}$.

2. Since abundant spins are strongly dipolar coupled, they are therefore subject to large fluctuating magnetic fields resulting from motion. This induces rapid spin-lattice relaxation at the abundant nuclei. The end result is that one does not have to wait for slowly relaxation dilute nuclei to relax, rather the recycle delay is dependent upon the $T_{1}$ values of protons, fluorine, which is shorter than dilute nuclei.

The cross polarization pulse sequence is:

$$
\begin{array}{lr}
{ }^{1} \mathrm{H} & (\pi / 2)_{\mathrm{x}}-(\mathrm{CP})_{\mathrm{y}}-\mathrm{CW} \\
{ }^{13} \mathrm{C} & (\mathrm{CP})-\text { Acquire }
\end{array}
$$

The sequence is more easily seen in Figure 1.38

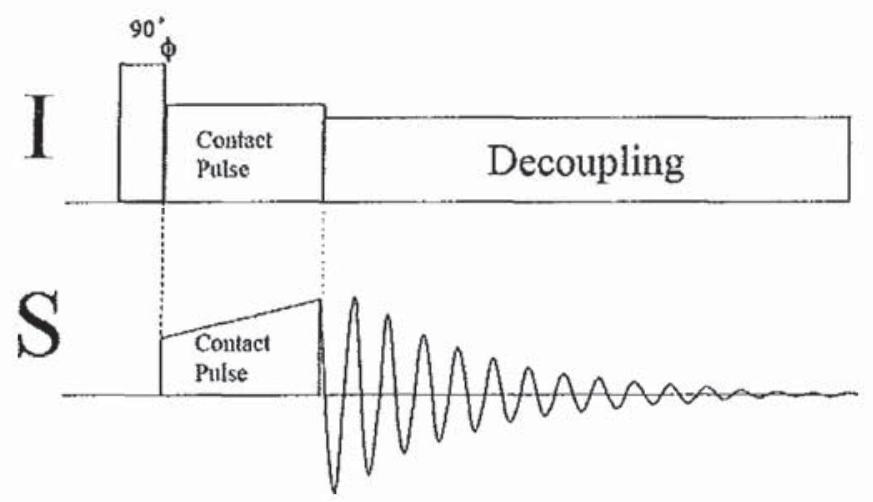

Figure 1.38: cross-polarization pulse sequence

The first $\pi / 2$ pulse of ${ }^{1} \mathrm{H}$ along $\mathrm{x}$ will generate a magnetization vector along the $\mathrm{y}$-axis, the phase shift of the ${ }^{1} \mathrm{H}$ radio frequency field means that, the magnetization becomes spin locked and precesses about the $y$-axis in the rotating frame at an angular frequency $\omega_{1}$ :

$$
\omega_{1 H}=\gamma_{H}\left(B_{1}\right)_{H}
$$


If we apply another pulse on ${ }^{13} \mathrm{C}$, then its precession frequency at its Larmor frequency would be:

$$
\omega_{1 \mathrm{C}}=\gamma_{\mathrm{C}}\left(\mathrm{B}_{1}\right)_{\mathrm{C}}
$$

If the two frequency are the same, then mutual spin-flips may occur. This matching frame frequency is referred as the Hartmann-Hahn condition:

$$
\gamma_{\mathrm{H}}\left(\mathrm{B}_{1}\right)_{\mathrm{H}}=\gamma_{\mathrm{C}}\left(\mathrm{B}_{1}\right)_{\mathrm{C}}
$$

In their respective rotating frames, the protons and carbons are in an effective field that produces the same precession frequency in the rotating frame. The ${ }^{1} \mathrm{H}$ magnetization is larger than the ${ }^{13} \mathrm{C}$ magnetization so cross polarization will cause the ${ }^{13} \mathrm{C}$ magnetization to increase.

In the $\mathrm{CP}$ process, the proton spins are irradiated during acquisition to provide dipolar decoupling. The combined $\mathrm{CP} /$ dipolar decoupling sequence may be applied to a sample that is spinning at the magic angle, to give what is commonly called the CP/MAS experiment, which has wide application in the study of polymers.

Normally, the behavior of ${ }^{13} \mathrm{C}$ magnetization as a function of contact time ( $\left.\mathrm{t}_{\mathrm{cp}}\right)$ during contact in the $\mathrm{H} \rightarrow \mathrm{C}$ CP experiment is fitted to a double exponential function as follows:

$$
M_{x}(t)=A\left\{-\exp \left(-\frac{t_{c p}}{T_{H C}}\right)+\exp \left(-\frac{t_{c p}}{T_{1 p}^{H}}\right)\right\}
$$

The $\mathrm{CP}$ dynamics of the rare weak ${ }^{13} \mathrm{C}$ spin is governed by the cross polarization time constant $T_{H C}$ and the $T_{1 \rho}$ values of the strong abundant ${ }^{1} \mathrm{H}$ nucleus. When the cross polarization occurs between 2 abundant strong spins, such as ${ }^{1} \mathrm{H},{ }^{19} \mathrm{~F}$ or ${ }^{31} \mathrm{P}$, the behavior of the $\mathrm{X}$ magnetization during contact in the $\mathrm{H} \rightarrow \mathrm{X}$ CP experiment as a function of contact time is described using a similar expression to equation 1.91 (a) below: 


$$
M_{x}(t)=A\left\{-\exp \left(-\frac{t_{c p}}{T_{H X}^{\prime}}\right)+\exp \left(-\frac{t_{c \rho}}{T_{i \rho}^{*}}\right)\right\}
$$

where $T_{H F}^{*}, T_{1 \rho}^{*}$, are effective cross polarization time constant and spin-lattice relaxation time in the rotating frame, and $\mathrm{A}$ is the fitting parameter determined by the maximum signal intensity.

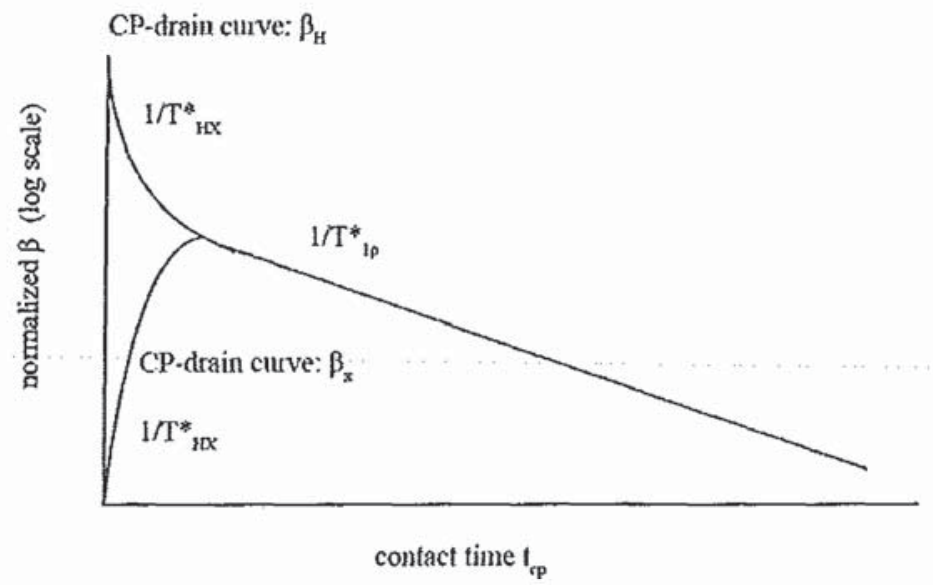

Figure 1.39: A schematic diagram of $\mathrm{H} \rightarrow \mathrm{X} \mathrm{CP}$ and $\mathrm{CP}$ drain curves

The values of $\mathrm{T}^{*}{ }_{\mathrm{HF}}, \mathrm{T}_{\mathrm{HF}}, \mathrm{T}^{*}{ }_{1 \rho}$ and $\mathrm{T}^{\mathrm{H}}{ }_{1 \rho}$ can be found using the expressions below:

$$
\begin{aligned}
& T_{H X}^{*}=\frac{T_{H X}}{a_{0}+\sqrt{a_{0}^{2}-b}}=f_{1} T_{H X} \\
& T_{1 \rho}^{*}=\frac{1}{a_{0}-\sqrt{a_{0}^{2}-b}} \frac{T_{H X}}{T_{1 \rho}^{H}} T_{1 \rho}^{H}=f_{2} T_{1 \rho}^{H}
\end{aligned}
$$

where

$$
\mathrm{a}_{0}=\frac{1}{2}\left(1+\varepsilon+\frac{\mathrm{T}_{\mathrm{HX}}}{\mathrm{T}_{1 \rho}^{\mathrm{H}}}+\frac{\mathrm{T}_{\mathrm{HX}}}{\mathrm{T}_{1 \rho}^{\mathrm{X}}}\right)
$$

And

$$
\mathrm{b}=\frac{\mathrm{T}_{H \mathrm{X}}}{\mathrm{T}_{1 \rho}^{\mathrm{H}}}\left(1+\frac{\mathrm{T}_{\mathrm{HX}}}{\mathrm{T}_{1 \rho}^{\mathrm{X}}}\right)+\varepsilon \frac{\mathrm{T}_{\mathrm{HX}}}{\mathrm{T}_{1 \rho}^{\mathrm{X}}}
$$

The value of $f_{1}$ and $f_{2}$ can be calculated using three parameter, $\varepsilon, T_{H X} / T^{X}{ }_{1 \rho}, T_{H X} / T^{H_{1 \rho}}{ }^{23}$. 
The cross polarization time constant is determined by the dipolar coupling between the two nuclei involved, which is a function of the internuclear distance, and is furthermore scaled by relative motion. Therefore the local internuclear environment can be probed for distance and motional information using cross polarization analysis supplementing dynamics information obtained from relaxation analysis. 


\section{References}

1. Bloch, F.; Hansen, W. W.; Packard, M., Physical Review 1946, 69: 127-127.

2. Purcell, E. M.; Torrey, H. C.; Pound, R. V., Physical Review 1946, 69: 37-38.

3. Ashbrook, S. E., Physical Chemistry Chemical Physics 2009, 11: 6892-6905.

4. Geppi, M.; Borsacchi, S.; Mollica, G.; Veracini, C. A., Applied Spectroscopy Review 2009, 44: 1-89.

5. Lesage, A., Physical Chemistry Chemical Physics 2009, 11: 6876-6891.

6. Widdifield, C. M.; Chapman, R. P.; Bryce, D. L., Annual Reports on Nmr Spectroscopy 2009, 66: 195-326.

7. Judge, P. J.; Watts, A., Current Opinion in Chemical Biology 2011, 15: 690-695.

8. Ketchem, R. R.; Hu, W.; Cross, T. A., Science 1993, 261: 1457-1460.

9. Schmidt-Rohr, K.; Spiess, H. W. Multidimensional solid-state NMR and Polymersm 1994.

10. Fitzgerald, J. J. Solid State NMR Spectroscopy of Inorganic Material. 1999, 717: $2-133$.

11. Smith, L. J.; Zanotti, J. M.; Sandi, G.; Carrado, K. A., Materials Research Society Symposium Proceeding 2003, 756.

12. Levitt, M. H., John Wiley and Sons: Chinchester, 2008.

13. Keeler, J., Understanding NMR Spectroscopy. University of Cambridge: Cambridge, 2005.

14. Duer, M. J., Solid-State NMR Spectroscopy Principal and Applications. University of Cambridge: Cambridge, 2002.

15. Levitt, M. H., Spin Dynamics: Basics of Nuclear Magnetic Resonance Second edition University of Southampton: UK, 2008.

16. Andrew, E. R.; Bradbury, A.; Eades, R. G., Natural 1958, 182: 1659

17. Lowe, I. J., Physical Review letters 1959, 2: 285-285

18. Andrew, E. R.; Bradbury, A.; Eades, R. G., Natural 1959, 183: 1802-1803

19. Hennel, J. H.; Klinowski, J., New techniques in Solid-state NMR. 2005.

20. Aue, W. P.; Bartholdi, E.; Ernst, R. R., Journal of Chemistry Physics 1976, 64: 2229-2246

21. Martin, G. E.; Zekter, A. S., Two-Dimensional NMR methods for Establishing Molecular Connectivity. VCH Publisher, INC: New York, 1988.

22. Bodenhausen, G.; Ruben, D. J., Chemical Physics Letters 1980, 69: 185-289

23 Shinji, A.; Robin, K. H.; Stefan, A. R., Magnetic Resonance in Chemistry 2002, 40: 97-106. 


\section{CHAPTER 2}

\section{POLYPHOSPHAZENE}

\subsection{INTRODUCTION}

Polyphosphazenes (phosphontrile-based polymer) are the most interesting and commercially promising groups of inorganic polymer and are by far the largest class of inorganic macromolecules. At least 700 different polymers in this class have been synthesized $^{1}$; comprising a broad range of cyclic or linear small-molecules and high molecular weight polymers, with a broad range of physical and chemical properties. Most polyphosphazenes have the general molecular structure shown in Figure $2.1^{2}$.

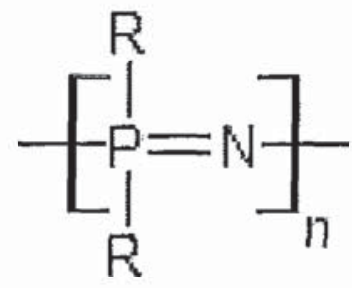

Figure 2.1: $\quad$ Polyphosphazene molecular structure

The polymer backbone consists of alternating phosphorus and nitrogen atoms, with two side groups, $R_{1}$, and $R_{2}$ being attached to the phosphorus. No substituents are present on the nitrogen atom, providing unsaturation within the skeletal structure. Cis- and transconformers both exist and the barrier to rotation about the P-N bond is very small. Although theories have been discussed since $1960^{3 \cdot 6}$, the bonding in phosphazene is still not well understood.

Despite unsaturation within the backbone, polyphosphazenes generally have low glass transition temperatures due to the bonding characteristics. The $d_{R}(P)-p_{\pi}(N)$ bond in the polyphosphazene backbone is believed to lower the barrier to rotation due to the spatial diffusion of the d-orbital and the ability of the nitrogen $p_{z}$ orbital to interact favorably with 
several different phosphorus $3 \mathrm{~d}$ orbitals as the bond undergoes torsion ${ }^{3}$.

Polyphosphazenes are synthesized in most case from a reactive macromolecular intermediate, poly(dichlorophosphazene), by substitution of the chlorine atoms with soluble nucleophiles. The most important feature of polyphosphazene chemistry is the unusual method of synthesis that allows side groups, $R_{1}, R_{2}$ to be varied over a very broad range, as shown in Figure 2.2 .

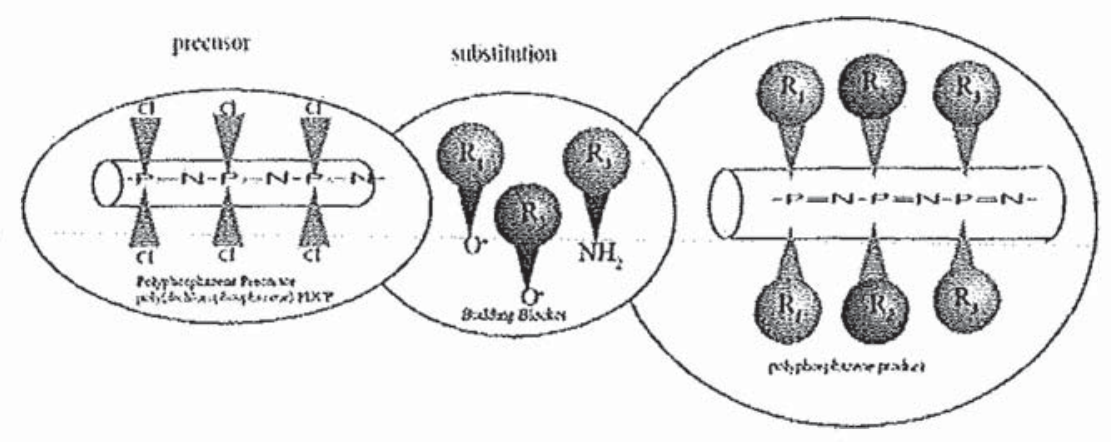

Figure 2.2: Side group substitution of poly(dichlorophosphazene)

A wide range of $\mathrm{R}$ groups are available, such as alkoxy, aryloxy, alkyl, aryl, amine, carboranyl, metallocenyl, or organosilicon groups ${ }^{7}$. Different side groups generate tunable properties making polyphosphazene suitable for many applications.

The wide ranges of unique properties that can be generated from the side groups have made these polymers the focus of interest for many advanced applications, especially when no alternating materials exist with the required combinations of characteristics. The applications of polyphosphazenes include several promising areas of research, such as ion conductive membranes for rechargeable polymer-based lithium batteries ${ }^{8}$, fuel cell membranes ${ }^{9}$, advanced elastomers for applications in the aerospace engineering ${ }^{10}$, photonic materials $^{11}$, and fire-resistant materials ${ }^{12}$. 


\subsection{HISTORY OF POLYPHOSPHAZENE}

The first report of small molecular weight phosphazenes can be traced to 1834 when Liebig $^{13}$ and Rose $^{14}$ independently reported that phosphorus pentachloride react with ammonia. The empirical formula $\left(\mathrm{NPCl}_{2}\right)$ of the resulting compound was confirmed by Gerhard $^{15}$, Laurent ${ }^{16}$, Gladstone and Holmes ${ }^{17}$, and Wichelhaus ${ }^{18}$. A major contribution was made by Stokes, who proposed the trimer cyclic structure of $\left(\mathrm{NPCl}_{2}\right)_{3}{ }^{19-22}$. Preparation protocols for cyclic oligomers were improved by Schenk and Römer ${ }^{23}$ in 1924 by conducting a reaction between phosphorus pentachloride and ammonium chloride in high boiling solvents, yielding the cyclic trimer $\left(\mathrm{NPCl}_{2}\right)_{3}$ and tetramer $\left(\mathrm{NPCl}_{2}\right)_{4}$ as well as small amounts of higher oligomers as shown in Figure 2.3.

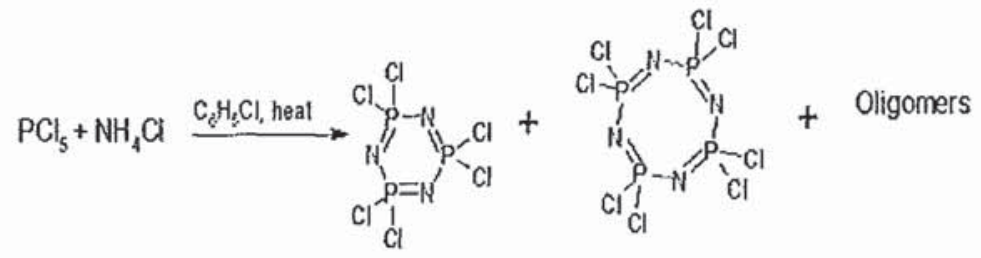

Figure 2.3: $\quad$ Synthesis of chlorophosphazene

Very little interest was paid to these materials the next 6 decades, mainly due to their insolubility in most solvents and the fact that they are hydrolytically unstable. Furthermore, the hydrolysis of the polymer releases phosphate, ammonia, and hydrochloride acid when exposed to the atmosphere. The first soluble linear poly(dichlorophophazene) polymer was obtained in 1965 by Allcock ${ }^{24}$, using thermal ring opening polymerization when melting highly purified $\mathrm{N}_{3} \mathrm{P}_{3} \mathrm{Cl}_{6}$ at $250{ }^{\circ} \mathrm{C}$ in a sealed evacuated glass tube, and many classes of polyorganophosphazene were made via nucleophilic substitution of the chlorines with organic nucleophiles, such as sodium salts of alcohols or phenols, or with primary or secondary amines ${ }^{24-26}$, as presented in Figure 2.4. 


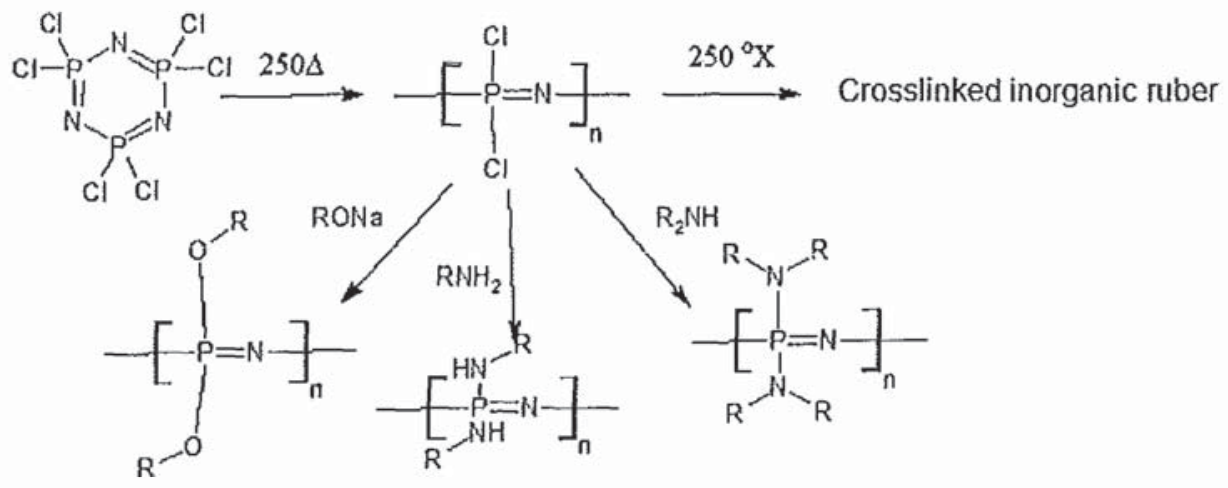

Figure 2.4: Synthesis of Polyphosphazenes

Polymer properties, such as glass transition and melting temperatures, polymer solubility and surface properties, can vary based on differences in side groups.

\subsection{SYNTHETIC ROUTES FOR POLYPHOSPHAZENE}

\subsubsection{Ring opening polymerization (ROP)}

ROP substitution methods seem to be the most popularly used route for polyphosphazene preparation. Allcock and co-workers first discovered that the cyclic trimer $\left(\mathrm{N}_{3} \mathrm{P}_{3} \mathrm{Cl}_{6}\right)$ can be thermally opened to yield high molecular weight, soluble poly(dichlorophosphazene); the trimer is synthesized from phosphorus pentachloride and ammonium chloride in an organic solvent such as chlorobenzene. The resulting compound, after careful purification and protection from moisture, was heated to molten state in a sealed glass tube at temperatures between $210^{\circ} \mathrm{C}$ to $250^{\circ} \mathrm{C}^{25.28}$, to induce polymerization (see Figure 2.5). Between $230^{\circ} \mathrm{C}$ and $300^{\circ} \mathrm{C}$, as the temperature increases, the rate of polymerization increases, the rate of crosslinking formation also increases. Certain additives or impurities, such as water, $\mathrm{BCl}_{3}$, or $\mathrm{AlCl}_{3}$ often exert a catalytic effect on the polymerization process ${ }^{29-30}$ by extracting chloride from phosphorus. Unfortunately, crosslinking can also be accelerated by the presence of these or other impurities. As the 
polymerization process continues, the polymerization mixture becomes more viscous. The reaction can be quenched after the contents become so viscous that little or no flow occurs by cooling the reaction tube to below $200^{\circ} \mathrm{C}$. Depending on different reaction environment, such as the purity of the trimer, polymerization temperature, and reaction time, up to $75 \%$ yields may be obtained.

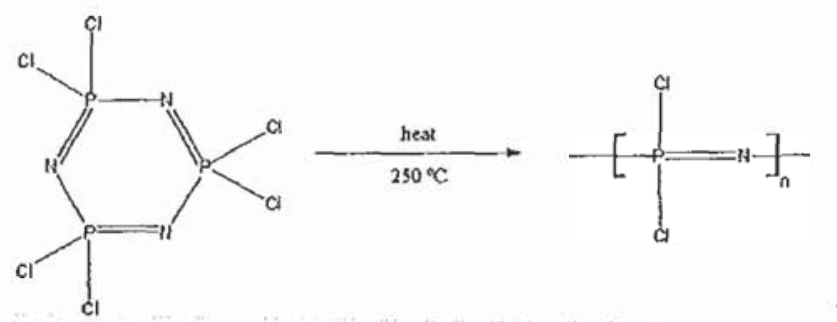

Figure 2.5: ROP method for synthesis poly(dichlorophosphazene)

Once prepared, poly(dichlorophosphazene) has a high solubility in several organic solvents. This species have become a prime candidate for chloride replacement, for the reasons of high solubility and high reactivity of the polar $\mathrm{P}-\mathrm{Cl}$ bond, to produce a variety of stable poly(organophosphazenes) (Figure 2.4). Preparing copolymer with two or more different side groups on the same backbone is also possible (Figure 2.6).

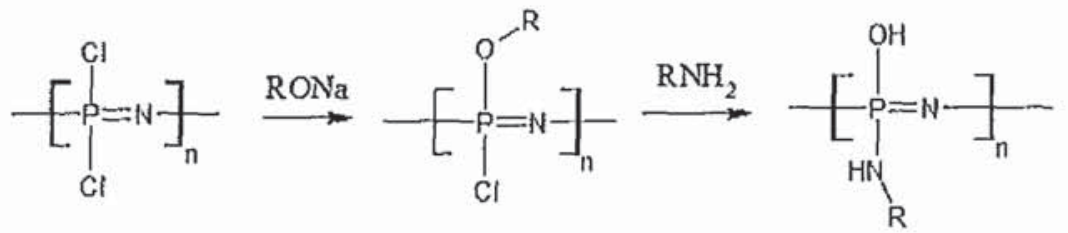

Figure 2.6: Polyphosphazene with two different side groups

To summarize, the synthesis of polyphosphazene often involves a ring opening polymerization of the cyclic trimer followed by direct nucleophilic substitution of the chloride atoms. This approach is at present the most extensively developed route to polyphosphazene polymer formation, and is very versatile in the preparation of alkoxy, aryloxy, amino and partially alkyl/aryl-substituted polymers. 


\subsubsection{Condensation Polymerization}

The methods discussed above are processes that produce reactive intermediates which undergo nucleophilic substitution to yield stable products. However, it is also possible to utilize one of the most commonly used polymerization reactions, condensation. As we mentioned above, one type of condensation reaction forms the cyclic trimer $\left(\mathrm{NPCl}_{2}\right)_{3}$. Phosphorus pentachloride and ammonia or ammonium chloride react in stepwise order, as shown in Figure 2.7, to form a monomer, then a dimer, trimer, tetramer and so on by eliminating hydrogen chloride each time. Cyclization could happen to give a cyclic structure at any step beyond the dimer, but in practice is most likely to occur before the chains grow beyond a certain length. It has been proven by several authors that this process also produces relatively low molecular weight poly(dichlorophosphazene) ${ }^{31-34}$

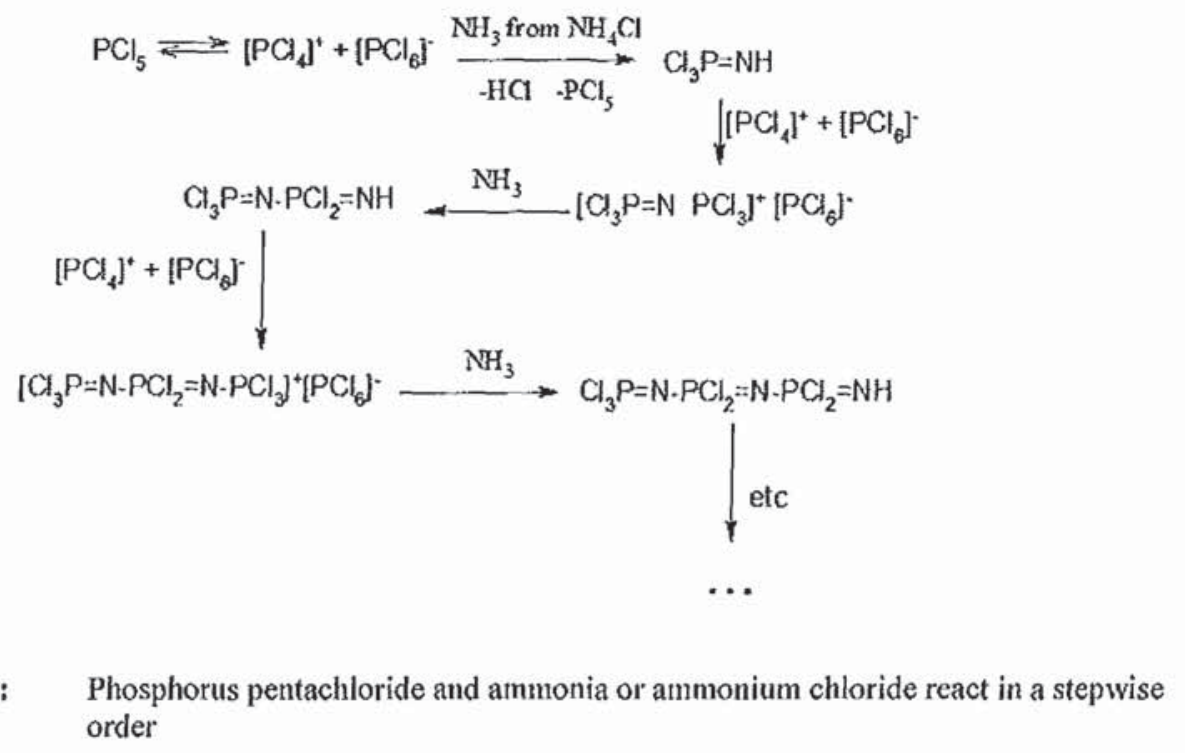




\subsubsection{Condensation Reaction of $\mathrm{OCl}_{2} \mathrm{PN}=\mathrm{PCl}_{3}$}

The commercially used condensation reaction of $\mathrm{OCl}_{2} \mathrm{PN}=\mathrm{PCl}_{3}$ was first carried out by De Jaeger and coworkers ${ }^{35-38}$. The monomer can be prepared in 3 different ways (a) from $\mathrm{PCl}_{5}$ and ammonium sulfate, (b) from phosphoroyl chloride and ammonium chloride, or (c) from $\mathrm{PCl}_{5}$ and ammonium chloride under oxidative condition ${ }^{2}$. This monomer loses phosphoroyl chloride, $\mathrm{POCl}_{3}$, under atmospheric pressure, at $240-290{ }^{\circ} \mathrm{C}$ to yield poly(dichlorophosphazene). The scheme of the overall process is presented in Figure 2.8.

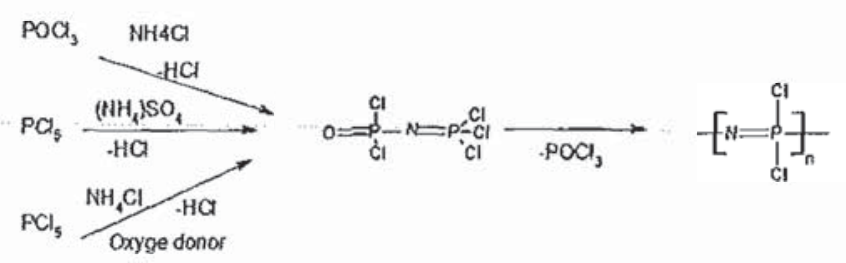

Figure 2.8: $\quad$ Overall process of Condensation reaction of $\mathrm{OCl}_{2} \mathrm{PN}=\mathrm{PCl}_{3}$

The principal advantage of this method is low cost. Disadvantages include the broad molecular weight distribution and total lower polymer molecular weight compared with products from ROP method.

\subsubsection{Living Cationic Condensation Polymerization of $\mathrm{Me} 3 \mathrm{SiN}=\mathrm{PCl}_{3}$}

At ambient temperature, living cationic polymerization of phosphoroanimine in the presence of trace Lewis acids as initiators is one of the newest techniques to prepare poly(dichlorophosphazene), and a large number of block copolymers have been developed by Allcock, Manners and their coworkers ${ }^{39-42}$ in 1995. Phosphorus pentachloride is utilized as a cationic initiator in the process to polymerize trichlorophosphoranimines $\left(\mathrm{Cl}_{3} \mathrm{P}=\mathrm{SiMe}_{3}\right)$, which was first synthesized by Niecke and Bitter from $\mathrm{PCl}_{5}$ and $\mathrm{LiN}\left(\mathrm{SiMe}_{3}\right)_{2}$ in $1973^{43}$ at $10^{\circ} \mathrm{C}$ with $20 \%$ yield, which was improved to $60 \%$ by Manners 
and coworkers by carrying out the reaction at $-78^{\circ} \mathrm{C}^{44}$. Motivated by the Wisian-Neilson approach concerning about phosphoranimines $\mathrm{BrR}_{2} \mathrm{P}=\mathrm{NSiMe}$ which itself was prepared via halogenation of a phosphorus (III) precursor, $\mathrm{R}_{2} \mathrm{PN}\left(\mathrm{SiMe}_{3}\right)_{2}$, using bromine ${ }^{45}$, Manners and coworkers discovered a new method for preparing $\mathrm{Cl}_{3} \mathrm{P}=\mathrm{NSiMe} \mathrm{N}_{3}$ using $\mathrm{PCl}_{3}$ and $\mathrm{SO}_{2} \mathrm{Cl}_{2}{ }^{46}$ (Figure 2.9). All three synthetic methods for $\mathrm{Cl}_{3} \mathrm{P}=\mathrm{NSiMe}_{3}$ are summarized in Figure 2.10.

$$
\begin{aligned}
& \mathrm{LiN}\left(\mathrm{SiMe}_{3}\right)_{2}+\mathrm{PCl}_{3} \stackrel{\mathrm{Et}_{2} \mathrm{O}}{\longrightarrow} \mathrm{Cl}_{2}-\mathrm{N}\left(\mathrm{SiMe}_{3}\right)_{2}+\mathrm{LiCl} \\
& \left.\mathrm{Cl}_{2}-\mathrm{N}_{2} \mathrm{SiHe}_{3}\right\}_{2}+\mathrm{SO}_{2} \mathrm{Cl}_{2} \underset{\underset{-\mathrm{ClSiMe}_{3}}{\stackrel{0^{\circ} \mathrm{C}}{\longrightarrow}}}{\stackrel{\mathrm{Et}_{2} \mathrm{O}}{\longrightarrow}} \mathrm{Cl}_{3} \mathrm{P}=\mathrm{NSiMe}_{3}+\mathrm{SO}_{2} \\
& \mathrm{Cl}_{3} \mathrm{P}=\mathrm{NSiMe}_{3} \frac{2 \mathrm{~S}^{\circ} \mathrm{C}}{\mathrm{Me}_{3} \mathrm{SiC}} \quad\left[\mathrm{N}=\int_{\mathrm{Cl}}^{\mathrm{Cl}}\right]_{\mathrm{n}}
\end{aligned}
$$

Figure 2.9: Methods for preparing $\mathrm{Cl}_{3} \mathrm{P}=\mathrm{NSiMe}_{3}$ using $\mathrm{PCl}_{3}$ and $\mathrm{SO}_{2} \mathrm{Cl}_{2}$

The initiation polymerization reaction involves the interaction of one molecule of monomer with two phosphorus pentachloride molecules. Cationic chain propagation continues until all the trichlorophosphoranimines monomer are reacted. Further additions of trichlorophosphoranimines to the propagating ionic species eventually lead to a living poly(dichlorophosphazene) chain (Figure 2.11).

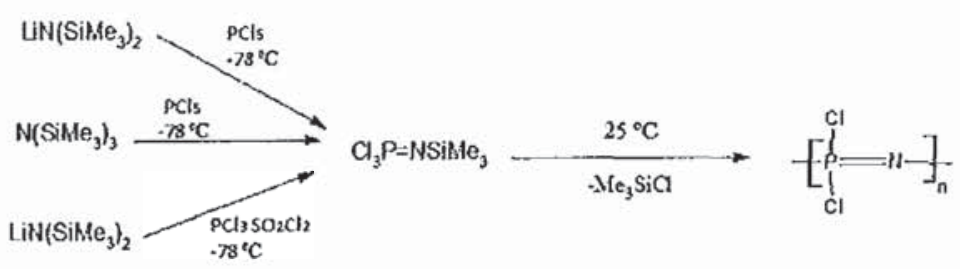

Figure 2.10: different routes for synthesis $\mathrm{Cl}_{3} \mathrm{P}=\mathrm{NSiMe}_{3}$ 


$$
\begin{aligned}
& \text { Initiation reaction } \quad 2 \mathrm{PCl}_{5} \\
& \mathrm{Cl}_{3} \mathrm{P}=\mathrm{NSiMe}_{3} \frac{\mathrm{CH}_{2} \mathrm{Cl}_{2}}{\mathrm{RT}} \rightarrow \mathrm{Cl}_{3} \mathrm{P}=\mathrm{NPCl}_{3}{ }_{3} \mathrm{PCO}^{\mathrm{R}}=\mathrm{NSIMC}_{3} \\
& \mathrm{Cl}_{3} \mathrm{P}=\mathrm{N} \cdot \mathrm{PCl}_{3} \cdot \mathrm{PCl}_{6} \cdot \frac{\mathrm{CH}_{2} \mathrm{Cl}_{2}}{\mathrm{RT}}-\mathrm{Cl}_{3} \mathrm{P}=\mathrm{NPCl}_{2} \cdot \mathrm{PCl}_{3} \cdot \mathrm{PCl}_{6} \cdot
\end{aligned}
$$

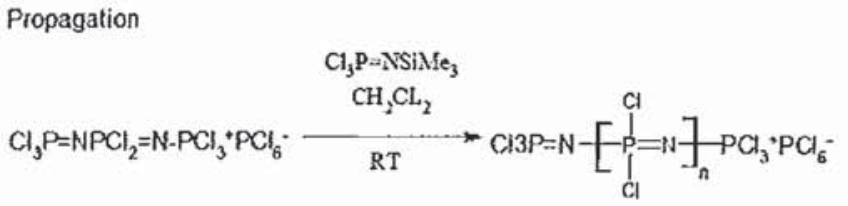

Figure 2.11: Cationic polymerization of trichlorophosphoranimines

The living cationic chain ends produced may be terminated by addition of a phosphoranimine that bears a functional group. The living nature of this polymerization allows the synthesis of block copolymers with other phosphazenes, with organic polymers, or silicones.

Physical and chemical properties and molecular weight are influenced by reaction condition, such as solvent, concentration, and monomer to initiator ratio. This route overcomes many drawbacks of other processes, and can yield products with a very narrow molecular weight distribution $(\mathrm{Mw} / \mathrm{Mn}=1.02-1.06)$ compared with $(\sim 2)$ from ROP route, with higher total molecular weight, providing access to block copolymerization ${ }^{47}$, and makes easier control of chain length.

\subsubsection{Preparation of Polyphosphazene by Ring Opening Polymerization Process of Substituted or Partially Substituted Cyclophosphazene}

Organometallic macromolecular substitution reactions allow the introduction of alkyl, aryl, or organometallic groups, with organic side groups linked to the skeleton through carbon-phosphorus bonds. However, this route is at the expense of possible backbone cleavage or incomplete halogen substitution. 
Several authors have considered the thermally induced polymerization reaction of partially or completely substituted cyclophosphazene. This is because it is much easier to prepare an organo-substituted small-molecule cyclic trimer phosphazene by organometallic substitution than it is to prepare the analogous high polymers, and the lack of any required additional macromolecular substitution process on the poly(organophosphazene) obtained by the ROP process of fully saturated trimer.

This approach involves the introduction of organic (or organometallic) side groups at the cyclic trimer level, followed by ring-opening polymerization of the substituted cyclic trimer to the high polymer. Substituent groups such as alkyl- ${ }^{48-49}$, aryl $-^{50}$, trifluoroethoxy ${ }^{51}$, phenoxy $^{52}$, organosilicon ${ }^{53}$, carboranyl ${ }^{54}$, ruthenocenyl, and ferrocenyl groups for cyclophosphazene have been studied in great detail.

The investigation of this method showed that the polymerization behavior of the phosphazene ring depends greatly on the number of halogen atoms and the number of organic side groups.

In general terms it appears that:

a. The higher the number of organic groups inserted onto the cyclophosphazene, the lower is the probability of a polymerization process and the higher is the possibility that a ring-expansion reaction takes place instead

b. The greater the bulkiness of the substituent organic groups on the trimer, the higher is the probability of a ring-expansion polymerization process

c. The presence of non-geminal substituent groups on the trimer favors polymerization processes, while geminal substitution may favor side reactions

d. Substituent groups on the cyclophosphazene that introduce strained structures or ring 
distortions favor polymerization processes

e. Cyclophosphazenes are not able to polymerize thermally by themselves for any reason may open their cycle and form polymer chains during thermal treatments in the presence of variable amounts of $\mathrm{N}_{3} \mathrm{P}_{3} \mathrm{Cl}_{6}$

f. Hexa-substituted cyclophosphazenes can polymerize under particular conditions to the corresponding fully substituted polyphosphazenes, even though this event is rather unusual

g. Thermal and/or chemical instability of the cyclophosphazene side substituents usually prevent both polymerization and ring-expansion processes from taking place, favoring the onset of decomposition phenomena

h. The presence of chlorine instead of fluorine atoms in the cyclophosphazene usually depress the polymerization temperature of the cyclophosphazene

\subsubsection{Condensation Polymerization of Organic Substitution Phosphoroanimines}

The first organo-phosphoranimine polymerization was reported by Flindt, Rose, and Marsmann ${ }^{55}$ in 1977 that tris(trifluoroethoxy) $\mathrm{N}$-(trimethylsily) phosphoranimine could be polymerized at $200{ }^{\circ} \mathrm{C}$ to produce poly[bis(trifluoroethoxy)phosphazene] (M $\mathbf{M}_{w} 4000$ 10,000). In 1980s, Wisian-Neilson, Neilson, and their coworkers showed that a variety of alky, aryl and alkyl/aryl phosphazene polymer and copolymers ${ }^{56-61}$ could be prepared in the same way, later refined by Matyjaszewski and coworkers ${ }^{62-65}$.

Finally, in 1995, I. Manners and H. R. Allcock ${ }^{66.68}$ put forward the room temperature polymerization approach allowing the cationic polymerization of variety of substituted phosphoroanimines, as shown in Figure 2.12. 


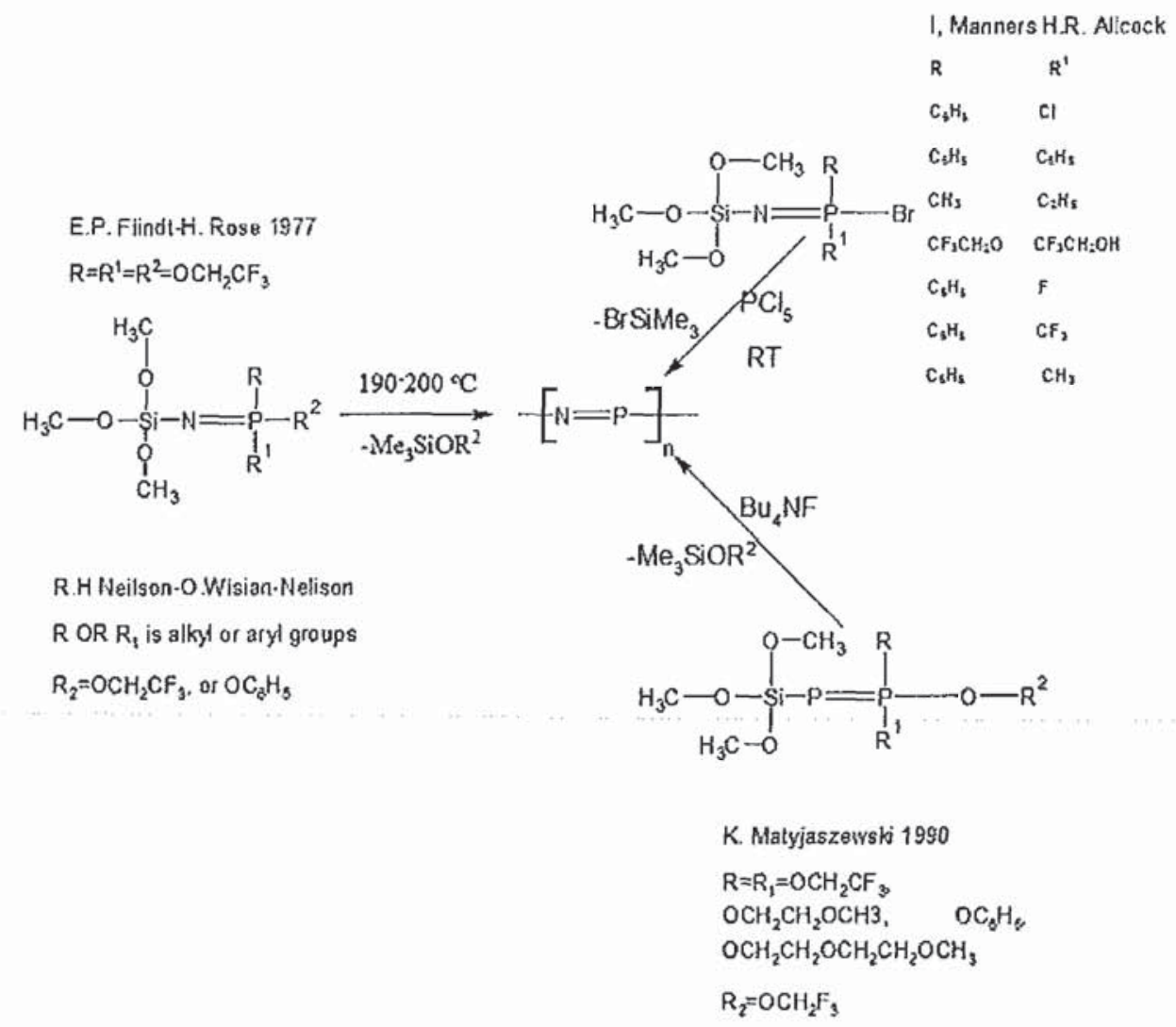

Figure 2.12: polymerization of variety of organophosphoranimine

The experimental approaches for preparation polyphosphazene are very numerous and give access to phosphazene polymer with different and structural properties, and these materials can find different potential practical applications. Polyphosphazene can be considered as one of the most important classed of macromolecules ever invented in human history.

\subsection{POLYPHOSPHAZENE PROPERTIES}

\subsubsection{Skeleton Property}

To understand the properties of polyphosphazene, it is necessary to analyze the structure-property relationships of these compounds. The basic structure properties of 
polyphosphazenes are composed of two main contributions. The first one is the intrinsic properties associated with $-\mathrm{P}=\mathrm{N}$ - inorganic backbone, the second one is connected to the chemical and physical characteristic of the substituent groups.

\subsubsection{Chain Flexibility}

Macromolecular flexibility is often defined in terms of the glass transition temperature, $\mathrm{T}_{\mathrm{g}}$. A polymer with a high $\mathrm{T}_{\mathrm{g}}$ is likely to have a backbone that offers more resistance to bond torsion than a polymer with a low $\mathrm{T}_{\mathrm{g}}$.

Polyphosphazenes are highly unusual macromolecules for the reason that specific ones have some of the lowest $\mathrm{T}_{\mathrm{g}}$ known in polymer chemistry. This is basically due to the inherent features of the "island" $\mathrm{d} \pi-\mathrm{p} \pi$ bond structure in phosphazenes, which allow the permanent overlapping of the $2 \mathrm{p}_{\mathrm{z}}$ orbital of the nitrogen with any one of the $3 \mathrm{p}$ orbitals of the phosphorus atoms ${ }^{69}$. Calculations suggests that the inherent torsional barrier in the backbone may be as low as $0.1 \mathrm{kcal}$ per bond ${ }^{70}$.

\subsubsection{Thermal stability}

The phosphazene backbone $-\mathrm{P}=\mathrm{N}$ - has a particularly high resistance to thermal treatment due to the high strength of phosphazene bond and its remarkable ionic character ${ }^{71}$. As a consequence, the onset of thermal decomposition phenomena are generally observed at very high temperatures. For example, for poly[bis(trifluoroethoxy)phosphazene], for polyphosphazenes substituted with 2,2-dihydroxybiphenyl groups and for poly(alkyl/aryl) phosphazenes. 


\subsubsection{Fire and Flam Resistance Properties}

The polyphosphazene skeleton $-\mathrm{N}=\mathrm{P}$ - has the ability to resist fire and combustion due to the inorganic elements that constitute of its structure ${ }^{72-74}$. The intrinsic limiting oxygen index (LOI) for certain polyphosphazenes could be up to about 60 , but will decrease considerably as the organic compound content in the side groups is enhanced ${ }^{72-73}$. However, the LOI can be further enhanced by inserting additional inorganic elements $(\mathrm{F}$, $\mathrm{Cl}, \mathrm{Br}, \mathrm{B}$, metals, etc.) in the substituent groups.

\subsection{APPLICATIONS OF POLYPHOSPHAZENES}

Applications based on structure-property correlations in the technological or medical industries are as follows:

\subsubsection{Polyphosphazenes as Ionic Conductor}

Polyphosphazenes with oligoethyleneoxy side groups have received considerable attention because of their potential for lithium ion conduction ${ }^{75}$, because they have high side chain flexibility and their ability to coordinate weakly to lithium and other monovalent ions $^{76}$. One of the most extensively studied examples of this group is poly[bis(methoxyethoxyethoxy)phosphazene], (MEEP). MEEP has been applied as a solid solvent for lithium triflate in lithium battery.

Aryloxyphosphazenes with acidic functional groups on the aryl ring, are examples of polyphosphazenes that function as excellent proton conductors, since their performance is comparable to that of Nafion and they have higher resistance to methanol crossover. 


\subsubsection{Polyphosphazene Biomaterials}

Polyphosphazenes can be considered as "biomaterials" under certain types of utilization. A significant advantage of polyphosphazenes is the ease to change the types of side groups to tune any combination of properties needed for a specific biomedical application.

Water-soluble polyphosphazenes have been extensively investigated because of their potential application for transporting molecules of therapeutic relevance in vivo. Some water-soluble polyphosphazenes have been used to prepare hydrogels by controlled reticulation under a variety of conditions in tissue engineering or in drug delivery. For example, water-soluble polyphosphazenes containing pendant imidazolyl groups were reported being able to complex $\mathrm{Fe}(\mathrm{II})$ and $\mathrm{Fe}$ (III) porphyrins in an attempt to produce synthetic analogues of myoglobins and hemoglobins ${ }^{77.78}$. Bioerodible, poly[bis(methylamino)phosphazenes] have been radiatively cross-linked to prepare phosphazene based membranes ${ }^{79}$.

Another polyphosphazene-based drug delivery system deals with hydrolytically unstable substrates, which are able to degrade in a controlled way under physiological conditions in human body to attain slow and controlled drug delivery, and one of the common examples is polyphosphazene matrices with amino acid esters or imidazole groups, which are both of great importance in tissue engineering and in bone regeneration ${ }^{80}$, treatment of periodontal disease ${ }^{81}$ and nerve reconstruction ${ }^{82-83}$. Another example is poly[bis(4-carboxylatophenoxy)phosphazene] which is used in preparation composites for bone or tooth regeneration ${ }^{84}$ and as a micro-encapsulant of drugs ${ }^{85-86}$, vaccines ${ }^{87}$, and proteins $^{88}$. 
The third aspect of polyphosphazenes used as biomaterials concerns surface property implication. The modification of surface properties can be achieved in different ways; some of most important types of modifications are reported in Table 2.1.

Table 2.1. examples of some types of polyphosphazene surface modification

\begin{tabular}{|c|c|c|}
\hline $\begin{array}{c}\text { Phosphazene } \\
\text { Substituent groups }\end{array}$ & $\begin{array}{c}\text { Substituent } \\
\text { Modification Reaction }\end{array}$ & $\begin{array}{l}\text { Features of the Resulting } \\
\text { Polymer Film Surfaces }\end{array}$ \\
\hline $\mathrm{P}-\mathrm{OCH}_{2} \mathrm{CF}_{3}$ & $\mathrm{P}-\mathrm{OH}$ or $\mathrm{P}-\mathrm{O}^{-} \mathrm{NBu}_{4}{ }^{+}$ & $\begin{array}{l}\text { Hydrophilic surface with } \\
\text { enhanced biocompatibility } \\
\text { and adhesion properties }\end{array}$ \\
\hline $\mathrm{P}-\mathrm{O}-\mathrm{C}_{6} \mathrm{H}_{5}$ & $\mathrm{P}-\mathrm{O}-\mathrm{C}_{6} \mathrm{H}_{4}-\mathrm{SO}_{3} \mathrm{H}$ & $\begin{array}{l}\text { Surface hydrophilicity and } \\
\text { surface hydrogels }\end{array}$ \\
\hline \multirow[b]{2}{*}{$\mathrm{P}-\mathrm{O}-\mathrm{C}_{6} \mathrm{H}_{5}$} & $\mathrm{P}-\mathrm{O}-\mathrm{C}_{6} \mathrm{H}_{4}-\mathrm{NO}_{2}$ & \multirow{2}{*}{$\begin{array}{l}\text { Hydrophilic surface suitable } \\
\text { for additional } \\
\text { functionalization reaction }\end{array}$} \\
\hline & $\mathrm{P}-\mathrm{O}-\mathrm{C} 6 \mathrm{H} 4-\mathrm{NH} 2$ & \\
\hline \multirow{2}{*}{ P-O- $\mathrm{C}_{6} \mathrm{H}_{4}-\mathrm{CH}_{3}$} & $\mathrm{P}-\mathrm{O}-\mathrm{C}_{6} \mathrm{H}_{4}-\mathrm{COOH}$ & \multirow{2}{*}{ Antithrombogenic surfaces } \\
\hline & $\mathrm{P}-\mathrm{O}-\mathrm{C}_{6} \mathrm{H}_{4}-\mathrm{CH}_{2} \mathrm{NEt}_{3}^{+}$ & \\
\hline
\end{tabular}

Polyphosphazenes can also be modified very easily by grafting organic polymers onto their surfaces using chemical, photochemical or $\gamma$-radiolytic processes, leading to an increase in the surface hydrophilicity and biocompatibility of the phosphazene films without destroying their bulk features. For example, MEEP can be grafted onto the surface if many biorelevent outstanding organic macromolecules using $\gamma$-rays to decrease their surface hydrophobicity.

\subsubsection{Optical and Photonic Polymers}

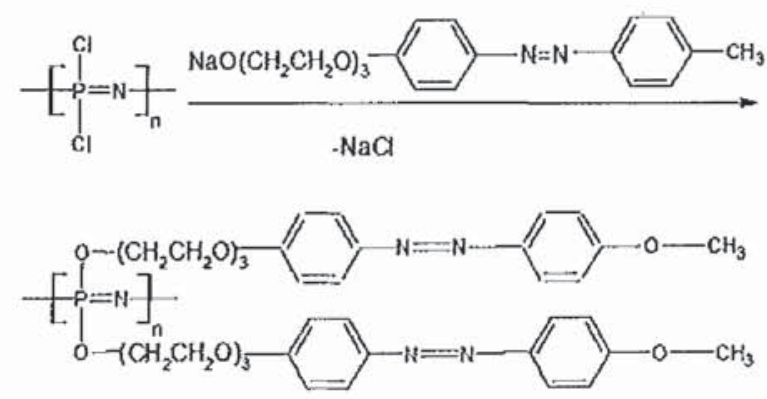

Figure 2.13: An example of a liquid-crystalline polyphosphazene reaction process 
Polyphosphazenes with large, rigid aryl side groups, have potential for use as liquid crystalline materials, as non-linear optical polymers, and as corrective lenses ${ }^{2}$. One example of a liquid crystalline polyphosphazene is illustrated in Figure: $2.13^{89-92}$. Heattreatment of this orange polymer across its glass transition temperature $\left(\mathrm{T}_{\mathrm{g}}\right)$ at $79^{\circ} \mathrm{C}$ makes it change from a glassy to a rubbery microcrystalline material.

The polyphosphazene shown in Figure 2.13 will undergo aromatic side chain reorientation when the films are heated above $\mathrm{T}_{\mathrm{g}}$, and subjected to a kilovolt-level electric field. The reorientation is in the direction of the field. Such a film will double the frequency of a laser beam that passes through it, this effect is almost as large as an inorganic NLO crystal $^{2}$. Experiments show that polyphosphazenes with higher $T_{\mathrm{g}}$ will retain this phenomenon for a longer period of time, but it will eventually lost as the side groups lose preferential reorientation due to thermal motion.

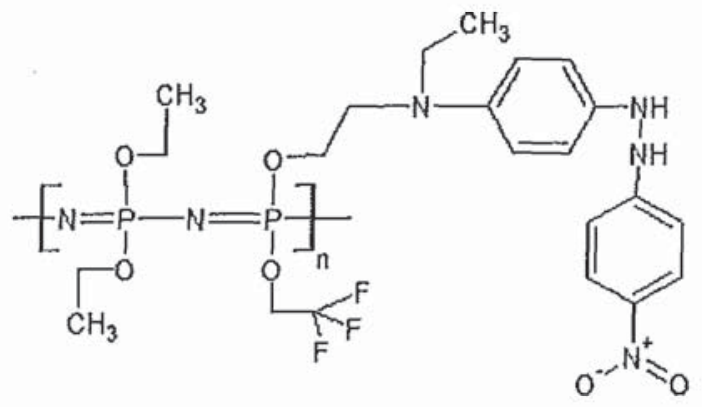

Figure 2.14: An example of polyphosphazene with controlled refractive index

Polyphosphazenes with electron-rich side groups, such as multi-aromatic side units or with halogen atoms in the side groups (see Figure 2.14), offer properties of controlled refractive index, and especially as high refractive lenses coupled with low optical dispersion. Some of the latest work indicates that polyphosphazenes that contain both fluoroalkoxy and di- or tri- chlorophenoxy side groups ${ }^{93}$ show a large variation of refractive index with changes in temperature, the refractive index vary from 1.39-1.56 depending on 
composition, making them candidates for thermo-optical switching devices.

\subsubsection{Advanced Elastomers}

Polyphosphazenes have single atoms (such as, $\mathrm{F}, \mathrm{Cl}$ ), or organic side groups ate sufficiently flexible, or have two or more different alkoxy, aryloxy, or organosilicon unit as the side groups will give rise to rubbery or elastomeric properties.

\subsection{POLY[BIS(TRIFLUOROETHOXY)PHOSPHAZENE}

This thesis deals with poly(bis(trifluoroethoxy)phosphazene) (PBFP) ${ }^{94}$ (Figure 2.15), which can be obtained through (a) nucleophilic substitution of chlorines in the poly(dichlorophosphazene) side groups upon reaction with sodium trifluoroethoxide. (b) by heating tris(trifluoroethoxy)- $\mathrm{N}$-trimethylsilylphosphoranimine, $\quad\left(\mathrm{CF}_{3} \mathrm{CH}_{2} \mathrm{O}\right) \mathrm{P}=\mathrm{N}$ $\mathrm{Si}\left(\mathrm{CH}_{3}\right)_{3}$, at $200^{\circ} \mathrm{C}$ under vacuum, however, in some cases, in the presence of fluorinated catalysts (e.g. tetrabutylammonium fluoride), the reaction temperature can be decreased to $95^{\circ} \mathrm{C}$, (c) by reaction of hexabis(trifluoroethoxy)cyclotriphosphazene with $\mathrm{BCl}_{3}$, or (d) by living cationic polymerization of bromo-bis(trifluoroethoxy)Ntrimethylsilylphosphoranimine $\left(\mathrm{CF}_{3} \mathrm{CH}_{2} \mathrm{O}\right)_{2} \mathrm{BrP}=\mathrm{NSi}\left(\mathrm{CH}_{3}\right)_{3}$. More specifically, route (a) is by far the simplest and mostly commonly used route for synthesis of this material, as shown in Figure 2.15 .

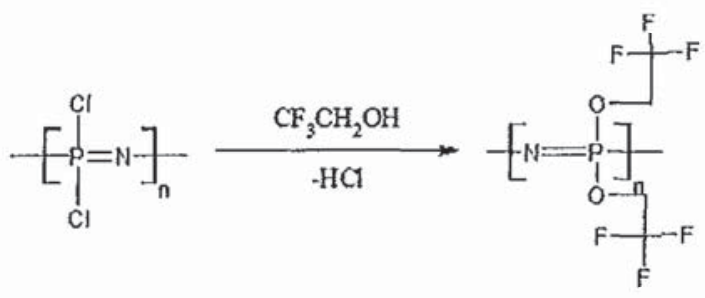

Figure 2.15: mostly common synthetic route for PBFP 
This polymer is important because it is one of the first macromolecules of this class initially synthesized by H. R. Allcock in $1965^{26,95}$, and also because of its extremely interesting properties ${ }^{96}$. PBFP has a high molecular weight polymer $\left(\mathrm{Mw}>10^{4}\right)$ with a large polydispersity ( $\mathrm{Mw} / \mathrm{Mn}>10)$, its morphologies are largely dependent on the preparation route and normally can range from white fibers to non-transparent flexible material that can form both films and fibers ${ }^{97}$. Parameters of PBFP in solution suggest a high degree of flexibility and coiling of the polymer backbone with a cis-, or trans- planar conformation. This polymer is extremely hydrophobic and chemically stable, and has been shown to be inert to alcohols, pyridine and concentrated $\mathrm{NaOH}$ solutions ${ }^{94}$, some decomposition, however, can occur in triethylamine and concentrated $\mathrm{H}_{2} \mathrm{SO}_{4}$. PBFP is soluble in many common organic solvents, such as THF, acetone, or methylehylketone. TGA studies suggest that PBFP has high thermal stability, will not volatilize below $500^{\circ} \mathrm{C}$. Tests on the flame resistance of the polymer show a Limiting Oxygen Index (LOI) of 48.0, resulting in a high resistance to combustion and it burns cleanly leaving little char or residue. PBFP is a highly versatile material that can be used for many applications, such as membranes for fuel cells, biomedical materials and passive membranes.

PBFP is a semicrystalline polymer that contains both rigid and ordered chains that make up the crystalline component, and randomly coiled and entangled chains make up for amorphous regions. This semicrystalline polymer can be characterized by the percentage of crystallinity and size and structure of the crystallite components, and combines both strength and flexibility. The boundaries between two types of components are insignificant; hence, these interfacial regions are often neglected; as illustrated in Figure 2.16. 


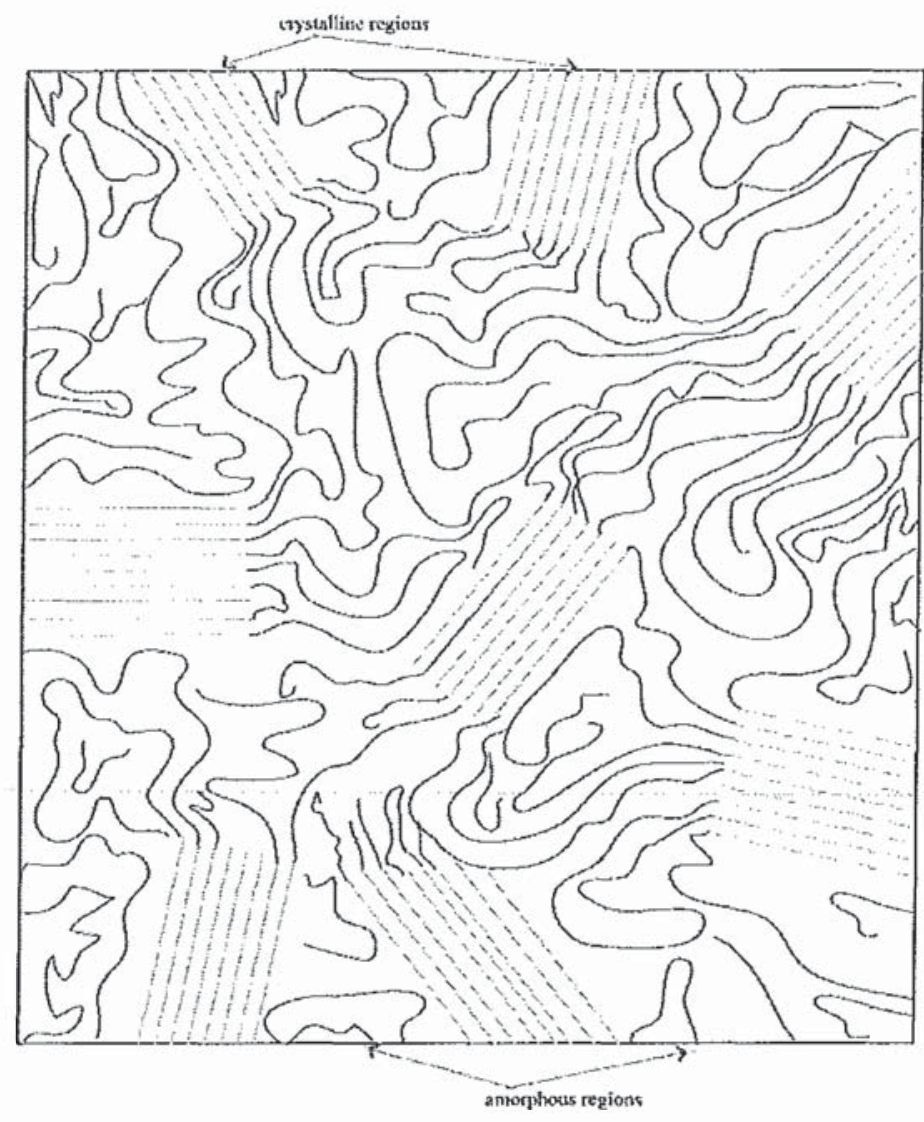

Figure 2.16: Domain structure of semi-crystalline materials

Investigations indicate that PBFP exhibits complex thermal and morphological behaviors, characterized by three transition temperatures that vary depending on the thermal history, molecular weight and crystallinity of the polymer. In semicrystalline PBFP three transitions have been established: glass transition, thermotropic (mesophase) transition and melting point, are $\mathrm{T}_{\mathrm{g}}=-66^{\circ} \mathrm{C}^{98}, \mathrm{~T}(1)=66-90^{\circ} \mathrm{C}$, and $\mathrm{T}_{\mathrm{m}} \approx 242{ }^{\circ} \mathrm{C}^{96}$, respectively.

PBFP tends to form large spherulites after solvent casting, composed of lamellae with either mostly a 3D chain folded $\alpha$-orthorhombic crystal form with unit cell dimensions corresponding to $a=10.16 \AA, b=9.35 \AA, c=4.86 \AA$ and having 2 monomer units ${ }^{99}$, a 3D chain-folded $\beta$-monoclinic form that has unit cell dimensions as $a=10.03 \AA, b=9.37 \AA, c$ 
$=4.86 \AA$ and $\gamma=0.91^{\circ}$ also being not much different from $\alpha$ cell ${ }^{99}$; or a mixture of two, depending on the initial crystallinity and polymorphism. The crystallinity of these specimens is surprisingly low $(<50 \%)$ and the crystal structure is monoclinic. Once heating through $\mathrm{T}(1)$, the spherulites are unchanged, however, PBFP transforms to a $2 \mathrm{D}$ pseudohexagonal meso-phase. In this condition, they have considerable fluidity as creep and other measurements substantiate. By keeping heating this polymer above $\mathrm{T}_{\mathrm{m}}$, the spherulitic arrangement is destroyed, and the melt polymer becomes isotropic with zero birefringence. If the temperature is cooled below $T_{m}$, the isotropic melt is reverted to a $2 \mathrm{D}$ pseudohexagonal mesophase, where needle like batonnet morphology is present. Cooling below $\mathrm{T}(1)$ leads to polymer crystallization into a chain-extended 3D $\gamma$-orthorhombic structure with unit cell dimensions as $a=20.60 \AA, b=9.40 \AA$ and $c=4.86 \AA$ and corresponding to four monomer units ${ }^{99}$, which is different from the original monoclinic form. The crystallinity of PBFP returned to room temperature is much higher than the starting material, as indicated by higher $T(1)$ value after each thermal cycling. Additional important practical applications of PBFP are:

(a). Biocompatible materials for implantation purposes, theromobio-, and biologicalresistant substrates, and as carriers for enzyme immobilization;

(b). Membranes for solvent separation, ion separation, gas permeation, and gas transportation problems; and,

(c). Hybrid materials prepared through the sol-gel technique, and

(d). Candidate electrolytes for dye sensitized solar cells 


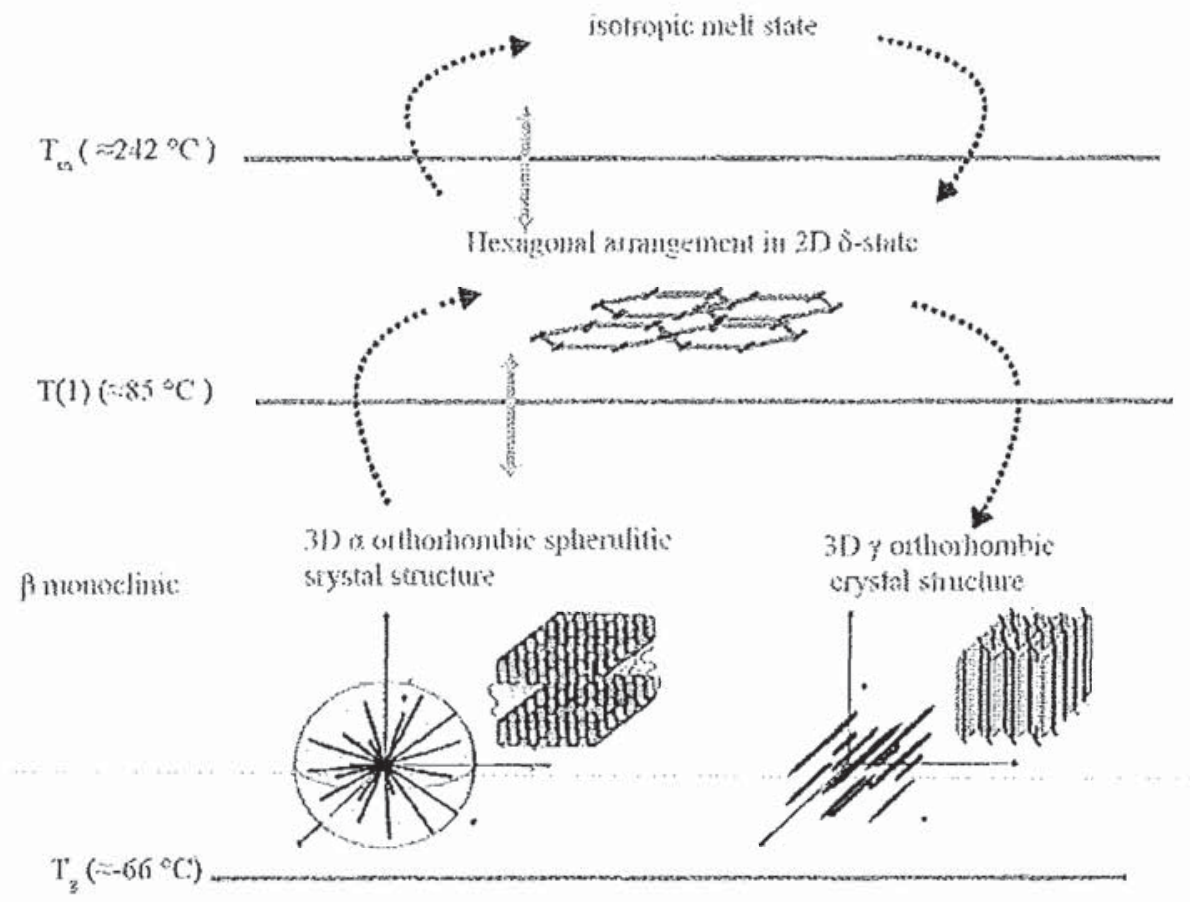

Figure 2.17: $\quad$ Phase transition diagram for solvent-cast PBFP between $T_{8}$ and $T_{m}$

\subsection{Conclusion}

The polyphosphazene system comprises some of the most diverse macromolecules discovered so far. A variety of synthetic procedures have been described based upon the ring opening polymerization process of $\left(\mathrm{NPCl}_{2}\right)_{3}$ to $\left(\mathrm{NPCl}_{2}\right)_{\mathrm{n}}$ followed by the nucleophilic replacement of the reactive chlorines with carefully selected nucleophiles, and condensation polymerization. Polyphosphazene features are a resultant combination of the inorganic backbone (-P $=\mathrm{N}-)$ and nucleophilic substituents. Thus, polyphosphazene with different side groups can be applied in energy related research, biomaterials, optical and photonic fields, and advanced elastomers. 


\section{References}

1. Allcock, H. R. Chemistry and Application of Polyphosphazene Hoboken NJ: Wiley-Interscience; 2003.

2. Mark, J. E.; Allcock, H. R.; West, R., Inorganic Polymer. Oxford Universuty Press: Oxford New York, 2005.

3. Allcock, H. R. Phosphorus-Nitrogen compounds New York: Academic press 1972.

4. Dewar, M. J. S.; Lucken, E. A. C.; Whitehead, M. J., Journal of American Chemistry Socity 1960. 2423-2429

5. $\quad$ Craig, D. P.; Paddock, N. L., Journal of Chemistry Society 1962.

6. Paddock, N. L., Quaterly Review Chemistry Society 1962, 18: 168

7. Stevens, M. P., Inorganic-Organic Polymers 1975.

8. Alamgir, M.; Reynolds, R. K.; Abraham, K. M., Journal of the Electrochemical Society 1988, 135, C342-C342.

9. Carter, R.; Evilia, R. F.; Pintauro, P. N., Journal of Physical Chemistry B 2001, 105, 2351-2355.

10. Allcock, H. R.; Nelson, J. M.; de Denus, C. R.; Manners, I., Design, synthesis, and uses of phosphazene high polymers. 2000: 165-173.

11. Allcock, H. R., Current Opinion in Solid State \& Materials Science 2006, 10, 231240.

12. Lyon, R. E.; Speitel, L.; Walters, R. N.; Crowley, S., Fire and Materials 2003, 27, 195-208.

13. Liebig, J., Annaden der chemie 1834, 11: 139

14. Rose, H., Annaden der chemie 1834, 11: 131

15. Gerhardt, C., Annales de Chimeie et de Physique 1846, 18: 188-188

16. Laurent, A., Comptes rendus de l'Académie des sciences 1850, 31: 356

17. Gladstone, J. H.; Holmes, J. D., Journal of Chemistry Science 1864, 17: 225

18. Wichelhaus, H., Chemische Berichte 1870, 163: 163

19. Stokes, H., N, American Chemistry Journal 1895, 17: 275

20. Stokes, H., N, American Chemistry Journal 1896, 18: 629

21. Stokes, H., N, Chemische Berichte 1895, 28: 437

22. Stokes, H., N, American Chemistry Journal 1897, 19: 782

23. Schenk, R.; Romer, G., Chemische Berichte 1924, 578: 1343

24. Allcock, H. R.; L., K. R., Journal of American Chemistry Society 1965, 87: 4216

25. Allcock, H. R.; Kugel, R. L.; Valan, K., Journal of Inorganic Chemistry 1966, 5: 1709

26. Allcock, H. R.; Kugel, R. L., Inorganic Chemistry 1966, 5: 1716

27. Allcock, H. R.; Kugel, R. L., Journal of American Chemistry Socity 1965, 87421: 4216-4217

28. Allcock, H. R.; Mack, D. R., Journal of Chemistry Society 1970, 11: 685

29. Allcock, H. R.; Gardner, J. E.; Smeltz, K. M., Macromolecules 1975, 8: 36-42

30. Sennett, M. S.; Hagnauer, G. L.; Singler, R. E., Polymeric Materials: Science and Engineering 1983, 49: 297-300 
31. Allen, G.; Lewis, C. J.; Todd, S. M., Polymer 11: 31-43

32. Kireev, V. V.; Lomonosov, A. V.; Skorovarov, D., I.; Filoppov, E. A. U. S. S. R., Patent SU 3859801973.

33. Kinoshita, T.; Ogata, Y.; Nishiuchi, K.; Masuda, S., Japanese Patent 80/25475 1980.

34. Hornbaker, E. D.; li, H. M., U.S. Patents 9356291980.

35. De Jaeger, R.; Helioui, M.; Puskaric, E., U.S. Patent 4,337,558 1983.

36. Helioui, M.; De, J., R.; Puskaric, E.; Heubel, J., Makromolecule Chemistry 1982, 183: 1137

37. De Jaeger, R.; Poton, P., Phosphorus, Sulfur; Silicon, and the Related Elements 1993, 76: 483

38. De Jaeger, R., NOVA Science Publishers 2003.

39. Honeyman, C. H.; Manners, I.; Morrissey, C. T.; Allcock, H. R., Journal of American Chemistry Socity 1995, 117: 7035

40. Allcock, H. R.; Crane, C. A.; Morrissey, C. T.; Nelson, J. M.; Reeves, S. D.; Honeyman, C. H.; Manners, I., Macromolecules 1996, 29: 7740-7747

41. Allcock, H. R.; Nelson, J. M.; Reeves, S. D.; Honeyman, C. H.; Manners, I., Macromolecules 1997, 30: 50-56.

42. Allcock, H. R.; Reeves, S. D.; de Denus, C. R.; Crane, C. K., Macromolecules 2001, 34: 748-754.

43. Niecke, E.; Bitter, W., Inorganic Nuclear Chemistry Letters 1973, 9: 127

44. Honeyman, C. H.; Lough, A. J.; Manners, I., Inorganic Chemistry 1994, 33: 2988

45. Wisian-Neilson, P.; Neilson, R. H., Journal of American Chemical Society 1980, 102: 2848

46. March, J., Advanced Organic Chemistry, 4th ed. Wiley-Interscience: Toronto, 1992.

47. Prange, R.; Reeves, S. D.; Allcock, H. R., Macromolecules 2000, 33: 5763

48. Prons, V. N.; Grinblat, M. P.; Klebanskii, A. L., Journal of General Chemistry USSR 1971, 41: 475

49. Ritchie, R. J.; Harris, P. J.; Alicock, H. R., Journal of the Chemical Socity, Chemical Communication 1980, 4: 198-199

50. Allcock, H. R.; Moore, G. Y., Macromolecules 1975, 8: 377-382

51. Allcock, H. R.; Schmultz, J. L.; Kosydar, K. M., Macromolecules 1978, 11: 179

52. Gibeau, T. E.; Marcus, R. K., Journal of Applied Polymer Science 2000, 76, 954 961.

53. Manners, I.; Riding, H.; Dodge, J. A.; Allcock, H. R., Journal of the American Chemical Society 1989, 111: 3067

54. Allcock, H. R.; Scopelianos, A. G.; Whittle, R. R.; Tellefson, N. M., Journal of the American Chemical Society 1983, 105: 1316.

55. Flindt, E. P.; ROSE, H., Zeitschrift für anorganische und allgemeine Chemi 1977, 428: 204

56. Wisian-Neilson, P.; Neilson, R. H., Journal of the American Chemical Society 1980, 102:2848 
57. Neilson, R. H.; Wisian-Neilson, P., Journal of Macromolecular Science Chemistry Part A 1981, 16: 425

58. Wisian-Neilson, P.; Ford, R., Phosphorus, Sulfur, Silicon, and the Related Elements 1987, 30:315

59. Gruneich, J. A.; Wisian-Neilson, P., Macromolecules 1996, 29: 5511

60. Wisian-Neilson, P.; Neilson, R. H.; Allcock, H. R., Inorganic Synthesis 1989, 25: 69

61. Wisian-Neilson, P., Contemporary Topics in Polymer Science 1992, 7: 333

62. Montague, R. A.; Matyjaszewski, K., Journal of the American Chemical Society 1990, 112: 6721

63. Matyjaszewski, K., Journal of Inorganic and Organometallic Polymers 1992, 2:

64. Matyjaszewski, K.; Cypryk, M.; Dauth, J.; Montague, R. A.; White, M., Makromol Chem Macromol Symp - Makromolekulare Chemie, Macromolecular Symposia 1992, 54/55: 13.

65. Matyjaszewski, K., Polymer 1994, 35: 5005

66. Allcock, H. R.; Nelson, J. M.; Reeves, S. D.; Honeyman, C. H.; Manners, I., Macromolecules 1997, 30: 50

67. Nelson, J. M.; Allcock, H. R.; Manners, I., Macromolecules 1997, 30: 50

68. Allcock, H. R.; de Denus, C. R.; Prange, R.; Nelson, J. M., Macromolecules 1999, 31: 7999

69. Allcock, H. R., Contemporary Topics in Polymer Science 1979, 3: 55-78

70. Singler, R. E.; Schneider, N. S.; Hagnauer, G. L., Polymer Engineering and Science 1975, 15: 321

71. Allcock, H. R., Chemistry and Application of Polyphosphazene, Wiley Interscience: Hoboken, New Jersey, 2002: P407.

72. Lawson, D. F.; Cheng, T. C., Organic Coating and Plastic Chemistry 1978, 38: 694

73. Lawson, D. F., Fire Research and Safety 1977, 1

74. Allen, C. W., Journal of Applied Fire Science 1993, 11: 320

75. Allcock, H. R.; Walters, R. N.; Laredo, E., Macromolecules 2002, 34: 787

76. Blonsky, P. M.; Shrlver, D. F.; Austin, P. E.; Allcock, H. R., Solid State Ionics 1986, 18: 258

77. Allcock, H. R.; Greigger, P. P.; Gardner, J. E.; Schmultz, J. L., Journal of the American Chemical Society 1979, 101: 606

78. Alicock, H. R.; Neenan, T. X.; Boso, B., Inorgnic Chemistry 1985, 24: 2756

79. Allcock, H. R.; Gebura, M.; Kwon, S.; Neenan, T. X., Biomaterials 1988, 9: 500

80. Laurencin, C. T.; Norman, M. E.; Elgendy, H. M.; EL-Amin, S. F.; Allcock, H. R.; Pucher, S. R.; Ambrosio, A. M. A., Journal of Biomedical Materials Ressearch 1993, 27: 963

81. Veronese, F. M.; Marsilio, f.; Lora, S.; Passi, P.; Orsolini, P., Biomaterials 1999, 20: 91

82. Langone, F.; Lora, S.; Veronese, F. M.; Caliceti, P.; Parnogotto, P. P.; Valenti, F.; Palma, G., Biomaterials 1995, 16: 347 
83. Aldini, N. N.; Fini, M.; Rocca, M.; Martini, L.; Giardino, R.; Caliceti, P.; Veronese, F. M.; Lora, S.; Maltarello, M. C., Journal of Bioactve and Compatible Polymers 1997, 12: 3

84. Reed, C. S.; Tenhuisen, K. S.; Brown, P. W.; Allcock, H. R., Chemsitry of Materials 1996, 8: 440

85. Andrianow, A. K.; Cohen, S.; Visscher, K. B.; Paynel, L. G.; Allcock, H. R.; Langer, R., Journal of Controlled Release 1993, 27: 69

86. Andrianow, A. K.; Payne, L. G.; Visscher, K. B.; Allcock, H. R.; Langer, R., Journal of Applied Polymer Science 1994, 53: 1573

87. Nelson, F. E.; Srinivas, G.; Ken, L.; Volker, G.; George, K., The Open Vaccine Journal 2009, 2: 134-143

88. Andrianow, A. K.; Payne, L. G., Microparticulate System for the Delivery of Propetins and Vaccines. Dekker New York, 1996; P 127

89. Kim, C.; Allcock, H. R., Macromolecules 1987, 20: 1726-1727

90. Singler, R. E.; Willingham, R. A.; Lenz, R. W.; Furukawa, A.; Finkelmann, H., Macromolecules 1987, 20: 1727-1728

91. Allcock, H. R.; Kim, C., Macromolecules 1989, 22: 2596-2602

92. Allcock, H. R.; Klingenberg, E. H., Macromolecules 1995, 28: 4351-4360

93. Allcock, H. R.; Bender, J.; Chang, Y., Chemistry of Materials 2003, 15: 473-477

94. Gleria, M.; Bertani, R.; De Jaeger, R.; Lora, S., Journal of Fluorine Chemistry 2004, 125: 329-337

95. Allcock, H. R.; Kugel, R. L., Journal of the American Chemical Society 1965, 87: 4216

96. Gleria, M.; Bertani, R.; De Jaeger, R., Journal of Inorganic and Organometallic Polymers 2004, 14: 1

97. Antipov, E. M.; Kulichikhin, V. G., Vysokomolek. Soedin 1990, 32: 118-115

98. Allcock, H. R., Phosphorus-nitrogen compounds. Cyclic, linear, and high polymeric system. New York, 1972; p 358.

99. Kojima, H.; Magill, J, H., Makromolecular Chemistry 1985, 186: 649-663

100. Kojima, H.; Magill, J, H., Polymer 1971, 26: 1971-1975 


\section{Chapter 3}

\section{NANOCOMPOSITE}

\subsection{INTRODUCTION}

Nanotechnology can be defined as the creation, processing, characterization and utilization of materials, devices, and systems with one dimension in the range of $0-100 \mathrm{~nm}^{1}$. The field of nanotechnology is one of the most popular areas for current research and development. Within the broad field of nanotechnology, polymer matrix based nanocomposites have become a prominent area of research in basically all technical disciplines ${ }^{2}$. Polymer nanocomposites are polymer resins that have been modified with small amounts ( $0-20 \%$ by weight) of nano-fillers, where at least one dimension of the nanofiller material is on the order of nanometer. Since the dimension of polymer resins and fillers are at the same order of magnitude, molecular interaction between them will give the polymer nanocomposites unusual material properties that conventional polymers do not

possess ${ }^{3}$, which have broadened the scope of polymer chemistry to great benefit of many different industries. These include composite reinforcement, barrier properties, flam resistance, electro-optical properties, and cosmetic applications.

\subsection{AN OVERVIEW OF NANOPARTICLES}

Depending on the choice of applications, there are many possible types of nanoparticles that can be incorporated into the polymer matrix to form polymer nanocomposites. Commonly encountered nanoparticles in literature may include:

- Montmorillonite organoclays (MMT) ${ }^{4}$

- Carbon nanotubes and carbon nanofibers (CNFs) ${ }^{5}$ 
- Nanopowders ${ }^{6}$ [metal (Au, Pt, Cu, etc.), semiconductors (Zns, CdS, CDSe, etc.), and metal oxides $\left(\mathrm{SiO}_{2}, \mathrm{AL}_{2} \mathrm{O}_{3}, \mathrm{TiO}_{2}, \mathrm{ZrO}_{2}, \mathrm{Fe}_{2} \mathrm{O}_{3}\right.$, etc. $\left.)\right]$

\subsubsection{Montmorillonite Clay}

The dominant constituent of montmorillonite is silica $\left(\mathrm{SiO}_{2}\right)$, with alumina $\left(\mathrm{Al}_{2} \mathrm{O}_{3}\right)$ being essential. The chemical structure of montmorillonite clay is composed of a sheet structure consisting of layers of tetrahedral silicate layer and octahedral alumina layer. Within one slab, two tetrahedral silicate layers sandwich an octahedral alumina layer. These three layers form one repeat unit that has a thickness of $0.96 \mathrm{~nm}$. The chemical formula of this montmorillonite clay sheet repeat unit is $\mathrm{Na}_{1 / 3}\left(\mathrm{Al}_{5 / 3} \mathrm{Mg}_{1 / 3}\right) \mathrm{Si}_{4} \mathrm{O}_{10}(\mathrm{OH})_{2}{ }^{3}$.

\subsubsection{Nanofibers and Nanotubes}

Carbon nanofibers are cylindrical nanostructures with graphene layers arranged as stacked cones, cups, or plates. Carbon nanofibers with graphene layers wrapped into perfect cylinders are called carbon nanotubes.

Carbon nanofibers have moderate electrochemical properties and incorporating carbon nanofibers into a polymer matrix is easy because the fibers are relatively large. Carbon nanofibers have the capability to incorporate the advantages of other forms of carbon into the applications of reinforcement in commodities and high performance engineering polymers. Furthermore, carbon nanofibers have transport and medicinal properties that are similar to other forms of carbon, such as single crystal but at lower cost ${ }^{3}$. Carbon nanofibers-based nanocomposites can offer multifunctional performance for several potential aerospace and other commercial applications, such as electrostatic painting, electronics, improved polymer mechanical properties etc. 


\subsubsection{Carbon Nanotubes}

Carbon nanotubes have been attracting increasing attention over the past several

years because of their extraordinary structural, electrical, and mechanical properties? ${ }^{7}$ As an example, carbon nano tubes are one of the strongest molecular materials known due to unique $\mathrm{C}$ - $\mathrm{C}$ covalent bonding and the seamless hexagonal network. The nanotubes also have substantial electrical conductivity or semiconductivity, in addition to high thermal conductivity in the axial direction.

\subsubsection{Nano titanium dioxide $\left(\mathrm{TiO}_{2}\right)$}

Among all the fillers mentioned previously, $\mathrm{TiO}_{2}$ nanopowder is attracting the most attention for the reasons of low-toxicity, chemically inertness, low cost, high refractive index, broadband UV filtering, corrosion resistance and high hardness. Literatures indicate that nanoscaled $\mathrm{TiO}_{2}$ reinforcement brings new optical, electrical, and physiochemical properties which are attained at very low $\mathrm{TiO}_{2}$ content, making polymer $\mathrm{TiO}_{2}$ nanocomposites a promising new class of materials.

The $\mathrm{TiO}_{2}$ surface is complex and extremely sensitive to thermo chemical history, such as temperature, pressure, cooling rate, and impurities. $\mathrm{TiO}_{2}$ surface is capable of absorbing, dissociating or reacting with a wide variety of inorganic and organic molecules and atoms under certain conditions. Among them, the most important one is the $\mathrm{H}_{2} \mathrm{O}$ dissociative or nondissociative adsorption behavior, which determines $\mathrm{TiO}_{2}$ wetting and dispersing capabilities in aqueous or non-aqueous mediums and other surface properties. Thus, the $\mathrm{TiO}_{2}$ surface plays an essential role in a wide range of applications. 


\subsection{METHODS FOR MAKING NANOCOMPOSITES}

The properties of nanocomposites depend greatly on the nanoparticle type, aspect ratio, and polymer matrix properties ${ }^{8}$. Besides, the preparation methods for producing the composites, which will affect the orientation ${ }^{9}$, dispersion and morphology of the polymer resins ${ }^{10}$, will also determine the polymer properties to some extent. Many synthetic approaches have been published concerning preparation of nanocomposites, which can be classified in different ways because of the overlapping of techniques. In the following sections, we will describe the three most used polymer nanocomposites synthetic approaches: In situ polymerization, solution mixing, and melt blending.

\subsubsection{In-situ Polymerization}

In in-situ polymerization, fillers are swollen within the liquid monomer or monomer solution, followed by the polymerization of the monomers ${ }^{11}$. The polymer formation occurs between the intercalated filler sheets. Polymerization can be initiated by heat or radiation, or by introduction of an initiator or catalyst before the swelling step of the monomer.

This preparation strategy in essence involves two steps: a) dispersion of nanoparticles in the monomers and b) polymerization of the monomers. The first polymer/nanoclay nanocomposite that attracted tremendous attention was (Nylon-6/MMT) which is prepared by in-situ polymerization by the Toyota research group. The application of this method for the preparation of carbon nanotubes-based nanocomposites has also been studied extensively for the past few years. Many epoxy ${ }^{12-13}$, polyurethane ${ }^{14-15}$, and polyester ${ }^{16-17}$ based nanocomposites were prepared using this method. The polymerization process can be done in bulk ${ }^{18}$, solution ${ }^{19}$, emulsion $^{20}$ or suspension ${ }^{21}$. 
The principal advantage of in-situ polymerization is that the monomer can diffuse into the galleries more efficiently than a high molecular mass polymer. The use of monomer and occasionally solvent offers the additional advantage of adjusting the competitive interactions among the components, making interactions stronger and resulting in exfoliation.

\subsubsection{Solution mixing}

Solution mixing is the most commonly used method for preparing nanocomposites. In this method, three major steps are usually involved: a) dispersion of nanoparticles in a suitable solvent or polymer solution, b) mixing of the nanoparticles and polymer in the solution, and c) recovery of the nanocomposite by precipitation or casting a membrane.

Many water-soluble polymers, such as $\mathrm{PEO}^{22-25}, \mathrm{PVA}^{26}, \mathrm{PVP}^{27}$, and $\mathrm{PEVA}^{25}$, have been made into clay nanocomposites using this method. Non-aqueous polymers, such as PCL, or PLA, can also be made into clay nanocomposites using this method with chloroform as a co-solvent. Nematic liquid crystal PLS nanocomposites have been prepared using this method in different organic solvents, such as toluene and DMF.

The primary limitation of this method is the requirement of a suitable solvent that must be able to expand the nanoparticle spacing as well as dissolve the polymer chains. The extent to which the solvent swells determines the final morphology of the nanocomposites. 


\subsubsection{Melt Blending}

The melt blending process usually involves the melting of the polymer matrix to form a viscous liquid, after which the nanoparticles are dispersed into the polymer melt by high shear rate and diffusion at high temperature. Compression molding, injection molding or fiber production techniques can also be applied to fabricate the final samples.

Melt blending methods can be applied to make nanocomposites from styrene polymers $^{28-29}$, natural rubbers ${ }^{30}$, polyamides ${ }^{31-35}$. Thermoplastic elastomer nanocomposites that possess enhanced ablation and fire retardancy characteristics were developed by the Air Force Research Laboratory/polymer Working Group at Edwards AFB, CA.

There are many advantages of melt blending over the other two routes. For instance, melt blending is highly specific to the polymer, leading to new hybrids that were previously inaccessible. Furthermore, the absence of a solvent makes melt blending an environmentally favorable alternating method for industries from a waste perspective.

\subsection{NANOPARTICLE DISPERSION AND THE INTERPHASE}

\subsubsection{Dispersion}

The properties of polymer nanocomposites are strongly influenced by the morphology in the polymer matrix. The uniform dispersion of nanoparticles is the first major hurdle scientists face when making a nanocomposites, because Van der Waals forces and differences in polymer/nanoparticle surface energies often cause nanoparticles to have greater affinity towards each other than to polymer matrix ${ }^{36}$. Even if a good dispersion is achieved initially, the nanoparticles may agglomerate during further processing, leaving a poor dispersion in the matrix. The high affinity between nanoparticles causes the problems 
of agglomeration. There are three commonly considered levels dispersion as illustrated in Figure 3.1.

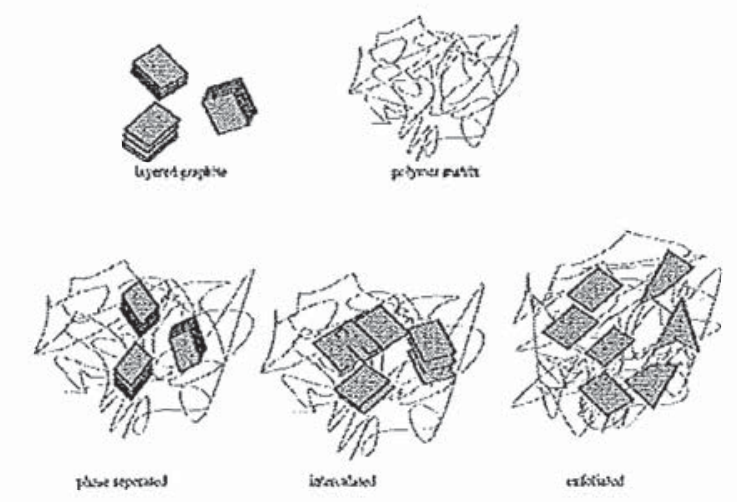

Figure 3.1: Illustration of three terms to describe dispersion quality

The first level of dispersion is phase-separated, where the polymer chains are unable to penetrate between the individual grapheme sheets. In this state, the material may be characterized as a macro-composite because the effective particle size can be greater than $100 \mathrm{~nm}$.

The second level of dispersion is nanocomposite exhibiting intercalated morphology. Intercalated clay morphology occurs when polymer chains are able to diffuse into the gallery spacing of the nano particles ${ }^{37}$. Intercalation is a physical process by which a macromolecule is inserted into the gallery, with gallery distance being typically on the order of a few nanometers. This material may be characterized as a nanocomposite because the effective particle sizes are below $100 \mathrm{~nm}$. The reinforcement efficiency of intercalated nanocomposites is improved compared to a phase-separated microcomposite.

The third level of dispersion is fully exfoliated, where the polymer chains are able to penetrate between the individual graphene sheets and completely disperse the graphene sheets in a continuous polymer matrix by an average distance that depends on clay loading ${ }^{37}$. 
Exfoliated morphology is often preferred because it usually provides better property improvements than intercalated morphology. The benefit of an exfoliated structure is the ability to take advantage of the high aspect ratio of individual filler layers. And the nanoclay content of an exfoliated nanocomposite is usually much lower than that of an intercalated nanocomposite.

\subsubsection{Interphase}

The interface between the polymer matrix and the filler ${ }^{38-44}$ is the most intensively studied area of polymer composite structure, because the interphase is so important to the mechanical properties of the composite and for its distinction. All polymer composite systems possess a phase border between the matrix and the filler with the formation of an interphase between them ${ }^{39,41,44-45}$.

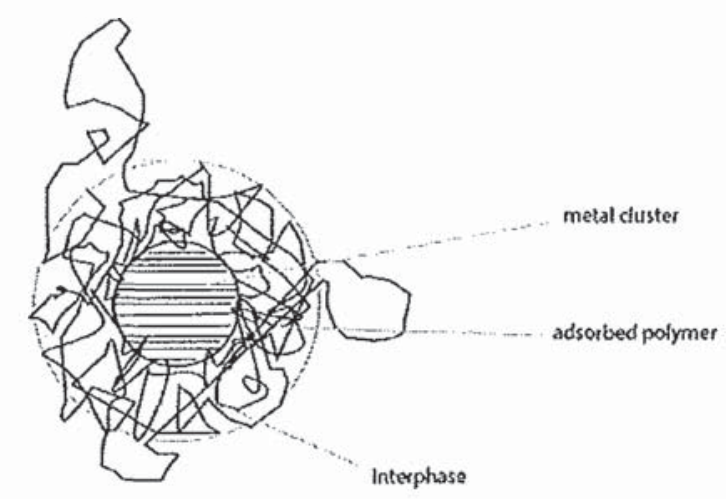

Figure 3.2: $\quad$ Schematic of interphase area between filler and the polymer matrix ${ }^{46}$

As shown in Figure 3.2, the interphase consists of flexible polymer chains, typically in a sequence of adsorbed segments, loops, and tails. There is no specific value for the thickness of the interphase, because for different nanocomposite systems, it depends on chain flexibility and on the energy of adsorption, which is determined by the surface energy of the polymer and the solid. For composite systems in which the polymer chain is 
sufficiently flexible, segments will be readily adsorbed, and if all areas of the surface are capable of adsorption, the polymer loops will be short and the macromolecule will form a dense layer close to the surface, as seen in Figure 3.3(a). If, on the other hand, the polymer chain has weak interactions with the surface, or if the chain is rigid, the loops and tails will extend father into the matrix and form a region of lower density, as shown in Figure 3.3 (b).

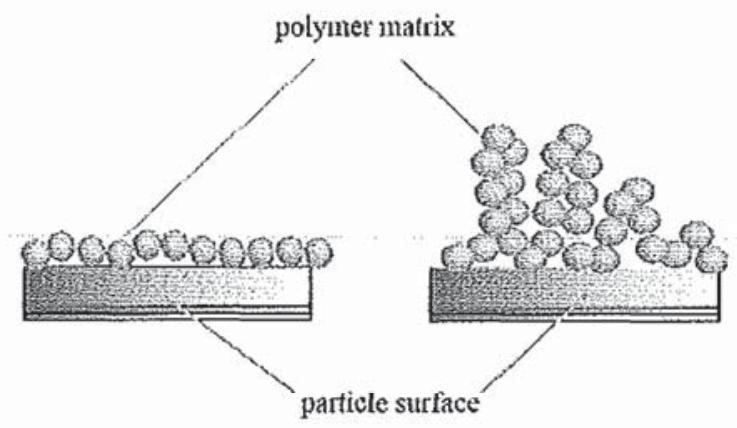

Figure3.3: Schematic representation adsorption characteristics of a metal-polymer nanocomposite (a) a strongly-binding polymer adheres to the surface (b) weaklybinding polymer adheres to the surface where most of the segments reside in loops. ${ }^{47}$

The concept of an interphase is widely accepted, even though the influence of the interphase on the specific properties of a polymer composite has not yet been quantitatively established $^{48}$. Many studies have identified the interphase as an important factor in determining the mechanical properties of composites.

The detection and characterization of the interphase is difficult with either direct or indirect methods. This is because the interphase is not present in sufficient amounts to detect if the extent of exfoliation is small, which occurs quite frequently. Spectroscopy cannot see changes in properties in the absence of chemical reaction. One of the few possibilities for the detection and analysis of interphase is to detect molecular morphology changes by nuclear magnetic resonance spectroscopy (NMR). 


\subsection{NANOCOMPOSITES PROPERTIES AND ANAYTICAL METHODS}

Despite the fact that there is no consensus on how nanoparticles affect material properties due to the novelty of this area, the challenges in processing nanocomposites, lack of systematic experimental result, and scarcity of theoretical studies, some properties of nanocomposites have been studied in-depth using different characterization and analytical methods.

\subsubsection{Mechanical Properties and Characterization Techniques}

\subsubsection{Tensile testing}

Tensile testing can be performed to determine elastic modulus, Poisson's ratio, yield strength, strain hardening, ultimate stress, and ultimate strain for composite systems. Mechanical properties are measured from a stress vs. strain plot of the load and elongation data. Tensile testing is a destructive characterization technique.

The tensile modulus of a polymer material is remarkably improved when nanocomposites are formed with nano-fillers. For instance, Nylon-6 nanocomposites exhibit a dramatic improvement in tensile properties at rather low filler content because the strong interaction between the matrix and silicate layers via formation of hydrogen bonds. Zhu et al. also found that tenacity at break and the initial modulus of PA6 with modified nano- $\mathrm{TiO}_{2}$ composites were improved by about $10 \%$, and $20 \%$, respectively, as compared to the pure $\mathrm{PA}^{49}$. 


\subsubsection{Dynamic mechanical analysis}

Dynamic mechanical analysis (DMA) can be used to collect information about elastic modulus, loss modulus, and damping coefficient as a function of temperature, frequency, or time. Also, DMA can be used to measure glass transition temperatures. DMA applies a torsional oscillation to samples while slowly moving through specific temperature ranges. Results are typically recorded plots of elastic modulus, loss modulus or damping coefficient versus temperature. For example, DMA has been used to study the temperature dependence of the storage modulus of PMMA upon nanocomposite formation under different experimental conditions ${ }^{50}$.

\subsubsection{Nanoindentation}

Nanoindentation can be used as another approach to gather comparative elastic modulus and hardness data. Indentation testing is based on the concept of touching a material whose mechanical properties are of interest with a material whose properties are known. Unlike tensile testing and DMA, nanoindentation is considered as a non-destructive technique.

\subsubsection{Analytical Methods}

\subsubsection{Thermal gravimetric analysis}

The thermal stability of a composite material can be usually studied by thermogravimetric analysis (TGA). TGA continuously measures the weight of a sample as a function of temperature and time. Changes in weight at specific temperatures correspond to reactions or changes in sample state such as decomposition. Generally, the incorporation of clay into a polymer matrix enhances its thermal stability by acting as an insulator and 
mass transport barrier to the volatile products generated during decomposition. For example, Blumstein reported improved thermal stability of a PLS nanocomposite that combined PMMA and MMT clay ${ }^{5 !}$.

\subsubsection{Scanning electron microscopy}

Scanning electron microscopy (SEM) can be used to determine particle size and

distribution and for examination of fracture surface ${ }^{52}$. SEM is capable of differentiating particle details as small as $1 \mathrm{~nm}$ depending on elemental contrast and other parameters. While there are several disadvantages associated with SEM. For one thing, SEM requires fine-tuning to get high resolution. In addition, non-conductive polymers often suffer variations in surface potential, which will introduce astigmatism, instabilities, and false xray signal. Finally, charging conditions often occur making it difficult to obtain high quality images.

\subsubsection{Fourier transform infrared spectroscopy}

Fourier transform infrared spectroscopy (FT-IR) can be used to analyze bonding between the polymer matrix and the nanoparticles. The signal detected is processed using a Fourier transform to provide infrared absorption spectra, presented as plots of intensity versus wavenumber (in $\mathrm{cm}^{-1}$ ). The infrared wavelengths absorbed by a material help to identify its molecular structure. 


\subsubsection{X-ray diffraction}

Wide-angle $\mathrm{x}$-ray diffraction is one of the most commonly used techniques to characterize the degree of nanoparticle dispersion within a specific polymer. It can be used to identify the specimen's crystalline phases and to measure its structural properties ${ }^{50}$. Xray diffraction is widely used in materials characterization for the reasons that it is nondestructive and does not require elaborate sample preparation.

\subsubsection{Transmission electron microscopy}

Transmission electron microscopy (TEM) ${ }^{53}$ is the only method that can used to get direct imaging of individual nanoparticles, which can provide a real space image of the atom distribution in the nano crystal and on its surface. TEM can provide not only atomic resolution lattice images, but also chemical information at a spatial resolution of $1 \mathrm{~nm}$ or better, allowing direct identification of the chemistry of a single nano-crystal.

\subsubsection{Nuclear magnetic resonance spectroscopy}

In addition to the techniques discussed, differential scanning calorimetry can be used to understand the nature of crystallization taking place in the matrix, and resonance Raman spectroscopy can be used for structural studies. However, few analytical methods can give insight about morphological changes at the molecular level for composites. On the other hand, solid-state nuclear magnetic resonance spectroscopy (SSNMR), has been proved to be a versatile and robust tool for providing valuable information about morphology, surface chemistry, and to a limited extent, the quantification of the level of exfoliation in polymer nanocomposites. 


\section{References}

1. Thostenson, E. T.; Li, C.; Chou, T. W., Nanocomposites in context, Composite. Science and Technology 2005, 65: 491-516

2. Paul, D. R.; Robeson, L. M., Polymer 2008, 49: 3187-3204

3. Koo, J. H., Polymer Nanocomposites: Processing, Characterization and Applcation. The McGraw-Hill Companies, Inc.: United States of America, 2006.

4. Xi, Y. F.; Ding, Z.; He, H.; Frost, R. L., Journal of Colloid and Interfce Science 2004, 277: 116

5. Rakov, E. T., Khimicheskaya Tekhnologiya 2003, 10:2-7

6. B., S. A.; Sudhakara; Aamanchi, R. B.; Karthik 2013.

7. Dresselhaus, M. S.; G., D.; Eklund, P. C., Science of Fullerenes and Carbon Nanotubes. Academic Press: San Diego, 1996.

8. Treacy, M. M. J.; Ebbesen, T. W.; Gibson, J. M., Nautre 1996, 381: 678-680

9. Wong, E. W.; Sheehan, P. E.; Lieber, C. M., Science 1997.

10. Salvetat, J. P.; Bonard, J. M.; Thomson, N. H.; Kulik, A. J.; Forro, L.; Benoit, W.; Zuppiroli, L., Applied Physics A 1999.

11. Park, S. S.; Bernet, N.; De la Roche, S.; Hahn, H., T., Journal of Conposite Materials 2003, 37: 465-476

12. Zilg, C.; Mulhaupt, R.; Finter, J., Journal of Macromolecular Chemistry and Physics 1999, 200: 661-670

13. Liu, W.; Hoa, S. V.; Pugh, M., Composites Science and Technology 2005, 65: 23642373

14. Wang, Z.; Pinnavaia, T. J., Chemistry of Materials 1998, 10: 3769-3771

15. Berta, M.; Lindsay, C.; Pans, G.; Camino, G., Polymer Degradation and Stability 2006, 91: 1179-1191

16. Sun, D.; Lim, Y.; Park, O., Polymer 2000, 41: 8557-8563

17. Bharadwaj, R. K.; Mehrabi, A. R.; Hamilton, C.; Trujillo, C.; Murga, M. F.; Chavira, A., Polymer 2002, 43: 3699-3705

18. Song, M.; Xia, H., S.; Yao, K. J.; Hourston, D. J., European Polymer Journal 2005, 41: $259-266$

19. Uthirakumar, P.; Kim, H. J.; Hong, C. H.; Suh, E. K.; Lee, Y. S., Polymer Composites 2008, 29, 142: 142-148

20. Kong, Q. H.; Hu, Y.; Yang, L.; Fan, W. C.; Chen, Z., Polymer Composites 2006, 27: $49-54$

21. Lei, X. P.; Liu, Y. S.; Su, Z. X., Polymer nanocomposites 2008, 29: 239-244

22. Aranda, P.; Ruiz-Hitzky, E., Chemistry of Materials 1992, 4: 1395-1403

23. Ogata, N.; Kawakage, S.; Orgihara, T., Journal of Applied Polymer Science 1997, 66: 573-581

24. Parfitt, R. L.; Greenland, D. L., Clay Mineral 1970, 8: 305-323

25. Zhao, X.; Urano, K.; Ogasawara, S., Colloid and Polymer Science 1989, 267: 899. 906

26. Greenland, D. J., Journal of Colloid Science 1963, 18: 647-664 
27. Francis, C. W., Soil Science 1973, 115: 40-54

28. Yoon, J. T.; Jo, W. H.; Lee, M. S.; Ko, M. B., Polymer 2001, 42: 329-336

29. Sorrentino, A.; Pantani, R.; Brucato, V., Polymer Engineering and Science 2006, 46: $1768-1777$

30. Ramorino, G.; Bignotti, F.; Conzatti, L.; Ricco, T., Polymer Engineering and Science 2007, 47: 1650-1657

31. Poisson, C.; Guerengomba, J.; Lacrampe, M. F.; Krawczak, P.; Gupta, B.; Miri, V.; Lefebvre, J. M., Polymer and Polymer Composites 2008, 16: 349-358

32. Fornes, T. D.; Yoon, P. J.; Keskkula, h.; Paul, D. R., 2001, Polymer.

33. Wu, S. S.; Jiang, D. J.; Ouyang, X. D.; Wu, F.; Shen, J., Polymer Engineering and Science 2004, 44: 2070-2074

34. Isik, I.; Yilmazer, U.; Bayram, G., Polymer Composites 2008, 29: 133-141

35. Yuan, M. J.; Song, Q.; Turng, L. S., Polymer Engineering and Science 2007, 47:

36. Thostenson, E. T.; Li, C.; Chou, T. W., Composites Science and Technology 2005, 65: 491-516

37. Ray, S. S.; Okamoto, M., Progree in Polymer Science 2003, 28: 1539-1641

38. Vollenberg, P. H. T.; Heikens, D., Polymer 1989, 30: 1656-1662

39. Wu, C. L.; Zhang, M. Q.; Rong, M. Z.; Friedrich, K., Composites Science and Technology 2002, 62: 1327-1340

40. Rong, M. Z.; Zhang, M. Q.; Pan, S. L.; Lehmann, B.; Friedrich, K., Polymer International 2003, 53: 176-183

41. Shang, S. W.; William, J. W.; Soderholm, K.-J. M., Polymer Composiles 1994.

42. Zhu, L.; Narh, K. A., Journal of Polymer Science Part B: Polymer Physics 2004, 42 .

43. Kovacevic, V.; Lucic, S.; Leskovac, M., Journal of Adhension Scence and Technology 2002, 16: 1343-1365

44. Kovacevic, V.; Leskovac, M.; Lucic, S., Journal of Adhension Scence and Technology 2002, 16: 1915-1926

45. Lipatov, Y. S., ChemTec Publishing 1995.

46. Aubouy, M.; Raphael, E., Macromolecules 1998, 31: 4357-4363

47. Tannenbaum, R.; Zubris, M.; David, K.; Jacob, K.; Jasiuk, I.; Dan, N., Chaeacterization of Metal-Polymer Interfaces in Nanocomposites and the Implications to Mechanical Properties. Georgia Institute of Technology.

48. Vollenberg, P. H. T.; Heikens, D., Polymer 1989, 30: 1656-1662

50. Manias, E.; V, K., Colloids and Surfaces 2001.

51. Blumstein, A., Journal of Polymer Science Part A. 1965, 3: 2665-2673

52. Carretti, E.; Chelazzi, D.; G., R.; Baglioni, P.; Poggi, G.; Dei, L., Online Computer File 2013, 29: 9881-9890

53. Chen, S. K.; Tsai, J. L.; Chin, T. S., Journal of Applied Physics 1997, 81: 56315633 


\section{Chapter 4}

\section{SOLID-STATE NUCLEAR MAGNETIC RESONANCE SPECTROSCOPY OF POLYMERS AND NANOCOMPOSITES}

\subsection{INTRODUCTION}

The quest for understanding the relationship between physical properties and molecular motions in solid polymers has intrigued the interest of scientists since the founding of polymer science ${ }^{1-3}$. The properties of solid polymers are governed partly by their molecular structure, and also strongly depend on the organization of the macromolecules in the solid state, eg. their phase behavior, morphology, molecular order and molecular dynamics. In order to have a detail understanding of polymer properties, one has to understand how these properties relate to structural features at the atomic level. A variety of analytical and characterization techniques can be applied to study polymers, such as X-ray diffraction, DSC, TGA, and GPC. However, most techniques are limited in the ability to provide the most important and determining structural and dynamic characteristics at the atomic level. Nuclear magnetic resonance (NMR) is regarded as the most valuable tool for elucidating molecular structure, and dynamics for the characterization of macromolecules. ${ }^{4}$

The rapid methodological development of solid-state NMR has opened new possibilities in this area. The most important aspects of NMR in this field are achieved in two ways. One route is through high resolution NMR of solids, in particular of ${ }^{13} \mathrm{C}$ at natural abundance. The improved resolution together with an enhancement of signal intensity is achieved by a combination of ${ }^{1} \mathrm{H} \rightarrow{ }^{13} \mathrm{C}$ Cross Polarization, high power proton decoupling and Magic Angle Spinning (CPMAS-NMR) ${ }^{5-7}$. The other route is that line 
shape and spin relaxation analysis can be exploited to yield valuable information about phase behavior in addition to the molecular dynamics ${ }^{7}$.

More recently, new methods involving two-dimensional $\mathrm{NMR}^{8-11}$ have been developed that yield previously inaccessible information. The macroscopic behavior of polymers are frequently related to molecular properties, and polymer conformations have been studied using a variety of 2D NMR techniques to measure the through-bond Jcoupling in solution or through space dipolar coupling in the solid state.

Another important development concerns the use of spin diffusion ${ }^{12}$ for the study of the phase behavior in semicrystalline as well as block copolymers and polymer blends ${ }^{13-15}$.

\subsection{MOLECULAR STRUCTURAL CONNECTIVITY, DYNAMICS AND MORPHOLOGY STUDIES OF MACROMOLECULES USING NMR SPECTROSCOPY}

\subsubsection{Relaxation}

NMR relaxation times play an important role in the characterization of macromolecules for the reason that it is a powerful tool for probing the chain dynamics within polymers, in both amorphous and crystalline phase ${ }^{16}$. Molecular dynamics have a profound impact on material properties, such as flexibility, elasticity, permeability. In contrast to solution where orientation dependent spectral parameters are averaged out by fast motion, in solids relaxation, linewidths and dipolar interactions, which depend strongly on the molecular motion, can provide detailed insight into chain dynamics. 


\subsubsection{Spin-Iattice relaxation $\left(\mathrm{T}_{\mathrm{I}}\right)$ and molecular motion}

Spin-lattice relaxation times are sensitive to motions that fall into the frequency regime of the Larmor frequency of the spectrometer. Therefore, $T_{1}$ experiments are particularly important for providing local motion information about stereo-chemical and morphological differences among materials.

The Larmor frequencies of nuclei can range from 60 to more than $600 \mathrm{MHz}^{17}$ making $T_{1}$ values particularly sensitive to high-frequency side chain motion. This is demonstrated by Edzes and Veeman in their studies of the effect of plasticizer and tactility on the spinlattice relaxation time of carbon nuclei in solid poly(methyl methacrylate) (PMMA) ${ }^{18}$.

In addition, $T_{1}$ measurements can be applied to offer information about different phases, thermal histories, and dynamics. As examples, Koening et al, used $\mathrm{T}_{1}$ spin-lattice relaxation time to characterize the motional difference between the alpha and beta phase of poly(butylene terephthalate) (PBT); Schaefer et al ${ }^{19}$ have shown that $T_{1}$ values can be used to study the effects of thermal histories on molecular motion in solid polymers. Schilling et al. ${ }^{20}$ used $T_{1}$ relaxation times to study the dynamic characteristics of solid state phase transition that occurs in the crystalline domains of trans-poly(butadiene) (TPBD).

In addition to being used to study neat polymers or polymer blends, $T_{1}$ relaxation times can also be used to study plasticized systems. For example, Schaefer et al. ${ }^{21}$ have shown that in certain systems, a plasticizer seems to have no effect on the $\mathrm{MHz}$ regime relaxation rate of main chain carbons of poly(vinyl butyral-co-vinyl alcohol) (PVB-VA), suggesting that plasticizers tend to enhance lower frequency motions ( $\mathrm{kHz}$ range or lower), and have little or no effect on the $\mathrm{MHz}$ range motion, the latter of which controls the $\mathrm{T}_{1}$ spin relaxation process. $T_{1}$ experiments have also used to study the adsorption of polymers 
onto the surface of a metal oxide. For example, $\mathrm{T}_{1}$ relaxation time of a composite containing $\mathrm{Al}_{2} \mathrm{O}_{3}$ and poly(vinylpyrrolidone-co-vinyl acetate ) revealed that the VA carbonyl carbon exhibits decreased mobility in the presence of $\mathrm{Al}_{2} \mathrm{O}_{3}$, while the PVP carbonyl carbon remains unaffected ${ }^{22}$.

\subsubsection{Spin-lattice relaxation in the rotating frame $\left(\mathrm{T}_{1 \rho}\right)$}

$\mathrm{T}_{\mathrm{l} \rho}$ relaxation is sensitive to molecular motions that occur in the $\mathrm{kHz}$ range. These $\mathrm{kHz}$ regime motions are typically associated with cooperative polymer backbone rearrangements that involve the collective motions of a large number of monomer units ${ }^{3}$. This kind of long range, cooperative motion is considered to be the type of motion that defines a polymer's response to mechanical perturbations ${ }^{23-24}$.

\subsubsection{Spin-spin relaxation $\left(\mathrm{T}_{2}\right)$}

The last type of spin relaxation to be considered is due to the spins precessing in the transverse plane following a RF pulse. This process is characterized by the time constant $T_{2}$, and similar to $T_{l \rho}$ is sensitive to $\mathrm{kHz}$ range molecular motions. $T_{2}$ is reflected in the

time domain NMR signal, the FID, and in the spectral line-width. The rigid components of a polymer will have a shorter $T_{2}$. It is also a routine to use a "T $T_{2}$ filter" in an NMR experiment to obtain spectra of mobile components while suppressing more rigid ones, where only the magnetization from the mobile components will remain for detection by inserting a short free evolution delay in a pulse sequence prior to acquisition. 


\subsubsection{Conclusion}

In summary, $T_{1}$ spin-lattice relaxation, $T_{1 \rho}$ and $T_{2}$ can be used to provide dynamic, structural, and morphological information about many types of polymer systems. This information can be used to elucidate important structural-property relationships for the purpose of widening our fundamental understanding of macromolecules.

\subsubsection{D NMR Studies of Polymer}

Solution and solid state two dimensional NMR is an important tool for material characterization. The NMR analysis of polymers has historically been limited by poor spectral resolution due to the repeating structure of monomers in the polymer matrix. However, the resolution is increased on proceeding from ID to 2D NMR, which makes it possible to obtain more precise and detailed information about structure-function relationships in polymers.

2D NMR is an effective tool to study the molecular dynamics of polymers; where several experiments have been designed to probe the dynamics over a wide range of time scales. Solution NMR experiments such as NOESY are utilized to directly measure polymer dynamics from the time dependence of the intensity of cross peaks ${ }^{25}$. Other approaches are two-dimensional Exchange Spectroscopy (EXSY) ${ }^{16}$ and Centerband Only Detection of Exchange $(\mathrm{CODEX})^{26}$, allowing the monitoring of molecular motion occurring at much lower frequencies $\left(10^{-1}-10^{4} \mathrm{~Hz}\right)$, which are particularly useful for investigating very slow dynamics of polymers near their glass-transition temperatures. Examples include 2D EXSY NMR studies of the conformational exchange dynamics in poly(ethylene glycol) substituted cyclodextrins ${ }^{27}$ and application of CODEX experiments 
to elucidate slow chain motion in (ring-fluoro)polycarbonate ${ }^{28}$, isotacticpoly(4-methyl-1pentene) crystallites ${ }^{29}$ and poly(alkyl methacrylate) ${ }^{30}$. Also $2 \mathrm{D}$ deuterium and CSA exchange spectroscopy are ideal for investigating the "ultra-slow" motions in polymers ${ }^{31-}$ 32. 2D spin exchange experiments can also provide direct evidence for chain diffusion between crystalline and amorphous regions of semicrystalline polymers. For example, chain diffusion has been suggested as the process giving rise to a transition reflected in the NMR spectrum of polyethylene ${ }^{33}$.

2D NMR has made important contributions to many areas of polymer science, ranging from materials characterization to understanding structure-function relationships in polymers. The higher resolution provided by $2 \mathrm{D}$ NMR has allowed a more detailed characterization of polymers that is important for not only structure-property relationships but also for understanding polymerization mechanisms. With the development of new experiments and the application to more materials, 2D NMR will continue to have a significant impact on material science.

\subsection{STRUCTURAL STUDIES OF POLYMER NANOCOMPOSITES USING SOLID-STATE NUCLEAR MAGNETIC RESONANCE}

Solid-state NMR spectroscopy is a technique that allows the investigation of nanocomposites at a molecular level, and provides information about local motion and dynamics ${ }^{34-35}$. For example, applications of ${ }^{1} \mathrm{H}$ and ${ }^{13} \mathrm{C}$ SSNMR for determining the structural and dynamic characteristics of polymer/clay nanocomposites were reported by Vanderhart et al. in $2001^{36}$, Mathias and colleagues used SSNMR to probe the interaction between nylon- 6 chains with the lamellae of aluminosilicate clay ${ }^{37}$, and variable 
temperature ${ }^{13} \mathrm{C}$ SSNMR was used to study the conformation and dynamics of surfactant molecules within the MMT by Wang et al. ${ }^{38}$.

Various relaxation parameters are of great importance in elucidating molecular dynamics in the solid state. Including spin-lattice relaxation in the laboratory frame, and spin-lattice relaxation in the rotating frame. Relaxation parameters are related to a system's morphology. Consequently, relaxation time measurement can be used to evaluate the dispersion of fillers in the polymer matrix, and the interactions between the nanoparticles and the polymer matrix.

2D SSNMR can be used for detailed studies of polymer-filler contacts, leading to understanding of the interaction mechanism at the molecular level. An important application concerning the application of 2D NMR in nanocomposites was made by Hou and Schmidt-Rohr where $2 \mathrm{D}^{1} \mathrm{H}^{-29} \mathrm{Si}$ NMR correlation experiment was applied to monitor the PS and PMMA nanocomposite with hectorite $(\mathrm{HCT})^{39}$. Another example was illustrated by Bordia and co-workers using $2 \mathrm{D}{ }^{1} \mathrm{H}_{-}{ }^{13} \mathrm{C}$ WISE NMR under various temperatures for elucidating the conformational heterogeneity and mobility of an ODA surfactant intercalated in $\mathrm{MMT}^{40}$.

In summary, different NMR methodological approaches, including, but not limited to 1D MAS spectra, relaxation time measurement and 2D NMR methods, can be employed to study the structural and dynamical characterizations of polymer matrices, nano-fillers, and the interaction between these two. 


\subsection{SOLID-STATE NMR STUDY OF POLYPHOSPHAZENES AND THEIR 5\% NANO-TiO 2 NANOCOMPOSITES}

The goal of material science is to make materials with desired properties for specific applications. Properties of materials are primarily determined by its phase composition, i.e. its morphology. Therefore, to design materials with desired properties implies the development of methodologies to process materials to attain desired morphologies. Morphological changes can be induced through heat treatment, mechanical stress, exposure to externally applied fields, or nano-filling. These processes lead to changes in the distribution of the crystalline and amorphous phases; ideally in a controlled manner. Most processes rely on external perturbations on the system to affect morphological changes. Nano-filling is inherently different, relying on the polymer-filler interactions to achieve the same goal. The question remains: which particular interactions are important to induce the changes of interest. What are the underlying principles relating the properties of the filler to the induction of phase changes. Detailed structural and dynamic information is required at the molecular level to answer such fundamental questions regarding the mechanism of phase transformations.

Much work has been done to study organic polymers, which are used extensively in industrial applications. However, very little is understood about the morphology of inorganic polymers, such as polyphosphazenes, which have a variety of properties of interest in various fields, such as ionic conductors, biomaterials, or optical and photonic polymers. However, the effect of heat treatment and nano-filling have seen little attention, despite the wide series properties this material offers to material science. Therefore, it is of great interest to develop the fundamental bases behind changing morphologies in these systems and identify the factors that govern them. 
In order to understand how various sample treatments and addition of fillers can achieve a desired morphology, one must understand the phase transformation they introduced at the molecular of detail. Most characterizing methods available are not able to give insight of information at molecular level. X-ray diffraction can be used to obtain information about crystal structures and unit cell dimensions. Differential scanning calorimetry (DSC) can be used to give insights into the kinetics of the phase transitions. However, both methods cannot complete the picture at the required level of detail. NMR spectroscopy can fill this gap by offering knowledge of structural information regardless of phases and mobility. The first objective of this study is to use solid-state NMR spectroscopy as a way to assess the phase structure and transitions in the proposed materials. The second objective is to use the insights from the first part to get information of thermally induced phase transitions of solvent cast materials. Lastly, the effect of nano-filling is investigated on both heat treated and non-heat treated materials to study the effect nanofiller polymer interaction on the morphology in solvent-cast and heat treated materials. Poly[bis(trifluoroethoxy)phosphazene] was chosen for its extremely hydrophobic nature resulting from its hydrophobic side groups. $\mathrm{Nano}_{-} \mathrm{TiO}_{2}$ was choose due to its hydrophilic surface, which is presumed to undergo strong interactions with the polymer backbone. 


\section{References}

1. Flory, P., Principal of Polymer Chemistry. Cornell University: Ithaca, NY, 1953.

2. Volkenstein, M. V., Configuration Statistics of Polymer Chains. Interscience: New York, 1963.

3. Boyer, R. F., Polymer Engineering and Science 1968, 8: 161-185

4. Kroschwith, J. J., Concise Encyclopedia of Polymer Science and Technology. Wiley: New York, 1990.

5. Veeman, W. S., Progress in Nuclear Magnetic Resonance Spectroscopy 1984, 16: 193-235

6. Möller, M., Advances in Polymer Science 1985, 66: 59-80

7. $\quad$ Voelkel, R., Angewandte Chemie 1988, 27:1468-1483

8. Einst, R. R.; Bodenhausen, G.; Wokaun, A., Principles of Nuclear Magnetic Resonance in One and Two Dimensions. Clarendon: Oxford, 1987.

9. $\quad$ Schaefer, J.; Sefcik, M. D.; Stejskal, E. O.; Mckay, R. A.; Dixon, W. T.; Cais, R. E., Macromolecules 1984, 17: 1107-1117

10. BlÜmich, B.; Spiess, H. W., Angewandte Chemie 1988, 27: 1655-1672

11. Hagemeyer, A.; Schmidt-Rohr, K.; Spiess, H. W., Magnetic Resonance Chemistry in Chemistry 1989, 13: 183-194

12. Abragam, A., The Principles of Nuclear Magnetism. Clarendon: Oxford, 1961.

13. Havens, J. R.; VanderHart, D. L., Macromolecule 1985, 18: 1663-1673

14. Caravatti, P.; Neuenschwander, P. E., R. R., Macromolecule 1986, 19: 1889-1895

15. Schmidt-Rohr, K.; Clauss, J.; Bittmich, B.; Spiess, H. W., Magnetic Resonance Chemistry in Chemistry 1990, 28: 53-59

16. Schmidt-Rohr, K., Abatracts of Papers of American Chmical Society 1994, 208: 111

17. Ritchey, W. M., Private Communications 1987, Cleveland, Ohio, September.

18. Edzes, H. T.; Veeman, W. S., Polymer Bulletin 1981, 5: 255-261

19. Schilling, F. C.; Bovey, F. A.; Tonelli, A. E., Macromolecules 1987, 20: 2954-2956

20. Scheaefer, J.; Garlow, J. R.; Stejskal, E. O., Macromolecules 1987, 20: 1271-1278

21. Flemming, W. W.; Lyerla, J. R.; Yannoni, C. S., NMR and Macromolecules Sequence Dynamics and Domain Structure 1984, American Chemistry Society.

22. Parker, A. A.; Armstrong, G. H.; Hedrick, D. P., Journal of Polymer Science 1993, 47: 1999

23. Farrar; Becker, Pulse and FoUrier Transform NMR. Academic Press: New York, 1971.

24. Cmpbell; Dwek, Biological Spectroscopy. The Benjamin Cummings Publishing Co., Inc: Menlo Park, CA, 1984.

25. Mirau, P.; Bovey, F., Journal of American Chemistry Socience 1986, 108: 5130

26. Grant, D. M.; Harris, R. K., Encyclopedia of Nuclear Magnetic Resonance 2002, Eds, Wiley.

27. Inoue, Y.; Miyauchi, M.; Nakajima, H.; Takashima, Y.; Yamaguchi, H.; Harada, A., Macromolecules 2007, 40: 3256-3262

28. Weldeghiorghis, T. K.; Stueber, D.; Schaefer, J., Journal of Polymer Science B 2008, 46: 1062-1066 
29. Miyoshi, T.; Pascui, O.; Reichert, D., Macromolecules 2004, 37: 6460-6471

30. Becker-Guedes, F.; de Azevedo, E. R.; Bonagamba, T. J.; Schmidt-Rohr, K., Applied Magnetics Resonance 2004, 27: 383-400

31. Spiess, H., Advanced Polymer Science 1985, 66: 23

32. Blumich; Speiss, H., Angewandte Chemie 1988, 27: 1655

33. Hoffmann, J.; Williams, G.; Passaglia, E., Journal of Polymer Science 1966, C14.

34. McBrierty, V. J.; Packer, K. J., Nuclear Magnetic Resonance in Solid Polymers. Cambridge University Press: 1993.

35. Bourbigot, S.; VanderHart, D. L.; Gilman, J. W.; Awad, W. H.; Davis, R. D.; Morgan, A. B.; Wikie, C. A., Journal of Polymer Science Part B: Polymer Physics 2003, $41: 3188$

36. VanderHart, D. L.; A., A.; Gilman, J. W., Macromolecules 2001, 34: 3819

37. Mathias, L. J.; Davis, R. D.; Jarrerr, W. L., Macromolecules 1999, 32: 7958

38. wang, L. Q.; Exarhos, G. J.; Liu, J., Advanced Material 1999, 11: 1331

39. Hou, S. S.; Schmidt-Rohr, K., Chemical Material 2003, 15: 1938

40. wang, L. Q.; Liu, J.; Exarhos, G. J.; Flanigan, K. Y.; Bordia, R., Journal of Physical Chemistry $B$ 2000, 104: 2810 


\section{Chapter 5}

\section{EXPERIMENTAL SECTION}

\subsection{MATERIAL}

Hexachlorocyclotriphosphazene $\left(\mathrm{N}_{3} \mathrm{P}_{3} \mathrm{Cl}_{6}\right)$, trifluoroethanol $\left(\mathrm{CF}_{3} \mathrm{CH}_{2} \mathrm{OH}\right)$, diethylene glycol methyl ether/methoxyethoxy ethanol $\left(\mathrm{CH}_{3} \mathrm{OCH}_{2} \mathrm{CH}_{2} \mathrm{OCH}_{2} \mathrm{CH}_{2} \mathrm{OH}\right)$, and nano- $\mathrm{TiO}_{2}(20 \mathrm{~nm})$ were purchased from Sigma-Aldrich and used as received. Sodium metal was obtained from Sigma-Aldrich and was stored in an MBraun glovebox under an argon atmosphere to avoid surface oxidation. All solvents were dried and purified via an MBraun MB SPS solvent purification system. All moisture or air sensitive reactions were carried out using common Schlenk apparati, high vacuum line or in an MBraun glovebox. The custom-made double vacuum line was equipped with an Edwards RV 12 pump and was operated with dry argon.

\subsection{SAMPLE PREPARATION}

\subsubsection{Synthesis of PBFP via Ring-Opening Polymerization of Hexachlorocyclotriphosphazene}

Hexachlorocyclotriphosphazene $\mathrm{e}^{1-2}(0.5 \mathrm{~g})$ was recrystallized from heptane $(3 \mathrm{~mL})$ in a glovebox and then filtered and recrystallized at $-35^{\circ} \mathrm{C}$ for 12 hours. This process was repeated 3 times. The recrystallized trimer was then dried under dynamic vacuum and sublimed under vacuum at a temperature between 40 to $60^{\circ} \mathrm{C}$ for 8 hours.

Purified hexachlorocyclotriphosphazene $(1.23 \mathrm{~g})$ was loaded into a Pyrex polymerization tube in a glovebox. The tube was then evacuated for 30 minutes, flame sealed and placed in an oven which had been preheated to $250^{\circ} \mathrm{C}^{3}$. The oven configuration is depicted in Figure 5.1, the set-up was specifically designed to accommodate this type of 
polymerization. The polymerization tube was clamped onto the platform which was constantly agitated throughout the entire polymerization process to ensure homogeneity of the propagating polymeric chains and to minimize cross-linking. The speed of the electric motor was regulated with a rheostat.

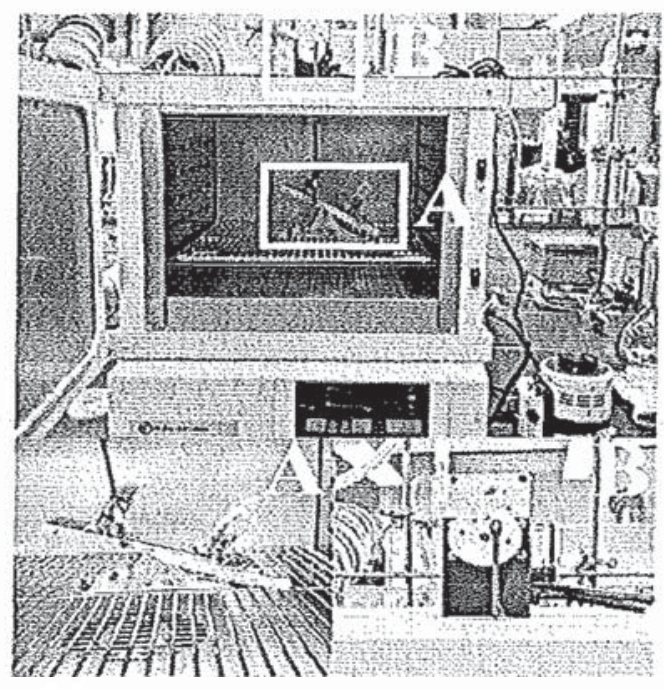

Figure 5.1. Design of the oven used for the thermal ring-opening polymerization. Expansions show a platform with a Pyrex polymerization tube containing poly[dichlorophosphazene] (A) and an electric motor (B)

As mentioned in the background, chain propagation during ring-opening polymerization is a very complicated process ${ }^{4}$. The reaction has to be terminated before cross-linking occurs $^{5}$. Thus, the ROP process must be conducted under constant observation. Since the ROP is controlled via the changing of viscosity of the phosphazene melt, changes viscosity were visually monitored every hour for the first 3 hours, followed by 30 minute intervals for 2 hours, then at 15 minute intervals, and finally at 5 minute intervals. The reaction was terminated when there was almost no fluidity, forming a mixture of unreacted cyclic trimer, oligomers, high molecular weight polymer and some cross-linked species. 
The polymerization tube was then cooled down to ambient temperature and brought into a glove box. The mixture was then transferred to a $250 \mathrm{~mL}$ three-necked roundbottomed flask equipped with a stir bar. This round bottomed flask was then brought out of the glove box and connected to the vacuum line. Dichloromethane $(20 \mathrm{~mL})$ was transferred to this flask under vacuum. This mixture of polymer and dichloromethane was then allowed to dissolve with stirring for 4 hours $^{6}$.

This resulted poly(dichlorophosphazene), which is a translucent, rubbery, air sensitive material which undergoes rapid hydrolysis of the $\mathrm{P}-\mathrm{Cl}$ bonds if it comes into contact with moisture, resulting in a cross-linked polymer matrix which is highly insoluble in organic media and therefore unsuitable for further substitutions ${ }^{7}$. To avoid this, chlorinated polymer side-chains were substituted with trifluoroethoxy units under dry atmosphere without delay, via reacting the polymer with a solution of sodium trifluoethoxide in 1,4 -dioxane at $0^{\circ} \mathrm{C}$.

The sodium trifluoroethoxide was prepared by cutting off the thin layer of oxidized surface of sodium metal $(0.5 \mathrm{~g}$ pure metal) and then loading it into a $150 \mathrm{~mL}$ two-neck round-bottomed flask equipped with a stirring bar. This was done inside a glove-box. This flask was then brought out and connected to a vacuum line, to which 1,4 dioxane was added under reduced. To this stirred mixture, 2,2,2-trifluoroethanol $(1.6 \mathrm{~mL})$ was slowly added dropwise using a syringe in order to keep the reaction temperature from going too high. After the addition was complete, the reaction mixture was left to stir for 10 hours under argon.

This mixture was then transferred to the polymer solution via cannula. This mixture was left to react for 10 hours. After this point, the solvent was removed under rotary 
evaporation to thicken the product. The resulting thick solution was washed with water to remove occluded sodium chloride by filling an Erlenmeyer flask three quarters full of distilled water. With vigorous stirring, the thick solution was transferred to this flask using a Pasteur pipette. The polymer membrane was then brought out and put into a new flask, and dried under vacuum for 30 minutes. The polymer was then dissolved in $5 \mathrm{~mL}$ of THF and precipitated via continuous stream addition into $200 \mathrm{~mL}$ of stirring hexane. Further removal of excess sodium trifluoroethoxide, low molecular weight oligomers and residue organic solvents was achieved by repeating the precipitation twice with water and twice more with hexane. The resulting product was then dried under vacuum for 1 day, resulting in a yellow-white flexible polymer.

\subsubsection{Preparation of The Solvent-Cast Sample}

A viscous solution of PBFP $(0.2 \mathrm{~g})$ in THF $(5 \mathrm{~mL})$ was poured onto a $10 \times 10 \mathrm{~cm}$ glass plate (the glass plates had been cleaned thoroughly prior to use. The plate was rinsed

in ethanol, distilled water, and finally acetone and dried in air inside a fumehood). The THF solvent was then allowed to evaporate under ambient conditions inside the fumehood, the resulting polymer film was then removed from the glass plate using a razor blade and then cut into pieces to fit into a $2.5 \mathrm{~mm}$ Vespel rotor. The same procedure was performed for all PBFP samples studied.

\subsubsection{Preparation of $\mathrm{NanoTiO}_{2} / \mathrm{PBFP}$ Nanocomposites}

Nanocomposite $\left[\mathrm{PBFP} /\left(\mathrm{TiO}_{2}\right) \mathrm{x}\right]$ membranes with $\mathrm{TiO}_{2}$ weight percent at $5 \%$ were prepared by the following general solvent casting procedure ${ }^{8}$. 
PBFP (0.1094) was dissolved in THF (5 mL) to make a viscous mixture, then nano$\mathrm{TiO}_{2}(0.0006 \mathrm{~g}, 5 \%)$, was dissolved in THF $(5 \mathrm{~mL})$. The two mixtures were mixed together and homogenized under fast stirring for $10 \mathrm{~h}$. Then the resulting mixture was poured into a Petri dish, and the solvent was evaporated inside a fumehood to afford thin membranes. The resulting nanocomposite was cut to fit a $2.5 \mathrm{~mm}$ Vespel rotor.

\subsubsection{Preparation of Heat-Treated Samples}

Annealed $\mathrm{PBFP}$, and $\mathrm{PBFP} / \mathrm{TiO}_{2}$ nanocomposites were prepared through solvent casting and then heated in a preheated stove at $220^{\circ} \mathrm{C}$ for 2 hours.

\subsection{NMR EXPERIMENTS}

\subsubsection{Solution NMR}

All solution-state NMR spectra were acquired at ambient temperature $\left(25^{\circ} \mathrm{C}\right)$ using a Bruker Avance 300 NMR spectrometer operating at a magnetic field strength of 7.05 Tesla, giving a Larmor frequency of $300.131 \mathrm{MHz}$ for ${ }^{1} \mathrm{H}$ and $75.468 \mathrm{MHz}$ for ${ }^{13} \mathrm{C}$, outfitted with a $5 \mathrm{~mm} \mathrm{HX}$ PABBO $\mathrm{BB}$ probe. $\mathrm{PBFP}$ was dissolved in deuterated tetrahydrofuran (THF-d8) to a concentration of $1 \mathrm{mg} / \mathrm{mL}$ using tetramethylsilane (TMS) as first order reference for both ${ }^{13} \mathrm{C}$ and ${ }^{1} \mathrm{H}$ nuclei. The $1 \mathrm{D}{ }^{1} \mathrm{H}$ spectrum was recorded with a $90^{\circ}$ pulse width of $12.4 \mu \mathrm{s}$, a recycle delay of $1.0 \mathrm{~s}$, and 128 transients. The $1 \mathrm{D}{ }^{13} \mathrm{C}$ DEPT 135 spectrum was recorded with a $90^{\circ}$ pulse width of $7.6 \mu \mathrm{s}$, a recycle delay of $2.0 \mathrm{~s}$, and 8464 transients. 


\subsection{2 ${ }^{1} \mathrm{H},{ }^{19} \mathrm{~F},{ }^{31} \mathrm{P}$ Solid-State NMR}

All samples were cut into small pieces before loaded into rotors to ensure homogeneous distribution and stable spinning. All soli-state NMR spectral and relaxation data were obtained using a Varian INOVA 500 spectrometer operating at $499.84 \mathrm{MHz}$, $470.332 \mathrm{MHz}$ and $202.34 \mathrm{MHz}$ for ${ }^{1} \mathrm{H},{ }^{19} \mathrm{~F}$ and ${ }^{31} \mathrm{P}$, respectively, equipped with a fourchannel HFXY MAS NMR probe with a $2.5 \mathrm{~mm}$ outer rotor diameter for room temperature experiments, and a probe with $4 \mathrm{~mm}$ rotor for variable temperature experiments. All DP NMR experiments were carried out at an MAS rate of $23 \mathrm{kHz}$. Variable-temperature experiments were carried out at a $10 \mathrm{kHz}$ spinning rate. The ${ }^{19} \mathrm{~F}$ NMR spectra were referenced to external hexafluorobenzene by setting this signal to $-166.4 \mathrm{ppm} .{ }^{1} \mathrm{H}$ and ${ }^{13} \mathrm{C}$ were referenced externally to adamantane by setting the ${ }^{1} \mathrm{H}$ peak to $1.63 \mathrm{ppm},{ }^{13} \mathrm{C}$ to 38.5 ppm. ${ }^{31} \mathrm{P} \mathrm{NMR}$ spectra were referenced to $85 \% \mathrm{H}_{3} \mathrm{PO}_{4}$ by setting its signal to $0.0 \mathrm{ppm} .90^{\circ}$

pulse lengths for ${ }^{1} \mathrm{H},{ }^{19} \mathrm{~F}$, and ${ }^{31} \mathrm{P}$ direct polarization (DP) experiments were calibrated with relaxation delays of 5,5 , and $60 \mathrm{~s}$, respectively 9

All NMR spectra were processed and deconvolved using Mestrelab Research S.L. MestReNova 5.2.5. Chemical structures and reaction schemes were created in CambridgeSoft ACD/labs Chemsketch. Schemes and figures were created in Adobe Illustrator CS4. Numerical data processing and plotting was processed using Microsoft Excel 2011. The references were created with EndNote $\mathrm{X}_{6}$ in Microsoft word.

\subsubsection{1 ${ }^{1}$ H DP MAS NMR spectroscopy of PBFP, annealed PBFP, NC 5\% from ring opening polymerization and PBFP from ambient temperature polymerization}

${ }^{1} \mathrm{H}$ DP solid-state NMR experiments were executed on a Bruker AV 500 spectrometer at a $\mathrm{H}$ Larmor frequency of $500.13 \mathrm{MHz}$. A $2.5 \mathrm{~mm}$ diameter $\mathrm{ZrO}_{2}$ rotor was 
used for four samples spun at $23 \mathrm{kHz}$. A recycle delay of $4 \mathrm{~s}$ was used. The spectra were externally referenced to adamantane (1.63 ppm for $\left.{ }^{1} \mathrm{H}\right)$. Solid state ${ }^{1} \mathrm{H}$ NMR spectra were acquired with a $90^{\circ}$ pulse length of $2.5 \mu \mathrm{s}$.

\subsubsection{2 ${ }^{19}$ F DP MAS NMR spectroscopy of PBFP, annealed PBFP, NC 5\% from ring} opening polymerization and PBFP from ambient temperature polymerization

${ }^{19} \mathrm{~F}$ DP solid-state NMR experiments were acquired with a $90^{\circ}$ pulse length of 3.5 $\mu \mathrm{s}$, and referenced to $\mathrm{C}_{6} \mathrm{~F}_{6}$ (166.4 $\mathrm{ppm}$ for ${ }^{19} \mathrm{~F}$ NMR). The spectra were collected with 16 scans. The sweep width was $500 \mathrm{kHz}$ and recycle delay was $5 \mathrm{~s}$.

\subsubsection{3 ${ }^{31}$ P DP MAS NMR spectroscopy of PBFP, annealed PBFP, NC 5\% from ring} opening polymerization and PBFP from ambient temperature polymerization

${ }^{31} \mathrm{P}$ DP solid-state NMR data were acquired using a $90^{\circ}$ pulse length of $5.5 \mu \mathrm{s}$, and referenced to $\mathrm{H}_{3} \mathrm{PO}_{4}\left(0 \mathrm{ppm}\right.$ for $\left.{ }^{31} \mathrm{P}\right)$. The spectra were acquired with $16 \mu$ s scans with a recycle delay of $60 \mathrm{~s}$, the sweep width was set at $500 \mathrm{kHz}^{10}$.

\subsubsection{VT Solid-State NMR Relaxation Times of PBFP/ Annealed PBFP/NC 5\%/ Annealed NC 5\%}

\subsubsection{1 ${ }^{19} \mathrm{~F}$ V'T relaxation data}

${ }^{19} \mathrm{~F}$ VT NMR relaxation data were obtained using a VARIAN INOVA $500 \mathrm{MHz}$ spectrometer operating in a triple channel HFP mode using a $4 \mathrm{~mm}$ probe. Vespel rotors were equipped with Kel-F turbine caps, inserts, and end caps. Samples were spun at a magic angle spinning rate of $10 \mathrm{kHz}$. All spectra were obtained using a sweep width of $500 \mathrm{kHz}$ wide, data points were zero-filled to $64 \mathrm{k}$ data points, unless stated otherwise.

All relaxation data were acquired over a temperature range from 20 to $80^{\circ} \mathrm{C}$. $\mathrm{T}_{1}$ 
relaxation times were obtained using the inversion recovery pulse sequence with a 90 degree pulse length of $3 \mu$ s and $P_{180}$ pulse length of $6 \mu$ s, the recycle delay used was $4 \mathrm{~s}$. The delay array used was $0,0.05,0.1,0.15,0.2,0.25,0.3,0.35,0.4,0.45,0.5,0.6,0.7,0.8$, $0.9,1,1.5,2,2.5,3,4,5$ in $\mathrm{s}$ with 8 scans at each delay.

${ }^{19} \mathrm{~F} \mathrm{~T}_{2}$ relaxation data were obtained using the Hahn-echo pulse sequence with a $3 \mu \mathrm{s}$ 90 degree pulse. The recycle delay was $4 \mathrm{~s}$ and with total 8 scans at each delay. The delay array used was: $0,300,500,800,1000,1300,1500,1800,2000,2500,3000,4000,5000$, 6000,7000 , and 8000 in micros.

${ }^{19} \mathrm{~F} \mathrm{~T}_{1 \rho}$ relaxation data were obtained using a $3 \mu \mathrm{s} 90$ degree pulse and a spin locking power of $71 \mathrm{~Hz}$. The recycle delay was $4 \mathrm{~s}$ and with total 8 scans. The delay array used were: $0,100,300,500,700,900,1000,1100,1300,1500,1700,1900,2000,2500,3000$, $4000,5000,6000,7000$, and 8000 in micros.

\subsubsection{2 ${ }^{31} \mathrm{P}$ VT relaxation data of four samples}

${ }^{31} \mathrm{P}$ VT NMR relaxation data were obtained using a VARIAN INOVA $500 \mathrm{MHz}$ spectrometer operating using a triple channel HFP mode using a $4 \mathrm{~mm}$ probe. Vespel rotors equipped with Kel-F turbine caps, inserts, and end caps. Samples were spun at magic angle at a spinning rate of $10 \mathrm{kHz}$. All spectra were obtained using a sweep width of $500 \mathrm{kHz}$ wide; data points were zero-filled to $64 \mathrm{k}$ data points, unless stated otherwise. All relaxation data were acquired over the temperature range from $20^{\circ} \mathrm{C}$ to $80^{\circ} \mathrm{C}$.

${ }^{31} \mathrm{P} \mathrm{T}_{1}$ relaxation was obtained using the inversion recovery pulse sequence with a 90 degree pulse at $3.2 \mu \mathrm{s}$ and $\mathrm{P}_{180}$ pulse length at $6.5 \mu \mathrm{s}$, the recycle delay used was $60 \mathrm{~s}$. delay array used was $0,0.2,0.4,0.6,0.8,1,1.5,2,2.5,3,4,5,6,7,8,9,10,15,20,30,40$, and 60 in s with 8 scans at each delay. 
${ }^{31} \mathrm{P}_{2}$ relaxation data were obtained using the Hahn-echo pulse sequence with a 3.2 $\mu$ s 90 degree pulse. The recycle delay was $60 \mathrm{~s}$ and with total 8 scans. The delay array was: $0,100,200,400,500,700,1000,1200,1400,1600,1800,2000,2200,2500,3000,4000$, 6000 , and 8000 in micros.

${ }^{31} \mathrm{P}_{1 \rho}$ relaxation data were obtained using a $3.2 \mu$ s 90 degree pulse and spin locking power of $77 \mathrm{~Hz}$. The recycle delay was $60 \mathrm{~s}$ and with total $8 \mathrm{scans}$. The delay for the sharp signal's tof was set at $3340.0 \mathrm{~Hz}$ with an array: $0,50,100,200,400,600,800,1000,1500$, $2000,3000,4000,6000$, and $8000 \mu$ s. The time delay for the broad signal's tof was set at $3340.0 \mathrm{~Hz}$ with an array: $0,10,20,40,60,80,1000,150,200,250,300,350,400$, and 500 in micros.

\subsubsection{Solid state ${ }^{1} \mathrm{H} \rightarrow{ }^{31} \mathrm{P} /{ }^{19} \mathrm{~F} \rightarrow{ }^{31} \mathrm{P}$ CP MAS NMR spectroscopy study of PBFP/ anmealed PBFP and NC 5\%/annealed NC 5\%}

All CP data were obtained using INOVA $500 \mathrm{MHz}$ NMR spectrometer operating in a triple channel HFP mode using a $4 \mathrm{~mm}$ probe. Vespel rotors were equipped with KelF turbine caps, inserts, and end caps. Samples were spun at the magic angle at a spinning rate of $10 \mathrm{kHz}$. All spectra were obtained using a sweep width of $500000.0 \mathrm{~Hz}$ wide, data points were zero-filled to $64 \mathrm{k}$ data points.

The NMR $\mathrm{T}_{\mathrm{I} \rho}$ values of ${ }^{\mathrm{I}} \mathrm{H}$ and ${ }^{19} \mathrm{~F}$ were measured by variable spin-lock time experiments. All CP experiments were carried at $20^{\circ} \mathrm{C}$, the ${ }^{19} \mathrm{~F} \rightarrow{ }^{31} \mathrm{P} \mathrm{CP}$ experiments were acquired without proton decoupling, and also, ${ }^{1} \mathrm{H} \rightarrow{ }^{31} \mathrm{P}$ experiments were acquired without fluorine decoupling. ${ }^{19} \mathrm{~F}$ NMR spectra were recorded with $\pi / 2$ pulses of $4.5 \mu$ s, and a relaxation delay of $4 \mathrm{~s}$ was used. The ${ }^{19} \mathrm{~F}$ to ${ }^{31} \mathrm{P}$ contact time array used was: $200,300,400$, $500,750,1000,1500,2000,3000,4000,5000,6000,7000$, and $8000 \mu$ s for all four samples. 
Similarly, ${ }^{1} \mathrm{H}$ spectra were recorded with $\pi / 2$ pulses of $3 \mu \mathrm{s}$, and a relaxation delay of $4 \mathrm{~s}$ was used. The ${ }^{1} \mathrm{H}$ to ${ }^{31} \mathrm{P}$ CP contact time array used was: $200,300,400,500,750,1000$, $1500,2000,3000,4000,5000,6000,7000$, and $8000 \mu$ s for all four samples. The evolutions of fluorine and proton magnetizations were observed as a function of the contact time, $t_{c p}$, in each experiments, separately.

\subsection{CALCULATIONS AND DECONVOLUTIONS}

The models for all ${ }^{19} \mathrm{~F}$ and ${ }^{31} \mathrm{P}$ NMR spectra were found and analyzed using MestreNova. The percentage areas of components were calculated using percentage of the total areas of all components. The plots of the each component percentage were plotted in Excel. All the $\mathrm{T}_{1}$ relaxation times were calculated using the equations below:

$$
\begin{gathered}
M_{\tau}=M_{0}\left(1-2 \exp \left(-\tau / T_{1}\right)\right) \\
\text { Slope }=-1 / T_{1}
\end{gathered}
$$

all the $\mathrm{T}_{2}$ were calculated using:

$$
\begin{array}{r}
M_{\tau}=M_{0}\left[\exp \left(-\tau / T_{2}\right)\right. \\
\text { Slope }=-1 / T_{2}
\end{array}
$$

And the $\mathrm{T}_{\mathrm{l} \rho}$ were calculated using:

$$
\begin{array}{r}
M_{\tau}=M_{0}\left[\exp \left(-\tau / T_{1 \rho}\right)\right. \\
\text { Slope }=-1 / T_{1 \rho}
\end{array}
$$

All of those calculations were done in Excel, the error analysis for $T_{1}, T_{2}, T_{1 p}$ are done in Excel using linear regression.

The activation energies were calculated using the equation of:

$$
\ln \frac{\mathrm{k}_{2}}{\mathrm{k}_{1}}=-\frac{\mathrm{B}_{\mathrm{a}}}{\mathrm{R}}\left(\frac{1}{\mathrm{Temp}_{2}}-\frac{1}{\mathrm{Temp}_{1}}\right)
$$


where $\mathrm{T}_{\text {empl }}$ is the temperature at one time point, $\mathrm{T}_{\text {emp2 }}$ is the temperature another time point with a unit of $\mathrm{K} . \mathrm{k}_{\mathrm{l}}$ equal $1 / \mathrm{T}_{1}\left(\mathrm{~T}_{1}\right.$ is the relaxation time constant at the first temperature), $k_{2}$ is equal to $1 / T_{2}\left(T_{2}\right.$ is the same relaxation time constant at the second temperature) in unit of $s^{-1}$.

The relaxation time constants $\left(\mathrm{T}_{1}, \mathrm{~T}_{2}, \mathrm{~T}_{1 \rho}\right)$ are used as the rate $\mathrm{k}$ in the activation equation5.4. For relaxation time constants that increase with temperature, positive activation energies result; while for relaxation time constants that decrease with temperature give negative activation energies. Since all activation energies must be positive, a positive value means the motion that drives the relaxation of the nucleus is in the fast motion regime, while negative values suggests the motion is in the slow motion regime with respect to its Larmor frequency.

The error for activation energy is computed from the errors in the relaxation times as follows:

$$
\Delta \mathrm{E}_{\mathrm{a}}=\sqrt{\left(\frac{\Delta \mathrm{T}_{1}}{\mathrm{~T}_{1}}\right)^{2}+\left(\frac{\Delta \mathrm{T}_{2}}{\mathrm{~T}_{2}}\right)^{2}} * 0.4343 * \mathrm{E}_{\mathrm{a}}
$$

Where $T_{1}$, and $T_{2}$ are the relaxation times, the $\Delta T_{1}$ and $\Delta T_{2}$ are the corresponding relaxation time constants errors. 


\section{Reference}

I Allcock, H. A., Journal of Inorganic and Organometalic polymer and Materials 2006, 16: 277-294.

2 Allcock, H. R.; Kugel, R. L.; Valan, K. J., Inorganic chemistry 1966, 5: 1709-1715.

3 Kilic, A., Journal of Qafqaz University 2000, 3: 133-148.

4 Allcock, H. R.; Kugel, R. L.; Valan, K. J., Inorganic Chemistry 1966, 5: 1711-1715.

5 Bose, S.; Pramanik, N.; Das, C. K.; Ranjan, A.; Saxena, A. K., Material and Design 2010, 31: 1148-1155.

6 Michael, L. W.; Krzysztof, M., Macromolecular Chemistry and Physics 1996, 198: 665-671.

7 A. S. Borisov, P. Hazendonk, P. G. Hayes, Journal of Inorganic and Organometalic. Polymer, Materials 2010, 20: 395-398

8 Allcock, H. R., Applied Organometallic Chemistry 1998, 12: 659-666.

9 Ghassemzadeh, L.; Pace, G.; Noto, V. D.; Muller, K., Physical Chemistry Chemical Physics 2011, 12: 9327-9334.

0 C. C. Sun; P. Hazendonk; A. S. Borisov; P. G. Hayes, Material Science and Technology 2013, 704: 229-234 


\section{Chapter 6}

\section{SOLUTION NMR SPECTROSCOPY OF POLY[BIS(TRIFLUOROETHOXY)PHOSPHAZENE]}

Previous work in characterizing the structure of PBFP was mainly dependent on proton solid-state NMR spectroscopy, which gave broad resonance due to slow motion in the solid state. Very limited work has been done on solution state. In this study, both 1D solution NMR spectra of PBFP in THF $\mathrm{d} 8$ and trifluoroethanol in $\mathrm{CDCl}_{3}$ were acquired to determine the chemical shift of the NMR signal in the solution state'.

\subsubsection{Solution NMR Spectra of $\mathrm{CF}_{3} \mathrm{CH}_{2} \mathrm{OH}$ in $\mathrm{CDCl}_{3}$}

In Figure 6.1 appears the ${ }^{1} \mathrm{H}$ DP NMR spectrum of trifluoroethanol. The peak at 2.8 ppm is assigned to the $-\mathrm{OH}$ group, the other peak near $3.95 \mathrm{ppm}$ is assigned to the $-\mathrm{CH}_{2}$ group, it appears as a quartet $(1: 3: 3: 1)$ due to $\mathrm{J}$-coupling to the three fluorines of the $\mathrm{CF}_{3}$ group, with a coupling constant of $8.64 \mathrm{~Hz}$, which is in agreement with in literature ${ }^{2}$.

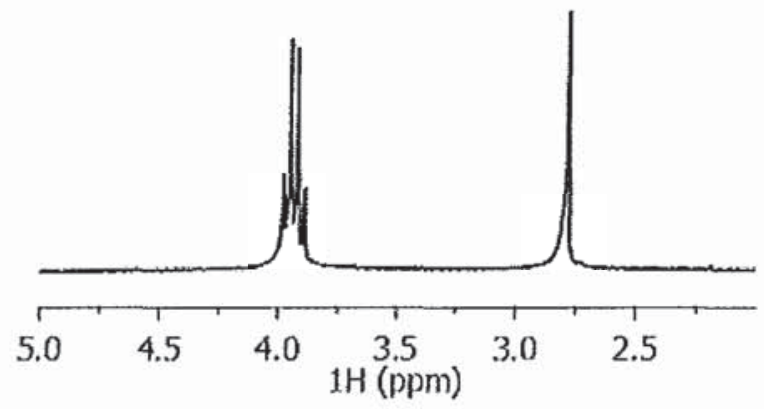

Figure 6.1: $\quad$ ' $\mathrm{H}$ DP NMR spectrum of $\mathrm{CF}_{3} \mathrm{CH}_{2} \mathrm{OH}$ in $\mathrm{CDCl}_{3}$

Figure 6.2 illustrates the ${ }^{1} \mathrm{H}$ NMR solution spectrum of trifluoroethanol with ${ }^{19} \mathrm{~F}$ decoupling. The peak at $2.8 \mathrm{ppm}$ is again from the $-\mathrm{OH}$ group. The other peak at $3.95 \mathrm{ppm}$ is a singlet now due to ${ }^{19} \mathrm{~F}$ decoupling. This proves that the quartet in Figure 6.1 is due to 
$\mathrm{J}$-coupling between ${ }^{1} \mathrm{H}$ and ${ }^{19} \mathrm{~F}$, because it collapses to a singlet when ${ }^{19} \mathrm{~F}$ decoupling is applied.

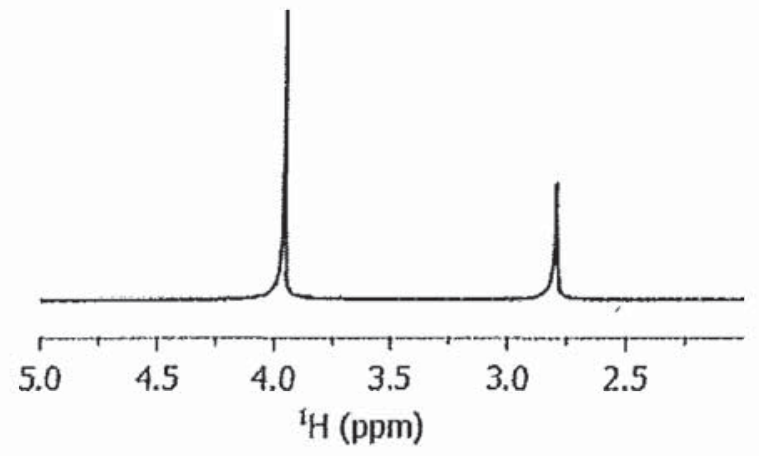

Figure 6.2: $\quad{ }^{1} \mathrm{H}\left\{{ }^{19} \mathrm{~F}\right\} \mathrm{NMR}$ spectrum of $\mathrm{CF}_{3} \mathrm{CH}_{2} \mathrm{OH}$ in $\mathrm{CDCl}_{3}$

As shown in Figure 6.3, ${ }^{19} \mathrm{~F}$ DP NMR spectrum has one signal which appears as a (1:2:1) triplet at $-76.42 \mathrm{ppm}$ due to $\mathrm{J}$ coupling to the protons of the $-\mathrm{CH}_{2}$ group with a scalar coupling constant of $8.64 \mathrm{~Hz}$, which is the same value obtained from the ${ }^{1} \mathrm{H}$ DP NMR spectrum.

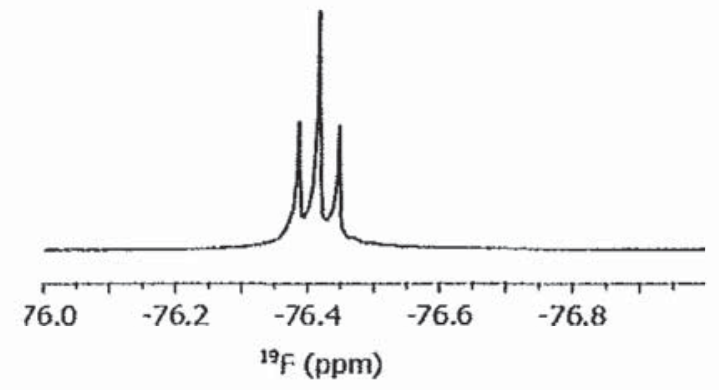

Figure 6.3: $\quad{ }^{19} \mathrm{~F}$ DP NMR spectrum of $\mathrm{CF}_{3} \mathrm{CH}_{2} \mathrm{OH}$ in $\mathrm{CDCl}_{3}$

When proton decoupling is applied, the triplet in the ${ }^{19} \mathrm{~F}$ DP NMR spectrum of Figure 6.3 collapses to a singlet (as depicted in Figure 6.4). It shows that the triplet in Figure 6.3 is due to $\mathrm{J}$ coupling between the fluorines and the two protons of the $-\mathrm{CH}_{2}$ group. 


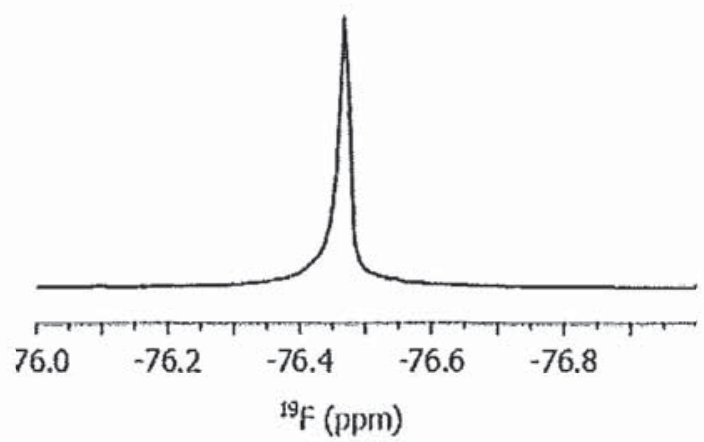

Figure 6.4: $\quad{ }^{19} \mathrm{~F}\left\{{ }^{1} \mathrm{H}\right\}$ NMR spectrum of $\mathrm{CF}_{3} \mathrm{CH}_{2} \mathrm{OH}$ in $\mathrm{CDCl}_{3}$

\subsubsection{Solution NMR Spectra of PBFP in THF-d8}

As mentioned earlier, solution-state NMR of PBFP were not done before, all the spectra are determined for the first time. The ${ }^{1} \mathrm{H}$ DP NMR spectrum of PBFP in THF-d8 is shown in Figure 6.5, the peaks from residual THF appear at $3.58 \mathrm{ppm}$ and $2.00 \mathrm{ppm}^{3}$, the signal at $1.73 \mathrm{ppm}$ is assigned to residual water, and the multiplet at $1.29 \mathrm{ppm}$ is from hexane $\mathrm{s}^{4}$. The peak appears at $-2.80 \mathrm{ppm}$ arises from the acetone used to clean the NMR tube. The ${ }^{1} \mathrm{H}$ signal from PBFP appears at $4.41 \mathrm{ppm}$, with coupling to three fluorines from the trifluoromethyl group resulting in a quartet.

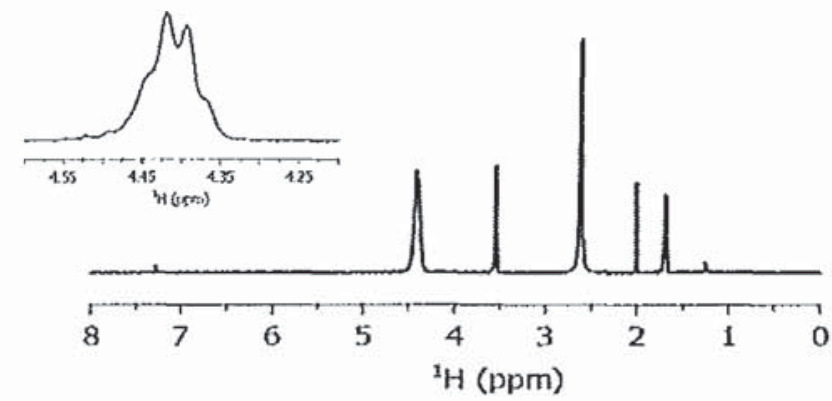

Figure 6.5: $\quad$ 'H DP NMR spectrum of PBFP in THF-d8 
When ${ }^{19} \mathrm{~F}$ decoupling was applied, the quartet at $4.41 \mathrm{ppm}$ in Figure 6.5 collapsed to a singlet as presented in Figure 6.6. This proves that this signal is from the $-\mathrm{CH}_{2}$ group of PBFP and that the quartet splitting is due to J-coupling to the three fluorines of the $-\mathrm{CF}_{3}$ group.

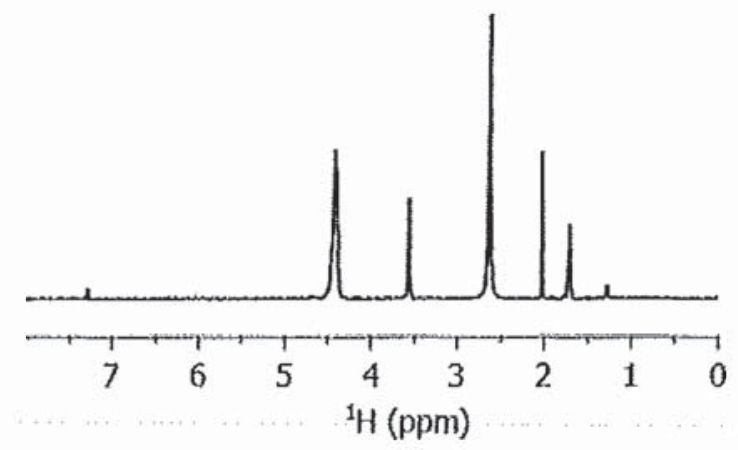

Figure 6.6: $\quad{ }^{1} \mathrm{H}\left\{{ }^{19} \mathrm{~F}\right\}$ spectrum of PBFP in THF-d8

As shown in Figure 6.7, this ${ }^{19} \mathrm{~F}$ DP NMR spectrum has one signal which appears as a (1:2:1) triplet at $-76.9 \mathrm{ppm}$ due to $\mathrm{J}$ coupling to the protons of the $-\mathrm{CH}_{2}$ group. The coupling constant is $8.11 \mathrm{~Hz}$, which is slightly different from the value in trifluoroethanol because the chemical environment is slightly different. In addition, the peaks are broader for PBFP than for trifluoroethanol, because PBFP is a polymer with a broader distribution of environments than trifluoroethanol.

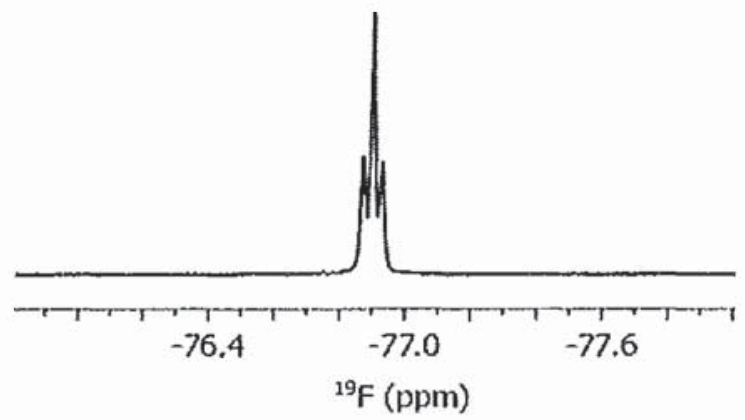

Figure 6.7: $\quad{ }^{19} \mathrm{~F}$ DP NMR spectrum of PBFP in THF- $d 8$ 
When proton decoupling is applied, the triplet in the ${ }^{19} \mathrm{~F}$ DP NMR spectrum of Figure 6.7 collapses to a singlet (as depicted in Figure 6.8). It proves that the triplet in Figure 6.7 is due to $\mathrm{J}$ coupling between the fluorines and the two protons of the $-\mathrm{CH}_{2}$ group.

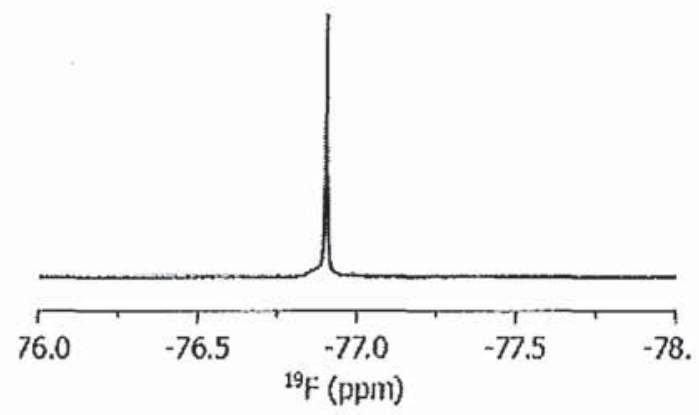

Figure 6.8: $\quad{ }^{19} \mathrm{~F}\left\{{ }^{1} \mathrm{H}\right\}$ NMR spectrum of PBFP in THF-d8

The ${ }^{31} \mathrm{P}$ NMR spectrum of PBFP appears in Figure 6.9. The signal appears as a singlet at $-7.44 \mathrm{ppm}$, and is likely because it is too far away from ${ }^{1} \mathrm{H}$ ( 3 bonds) and ${ }^{19} \mathrm{~F}(4$ bonds) to observe $\mathrm{J}$ coupling to these nuclei.

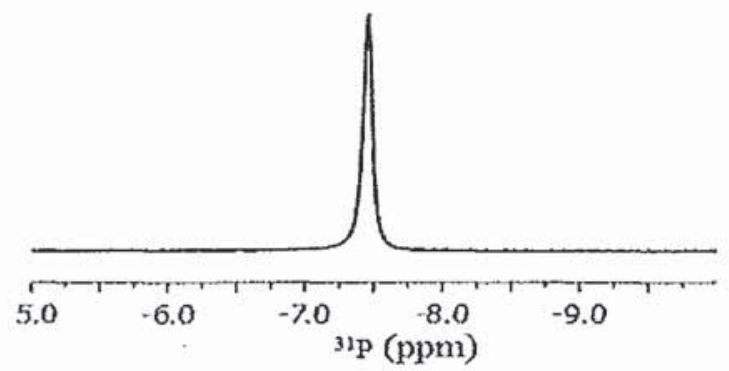

Figure 6.9: $\quad{ }^{31} \mathrm{P}$ DP spectrum of PBFP in THF-d8 


\section{Reference}

1 Stewart, F. F.; Peterson, E. S.; Busse, S. C., Chemistry of Materials 1997, 9: 155-163

2 Albert, M.; Seebach, D.; Duchardt, E.; Schwalbe, H., Helvetica Chimica Acta 2002, 85: 633-658.

3 Gottlieb, H. E.; Kotlyar, V.; Nudelman, A., The Journal of Organic Chemistry 1997, 62: 7512-7515.

4 Fulmer, G. R.; Miller, A. J. M.; Sherded, N. H., Organometallics 2010, 29: 21762179. 


\section{CHAPTER 7}

\section{SOLID-STATE NMR SPECTROSCOPY OF PBFP/NANOCOMPOSITES}

\subsection{Solid-State NMR Spectroscopy of Samples Acquired at an MAS Rate of $23 \mathbf{~ k H z}$ at $20^{\circ} \mathrm{C}$}

${ }^{1} \mathrm{H},{ }^{19} \mathrm{~F},{ }^{31} \mathrm{P}$ MAS NMR spectra of a series of solvent-cast (SC) ring opening polymerization (ROP) PBFP, heat-treated (HT) PBFP, ambient temperature polymerized (ATP) PBFP and nanocomposites of $5 \%$ nano- $\mathrm{TiO}_{2}$ PBFP (NC 5\%) were acquired with an MAS rate at $23 \mathrm{kHz}^{1-2}$. For all spectra, the signals are composed of overlapping signals. Quantitative information about parameters for the signals need to be extracted, via signal deconvolution analysis. The deconvolved components have their individual chemical shifts, line widths, signal intensities, areas and Gaussian/Lorentzian (G/L) fractions. In order to obtain reliable and consistent analyses of the 4 samples, I tried to use the same chemical shift, and line width, and the G/L ratio unless the morphology is significantly changed.

\subsection{1 ${ }^{1} \mathrm{H}$ Solid-State NMR Spectra of Solvent Cast PBFP/ Ambient Temperature $\begin{array}{llll}\text { Polymerization PBFP/Heat Annealed PBFP/5\% Nano-TiO } & \text { PBFP }\end{array}$ Nanocomposite with an MAS $23 \mathrm{kHz}$}

Proton solid-state NMR is a versatile and commonly used NMR experiment to study polymers that contain protons and provide several advantages over that of other nuclei. For one thing, ${ }^{1} \mathrm{H}$ is almost $100 \%(99.985 \%)$ abundant and has the biggest gyromagnetic ratio among all $I=1 / 2$ or stable nuclei, with a value at $267.522 \mathrm{~s}^{-1} \mathrm{~T}^{-1}$. Proton is the nucleus that is most sensitive to NMR signals (except for ${ }^{3} \mathrm{H}$ which is instable and is radioactive and is rarely used). It is common for protons to have strong homonuclear dipolar coupling that is difficult to be efficiently removed by MAS spinning and result in low spectral resolution. 

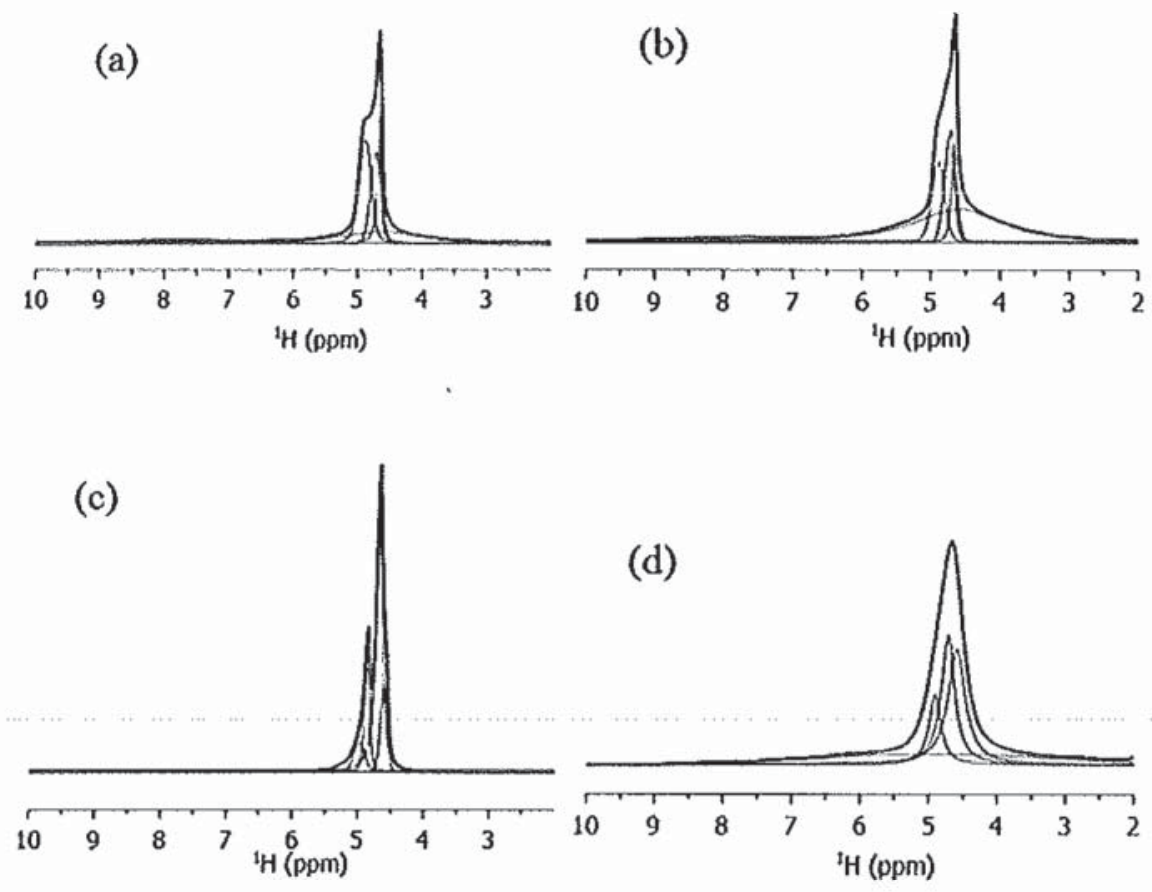

Figure 7.1: (a) SC: $500 \mathrm{MHz}$ 'H NMR spectra of solvent cast ROP PBFP; (b) HT: $500 \mathrm{MHz}$ 'H MAS NMR spectra of heat-treated ROP PBFP; (c) AT: 500MHz 1H MASNMR spectra of AT polymerization PBFP; (d) NC: $500 \mathrm{MHz}{ }^{1} \mathrm{H}$ MAS NMR spectra of ROP PBFP doped with $5 \%$ nc- $\mathrm{TiO}_{2}$. AlI NMR spectra were recorded with an MAS rate of $23 \mathrm{kHz}$.

The ${ }^{1} \mathrm{H}$ MAS NMR spectra of solvent-cast, heat-treated, ambient temperature polymerization, and nano- $\mathrm{TiO}_{2}$ doped $\mathrm{PBFP}$ (NC 5\%) were acquired at an MAS rate of 23 $\mathrm{kHz}$ and the results from the deconvolution are shown in Figure 7.1 and Table $7.1^{1}$, as what already published ${ }^{1}$. The NMR signals from mobile domains tend to give rise to narrow Lorentzian features, while signals from rigid environments result in broad Gaussian lines that are characteristic of crystalline phases. Peaks 2, 3 and 4 in the NMR spectra of the SC and HT samples are narrow Lorentzian signals whose peak areas decrease with heat treatment and therefore correspond to amorphous ${ }^{1} \mathrm{H}$ environments of PBFP. Peak 1 and peak 5 are broad Gaussian signals and increase in area with heat treatment, and thus correspond to crystalline ${ }^{1} \mathrm{H}$ environments. 
The ${ }^{1} \mathrm{H}$ NMR spectrum of ATP PBFP contains four Lorentzian signals (6 to 9) with line widths falling in the range of $40-170 \mathrm{~Hz}$. These siguals are thus assumed to correspond to mobile ${ }^{1} \mathrm{H}$ environments. Peak 10 is Gaussian in shape and $1870 \mathrm{~Hz}$ wide. The total relative area of this crystalline signal is $11.75 \%$, which is significantly reduced compared to SC PBFP, indicating that ATP conditions greatly favour amorphous phase compositions.

The ${ }^{~} \mathrm{H}$ NMR spectrum of the NC $5 \%$ sample contains a broad Gaussian line (Peak 13) with linewidth of $2058 \mathrm{~Hz}$, comprising $31.1 \%$ of the total area. The narrow Lorentzian contributions, Peaks 11, 12 and 14, have widths of 229,117 and $103 \mathrm{~Hz}$, respectively. The crystallinity is significantly lower compared with SC PBFP, suggesting that the crystallization process is dramatically affected by the presence of nc- $\mathrm{TiO}_{2}$.

Table 7.1: $\quad$ Peak parameters of 'H $\quad$ NMR spectra of solvent cast (SC), heat treated (HT), ambient temperature polymerized (ATP) PBFP and PBFP doped with $5 \%$ nano- $\mathrm{TiO}_{2}$ (NC $5 \%$ ) samples obtained with an MAS rate of $23 \mathrm{kHz}$

\begin{tabular}{|c|c|c|c|c|c|c|c|c|c|c|}
\hline Samples & \multicolumn{5}{|c|}{ SC } & \multicolumn{5}{|c|}{ HT } \\
\hline Peaks & 1 & 2 & 3 & 4 & 5 & 1 & 2 & 3 & 4 & 5 \\
\hline $\begin{array}{c}\mathrm{CS} \\
(\mathrm{ppm})\end{array}$ & 4.60 & 4.69 & 4.73 & 4.91 & 7.69 & 4.60 & 4.69 & 4.73 & 4.91 & 7.69 \\
\hline $\begin{array}{c}\text { Width } \\
\text { (Hz) }\end{array}$ & 783 & 33 & 96 & 107 & 1514 & 783 & 33 & 96 & 107 & 1514 \\
\hline Area (\%) & 26.7 & 13.1 & 22.0 & 29.8 & 8.5 & 50.5 & 6.4 & 17.6 & 14.9 & 10.9 \\
\hline Samples & \multicolumn{5}{|c|}{ ATP } & \multicolumn{4}{|c|}{ NC $5 \%$} & \\
\hline Peaks & 6 & 7 & 8 & 9 & 10 & 11 & 12 & 13 & 14 & \\
\hline $\begin{array}{c}C S \\
(p p m)\end{array}$ & 4.60 & 4.69 & 4.86 & 4.89 & 6.58 & 4.60 & 4.73 & 4.90 & 4.91 & \\
\hline $\begin{array}{c}\text { Width } \\
(\mathbf{H z})\end{array}$ & 52.9 & 43.6 & 39.3 & $\begin{array}{c}165 . \\
6 \\
\end{array}$ & 1870 & 152 & 117 & 2058 & 103 & \\
\hline Area (\%) & 18.2 & 36.0 & 15.0 & 19.1 & 11.8 & 30.5 & 26.0 & 31.1 & 12.5 & \\
\hline \multicolumn{10}{|c|}{$\begin{array}{l}\text { Note: } \mathrm{SC}=\text { solvent cast of poly[bis(trifluoroethoxy)phosphazene (PBFP); } \mathrm{HT}=\text { heat } \\
\text { treated solvent cast PBFP; ATP = ambient temperature polymerized PBFP; NC= } \\
\text { solvent casted PBFP with } 5 \% \text { (in wcight) nano-TiO } \mathrm{O}_{2} \text { CS = chemical shift in ppm; } \\
\text { Width = spectral linewidth at half height; Area = percentage area of total spectuum }\end{array}$} & \\
\hline
\end{tabular}




\subsection{2 ${ }^{19} \mathrm{~F}$ Solid-State NMR Spectra of Solvent Cast PBFP/ Ambient Temperature Polymerization PBFP/Heat Annealed PBFP/5\% Nano-TiO 2 PBFP Nanocomposite at an MAS Rate of $23 \mathrm{kHz}$}

Fluorine-19 solid-state NMR spectroscopy is commonly used to study fluorinecontaining polymers for the reason that ${ }^{19} \mathrm{~F}$ is $100 \%$ abundant and has a large gyromagnetic ratio which is only little smaller than ${ }^{1} \mathrm{H}$ with a value of $251.815 \mathrm{~s}^{-1} \mathrm{~T}^{-1}$. Fluorine-19 is a commonly measured spin $\mathrm{I}=\mathrm{I} / 2$ nucleus beside ${ }^{1} \mathrm{H}$ and ${ }^{13} \mathrm{C}$, and it has similar sensitivity as 'H. Fluorine also has strong homonuclear dipolar interaction that requires high MAS spinning (over $23 \mathrm{kHz}$ ) to be efficiently removed. The advantage of ${ }^{19} \mathrm{~F}$ over ${ }^{1} \mathrm{H}$ NMR spectroscopy is the larger ${ }^{19} \mathrm{~F}$ chemical shift range, as a consequence, ${ }^{19} \mathrm{~F}$ is more sensitive to its local environment ${ }^{2}$.
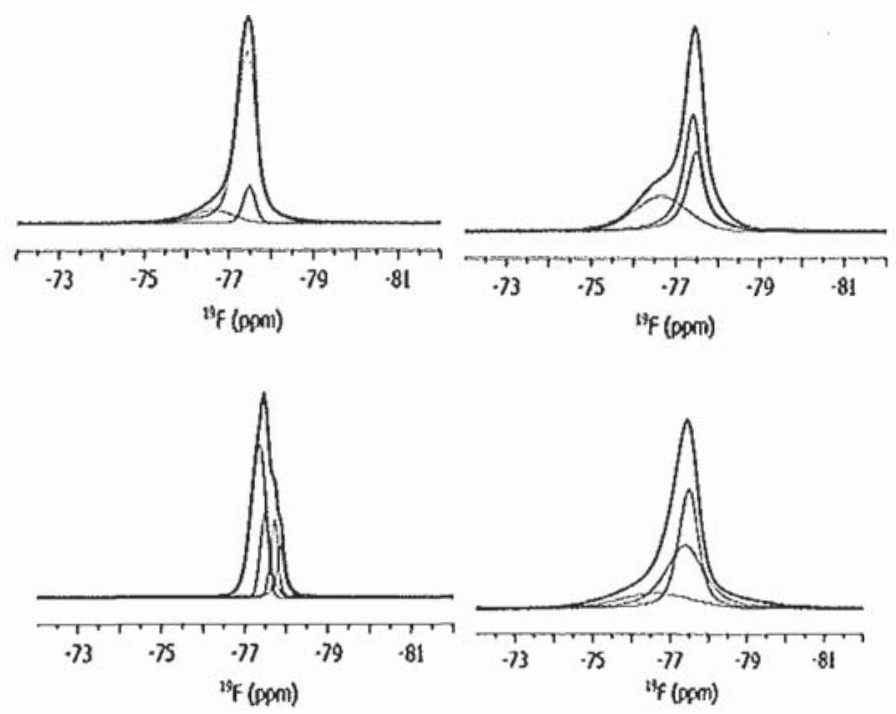

Figure 7.2: $\quad 500 \mathrm{MHz}{ }^{19} \mathrm{~F}$ DP NMR spectrum of (top left) Solvent cast ROP PBFP; (top right) Heat-treated ROP PBFP; (bottom left) Ambient temperature polymerized PBFP; (bottom right) ROP PBFP with $5 \%$ nc- $\mathrm{TiO}_{2}$ obtained at an MAS rate of $23 \mathrm{kHz}$

Fluorine-19 MAS NMR spectra of solvent-cast, heat-treatment, ATP, and nano-TiO 2 doped PBFP are acquired at an MAS rate of $23 \mathrm{kHz}$ and the deconvolution parameters are shown in Figure 7.2. ${ }^{19} \mathrm{~F}$ NMR spectra of the ATP PBFP material was deconvolved into four narrow Lorentzian contributions, indicating that no signal is observed from crystalline 
components. This observation is consistent with the conclusion from ${ }^{1} \mathrm{H}$ NMR spectra that ATP polymerization favors the production of amorphous material. The spectrum of the NC $5 \%$ contains amorphous components, i.e., peaks 2 and 3, which exhibit Lorentzian line shapes and whose combined area comprises $83.1 \%$ of the total area. This proportion is significantly reduced compared to the $\mathrm{SC}$ material. This finding is also consistent with the observation in the ${ }^{1} \mathrm{H}$ NMR spectra that crystallinity is reduced in the presence of nc-TiO2.

Table 7.2. Peak parameters of ${ }^{i \varphi} \mathrm{FNMR}$ spectra of solvent cast (SC), heat treated (HT), ambient temperature polymerized (ATP) PBFP and PBFP doped with $5 \%$ nano- $\mathrm{TiO}_{2}$ (NC $5 \%$ ) samples obtained at an MAS rate of $23 \mathrm{kHz}$

\begin{tabular}{|c|c|c|c|c|c|c|c|}
\hline Samples & \multicolumn{4}{|c|}{ SC } & \multicolumn{3}{|c|}{ HT } \\
\hline Peaks & 1 & 2 & \multicolumn{2}{|c|}{3} & 1 & 2 & 3 \\
\hline CS (ppm) & -76.64 & -77.37 & \multicolumn{2}{|c|}{-77.46} & -76.64 & -77.37 & -77.46 \\
\hline Width (Hz) & 514 & 261 & \multicolumn{2}{|c|}{158} & 697 & 230 & 229 \\
\hline Arca (\%) & 10.6 & 79.9 & \multicolumn{2}{|c|}{9.6} & 30.4 & 41.4 & 28.2 \\
\hline Samples & \multicolumn{4}{|c|}{ AT } & \multicolumn{3}{|c|}{ NC 5\% } \\
\hline Peaks & 1 & 2 & 3 & 4 & 1 & 2 & 3 \\
\hline CS (ppm) & -77.28 & -77.46 & -77.69 & -77.88 & -76.64 & -77.37 & -77.46 \\
\hline Width (Hz) & 197 & 117 & 106 & 84 & 1050 & 558 & 290 \\
\hline Area (\%) & 54.2 & 20.8 & 15.6 & 9.4 & 16.9 & 42.2 & 40.9 \\
\hline
\end{tabular}

Note: $\mathrm{SC}=$ solvent cast of poly[bis(trifluorocthoxy)phosplazene (PBFP); HT = heat treated solvent cast $\mathrm{PBFP} ; \mathrm{ATP}=$ ambient temperature polymerized $\mathrm{PBFP} ; \mathrm{NC}=$ solvent casted PBFP with $5 \%$ (in weight) nano- $\mathrm{TiO}_{2} ; \mathrm{CS}=$ chemical shift in ppm; Width $=$ spectral linewidth at half height; Area = percentage area of total spectrum

\subsection{3 ${ }^{31}$ P Solid-State NMR Spectra of Solvent Cast PBFP/ Ambient Temperature Polymerization PBFP/Heat Annealed PBFP/ 5\% Nano-TiO2 PBFP Nanocomposite at an MAS rate of $23 \mathrm{kHz}$}

${ }^{31} \mathrm{P}$ is another commonly used spin $\mathrm{I}=1 / 2$ nucleus, with a $100 \%$ natural abundance and a high gyromagnetic ratio value at $108.394 \mathrm{~s}^{-1} \mathrm{~T}^{-1} \cdot{ }^{31} \mathrm{P}$ is less sensitive than ${ }^{1} \mathrm{H}$ and ${ }^{19} \mathrm{~F}$ as a medium sensitive nucleus, but its wide chemical shift range makes it sensitive to it local environment ${ }^{3-4}$. 

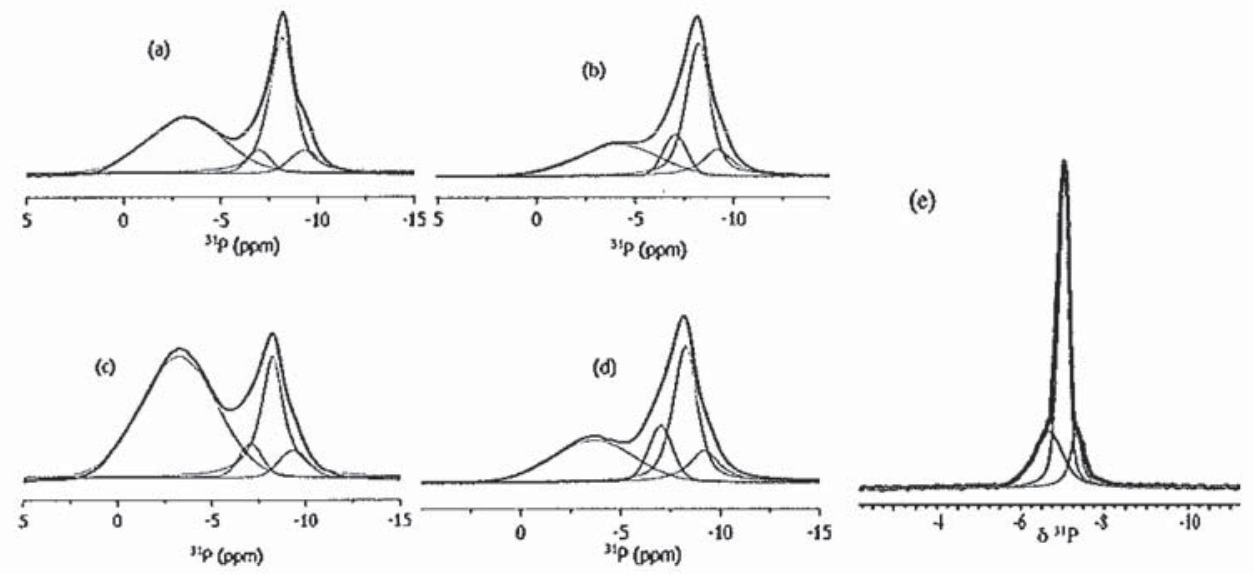

$500 \mathrm{MHz}{ }^{31}$ P DP MAS $23 \mathrm{kHz}$ NMR spectnum of (a) SC: solvent cast ROP PBFP; (b) NC: ROP PBFP with $5 \%$ nc-TiO 2 ; (c) HT: Heat treated ROP PBFP; (d) Heat treated ROP PBFP with $5 \%$ nc-TiO ${ }_{2}$; (c) ATP: ambient temperature polymerized PBFR.

Phosphorus-31 MAS NMR spectra of solvent cast, heat treatment, ATP, and nano$\mathrm{TiO}_{2}$ doped PBFP were acquired at an MAS rate of $23 \mathrm{kHz}$ and deconvolved as shown in Figure 7.3. All of the spectra have poor resolution in comparison to ${ }^{1} \mathrm{H}$ and ${ }^{19} \mathrm{~F}$, due to the high degree of heterogeneity in the backbone environment and residual quadrupolar coupling to ${ }^{14} \mathrm{~N}$. As a result, the ${ }^{31} \mathrm{P}$ NMR spectra of PBFP, HT PBFP, $5 \% \mathrm{nc}^{-\mathrm{TiO}_{2}}$ and HT $5 \% \mathrm{nc}^{-\mathrm{TiO}_{2}}$ reveal 4 components: one broad Gaussian peak between -3.18 and $-4.16 \mathrm{ppm}$ and a set of three closely spaced narrow Lorentzian peaks between -6.63 and $-9.31 \mathrm{ppm}$. The deconvolution results are provided in Table 7.3. The crystallinity is seen to increase from $45 \%$ to $65 \%$ in the SC and HT samples and is much reduced in the ATP material. 
Table 7.3: $\quad$ Peak parameters of ${ }^{31} \mathrm{P}$ NMR spectra of solvent cast PBFP (SC-PBFP), heat treated (HT), ambient temperature polymerized (ATP) PBFP, PBFP doped with $5 \%$ nano- $\mathrm{TiO}_{2}(5 \%$ uc-TiO $)$ and PBFP doped with $5 \%$ nano- $\mathrm{TiO}_{2}$ (NC 5\%) annealed samples obtained at an MAS rate of $23 \mathrm{kHz}$

\begin{tabular}{|c|c|c|c|c|c|c|c|c|}
\hline Samples & \multicolumn{4}{|c|}{ SC-PBFP } & \multicolumn{4}{|c|}{ NC $5 \%$} \\
\hline Peaks & 1 & 2 & 3 & 4 & 1 & 2 & 3 & 4 \\
\hline CS (ppm) & -3.18 & -8.12 & -6.93 & -9.31 & -4.16 & -8.12 & -6.99 & -9.14 \\
\hline Width (Hz) & 968 & 289 & 290 & 368 & 968 & 289 & 290 & 368 \\
\hline Area (\%) & 44.9 & 38.6 & 7.2 & 9.4 & 29.2 & 46.1 & 13.1 & 11.6 \\
\hline Samples & \multicolumn{4}{|c|}{ Heat Treated PBFP } & \multicolumn{4}{|c|}{ Heat Treated NC 5\% } \\
\hline Peaks & 1 & 2 & 3 & 4 & 1 & 2 & 3 & 4 \\
\hline CS (ppm) & -3.20 & -8.15 & -7.03 & -9.30 & -3.61 & -8.14 & -6.95 & -9.17 \\
\hline Width (Hz) & 968 & 289 & 290 & 368 & 968 & 289 & 290 & 368 \\
\hline Area $(\%)$ & 65.0 & 21.0 & 7.5 & 6.5 & 35.6 & 39.6 & 15.2 & 9.7 \\
\hline Samples & \multicolumn{3}{|c|}{ ATP } & & & & & \\
\hline Peaks & 1 & 2 & 3 & & & & & \\
\hline CS (ppm) & -6.63 & -6.99 & -7.38 & & & & & \\
\hline Width (Hz) & 412 & 133 & 177 & & & & & \\
\hline Area $(\%)$ & 28 & 57.8 & 14.2 & & & & & \\
\hline \multicolumn{4}{|c|}{$\begin{array}{l}\text { Note: SC }=\text { solvent cast of } \\
\text { poly[bis(trifluoroethoxy)phosphazene } \\
\text { (PBFP); HT = heat treated solvent cast } \\
\text { PBFP; ATP = ambient temperature } \\
\text { polymerized PBFP; NC } 5 \%=\text { solvent casted } \\
\text { PBFP with } 5 \% \text { (in wcight) nano-TiO ; } \\
\text { annealed NC } 5 \%=\text { annealed PBFP } \mathrm{TiO}_{2} 5 \% \text {; } \\
\mathrm{CS}=\text { chemical shift in ppm; Width = } \\
\text { spectral linewidth at half height; Area = } \\
\text { percentage area of total spectrum }\end{array}$} & & & & & \\
\hline
\end{tabular}

In the NC 5\% material the broadest signal indicated a crystalline composition of $29.2 \%$, which is markedly less than the $45 \%$ observed in the SC sample and is consistent with the findings in the ${ }^{1} \mathrm{H}$ and ${ }^{19} \mathrm{~F}$ NMR spectra. Such information consistently suggests that the presence of the filler suppressed crystal growth in cast samples. Upon heat treatment the crystallinity in the $5 \%$ nano- $\mathrm{TiO}_{2}$ PBFP NC sample increased from $29.2 \%$ to $35.6 \%$, which is far less than that observed $(65 \%)$ in the HT sample. Clearly, that nc- $\mathrm{TiO}_{2}$ also suppresses crystal growth during heat treatment. 
In conclusion, the ${ }^{1} \mathrm{H},{ }^{19} \mathrm{~F}$ and ${ }^{31} \mathrm{P}$ solid-state NMR data of the five samples: SC-PBFP, HT-PBFP, NC 5\% and annealed NC 5\% and ATP PBFP are presented above, demonstrating that preparation methodology and polymer treatment afford different polymer phase, and hence, distinct polymer morphology. Heat treatment facilitates the transformation from amorphous to crystalline. Ambient temperature polymerization yields polyphosphazene (PBFP) with a greater proportion of amorphous constitution compared to the thermal ring-opening approach. Addition of nc- $\mathrm{TiO}_{2}$ particles decreases the percentage of crystalline components compared to the virgin polymer in both cast and heat-treated preparations, indicating that the presence of $\mathrm{nc}^{-\mathrm{TiO}_{2}}$ suppresses crystal growth.

7.2 The Studies of Morphological Changes of PBFP, Annealed PBFP, $5 \%$ Nano-TiO2 PBFP nanocomposite (NC 5\%) and Annealed NC 5\% under Variable Temperature by Solid-State NMR Spectroscopy Using an MAS rate of $10 \mathrm{kHz}$

\subsection{1 ${ }^{19} \mathrm{~F}$ and ${ }^{31} \mathrm{P}$ solid-State NMR Spectroscopy under Variable Temperature at an MAS rate of $10 \mathrm{kHz}$}

Variable temperature ${ }^{19} \mathrm{~F}$ and ${ }^{31} \mathrm{P}$ MAS NMR spectra were obtained for PBFP, annealed PBFP, nanocomposites with 5\% nano- $\mathrm{TiO}_{2}$ (NC 5\%) and annealed $\mathrm{NC} 5 \%$ at an MAS rate of $10 \mathrm{kHz}$ between $20^{\circ} \mathrm{C}$ and $80^{\circ} \mathrm{C}$. The corresponding results are shown in Figure 7.4 and Figure 7.5. The ${ }^{19}$ F NMR signals are used to observe morphological changes via the side group environment and the ${ }^{31} \mathrm{P}$ NMR spectra to report on the polymer backbone.

Figure 7.4 shows that in the ${ }^{19} \mathrm{~F}$ NMR spectra between 20 and $40^{\circ} \mathrm{C}$, the broad component at the bottom of the signal decreases in all four samples. However, the initial contribution from the broad component varies greatly reflecting their different preparation methods. The annealed PBFP has the highest proportion of the broad signal, presumably reflecting the highest degree of crystallinity. Similarly, the annealed NC $5 \%$ has much 
increased broad signal compared to NC $5 \%$, however, significantly smaller than the annealed PBFP. Furthermore the NC 5\% has a slightly lower level of the broad component than PBFP.
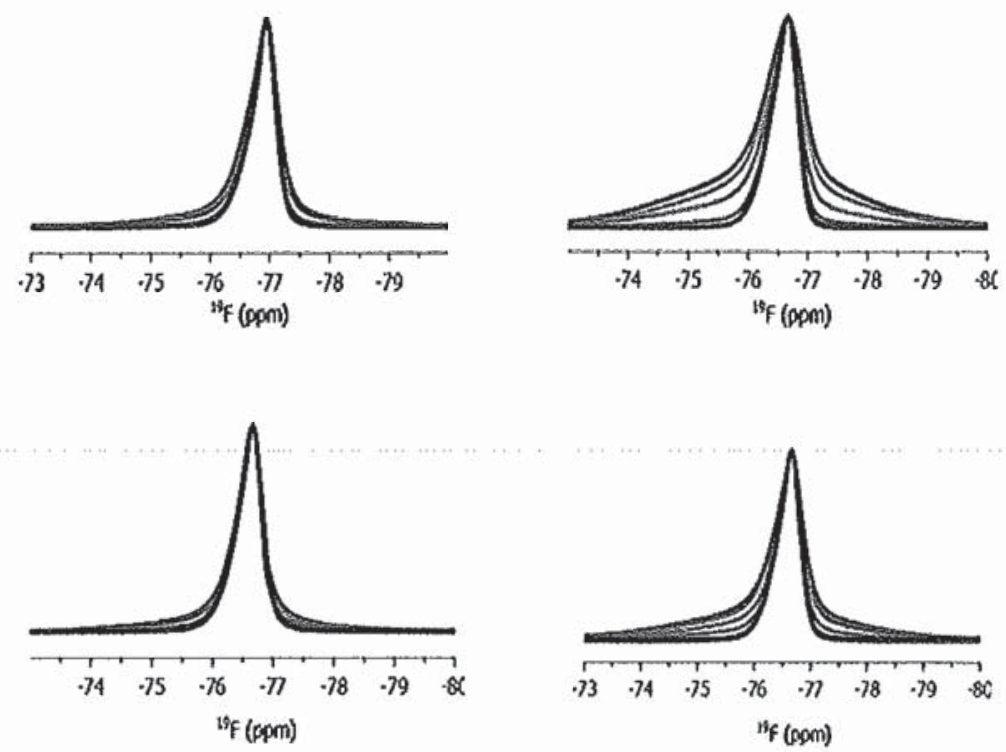

$20^{\circ} \mathrm{C} 30^{\circ} \mathrm{C} 40^{\circ} \mathrm{C} 50^{\circ} \mathrm{C} 60^{\circ} \mathrm{C} 70^{\circ} \mathrm{C} 80^{\circ} \mathrm{C}$

Figure 7.4: $\quad{ }^{19} \mathrm{~F}$ solid-state NMR spectra of 4 samples under variable temperature with an MAS rate of $10 \mathrm{kHz}$ (top left) non-annealed PBFP; (top right) annealed PBFP; (bottom left) non anncaled $\mathrm{NC} 5 \%-\mathrm{TiO}_{2}$; (bottom right) annealed NC 5\%-TiO

Figure 7.5 shows the intensity of the broad signal at $-4 \mathrm{ppm}$ in the ${ }^{31} \mathrm{P}$ NMR spectra decreasing in all four samples, with the temperature increasing from $20-40^{\circ} \mathrm{C}$. However, each sample has a different rate of intensity decrease, similarly to the behavior observed in the corresponding ${ }^{19} \mathrm{~F}$ NMR spectra in Figure 7.4. The annealed PBFP sample has the highest ratio of the broad components appearing in the -2 to $-6 \mathrm{ppm}$ range, which corresponds to the crystalline components. The annealed NC 5\% samples also has a high ratio of the broad signal but slightly less than the annealed PBFP sample. Furthermore, the 
non-annealed PBFP has a higher ratio of broad component as compared to the NC 5\% nonannealed.
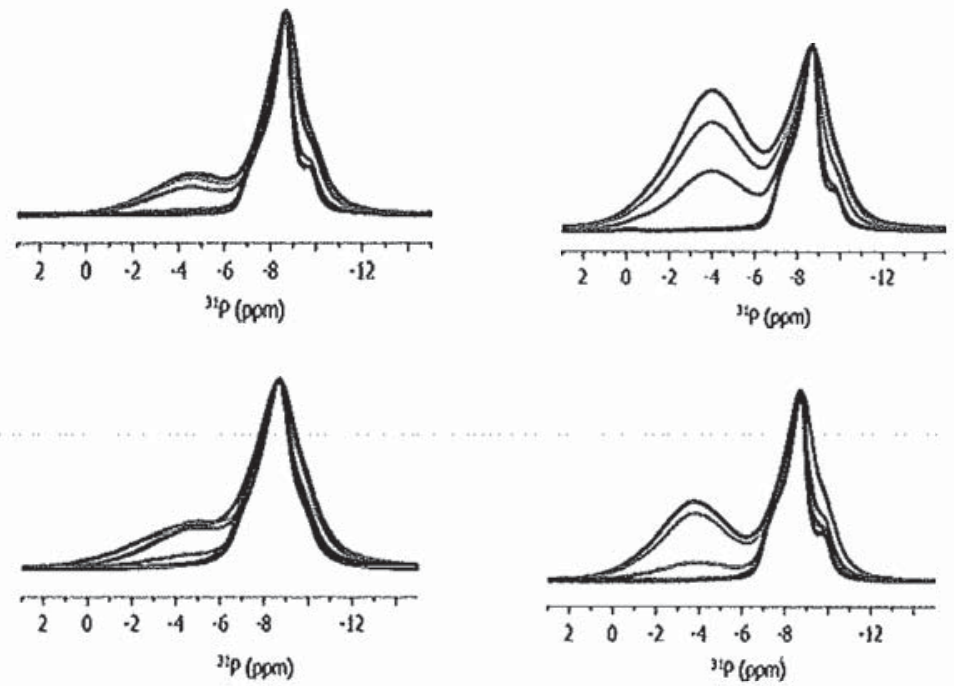

$20^{\circ} \mathrm{C} 30^{\circ} \mathrm{C} 40^{\circ} \mathrm{C} 50^{\circ} \mathrm{C} 60^{\circ} \mathrm{C} 70^{\circ} \mathrm{C} 80^{\circ} \mathrm{C}$

Figure 7.5: $\quad{ }^{31} \mathrm{P}$ solid-state NMR spectra of 4 samples under variable temperature with an MAS rate of $10 \mathrm{kHz}$ (top left) non-annealed PBFP; (top right) annealed PBFP; (bottom left) non annealed $\mathrm{NC} 5 \%-\mathrm{TiO}_{2}$; (bottom right) annealed $\mathrm{NC} 5 \%-\mathrm{TiO}_{2}$.

All ${ }^{19} \mathrm{~F}$ and ${ }^{31} \mathrm{P}$ NMR spectra obtained at an MAS spinning rate of $10 \mathrm{kHz}$ showed the intensity of broad components decrease with increasing temperature from $20-50{ }^{\circ} \mathrm{C}$. When the temperature approaches the $\mathrm{T}(1)$ transition temperature, around $60^{\circ} \mathrm{C}$, no significant changes are observed with continually increasing temperature for both nuclei (Figure 7.4 and Figure 7.5). Samples with different preparations, such as nano-filling and annealing, exhibit distinct changes with temperature, thus reflect their different morphologies. To gain a deeper understanding into the polymer morphological behaviour with changes in sample treatment and temperature, a combined deconvolution and relaxation analysis of the ${ }^{19} \mathrm{~F}$ and ${ }^{31} \mathrm{P}$ NMR spectra will be necessary ${ }^{5.6}$. 


\subsubsection{Deconvolution Analysis of ${ }^{19} \mathrm{~F}$ and ${ }^{31} \mathrm{P}$ Solid-State NMR Spectra at an MAS 10 $\mathrm{kHz}$ and Variable Temperature}

\subsubsection{Deconvolution analysis for ${ }^{31} \mathrm{P}$ DP MAS NMR Spectra for four samples at the temperature range of $20-80^{\circ} \mathrm{C}$}

Relaxation behaviour characterized by the $T_{1}$ and $T_{2}$ relaxation times are reflected in their distinct sensitivities to the time scales of a variety of motional processes taking place in the polymer? It is strongly indicative of differences in local environments within the polymer and hence an invaluable tool to investigate polymer morphologies ${ }^{8}$. To ensure accurate results, it is essential that a reliable model is established before relaxation analysis can be performed.

Figure 7.6 contains an example of a series Hahn-echo experiments for the determination of $\mathrm{T}_{2}$ values of ${ }^{31} \mathrm{P}$ in $\mathrm{PBFP}$ at $20^{\circ} \mathrm{C}$. By inspection one can pick out one broad component around -1 to $-6 \mathrm{ppm}$. A narrow signal appears from -6 to $-11 \mathrm{ppm}$, which is made up of at least three components, which differ slightly in the rate of decay with increasing relaxation delay. The $T_{1}$ and $T_{1 p}$ relaxation series are consistent with these observations. Consequently, the ${ }^{31}$ P NMR spectra were deconvolved into 4 components by adjusting the line-width and Lorentz to Gauss ratio $(L / G)$ and frequency. The resulting model is subsequently applied to the relaxation series by keeping the frequency, width, and L/G fixed varying only the intensities. If the model starts to phase out at certain points when going from spectrum to spectrum over the relaxation array, I need to re-adjust the chemical shift and line-width and G/L until the model fits all spectra in the array. Ideally, the same model would apply to all four samples over the entire temperature range of interest. For the ${ }^{31} \mathrm{P}$ NMR spectra from $20-40^{\circ} \mathrm{C}$ the 4 line model was used for all 4 samples. 
For temperatures over $50-80^{\circ} \mathrm{C}$, another model was used as the broad component at -2 to $-6 \mathrm{ppm}$ disappeared, leaving only the narrow signals. This is likely caused by the crystalline phase being slowly transformed into liquid crystalline phase which has a faster motion resulting in a narrower signal. As a result, there are additional narrow components considered at this temperature range compared with lower temperature from 20 to $40^{\circ} \mathrm{C}$. The relaxation series from 50 to $80^{\circ} \mathrm{C}$ were therefore subjected to the five-component model that contains only narrow components.

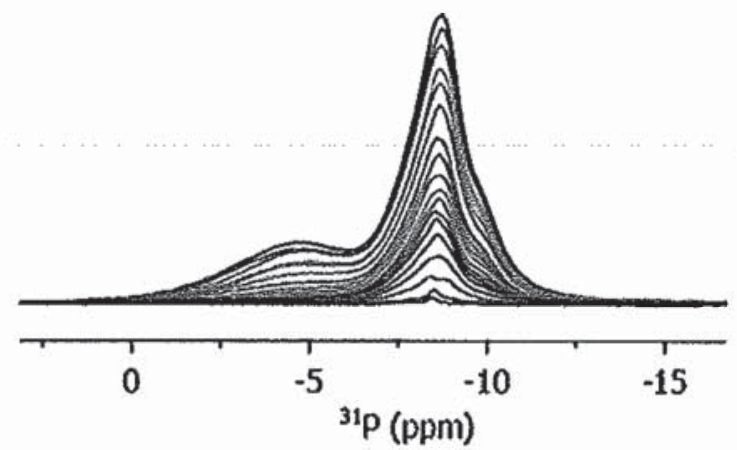

Figure 7.6 ${ }^{31} \mathrm{P} \mathrm{T}_{2}$ solid-state NMR relaxation array spectra of $\mathrm{PBFP}$ at $20^{\circ} \mathrm{C}$ at an MAS rate of $23 \mathrm{kHz}$. The delay array is: $0,100,200,400,500,700,1000,1200,1400,1600,1800$, $2000,2200,2500,3000,4000,6000$, and 8000 in micros

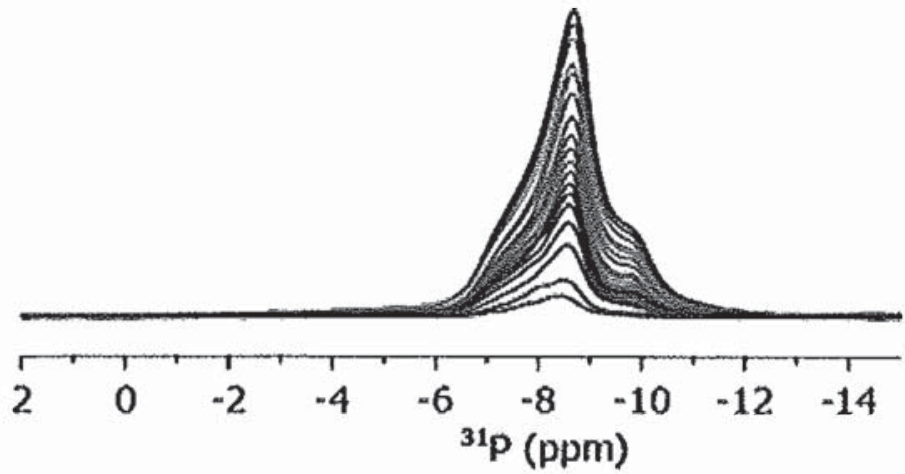

Figure 7.7 $\quad{ }^{31} \mathrm{P} \mathrm{T}_{2}$ solid-state NMR relaxation array spectra of $\mathrm{PBFP}$ at $50^{\circ} \mathrm{C}$ at an $\mathrm{MAS}$ rate of $23 \mathrm{kHz}$. The delay array was: $0,100,200,400,500,700,1000,1200,1400,1600$, $1800,2000,2200,2500,3000,4000,6000$, and 8000 in micros 
In Figure 7.8, the first ${ }^{31} \mathrm{P}$ NMR spectrum of PBFP of the $\mathrm{T}_{1}$ series at $20^{\circ} \mathrm{C}$ is shown, and deconvolved into 4 components at chemical shifts at $-4.55,-8.12,-8.68$ and $-9.88 \mathrm{ppm}$, with line-widths of $834,344,272$ and $242 \mathrm{~Hz}$, respectively. This deconvolution result is consistent with what I assumed earlier with four lines in total, including a broad signal composed of one broad component and a sharp signal composed with three components. The same deconvolution parameters are applied for ${ }^{31} \mathrm{P} T_{1}, T_{2}, T_{1 \rho}$ relaxation over the temperature range from $20-40^{\circ} \mathrm{C}$ for all 4 samples.

Figure 7.9 provides an example to show how the model beautifully fit the $T_{1}$ relaxation array of ${ }^{31} \mathrm{P}$ from $\mathrm{PBFP}$ at $20^{\circ} \mathrm{C}$, even when some component have recovered to the positive $\mathbf{z}$-axis, and some components are still in the negative $-\mathrm{z}$ axis, the residual error is kept to within a small range where the chemical shift and line-width is constrained and only the line intensities are allowed to vary.

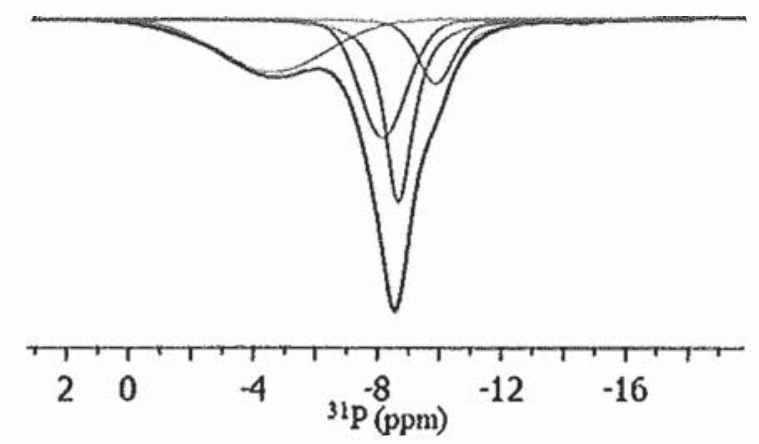

Figure 7.8: $\quad{ }^{31} \mathrm{P}$ solid-state NMR $\mathrm{T}_{1}$ relaxation deconvolution model of $\mathrm{PBFP}$ at $20^{\circ} \mathrm{C}$ fitted for the first spectrum in the relaxation array obtained at an MAS rate of $10 \mathrm{kHz}$ 


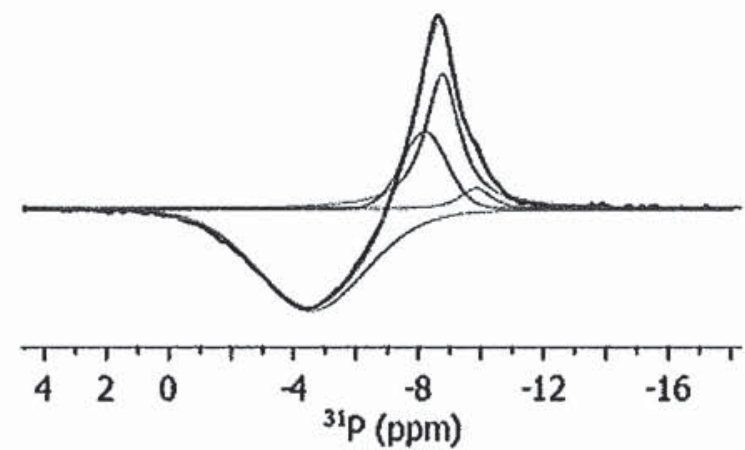

Figure 7.9: $\quad{ }^{31} \mathrm{P}$ NMR $\mathrm{T}_{1}$ relaxation deconvolution model of PBFP at $20^{\circ} \mathrm{C}$ fitted for the $8^{\text {th }}$ spectrum in the relaxation array obtained at an MAS rate of $10 \mathrm{kHz}$

The model for all ${ }^{31} \mathrm{P}$ solid-state NMR relaxation analysis at temperatures between 20 and $40^{\circ} \mathrm{C}$ is shown in Figure 7.10. The chemical shift and line width parameter of this ${ }^{31} \mathrm{P}$ model is shown in Table 7.4. The sharp components have a larger fraction of the Lorentzian shape, while the broader components have a more pronounced fraction of the Gaussian shape.

Table 7.4: Deconvolution parameters for ${ }^{31} \mathrm{P}$ NMR spectra of 4 samples over the temperature range of $20-40^{\circ} \mathrm{C}$ obtained at an MAS rate of $10 \mathrm{kHz}$

\begin{tabular}{ccc}
$\#$ & CS(ppm) & Width(Hz) \\
\hline $1(\circ)$ & -4.55 & 834 \\
$2(\theta)$ & -8.12 & 344 \\
$3(\bullet)$ & -8.68 & 272 \\
$4(\%)$ & -9.88 & 242
\end{tabular}

Note: $\mathrm{CS}=$ chemical shift in ppm; Width = linewidth at half height in $\mathrm{Hz} ; 4$ samples $=$ PBFP, annealed PBFP, nanocomposite $\mathrm{TiO}_{2} 5 \%$ 


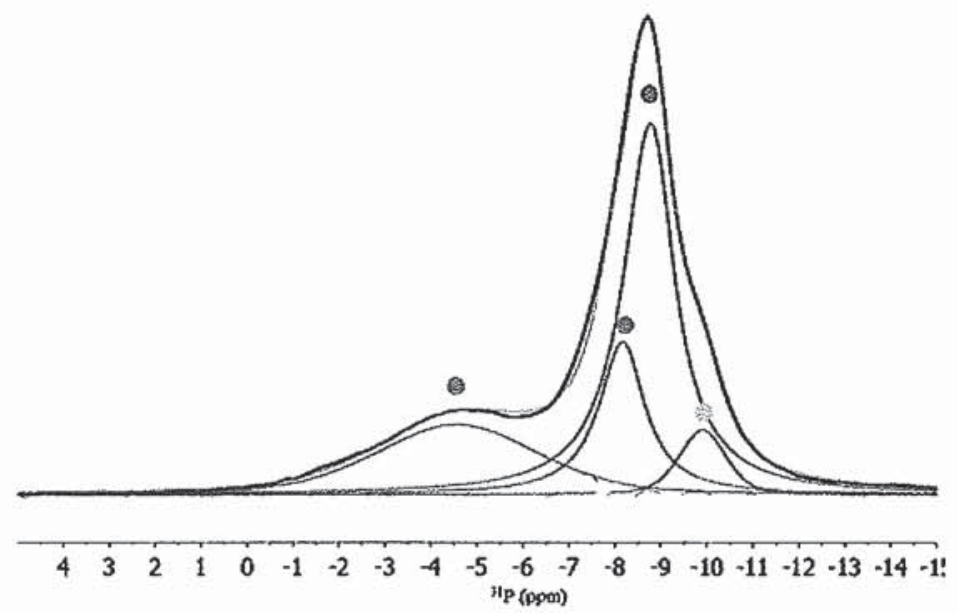

Figure 7.10: Deconvolution model for ${ }^{31} \mathrm{P} N \mathrm{NMR}$ spectra of 4 samples at the temperature range of $20-40^{\circ} \mathrm{C}$ obtained at an MAS ratc of $10 \mathrm{kHz}$

As mentioned earlier, at higher temperature, the polymer membrane sample transformed to liquid crystal, which will have different motional and dynamic properties, causing the model to change. In the Figure $7.11,{ }^{31} \mathrm{P} \mathrm{T}_{1}$ NMR relaxation model of PBFP at $50^{\circ} \mathrm{C}$ for the fir'st spectrum in the relaxation array is shown, with 5 components at chemical shifts at $-7.37,-8.13,-8.62,-9.06$ and $-9.79 \mathrm{ppm}$, the corresponding line widths are: 220 , $176,124,151$, and $205 \mathrm{~Hz}$, respectively. The deconvolution parameters are also used to deconvolve the other three samples over the temperature range from $50-80^{\circ} \mathrm{C}$ for all four samples. 


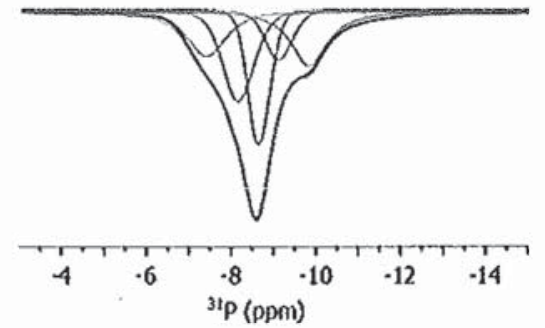

Figure 7.11: "P PBFP SSNMR $T_{1}$ relaxation deconvolution model at $50^{\circ} \mathrm{C}$ fitted for the first spectrum in the relaxation array at an MAS rate of $10 \mathrm{kHz}$

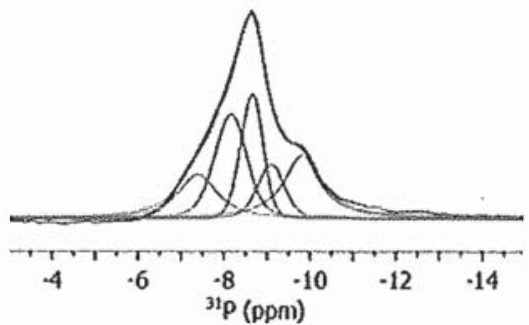

Figure 7.12: ${ }^{31} \mathrm{P}$ PBFP SSNMR $\mathrm{T}$, relaxation deconvolution model at $50{ }^{\circ} \mathrm{C}$ fitted for the 8th spectrum in the relaxation array at an MAS rate of $10 \mathrm{kHz}$

Figure 7.12 shows the same deconvolution parameter is applied to the spectra when

$\mathrm{d}_{2}$ equals $2 \mathrm{~s}$ of the $\mathrm{T}_{1}$ array for ${ }^{3 \prime} \mathrm{P} \mathrm{T}_{1} \mathrm{PBFP}$ at $50^{\circ} \mathrm{C}$. This indicates that the model fits well with the spectrum, even when all components have recovered to the positive $z$-axis.

The model for all ${ }^{31} \mathrm{P}$ NMR relaxation at the temperature range from $50-80{ }^{\circ} \mathrm{C}$ is shown in Figure 7.13. The chemical shift and line-width parameters are shown in Table 7.5.

Table 7.5: Deconvolution parameters for ${ }^{31} \mathrm{P}$ NMR spectra of 4 samples at the temperature range of $50-80^{\circ} \mathrm{C}$ obtained at an MAS rate of $10 \mathrm{kHz}$

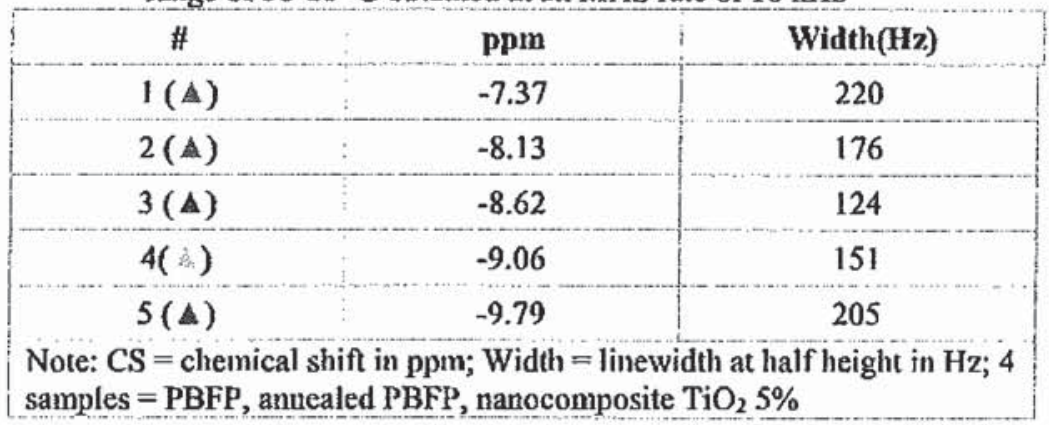




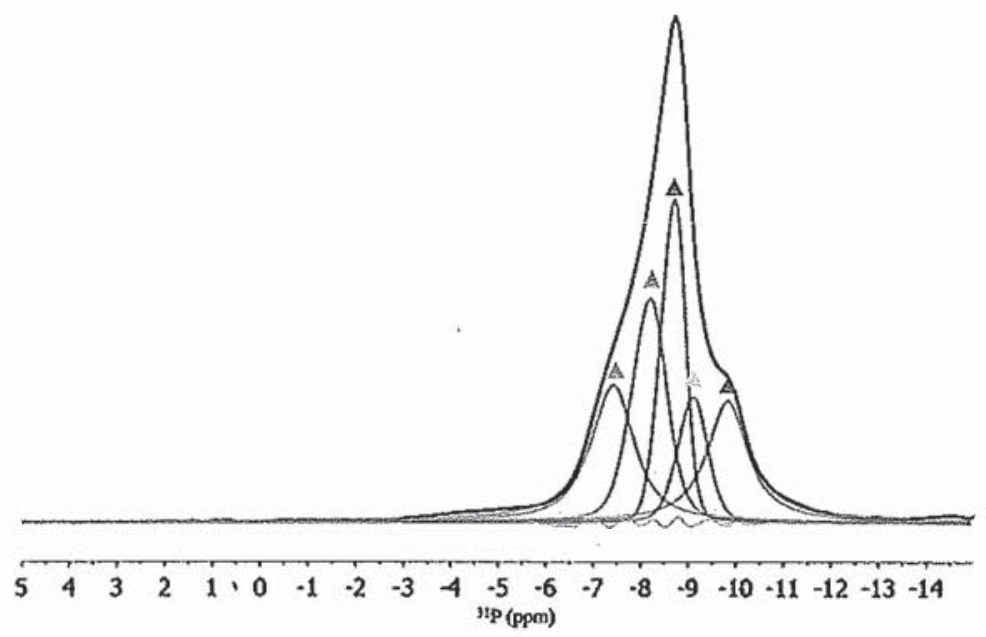

Figure 7.13: Deconvolution model for ${ }^{31} \mathrm{P}$ NMR spectra of 4 samples at the temperature range of $50-80^{\circ} \mathrm{C}$ obtained at an MAS rate of $10 \mathrm{kHz}$

In conclusion, the ${ }^{31} \mathrm{P}$ MAS NMR spectra of all the four samples were deconvolved using models obtained through relaxation series. The spectra were deconvolved into four lines at different chemical shift over the temperature range from $20-40^{\circ} \mathrm{C}$, when the temperature increased approach its transition temperature, the broad signal appears at -4.55 ppm disappeared, and two more small lines showed up. Thus, at the temperature range from $50-80^{\circ} \mathrm{C}$, the spectra are deconvolved into five lines, all of which are sharper compared to the line appears at $-4.55 \mathrm{ppm}$.

\subsubsection{2 ${ }^{31}$ P DP NMR spectra for four samples each deconvolution percentage over the temperature range from 20 to $80^{\circ} \mathrm{C}$ with an MAS at $10 \mathrm{kHz}$}

The two models for ${ }^{31} \mathrm{P}$ NMR spectra were used for all four samples over the temperature range $20-80^{\circ} \mathrm{C}$, the distribution of each component as a percentage of the total signal for all four samples was calculated. The results are discussed as following.

The ${ }^{31}$ P MAS NMR spectra of PBFP and annealed PBFP were collected at an MAS rate of $10 \mathrm{kHz}$ and were deconvolved using the model in Figure 7.10 and Table 7.4. For 
the temperature range from $20-40{ }^{\circ} \mathrm{C}$, Table 7.6 shows the calculated area of each component samples PBFP and amealed PBFP. Figure 7.14 shows the trends of each component with increasing temperature.

Table 7.6: $\quad{ }^{31} \mathrm{P}$ NMR spectrat deconvolution percentage area for the 4 components over the temperature range of $20-40^{\circ} \mathrm{C}$ for PBFP (left) and annealed PBFP (right) obtained at an MAS rate of $10 \mathrm{kHz}$

\begin{tabular}{|c|c|c|c|c|c|c|c|c|c|}
\hline CS/ppm & $\begin{array}{c}-4.55 \\
(\theta)\end{array}$ & $\begin{array}{c}-8.12 \\
\text { (a) }\end{array}$ & $\begin{array}{c}-8.68 \\
(0)\end{array}$ & $\begin{array}{c}-9.88 \\
(\text { ) }\end{array}$ & CS/ppm & $\begin{array}{c}-4.55 \\
\text { (e) }\end{array}$ & $\begin{array}{c}-8.12 \\
(0)\end{array}$ & $\begin{array}{c}-8.68 \\
(0)\end{array}$ & $\begin{array}{c}-9.88 \\
()\end{array}$ \\
\hline Width/Hz & 834 & 344 & 272 & 242 & Width/Hz & 834 & 344 & 272 & 242 \\
\hline $\begin{array}{l}\text { Temp } \\
\left({ }^{\circ} \mathrm{C}\right)\end{array}$ & \multicolumn{4}{|c|}{$\begin{array}{c}\text { PBFP } \\
\% \text { composition }\end{array}$} & $\begin{array}{l}\text { Temp } \\
\left({ }^{\circ} \mathrm{C}\right)\end{array}$ & \multicolumn{4}{|c|}{$\begin{array}{l}\text { PBFP annealed } \\
\% \text { composition }\end{array}$} \\
\hline 20 & 33 & 18 & 35 & 14 & 20 & 60 & 12 & 20 & 8 \\
\hline 30 & 29 & 18 & 40 & 13 & 30 & 54 & 14 & 25 & 7 \\
\hline 40 & 28 & 19 & 45 & 9 & 40 & 44 & 16 & 34 & 7 \\
\hline
\end{tabular}

Note: $\mathrm{CS}=$ chemical shift in ppm; Width $=$ linewidth at half height in $\mathrm{Hz} ; \%$ composition = percentage area of total spectra area
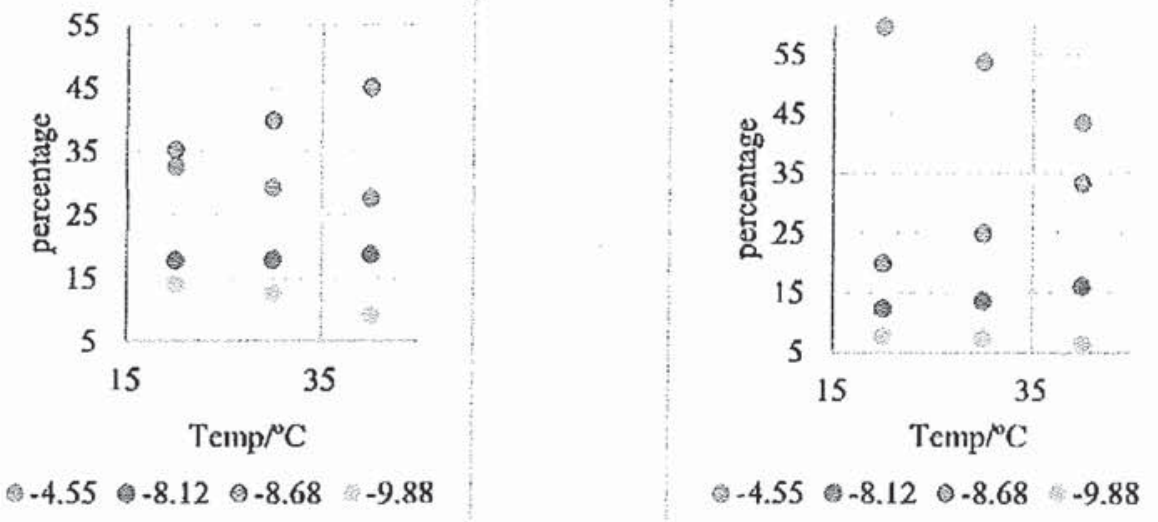

Figure 7.14: ${ }^{31} \mathrm{P}$ NMR spectral deconvolution percentage area changing trends for the 4 components over the temperature range of $20-40^{\circ} \mathrm{C}$ for PBFP (left) and annealed PBFP (right) obtained at an MAS rate of $10 \mathrm{kHz}$

The ${ }^{31} \mathrm{P}$ NMR spectrum model in Figure 7.10 and Table 7.4 is also applied to samples NC $5 \%$ and annealed NC $5 \%$, the percentage area for each component was calculated and listed in Table 7.7, and the corresponding trends of each component are shown in Figure 7.15 . 
Table 7.7: $\quad{ }^{31} \mathrm{P}$ NMR spectral deconvolution percentage area for the 4 components over the temperature range of $20-40{ }^{\circ} \mathrm{C}$ for NC $5 \%$ (left) and annealed NC $5 \%$ (right) obtaincd at an MAS rate of $10 \mathrm{kHz}$

\begin{tabular}{|c|c|c|c|c|c|c|c|c|c|}
\hline $\mathrm{CS} / \mathrm{ppm}$ & $\begin{array}{c}-4.55 \\
(0)\end{array}$ & $\begin{array}{c}-8.12 \\
(\bullet)\end{array}$ & $\begin{array}{c}-8.68 \\
(0)\end{array}$ & $\begin{array}{c}-9.88 \\
(3)\end{array}$ & CS/ppm & $\begin{array}{l}-4.55 \\
\text { (c) }\end{array}$ & $\begin{array}{c}-8.12 \\
(0)\end{array}$ & $\begin{array}{c}-8.68 \\
(0)\end{array}$ & $\begin{array}{c}-9.88 \\
(0)\end{array}$ \\
\hline Width/Hz & 834 & 344 & 272 & 242 & Width/Hz & 834 & 344 & 272 & 242 \\
\hline $\begin{array}{c}\text { Temp } \\
\left({ }^{\circ} \mathrm{C}\right)\end{array}$ & & $\begin{array}{r}\text { NC } \\
\% \text { com }\end{array}$ & $\begin{array}{l}\% \% \\
\text { osition }\end{array}$ & & $\begin{array}{c}\text { Temp } \\
\left({ }^{\circ} \mathrm{C}\right)\end{array}$ & & $\begin{array}{l}\text { Anneale } \\
\% \text { com }\end{array}$ & $\begin{array}{l}\text { NC 5\% } \\
\text { osition }\end{array}$ & \\
\hline 20 & 31 & 12 & 44 & 13 & 20 & 51 & 14 & 27 & 8 \\
\hline 30 & 29 & 14 & 45 & 12 & 30 & 46 & 17 & 29 & 8 \\
\hline 40 & 23 & 15 & 51 & 10 & 40 & 43 & 18 & 33 & 7 \\
\hline
\end{tabular}

Note: $\mathrm{CS}=$ chemical shift in $\mathrm{ppm}$; Width $=$ linewidth at half height in $\mathrm{Hz} ; \%$ composition $=$ percentage area of total spectra area
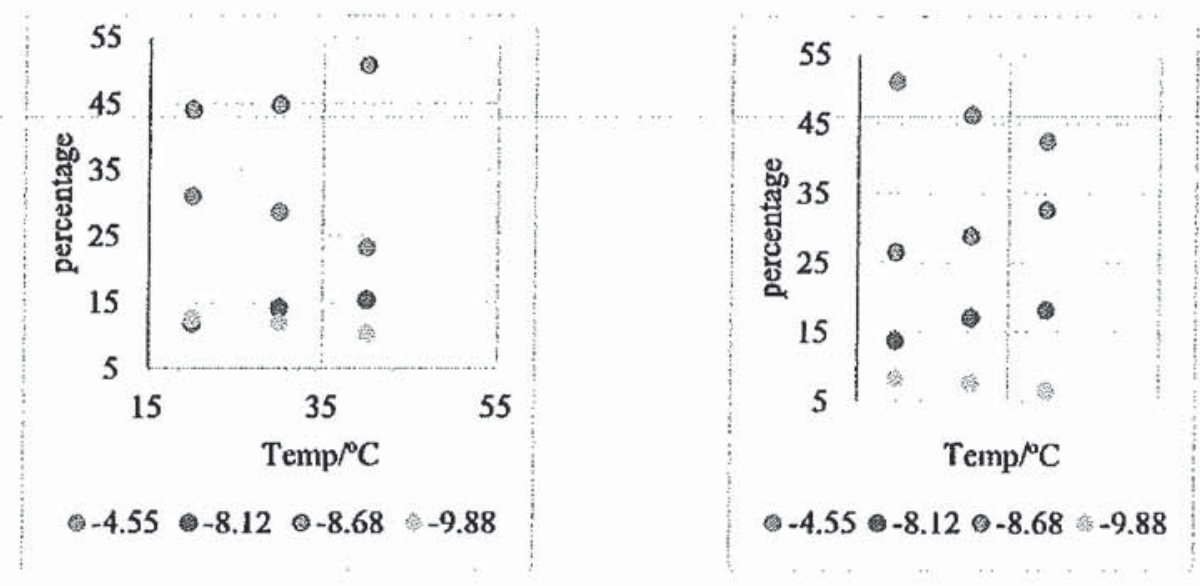

Figure 7.15: ${ }^{31} \mathrm{P}$ NMR spectral deconvolution percentage area changing trends for the 4 components over the temperature range of $20.40{ }^{\circ} \mathrm{C}$ for $\mathrm{NC} 5 \%-\mathrm{TiO}_{2}$ (left) and annealed $\mathrm{NC} 5 \%-\mathrm{TiO}_{2}$ (right) obtained at an $\mathrm{MAS}$ rate of $10 \mathrm{kHz}$

Figures $7.14,7.15$, and Tables $7.6,7.7$ show that the percentage area for the component at -4.55 and $-9.88 \mathrm{ppm}$ decrease as temperature increases from 20 to $40{ }^{\circ} \mathrm{C}$, which likely represent the rigid environment of the polymer. The percentage areas of the other two components appearing at -8.12 and $-8.68 \mathrm{ppm}$ increase with increasing temperature. Thus they likely correspond to the mobile environment. Components at -4.55 and -9.88 ppm make up a crystalline percentage of $68 \%$ for annealed PBFP, and $60 \%$ for annealed NC $5 \%$ at $20^{\circ} \mathrm{C}$. It is higher than the PBFP and NC 5\%, with a value of $47 \%$ for 
PBFP and $44 \%$ for NC $5 \%$ at $20^{\circ} \mathrm{C}$, individually. This phenomenon is also observed at 30 ${ }^{\circ} \mathrm{C}$, and $40^{\circ} \mathrm{C}$ NMR spectra.

Table 7.8: $\quad{ }^{31}$ P NMR spectral deconvolution percentage area for the 5 components over the temperature range of $50-80^{\circ} \mathrm{C}$ for PBFP and annealed PBFP obtained at an MAS rate of $10 \mathrm{kHz}$

\begin{tabular}{|c|c|c|c|c|}
\hline CS/ppm & $\begin{array}{l}-7.37 \\
(\triangle)\end{array}$ & $\begin{array}{r}-8.13 \\
\text { (A) }\end{array}$ & $\begin{array}{l}-8.62 \\
(\Delta)\end{array}$ & $\begin{array}{c}-9.06 \\
(\mathrm{)})\end{array}$ \\
\hline Width/Hz & 220 & 176 & 124 & 151 \\
\hline $\begin{array}{l}\text { Temp } \\
\text { ('C) }\end{array}$ & \multicolumn{4}{|c|}{ PBFP \% composition } \\
\hline 50 & 30 & 23 & 17 & 10 \\
\hline 60 & 25 & 25 & 21 & 19 \\
\hline 70 & 25 & 25 & 23 & 18 \\
\hline 80 & 25 & 25 & 24 & 18 \\
\hline
\end{tabular}

Note: $\mathrm{CS}=$ chemical shift in $\mathrm{ppm}$; Width $=$ lincwidth at half height in $\mathrm{Hz} ; \%$ composition = percentage area of total spectra area

$\begin{array}{ccccccc}\text { CS/ppm } & \begin{array}{c}-7.37 \\ (\Delta)\end{array} & \begin{array}{c}-8.13 \\ (\Delta)\end{array} & \begin{array}{c}-8.62 \\ (\Delta)\end{array} & \begin{array}{c}-9.06 \\ (\Delta)\end{array} & \begin{array}{c}-9.79 \\ (\Delta)\end{array} \\ \begin{array}{c}\text { Widih/Hz } \\ \text { Temp }\end{array} & 220 & 176 & 124 & 151 & 205 \\ \left(\begin{array}{c}\left.{ }^{\circ} \mathrm{C}\right) \\ \mathbf{5 0}\end{array}\right. & 25 & 22 & 14 & 10 & 19 \\ \mathbf{6 0} & 24 & 27 & 27 & 14 & 8 \\ \mathbf{7 0} & 25 & 28 & 26 & 14 & 8 \\ \mathbf{8 0} & 25 & 28 & 27 & 13 & 8\end{array}$

Note: $\mathrm{CS}=$ chemical shift in ppm; Width $=$ linewidth at half height in $\mathrm{Hz} ; \%$ composition = percentage area of total spectra area
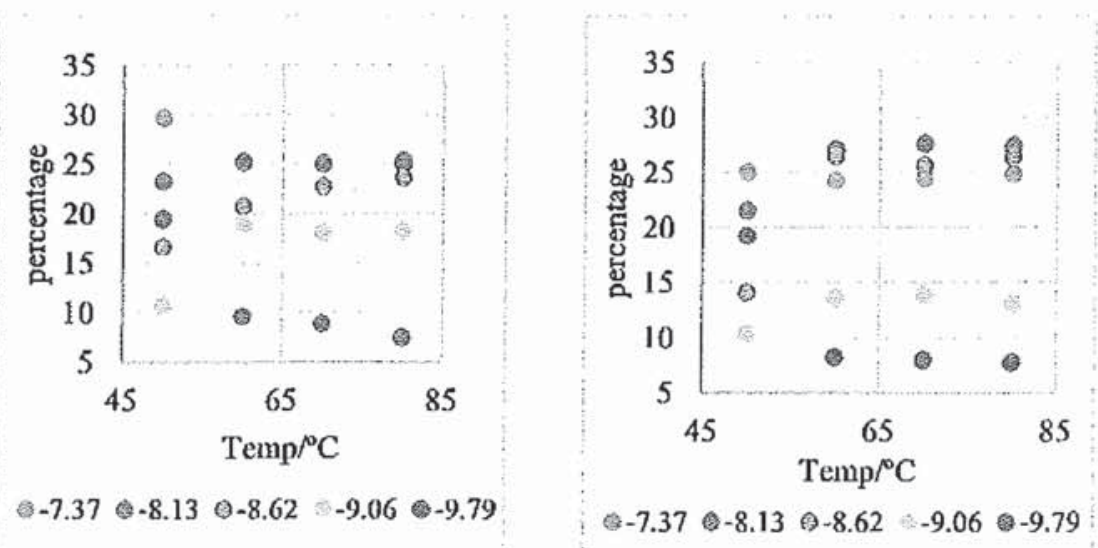

Figure 7.16: $\quad{ }^{31} \mathrm{P}$ deconvolution percentage area for the 5 components over $50-80{ }^{\circ} \mathrm{C}$ for PBFP and annealed PBFP obtained at an MAS rate of $10 \mathrm{kHz}$ 
Phosphorus-31 MAS NMR spectra of PBFP, annealed PBFP, NC 5\% and annealed $\mathrm{NC} 5 \%$ over the temperature range over $50-80^{\circ} \mathrm{C}$ were acquired at an MAS rate of $10 \mathrm{kHz}$ and are deconvolved using the model in Figure 7.13 and Table 7.5, and the area for each component was calculated and listed in Tables 7.8 and 7.9 , respectively. The corresponding percentage changing trends for each component under each temperature is plotted in Figure 7.16 and Figure 7.17.

Figures 7.16 and 7.17 indicate that at higher temperature, the percentage area of component at $-7.37 \mathrm{ppm}$ is decreased from $30 \%\left(50^{\circ} \mathrm{C}\right)$ to $25 \%\left(60^{\circ} \mathrm{C}\right)$. The value of component at -8.13 ppm increased from $22 \%$ to $27 \%$. Similarly, the value of component at -9.79 ppm decreased from $20 \%$ to $10 \%$ for sample PBFP when the temperature is increased from 50 to $60^{\circ} \mathrm{C}$. The area fractions of the other three components at $-8.13,-8.62$, and $9.06 \mathrm{ppm}$ increase with increasing temperature. This trend is also presented in samples annealed PBFP, NC 5\% and annealed NC 5\%. When the temperature is increased from 60 to $80^{\circ} \mathrm{C}$, the percentage of each component has very little changes. The same phenomenon is seen in samples annealed PBFP, NC 5\% and annealed NC $5 \%$. 
Table 7.9: $\quad 31 \mathrm{P}$ NMR spectral deconvolution percentage area for the 5 components over the temperature range of $50-80^{\circ} \mathrm{C}$ for NC $5 \%$ and annealed NC $5 \%$ obtained at an MAS rate of $10 \mathrm{kHz}$

\begin{tabular}{|c|c|c|c|c|c|}
\hline CS/ppm & $\begin{array}{l}-7.37 \\
\text { (A) }\end{array}$ & $\begin{array}{l}-8.13 \\
(\Delta)\end{array}$ & $\begin{array}{l}-8.62 \\
(\Delta)\end{array}$ & $\begin{array}{l}-9.06 \\
(k)\end{array}$ & $\begin{array}{l}-9.79 \\
(\Delta)\end{array}$ \\
\hline Width/Hz & 220 & 176 & 124 & 151 & 205 \\
\hline $\begin{array}{l}\text { Temp } \\
\left({ }^{\circ} \mathrm{C}\right)\end{array}$ & \multicolumn{5}{|c|}{ NC5 \% composition } \\
\hline 50 & 25 & 23 & 16 & 13 & 22 \\
\hline 60 & 20 & 25 & 27 & 18 & 11 \\
\hline 70 & 21 & 25 & 28 & 18 & 9 \\
\hline 80 & 20 & 25 & 29 & 17 & 9 \\
\hline
\end{tabular}

Note: $\mathrm{CS}=$ chemical shift in ppm; Width $=$ linewidth at half height in $\mathrm{Hz} ; \%$ composition $=$ percentage area of total spectra area

\begin{tabular}{|c|c|c|c|c|c|}
\hline CS/ppm & $\begin{array}{c}-7.37 \\
\text { (A) }\end{array}$ & $\begin{array}{c}-8.13 \\
(\mathrm{~A})\end{array}$ & $\begin{array}{c}-8.62 \\
(\Delta)\end{array}$ & $\begin{array}{c}-9.06 \\
(\leq)\end{array}$ & $\begin{array}{c}-9.79 \\
(\Delta)\end{array}$ \\
\hline Wilth/IIz & 220 & 176 & 124 & 151 & 205 \\
\hline $\begin{array}{l}\text { Temp } \\
\text { ('C) }\end{array}$ & \multicolumn{5}{|c|}{ NC5 $\%$ composition } \\
\hline 50 & 23 & 20 & 26 & 13 & 18 \\
\hline 60 & 20 & 26 & 26 & 18 & 10 \\
\hline 70 & 20 & 26 & 26 & 18 & 11 \\
\hline 80 & 20 & 26 & 26 & 18 & 10 \\
\hline
\end{tabular}

Note: $\mathrm{CS}=$ chemical shift in ppm; Width $=$ linewidth at half height in $\mathrm{Hz} ; \%$ composition $=$ percentage area of total spectra area
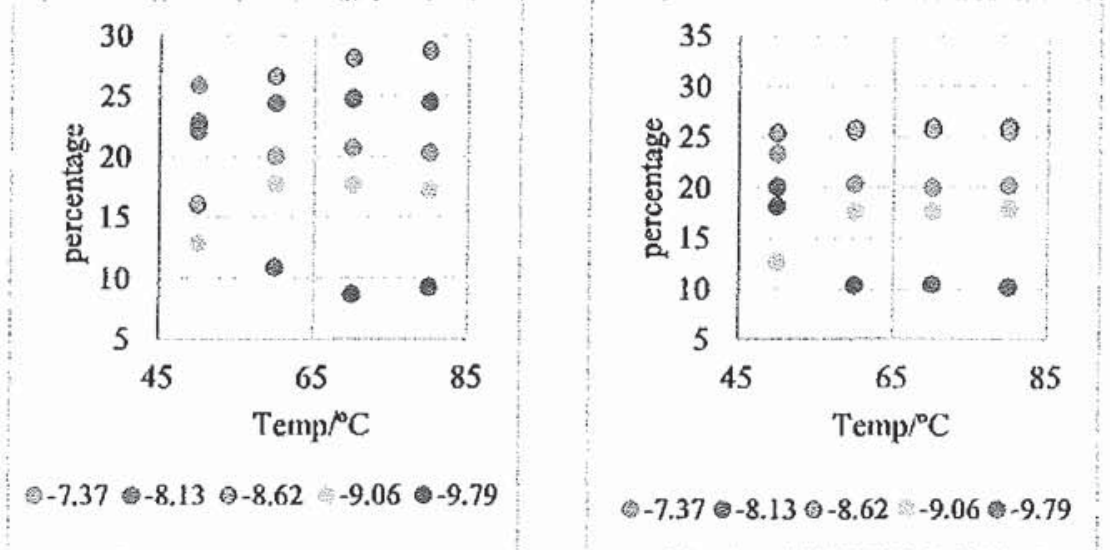

Figure 7.17: $\quad{ }^{31} \mathrm{P}$ deconvolution area for the 5 components from 50 to $80{ }^{\circ} \mathrm{C}$ for $\mathrm{NC} 5 \%$ and annealed NC $5 \%$ obtained at an MAS rate of $10 \mathrm{kHz}$

To conclude, each component area distribution is compared between all 4 samples. The percentage area for the two components at -4.55 and $-9.88 \mathrm{ppm}$ decrease with increasing temperature, most likely representing the rigid crystalline environment. The 
other two components at -8.12 and $-8.68 \mathrm{ppm}$ have increased percentage area with increased temperature, are most likely assigned to the mobile amorphous environment.

\subsubsection{3 ${ }^{19} \mathrm{~F}$ DP NMR spectra for four samples from $20-80^{\circ} \mathrm{C}$ at an MAS of $10 \mathrm{kHz}$}

The method for establishing the deconvolution model for ${ }^{31} \mathrm{P}$ NMR is also applied to find models for ${ }^{19} \mathrm{~F}$ NMR spectra. The non-annealed PBFP and non-annealed NC $5 \%$ can be fitted with the same model. The parameters are listed in Table 7.10 and the model is presented in Figure 7.18 .

Table 7.10: $\quad{ }^{19} \mathrm{~F}$ NMR spectral deconvolution parameters for PBFP and NC $5 \%$ over $20-40{ }^{\circ} \mathrm{C}$ obtained at an MAS rate of $10 \mathrm{kHz}$

$\begin{array}{ccc}\# & \text { ppm } & \text { Width(Hz) } \\ 1(\%) & -76.64 & 171 \\ 2(\%) & -76.73 & 538 \\ 3(\$) & -76.93 & 191\end{array}$

Note: $\mathrm{CS}=$ chemical shift in ppm; Width = linewidth at half height in $\mathrm{Hz}$; PBFP

$=$ poly $\left[\right.$ bis(trifluoroethoxy)phosphazene; $\mathrm{NC} 5 \%=\mathrm{PBFP} /$ nano- $\mathrm{TiO}_{2} 5 \%$

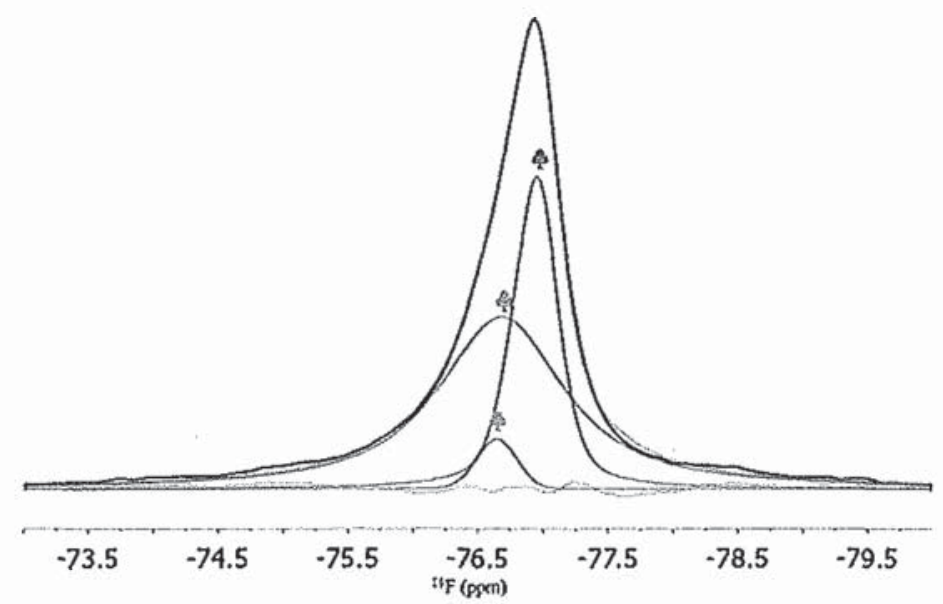

Figure 7.18: $\quad{ }^{19}$ F NMR spectral deconvolution model for PBFP and NC $5 \%$ samples over the temperature range of $20-40^{\circ} \mathrm{C}$ obtained at an MAS rate of $10 \mathrm{kHz}$ 
Table 7.11: $\quad{ }^{19} \mathrm{~F}$ NMR spectral deconvolution parameters for annealed PBFP, and annealed NC $5 \%$ for $20-40^{\circ} \mathrm{C}$ obtained at an MAS rate of $10 \mathrm{kHz}$

$$
\text { \# }
$$

1 (a)

2()

$3($ (घ)

4 (日)
Ppm

$-76.62$

$-76.71$

$-76.95$

$-77.20$
Width(Hz)

1647

266

166

127

Notc: $\mathrm{CS}=$ chemical shift in ppm; Width = linewidth at half height in $\mathrm{Hz}$; annealed PBFP = annealed poly[bis(trifluoroethoxy)phosphazene; annealed NC $5 \%=$ annealed $\mathrm{PBFP} /$ nano- $\mathrm{TiO}_{2} 5 \%$

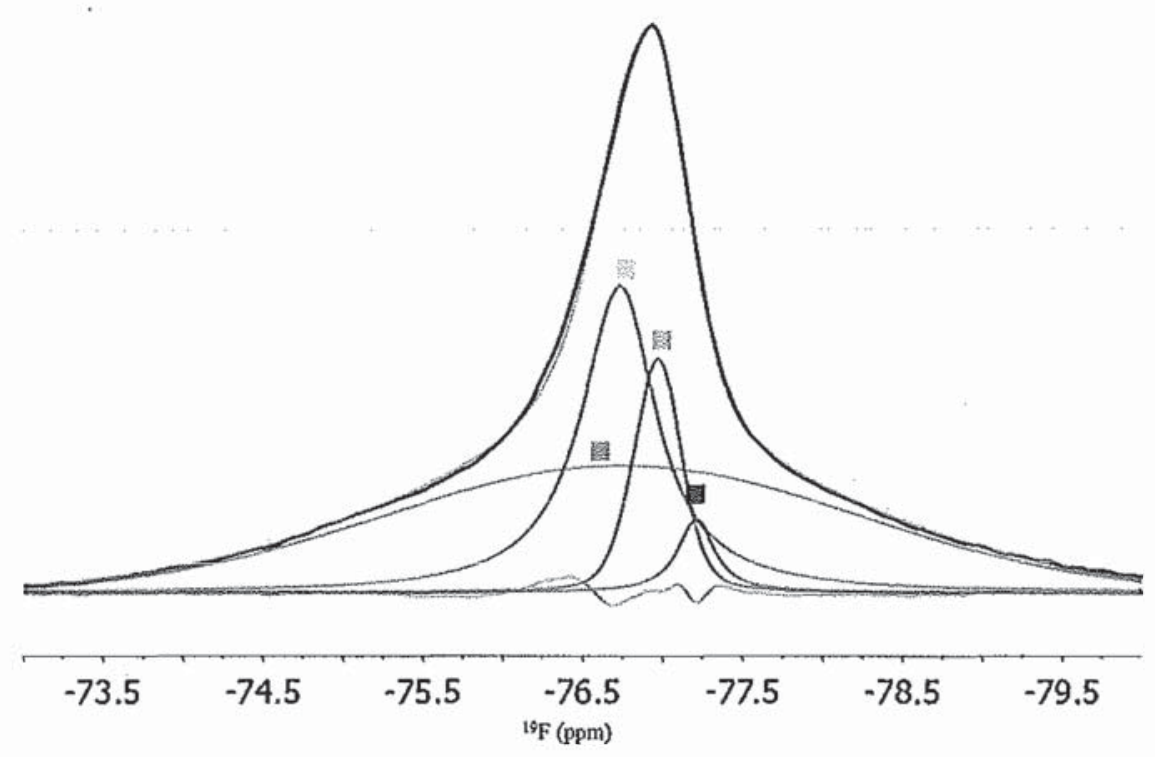

Figure 7.19: $\quad{ }^{14} \mathrm{~F}$ NMR spectral deconvolution model for annealed PBFP and annealed NC 5\% samples over the temperature range of $20-40^{\circ} \mathrm{C}$ obtained at an MAS rate of $10 \mathrm{kHz}$

Similarly, samples of annealed PBFP and anncaled NC 5\% can be fitted with a different model over the temperature range of $20-40^{\circ} \mathrm{C}$. The parameters are listed in Table 7.11 and the model is presented in Figure 7.19. The reason that the annealed samples cannot be fitted with the same model as for non-annealed (as was the case for ${ }^{31} \mathrm{P}$ ) is because fluorines are located at the side group and have fast motion, as a consequence, annealing treatment would have a more significant effect on ${ }^{19} \mathrm{~F}$ than it would for ${ }^{31} \mathrm{P}$ from the rigid backbone 
At higher temperature, all four samples can be fit with one model as listed in Table

\subsection{2 and Figure 7.20.}

Table 7.12: $\quad{ }^{19} \mathrm{~F}$ NMR spectral deconvolution parameters for 4 samples for $50-80^{\circ} \mathrm{C}$ obtained at an MAS rate of $10 \mathrm{kHz}$

$\#$

ppm

$-76.42$

$-76.76$

$-76.92$

$-77.10$
Width(Hz)

239

181

103

125

Note: $\mathrm{CS}=$ chemical shift in ppm; Width = linewidth at half height in $\mathrm{Hz}$; $\mathrm{PBFP}=$ poly[bis(trifluoroethoxy) phospltazene; NC 5\% = PBFP/nano-TiO 2 $5 \%$; annealed PBFP $=$ annealed poly[bis(trifluoroethoxy)phosphazene; annealed $\mathrm{NC} 5 \%=$ annealed PBFP/nano-TiO $2 \%$

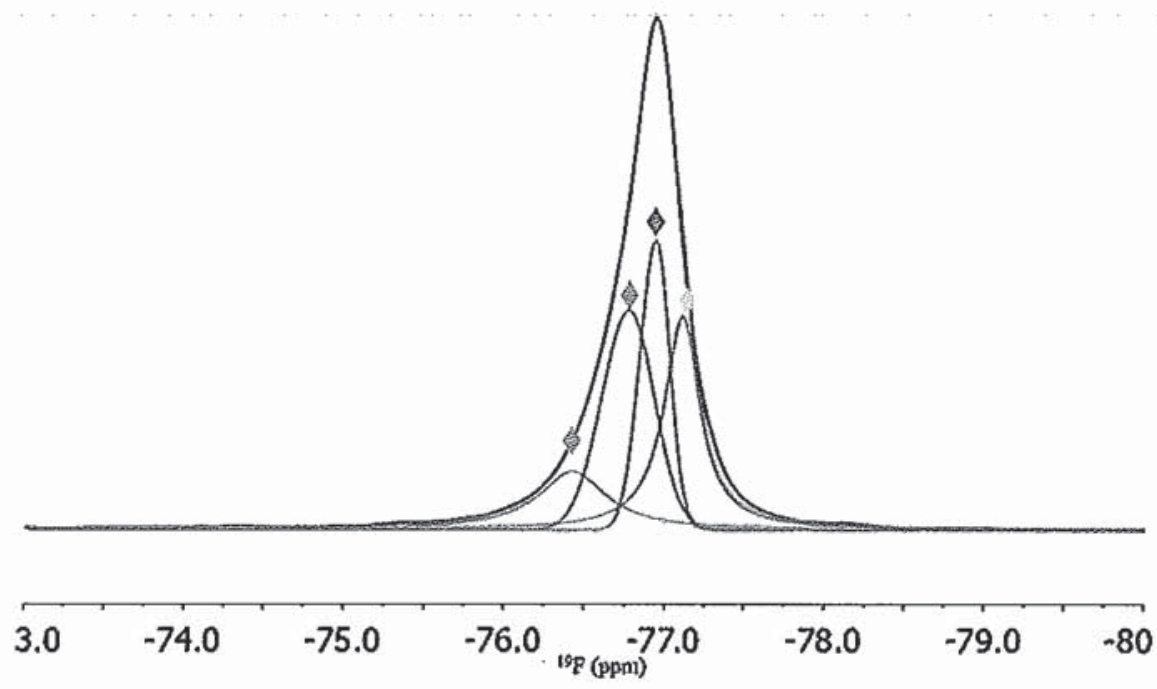

Figure 7.20: Deconvolution model for ${ }^{19} \mathrm{~F}$ NMR spectra of 4 samples at temperature of $50-80^{\circ} \mathrm{C}$ obtained at an MAS rate of $10 \mathrm{kHz}$

As discussed, ${ }^{19} \mathrm{~F}$ MAS NMR spectra of PBFP and NC $5 \%$ are deconvolved into 3 components over $20-40^{\circ} \mathrm{C}$. Samples annealed PBFP and annealed NC 5\% are deconvolved using a four-component model with a significantly broad component at $-76.62 \mathrm{ppm}$ with a line width of $1647 \mathrm{~Hz}$ over $20-40^{\circ} \mathrm{C}$. When the temperature is increased to $50^{\circ} \mathrm{C}$, all four samples are observed to have the same model with 4 relative narrow lines. 


\subsubsection{4 ${ }^{19}$ F DP NMR spectra of four samples each deconvolution percentage analysis over the temperature range over 20 to $80^{\circ} \mathrm{C}$ at an MAS rate at $10 \mathrm{kHz}$}

With the established model for ${ }^{19} \mathrm{~F}$ NMR spectra over the temperature range $20-80$ ${ }^{\circ} \mathrm{C}$, the distribution of each component as a percentage of the total signal for all four samples were calculated. These are tabulated along with the spectra throughout the following discussion.

Fluorine-19 MAS NMR spectra of PBFP and NC 5\% were acquired at an MAS rate of $10 \mathrm{kHz}$ over $20-40^{\circ} \mathrm{C}$ and are deconvolved using the model shown in Figure 7.18 and Table 7.10. The spectra corresponding to the annealed PBFP and annealed NC 5\% are deconvolved using the model shown in Figure 7.19 and Table 7.11. The area for each component is listed in Table 7.13 and Table 7.14. The trends in sample composition with temperature are shown in Figure 7.21 and Figure 7.22.

Table 7.13: ${ }^{19} \mathrm{~F}$ NMR spectral deconvolution percentage area for different conponents over the temperature range of $20-40^{\circ} \mathrm{C}$ for PBFP (left) and NC 5\% (right) obtained with an MAS rate of $10 \mathrm{kHz}$

\begin{tabular}{|c|c|c|c|c|c|c|c|}
\hline CS/ppm & $\begin{array}{c}-76.64 \\
\text { (ब) }\end{array}$ & $\begin{array}{c}-76.73 \\
\text { (क) }\end{array}$ & $\begin{array}{c}-76.93 \\
\text { (ब) }\end{array}$ & CS/ppm & $\begin{array}{c}-76.64 \\
(\Leftrightarrow)\end{array}$ & $\begin{array}{c}-76.73 \\
\text { (क) }\end{array}$ & $\begin{array}{c}-76.93 \\
\text { (ㅇ) }\end{array}$ \\
\hline Width/Hz & 171 & 538 & 191 & Width/Hz & 171 & 538 & 191 \\
\hline $\begin{array}{c}\text { Temp } \\
\left({ }^{\circ} \mathrm{C}\right)\end{array}$ & & $\begin{array}{l}\text { PBFP } \\
\text { omposit }\end{array}$ & & $\begin{array}{c}\text { Temp } \\
\left({ }^{\circ} \mathrm{C}\right)\end{array}$ & & $\begin{array}{l}\text { NC } 5 \% \\
\text { omposi }\end{array}$ & \\
\hline 20 & 7 & 59 & 34 & 20 & 14 & 26 & 43 \\
\hline 30 & 6 & 55 & 39 & 30 & 14 & 14 & 73 \\
\hline 40 & 5 & 48 & 47 & 40 & 10 & 10 & 81 \\
\hline
\end{tabular}

Note: $\mathrm{PBFP}=$ poly[bis(trifluoroethoxy)phosphazene; $\mathrm{CS}=$ chemical shift in $\mathrm{ppm}$; Width $=0$ linewidth at half height in $\mathrm{Hz}$; NC 5\%: $\mathrm{PBFP}_{\mathrm{TiO}}-5 \%$ \% composition: proportion this this component of the total spectra 

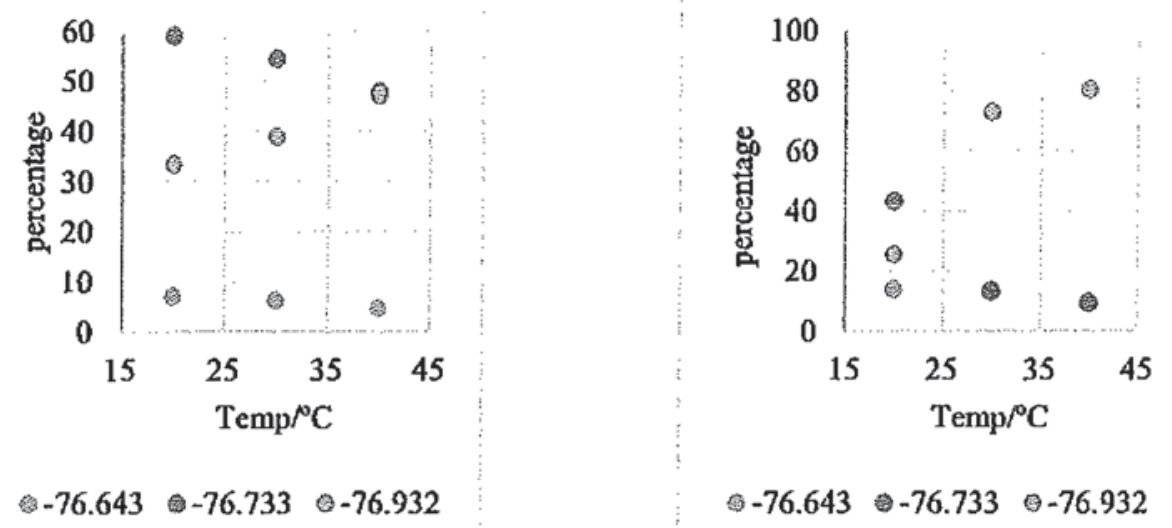

Figurc 7.21: ${ }^{19} \mathrm{~F}$ NMR spectral deconvolution percentage area changing trends for different components over the temperature range of $20-40^{\circ} \mathrm{C}$ for PBFP (left) and NC $5 \%$ $\mathrm{TiO}_{2}$ (right) obtained at an MAS rate of $10 \mathrm{kHz}$

Table 7.14: $\quad{ }^{19} \mathrm{~F}$ NMR spectral deconvolution percentage area for different components over the temperature range of $20-40^{\circ} \mathrm{C}$ for annealed PBFP (left) and annealed NC 5\% (right) obtained with an MAS rate of $10 \mathrm{kHz}$

\begin{tabular}{|c|c|c|c|c|c|c|c|c|c|}
\hline CS/ppm & $\begin{array}{c}-76.62 \\
\text { (细) }\end{array}$ & $\begin{array}{c}-76.71 \\
\text { ( })\end{array}$ & $\begin{array}{c}-76.95 \\
\text { (6) }\end{array}$ & $\begin{array}{c}-77.20 \\
\text { (E) }\end{array}$ & CS/ppm & $\begin{array}{c}-76.62 \\
\text { (a) }\end{array}$ & $\begin{array}{c}-76.71 \\
(\text { () }\end{array}$ & $\begin{array}{c}-76.95 \\
\text { (圆) }\end{array}$ & $\begin{array}{c}-77.20 \\
\text { (a) }\end{array}$ \\
\hline Width/Hz & 1647 & 265 & 166 & 127 & Width/Hz & 1647 & 265 & 166 & 127 \\
\hline \multicolumn{5}{|c|}{$\begin{array}{l}\text { Annealed PBFP } \\
\% \text { Composition }\end{array}$} & \multicolumn{5}{|c|}{$\begin{array}{l}\text { Annealed NC 5\% } \\
\% \text { Composition }\end{array}$} \\
\hline 20 & 50 & 32 & 15 & 4 & 20 & 52 & 30 & 14 & 3 \\
\hline 30 & 43 & 35 & 17 & 4 & 30 & 49 & 32 & 16 & 3 \\
\hline 40 & 30 & 43 & 24 & 3 & 40 & 40 & 35 & 21 & 3 \\
\hline
\end{tabular}

Note: PBFP $=$ poly[bis(trifluoroethoxy)phosphazene; $\mathrm{CS}=$ chemical shift in $\mathrm{ppm}$; Width $=$ linewidth at half height in $\mathrm{Hz}$; $\mathrm{NC} 5 \%$ : $\mathrm{PBFP} / \mathrm{TiO}_{2}-5 \%$; \% composition: proportion this this component of the total spectra
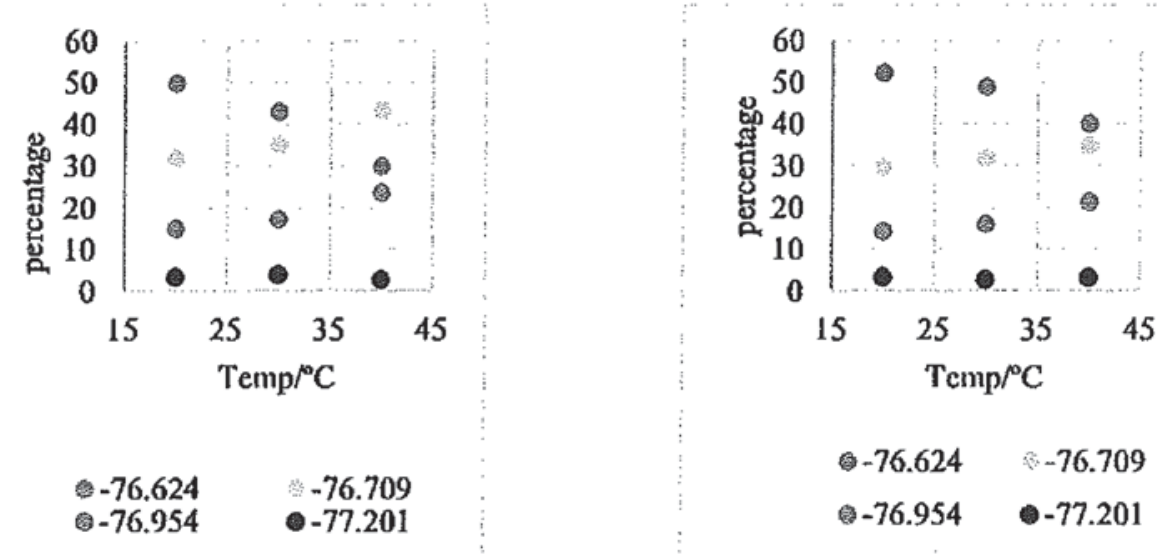

Figure 7.22: ${ }^{19} \mathrm{~F}$ NMR spectral deconvolution percentage area changing trends for different components over the temperature range over $20-40^{\circ} \mathrm{C}$ for annealed PBFP (left) and annealed $\mathrm{NC}_{5} \%-\mathrm{TiO}_{2}$ (right) obtained at an $\mathrm{MAS} 10 \mathrm{kHz}$ 
As seen in Figure 7.21, Table 7.13 and Table 7.14, the percentage area of PBFP and NC $5 \%$ for components at $-76.64-76.73 \mathrm{ppm}$ decrease as the temperature goes up from 20 to $40^{\circ} \mathrm{C}$, likely representing the crystalline phase. The proportion of the component at $76.93 \mathrm{ppm}$, with a line width of $191 \mathrm{~Hz}$ increases with increasing temperature, thus corresponds to the amorphous environment of this polymer

In Figure 7.22 , the percentage area of broad component at $-76.62 \mathrm{ppm}$ decreases as temperature increases, assuming representing the rigid crystalline environment. The percentage of this component for annealed NC $5 \%$ is lower than annealed PBFP at temperature 20,30 , and $40^{\circ} \mathrm{C}$.

For higher temperature from $50-80^{\circ} \mathrm{C},{ }^{19} \mathrm{~F}$ MAS NMR spectra of PBFP, annealed PBFP, NC 5\%, and annealed NC $5 \%$ were acquired at an MAS rate of $10 \mathrm{kHz}$ and deconvolved using the model in Figure 7.20 and Table 7.12. The deconvolution area for each component is tabulated in Table 7.15 and Table 7.16. The trend for each component with temperature is plotted in Figure 7.23 and Figure 7.24. 
Table 7.15: $\quad{ }^{19} \mathrm{~F}$ NMR spectral deconvolution percentage area for different components over the temperature range of $50-80^{\circ} \mathrm{C}$ for PBFP (left) and annealed PBFP (right) obtaincd with an MAS rate of $10 \mathrm{kHz}$

\begin{tabular}{|c|c|c|c|c|c|c|c|c|c|}
\hline CS/ppm & $\begin{array}{c}-76.42 \\
\text { (\%) }\end{array}$ & $\begin{array}{c}-76.76 \\
\text { (क) }\end{array}$ & $\begin{array}{c}-76.92 \\
\text { (॰) }\end{array}$ & $\begin{array}{c}-77.10 \\
(y\end{array}$ & CS/ppm & $\begin{array}{c}-76.42 \\
\text { (9) }\end{array}$ & $\begin{array}{c}-76.76 \\
(0)\end{array}$ & $\begin{array}{c}-76.92 \\
(8)\end{array}$ & $\begin{array}{c}-77.10 \\
()\end{array}$ \\
\hline Width/Hz & 239 & 181 & 103 & 125 & Width/Hz & 239 & 181 & 103 & 125 \\
\hline $\begin{array}{l}\text { Temp } \\
\left({ }^{\circ} \mathrm{C}\right)\end{array}$ & $\begin{array}{l}\text { Temip } \\
\text { ( } \mathrm{C} \mathrm{C})\end{array}$ & & $\begin{array}{l}\text { PBFP } \\
\text { composi }\end{array}$ & & $\begin{array}{l}\text { Temp } \\
\text { ("C) }\end{array}$ & & $\begin{array}{r}\text { PE } \\
\% \text { Com }\end{array}$ & $\begin{array}{l}\text { EP } \\
\text { position }\end{array}$ & \\
\hline 50 & 25 & 33 & 18 & 24 & 50 & 24 & 35 & 16 & 26 \\
\hline 60 & 23 & 37 & 19 & 21 & 60 & 20 & 38 & 18 & 24 \\
\hline 70 & 22 & 39 & 19 & 20 & 70 & 19 & 40 & 18 & 23 \\
\hline 80 & 21 & 40 & 19 & 21 & 80 & 18 & 41 & 19 & 22 \\
\hline
\end{tabular}

Note: $\mathrm{PBFP}=$ poly[bis(trifluoroethoxy)phosphazene; $\mathrm{CS}=$ chemical shift in $\mathrm{ppm}$; Width $=$ linewidth at half height in $\mathrm{Hz}$; NC 5\%: PBFP/TiO ${ }_{2}-5 \%$; \% composition: proportion this this component of the total spectra
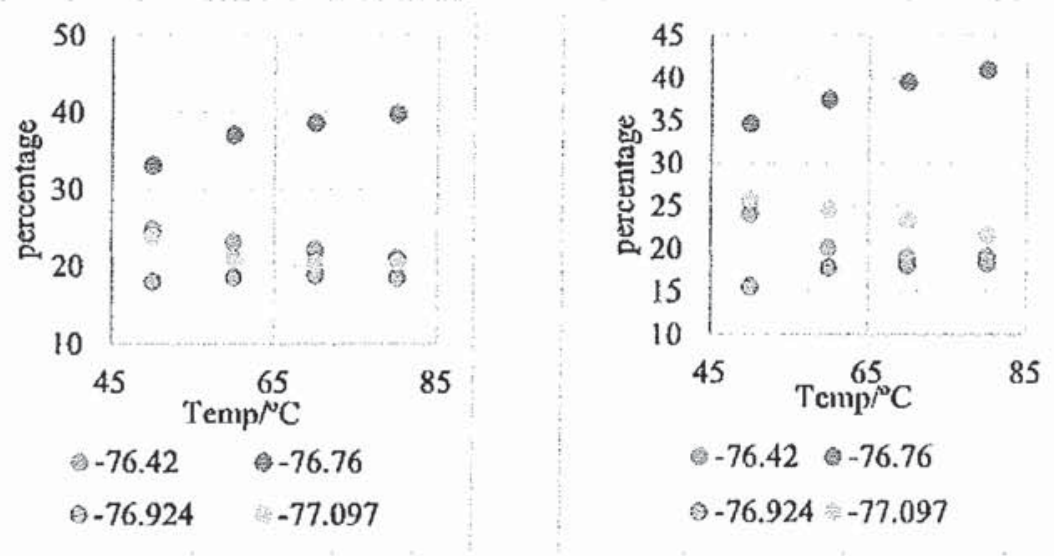

Figure 7.23: ${ }^{19} \mathrm{~F}$ NMR spectral deconvolution percentage arca changing trends for the 4 components over the temperature range of $50-80^{\circ} \mathrm{C}$ for PBFP (left) and annealed PBFP (right) obtained at an MAS $10 \mathrm{kHz}$ 
Table 7.16: ${ }^{19} \mathrm{~F}$ NMR spectral deconvolution percentage area for different components over the temperature range of $50-80^{\circ} \mathrm{C}$ for $\mathrm{NC} 5 \%$ (left) and annealed NC $5 \%$ (right) obtained with an MAS ratc of $10 \mathrm{kHz}$

\begin{tabular}{|c|c|c|c|c|c|c|c|c|c|}
\hline CS/ppm & $\begin{array}{c}-76.42 \\
(\phi)\end{array}$ & $\begin{array}{c}-76.76 \\
\text { ( })\end{array}$ & $\begin{array}{c}-76.92 \\
\text { (४) }\end{array}$ & $\begin{array}{c}-77.10 \\
()\end{array}$ & CS/ppm & $\begin{array}{c}-76.42 \\
(4)\end{array}$ & $\begin{array}{c}-76.76 \\
(\text { ) }\end{array}$ & $\begin{array}{c}-76.92 \\
(\diamond)\end{array}$ & $\begin{array}{c}-77.10 \\
(\varphi)\end{array}$ \\
\hline Width/Hz & 239 & 181 & 103 & 125 & Width/Hz & 239 & 181 & 103 & 125 \\
\hline $\begin{array}{l}\text { Temp } \\
\left({ }^{\circ} \mathrm{C}\right)\end{array}$ & \multicolumn{4}{|c|}{$\begin{array}{l}\text { Annealcd NC } 5 \% \\
\% \text { Composition }\end{array}$} & $\begin{array}{c}\text { Temp } \\
\left({ }^{\circ} \mathrm{C}\right)\end{array}$ & \multicolumn{4}{|c|}{$\begin{array}{l}\text { Annealed NC 5\% } \\
\% \text { Composition }\end{array}$} \\
\hline 50 & 19 & 31 & 21 & 29 & 50 & 24 & 35 & 16 & 25 \\
\hline 60 & 15 & 35 & 23 & 28 & 60 & 16 & 38 & 23 & 22 \\
\hline 70 & 13 & 36 & 26 & 25 & 70 & 17 & 39 & 23 & 21 \\
\hline 80 & 14 & 35 & 26 & 24 & 80 & 17 & 39 & 24 & 20 \\
\hline
\end{tabular}

Note: $\mathrm{PBFP}=$ poly[bis(tritluoroethoxy)phosphazene; $\mathrm{CS}=$ chemical shitt in ppm; Width = linewidth at half height in $\mathrm{Hz}$; NC 5\%: $\mathrm{PBFP} / \mathrm{TiO}_{2}-5 \%$; \% composition: proportion this this component of the total spectra
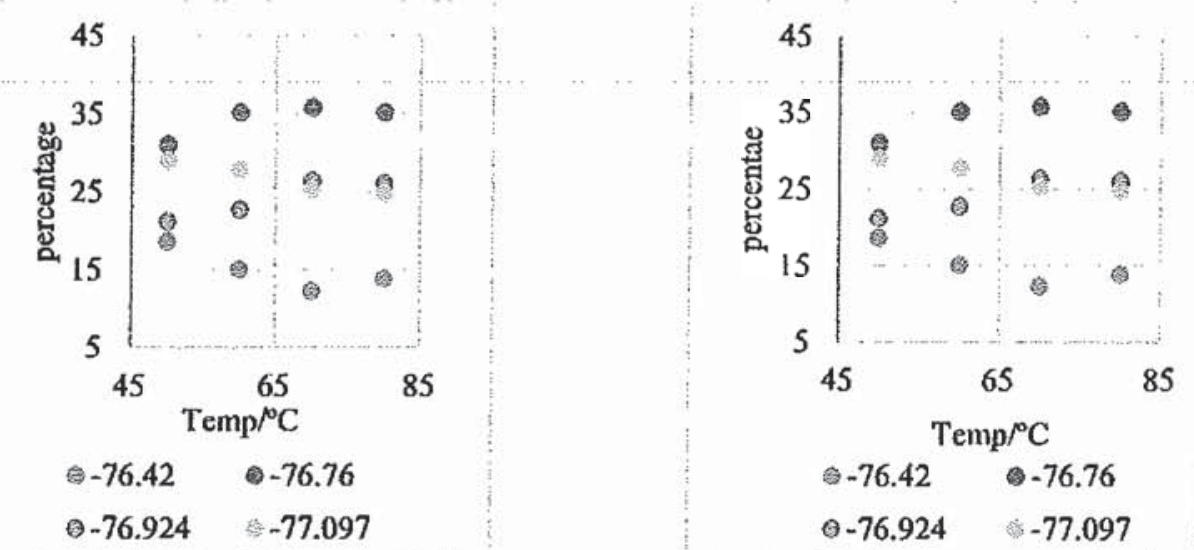

Figure 7.24: ${ }^{19} \mathrm{~F}$ NMR spectral deconvolution percentage area changing trends for the 4 components over the temperature range of 50 to $80^{\circ} \mathrm{C}$ for $\mathrm{NC}^{2} \%-\mathrm{TiO}_{2}$ (left) and annealed $\mathrm{NC} 5 \%-\mathrm{TiO}_{2}$ (right) obtained at an MAS $10 \mathrm{kHz}$

For PBFP, percentage area for components with chemical shift of -76.76 and -76.92 ppm, are increased from $33 \%, 18 \%$ to $37 \%$ and $19 \%$, respectively, with temperature increasing from 50 to $60^{\circ} \mathrm{C}$. When the temperature is increased from $60-80^{\circ} \mathrm{C}$, the percentage of each samples stays almost unchanged. The value for components at -76.42 , and $-77.10 \mathrm{ppm}$ with line width of $239 \mathrm{~Hz}$ and $125 \mathrm{~Hz}$, are decreased from $25 \%, 24 \%$ to $23 \%$ and $21 \%$, with temperature increasing from 50 to $60^{\circ} \mathrm{C}$. As temperature increased 
from $60-80^{\circ} \mathrm{C}$, the percentage areas for both components keep almost unchanged. Similar phenomena are observed for samples annealed PBFP, NC 5\% and annealed NC 5\%.

To conclude, over the temperature range from $20-40^{\circ} \mathrm{C}$, all components of ${ }^{19} \mathrm{~F}$ MAS NMR spectra are analyzed using their corresponding models. For PBFP and NC 5\% that have the same model, the percentage area for the contributions that appear at -76.64 and $76.73 \mathrm{ppm}$ decrease as the temperature increases, and are assigned to the crystalline components. The percentage area for the other component at $-76.93 \mathrm{ppm}$, increases with increasing temperature, representing the amorphous component. For annealed PBFP and annealed NC 5\% that share the same model, the percentage area for the broad component at -76.62 ppm decreases with increasing temperature. The percentage area for the other two components at -76.71 and $-76.95 \mathrm{ppm}$ increase as temperature increases, the small component appearing at $-77.20 \mathrm{ppm}$ has a slightly change.

At higher temperature from 50 to $80^{\circ} \mathrm{C}$, four samples have the same model. The area fraction for components at -76.42 and $-77.10 \mathrm{ppm}$ decrease with increasing temperature, while the components appear at -76.76 and $-76.92 \mathrm{ppm}$ increase with increasing temperature, besides, all four component have reached their maximum at certain temperature.

\subsection{Conclusion}

The ${ }^{31}$ P DP NMR spectra of PBFP, annealed PBFP, NC $5 \%$ and annealed NC $5 \%$ were acquired at an MAS rate of $10 \mathrm{kHz}$. The spectra were deconvolved to reveal morphological information about different domains within the polymer matrix. At low temperature from 20 to $40^{\circ} \mathrm{C}$, the ${ }^{31} \mathrm{P}$ NMR spectra of all four samples are deconvolved 
into four components, i.e., one very broad component at $-4.55 \mathrm{ppm}$ with linewidth at 834 $\mathrm{Hz}$, and three narrow components between -8.12 and $-9.88 \mathrm{ppm}$. At temperatures from 50 $80^{\circ} \mathrm{C}$, where the polymer transformed to a liquid crystal phase. The spectra were deconvolved using a five-component model all with narrower linewidth compared to those from the spectra recorded at the low temperature range.

Similarly, ${ }^{19} \mathrm{~F}$ DP NMR spectra of four samples were acquired at an MAS rate of 10 $\mathrm{kHz}$. At the low temperature range, the ${ }^{19} \mathrm{~F}$ NMR spectra of PBFP and NC $5 \%$ were deconvolved into 3 contributions based on the study of the relaxation arrays. For annealed PBFP and annealed NC 5\%, the spectra were deconvolved using a four-component model with a very broad contribution at $-76.62 \mathrm{ppm}$ with a linewidth of $1647 \mathrm{~Hz}$. At the higher temperature range, the ${ }^{19} \mathrm{~F}$ NMR spectra of all four samples were deconvolved using the same four-component model.

The proportion of the different components that make up all ${ }^{31} \mathrm{P}$ DP NMR spectra of four samples were studied over the two different temperature ranges. The proportion of the broad component at $-4.55 \mathrm{ppm}$ decreases with increasing temperature and the proportions of the two narrow components at -8.12 and -8.68 ppm increase with temperature. The small remaining component at $\mathbf{- 9 . 8 8} \mathrm{ppm}$ showed a slightly decrease in percentage. This suggests that the motion of the broad component is increased when the temperature approaches or is raised above its $\mathrm{T}(1)$ temperature, which is accompanied by a change in the backbone and sidechain environments resulting in a shift to higher field. The same phenomenon was also observed in the ${ }^{19} \mathrm{~F}$ NMR spectra. Since the sidechain undergoes a faster motion than the backbone, it is more difficult to attain confidence deconvolution in the corresponding 
separately. The component at $-8.68(\theta)$ ppm has the smallest $\mathrm{E}_{\mathrm{a}}$ at $2.99 \mathrm{~kJ} \cdot \mathrm{mol}^{-1}$, and should also be in its own phase category.

Table 8.3: $\quad{ }^{31} \mathrm{P} \mathrm{T}_{2}$ times of solid non-annealed PBFP over the temperature range of 20 to $40^{\circ} \mathrm{C}$ obtained with an MAS rate of $10 \mathrm{kHz}$

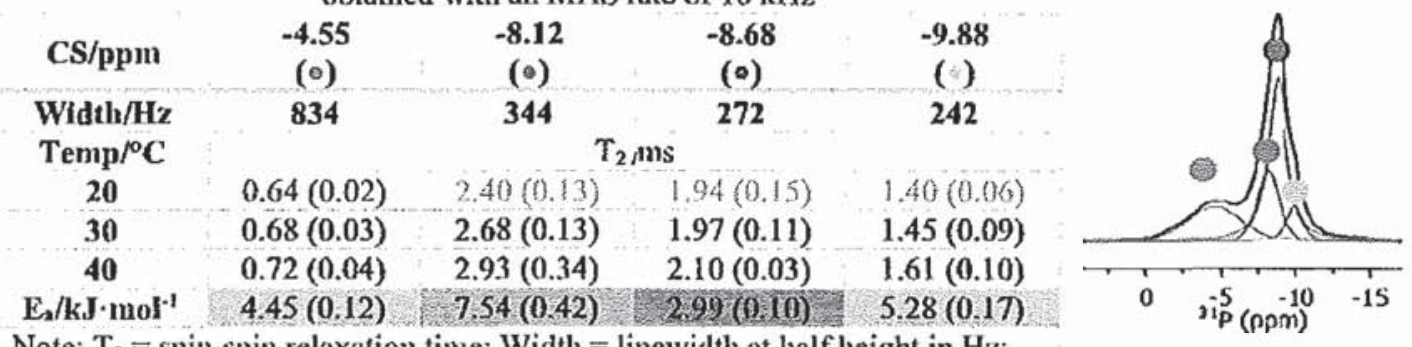

Note: $\mathrm{T}_{2}=$ spin-spin relaxation time; Width $=$ linewidth at half height in $\mathrm{Hz}$;

PBFP = poly[bis(trifluoroethoxy)phosphazenc; $\mathrm{CS}=$ chemical shift in ppm;

The standard deviations are listed in brackets

Table 8.4: $\quad{ }^{31} \mathrm{P} \mathrm{T}_{2}$ times of solid non-annealed PBFP over the temperature range of 50 to

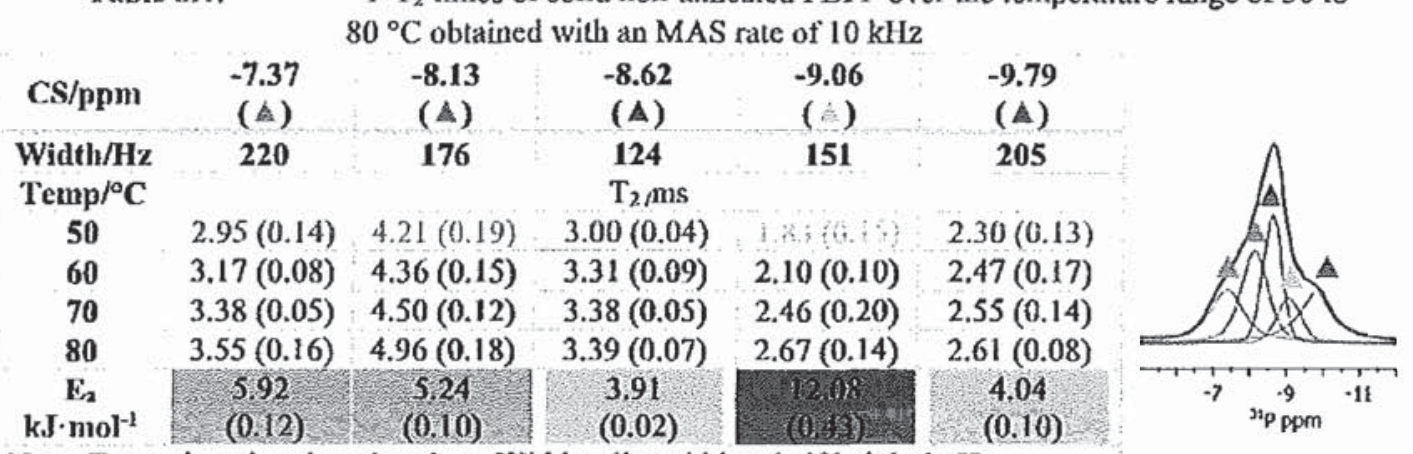

Note: $\mathrm{T}_{2}=$ spin-spin relaxation time; Width = linewidth at half height in $\mathrm{Hz}$;

PBFP = poly[bis(trifluoroethoxy)phosphazene; CS = chemical shift in ppm;

The standard deviations are listed in brackets

The ${ }^{3 i} \mathrm{P} \mathrm{T}_{2}$ parameters of non-annealed PBFP from $50-80^{\circ} \mathrm{C}$ are listed in Table 8.4, showing that all five components increase with temperature, similar to $20-40{ }^{\circ} \mathrm{C}$. The activation energies are different from each other, the components at $-7.37(\Delta)$ and -8.13 (今) ppm have similar $\mathrm{E}_{\mathrm{a}}$ values at 5.92 and $5.24 \mathrm{~kJ} \cdot \mathrm{mol}^{-1}$, and thus can be grouped together to the same phase group. The components at -8.62 (A) and -9.79 (A) ppm also have a similar $\mathrm{E}_{\mathrm{a}}$ values at 3.91 and $4.04 \mathrm{~kJ} \cdot \mathrm{mol}^{-1}$, and are considered together. The component at $-9.06(4)$ ppm has the largest $E_{\mathrm{a}}$ at $12.08 \mathrm{~kJ} \cdot \mathrm{mol}^{-1}$, and thus has a different phase. 


\subsection{3 ${ }^{31} \mathrm{P}$ NMR spin-lattice relaxation time in the rotating frame $\left(\mathrm{T}_{1 \rho}\right)$ of PBFP at an MAS rate of $10 \mathrm{kHz}$}

The ${ }^{31} \mathrm{P} \mathrm{T}_{I_{\mathrm{p}}}$ values of each component from sample PBFP are tabulated in Table 8.5 , showing that the $T_{1 \rho}$ values increase with temperature for the three components at -8.68 , 9.88 and $-8.12 \mathrm{ppm}$, implying that the backbone local motion in those environments is fast with respect to the time scale defined by the spin-locking field strength of $77 \mathrm{kHz}$, which is $13 \mu \mathrm{s}$. In contrast, the $T_{I_{\rho}}$ values of the signal at $-4.55 \mathrm{ppm}$, decrease with temperature, implying that in this environment backbone motion is slow on thel3 $\mu$ s time scale.

Table 8.5: $\quad{ }^{31} \mathrm{P} T_{\iota_{p}}$ times of solid non-annealed PBFP over the temperature range of 20 to

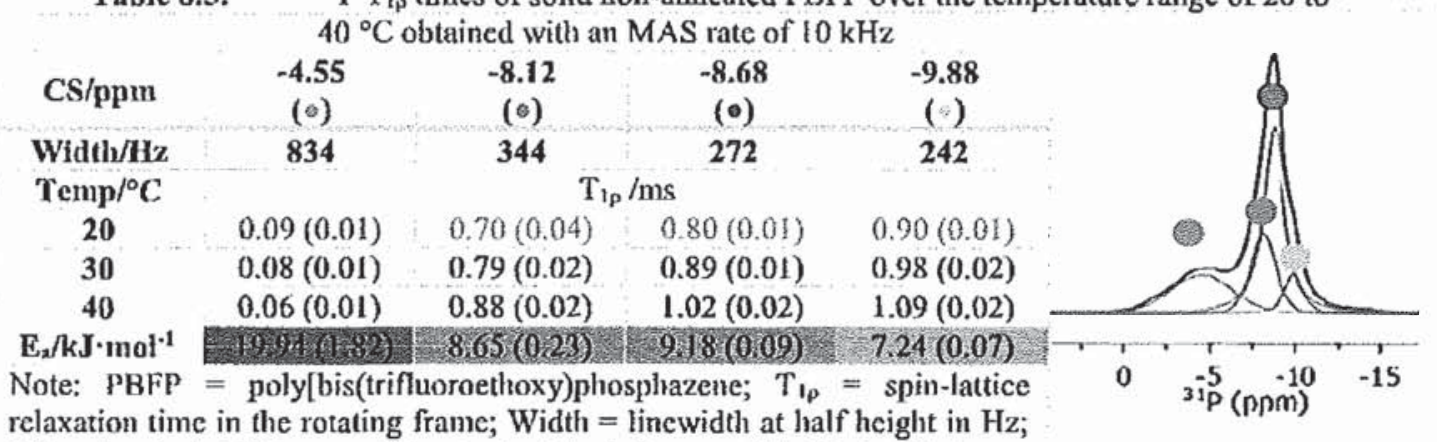
$\mathrm{CS}=$ chemical shift in ppm; The standard deviations are listed in brackets

In Table 8.5 , the component at $-4.55 \mathrm{ppm}$ has a uniquely small and negative $\mathrm{E}_{\mathrm{a}}$ at $19.94 \mathrm{~kJ} \cdot \mathrm{mol}^{-1}$ and thus is assigned to its own phase category. While the other three components at $-9.88,-8.12$ and $-9.18 \mathrm{ppm}$ are best considered together in their own phase group as their $\mathrm{E}_{\mathrm{a}}$ results are similar and are all positive.

At higher tcmperature, from $50-80^{\circ} \mathrm{C}$, the corresponding $\mathrm{T}_{1 \rho}$ results are listed in Table 8.6, showing that the $T_{1 \rho}$ values of all components increase with increasing temperature, similar to the three narrow components in Table 8.5. The two components at -7.37 and $-9.79 \mathrm{ppm}$ have a similar relative small $\mathrm{E}_{\mathrm{d}}$ values at 2.99 and $3.94 \mathrm{~kJ} \cdot \mathrm{mol}^{-1}$, thus putting them together into similar phase category. The two components at -8.13 and -8.62 
ppm have a distinct large $\mathrm{E}_{\mathrm{a}}$ at 15.36 and $11.03 \mathrm{~kJ} \cdot \mathrm{mol}^{-1}$, placing them in their own phase group. The component at $-9.06 \mathrm{ppm}$ has a different $\mathrm{E}_{\mathrm{a}}$ value at $8.67 \mathrm{~kJ} \cdot \mathrm{mol}^{-1}$, is mostly likely considered separate. The $\mathrm{T}_{10}$ values would place the components at -8.62 and -9.06 ppm together, furthermore those at -7.37 and -8.13 ppm would also be in their own phase group.

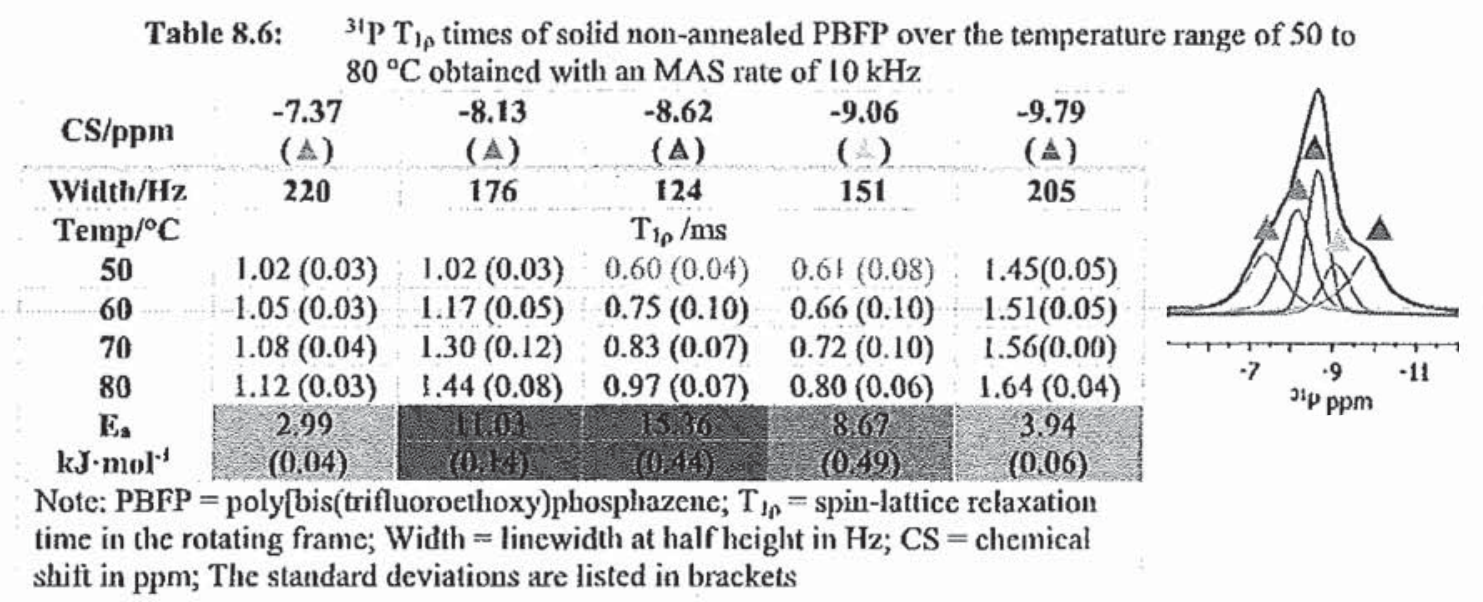

\subsection{4 ${ }^{19} \mathrm{~F}$ spin-lattice relaxation time $\left(\mathrm{T}_{1}\right)$ of $\mathrm{PBFP}$ at an MAS rate of $10 \mathrm{kHz}$}

Table 8.7 shows the $T_{1}$ values for each component from sample PBFP. As temperature increases, the $T_{1}$ values of all four components increase, meaning that the sidechain is in the fast motion regime, with respect to the ${ }^{19} \mathrm{~F}$ Larmor frequency. In the fast motion regime, the relaxation times increase with decreasing correlation times, hence with increasing temperature. The activation energies for all three components are positive also indicating fast motion, however they are different from each other, where the component at $-76.73 \mathrm{ppm}$ has the smallest $\mathrm{E}_{\mathrm{a}}$ value. 
Table 8.7: $\quad{ }^{19} \mathrm{~F} \mathrm{~T}_{1}$ times of solid non-annealed PBFP over the temperature range of 20 to $40^{\circ} \mathrm{C}$ obtained with an MAS rate of $10 \mathrm{kHz}$

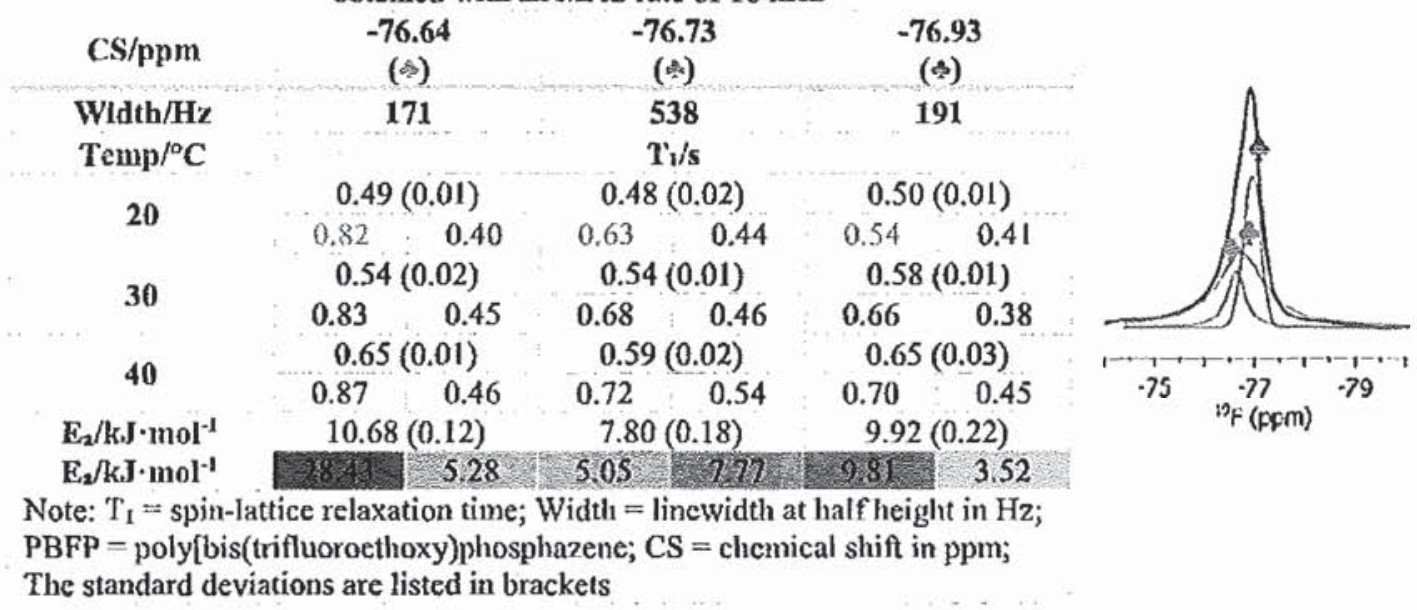

The ${ }^{19} \mathrm{~F} \mathrm{~T}_{1}$ values exhibit bi-exponential decay for all three components as indicated by two distinct relaxation times: one long $\mathrm{T}_{1}$ and one short $\mathrm{T}_{1}$. In addition, for both the fast and slow decaying components, the $T_{1}$ values consistently increase with temperature.

The ${ }^{19} \mathrm{~F} \mathrm{~T}_{1}$ data from sample PBFP over $50-80^{\circ} \mathrm{C}$ are listed in Table 8.8 , showing that with increasing temperature from 50 to $80^{\circ} \mathrm{C}$, the $\mathrm{T}_{1}$ values for all four components decrease, similarly to $20-40^{\circ} \mathrm{C}$, and thus are in the fast motion regime as indicated by positive activation energies. The broad component at -76.42 ppm has the largest $\mathrm{E}_{\mathrm{a}}$ at 10.17 $\mathrm{kJ} \cdot \mathrm{mol}^{-1}$ is assigned to its own phase group. Moreover, the components at $-76.76,-76.92$ and $-77.10 \mathrm{ppm}$ have a much smaller $\mathrm{E}_{\mathrm{a}}$ when compared to the broad component, thus can be assigned to their own phase group. 
Table 8.8: $\quad{ }^{19} \mathrm{~F} \mathrm{~T}_{1}$ times of solid non-annealed PBFP over the temperature range of 50 to $80^{\circ} \mathrm{C}$ obtained with an MAS rate of $10 \mathrm{kHz}$

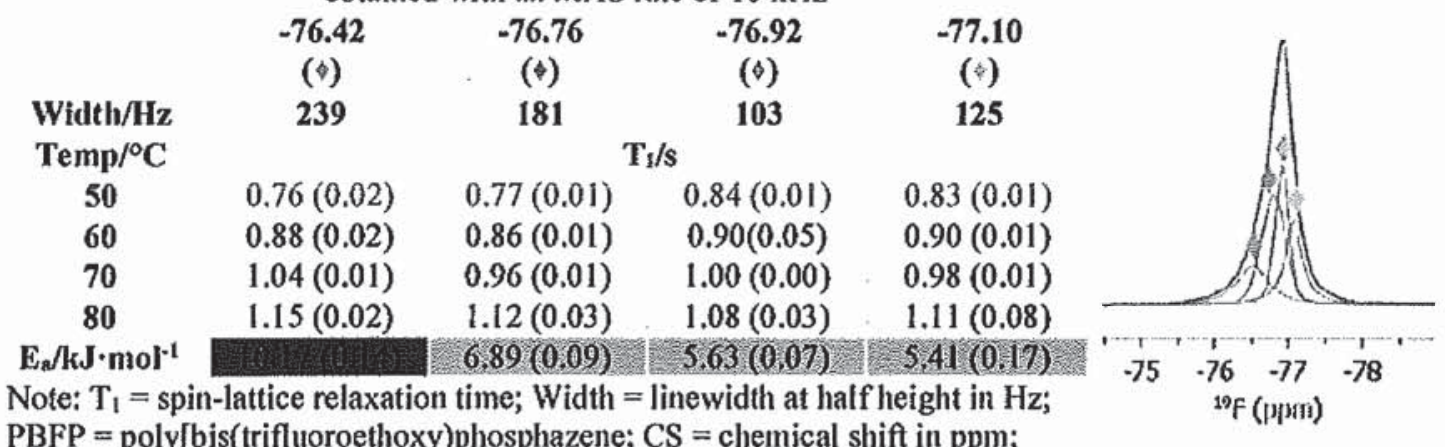

The standard deviations are listed in brackets

\subsection{5 ${ }^{19} \mathrm{~F}$ spin-spin relaxation time ( $\left.\mathrm{T}_{2}\right)$ of PBFP at an MAS rate of $10 \mathrm{kHz}$}

Over the low temperature range from 20 to $40{ }^{\circ} \mathrm{C}$, the ${ }^{19} \mathrm{~F} \mathrm{~T}_{2}$ values are listed in Table 8.9. For the higher temperature over $50-80^{\circ} \mathrm{C}$, the $\mathrm{T}_{2}$ results of each component are listed in Table 8.10.

Table 8.9: $\quad{ }^{19} \mathrm{~F} \mathrm{~T}_{2}$ times of solid non-annealed PBFP over the temperature range of 20 to $40{ }^{\circ} \mathrm{C}$

\begin{tabular}{|c|c|c|c|}
\hline \multicolumn{4}{|c|}{ obtained with an MAS rate of $10 \mathrm{kHz}$} \\
\hline & -76.64 & -76.73 & -76.93 \\
\hline CS/ppm & $(\omega)$ & («) & $(\%)$ \\
\hline Width/Hz & 171 & 538 & 191 \\
\hline Temp $/{ }^{\circ} \mathrm{C}$ & & $\mathrm{T}_{2} \mathrm{~ms}$ & \\
\hline 20 & $1.64(0.06)$ & $1.10(0.04)$ & $1.66(0.02)$ \\
\hline 30 & $1.65(0.07)$ & $1.16(0.08)$ & $1.68(0.02)$ \\
\hline 40 & $1.75(0.06)$ & $1.26(0.08)$ & $1.88(0.03)$ \\
\hline $\mathrm{Ga} / \mathrm{kJ} \cdot \mathrm{mol}^{-1}$ & $5.40 .03)$ & $5.13(0.16)$ & 3.22 (0.03) \\
\hline
\end{tabular}

Note: $\mathrm{T}_{2}=$ spin-spin relaxation time; Width $=$ linewidth at half height in $\mathrm{Hz}$; PBFP = poly[bis(trifluoroethoxy)phosphazene; $\mathrm{CS}=$ chemical shift in ppm; The standard deviations are listed in brackets

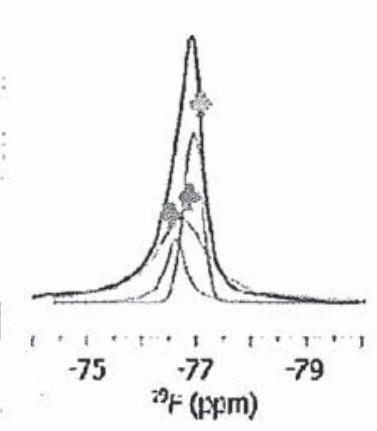

Both Table 8.9 and 8.10 show $\mathrm{T}_{2}$ increasing with temperature. In Table 8.9, the component at $-76.73 \mathrm{ppm}$ has a large $E_{\mathrm{a}}$ at $5.13 \mathrm{~kJ} \cdot \mathrm{mol}^{-1}$ and the smallest $T_{2}$ and should be considered in its own domain. The components at -76.64 and -76.93 ppm have similar $\mathrm{E}_{\mathrm{a}}$ values at 2.45 and $3.27 \mathrm{~kJ} \cdot \mathrm{mol}^{-1}$ and also have similar $\mathrm{T}_{2}$ values, hence they should be assigned to the same phase group. Likewise, in Table 8.10 , the component at $-77.10 \mathrm{ppm}$ 
has a large $E_{a}$ at $9.32 \mathrm{~kJ} \cdot \mathrm{mol}^{-1}$ when compared to the remaining components, and should be assigned to its own phase category. The components at -76.42 and $-76.92 \mathrm{ppm}$ have similar $E_{a}$ values at 6.90 and $6.34 \mathrm{~kJ} \cdot \mathrm{mol}^{-1}$ putting them into the same phase group. The $\mathrm{E}_{\mathrm{a}}$ of component at $-76.76 \mathrm{ppm}$ differs from the others at $7.50 \mathrm{~kJ} \cdot \mathrm{mol}^{-1}$, and thus could be placed in its own phase group. The components a -76.43 and $-77.10 \mathrm{ppm}$ could be considered together as they have similar $\mathrm{T}_{2}$ values.

Table 8.10 $\quad{ }^{19} \mathrm{~F} \mathrm{~T}_{2}$ times of solid non-annealed PBFP over the temperature range of 50 to $80^{\circ} \mathrm{C}$ obtained with an MAS rate of $10 \mathrm{kHz}$

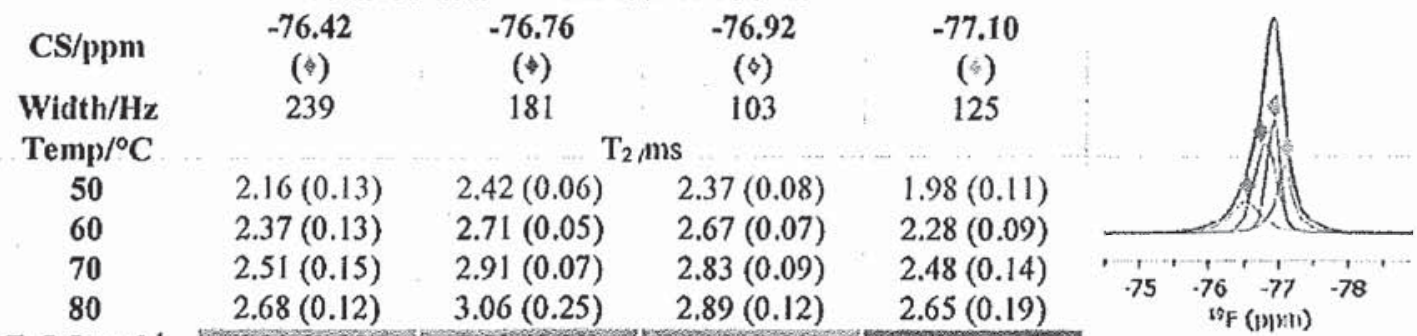

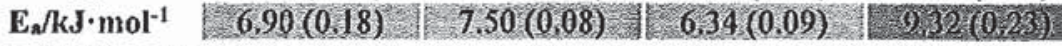

Note: $\mathrm{T}_{2}=$ spin-spin relaxation time; Width = linewidth at half height in $\mathrm{Hz}$; $\mathrm{PBFP}=$ poly $[$ bis(trifluoroethoxy)phosphazene; $\mathrm{CS}=$ clemical shift in ppm;

The standard deviations are listed in brackets

\subsection{6 ${ }^{19} \mathrm{~F}$ spin-lattice relaxation time in the rotating frame $\left(\mathrm{T}_{1 \rho}\right)$ of PBFP at an MAS rate of $10 \mathrm{kHz}$}

Table 8.11: $\quad{ }^{19} \mathrm{FT}_{1 \rho}$ times of solid non-annealed PBFP over the temperature range of 20 to $40^{\circ} \mathrm{C}$ obtained with an MAS rate of $10 \mathrm{kHz}$

$$
\begin{array}{ll}
-76.64 & -76.73
\end{array}
$$

CS/ppm

Width/Hz

Temp $/{ }^{\circ} \mathrm{C}$

20

30

40

$\mathrm{E}_{\mathrm{a}} / \mathrm{kJ} \cdot \mathrm{mol}^{-1}$
(눈)

171

$(\stackrel{n}{*})$

538

$\mathrm{T}_{1 \mathrm{p}} / \mathrm{ms}$

$1.06(0.01)$

$1.11(0.02)$

$1.21(0.02)$

$5.00(0.04)$
$-76.93$

(4)

191

$$
1.25(0.01)
$$

$1.36(0.02)$

$1.51(0.01)$

$7.14(0.03)$

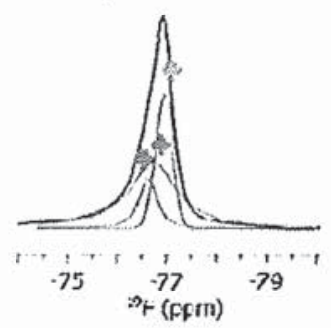

Note: $\mathrm{PBFP}=$ poly[bis(trifluoroethoxy)phosphazene; $\mathrm{T}_{1 \rho}=$ spin-lattice relaxation time in the rotating frame; Width = linewidth at half height in $\mathrm{Hz}$; $\mathrm{CS}=$ chemical shift in ppm; The standard deviations are listed in brackets

${ }^{19} \mathrm{~F} \mathrm{~T}_{1 \mathrm{p}}$ data of PBFP from $20-40{ }^{\circ} \mathrm{C}$ are shown in Table 8.11. At the higher temperature range, from $50-80^{\circ} \mathrm{C}$, the $\mathrm{T}_{1 \mathrm{p}}$ results are listed in Table 8.12. Both tables show 
that the $T_{1 p}$ values of all components increase with increasing temperature, meaning that the local sidechain motion of fluorine is on the fast motion regime, which is further indicated by positive activation energies for all components.

Table 8.12: $\quad{ }^{19} \mathrm{~F} \mathrm{~T}_{1 \rho}$ times of solid non-annealed PBFP over the temperature range of 50 to $80^{\circ} \mathrm{C}$ obtained with an MAS rate of $10 \mathrm{kHz}$

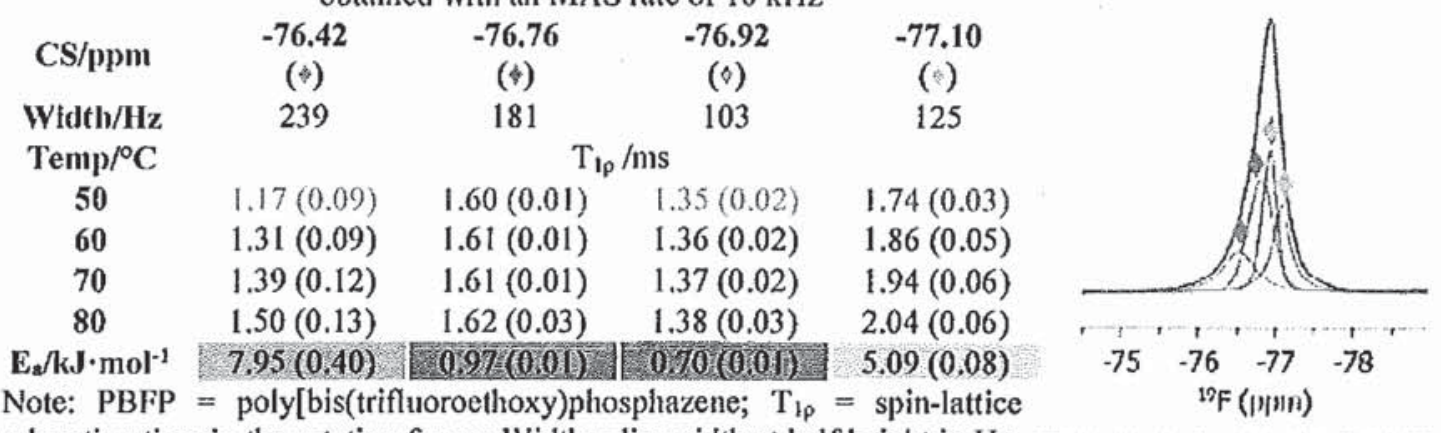
relaxation time in the rotating frame; Width = linewidth at half height in $\mathrm{Hz}$; $\mathrm{CS}=$ chemical shift in ppm; The standard deviations are listed in brackets

In Table 8.11 , the component at $-76.64 \mathrm{ppm}$ has a relative large $\mathrm{E}_{\mathrm{a}}$ at $5.09 \mathrm{~kJ} \cdot \mathrm{mol}^{-1}$, is assigned to its own phase group, The other two components at -76.73 and $-76.93 \mathrm{ppm}$ have relatively similar $\mathrm{E}_{\mathrm{a}}$ values and $\mathrm{T}_{\mathrm{l} p}$ results, and thus belong to the same phase group. Similarly, in Table 8.12 , the two components at -76.92 and $-76.76 \mathrm{ppm}$ have similar small $\mathrm{E}_{\mathrm{a}}$ at 0.70 and $0.97 \mathrm{~kJ} \cdot \mathrm{mol}^{-1}$, grouping them together. The components at -76.42 and -77.10 ppm have similar large $E_{a}$ at 7.95 and $5.09 \mathrm{~kJ} \cdot \mathrm{mol}^{-1}$, putting them being together. The $T_{1 \rho}$ times would group -7.92 and $-76.42 \mathrm{ppm}$ together, having the smaller values, while -77.10 and $76.76 \mathrm{ppm}$ would be in the same group as they have the longer values.

\subsubsection{Conclusion}

In summary, the ${ }^{31} \mathrm{P}$ NMR spectra of PBFP were deconvolved using a fourcomponent model over $20-40^{\circ} \mathrm{C}$, and a five-component model from $50-80^{\circ} \mathrm{C}$. At the low temperature range, the $T_{1}$ data show that component at $-4.55 \mathrm{ppm}$ is composed of two long $T_{1}$ contributions, the three narrow components all contain one long $T_{1}$ and one short $T_{1}$ 
contributions. The $T_{1 \rho}$ values of component at -4.55 are smaller than $0.009 \mathrm{~ms}$, which separate it from the other three components. At high temperature, the component at -7.37 ppm and $-8.13 \mathrm{ppm}$ are of similar motion as indicated by their similar $\mathrm{E}_{\mathrm{a}}$ values. Because of the increased motion above its $\mathrm{T}(1)$ transition temperature, the difference between each component becomes smaller and more difficult to distinguish.

A three-component model is used to deconvolve the ${ }^{19} \mathrm{~F}$ NMR spectra of PBFP over $20-40^{\circ} \mathrm{C}$, and a four-component model from $50-80^{\circ} \mathrm{C}$. At the low temperature range, the ${ }^{19} \mathrm{~F}_{1}$ data show bi-exponential behavior, each component is composed of one longer $\mathrm{T}_{1}$ and one shorter $T_{1}$. The $T_{2}$ results show that the broad component at -76.73 ppm has a distinct smaller $T_{2}$ compared to other two narrow components, indicating that it arises from different domains within the polymer. The $T_{1 \rho}$ data showed that all three components have similar $T_{1}$ values, suggesting that they should appear in the same motion regime with respect to the locking power. At higher temperature, the sidechain motion of all polymer domains is enhanced, the differences becomes smaller and more difficult to separate. 


\subsection{Solid-State NMR Relaxation Data of Annealed PBFP}

\subsection{1 ${ }^{31} \mathrm{P}$ spin-lattice relaxation time $\left(\mathrm{T}_{\mathrm{l}}\right)$ of annealed PBFP at an MAS rate of $10 \mathrm{kHz}$}

The ${ }^{31} \mathrm{P} \mathrm{T}_{1}$ values of annealed PBFP of each component at the temperature range from $20-40^{\circ} \mathrm{C}$ are listed in Table 8.13. The ${ }^{31} \mathrm{P}_{1}$ data from $50-80^{\circ} \mathrm{C}$ are listed in Table 8.14 .

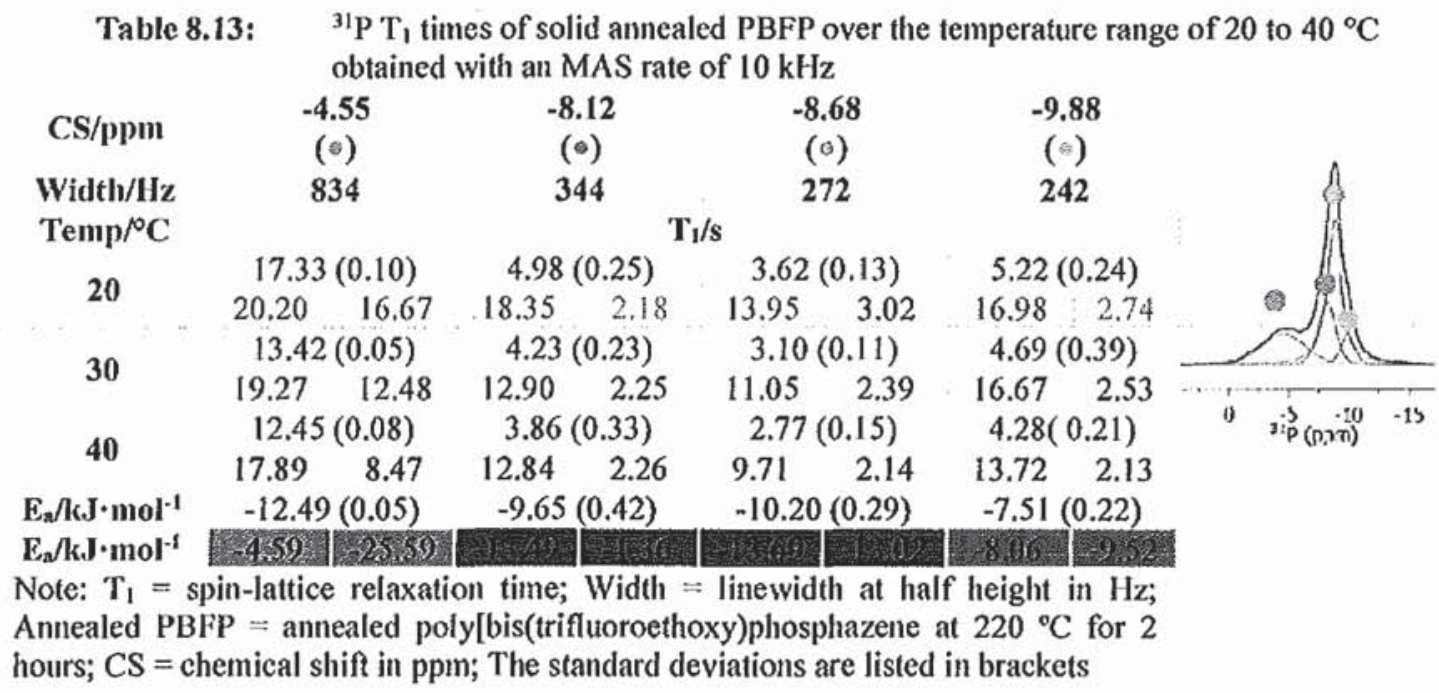

As indicated in Table 8.13 and Table 8.14 , all the $\mathrm{T}_{1}$ values for ${ }^{31} \mathrm{P}$ using an MAS rate of $10 \mathrm{kHz}$ for annealed PBFP are decreasing with increasing temperature, similar to non-annealed PBFP, with negative activation energies, indicating that the backbone dynamics in annealed PBFP are also in the slow motion regime. However, in Table 8.13, the component that appears at $-4.55 \mathrm{pm}$ has much larger $\mathrm{T}_{1}$ values than the other three remaining components. This difference is also reflected by a much large (small negative) $\mathrm{E}_{\mathrm{a}}$ value of $-12.49 \mathrm{~kJ} \cdot \mathrm{mol}^{-1}$. The $\mathrm{E}_{\mathrm{a}}$ for the component at -8.68 and -8.12 ppın have a similar value, therefore, are assigned together to one phase group with similar phase structure. The component at -9.88 has the smallest (largest negative) $\mathrm{E}_{\mathrm{a}}$ is mostly likely belongs to its own phase category. 
Table 8.14. $\quad{ }^{31} \mathrm{P} \mathrm{T}_{1}$ times of solid annealed PBFP over the temperature range of 50 to $80^{\circ} \mathrm{C}$ obtained with an MAS rate of $10 \mathrm{kHz}$

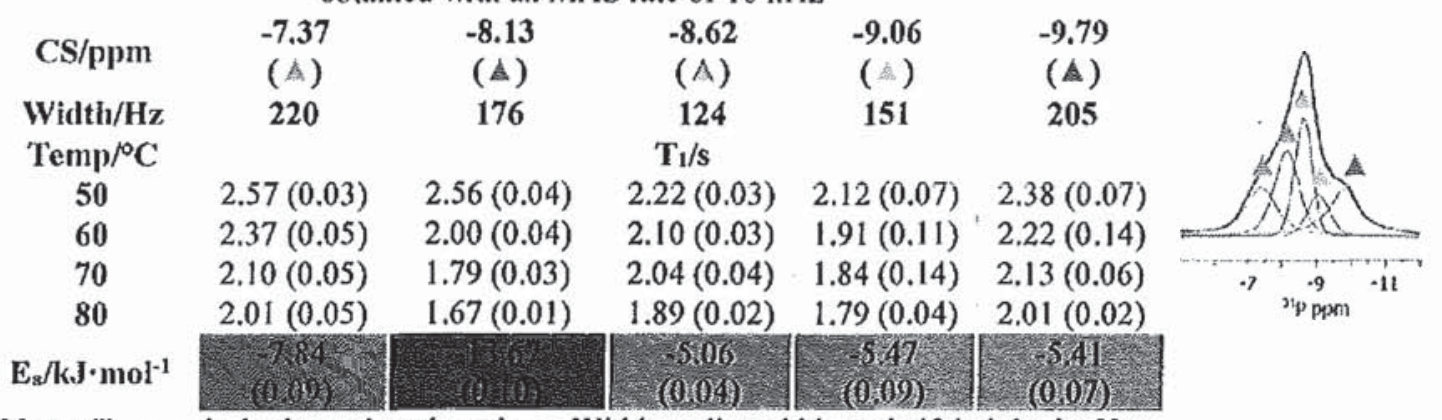

Note: $\mathrm{T}_{1}=$ spin-lattice relaxation time; Width $=$ linewidth at half height in $\mathrm{Hz}$; Annealed PBFP = annealed poly[bis(trifluoroethoxy)phosphazene at $220^{\circ} \mathrm{C}$ for 2 hours; $\mathrm{CS}=$ chemical shift in ppm; The standard deviations are listed in brackets

The $T_{1}$ values of all four components are bi-exponential as shown in Table 8.13 for the temperature range $20-40^{\circ} \mathrm{C}$. More specifically, the component at $-4.55 \mathrm{ppm}$ has longer $\mathrm{T}_{1}$ values at 20.20 and $16.67 \mathrm{~s}$ at $20^{\circ} \mathrm{C}$. The remaining three components are composed of one longer $T_{1}$ and one shorter $T_{1}$. In addition, for both the faster and slower decay composition, the $T_{1}$ values decrease as temperature increase indicating slow motion which is further supported by the negative activation energies.

In Table 8.14, the components at $-8.62,-9.06$ and $-9.79 \mathrm{ppm}$ have $\mathrm{E}_{\mathrm{a}}$ value at -5.06 , -5.47 and $-5.41 \mathrm{~kJ} \cdot \mathrm{mol}^{-1}$, respectively. They are so close that it is difficult to differentiate them, hence placing them in the same phase group. The component at $-7.37 \mathrm{ppm}$ has a large (small negative) $\mathrm{E}_{\mathrm{a}}$ at $-7.84 \mathrm{~kJ} \cdot \mathrm{mol}^{-1}$, could be considered separately from the others. The same is true for the component at $-8.13 \mathrm{ppm}$ with the largest (smallest negative) $\mathrm{E}_{\mathrm{a}}$ at $13.67 \mathrm{~kJ} \cdot \mathrm{mol}^{-1}$, which must considered separately from the remaining components. 


\subsection{2 ${ }^{31} \mathrm{P}$ spin-spin relaxation time $\left(\mathrm{T}_{2}\right)$ of annealed PBFP at an MAS rate of $10 \mathrm{kHz}$}

The ${ }^{31} \mathrm{P} \mathrm{T}_{2}$ values of annealed PBFP from $20-40^{\circ} \mathrm{C}$ are listed in Table 8.15. At

higher temperature, from $50-80^{\circ} \mathrm{C}$, the corresponding $\mathrm{T}_{2}$ results are shown in Table 8.16 .

Table 8.15: $\quad{ }^{31} \mathrm{P} \mathrm{T}_{2}$ times of solid annealed PBFP over the temperature range of 20 to $40^{\circ} \mathrm{C}$ obtained with an MAS rate of $10 \mathrm{kHz}$

\begin{tabular}{|c|c|c|c|c|}
\hline CS/ppm & $\begin{array}{c}-4.55 \\
(\bullet)\end{array}$ & $\begin{array}{c}-8.12 \\
(0)\end{array}$ & $\begin{array}{c}-8.68 \\
(0)\end{array}$ & $\begin{array}{c}-9.88 \\
(\Leftrightarrow)\end{array}$ \\
\hline Width/Hz & 834 & 344 & 272 & 242 \\
\hline Temp $/{ }^{\circ} \mathrm{C}$ & \multicolumn{4}{|c|}{$\mathrm{T}_{2} \mathrm{~ms}$} \\
\hline 20 & $0.58(0.01)$ & $1.62(0.07)$ & $1.18(0.02)$ & $1.02(0.04)$ \\
\hline 30 & $0.60(0.03)$ & $1.63(0.10)$ & $1.34(0.03)$ & $1.18(0.07)$ \\
\hline 40 & $0.67(0.02)$ & $1.68(0.03)$ & $1.40(0.17)$ & $1.20(0.13)$ \\
\hline $\mathrm{E}_{\mathrm{a}} / \mathrm{kJ} \cdot \mathrm{mol}^{-1}$ & $354(0.08)$ & $1.37(0.03)$ & $646(0) 28)$ & $86184(0,3)$ \\
\hline
\end{tabular}

Note: $\mathrm{T}_{2}=$ spin-spin relaxation time; $\mathrm{Width}=$ linewidth at half height in $\mathrm{Hz}$; Annealed PBFP = annealed poly[bis(trifluoroethoxy)phosphazene at $220^{\circ} \mathrm{C}$ for 2 hours; $\mathrm{CS}=$ chemical shift in ppm; The standard deviations are listed in brackets

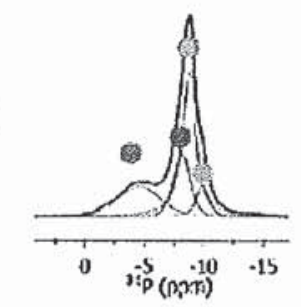

Table 8.16: $\quad{ }^{31} \mathrm{P} \mathrm{T}_{1}$ times of solid annealed PBFP over the temperature range of 50 to $80{ }^{\circ} \mathrm{C}$ obtained with an MAS rate of $10 \mathrm{kHz}$

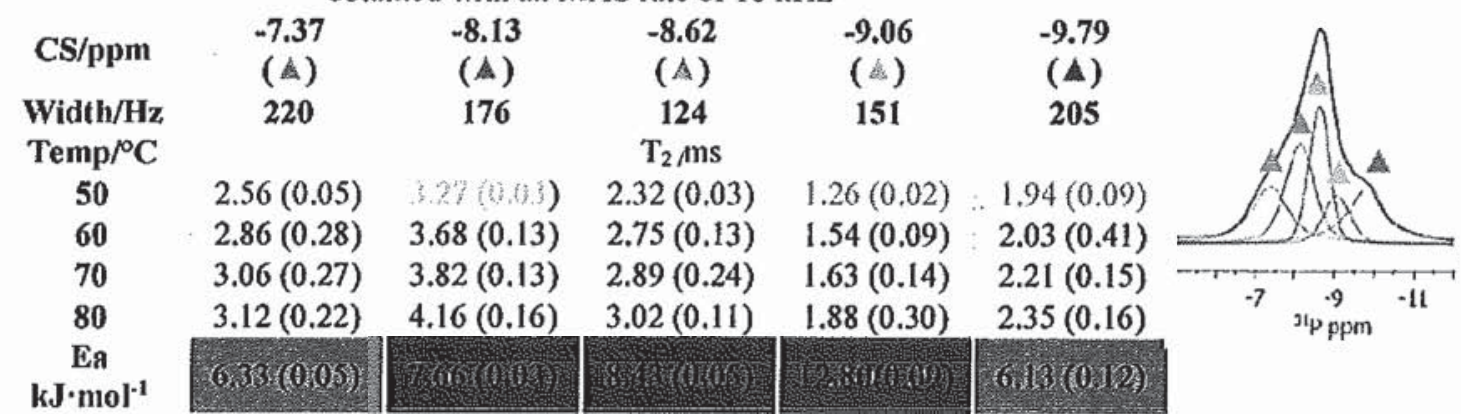

Note: $\mathrm{T}_{2}=$ spin-spin relaxation time; $\mathrm{Width}=$ linewidth at half height in $\mathrm{Hz} ;$ Annealed $\mathrm{PBFP}=$ annealed poly[bis(trifluoroethoxy)phosphazene at $220^{\circ} \mathrm{C}$ for 2 hours; $\mathrm{CS}=$ chemical shift in ppm; The standard deviations are listed in brackets

The $T_{2}$ values in both Tables 8.15 and 8.16 show that $T_{2}$ increases with temperature for all components in both temperature ranges. The component at $-4.55(\bullet) \mathrm{ppm}$ again has a distinctly small $T_{2}$, indicating the backbone is slow, similar to that in PBFP. The activation energies for components at $-4.55,-8.68(0)$ and $-9.88(*)$ ppm have similar values at $5.45,6.46$ and $6.14 \mathrm{~kJ} \cdot \mathrm{mol}^{-1}$, and thus are assigned to one phase group. The component at $-8.12(\bullet) \mathrm{ppm}$ has the smallest $\mathrm{E}_{\mathrm{a}}$ at $1.37 \mathrm{~kJ} \cdot \mathrm{mol}^{-1}$ and is considered separately. In Table 
8.16 , the one component at -9.06 ( $\mathrm{M}$ ) ppm has a distinctly large $\mathrm{E}_{\mathrm{a}}$ value at $12.80 \mathrm{~kJ} \cdot \mathrm{mol}^{-}$

1; however, the other four components have similar $\mathrm{E}_{\mathrm{a}}$ values.

\subsection{3 ${ }^{31} \mathrm{P}$ spin-lattice relaxation times in the rotating frame $\left(\mathrm{T}_{\mathrm{I}_{\rho}}\right)$ of annealed PBFP at an MAS rate of $10 \mathrm{kHz}$}

Table 8.17: $\quad{ }^{1} \mathrm{P} \mathrm{T}_{1 \rho}$ times of solid annealed PBFP over the temperature range of 20 to $40^{\circ} \mathrm{C}$

\begin{tabular}{|c|c|c|c|c|}
\hline CS/ppm & $\begin{array}{c}-4.55 \\
(\theta)\end{array}$ & $\begin{array}{c}-8.12 \\
(\theta)\end{array}$ & $\begin{array}{c}-8.68 \\
(0)\end{array}$ & $\begin{array}{c}-9.88 \\
\text { (e) }\end{array}$ \\
\hline Width/Hz & 834 & 344 & 272 & 242 \\
\hline Temp $/{ }^{\circ} \mathrm{C}$ & \multicolumn{4}{|c|}{$\mathrm{T}_{1 p} / \mathrm{ms}$} \\
\hline 20 & $0.27(0.01)$ & $0.68(0.04)$ & $0.73(0.01)$ & $0.86(0.01)$ \\
\hline 30 & $0.18(0.01)$ & $0.74(0.03)$ & $0.81(0.01)$ & $0.92(0.02)$ \\
\hline 40 & $0.12(0.01)$ & $0.83(0.02)$ & $0.93(0.02)$ & $1.03(0.02)$ \\
\hline
\end{tabular}

$\mathrm{E}_{\boldsymbol{n}} / \mathrm{kJ} \cdot \mathrm{mol}^{-\mathrm{t}}$

\begin{tabular}{|c|}
\hline $7.53(0,21)$ \\
\hline
\end{tabular}

Note: $\mathrm{T}_{1 \mathrm{p}}=$ spin-lattice relaxation time in the rotating frame; Width $=$ linewidth at half height in $\mathrm{Hz}$; Annealed PBFP = annealed poly[bis(trifluoroethoxy)phosphazene at $220^{\circ} \mathrm{C}$ for 2 hours; $\mathrm{CS}=$ chemical shift in ppm; The standard deviations are listed in brackets

Table 8.18: $\quad$ ' $P \mathrm{~T}_{1 \mathrm{p}}$ times of solid annealed PBFP over the temperature range of 20 to $40{ }^{\circ} \mathrm{C}$ obtained with an MAS rate of $10 \mathrm{kHz}$

\begin{tabular}{|c|c|c|c|c|c|}
\hline CS/ppm & $\begin{array}{c}-7.37 \\
(\mathrm{~A})\end{array}$ & $\begin{array}{l}-8.13 \\
(\mathrm{~A})\end{array}$ & $\begin{array}{c}-8.62 \\
(A)\end{array}$ & $\begin{array}{c}-9.06 \\
(\text { 埃) }\end{array}$ & $\begin{array}{c}-9.79 \\
(\mathrm{~A})\end{array}$ \\
\hline Width/Hz & 220 & 176 & 124 & 151 & 205 \\
\hline Temp $/{ }^{\circ} \mathrm{C}$ & & & $\mathrm{T}_{1 \rho} / \mathrm{ms}$ & & \\
\hline 50 & $0.90(0.02)$ & $0.93(0.05)$ & $0.54(0.06)$ & $0.53(0.07)$ & $1.35(0.02)$ \\
\hline 60 & $0.94(0.03)$ & $1.04(0.03)$ & $0.65(0.03)$ & $0.60(0.06)$ & $1.40(0.03)$ \\
\hline 70 & $0.97(0.02)$ & $1.18(0.02)$ & $0.72(0.04)$ & $0.67(0.06)$ & $1.45(0.02)$ \\
\hline 80 & $1.01(0.03)$ & $1.33(0.03)$ & $0.88(0.07)$ & $0.72(0.05)$ & $1.54(0.03)$ \\
\hline$\underset{\mathrm{kJ} \cdot \mathrm{mol}^{-1}}{\mathbf{E}_{\mathrm{a}}}$ & $\begin{array}{l}3169 \\
(066)\end{array}$ & & & $\begin{array}{l}9880 \\
(0.56)\end{array}$ & $\begin{array}{c}4.21 \\
(0.03)\end{array}$ \\
\hline
\end{tabular}

Note: $T_{1 \rho}=$ spin-lattice relaxation time in the rotating frame; Width $=$ linewidth at half height in Hz; Annealed PBFP = annealed poly[bis(trifluoroethoxy)phosphazene at $220^{\circ} \mathrm{C}$ for 2 hours; $\mathrm{CS}=$ chemical shift in ppm; The standard deviations are listed in brackets

The ${ }^{31} \mathrm{P} \mathrm{T}_{1 \mathrm{p}}$ values of annealed PBFP are listed in Table 8.17, showing that the $\mathrm{T}_{1 \mathrm{p}}$ increases with temperature for the components at $-8.68,-9.88$ and $-8.12 \mathrm{ppm}$, indicating that the backbone motion in those environments are fast with respect $13 \mu \mathrm{s}$. In contrast, the $T_{1 \rho}$ values decrease as temperature increases for the component at $-4.55 \mathrm{ppm}$, meaning that its backbone local motion is in the slow motion regime, which is similar as what is 
concluded for PBFP at low temperature. At higher temperature, from $50-80^{\circ} \mathrm{C}$, the ${ }^{31} \mathrm{P} \mathrm{T}_{1 \rho}$ data are listed in Table 8.18, showing that the $T_{1 p}$ values of all components increase with temperature.

The component at $-4.55 \mathrm{ppm}$ has a negative $\mathrm{E}_{\mathrm{a}}$ in PBFP, as seen in Table 8.17 , and thus is assigned to its own phase category. This negative activation energy value suggests that the backbone motion is in the slow motion regime. The components at -8.12 and -9.88 ppm have close positive $\mathrm{E}_{\mathrm{a}}$ values at 7.53 and $6.82 \mathrm{~kJ} \cdot \mathrm{mol}^{-1}$, and thus are grouped together in the fast motion regime.. The component at $-8.68 \mathrm{ppm}$ has the largest $\mathrm{E}_{\mathrm{a}}$ at $9.15 \mathrm{~kJ} \cdot \mathrm{mol}^{-1}$ can be being considered separately to its own phase group; however, the $T_{1 \rho}$ data would place it with the previous two components. In Table 8.18, components at -8.62 and -8.13 $\mathrm{ppm}$ have similar large $\mathrm{E}_{\mathrm{a}}$ at 15.62 and $11.44 \mathrm{~kJ} \cdot \mathrm{mol}^{-1}$ fall into the same phase category. The two components at -7.37 and $-9.79 \mathrm{ppm}$ have $\mathrm{E}_{\mathrm{a}}$ at 3.69 and $4.21 \mathrm{~kJ} \cdot \mathrm{mol}^{-1}$, which are relatively close to each other, are assigned to a separate phase group. The last component at $-9.06 \mathrm{ppm}$ has an $\mathrm{E}_{\mathrm{a}}$ value at $9.80 \mathrm{~kJ} \cdot \mathrm{mol}^{-1}$ can be putting into its own phase category; however; the $T_{1 \rho}$ data would place it together with $-8.62 \mathrm{ppm}$. 


\subsection{4 ${ }^{19} \mathrm{~F}$ spin-lattice relaxation time ( $\left.\mathrm{T}_{1}\right)$ of annealed PBFP at an MAS rate of $10 \mathrm{kHz}$}

Table 8.19: $\quad{ }^{19} \mathrm{~F} \mathrm{~T}_{1}$ times of solid annealed PBFP over the temperature range of 20 to $40^{\circ} \mathrm{C}$ obtained with an MAS rate of $10 \mathrm{kHz}$

\begin{tabular}{|c|c|c|c|c|c|}
\hline $\mathrm{CS} / \mathrm{ppm}$ & $\begin{array}{c}-76.62 \\
\text { (m) }\end{array}$ & $\begin{array}{c}-76.71 \\
(1)\end{array}$ & $\begin{array}{c}-76.95 \\
\text { (a) }\end{array}$ & $\begin{array}{c}-77.20 \\
\text { (日) }\end{array}$ & \\
\hline Width/Hz & 1647 & 266 & 166 & 127 & \\
\hline Temp $/{ }^{\circ} \mathrm{C}$ & \multicolumn{4}{|c|}{$\mathrm{T}_{1} / \mathrm{s}$} & \\
\hline 20 & $0.40(0.01)$ & $0.41(0.01)$ & $0.45(0.01)$ & $0.49(0.01)$ & 10 \\
\hline 30 & $0.47(0.01)$ & $0.45(0.01)$ & $0.50(0.01)$ & $0.54(0.02)$ & \\
\hline 40 & $0.52(0.01)$ & $0.52(0.02)$ & $0.53(0.01)$ & $0.61(0.02)$ & \\
\hline $\mathrm{E}_{a} / \mathbf{k J} \cdot \mathrm{mol}^{-1}$ & $926(0149)$ & 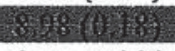 & $6.18(0.08)$ & 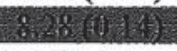 & 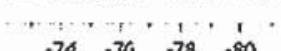 \\
\hline
\end{tabular}

Table 8.20: $\quad{ }^{19} \mathrm{~F} \mathrm{~T}_{1}$ times of solid annealed PBFP over the temperature range of 50 to $80{ }^{\circ} \mathrm{C}$ obtained with an MAS rate of $10 \mathrm{kHz}$

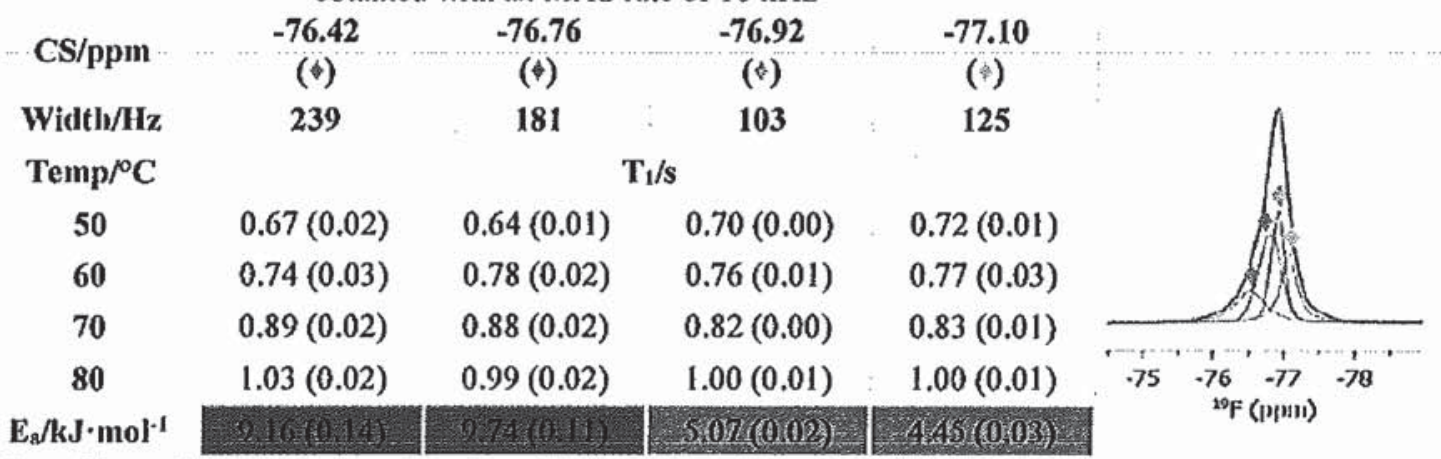

Note: $\mathrm{T}_{1}=$ spin-lattice relaxation time; Width $=$ linewidth at half height in $\mathrm{Hz}$; Annealed PBFP = annealed poly[bis(trifluoroethoxy)phosphazene at $220^{\circ} \mathrm{C}$ for 2 hours; $\mathrm{CS}=$ chemical shift in ppm; The standard deviations are listed in brackets

Fluorine-19 $\mathrm{T}_{1}$ values of annealed PBFP are shown in Table 8.19 for the low temperature range and 8.20 for high the temperature range. As indicated in both tables, all ${ }^{19} \mathrm{~F} \mathrm{~T}_{1}$ values for annealed PBFP increase with temperature from $20-80^{\circ} \mathrm{C}$, which is consistent with the $T_{1}$ values in non-annealed PBFP, and thus are also in the fast motion regime. Also, in Table 8.19 , the component at $-76.62 \mathrm{ppm}$ has a much large positive $\mathrm{E}_{\mathrm{a}}$ value at $9.92 \mathrm{~kJ} \cdot \mathrm{mol}^{-1}$ and thus should be considered distinct from the others. The $\mathrm{E}_{\mathrm{a}}$ for the component at -76.71 and -77.20 ppm have similar values, therefore, are assigned to one phase group. The component at $-76.95 \mathrm{ppm}$ has the smallest $\mathrm{E}_{\mathrm{a}}$ at $6.18 \mathrm{~kJ} \cdot \mathrm{mol}^{-1}$ mostly likely belongs to its own phase category. 
In Table 8.20, the two components at -76.42 and $-76.76 \mathrm{ppm}$ have close $\mathrm{E}_{\mathrm{a}}$ values at 9.16 and $9.74 \mathrm{~kJ} \cdot \mathrm{mol}^{-1}$, and thus should be placed in the same phase category. The components at -76.92 and $-77.10 \mathrm{ppm}$ also have similar $E_{\mathrm{a}}$ results at 5.07 and $4.55 \mathrm{~kJ} \cdot \mathrm{mol}^{-}$ ', and thus should also be assigned to their own phase group.

\subsection{5 ${ }^{19} \mathrm{~F}$ spin-spin relaxation time $\left(\mathrm{T}_{2}\right)$ of annealed PBFP at an MAS rate of $10 \mathrm{kHz}$}

Table 8.21: $\quad{ }^{19} \mathrm{~F} \mathrm{~T}_{2}$ times of solid annealed PBFP over the temperature range of 20 to $40{ }^{\circ} \mathrm{C}$ obtained with an MAS rate of $10 \mathrm{kHz}$

\begin{tabular}{|c|c|c|c|c|c|}
\hline CS/ppm & $\begin{array}{c}-76.62 \\
\text { (*) }\end{array}$ & $\begin{array}{c}-76.71 \\
\text { (㣙) }\end{array}$ & $\begin{array}{c}-76.95 \\
\text { (घ) }\end{array}$ & $\begin{array}{c}-77.20 \\
\text { (घ) }\end{array}$ & \\
\hline Width/Hz & 1647 & 266 & 166 & 127 & \\
\hline Temp $/{ }^{\circ} \mathrm{C}$ & & \multicolumn{3}{|c|}{$\mathrm{T}_{2} \mathrm{~ms}$} & \\
\hline 20 & $0.13(0.00)$ & $1.35(0.03)$ & $1.65(0.05)$ & $1.51(0.08)$ & \\
\hline 30 & $0.14(0.00)$ & $1.45(0.02)$ & $1.72(0.04)$ & $1.85(0.28)$ & \\
\hline 40 & $0.15(0.00)$ & $1.58(0.08)$ & $1.78(0.16)$ & $2.15(0.16)$ & \\
\hline $\mathrm{E}_{2} / \mathrm{kJ} \cdot \mathrm{mol}^{-1}$ & $4.39(0.00)$ & $5.95(0.14)$ & $2887(012)$ & $86.56(0,5)$ & $74-76 \quad-78$ \\
\hline
\end{tabular}

The ${ }^{19} \mathrm{~F} \mathrm{~T}_{2}$ results of annealed PBFP over the temperature range $20-40$ are listed in Table 8.21. At higher temperature from $50-80^{\circ} \mathrm{C}$, the $\mathrm{T}_{2}$ data are listed in Table 8.22 . Both tables show that the $\mathrm{T}_{2}$ values increase with temperature for all components. The distinctly

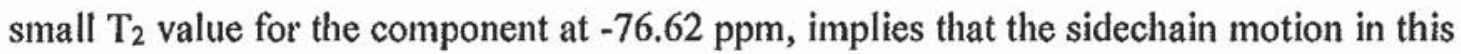
component is slower than in the others.

In Table 8.21 , the component at $\mathbf{- 7 7 . 2 0}$ ppm has a distinctly large $\mathrm{E}_{\mathrm{a}}$ and should be assigned to a separate structural domain. The two components at -76.71 and $-76.62 \mathrm{ppm}$ have similar $\mathrm{E}_{\mathrm{a}}$ values at 5.95 and $4.37 \mathrm{~kJ} \cdot \mathrm{mol}^{-1}$, and thus can be assigned together to one phase group. The component at $-76.95 \mathrm{ppm}$ has the smallest $\mathrm{E}_{\mathrm{a}}$ of $2.87 \mathrm{~kJ} \cdot \mathrm{mol}^{-1}$, and mostly likely should be considered on its own. In Table 8.22 , the $\mathrm{E}_{\mathrm{a}}$ values are vary less dramatically between components. For example, the component at $-76.42 \mathrm{ppm}$ has a 
relatively small $\mathrm{E}_{\mathrm{a}}$ of $4.88 \mathrm{~kJ} \cdot \mathrm{mol}^{-1}$, and thus is assigned to its own phase category. The three components at $-77.10,-76.76$ and -76.92 ppm have similar $E_{a}$ at $6.13,7.05$ and 8.51 $\mathrm{kJ} \cdot \mathrm{mol}^{-1}$, separately, putting them into the same phase category; however since the component at $-77.10 \mathrm{ppm}$ has a $\mathrm{T}_{2}$ close to $-76.42 \mathrm{ppm}$ they could be related.

Table 8.22: $\quad{ }^{19} \mathrm{~F} \mathrm{~T}_{2}$ times of solid annealed PBFP over the temperature range of 50 to $80{ }^{\circ} \mathrm{C}$

\begin{tabular}{|c|c|c|c|c|}
\hline \multirow[b]{2}{*}{ CS/ppm } & \multicolumn{4}{|c|}{ obtained with an MAS rate of $10 \mathrm{kHz}$} \\
\hline & $\begin{array}{c}-76.42 \\
(\phi)\end{array}$ & $\begin{array}{c}-76.76 \\
(\diamond)\end{array}$ & $\begin{array}{c}-76.92 \\
(\diamond)\end{array}$ & $\begin{array}{c}-77.10 \\
(\%)\end{array}$ \\
\hline Width/Hz & 239 & 181 & 103 & 125 \\
\hline Temp $/{ }^{\circ} \mathrm{C}$ & \multicolumn{4}{|c|}{$\mathrm{T}_{2} \mathrm{~ms}$} \\
\hline 50 & $1.70(0.07)$ & $2.00(0.10)$ & $2.15(0.03)$ & $1.61(0.07)$ \\
\hline 60 & $1.81(0.09)$ & $2.15(0.03)$ & $2.00(0.10)$ & $1.79(0.09)$ \\
\hline 70 & $1.89(0.08)$ & $2.51(0.04)$ & $2.20(0.12)$ & $1.89(0.10)$ \\
\hline 80 & $1.98(0.07)$ & $2.55(0.03)$ & $2.39(0.14)$ & $1.95(0.14)$ \\
\hline$a / k J \cdot m c$ & $4.88(0.09)$ & $8 \times 5140,00)$ & $7.05(0.34)$ & $613(0.12)$ \\
\hline
\end{tabular}

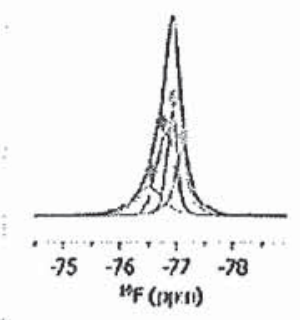

Note: $\mathrm{T}_{2}=$ spin-spin relaxation time; $\mathrm{Width}=$ linewidth at half height in $\mathrm{Hz}$; Annealed PBFP $=$ annealed poly[bis(trifluoroethoxy)phosphazene at $220^{\circ} \mathrm{C}$ for 2 hours; $\mathrm{CS}=$ chemical shift in ppm; The standard deviations are listed in brackets

\subsection{6 ${ }^{19} \mathrm{~F}$ spin-lattice relaxation time in the rotating frame $\left(\mathrm{T}_{1}\right)$ of annealed PBFP at an MAS rate of $10 \mathrm{kHz}$}

Table 8.23: $\quad{ }^{19} \mathrm{~F} \mathrm{~T}_{1 \rho}$ times of solid annealed PBFP over the temperature range of 20 to $40^{\circ} \mathrm{C}$ obtained with an MAS rate of $10 \mathrm{kHz}$

\begin{tabular}{|c|c|c|c|c|c|}
\hline CS/ppm & 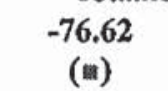 & $\begin{array}{c}-76.71 \\
(\approx)\end{array}$ & $\begin{array}{c}-76.95 \\
\text { (घ) }\end{array}$ & $\begin{array}{c}-77.20 \\
\text { (a) }\end{array}$ & \\
\hline Width/Hz & 1647 & 266 & 166 & 127 & \\
\hline Temp $/{ }^{\circ} \mathrm{C}$ & \multicolumn{4}{|c|}{$\mathrm{T}_{1 \rho} / \mathrm{ms}$} & \\
\hline 20 & $0.99(0.02)$ & $0,6,3(0,03)$ & $0.67(0.02)$ & $0.86(0.01)$ & \\
\hline 30 & $1.15(0.04)$ & $0.84(0.02)$ & $0.85(0.01)$ & $0.97(0.01)$ & \\
\hline 40 & $1.30(0.04)$ & $0.94(0.02)$ & $1.02(0.02)$ & $1.14(0.01)$ & \\
\hline $\mathrm{E}_{\mathrm{a}} / \mathbf{k J} \cdot \mathbf{m o l}^{-1}$ & 14: & 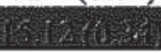 & 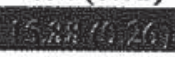 & 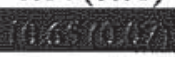 & 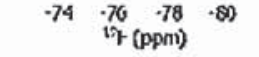 \\
\hline
\end{tabular}

Note: $\mathrm{T}_{\mathrm{ip}}=$ spin-lattice relaxation time in the rotating frame; $W$ idth $=$ linewidth at half height in $\mathrm{Hz}$; Annealed $\mathrm{PBFP}=$ annealed poly[bis(trifluoroethoxy)phosphazene at $220^{\circ} \mathrm{C}$ for 2 hours; $\mathrm{CS}=$ chemical shift in $\mathrm{ppm}$; The standard deviations are listed in brackets

${ }^{19} \mathrm{~F}_{1 \rho}$ data are listed in Table 8.23 for temperature from $20-40{ }^{\circ} \mathrm{C}$. For the higher temperature range from $50-80^{\circ} \mathrm{C}$, the $\mathrm{T}_{1_{\mathrm{p}}}$ values of all five components are listed in Table 8.24. Both tables indicate that the $T_{1 \rho}$ values of all components increase with increasing 
temperature giving rise to positive activation energies indicating that sidechain motion is in the fast regime in both cases.

Table 8.24: $\quad{ }^{19} \mathrm{~F} \mathrm{~T}_{1 \mathrm{p}}$ times of solid annealed PBFP over the temperature range of 50 to $80^{\circ} \mathrm{C}$ obtained with an MAS rate of $10 \mathrm{kHz}$

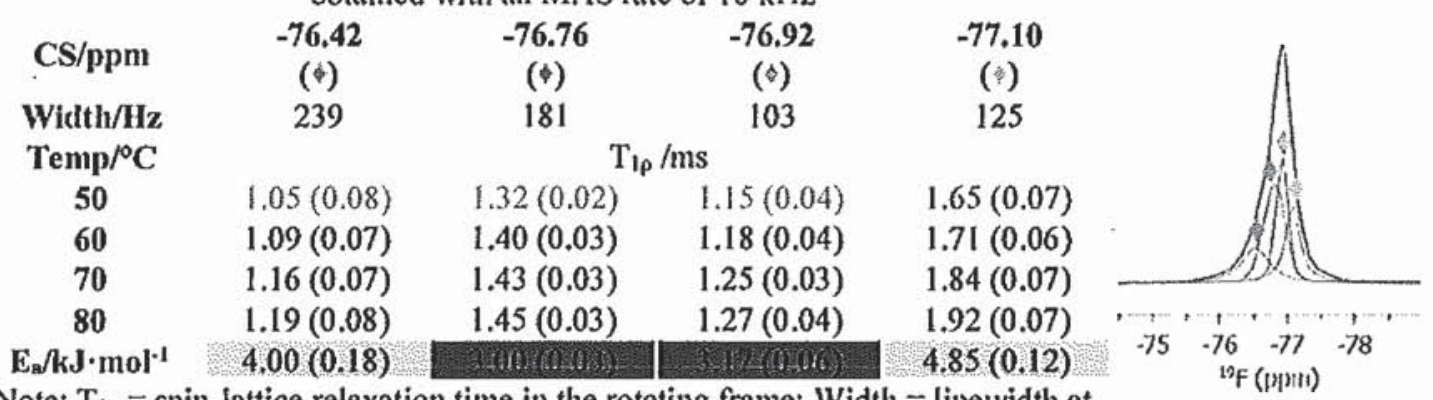

Note: $T_{1 \rho}=$ spin-lattice relaxation time in the rotating frame; Width $=$ linewidth at

half height in $\mathrm{Hz}$; $\mathrm{CS}=$ chemical shift in ppm; Annealed PBFP = annealed poly[bis(trifluoroethoxy)phosphazene at $220^{\circ} \mathrm{C}$ for 2 hours; The standard deviations are listed in brackets

In Table 8.23 , the two components at -76.71 and $-76.95 \mathrm{ppm}$ both have similarly large $E_{a}$ results and slightly longer $T_{1 p}$ values and thus likely belong to the same phase category. The two components at $\mathbf{- 7 7 . 2 0}$ and $-76.62 \mathrm{ppm}$ have a small $\mathrm{E}_{\mathrm{a}}$ values and smaller $\mathrm{T}_{i \rho}$ values putting them into their own phase category. In table 8.24 the $\mathrm{E}_{\mathrm{a}}$ results would place -76.76 and -76.92 ppm together, as well as -76.42 , and $-77.10 \mathrm{ppm}$ in their own phase group. The $T_{1 \rho}$ values would suggest a different arrangement putting $\mathbf{- 7 6 . 4 2}$ and $\mathbf{- 7 6 . 9 2}$ together, and -77.10 and $-76.76 \mathrm{ppm}$ in the same phase group.

\subsubsection{Conclusion}

To conclude, a four-component model is used to deconvolve the ${ }^{31} \mathrm{P}$ NMR spectra of annealed PBFP over $20-40^{\circ} \mathrm{C}$, and a five-component model from $50-80^{\circ} \mathrm{C}$. At the low temperature range, the $T_{1}$ data show bi-exponential behavior for all four components. The component at $-4.55 \mathrm{ppm}$ is composed of one very long contribution which is around $20 \mathrm{~s}$ and another long $\mathrm{T}_{1}$ with value around $16 \mathrm{~s}$, the three narrow components are all contain 
one long $T_{1}$ contribution around $15 \mathrm{~s}$ and one short $T_{1}$ contribution with value about $3 \mathrm{~s}$. The $T_{1}$ values of component at -4.55 are smaller than the other three components. At high temperature, the increased backbone motion above its $\mathrm{T}(1)$ transition temperature makes the difference between each component become smaller and more difficult to distinguish. A four-component model is used to deconvolve the ${ }^{19} \mathrm{~F}$ NMR spectra of annealed PBFP over $20-40^{\circ} \mathrm{C}$, and a four-component model from $50-80^{\circ} \mathrm{C}$. At the low temperature range, the ${ }^{19} \mathrm{~F} T_{1}$ data show mono-exponential behavior, each component has similar $T_{1}$ around $0.4-0.5 \mathrm{~ms}$. The $\mathrm{T}_{2}$ results suggest that the broad component at $-76.62 \mathrm{ppm}$ with linewidth of $1647 \mathrm{~Hz}$ has a distinct smaller $\mathrm{T}_{2}$ around $0.2 \mathrm{~ms}$ compared to other three narrow components, indicating that it arises from different domains within the polymer. The $T_{1 \rho}$ data showed that all four components have similar $T_{1 \rho}$ values, suggesting that they should appear at the same regime with respect to the locking power. At higher temperature, the sidechain motion of all polymer domains is enhanced, the differences becomes smaller and more difficult to separate. Extra data are necessary to make a confidence assignment. 


\subsection{Solid-State NMR Relaxation Data of NC 5\% (5\% Nano-TiO 2 PBFP Nanocomposite)}

\subsection{1 ${ }^{31} \mathrm{P}$ spin-lattice relaxation time $\left(\mathrm{T}_{1}\right)$ of $\mathrm{NC} 5 \%$ at an MAS rate of $10 \mathrm{kHz}$}

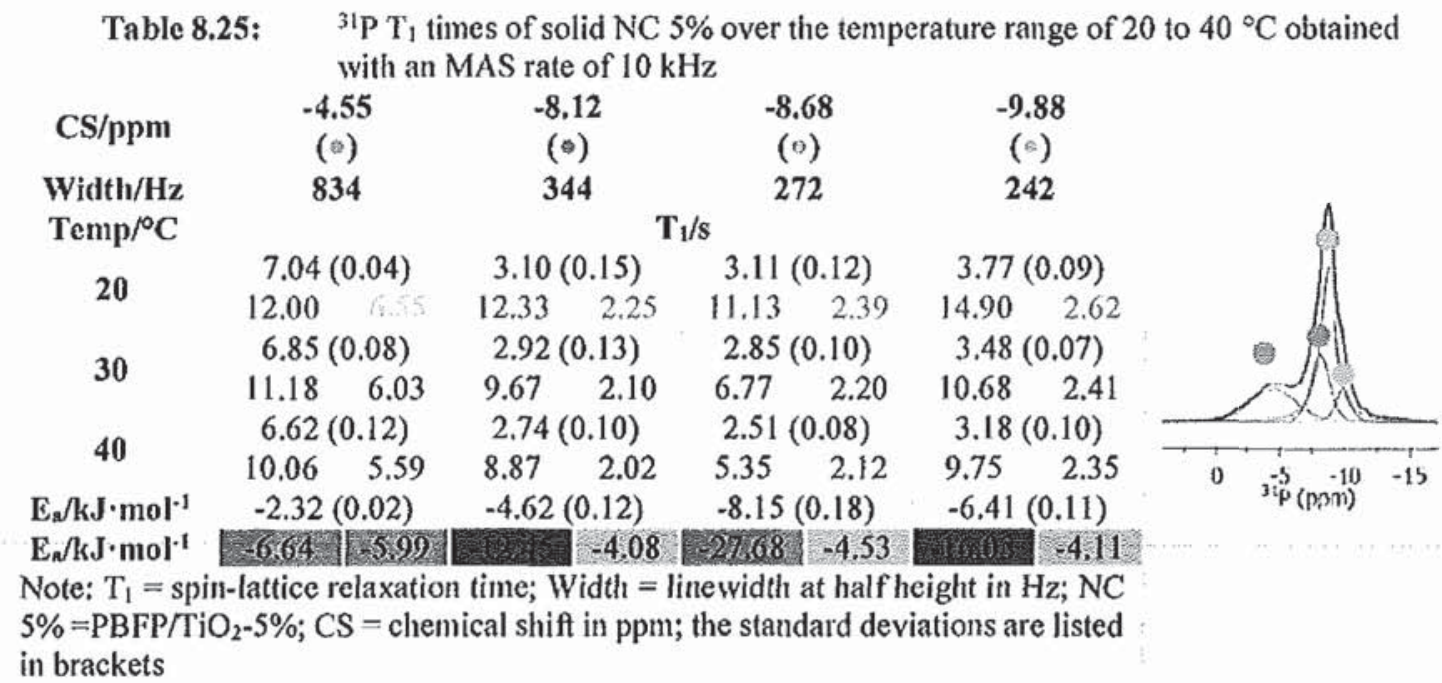

The ${ }^{31} \mathrm{P} \mathrm{T}_{1}$ data of $\mathrm{NC} 5 \%$ at the temperature range from $20-40^{\circ} \mathrm{C}$ are listed in Table 8.25. At the higher temperature range from $50-80^{\circ} \mathrm{C}$, the corresponding results are shown in Table 8.26. Both tables suggest that as temperature goes up, the $T_{1}$ values decrease for both models, together with the positive activation energies, showing that the molecule is in the slow motion regime as what is presented for PBFP and sample annealed PBFP. As shown in Table 8.25 , the component that appears at $-4.55 \mathrm{ppm}$ is different from the remaining three components by having a much larger $T_{1}$ value. Furthermore, its $E_{a}$ value is small (large negative) compared to the $\mathrm{E}_{\mathrm{a}}$ values of the other components. In Table 8.26, the components at -7.37 and $-8.13 \mathrm{ppm}$ also have a smaller $\mathrm{E}_{\mathrm{a}}$ than the other three components, despite that the $\mathrm{T}_{1}$ values are quite similar between all five components. 
Table 8.26: $\quad{ }^{31} \mathrm{P}_{1}$ times of solid $\mathrm{NC} 5 \%$ over the temperature range of 50 to $80^{\circ} \mathrm{C}$ obtained

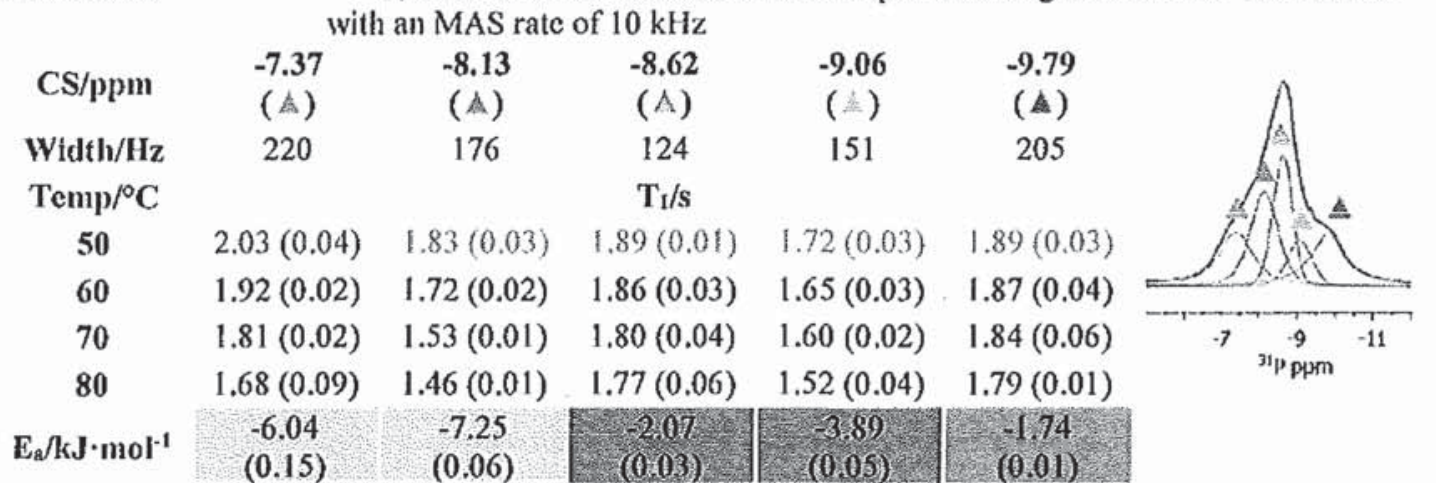

Note: $\mathrm{T}_{1}=$ spin-lattice relaxation time; $\mathrm{Width}=$ linewidth at half height in $\mathrm{Hz} ; \mathrm{NC}$ $5 \%=\mathrm{PBFP} / \mathrm{TiO}_{2}-5 \% ; \mathrm{CS}=$ chemical shift in ppm; the standard deviations are listed in brackets

\subsection{2 ${ }^{31} \mathrm{P}$ spin-spin relaxation time $\left(\mathrm{T}_{2}\right)$ of $\mathrm{NC} 5 \%$ at an MAS rate of $10 \mathrm{kHz}$}

The ${ }^{31} \mathrm{P} \mathrm{T}_{2}$ values of NC $5 \%$ from $20-40^{\circ} \mathrm{C}$ of each component are tabulated in Table 8.27. As the temperature goes up, the $\mathrm{T}_{2}$ values of all four components increase. At higher temperature, from $50-80^{\circ} \mathrm{C},{ }^{31} \mathrm{P} \mathrm{T}_{2}$ results are listed in Table 8.28 , showing that increase with temperature as for low temperature.

Table 8.27: $\quad{ }^{31} \mathrm{P} \mathrm{T}_{2}$ times of solid NC $5 \%$ over the temperature range of 20 to $40^{\circ} \mathrm{C}$ obtained

\begin{tabular}{ccccc}
\multicolumn{5}{c}{ with an MAS rate of $10 \mathrm{kHz}$} \\
CS/ppm & -4.55 & -8.12 & -8.68 & -9.88 \\
Widith $/ \mathrm{Hz}$ & $(\%)$ & $(\bullet)$ & $(0)$ & $(6)$ \\
Temp $/{ }^{\circ} \mathrm{C}$ & 834 & 344 & 272 & 242
\end{tabular}

Temp/ $/{ }^{\circ} \mathrm{C}$

20

$0.69(0.01)$

$2.55(0.21)$

$2.76(0.38)$

$0.73(0.02)$

$2.87(0.42)$

$3.00(0.37)$

$1.48(0.06)$

$0.78(0.03)$

$3.30(0.33)$

$3.25(0.09)$

$1.52(0.20)$

40

$463-0.08)$

$\mathrm{E}_{\mathrm{a}} / \mathbf{k J} \cdot \mathrm{mol}^{-1}$

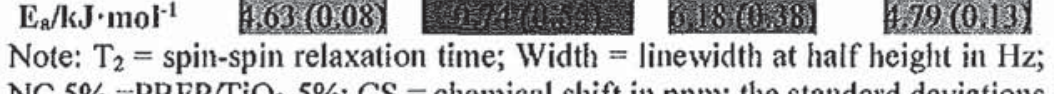
$\mathrm{NC} 5 \%=\mathrm{PBFP} / \mathrm{TiO}_{2}-5 \%$; $\mathrm{CS}=$ chemical shift in ppm; the standard deviations are listed in brackets

In Table 8.27 , the component at $-4.55 \mathrm{ppm}$ has a smaller $\mathrm{T}_{2}$ compared to the remaining three components, implying that the polymer backbone motion of this component is slower. The two components at $-4.55(0)$ and $-9.88(\%)$ ppm have similar $E_{a}$ 
values at 4.63 and $4.79 \mathrm{~kJ} \cdot \mathrm{mol}^{-1}$, should be assigned into one phase category. The component at $-8.68(\circ) \mathrm{ppm}$ has a large $\mathrm{E}_{\mathrm{a}}$ at $-6.18 \mathrm{~kJ} \cdot \mathrm{mol}^{-1}$, it should be considered separately. While the components at $-8.12(0) \mathrm{ppm}$ has the largest $\mathrm{E}_{\mathrm{s}}$ value amongst all four components, it should be assigned to its own phase category. In Table 8.28, the components at $-8.62(\hat{A})$ and $-9.06(\mathrm{~s})$ ppm have relatively small and similar $E_{a}$ at 2.42 and 1.71 $\mathrm{kJ} \cdot \mathrm{mol}^{-1}$, and thus should be grouped together. The component at $-8.13(\mathbb{A}) \mathrm{ppm}$ has the largest $\mathrm{E}_{\mathrm{a}}$ with a value at $7.02 \mathrm{~kJ} \cdot \mathrm{mol}^{-1}$, it should be assigned to its own phase group. The two components at $-7.37(\mathrm{~A})$ and -9.79 (A) ppm have similar $E_{\mathrm{n}}$ at 3.74 and $4.41 \mathrm{~kJ} \cdot \mathrm{mol}^{-}$ ${ }^{1}$ thus should be assigned together.

\begin{tabular}{|c|c|c|c|c|c|c|}
\hline \multicolumn{2}{|c|}{ Table 8.28: } & \multicolumn{5}{|c|}{$\begin{array}{l}{ }^{11} \mathrm{P} \mathrm{T}_{2} \text { times of solid } \mathrm{NC} 5 \% \text { over the temperature range of } 20 \text { to } 40^{\circ} \mathrm{C} \text { obtained } \\
\text { with an MAS rate of } 10 \mathrm{kHz}\end{array}$} \\
\hline $\mathrm{CS} / \mathrm{ppm}$ & $\begin{array}{c}-7.37 \\
(\mathrm{~A})\end{array}$ & $\begin{array}{c}-8.13 \\
(\Delta)\end{array}$ & $\begin{array}{c}-8.62 \\
(\mathrm{~A})\end{array}$ & $\begin{array}{c}-9.06 \\
\text { (A) }\end{array}$ & $\begin{array}{c}-9.79 \\
(\mathbb{A})\end{array}$ & \\
\hline Width/Hz & 220 & 176 & 124 & 151 & 205 & \\
\hline Temp/ $/{ }^{\circ} \mathrm{C}$ & & & $\mathrm{T}_{2 / \mathrm{ms}}$ & & & \\
\hline $\mathbf{5 0}$ & $3.55(0.25)$ & $4.68(0.19)$ & $3.82(0.06)$ & $2.10(0.13)$ & $2.64(0.12)$ & \\
\hline 60 & $3.76(0.19)$ & $5.12(0.47)$ & $3.91(0.15)$ & $2.70(0.25)$ & $2.80(0.18)$ & \\
\hline 70 & $3.97(0.39)$ & $5.57(0.17)$ & $4.00(0.08)$ & $3.01(0.25)$ & $2.88(0.17)$ & \\
\hline 80 & $3.99(0.05)$ & $5.83(0.21)$ & $4.12(0.09)$ & $3.59(0.39)$ & $3.03(0.17)$ & \\
\hline$\underset{\mathrm{kJJ} \cdot \mathrm{mol}^{-1}}{\mathrm{E}_{\mathrm{a}}}$ & $374(0.11)$ & $2002(0) 12)$ & $2.42(0.02)$ & $1.71(0.05)$ & $4.41(0.09)$ & $-7 \quad \begin{array}{ll}-9 \\
34 p p m\end{array}$ \\
\hline
\end{tabular}

\subsection{3 ${ }^{31} \mathrm{P}$ spin-lattice relaxation time in the rotating frame $\left(\mathrm{T}_{1_{\rho}}\right)$ of $\mathrm{NC} 5 \%$ at an MAS rate of $10 \mathrm{kHz}$}

${ }^{31} \mathrm{P} \mathrm{T}_{1 \mathrm{p}}$ results of $\mathrm{NC} 5 \%$ from $20-40^{\circ} \mathrm{C}$ are listed in Table 8.29 . The $\mathrm{T}_{1 \mathrm{p}}$ values increase with temperature for those components appearing at $-8.68,-9.88$ and $-8.12 \mathrm{ppm}$. While, for component at $-4.55 \mathrm{ppm}$, its $\mathrm{T}_{1 \mathrm{p}}$ decreases as temperature increases, and its activation has a negative value, suggesting that its motion is on the slow motion regime 
compared to the locking power. For higher temperatures, from $50-80^{\circ} \mathrm{C}$, the $\mathrm{T}_{1 \mathrm{p}}$ values of all five components in Table 8.30 show that the $T_{1 p}$ values of all components increase with temperature.

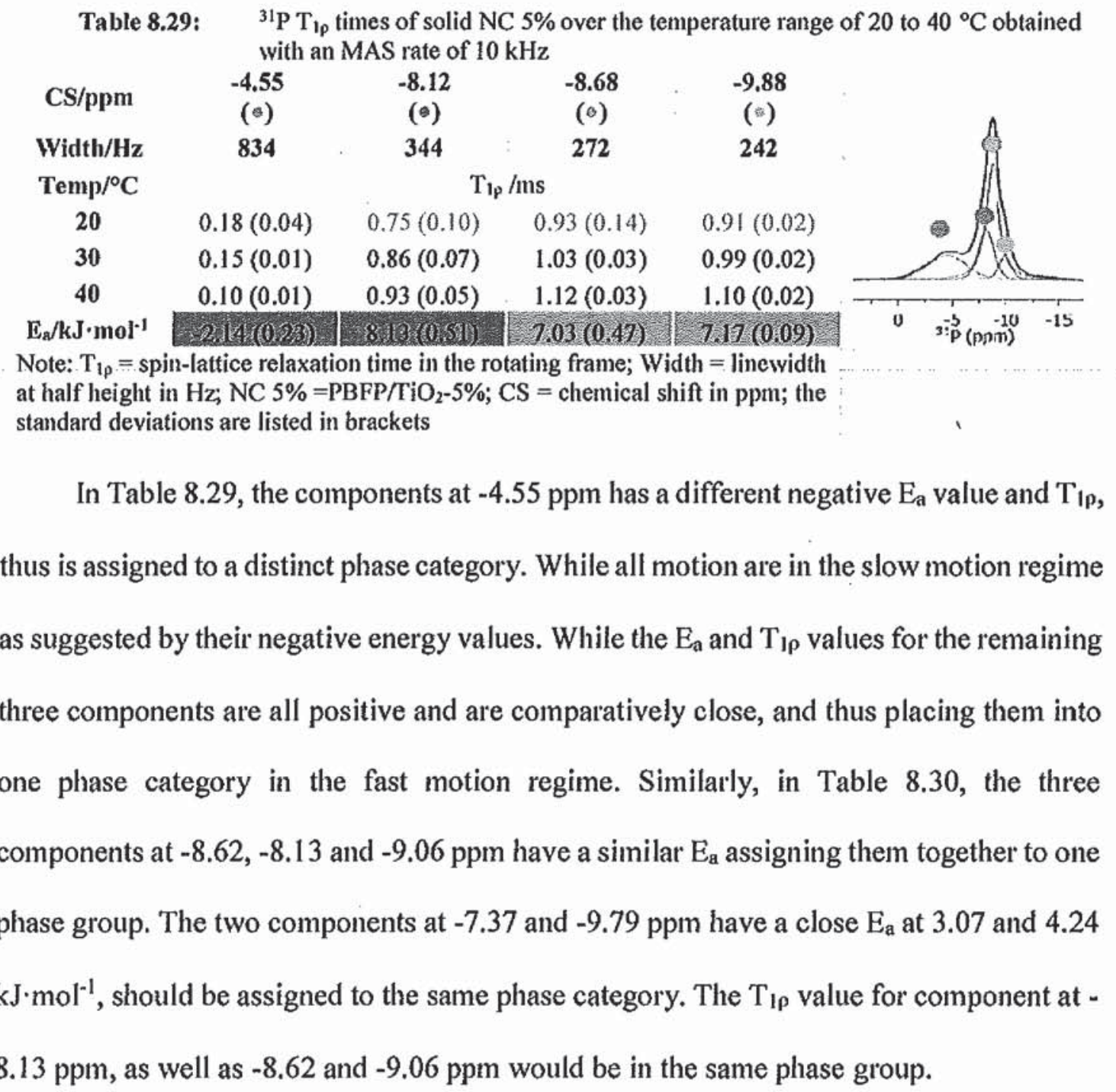


Table 8.30: $\quad{ }^{31} \mathrm{P} \mathrm{T}_{1 p}$ times of solid NC $5 \%$ over the temperature range of 50 to $80^{\circ} \mathrm{C}$ obtained with an MAS rate of $10 \mathrm{kHz}$

\begin{tabular}{|c|c|c|c|c|c|c|}
\hline CS/ppm & $\begin{array}{c}-7.37 \\
(\Delta)\end{array}$ & $\begin{array}{c}-8.13 \\
(A)\end{array}$ & $\begin{array}{l}-8.62 \\
(A)\end{array}$ & $\begin{array}{c}-9.06 \\
(B)\end{array}$ & $\begin{array}{c}-9.79 \\
\text { (A) }\end{array}$ & $\Lambda$ \\
\hline Width/Hz & 220 & 176 & 124 & 151 & 205 & \\
\hline Temp ${ }^{\circ} \mathrm{C}$ & & & $\mathrm{T}_{1 \rho} / \mathrm{ms}$ & & & \\
\hline 50 & $1.09(0.01)$ & $1.09(0.03)$ & $0.64(0.04)$ & $0.64(0.06)$ & $1.48(0.04)$ & \\
\hline 60 & $1.12(0.06)$ & $1.23(0.10)$ & $0.82(0.08)$ & $0.78(0.09)$ & $1.59(0.07)$ & \\
\hline 70 & $1.15(0.03)$ & $1.37(0.07)$ & $0.92(0.04)$ & $0.83(0.11)$ & $1.62(0.04)$ & $\begin{array}{lc}-7 & -9 \\
& -9 \\
p p m\end{array}$ \\
\hline 80 & $1.20(0.08)$ & $1.52(0.20)$ & $1.05(0.10)$ & $0.92(0.01)$ & $1.69(0.11)$ & \\
\hline $\begin{array}{c}\mathbf{E}_{\mathrm{a}} \\
\mathrm{kJ} \cdot \mathrm{mol}^{-1}\end{array}$ & $\begin{array}{r}3.07 \\
(001)\end{array}$ & $(0)=$ & (10.49 & $(1,29)$ & $\begin{array}{c}4.24 \\
(0.05)\end{array}$ & \\
\hline
\end{tabular}

Note: $\mathrm{T}_{1 p}=$ spin-lattice relaxation time in the rotating frame; Width = linewidth at half height in $\mathrm{Hz}$; $\mathrm{NC} 5 \%=\mathrm{PBFP}^{\mathrm{TiO}} \mathrm{T}_{2}-5 \%$; $\mathrm{CS}=$ chemical shift in ppm; the standard deviations are listed in brackets

\subsection{4 ${ }^{19} \mathrm{~F}$ spin-lattice relaxation time $\left(\mathrm{T}_{1}\right)$ of $\mathrm{NC} 5 \%$ at an MAS rate of $10 \mathrm{kHz}$}

Table 8.31: $\quad{ }^{19} \mathrm{~F} \mathrm{~T}$, times of solid NC $5 \%$ over the temperature range of 20 to $40^{\circ} \mathrm{C}$ obtained with an MAS rate of $10 \mathrm{kHz}$

\begin{tabular}{|c|c|c|c|c|c|c|}
\hline CS/ppm & & & & & \multicolumn{2}{|c|}{$\begin{array}{c}-76.93 \\
(\%)\end{array}$} \\
\hline Width/Hz & & & & & & \\
\hline Temp $/{ }^{\circ} \mathrm{C}$ & \multicolumn{6}{|c|}{$\mathrm{T}_{1} / \mathrm{s}$} \\
\hline \multirow{2}{*}{20} & \multicolumn{2}{|c|}{$0.56(0.01)$} & \multicolumn{2}{|c|}{$0.54(0.00)$} & \multicolumn{2}{|c|}{$0.56(0.01)$} \\
\hline & 0.62 & 0.35 & 0.57 & 0.48 & 0.65 & 0.49 \\
\hline \multirow{2}{*}{30} & \multicolumn{2}{|c|}{$0.63(0.01)$} & \multicolumn{2}{|c|}{$0.58(0.02)$} & \multicolumn{2}{|c|}{$0.62(0.01)$} \\
\hline & 0.73 & 0.41 & 0.64 & 0.57 & 0.66 & 0.58 \\
\hline \multirow{2}{*}{40} & \multicolumn{2}{|c|}{$0.71(0.03)$} & \multicolumn{2}{|c|}{$0.65(0.01)$} & \multicolumn{2}{|c|}{$0.70(0.01)$} \\
\hline & 0.77 & 0.61 & 0.70 & 0.56 & 0.74 & 0.63 \\
\hline $\mathrm{E}_{\mathrm{a}} / \mathrm{kJ} \cdot \mathrm{mol}^{-1}$ & \multicolumn{2}{|c|}{$8.43(0.17)$} & \multicolumn{2}{|c|}{$7.01(0.05)$} & \multicolumn{2}{|c|}{$8.43(0.08)$} \\
\hline $\mathrm{E}_{\mathrm{a}} / \mathrm{kJ} \cdot \mathrm{mol}^{-1}$ & (n) & 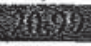 & 776 & 5.83 & 4.90 & 9.50 \\
\hline
\end{tabular}

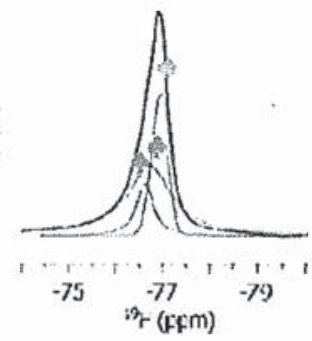

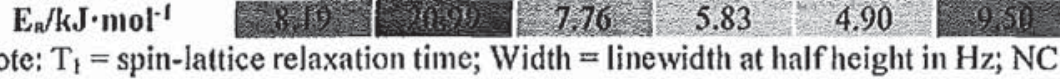
$5 \%=\mathrm{PBFP} \mathrm{TiO} \mathrm{O}_{2}-5 \%$; $\mathrm{CS}=$ chemical shift in $\mathrm{ppm}$; the standard deviations are listed in brackets

The ${ }^{19} \mathrm{~F}$ T, results of $\mathrm{NC} 5 \%$ over the temperature range $20-40{ }^{\circ} \mathrm{C}$ are listed in Table 8.31. The $T_{1}$ values of all three components are bi-exponential as indicated in Table 8.31. In the higher temperature range from $50-80^{\circ} \mathrm{C}$, the corresponding results are shown in Table 8.32.The $\mathrm{T}_{1}$ results of each component in Table 8.31 and Table 8.32 increase in both temperature ranges, together with the positive energies, indicating that sidechain motion is 
in the fast motion regime as seen previously for PBFP and annealed PBFP. The $T_{1}$ values for all components in Table 8.32 are statistically different; however, the differences are not as large as seen at lower temperature in Table 8.31. In Table 8.31, the component at $\mathbf{- 7 6 . 7 3}$ ppm has a larger $E_{a}$ at $7.01 \mathrm{~kJ} \cdot \mathrm{mol}^{-1}$ as compared to the other two components at -76.64 ppm and $-76.93 \mathrm{ppm}$. In Table 8.32 , the components at -76.42 and $-76.76 \mathrm{ppm}$ have similar $\mathrm{E}_{\mathrm{a}}$ results at 8.55 and $8.12 \mathrm{~kJ} \cdot \mathrm{mol}^{-1}$ and should be considered together, while the component at $-77.10 \mathrm{ppm}$ has a $\mathrm{E}_{\mathrm{a}}$ value at $6.97 \mathrm{~kJ} \cdot \mathrm{mol}^{-1}$, which is distinct from other three components and thus should be considered on its own phase. Additionally, the component at -76.92 ppm has the smallest $\mathrm{E}_{\mathrm{a}}$ at $4.93 \mathrm{~kJ} \cdot \mathrm{mol}^{-1}$, and hence must considered separately.

Table 8.32: $\quad{ }^{19} \mathrm{~F} \mathrm{~T}_{1}$ times of solid NC $5 \%$ over the temperature range of 50 to $80^{\circ} \mathrm{C}$ obtained

\begin{tabular}{|c|c|c|c|c|}
\hline $\mathrm{CS} / \mathrm{ppm}$ & $\begin{array}{c}-76.42 \\
(\diamond)\end{array}$ & $\begin{array}{c}-76.76 \\
(\diamond)\end{array}$ & $\begin{array}{c}-76.92 \\
(\hat{8})\end{array}$ & $\begin{array}{c}-77.10 \\
(5)\end{array}$ \\
\hline Width/Hz & 239 & 181 & 103 & 125 \\
\hline Temp $/{ }^{\circ} \mathrm{C}$ & \multicolumn{4}{|c|}{$\mathbf{T}_{1} / \mathbf{s}$} \\
\hline 50 & $0.91(0,0,3)$ & $0.89(0.03)$ & $1.08(0.01)$ & $0.89(0.02)$ \\
\hline 60 & $1.07(0.04)$ & $1.05(0.03)$ & $1.15(0.01)$ & $1.05(0.02)$ \\
\hline 70 & $1.19(0.03)$ & $1.15(0.03)$ & $1.26(0.02)$ & $1.11(0.01)$ \\
\hline 80 & $1.32(0.07)$ & $1.22(0.02)$ & $1.36(0.01)$ & $1.18(0.02)$ \\
\hline $\mathrm{Ea} / \mathrm{kJ} \cdot \mathrm{mol}^{-1}$ & $8.55(0.23)$ & $8,12(0,13)$ & $493(003)$ & $6.97(0.09)$ \\
\hline
\end{tabular}

\subsection{5 ${ }^{19} \mathrm{~F}$ spin-spin relaxation time $\left(\mathrm{T}_{2}\right)$ of $\mathrm{NC} 5 \%$ at an MAS rate of $10 \mathrm{kHz}$}

The ${ }^{19} \mathrm{~F} \mathrm{~T}_{2}$ parameters of $\mathrm{NC} 5 \%$ from $20-40^{\circ} \mathrm{C}$ are listed in Table 8.33. For higher temperature, from $50-80^{\circ} \mathrm{C}$, the ${ }^{19} \mathrm{~F} \mathrm{~T}_{2}$ data are listed in Table 8.34. Both tables indicate that the $T_{2}$ values increases with increasing temperature for all components. 
Table 8.33: $\quad{ }^{19} \mathrm{~F} \mathrm{~T}_{2}$ times of solid NC $5 \%$ over the temperature range of 20 to $40^{\circ} \mathrm{C}$ obtained with an MAS rate of $10 \mathrm{kHz}$

\begin{tabular}{|c|c|c|c|}
\hline CS/ppm & $\begin{array}{c}-76.64 \\
(*)\end{array}$ & $\begin{array}{c}-76.73 \\
(4)\end{array}$ & $\begin{array}{c}-76.93 \\
\left(\hat{a}^{2}\right)\end{array}$ \\
\hline Width/Hz & 171 & 538 & 191 \\
\hline Temp/ $/{ }^{\circ} \mathrm{C}$ & & $\mathrm{T}_{2}$ Ams & \\
\hline 20 & $1.73(0.24)$ & $1.17(0.08)$ & $1.70(0.10)$ \\
\hline 30 & $1.79(0.10)$ & $1.23(0.11)$ & $1.77(0.14)$ \\
\hline 40 & $1.83(0.21)$ & $1.29(0.07)$ & $1.84(0.17)$ \\
\hline $\mathrm{Ea} / \mathrm{kJ} \cdot \mathrm{mol}^{-1}$ & $2 \pi 12(0) 190)$ & $3609(0.14)$ & $2929(0.14)$ \\
\hline
\end{tabular}

Note: $\mathrm{T}_{2}=$ spin-spin relaxation time; Width $=$ linewidth at half height in $\mathrm{Hz}$; NC $5 \%=\mathrm{PBFP} / \mathrm{TiO}_{2}-5 \%$; $\mathrm{CS}=$ chemical shift in $\mathrm{ppm}$; the standard deviations are listed in brackets

In Table 8.33 , the $\mathrm{E}_{\mathrm{a}}$ values for all three components are very similar ranging from 2.12 to $3.69 \mathrm{~kJ} \cdot \mathrm{mol}^{-1}$, and therefore are assigned to one phase category. Alternatively as the $E_{a}$ is -76.73 ppm being somewhat larger, and its $T_{2}$ is different, from the others, it could be considered to be on its own. In Table 8.34 , the two components at -76.76 and -76.42 ppm have close $E_{a}$ values at 6.75 and $7.96 \mathrm{~kJ} \cdot \mathrm{mol}^{-1}$, thus should put them into the same phase category. The two components at -76.92 and $-77.10 \mathrm{ppm}$ have similar $\mathrm{E}_{\mathrm{a}}$ at 2.88 and $3.95 \mathrm{~kJ} \cdot \mathrm{mol}^{-1}$ and close $\mathrm{T}_{2}$ values, and should be considered together.

Table 8.34: $\quad{ }^{19} \mathrm{~F} \mathrm{~T}_{2}$ times of solid $\mathrm{NC} 5 \%$ over the temperature range of 50 to $80{ }^{\circ} \mathrm{C}$ obtained with an MAS rate of $10 \mathrm{kHz}$

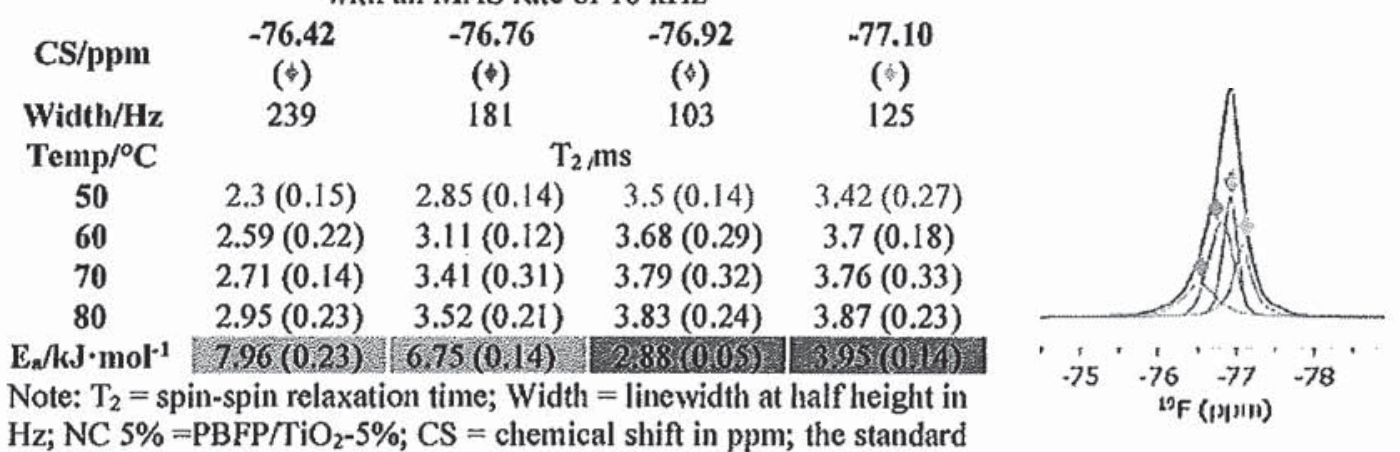

$\mathrm{Hz} ; \mathrm{NC} 5 \%=\mathrm{PBFP} / \mathrm{TiO}_{2}-5 \%$; $\mathrm{CS}=$ chemical shift in ppm; the standard deviations are listed in brackets 


\subsection{6 ${ }^{19} \mathrm{~F}$ spin-lattice relaxation time in the rotating frame $\left(\mathrm{T}_{1_{\wp}}\right)$ of $\mathrm{NC} 5 \%$ at an MAS rate of $10 \mathrm{kHz}$}

Table 8.35: $\quad{ }^{19} \mathrm{~F} \mathrm{~T}_{1 p}$ times of solid NC $5 \%$ over the temperature range of 20 to $40^{\circ} \mathrm{C}$ obtained with an MAS rate of $10 \mathrm{kHz}$

\begin{tabular}{cccc} 
CS/ppm & -76.64 & -76.73 & -76.93 \\
Width/Hz & $(\%)$ & $(\%)$ & $(\%)$ \\
Temp/ $/{ }^{\circ} \mathrm{C}$ & $\mathbf{1 7 1}$ & $\mathbf{5 3 8}$ & 191 \\
$\mathbf{2 0}$ & & $\mathrm{T}_{1 \mathrm{p}} / \mathrm{ms}$ & \\
$\mathbf{3 0}$ & $1.19(0.03)$ & $1.50(0.01)$ & $1.38(0.02)$ \\
$\mathbf{4 0}$ & $1.32(0.03)$ & $1.58(0.01)$ & $1.49(0.01)$ \\
$\mathbf{E}_{\mathrm{a}} / \mathbf{k J} \cdot \mathbf{m o l}^{-1}$ & $1.47(0.04)$ & $1.66(0.02)$ & $1.65(0.01)$ \\
\hline
\end{tabular}

Note: $\mathrm{T}_{1 \rho}=$ spin-lattice relaxation time in the rotating frame; Width $=$ linewidth at half height in $\mathrm{Hz} ; \mathrm{NC} 5 \%=\mathrm{PBFP}_{\mathrm{TiO}}-5 \% ; \mathrm{CS}=$ chemical shift in ppm; the standard deviations are listed in brackets

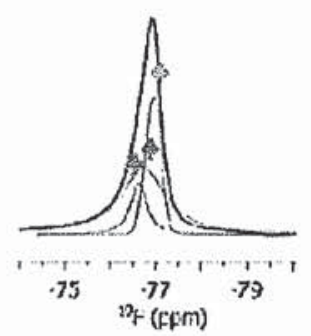

${ }^{19} \mathrm{~F} \mathrm{~T}_{1 p}$ values of NC $5 \%$ from $20.40{ }^{\circ} \mathrm{C}$ are shown in Table 8.35 . For the temperature range from $50-80^{\circ} \mathrm{C}$ the $\mathrm{T}_{1 p}$ data are presented in Table 8.36. Both tables show that the $T_{1 \rho}$ data of all components increase with increasing temperature, similar as observed for PBFP and annealed PBFP, the activation energies are all positive, suggesting that sidechain motion is in the fast regime in both cases.

Table 8.50: $\quad{ }^{19} \mathrm{~F} \mathrm{~T}_{1 \rho}$ times of solid NC $5 \%$ over the temperature range of 50 to $80^{\circ} \mathrm{C}$ obtained

\begin{tabular}{|c|c|c|c|c|}
\hline \multirow[b]{2}{*}{ CS/ppm } & \multicolumn{4}{|c|}{ with an MAS rate of $10 \mathrm{kHz}$} \\
\hline & $\begin{array}{c}-76.42 \\
(\phi)\end{array}$ & $\begin{array}{c}-76.76 \\
(\$)\end{array}$ & $\begin{array}{c}-76.92 \\
\text { (今) }\end{array}$ & $\begin{array}{c}-77.10 \\
(\varphi)\end{array}$ \\
\hline Width/Hz & 239 & 181 & 103 & 125 \\
\hline $\mathrm{Temp} /{ }^{\circ} \mathrm{C}$ & \multicolumn{4}{|c|}{$\mathrm{T}_{\mathrm{I} \rho} / \mathrm{ms}$} \\
\hline 50 & $1.47(0.11)$ & $1.68(0.02)$ & $1.50(0.03)$ & $1.91(0.18)$ \\
\hline 60 & $1.60(0.16)$ & $1.70(0.03)$ & $1.55(0.03)$ & $2.27(0.12)$ \\
\hline 70 & $1.73(0.17)$ & $1.72(0.02)$ & $1.56(0.06)$ & $2.43(0.11)$ \\
\hline 80 & $1.89(0.22)$ & $1.73(0.02)$ & $1.61(0.07)$ & $2.50(0.07)$ \\
\hline $\mathbf{E}_{\mathrm{a}} / \mathrm{kJ} \cdot \mathrm{mol}^{-1}$ & $804(0.4)$ & $0.94(0.01)$ & $2.26(0.05)$ & $868(0.37)$ \\
\hline
\end{tabular}

Note: $\mathrm{T}_{1 \mathrm{p}}=$ spin-lattice relaxation time in the rotating frame; Width $=$

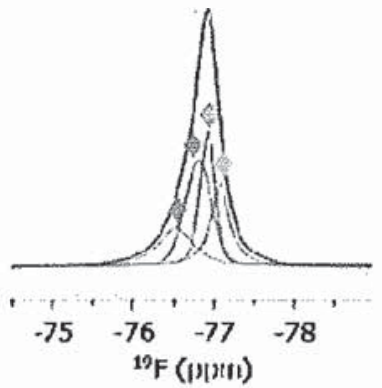
linewidth at half height in $\mathrm{Hz} ; \mathrm{NC} 5 \%=\mathrm{PBFP} / \mathrm{TiO}_{2}-5 \% ; \mathrm{CS}=$ chemical shift in ppm; the standard deviations are listed in brackets

In Table 8.35, the component at $-76.73 \mathrm{ppm}$ has the smallest $\mathrm{E}_{\mathrm{a}}$ at $3.83 \mathrm{~kJ} \cdot \mathrm{mol}^{-1}$ and longest $T_{1 p}$ and thus is mostly likely assigned to its own phase category. The $E_{a}$ values of the other two components are similar, with shorter $\mathrm{T}_{1 p}$ values, and thus likely belonging to 
the same phase category. In Table 8.36 , the components at -76.42 and $-77.10 \mathrm{ppm}$ have larger $\mathrm{E}_{\mathrm{a}}$ values, putting them together into one phase group. The components at -76.92 and $-76.76 \mathrm{ppm}$ have the smallest $\mathrm{E}_{\mathrm{a}}$ values hence they fall into the same phase category. The $\mathrm{T}_{1 \mathrm{p}}$ results would put $-\mathbf{- 7 6 . 4 2}$ and $-\mathbf{- 7 6 . 9 2} \mathrm{ppm}$ together, and $-\mathbf{7 6 . 7 5} \mathrm{ppm}$ with $\mathbf{- 7 7 . 1 0}$ ppm.

\subsubsection{Conclusion}

In summary, the ${ }^{31} \mathrm{P}$ NMR spectra of NC $5 \%$ were deconvolved using a fourcomponent model over $20-40^{\circ} \mathrm{C}$, and a five-component model from $50-80^{\circ} \mathrm{C}$. At the low temperature range, the $T_{1}$ data show that component at $-4.55 \mathrm{ppm}$ is composed one very long $T_{1}$ contribution with values around $10-12 s$ and another long $T_{1}$ contribution around 6 $\mathrm{s}$, the three narrow components are all contain one long $T_{1}$ around $12 \mathrm{~s}$ and one short $T_{1}$ contribution around $3 \mathrm{~s}$. The $\mathrm{T}_{2}$ data show that the component at $-4.55 \mathrm{ppm}$ has a comparably short $T_{2}$ than the remaining three components. The $T_{1 \rho}$ values of component at -4.55 is smaller than the three narrow components, which separate it from the other three components. At high temperature, because of the increased motion above its $\mathrm{T}(1)$ transition temperature, the difference between each component becomes smaller and more difficult to distinguish.

A three-component model is used to deconvolve the ${ }^{19} \mathrm{~F}$ NMR spectra of PBFP over $20-40^{\circ} \mathrm{C}$, and a four-component model from $50-80^{\circ} \mathrm{C}$. At the low temperature range, the

${ }^{19} \mathrm{~F} \mathrm{~T}_{1}$ data show bi-exponential behavior, each component is composed of one longer $\mathrm{T}_{1}$ and one shorter $T_{1}$. The $T_{2}$ results suggest that the broad component at $-76.73 \mathrm{ppm}$ with linewidth of $538 \mathrm{~Hz}$ has a smaller $\mathrm{T}_{2}$ compared to other two narrow components, indicating 
that it arises from a different phase domain. The $T_{1 \rho}$ data showed that all three components have similar $T_{1_{p}}$ values around $1.2-1.5 \mathrm{~ms}$, suggesting that they should appear in the same regime with respect to the locking power. At higher temperature, the sidechain motion of all polymer domains is enhanced, the differences becomes smaller and more difficult to separate. 


\subsection{Solid-State NMR Relaxation Data of Annealed NC 5\%}

\subsection{1 ${ }^{31} \mathrm{P}$ spin-lattice relaxation time $\left(\mathrm{T}_{1}\right)$ of annealed $\mathrm{NC} 5 \%$ at an MAS rate of 10 $\mathrm{kHz}$}

The $\mathrm{T}_{1}$ relaxation time of ${ }^{31} \mathrm{P}$ of annealed $\mathrm{NC} 5 \%$ from $20-40^{\circ} \mathrm{C}$ is listed in Table 8.37, the numbers indicate that as temperature goes up, the $T_{1}$ values decrease. The phosphorus $\mathrm{T}_{1}$ results of annealed $\mathrm{NC} 5 \%$ at the temperature range $50-80{ }^{\circ} \mathrm{C}$ were calculated and listed in Table 8.38. The negative energy values in both tables suggest that the backbone motion is in the slow motion regime with respect to the Larmor frequency.

Table 8.37: $\quad{ }^{31} \mathrm{P} \mathrm{T}_{1}$ times of solid annealed NC $5 \%$ over the temperature range of 20 to $40{ }^{\circ} \mathrm{C}$ obtained with an MAS rate of $10 \mathrm{kHz}$

\begin{tabular}{|c|c|c|c|c|c|c|}
\hline $\mathrm{CS} / \mathrm{ppm}$ & & & $\begin{array}{l}-8.12 \\
(0)\end{array}$ & $\begin{array}{c}-8.68 \\
(0)\end{array}$ & $\begin{array}{c}-9.88 \\
(\%)\end{array}$ & \\
\hline Width/Hz & & & 344 & 272 & 242 & 1 \\
\hline $\mathrm{Temp} /{ }^{\circ} \mathrm{C}$ & \multicolumn{5}{|c|}{$T_{1} / s$} & \\
\hline \multirow{2}{*}{20} & \multicolumn{2}{|c|}{$12.48(0.06)$} & $4.13(0.18)$ & $3.36(0.08)$ & $4.13(0.18)$ & \\
\hline & 15.46 & 12.25 & $22.22 \quad 2.08$ & $17.02 \quad 2.41$ & $12.93 \bigcirc 2.47$ & \\
\hline \multirow{2}{*}{30} & \multicolumn{2}{|c|}{$11.25(0.14)$} & $3.73(0.14)$ & $3.22(0.09)$ & $3.73(0.14)$ & \\
\hline & 14.66 & 10.42 & $19.08 \quad 2.10$ & $16.03 \quad 2.36$ & $10.79 \quad 2.30$ & \\
\hline \multirow{2}{*}{40} & \multicolumn{2}{|c|}{$10.00(0.03)$} & $3.29(0.12)$ & $2.61(0.09)$ & $3.29(0.12)$ & ${ }_{3 i p(m i n)}^{5}-10$ \\
\hline & 12.15 & 9.45 & $16.21 \quad 2.05$ & $16.69 \quad 2.39$ & \multirow{2}{*}{$\begin{array}{ll}8.70 & 2.24 \\
-8.62 & (0.21)\end{array}$} & \\
\hline $\mathbf{E}_{\mathrm{a}} / \mathrm{kJ} \cdot \mathrm{mol}^{-1}$ & \multicolumn{2}{|c|}{$-8.39(0.02)$} & $-8.62(0.21)$ & $-9.46(0.17)$ & & \\
\hline $\mathrm{E}_{\mathrm{A}} / \mathrm{kJ} \cdot \mathrm{mol}^{-1}$ & 2010 & -928 & 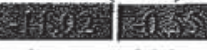 & 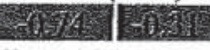 & 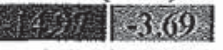 & \\
\hline
\end{tabular}

As presented in Table 8.37 and 8.38 , as temperature goes up, the $T_{1}$ values decrease for both temperature range $20-40^{\circ} \mathrm{C}$ and $50-80^{\circ} \mathrm{C}$, showing that the molecule is in the slow motion regime as what is present for PBFP and sample PBFP-annealed. In Table 8.37, the broad component that appears at $-4.55 \mathrm{ppm}$ has a much larger $\mathrm{T}_{1}$ value, making it separate from other reaming constitutions. While the component at $-9.88 \mathrm{ppm}$ obviously has a smaller $\mathrm{T}_{1}$ value than the other three components, which would distinguish it from the other components. 
All $\mathrm{T}_{1}$ values have a bi-exponential decay for all four components as indicated by two different relaxation times in Table 8.37 at the temperature range $20-40^{\circ} \mathrm{C}$. More specifically, the component at $-4.55 \mathrm{ppm}$ has two $\mathrm{T}_{1}$ values at 15.46 and $12.25 \mathrm{~s}$, respectively. The other three components are composed of one long $T_{1}$ and one short $T_{1}$ value. Furthermore, the slower $T_{1}$ with $12.25 \mathrm{~s}$ is considerably longer than the shorter T,value of the remaining three components. In addition, for both the faster and slower relaxation times of all four components, The $T_{1}$ values decrease with increasing temperature.

Table 8.38: $\quad{ }^{31} \mathrm{P}_{1}$ times of solid annealed $\mathrm{NC} 5 \%$ over the temperature range of 50 to $80^{\circ} \mathrm{C}$ obtained with an MAS rate of $10 \mathrm{kHz}$

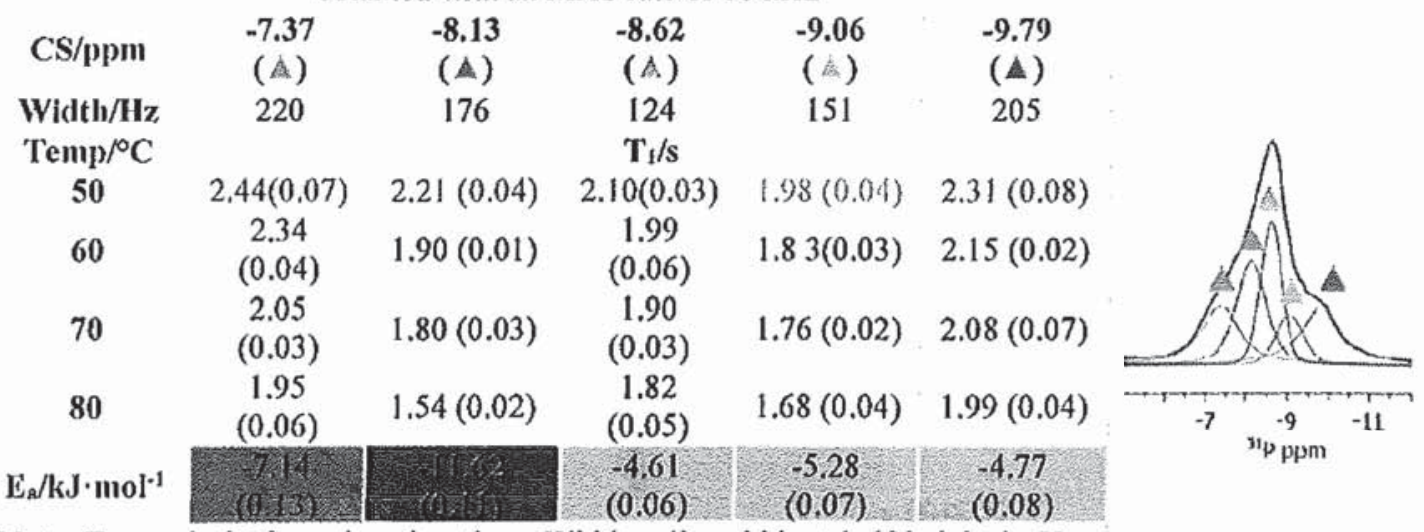

Note: $\mathrm{T}_{1}=$ spin-lattice relaxation time; Width $=$ linewidth at half height in $\mathrm{Hz}$; annealed $\mathrm{NC} 5 \%=$ annealed $\mathrm{PBFP} / \mathrm{TiO}_{2}-5 \%$ at $220^{\circ} \mathrm{C}$ for 2 hours; $\mathrm{CS}=$ chemical shift in ppm; the standard deviations are listed in brackets

In Table 8.38, all four components have very similar ' $\mathrm{T}_{1}$ values, while the component at $-8.13 \mathrm{ppm}$ has a smallest $\mathrm{E}_{\mathrm{a}}$ at $-11.62 \mathrm{~kJ} \cdot \mathrm{mol}^{-1}$, which is assigned to a separate phase group. The components at $-8.62,-9.79$, and $-9.06 \mathrm{ppm}$ have $\mathrm{E}_{\mathrm{a}}$ values at $-4.61,-4.77$ and $5.28 \mathrm{~kJ} \cdot \mathrm{mol}^{-1}$, that are attributed to one phase group. The remaining component at -7.37 ppm has a medium value at $-7.14 \mathrm{~kJ} \cdot \mathrm{mol}^{-1}$ is allocated to another distinct phase group. Therefore, all five components in Table 8.8 are presumed to fall into three separate phase categories. 


\subsection{2 ${ }^{31} \mathrm{P}$ spin-spin relaxation time $\left(\mathrm{T}_{2}\right)$ of annealed $\mathrm{NC} 5 \%$ at an MAS rate of $10 \mathrm{kHz}$}

${ }^{31} \mathrm{P} \mathrm{T}_{2}$ results of annealed NC $5 \%$ over $20-40^{\circ} \mathrm{C}$ are shown in Table 8.39 ; for higher temperature, from $50-80^{\circ} \mathrm{C}$, the corresponding $\mathrm{T}_{2}$ data are listed in Table 8.40 .

Table 8.39: $\quad{ }^{31} \mathrm{P}_{2}$ times of solid annealed NC 5\% over the temperature range of 20 to $40{ }^{\circ} \mathrm{C}$

\begin{tabular}{|c|c|c|c|c|}
\hline \multirow[b]{2}{*}{ CS/ppm } & \multicolumn{4}{|c|}{ obtained with an MAS rate of $10 \mathrm{kHz}$} \\
\hline & $\begin{array}{c}-4.55 \\
(c)\end{array}$ & $\begin{array}{c}-8.12 \\
\text { (e) }\end{array}$ & $\begin{array}{c}-8.68 \\
(0)\end{array}$ & $\begin{array}{c}-9.88 \\
(\%)\end{array}$ \\
\hline Width/Hz & 834 & 344 & 272 & 242 \\
\hline Temp/ $/ \mathrm{C}$ & \multicolumn{4}{|c|}{$\mathrm{T}_{2, \mathrm{~ms}}$} \\
\hline 20 & $0,60(0.01)$ & $1.73(0.03)$ & $1.40(0.09)$ & $1.21(0.11)$ \\
\hline 30 & $0.65(0.01)$ & $1.81(0.13)$ & $1.55(0.04)$ & $1.27(0.10)$ \\
\hline 40 & $0.70(0.03)$ & $1.94(0.03)$ & $1.68(0.08)$ & $1.34(0.03)$ \\
\hline $\mathrm{E}_{2} / \mathrm{kJ} \cdot \mathrm{mol}^{-1}$ & $5.83(0.12)$ & $4.33(0.05)$ & $6.89(0.24)$ & $3.86(0.16)$ \\
\hline
\end{tabular}

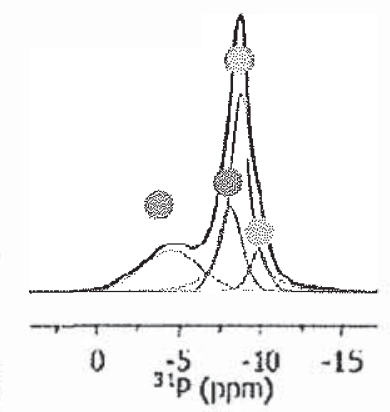

Note: $\mathrm{T}_{2}=$ spin-spin relaxation time; Width $=$ linewidth at half height in $\mathrm{Hz}$; annealed $\mathrm{NC} 5 \%=$ annealed $\mathrm{PBFP} / \mathrm{TiO}_{2}-5 \%$ at $220^{\circ} \mathrm{C}$ for 2 hours; $\mathrm{CS}=$ chemical shift in ppm; the standard deviations are listed in brackets

Table 8.40: $\quad{ }^{31} \mathrm{P} \mathrm{T}_{2}$ times of solid annealed NC $5 \%$ over the temperature range of 50 to $80{ }^{\circ} \mathrm{C}$ obtained with an MAS rate of $10 \mathrm{kHz}$

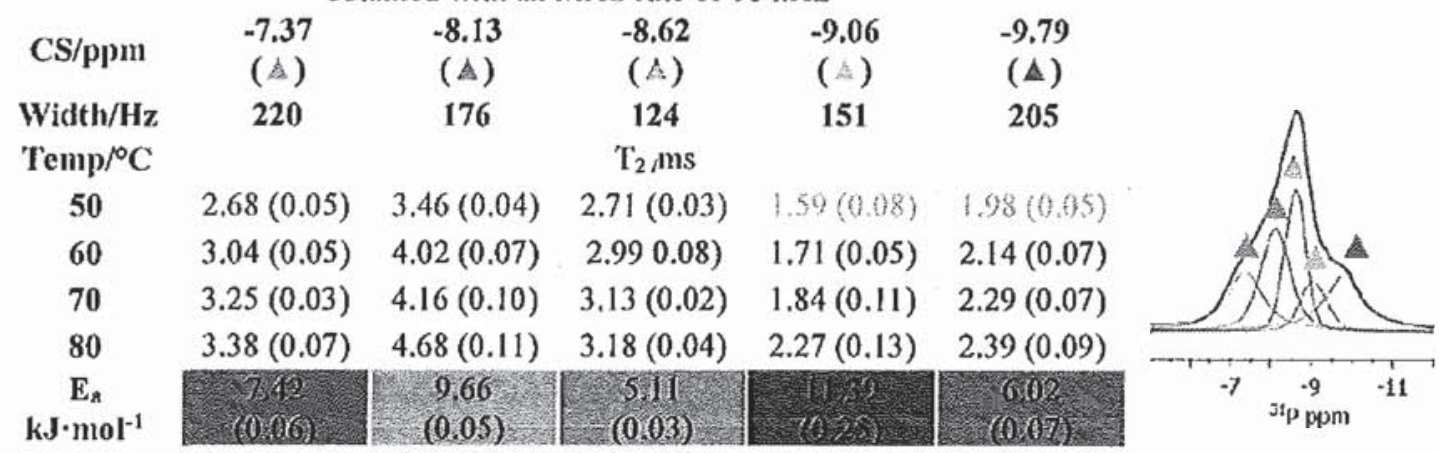

Note: $\mathrm{T}_{2}=$ spin-spin relaxation time; Width = linewidth at half height in $\mathrm{Hz}$; annealed $\mathrm{NC} 5 \%=$ annealed $\mathrm{PBFP} / \mathrm{TiO}_{2}-5 \%$ at $220^{\circ} \mathrm{C}$ for 2 hours; $\mathrm{CS}=$ chemical shift in ppm; the standard deviations are listed in bracket

The $\mathrm{T}_{2}$ values in both Table 8.39 and 8.40 , show $\mathrm{T}_{2}$ increase with temperature, for all components in both temperature ranges. The component at $-4.55 \mathrm{ppm}$ has a distinctly small $\mathrm{T}_{2}$ compared to the remaining three components, indicating that the backbone motion in this component is slower. Furthermore, in Table 8.39 , the $E_{a}$ for four components are very distinguishable, where the components at $-9.88(\lesssim)$ and $-8.12(\bullet) \mathrm{ppm}$ have similar $\mathrm{E}_{\mathrm{a}}$ values at 3.86 and $4.33 \mathrm{~kJ} \cdot \mathrm{mol}^{-1}$, thus should be considered together. The two components 
at $-4.55(\odot)$ and $-8.68(\circ) \mathrm{ppm}$ also have close $\mathrm{E}_{\mathrm{a}}$ values at 5.83 and $6.89 \mathrm{~kJ} \cdot \mathrm{mol}^{-1}$, and thus should be assigned to one phase category. In addition, in Table 8.40 , the activation energies for all five components are different between components. Components at $-9.79(\mathbb{\Delta})$ and 7.37 (A) ppm have close $\mathrm{E}_{\mathrm{a}}$ at 6.02 and $7.42 \mathrm{~kJ} \cdot \mathrm{mol}^{-1}$, are mostly likely being grouped together. The component at -8.62 has $E_{a}$ at $5.11 \mathrm{~kJ} \cdot \mathrm{mol}^{-1}$, should be considered to its own phașe category. The remaining component at $-8.13(\mathrm{~A})$ and $-9.06(\mathrm{~A}) \mathrm{ppm}$, all have distinct bigger $\mathrm{E}_{\mathrm{a}}$ values at 9.66 and $11.39 \mathrm{~kJ} \cdot \mathrm{mol}^{-1}$, are considered to its own phase category.

\subsection{3 ${ }^{31} \mathrm{P}$ spin-lattice relaxation time in the rotating frame ( $\left.T_{1}\right)$ of annealed NC $5 \%$ at an MAS rate of $10 \mathrm{kHz}$}

The ${ }^{31} \mathrm{P} \mathrm{T}_{1 \rho}$ values of annealed $\mathrm{NC} 5 \%$ at the temperature range $20-40^{\circ} \mathrm{C}$ are listed

in Table 8.41, showing that as temperature increases, the $T_{1 \rho}$ values increase for those components appears at $-8.68,-9.88$ and $-8.12 \mathrm{ppm}$, however, for signal appears at -4.55 ppm, its $T_{1 p}$ value decreases with increasing temperature. And they have different activation energies, suggesting that the component at $-4.55 \mathrm{ppm}$ is in different motion regime compared to other three components. For the higher temperature range from 50-80 ${ }^{\circ} \mathrm{C}$, the $\mathrm{T}_{1 \mathrm{p}}$ values of all five components increase with temperature resulting in positive activation energies as shown in Table 8.42 , indicating that that the $T_{i_{p}}$ values for all components increase with increasing temperature. 
Table 8.41: $\quad{ }^{31} \mathrm{P} \mathrm{T}_{1 \mathrm{p}}$ times of solid annealed NC $5 \%$ over the temperature range of 20 to $40{ }^{\circ} \mathrm{C}$ obtained with an MAS rate of $10 \mathrm{kHz}$

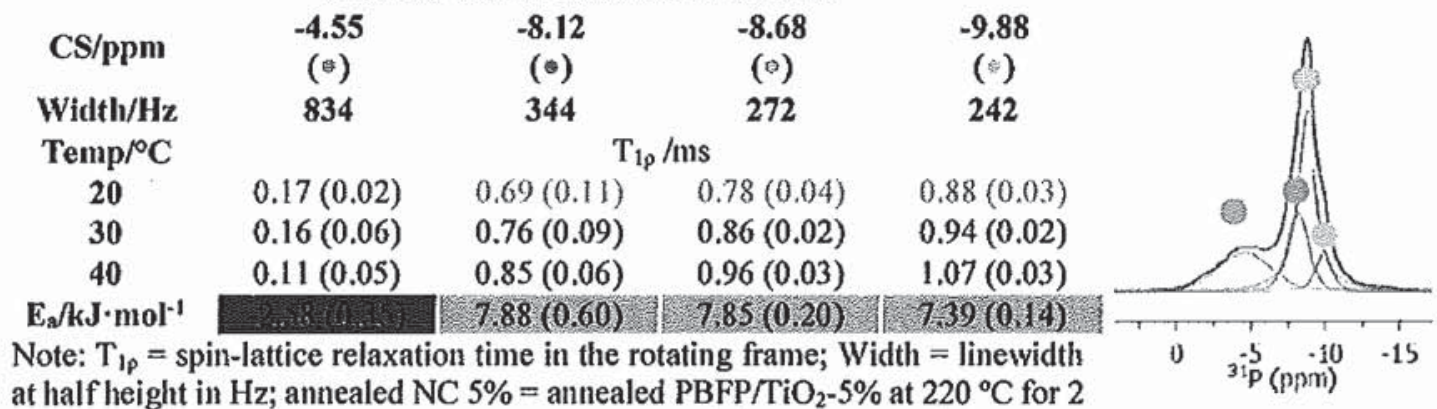
hours; $\mathrm{CS}=$ chemical shift in ppm; the standard deviations are listed in brackets

Table 8.42: $\quad{ }^{31} \mathrm{PT}_{1 \mathrm{p}}$ times of solid annealed NC $5 \%$ over the temperature range of 50 to $80^{\circ} \mathrm{C}$

\begin{tabular}{|c|c|c|c|c|c|c|}
\hline \multirow[b]{2}{*}{ CS/ppm } & \multicolumn{4}{|c|}{ obtained with an MAS rate of $10 \mathrm{kHz}$} & & \\
\hline & $\begin{array}{c}-7.37 \\
(\mathrm{~A})\end{array}$ & $\begin{array}{c}-8.13 \\
\text { (A) }\end{array}$ & $\begin{array}{l}-8.62 \\
(A)\end{array}$ & $\begin{array}{c}-9.06 \\
(\hat{A})\end{array}$ & $\begin{array}{l}-9.79 \\
(\mathbb{A})\end{array}$ & \\
\hline Widtli/Hz & 220 & 176 & 124 & 151 & 205 & A \\
\hline Temp $/{ }^{\circ} \mathrm{C}$ & & & $\mathrm{T}_{1 p} / \mathrm{ms}$ & & & \\
\hline 50 & $0.93(0.01)$ & $0.96(0.01)$ & $0.57(0.03)$ & $0.55(0.05)$ & $1.38(0.05)$ & \\
\hline 60 & $0.97(0.03)$ & $1.09(0.03)$ & $0.70(0.4)$ & $0.62(0.05)$ & $1.44(0.06)$ & \\
\hline 70 & $0.99(0.02)$ & $1.23(0.11)$ & $0.79(0.05)$ & $0.68(0.05)$ & $1.49(0.06)$ & \\
\hline 80 & $1.04(0.03)$ & $1.36(0.12)$ & $0.95(0.09)$ & $0.75(0.06)$ & $1.54(0.07)$ & \\
\hline$\underset{\mathrm{kJ} \cdot \mathrm{mol}^{-1}}{\mathbf{E}_{\mathrm{R}}}$ & $(0.02)$ & & & $\begin{array}{l}902 \\
(0) 39)\end{array}$ & $\begin{array}{c}3.51 \\
(0.06)\end{array}$ & $-7{ }_{10 \mathrm{ppm}}^{-9}-11$ \\
\hline
\end{tabular}

Note: $T_{1 \rho}=$ spin-lattice relaxation time in the rotating frame; Width = linewidth at half height in $\mathrm{Hz}$; annealed NC $5 \%=$ annealed $\mathrm{PBFP} / \mathrm{TiO}_{2}-5 \%$ at $220^{\circ} \mathrm{C}$ for 2 hours; $\mathrm{CS}=$ chemical shift in ppm; the standard deviations are listed in brackets

In Table 8.41 , the components at $-4.55 \mathrm{ppm}$ has a negative $\mathrm{E}_{\mathrm{a}}$ at $-2.58 \mathrm{~kJ} \cdot \mathrm{mol}^{-1}$, and by far the smallest $T_{1 p}$ hence making up its own phase category. Its distinct negative energy value also indicates its different motion compared to other components. The other three components at $-8.68,-9.88$ and $-8.12 \mathrm{ppm}$, have similar positive $E_{a}$ and $T_{1 \rho}$ values are assigned together to one phase group with fast motion. Likewise, in Table 8.42, the two components at -8.62 , and $-8.13 \mathrm{ppm}$ have comparatively close and large $\mathrm{E}_{\mathrm{a}}$ values at 16.34 , $11.14 \mathrm{~kJ} \cdot \mathrm{mol}^{-1}$, and thus are belonging to one phase category. The components at -7.37 and $-9.79 \mathrm{ppm}$ have similar $\mathrm{E}_{\mathrm{a}}$ at 3.58 and $3.51 \mathrm{~kJ} \cdot \mathrm{mol}^{-1}$, putting them into one phase group. In addition, the remaining component at $-9.06 \mathrm{ppm}$ has a relatively different $\mathrm{E}_{\mathrm{a}}$ value amongst 
all five components at $9.92 \mathrm{~kJ} \cdot \mathrm{mol}^{-1}$, should be considered separately. The $\mathrm{T}_{\mathrm{l} \rho}$ results would place those at -7.37 and $-8.13 \mathrm{ppm}$ together as well as those at -8.62 and $-9.06 \mathrm{ppm}$.

\subsection{4 ${ }^{19} \mathrm{~F}$ spin-lattice relaxation time $\left(\mathrm{T}_{1}\right)$ of amnealed NC $5 \%$ at an MAS rate of 10 $\mathrm{kHz}$}

The ${ }^{19} \mathrm{~F} \mathrm{~T}_{1}$ data of annealed NC $5 \%$ from $20-40{ }^{\circ} \mathrm{C}$ are listed in Table 8.43 . The ${ }^{19} \mathrm{~F}$ $\mathrm{T}_{1}$ results at higher temperature from $50-80^{\circ} \mathrm{C}$, are listed in Table 8.44.

Table 8.43: $\quad{ }^{19} \mathrm{~F} \mathrm{~T}_{1}$ times of solid annealed NC $5 \%$ over the temperature range of 20 to $40^{\circ} \mathrm{C}$

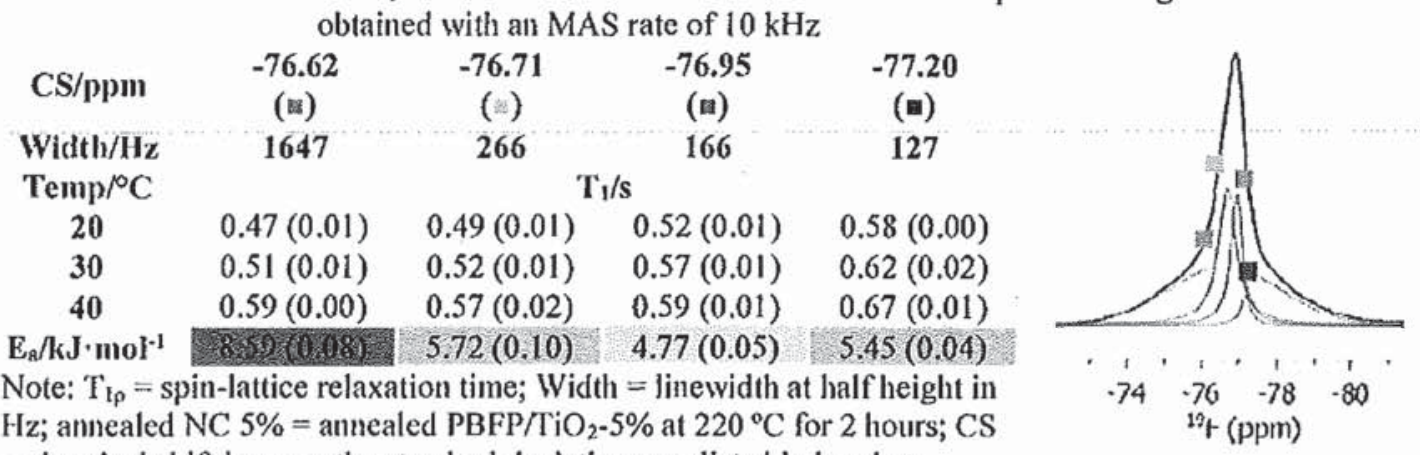

The $\mathrm{T}_{1}$ values of each component in Table 8.43 and Table 8.44 show that increase with temperature, together with positive activation energies, indicating that the sidechain dynamics is in the fast motion regimes as seen for the other three samples. In Table 8.43, the activation energy for all four components have obvious differences between them. The component at $-76.62 \mathrm{ppm}$ has a distinct small $\mathrm{E}_{\mathrm{a}}$ at $8.59 \mathrm{~kJ} \cdot \mathrm{mol}^{-1}$, setting it apart from the others. The remaining three components have similar $E_{a}$ at $4.77,5.45$, and $5.72 \mathrm{~kJ} \cdot \mathrm{mol}^{-1}$ for components at $-76.95,-77.10$ and $-76.71 \mathrm{ppm}$, respectively, and thus should be grouped together with similar phases. At higher temperature, the components at -76.42 and -76.76 ppm have a similar larger $E_{a}$ values at 14.02 and $13.45 \mathrm{~kJ} \cdot \mathrm{mol}^{-1}$ putting them together. While the remaining components at -76.92 and $-77.10 \mathrm{ppm}$ have $\mathrm{E}_{\mathrm{a}}$ at 10.16 and 9.24 $\mathrm{kJ} \cdot \mathrm{mol}^{-1}$ and thus are assigned to the same phase group. 
Table 8.44: $\quad{ }^{19} \mathrm{~F} \mathrm{~T}_{1}$ times of solid annealed NC $5 \%$ over the temperature range of 50 to $80^{\circ} \mathrm{C}$ obtained with an MAS rate of $10 \mathrm{kHz}$

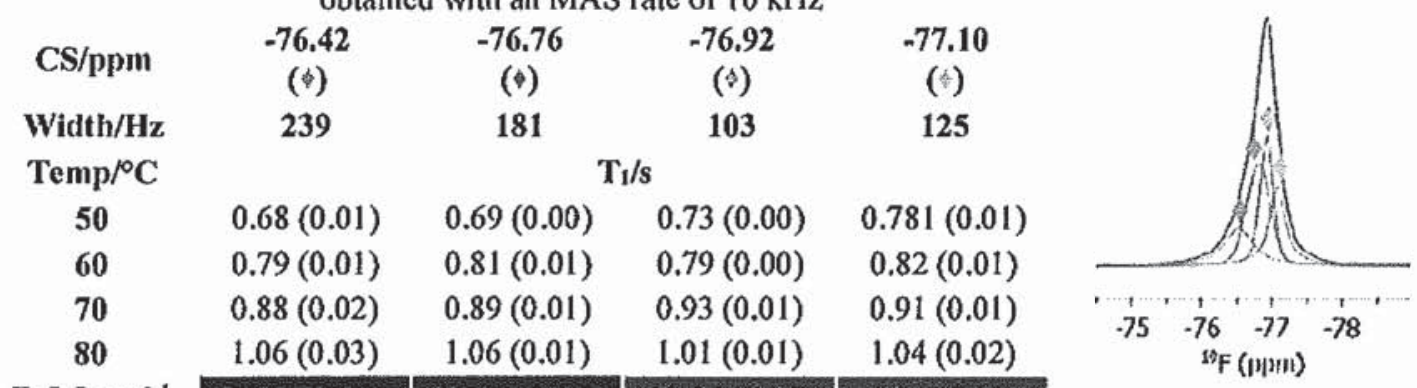

$\mathbf{E}_{2} / \mathbf{k J} \cdot \mathbf{m o l}^{-1}$

Note: $\mathrm{T}_{1}=$ spin-lattice relaxation time; $\mathrm{Width}=$ linewidth at half height in $\mathrm{Hz}$; annealed NC $5 \%=$ annealed $\mathrm{PBFP} / \mathrm{TiO}_{2}-5 \%$ at $220^{\circ} \mathrm{C}$ for 2 hours; $\mathrm{CS}=$ chemical shift in ppm; the standard deviations are listed in brackets

\subsection{5 ${ }^{19} \mathrm{~F}$ spin-spin relaxation time $\left(\mathrm{T}_{2}\right)$ of annealed NC $5 \%$ at an MAS rate of $10 \mathrm{kHz}$}

The ${ }^{19} \mathrm{~F} \mathrm{~T}_{2}$ data of annealed $\mathrm{NC} 5 \%$ from $20-40{ }^{\circ} \mathrm{C}$ are listed in Table 8.45. For the higher temperatures from $50-80^{\circ} \mathrm{C}$, the corresponding results are listed in Table 8.46. The $T_{2}$ values in both tables show that as temperature increases, the $T_{2}$ values increase.

Table 8.45: $\quad{ }^{19} \mathrm{~F} \mathrm{~T}_{2}$ times of solid annealed NC $5 \%$ over the temperature range of 20 to $40^{\circ} \mathrm{C}$ obtained with an MAS rate of $10 \mathrm{kHz}$

\begin{tabular}{|c|c|c|c|c|}
\hline CS/ppm & $\begin{array}{c}-76.62 \\
\text { (溸) }\end{array}$ & $\begin{array}{c}-76.71 \\
(\approx)\end{array}$ & $\begin{array}{c}-76.95 \\
\text { (日) }\end{array}$ & $\begin{array}{c}-77.20 \\
\text { (a) }\end{array}$ \\
\hline Width/Hz & 1647 & 266 & 166 & 127 \\
\hline Temp $/{ }^{\circ} \mathrm{C}$ & \multicolumn{4}{|c|}{$\mathrm{T}_{2} \mathrm{~ms}$} \\
\hline 20 & $0.18(0.00)$ & $1.48(0.02)$ & $1.82(0.03)$ & $1.67(0.04)$ \\
\hline 30 & $0.19(0.00)$ & $1.54(0.02)$ & $1.84(0.03)$ & $1.98(0.19)$ \\
\hline 40 & $0.20(0.00)$ & $1.68(0.05)$ & $1.86(0.03)$ & $2.45(0.32)$ \\
\hline $\mathrm{E}_{a} / \mathbf{k J} \cdot \mathrm{mol}^{-1}$ & $3.98(0.00)$ & $4.79(0.07)$ & $812(0.08)$ & 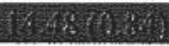 \\
\hline
\end{tabular}

Note: $\mathrm{T}_{2}=$ spin-spin relaxation time; Width $=$ linewidth at half height in $\mathrm{Hz}$; annealed NC $5 \%=$ annealed $\mathrm{PBFP} / \mathrm{TiO}_{2}-5 \%$ at $220^{\circ} \mathrm{C}$ for 2 hours; $\mathrm{CS}=$ chemical shift in ppm; the standard deviations are listed in brackets

In Table 8.45 , the component at $-77.20 \mathrm{ppm}$ has a distinctly large $\mathrm{E}_{\mathrm{a}}$ at $14.48 \mathrm{~kJ} \cdot \mathrm{mol}^{-}$

${ }^{1}$, thus is mostly likely assigning to its own phase category. The two components at -76.71 and $-76.62 \mathrm{ppm}$ have similar $\mathrm{E}_{\mathrm{a}}$ values at 4.79 and $3.98 \mathrm{~kJ} \cdot \mathrm{mol}^{-1}$, putting them into one separate phase category; however, their $\mathrm{T}_{2}$ values would indicate they represent different 
environments. The component at $-76.95 \mathrm{ppm}$ has an $\mathrm{E}_{\mathrm{a}}$ of $8.12 \mathrm{~kJ} \cdot \mathrm{mol}^{-1}$, and is best considered on its own. In Table 8.46, the activation energies vary to a lesser degree. The components at -76.42 and $-77.10 \mathrm{ppm}$, have similar $T_{2}$ and $E_{a}$ values and thus are best grouped together. Likewise the remaining two components belong together based on similarity of their $\mathrm{E}_{\mathrm{a}}$ and $\mathrm{T}_{2}$ values.

\begin{tabular}{|c|c|c|c|c|c|}
\hline \multirow[b]{2}{*}{ CS/ppm } & \multicolumn{5}{|c|}{$\begin{array}{l}{ }^{19} \mathrm{~F} \mathrm{~T}_{2} \text { times of solid annealed } \mathrm{NC} 5 \% \text { over the temperature range of } 50 \text { to } 80^{\circ} \mathrm{C} \\
\text { obtained with an MAS rate of } 10 \mathrm{kHz}\end{array}$} \\
\hline & $\begin{array}{c}-76.42 \\
(4)\end{array}$ & $\begin{array}{c}-76.76 \\
(\$)\end{array}$ & $\begin{array}{c}-76.92 \\
(\hat{v})\end{array}$ & $\begin{array}{c}-77.10 \\
(\%)\end{array}$ & \\
\hline Width/Hz & 239 & 181 & 103 & 125 & \\
\hline Temp $/{ }^{\circ} \mathrm{C}$ & \multicolumn{4}{|c|}{$\mathrm{T}_{2} \mathrm{~ms}$} & \\
\hline $\mathbf{5 0}$ & $1.79(0.06)$ & $2.33(0.05)$ & $2.15(0.07)$ & $1.67(0.05)$ & 10 \\
\hline 60 & $1.87(0.08)$ & $2.53(0.08)$ & $2.50(0.10)$ & $1.83(0.07)$ & \\
\hline 70 & $1.96(0.08)$ & $2.61(0.08)$ & $2.55(0.17)$ & $1.93(0.08)$ & \\
\hline 80 & $2.03(0.12)$ & $2.84(0.10)$ & $2.68(0.19)$ & $2.00(0.09)$ & , , , , , , , , , , \\
\hline $\mathbf{E}_{\mathrm{a}} / \mathbf{k J} \cdot \mathbf{m o l}^{-1}$ & $4.02(0.06)$ & $6.33(0.06)$ & $7.05(0.10)$ & $5.77(0.08)$ & $\begin{array}{cccc}-75 & -76 & -77 & -78\end{array}$ \\
\hline
\end{tabular}

\subsection{6 ${ }^{19} \mathrm{~F}$ spin-lattice relaxation time in the rotating frame $\left(T_{1_{\rho}}\right)$ of annealed $\mathrm{NC} 5 \%$ at an MAS rate of $10 \mathrm{kHz}$}

${ }^{19} \mathrm{~F} \mathrm{~T}_{1 \rho}$ values of each component from annealed $\mathrm{NC} 5 \%$ at the temperature range 20 $40^{\circ} \mathrm{C}$ is calculated and listed in Table 8.47 , showing that as temperature goes up, the $\mathrm{T}_{1 p}$ values increase for all four components resulting in positive activation energies. 
Table 8.47: $\quad{ }^{19} \mathrm{~F} \mathrm{~T}_{1 \rho}$ times of solid annealed NC $5 \%$ over the temperature range of 20 to $40{ }^{\circ} \mathrm{C}$ obtained with an MAS rate of $10 \mathrm{kHz}$

\begin{tabular}{|c|c|c|c|c|c|}
\hline CS/ppm & $\begin{array}{c}-76.62 \\
(n)\end{array}$ & $\begin{array}{c}-76.71 \\
(\Leftrightarrow)\end{array}$ & $\begin{array}{c}-76.95 \\
\text { (घ) }\end{array}$ & $\begin{array}{c}-77.20 \\
(\text { a) }\end{array}$ & A \\
\hline Width $/ \mathrm{Hz}$ & 1647 & 266 & 166 & 127 & l \\
\hline Temp $/{ }^{\circ} \mathrm{C}$ & \multicolumn{4}{|c|}{$\mathrm{T}_{1 p} / \mathrm{ms}$} & if \\
\hline 20 & $1.36(0.04)$ & $0.86(0.02)$ & $0.96(0.01)$ & $1.24(0.01)$ & \\
\hline 30 & $1.48(0.03)$ & $0.94(0.02)$ & $1.07(0.01)$ & $1.28(0.01)$ & \\
\hline 40 & $1.64(0.08)$ & $1.00(0.03)$ & $1.14(0.01)$ & $1.38(0.01)$ & $\cdots, \ldots$ \\
\hline $\mathrm{i} / \mathbf{k J} \cdot \mathbf{m o l}^{-1}$ & $7.07(0.17)$ & $5.70(0.09)$ & $6.49(0.04)$ & $4.04(0.02)$ & $\begin{array}{llll}74 & 76 & 78 & -80\end{array}$ \\
\hline
\end{tabular}
half height in Hz; annealed NC $5 \%=$ annealed $\mathrm{PBFP} / \mathrm{TiO}_{2}-5 \%$ at $220^{\circ} \mathrm{C}$ for 2 hours; $\mathrm{CS}=$ chemical shift in ppm; the standard deviations are listed in brackets

For the higher temperature range from $50-80^{\circ} \mathrm{C}$, the $\mathrm{T}_{t \rho}$ values are listed in Table 8.48 showing that the $T_{1 \rho}$ values of all components increases with increasing temperature; giving positive activation energies; therefore, the sidechain motion is in the fast motion regime in both temperature ranges.

Table 8.48

\section{CS/ppm}

Width/Hz Temp $/{ }^{\circ} \mathrm{C}$ $\mathbf{5 0}$

60

70

80

$\mathbf{E}_{a} / \mathbf{k J} \cdot \mathrm{mol}^{* 1}$ $5.25(0.28)$ ${ }^{19} \mathrm{~F} \mathrm{~T}_{1 \rho}$ times of solid annealed $\mathrm{NC} 5 \%$
obtained with an MAS rate of $10 \mathrm{kHz}$ $-76.42$ (4) 239

\section{$1.12(0.07)$}

$1.20(0.08)$

$1.28(0.06)$

$1.32(0.14)$
$-76.76$
(b)
181
$-76.92$
(४)
103

\begin{tabular}{ll}
\multicolumn{2}{c}{$\mathrm{T}_{1 \rho} / \mathrm{ms}$} \\
$1.41(0.02)$ & $1.24(0.02)$ \\
$1.51(0.01)$ & $1.29(0.02)$ \\
$1.51(0.01)$ & $1.32(0.02)$ \\
$1.57(0.01)$ & $1.39(0.04)$
\end{tabular}

$3,44(0.02)$

$1.39(0.04)$

-lattice relaxati

in the rotating frame; $W_{i d t h}=$ linewidth at haif height in $\mathrm{Hz}$; annealed $\mathrm{NC} 5 \%=$ annealed $\mathrm{PBFP} / \mathrm{TiO}_{2}-5 \%$ at $220^{\circ} \mathrm{C}$ for 2 hours; $\mathrm{CS}=$ chemical shift in ppm; the standard deviations are listed in brackets

In Table 8.47, the components at $\mathbf{- 7 6 . 6 2}$ and $-76.95 \mathrm{ppm}$ have larger $\mathrm{E}_{\mathrm{a}}$ values and those at -76.71 and $-77.20 \mathrm{ppm}$ have the small $\mathrm{E}_{\mathrm{a}}$ values, putting them into their own phase group,. The $\mathrm{T}_{1 \rho}$ data leads to the opposite categorization. In Table 8.48, the two components at -76.42 and $-77.10 \mathrm{ppm}$ have larger $E_{\mathrm{a}}$ values, while those at -76.92 and $-76.76 \mathrm{ppm}$ have smaller $\mathrm{E}_{\mathrm{a}}$ values and thus are grouped accordingly. 


\subsubsection{Conclusion}

To conclude, a four-component model is used to deconvolve the ${ }^{31} \mathrm{P}$ NMR spectra of annealed PBFP over $20-40^{\circ} \mathrm{C}$, and a five-component model from $50-80^{\circ} \mathrm{C}$. At low the temperature range, the $T_{1}$ data show bi-exponential behavior for all four components. The component at $-4.55 \mathrm{ppm}$ is composed of one very long contribution which is around $15 \mathrm{~s}$ and another long $T_{1}$ with value around $12 \mathrm{~s}$, the three narrow components are all contain one long $T_{1}$ contribution and one short $T_{1}$ contribution with value about $2 \mathrm{~s}$. The $T_{2}$ data indicate that the broad component at $-4.55 \mathrm{ppm}$ has a smaller $\mathrm{T}_{2}$ than the remaining three narrow components, suggesting that it is generated from a different phase. $T$ he $T_{1 p}$ values of component at -4.55 are smaller than the other three components, indicating that it arises from a different polymer domain At high temperature, the increased backbone motion above its $T(1)$ transition temperature makes the difference between each component become smaller and more difficult to distinguish.

A four-component model is used to deconvolve the ${ }^{19} \mathrm{~F}$ NMR spectra of annealed PBFP over $20-40^{\circ} \mathrm{C}$, and a four-component model from $50-80^{\circ} \mathrm{C}$. At the low temperature range, the ${ }^{19} \mathrm{~F} \mathrm{~T}_{1}$ data show mono-exponential behavior, each component has similar $\mathrm{T}_{1}$ around $0.47-0.58 \mathrm{~ms}$. The $T_{2}$ results suggest that the broad component at $-76.62 \mathrm{ppm}$ with linewidth of $1647 \mathrm{~Hz}$ has a distinct smaller $\mathrm{T}_{2}$ around $0.2 \mathrm{~ms}$ compared to other three narrow components, indicating that it arises from different domains. The $T_{1 p}$ data showed that all four components have similar $\mathrm{T}_{1 \mathrm{p}}$ values, suggesting that they should appear at the same regime with respect to the locking power. At higher temperature, the sidechain motion of all polymer domains is enhanced, the differences becomes smaller and more difficult to separate. Extra data are necessary to make a confidence assignment. 


\section{6 ${ }^{31}$ P Solid-State NMR Relaxation Times of Different Preparation Methods}

\subsection{1 ${ }^{31} \mathrm{P}$ solid-state $\mathrm{NMR} \mathrm{T}_{1}$ relaxation time of samples with different preparation methods}

The $T_{1}$ values of each component in the ${ }^{31} \mathrm{P}$ NMR spectrum for the PBPF, annealed PBFP NC 5\%, and annealed NC 5\% samples over the temperature range $20-80{ }^{\circ} \mathrm{Care}$ compared to show how the different sample preparation methods affect the polymer backbone dynamics. Figure 8.1 shows the $T_{1}$ values over the temperature range of $20-40$ ${ }^{\circ} \mathrm{C}$ at $-4.55(\circ),-8.68(0),-9.88(\odot)$ and $-8.12 \mathrm{ppm}(\%)$ with the corresponding linewidths of 834, 272, 242 and $344 \mathrm{~Hz}$, respectively. Figure 8.2 presents the $\mathrm{T}_{1}$ values of the five components over the temperature range $50-80^{\circ} \mathrm{C}$ for all four samples.

As shown in Figure 8.1, the annealed PBFP sample always has the longest $T_{1}$ values for all 4 components. Those of the annealed NC 5\% have the second longest values, followed by those of the PBFP and NC 5\% samples having the smallest values. Samples with nano- $\mathrm{TiO}_{2}$ filling always have smaller $\mathrm{T}_{1}$ values than the pure polymer counterpart and furthermore the annealed samples always have a longer $T_{1}$ values than the corresponding non-annealed samples. This is particularly obvious for the component at $4.55 \mathrm{ppm}(\Theta)$, which in annealed PBFP has a bigger $\mathrm{T}_{1}$ values than in PBFP.

In Figure 8.1, the $\mathrm{T}_{1}$ values for the component at $-4.55 \mathrm{ppm}(e)$ are distinguishable between samples as indicated in Figure 8.1, while for component at $-8.68(0)$ ppm, the $T_{1}$ value are so close to each other that it is difficult to distinguish between them. While for the component at $-9.88 \mathrm{ppm}(\bullet)$, the $\mathrm{T}_{1}$ values of annealed PBFP are different from that of annealed $\mathrm{NC} 5 \%$, however, for PBFP and $\mathrm{NC} 5 \%$, the $\mathrm{T}_{1}$ values are very similar. For the component at $-8.12 \mathrm{ppm}(\bullet)$, there is a difference between the annealed PBFP and annealed NC $5 \%$ samples, which is not as pronounced for the component at $-9.88 \mathrm{ppm}$, yet still 
distinct from each other; however, for PBFP and NC 5\% their $\mathrm{T}_{1}$ values are very close to each other.
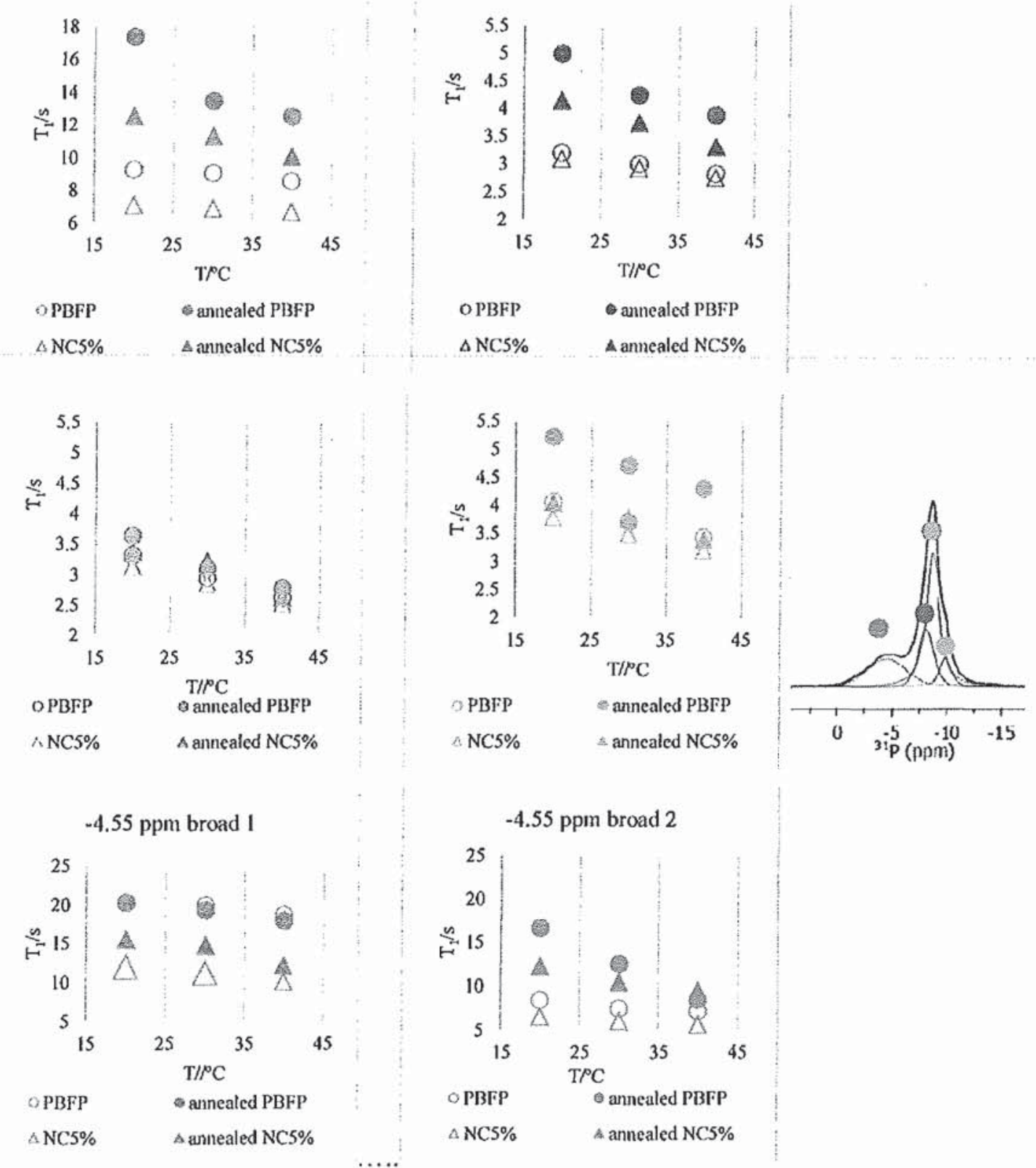

Figure 8.1: $\quad{ }^{31} \mathrm{P}$ spin-lattice relaxation time $\mathrm{T}_{1}$ for the four components obtained by deconvolution of the ${ }^{31} \mathrm{P}$ solid-state NMR spectra of PBFP, annealed PBFP, PBFP with $5 \%$ of nano- $\mathrm{TiO}_{2}$, and annealed sample of PBFP with $5 \%$ of nano- $\mathrm{TiO}_{2}$ over the temperature range of 20 to $40^{\circ} \mathrm{C}$; the biexponential behavior of the two contributions at $-4.55 \mathrm{ppm}$ (bottom) 
For the broad contribution at $-4.55 \mathrm{ppm}$, the $\mathrm{T}_{1}$ values for non-filled samples are larger than those of nano-filled samples, and the $T_{1}$ values change very subtlety, implying that the non-filled samples are at certain size and that the increasing temperature has little effect on its $T_{1}$ motion. The nano-filled samples are smaller and are more sensitive to temperature changes. For the second broad contribution at $-4.55 \mathrm{ppm}$, the $T_{1}$ values for the annealed ones are larger than those of the corresponding non annealed samples and are sensitive to temperature changes, indicating that their size are still relative smaller. Those two phenomena imply that the two broad components at $-4.55 \mathrm{ppm}$ have different contributions and thus different morphology.

In Figure 8.2, at the high temperature range from $50-80^{\circ} \mathrm{C}$, the $\mathrm{T}_{1}$ values in all four samples are very small, however, the behavior observed in the low temperature range (Figure 8.1) still continues: where the annealed PBFP sample always has the longest $\mathrm{T}_{1}$ values for all 4 components. Those of the annealed NC $5 \%$ have the second longest values, followed by those of the PBFP and NC 5\% samples having the smallest values. Samples with nano- $\mathrm{TiO}_{2}$ filling always have a smaller $\mathrm{T}_{1}$ value than their pure polymer counterparts, and the annealed samples always have a longer $T_{1}$ value than the corresponding nonannealed samples.

In the low temperature range, the broad component at -4.55 ppm actually consists of two contributions that are similar in proportion and mobility. The three narrow components overlap at least one broad component not previously observed gives rise to the small contribution from a long $\mathrm{T}_{1}$. 

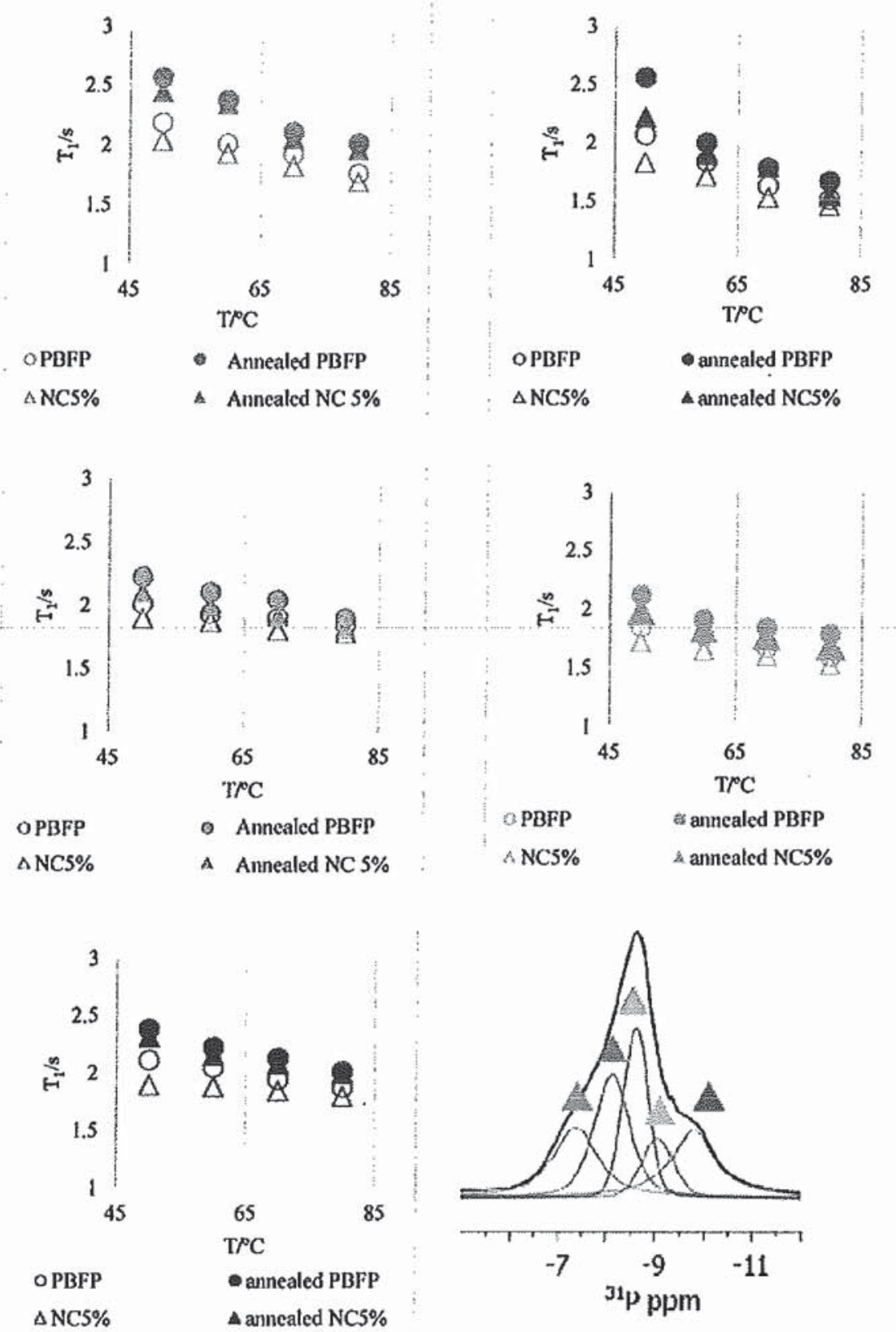

Figure 8.2: ${ }^{31} \mathrm{P}$ spin-lattice relaxation time $\mathrm{T}_{1}$ for the five components obtained by deconvolution of the ${ }^{31} \mathrm{P}$ solid-state NMR spectra of PBFP, annealed PBFP, PBFP with $5 \%$ of nano- $\mathrm{TiO}_{2}$, and annealed sample of PBFP with $5 \%$ of nano- $\mathrm{TiO}_{2}$ over the temperature range of 50 to $80^{\circ} \mathrm{C}$

In both temperature ranges, the $T_{1}$ values decrease with increasing temperature for all components, broad and narrow, which implies that the polymer backbone undergoes a slow motion on a time scale larger than $5 \mathrm{~ns}$. As the broad signals have much larger $\mathrm{T}_{1}$ 
values than the narrow signals, its motion must be significantly slower. This is also seen in the activation energies which tend to be significantly larger for the broad components. The ${ }^{31} \mathrm{P} \mathrm{T}_{1}$ 's over from $20-40^{\circ} \mathrm{C}$ for the two broad signals around $-4.55 \mathrm{ppm}$ increase after annealing for both PBFP and NC 5\%, which implies that the mobility decreases upon annealing. Furthermore, after nano-filling the $T_{1}$ values increase suggesting that the presence of nano- $\mathrm{TiO}_{2}$ increases the backbone mobility to some extent

At higher temperature, from $50-80^{\circ} \mathrm{C}$, upon annealing and nano-filling, the $\mathrm{T}_{1}$ values see the most significant change in the component at -7.37 and $-8.13 \mathrm{ppm}$ indicating that they likely originate from the two broad components at $-4.55 \mathrm{ppm}$, which is further supported by their significantly larger activation energies. The component at $-9.79 \mathrm{ppm}$ is proposed to originate from the broad component that sits underneath of the three narrow components, which was not previously observed, at lower temperature, as it is the only component not decreasing significantly in linewidth with temperature. This is further supported by the $T_{1}$ values themselves, as the contributions proposed to arise from the narrow lines at low temperature consistently have smaller values at high temperature than those proposed to arise from the broad contributions at low temperature.

To conclude, at low temperature there are three broad and three narrow components. Two of the broad component appear around $-4.55 \mathrm{ppm}$, the third broad component appears somewhere near $-9.00 \mathrm{ppm}$. At higher temperature, the two broad components at -4.55 are shifted to -7.37 and $-8.13 \mathrm{ppm}$ and become narrower due to increased backbone motion. The remaining broad signal that overlaps with the narrow signals is shifted to $-9.79 \mathrm{ppm}$ and also becomes narrower. All three broad components remain broader at higher temperature relative to the narrow components. However, the linewidths of all five 
components are close enough to suggest that their backbone dynamics are approaching the same time scale. It is likely that the three narrow components at low temperature coalesce into two narrow ones, hence only a total of five components are observed.

\subsection{2 ${ }^{31} \mathrm{P}$ solid-state $\mathrm{NMR} \mathrm{T}_{2}$ relaxation times of samples with different preparation methods}

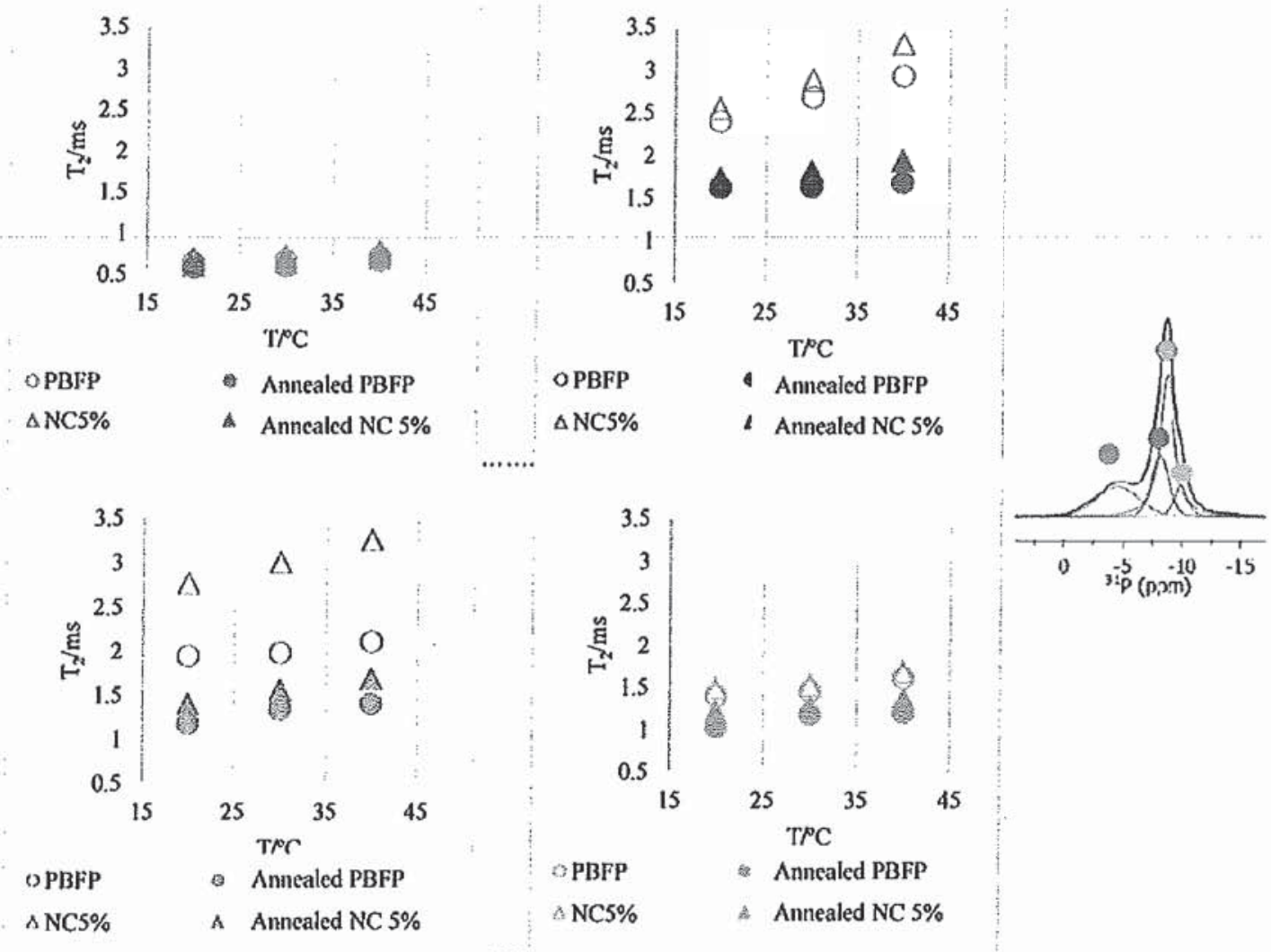

Figure 8.3: $\quad{ }^{31} \mathrm{P}$ transverse relaxation time $\mathrm{T}_{2}$ for the four components obtained by deconvolution of the ${ }^{31} \mathrm{P}$ solid-state NMR spectra of PBFP, annealed PBFP, PBFP with $5 \%$ of nano$\mathrm{TiO}_{2}$, and annealed sample of $\mathrm{PBFP}$ with $5 \%$ of nano- $\mathrm{TiO}_{2}$ over the temperature range of 20 to $40^{\circ} \mathrm{C}$

The ${ }^{31} \mathrm{P} \mathrm{T}_{2}$ values of each component in the ${ }^{31} \mathrm{P}$ NMR spectrum for PBFP, annealed PBFP, NC 5\%, and annealed NC 5\% over temperature range $20-80^{\circ}$ Care plotted to show how the different sample preparation methods affect the polymer backbone dynamics. Figure 8.3 shows the ${ }^{31} \mathrm{P}_{2}$ values for all four samples from $20-40^{\circ} \mathrm{C}$ at $-4.55(\bullet),-8.68$ 
$(\odot),-9.88(\odot)$ and $-8.12 \mathrm{ppm}(\odot)$ with the corresponding linewidths of $834,272,242$ and $344 \mathrm{~Hz}$, respectively. Figure 8.4 presents the $\mathrm{T}_{2}$ values of the five components at -7.37 (太), $-8.13(\Delta),-8.62(\mathrm{~A}),-9.06(\mathrm{~s})$ and -9.79 (A) ppm, individually, over $50-80^{\circ} \mathrm{C}$ for all four samples.

In Figure 8.3, samples with nano- $\mathrm{TiO}_{2}$ filling always have a longer $\mathrm{T}_{2}$ value than their pure polymer counterparts and the annealed samples have shorter $T_{2}$ compared with the corresponding non-annealed samples. For example, the NC $5 \%$ has longer $\mathrm{T}_{2}$ than PBFP and annealed PBFP have shorter $\mathrm{T}_{2}$ than PBFP. There is an obvious $\mathrm{T}_{2}$ difference between NC 5\% and PBFP for the component at $-8.68 \mathrm{ppm} \mathrm{(0),} \mathrm{and} \mathrm{so} \mathrm{is} \mathrm{also} \mathrm{true} \mathrm{for} \mathrm{the}$ components at $-4.55 \mathrm{ppm}(*)$ and $-9.88 \mathrm{ppm}(\odot)$, despite the differences being much smaller.

In Figure 8.3 , the component at $-4.55 \mathrm{ppm}$ has similar behavior as the component at $-9.88 \mathrm{ppm}$ where the four samples have similar small changes upon annealing and nanofilling and the sensitivity to $T_{2}$ is weak. This implies that the component at $-4.55 \mathrm{ppm}$ is rigid, and the component at $-9.88 \mathrm{ppm}$ is more dominated by underlying broad contribution. This is further supported by their smaller $T_{2}$ values compared to the two narrow components. Similarly, one can appreciate that the narrow components at -8.12 and -8.68 ppm are also superimposed on the same broad contribution, which is evidenced upon annealing where their $T_{2}$ values become insensitive to temperature. 

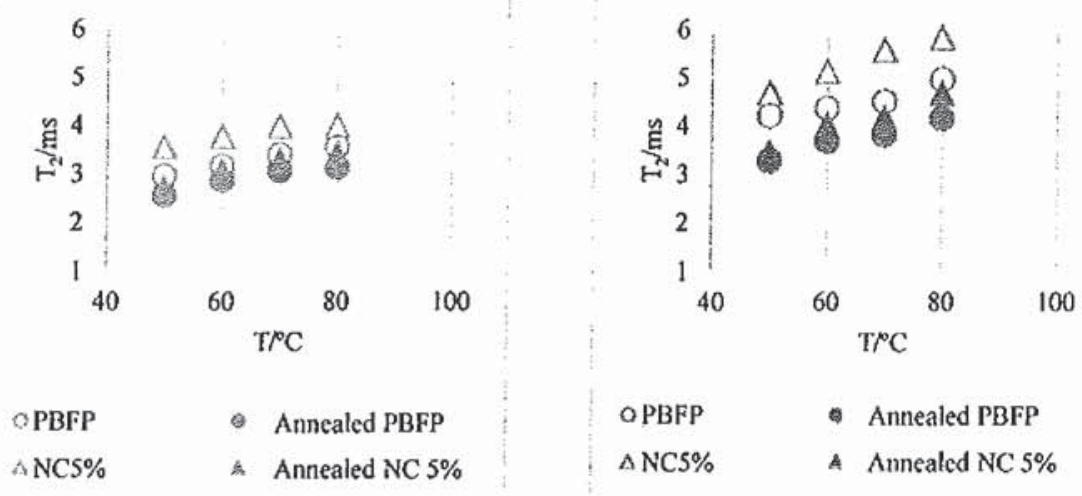

$$
\begin{array}{ll}
\text { OPBFP } & \text { Annealed PBFP } \\
\triangle \mathrm{NC} 5 \% & \text { A Annealed NC 5\% }
\end{array}
$$
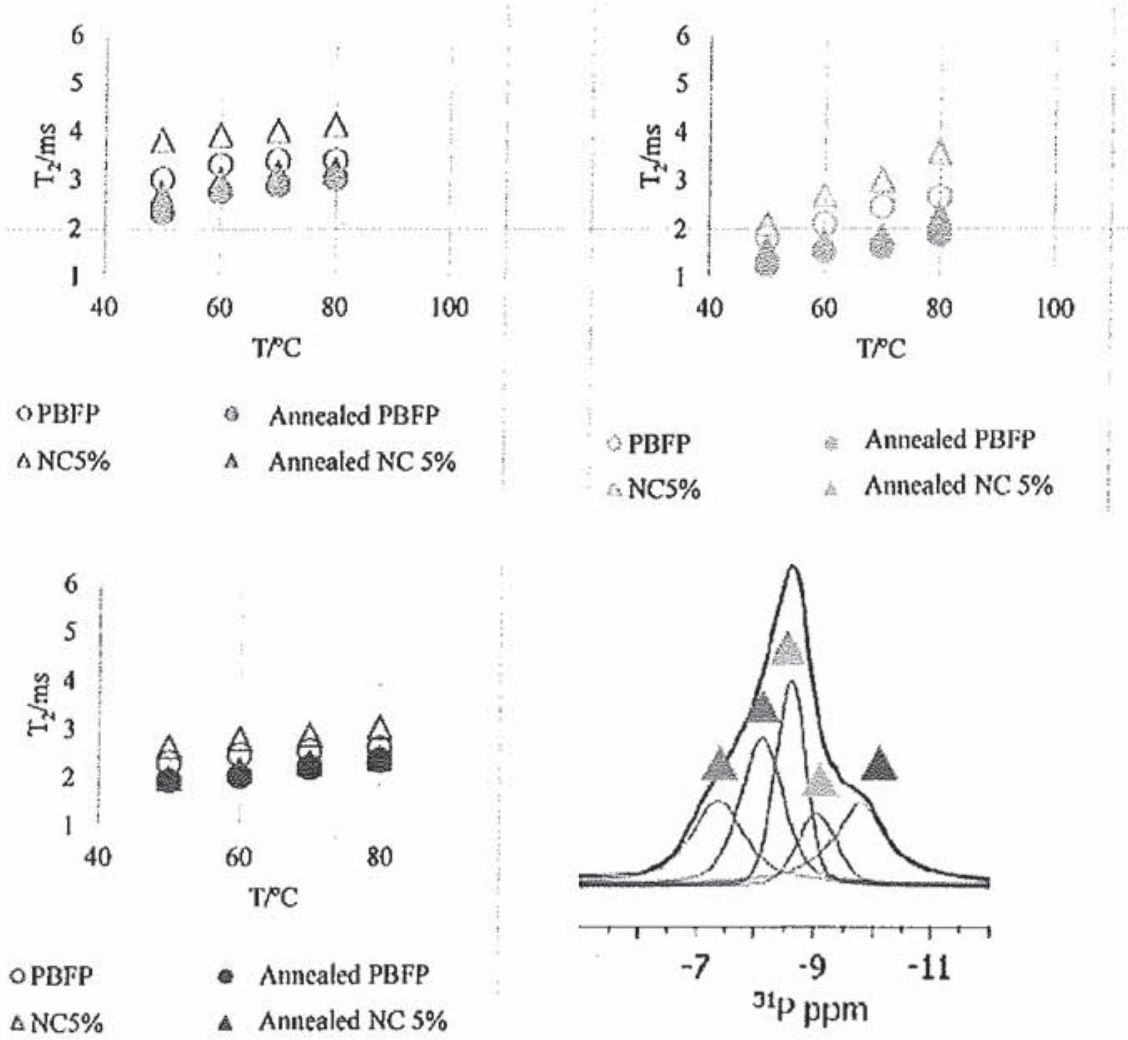

Figure 8.4: $\quad{ }^{31} \mathrm{P}$ transverse relaxation time $\mathrm{T}_{2}$ for the four components obtained by deconvolution of the ${ }^{31} \mathrm{P}$ solid-state NMR spectra of PBFP, annealed PBFP, PBFP with $5 \%$ of nano$\mathrm{TiO}_{2}$, and annealed sample of PBFP with $5 \%$ of nano- $\mathrm{TiO}_{2}$ over the temperature range of 20 to $40^{\circ} \mathrm{C}$

Each component of ${ }^{31} \mathrm{PT}_{2}$ for four samples over the temperature range $50-80^{\circ} \mathrm{C}$ are presented in Figure 8.4. Samples with nano- $\mathrm{TiO}_{2}$ filling always have a longer $\mathrm{T}_{2}$ than their pure polymer counterparts. The annealed samples would have a smaller $\mathrm{T}_{2}$ value compared with the corresponding non-annealed samples as concluded from the low temperature 
range. Furthermore, there is a distinctive difference in $\mathrm{T}_{2}$ between $\mathrm{NC} 5 \%$ and PBFP for the three components at $-8.62(\mathrm{~A}),-8.13(\mathrm{~A})$ and $-9.06 \mathrm{ppm}(\mathrm{A})$, while the $\mathrm{T}_{2}$ values for annealed PBFP and annealed NC 5\% are small. For the two remaining components at-7.37 (A) and $-9.79 \mathrm{ppm}(\mathbf{A})$, the $T_{2}$ are so similar that it is difficult to distinguish between samples.

At higher temperature, from $50-80^{\circ} \mathrm{C}$, the broad component at $-4.55 \mathrm{ppm}$ disappears and additional narrow components are observed. The component at $-7.37 \mathrm{ppm}$ and $\mathbf{- 8 . 1 3}$ ppm have similar $\mathrm{E}_{\mathrm{a}}$ values at 5.92 and $5.24 \mathrm{~kJ} \cdot \mathrm{mol}^{-1}$, are assigned to the same phase category: the rigid motion environment. This is further supported by their broad line-widths of 220 and $176 \mathrm{~Hz}$, respectively, which are proposed to originate from the broad component at $-4.55 \mathrm{ppm}$ at lower temperature. The component at $-9.79 \mathrm{ppm}$ is postulated to arise from the broad component underneath the narrow components at $20-40^{\circ} \mathrm{C}$, which is further supported by its $\mathrm{E}_{\mathrm{a}}$ value being similar to the two broad components at -7.37 and $-8.13 \mathrm{ppm}$.

In Figure 8.4, at higher temperature the differences in $T_{2}$ between samples are not as clearly indicated as at low temperature; therefore, the $T_{1} / T_{2}$ ratio is employed later to discuss structural changes with sample treatment in more detail. The ${ }^{31} \mathrm{P} \mathrm{T}_{2}$ values over from $20-80^{\circ} \mathrm{C}$ for all components decrease after annealing for both PBFP and NC $5 \%$, which implies that the mobility decreases upon annealing. Furthermore, after nano-filling the $\mathrm{T}_{2}$ values increase suggesting that the presence of nano- $\mathrm{TiO}_{2}$ increases the backbone mobility to some extent.

In addition to the analysis of $T_{1}, T_{2}$ data, the $T_{1} / T_{2}$ ratio study is used to estimate the correlation time of motion in the local environment represented by the component of interest. $1000 / \mathrm{T}$ is used instead of 'T, as it is directly proportional to the correlation time to 
first order, hence one can demonstrate a simple, essentially quadratic, relationship between $\tau_{\mathrm{c}}$ and $\mathrm{T}_{1} / \mathrm{T}_{2}$. The correlation time of ${ }^{31} \mathrm{P}$ is a direct indication of the mobility of the backbone in this environment, which is strongly determined by the local phase structure. Large $T_{1} / T_{2}$ values indicate rigidity, whereas small values imply mobility. Increases in correlation time indicate growth of crystallite size; while decreasing correlation time indicates melting of crystallites to smaller sizes.

The $T_{1} / T_{2}$ values and correlation times for all components with respect to temperature are presented in Table 8.49 and Table 8.50 to gain insight into the changes in polymer morphology with the variation in sample preparation and heat treatment. At low temperature annealed PBFP always has the largest $T_{1} / T_{2}$ values and the longest correlation times, followed by annealed NC 5\%, which in turn is followed by PBFP and NC 5\% with the smallest values for all components - both broad and narrow. Similarly, at higher temperature $50-80^{\circ} \mathrm{C}$, the same trends persist; where PBFP always has the largest value; the annealed NC $5 \%$ had the next largest values, followed by PBFP and NC $5 \%$ with the smallest values. 
Table 8.49: $\quad{ }^{31} \mathrm{P} \mathrm{T}_{1} / \mathrm{T}_{2}$ ratios of four samples with the change of temperature for samples over 20$40^{\circ} \mathrm{C}$ obtained with an MAS rate of $10 \mathrm{kHz}$

\begin{tabular}{|c|c|c|c|c|c|c|c|c|c|}
\hline \multirow{2}{*}{$\begin{array}{c}\text { CS/ppm } \\
\text { Width/Hz }\end{array}$} & \multirow[t]{2}{*}{$\underset{\Gamma e m p}{{ }^{\circ} \mathrm{C}}$} & \multirow{2}{*}{\multicolumn{2}{|c|}{$\begin{array}{c}-4.55 \\
(\theta) \\
834\end{array}$}} & \multirow{2}{*}{\multicolumn{2}{|c|}{$\begin{array}{l}-8.12 \\
(0) \\
344\end{array}$}} & \multirow{2}{*}{\multicolumn{2}{|c|}{$\begin{array}{c}-8.68 \\
(0) \\
272\end{array}$}} & \multicolumn{2}{|c|}{$\begin{array}{l}-9.88 \\
(e)\end{array}$} \\
\hline & & & & & & & & 2 & \\
\hline \multirow{3}{*}{ PBFP } & 20 & $31625(1015)$ & 13234 & 9320 & 950 & 4902 & $1345(210)$ & 10507 & 1629 \\
\hline & 30 & $20235(976)$ & 11000 & 5902 & 824 & 4411 & $1208(200)$ & 9089 & 1517 \\
\hline & 40 & $25861(918)$ & 9972 & 4781 & 774 & 4095 & $1000(190)$ & 5720 & 1286 \\
\hline \multirow{4}{*}{$\begin{array}{l}\text { Annealed } \\
\text { PBFP }\end{array}$} & 20 & $33667(1047)$ & 27783 & 11258 & 1337 & 10410 & $2254(270)$ & 14390 & 2686 \\
\hline & 30 & $32117(1022)$ & 20800 & 7914 & 1380 & 8246 & $1784(240)$ & 14127 & 2108 \\
\hline & 40 & $26701(933)$ & 12642 & 7643 & 1345 & 6936 & {$[529(220)$} & 11433 & 1775 \\
\hline & 20 & $17391(753)$ & 9492 & 4835 & 882 & 4033 & $866(170)$ & 10067 & 1770 \\
\hline \multirow[t]{2}{*}{ NC $5 \%$} & 30 & $15315(706)$ & 8260 & 3369 & 732 & 2257 & $733(150)$ & 7026 & 1586 \\
\hline & 40 & $12897(648)$ & 7166 & 2687 & 612 & 1646 & $6.52(150)$ & 5417 & 1306 \\
\hline \multirow{3}{*}{$\begin{array}{c}\text { Annealed } \\
\text { NC 5\% }\end{array}$} & 20 & $25766(916)$ & 20416 & 12843 & 1202 & 12157 & $1721(240)$ & 10686 & 2041 \\
\hline & 30 & $22553(857)$ & 16030 & 10541 & 1160 & 1035 & $1522(220)$ & 8496 & 1811 \\
\hline & 40 & $17357(752)$ & 13500 & 8355 & 1057 & 9935 & $1422(220)$ & 6492 & 1672 \\
\hline
\end{tabular}

Note: $T_{1}=$ spin-lattice relaxation time; $T_{2}=$ spin-spin relaxation time; four sample $=$ solvent cast PBFP, annealed solvent cast PBFP, $\mathrm{PBFP} / \mathrm{TiO}_{2}-5 \%$ and annealed $\mathrm{PBFP} / \mathrm{TiO}_{2}-5 \% ; \mathrm{CS}=$ chemical shift in ppm; Width $=$ linewidth at half height in $\mathrm{Hz}$; correlation times in nanosecond are shown in the brackets for the broad contribution at $-4.55 \mathrm{ppm}$

Table 8.50: $\quad{ }^{31} \mathrm{P} \mathrm{T}_{1} / \mathrm{T}_{2}$ ratios of four samples with the change of temperature for samples over

\begin{tabular}{|c|c|c|c|c|c|c|}
\hline & & $30^{\circ} \mathrm{C}$ obtaine & an MA & of $10 \mathrm{k}$ & & \\
\hline CS/ppm & Temp $/{ }^{\circ} \mathrm{C}$ & $\begin{array}{c}-7.37 \\
(\mathbb{A})\end{array}$ & $\begin{array}{l}-8.13 \\
(\Delta)\end{array}$ & $\begin{array}{l}-8.62 \\
(A)\end{array}$ & $\begin{array}{l}-9.106 \\
(\Delta)\end{array}$ & $\begin{array}{c}-9.79 \\
\text { (A) }\end{array}$ \\
\hline Widtlı/Hz & & 220 & 176 & 124 & 151 & 205 \\
\hline & 50 & $742(155)$ & 487 & 670 & $1005(181)$ & 917 \\
\hline & 60 & $634(144)$ & 420 & 583 & $843(166)$ & 826 \\
\hline PBसP & 70 & $568(136)$ & 362 & 562 & $679(149)$ & 761 \\
\hline & 80 & $493(127)$ & 304 & 543 & $596(1,39)$ & 716 \\
\hline & 50 & $1004(181)$ & 783 & 957 & $1683(234)$ & 1227 \\
\hline Annealed & 60 & $832(165)$ & 543 & 764 & $1240(201)$ & 1094 \\
\hline PBFP & 70 & $686(1.99)$ & 469 & 706 & $1129(192)$ & 964 \\
\hline & 80 & $644(145)$ & 401 & 626 & $952(176)$ & 855 \\
\hline & 50 & $572(136)$ & 391 & 495 & $819(163)$ & 716 \\
\hline & 60 & $511(129)$ & 336 & 476 & $61 !(14 !)$ & 668 \\
\hline NC $5 \%$ & 70 & $456(122)$ & 275 & 450 & $532(132)$ & 639 \\
\hline & 80 & $421(117)$ & 250 & 430 & $423(117)$ & 591 \\
\hline & 50 & $910(172)$ & 639 & 775 & $1245(201)$ & 1167 \\
\hline Annealed & 60 & $770)(158)$ & 473 & 666 & $1670(187)$ & 1005 \\
\hline NC $5 \%$ & 70 & $631(143)$ & 433 & 607 & $957(176)$ & 908 \\
\hline & 80 & $577(137)$ & 329 & 572 & $740(155)$ & 833 \\
\hline
\end{tabular}

Note: $T_{1}=$ spin-lattice relaxation time; $T_{2}=$ spin-spin relaxation time; four sample $=$ solvent cast PBFP, annealed solvent cast PBFP, PBFP/TiO $2-5 \%$ and annealed $\mathrm{PBFP}_{2} \mathrm{TiO}_{2}-5 \%$; $\mathrm{CS}=$ chemical shift in ppm; Width = linewidth at half height in $\mathrm{Hz}$; correlation times in nanosecond are shown in the brackets for the contributions at -7.37 and $-9.06 \mathrm{ppm}$ 
As an example, a broad $(4.55 \mathrm{ppm})$ and a narrow contribution $(8.68 \mathrm{ppm})$ are illustrated in detail, which are proposed to be the same contribution in the low and high temperature ranges respectively. In Figure 8.5 the $T_{1} / T_{2}$ for all four samples were plotted with $1000 /$ Temperature for the low temperature range; the corresponding values are highlighted in Table 8.49. The trends in the corresponding contributions in the high temperature range are shown for all four samples in Figure 8.6, and are also highlighted in Table 8.50 .
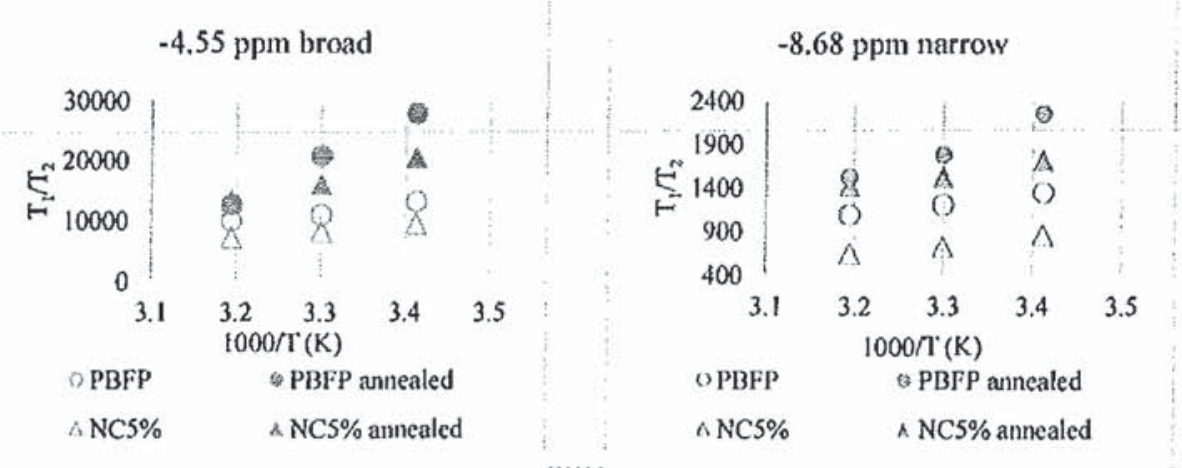

Figure 8.5: Four examples of ${ }^{31} \mathrm{P} \mathrm{T}_{1} / \mathrm{T}_{2}$ ratio with respect to the changes of temperature $(1000 / \mathrm{T})$ for one broad contribution (Left) and one narrow contribution (Right) obtained by deconvolution of the ${ }^{31} \mathrm{P}$ solid-state NMR spectra of PBFP, annealed PBFP, PBFP with $5 \%$ of nano- $\mathrm{TiO}_{2}$, and annealed sample of PBFP with $5 \%$ of nano- $\mathrm{TiO}_{2}$ over the temperature range of 20 to $40^{\circ} \mathrm{C}$
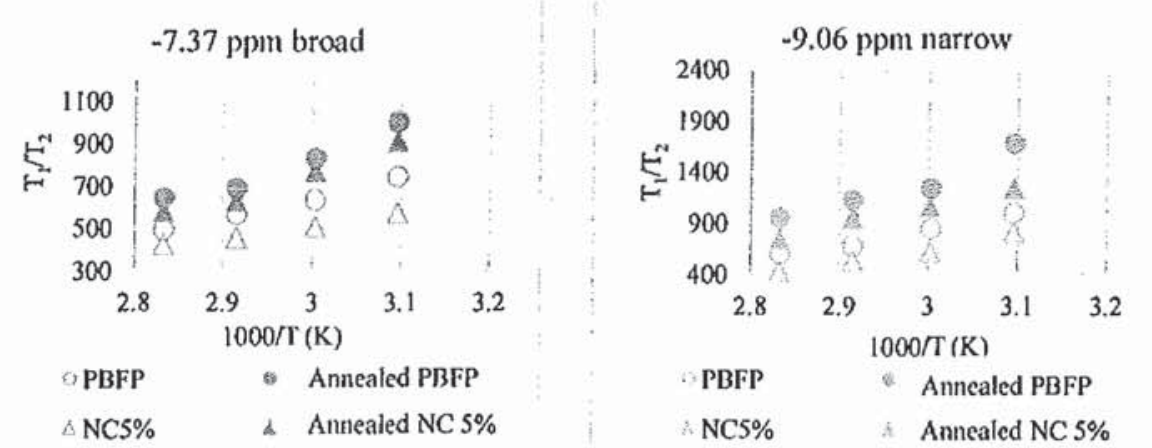

Figure 8.6: $\quad$ Four examples of ${ }^{31} \mathrm{P} \mathrm{T}_{1} / \mathrm{T}_{2}$ ratio with respect to the changes of temperature (1000/T) for one broad contribution (Left) and one narrow contribution (Right) obtained by deconvolution of the ${ }^{31} \mathrm{P}$ solid-state NMR spectra of PBFP, annealed PBFP, PBFP with $5 \%$ of nano- $\mathrm{TiO}_{2}$, and annealed sample of PBFP with $5 \%$ of nano- $\mathrm{TiO}_{2}$ over the temperature range of 50 to $80^{\circ} \mathrm{C}$ 
The correlation time values derived from the $T_{1} / T_{2}$ results start at their largest value and decrease with increasing temperature. The same behavior is observed in all four samples, indicating that the rigidity in this phase, which likely corresponds to crystallite size, decreases with temperature. The rate of decrease is strongly correlated to the size of the initial value, however, they all seem to converge to the same value at high temperature. For example, the $T_{1} / T_{2}$ value of the broad contribution at $-4.55 \mathrm{ppm}$ in annealed PBFP is initially extremely large, with a correlation time of $181 \mathrm{~ns}$, which decreases the most rapidly with temperature, whereas for annealed NC $5 \%$, the $T_{1} / T_{2}$ starts next largest, with $\tau_{\mathrm{c}}$ at $171 \mathrm{~ns}$, which deceases at a slightly slower rate than annealed PBFP. PBFP and NC $5 \%$ have initially the smallest correlation times and $\mathrm{T}_{1} / \mathrm{T}_{2}$ values and decrease the slowest with temperature. This behavior is consistent with annealing increasing the crystallite sizes where upon increasing temperature the crystallite size decreases at a rate proportional to the initial crystallite size, hence more dramatic changes are expected for larger crystallite sizes than those environments with smaller crystallite sizes making them more sensitive to temperature. As the initial correlation values are smaller in the nano-filled samples and as these values in the annealed NC 5\% change to lesser degree with temperature, one can propose that the nano-filler suppresses crystallite growth.

Note that the $T_{1} / T_{2}$ ratio for the broad contribution at $-4.55 \mathrm{ppm}$ in Figure 8.5 decreases to around $1200-2700$ at $40^{\circ} \mathrm{C}$ for all four samples, while the corresponding high temperature contribution at $-7.37 \mathrm{ppm}$, Figure 8.6 , starts around $500-1200$ at $50^{\circ} \mathrm{C}$ and continues to decrease with temperature. This discontinuity in $T_{1} / T_{2}$ with temperature suggests a phase change takes place around $50^{\circ} \mathrm{C}$. This discontinuity is also observed in the change of correlation time with temperature. For example for sample PBFP, the 
correlation time at $40^{\circ} \mathrm{C}$ is $918 \mathrm{~ns}$, while at $50^{\circ} \mathrm{C}$ its correlation time starts at $155 \mathrm{~ns}$. In contrast, for the narrow component at $-8.68 \mathrm{ppm}$, the $\mathrm{T}_{1} / \mathrm{T}_{2}$ ratios decrease to around 500 1500 , together with correlation time around 150-200 ns; the corresponding high temperature values continues from the same place, hence there is no phase change occurring for this contribution but only consistently crystallite size decreasing with temperature. Those two phenomena further prove that the components at -7.37 and -9.06 $\mathrm{ppm}$ at high temperature originate from the broad contribution at $-4.55 \mathrm{ppm}$ and the narrow component at $-8.68 \mathrm{ppm}$ at low temperature, respectively.

The component at $-4.55 \mathrm{ppm}$ has two large $T_{1} / T_{2}$ ratios on the order of $10^{4}$ and two different long correlation times, while the remaining three components have one large $\mathrm{T}_{1} / \mathrm{T}_{2}$ ratio on the order of $5 * 10^{3}-10^{4}$, and one small $\mathrm{T}_{1} / \mathrm{T}_{2}$ ratio on the order of $1 * 10^{3}$. This implies that the broad component at $-4.55 \mathrm{ppm}$ is composed of two broad contributions. The three narrow components are likely overlapped with at least one broad contribution. At low temperature, the three broad components have different $T_{1} / T_{2}$ values, amongst which, the component around $-4.55 \mathrm{ppm}$ with the largest $\mathrm{T}_{1} / \mathrm{T}_{2}$ ratio and longest correlation time is supposed to have the highest rigidity, thus the most slow motion. Another contribution at -4.55 ppm has similar $T_{1} / T_{2}$ values as the broad contribution at $-8.12,-8.68$ and $-9.88 \mathrm{ppm}$, thus are assumed to have a similar rigidity.

To conclude, the ${ }^{31} \mathrm{P} \mathrm{T}_{1} / \mathrm{T}_{2}$ data imply that the phosphorus spectrum is composed of three broad components and three narrow components at low temperature. Two of the broad components appear around $-4.55 \mathrm{ppm}$, the third broad component appears overlapped with the three narrow components. At higher temperature, the two broad components at $-4.55 \mathrm{ppm}$ are shifted to -7.37 and $-8.13 \mathrm{ppm}$, and become narrower due to 
increased backbone motion and phase changes. The remaining broad that is overlapped with the narrow signals is shifted to $-9.79 \mathrm{ppm}$ and also become narrower. All three broad components remain broader than the narrow components at high temperature where their backbone dynamics approach the same time scale. This assignment is in consistent with the conclusion drawn from the $\mathrm{T}_{1}$ and $\mathrm{T}_{2}$ data.

\subsection{3 ${ }^{31} \mathrm{P}$ solid-state $\mathrm{NMR} \mathrm{T}_{\mathbf{}_{\mathfrak{\rho}}}$ relaxation time of samples with different preparation methods}
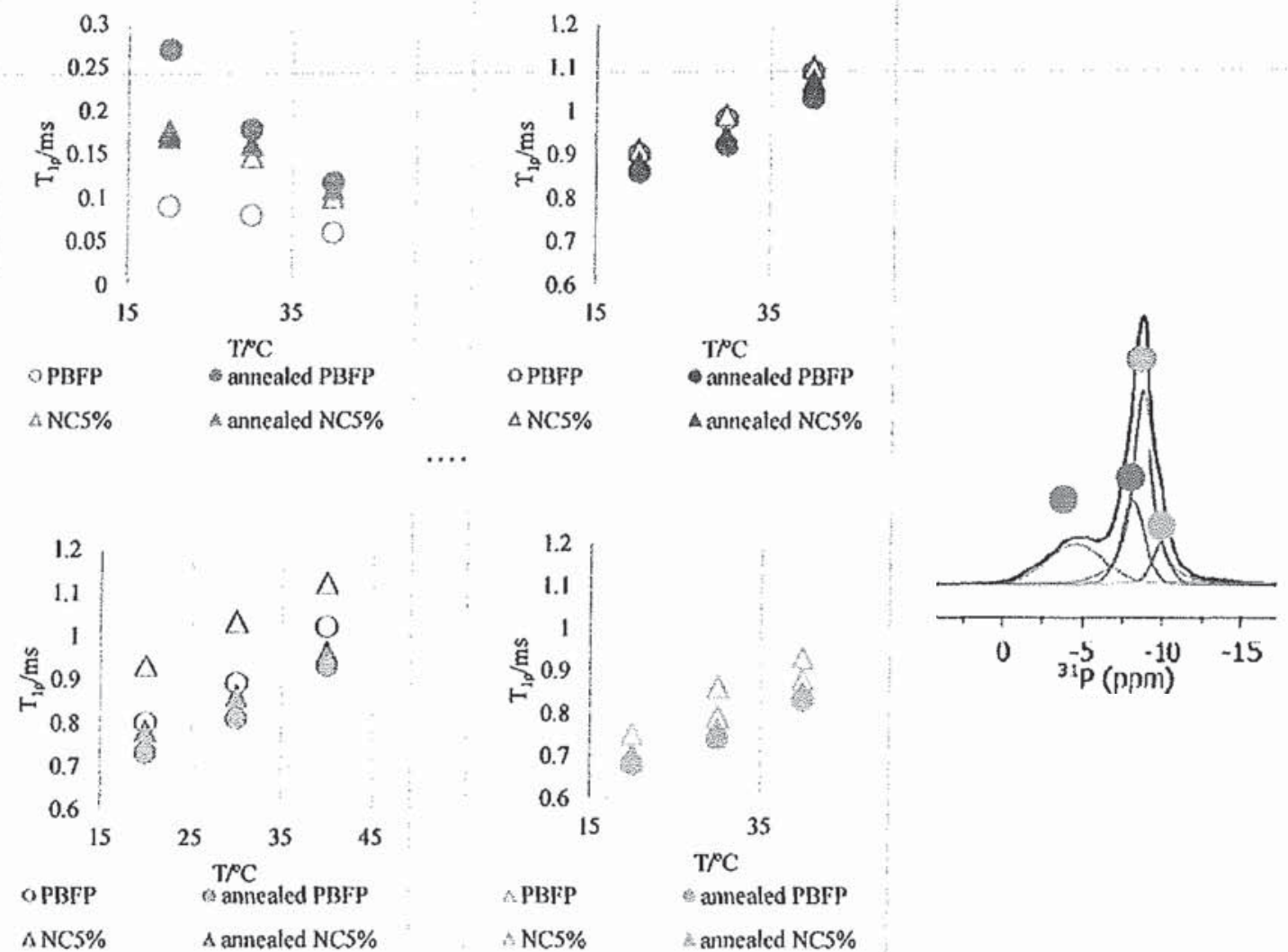

Figure 8.7: $\quad{ }^{31} \mathrm{P}$ spin-lattice relaxation time in the rotating frame for the four components obtained by deconvolution of the ${ }^{31} \mathrm{P}$ solid-state NMR spectra of PBFP, annealed PBFP, PBFP with $5 \%$ of nano- $\mathrm{TiO}_{2}$, and annealed sample of PBFP with $5 \%$ of nano$\mathrm{TiO}_{2}$ over the temperature range of 20 to $40^{\circ} \mathrm{C}$ 
The ${ }^{31} \mathrm{P} \mathrm{T}_{1 \mathrm{p}}$ results are compared between the four samples in Figures 8.7 for 20 $40^{\circ} \mathrm{C}$ and in Figure 8.8 for $50-80^{\circ} \mathrm{C}$. The $\mathrm{T}_{1 p}$ values for the four components at $-4.55\left({ }^{\circ}\right)$, $8.12(\bullet),-8.68(0)$ and $-9.88 \mathrm{ppm}(\odot)$ are compared in Figure 8.7. In general, the samples with nano- $\mathrm{TiO}_{2}$ always have a longer $\mathrm{T}_{1 \rho}$ than their neat polymer counterparts, and the annealed samples always have a smaller $\mathrm{T}_{t_{\rho}}$ than the corresponding non-annealed samples. The component at chemical shift $-4.55 \mathrm{ppm}$ is the exception to this trend, which has a distinct small $T_{1 \rho}$, which decreases $T_{1 \rho}$ with increasing temperature in all four samples. Furthermore, the annealed PBFP sample has the largest $T_{1 p}$ and the virgin PBFP has the smallest $T_{1 p}$ value. Also, for all four components, the differences in $T_{1 p}$ values between the annealed samples are very small, except at $20^{\circ} \mathrm{C}$ for the component at $-4.55 \mathrm{ppm}$. In fact, the difference in $T_{1 p}$ values between the annealed and non-annealed samples are relatively small for the components at -8.12 and $-9.88 \mathrm{ppm}$, suggesting that there are consistently small increases in backbone motion in these environments.

At $-8.68 \mathrm{ppm}$ the change in $T_{1 \rho}$ values are very pronounced between the annealed and non-annealed nano-filled samples implying that the presence of the filler strongly influences the dynamics of the backbone in this phase. In contrast the neat polymer samples exhibit the largest differences in $T_{1 \rho}$ values at $-4.55 \mathrm{ppm}$, and the nano-filled values lie in between. First, note that this phase has the lowest mobility of all the components, indicated by its decreasing trend with temperature. Lower values indicate relatively accelerated motion in the slow motion regime, thus the neat polymer is most mobile, becoming the most rigid upon annealing. The nano-filled materials have more restricted motion to begin with, which changes little upon annealing. 

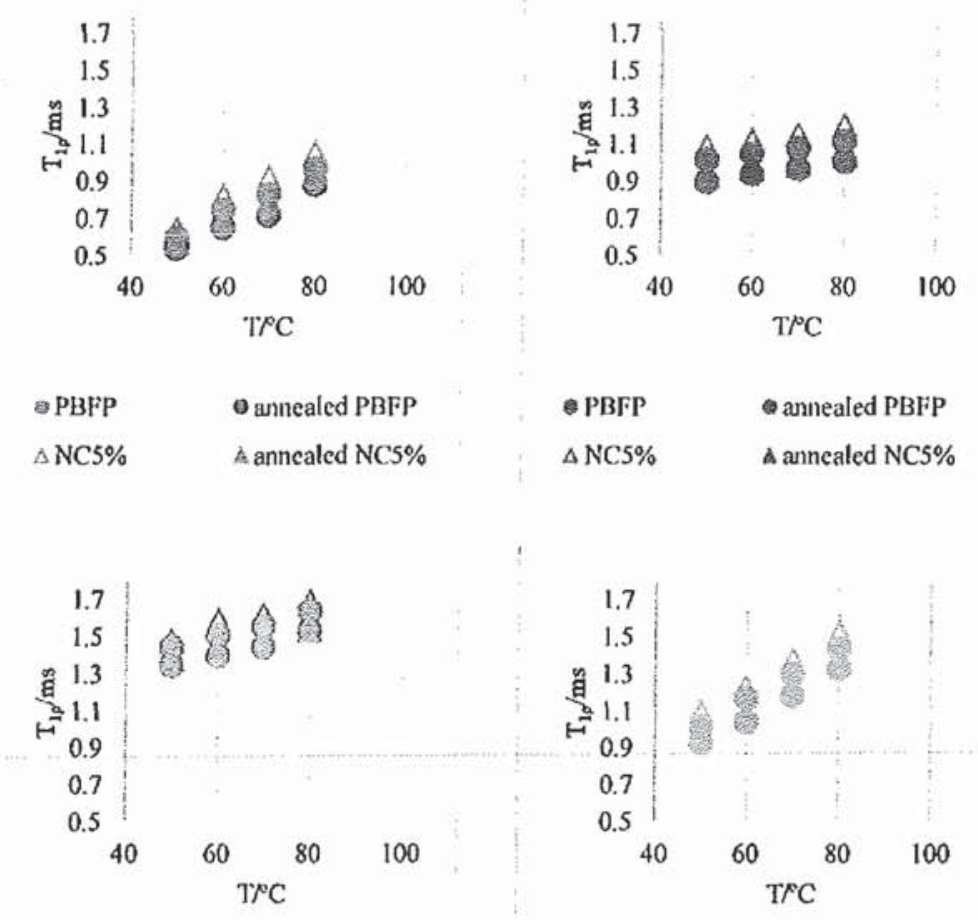

$$
\begin{array}{ll}
\triangle \mathrm{PBFP} & \quad 0 \text { annealed PBFP } \\
\wedge \mathrm{NC} 5 \% & \triangle \text { annealed NC5\% }
\end{array}
$$

$$
\begin{array}{ll}
\approx \text { PBFP } & \text { a annealed PBFP } \\
\therefore \mathrm{NC} 5 \% & \text { b annealed NC5\% }
\end{array}
$$

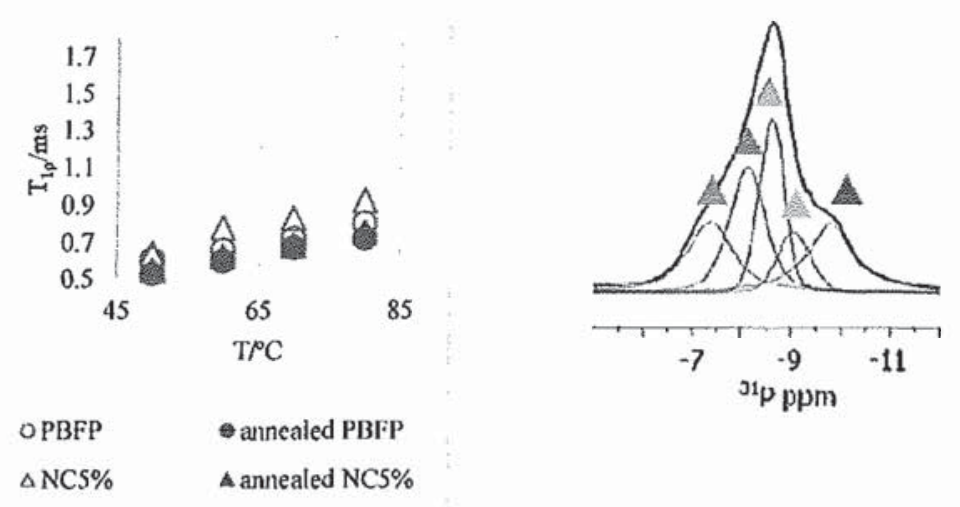

Figure 8.8: ${ }^{31} \mathrm{P}$ spin-lattice relaxation time in the rotating frame for the fiver components obtained by deconvolution of the ${ }^{31} \mathrm{P}$ solid-state NMR spectra of PBFP, annealed PBFP, PBFP with $5 \%$ of nano-TiO ${ }_{2}$, and annealed sample of PBFP with $5 \%$ of nano$\mathrm{TiO}_{2}$ over the temperature range of 50 to $80^{\circ} \mathrm{C}$ 
The $T_{I_{p}}$ values decrease with increasing temperature for the components at -8.12 , 8.68 and $-9.88 \mathrm{ppm}$, implies that the polymer backbone undergoes a fast motion. The ${ }^{31} \mathrm{P}$ $\mathrm{T}_{1 \rho}$ values from $20-40^{\circ} \mathrm{C}$, decrease after annealing for both PBFP and $\mathrm{NC} 5 \%$, which implies that the mobility decreases upon annealing because of increasing crystallite size. Furthermore, after nano-filling the $T_{1 p}$ values increase suggesting that the presence of nano $\mathrm{TiO}_{2}$ increases the mobility to some extent possibly due to suppression of crystallite growth.

Each component of phosphorus $T_{1 p}$ spectrum of the four samples at $-8.62(A),-7.37$ (A), -9.79 (A),$-8.13(\mathrm{~A})$ and $-9.06 \mathrm{ppm}$ ( are compared in Figure 8.8. Even though there are slight differences between samples, they are too small to be of note for all five components. The main differences appear to be between the components in all four samples in a similar fashion, where the component at 8.62 (A) has consistently longer values, however it is less sensitive to temperature just as $-8.13 \mathrm{ppm}(\mathrm{A})$ and $-9.79(\mathbf{A})$. The components at $-7.37(\mathrm{~A})$ and $-9.06 \mathrm{ppm}(\mathrm{A})$ are far more sensitive to temperature, which suggest that their mobility is increasing more significantly with temperature.

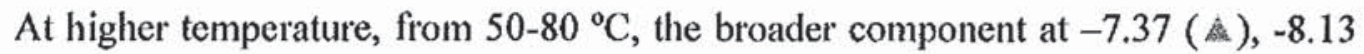
ppm ( $\mathbf{\Delta}$ ) and $-9.79(\mathbf{\Delta})$, consistently have the lowest $T_{1 p}$ values. Since at these temperatures all components are in the fast motion regime, this implies that the aforementioned components have the slowest motion, which is consistent with them originating from the broad components at low temperature. The remaining components have narrower linewidths and larger $T_{1 \rho}$ values and thus have significantly enhanced mobility. 
To conclude, at low temperature there are three broad and three narrow components. At higher temperature, the broad components at $-4.55 \mathrm{ppm}$ are transformed into two broad components at -7.37 and $-8.13 \mathrm{ppm}$ which become narrower due to increased backbone motion. The remaining broad component overlaps with the narrow signals at low temperature narrows and appear at $-9.79 \mathrm{ppm}$ at higher temperature. All three broad components remain relatively broad compared to the narrow components at high temperature; however, the difference is much smaller suggesting that their backbone dynamics are approaching the same time scale. It is likely that the three narrow components at low temperature coalesce into two narrow ones.

\section{7 ${ }^{19}$ F Solid-State NMR Relaxation Times of Different Preparation Methods}

\subsection{1 ${ }^{19} \mathrm{~F}$ solid-state $\mathrm{NMR} \mathrm{T}_{1}$ relaxation times of samples with different preparation methods}

The three models used for the ${ }^{19} \mathrm{~F}$ NMR spectra of PBFP have to be reconciled, especially the two at low temperature. Currently bases on ${ }^{19} \mathrm{~F} \mathrm{~T}_{1}$ relaxation, the nonannealed samples at low temperature are reconsidered to be composed of three contributions: one broad and two narrow; while the annealed samples are composed of four contributions: one broad and three narrow (one of which is very minor). At higher temperature, a single model with four contributions is considered which are all narrow. Two of these are significantly broader than the others and are proposed to originate from the broad components at low temperature. This would suggest that the broad components in the low temperature model actually contain two contributions.

For the non-annealed samples at low temperature the broad component at -76.73 exhibits bi-exponential $T_{1}$ relaxation with one relatively long $T_{1}$ at $1.06 \mathrm{~s}$, and one short $T_{1}$ 
at $0.44 \mathrm{~s}$. The remaining two narrow components also have biexponential decay where the $\mathrm{T}_{1}$ values range from 0.29 to 0.82 second, and one small $\mathrm{T}_{1}$. It seems that all three components have one contribution of $\mathrm{T}_{1}$ in common on the order of $0.4-0.5 \mathrm{~s}$. Furthermore, all three components also have a common activation energy ranging from -5 to $-10 \mathrm{~kJ} / \mathrm{mol}$. This indicates that a broad component lies underneath all three contributions that were not previously observed. Thus, the spectrum is composed of two narrow and two broad components at low temperature.
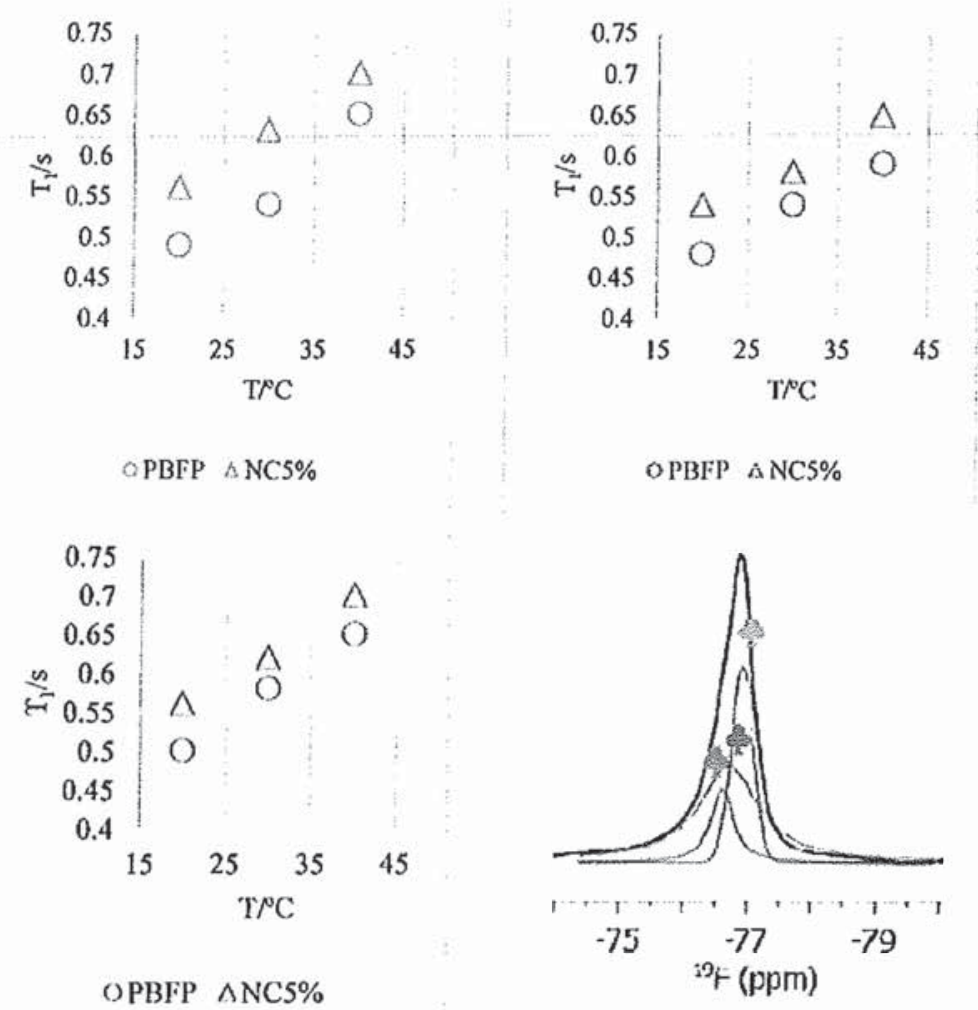

Figure 8.9: $\quad{ }^{19} \mathrm{~F}$ spin-lattice relaxation time $T_{1}$ for the three components obtained by deconvolution of the ${ }^{19} \mathrm{~F}$ solid-state NMR spectra of PBFP, and sample of PBFP with $5 \%$ of nano- $\mathrm{TiO}_{2}$ over the temperature range of 20 to $40^{\circ} \mathrm{C}$

In the annealed samples at low temperature all four components exhibit monoexponential decay. The broad component at -76.62 , with linewidth $1647 \mathrm{~Hz}$, undergoes slow sidechain motion. Note that the broad component in this case is nearly twice as wide 
as that in the non-annealed model, suggesting the possibility that it is actually made up of two components of comparable width to that seen in the non-annealed sample. This suggests that the model for the annealed samples has two broad and three narrow components.

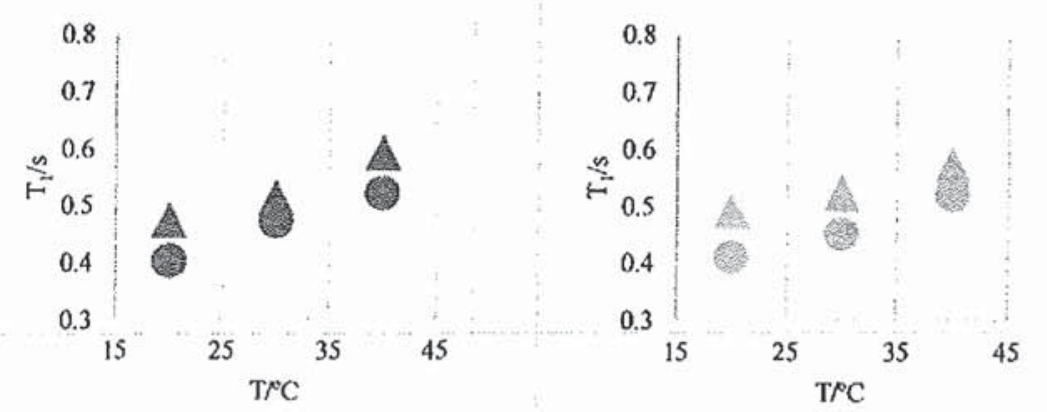
Annealed
- PBFP
Annealed
Anncaled
\& $\mathrm{PBFP}$
Annealed
s. NC $5 \%$
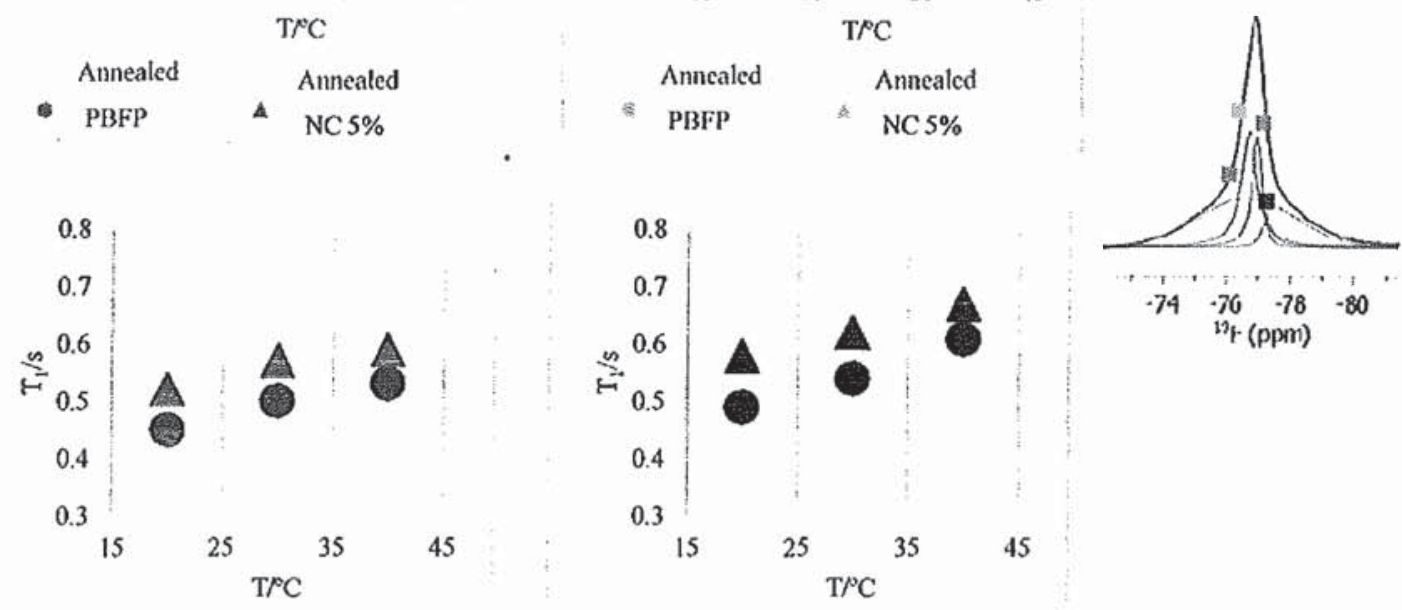

Annealed Annealed
PBFP
NC $5 \%$

Annealed

$\triangle \mathrm{NCS} \%$ annealed

Figure 8.10: ${ }^{19} \mathrm{~F}$ spin-lattice relaxation time $T_{1}$ for the three components obtained by deconvolution of the ${ }^{19} \mathrm{~F}$ solid-state NMR spectra of annealed PBFP, and annealed sample of PBFP with $5 \%$ of nano- $\mathrm{TiO}_{2}$ over the temperature range of 20 to $40{ }^{\circ} \mathrm{C}$

At higher temperatures for all samples the components at -76.42 and $-76.76 \mathrm{ppm}$, have similar $E_{\mathrm{a}}$ values, indicating that they originate from the broad components at lower temperature. This is also supported by their relatively large linewidths of 239 and $181 \mathrm{~Hz}$, respectively, when compare to the remaining narrow components at -76.92 and $-77.10 \mathrm{ppm}$ with linewidths under $125 \mathrm{~Hz}$. These lines are broader than any of the narrow components 
at low temperature, and thus cannot arise from them. Furthermore, the $T_{1}$ values of these two components are consistently shorter than the remaining narrow components for all four samples, suggesting that they are less mobile, which is consistent with originating from the broad components at low temperature

The ${ }^{19} \mathrm{~F} \mathrm{~T}_{\text {I data }}$ of each component are compared between samples over the temperature range from $20-80^{\circ} \mathrm{C}$ to show how different sample treatment methodologies such as annealing and nano-filling affect the sidechain mobility. Figure 8.9 summarize the $T_{1}$ values for the non-annealed samples from $20-40^{\circ} \mathrm{C}$ for the components at $-76.64(\AA)$, $76.73(\$)$ and $-76.93 \mathrm{ppm}\left({ }_{0}^{\circ}\right)$, which have corresponding linewidth of 171,538 and 191 $\mathrm{Hz}$, respectively. Figure 8.10 presents the $\mathrm{T}_{1}$ values of the four component from $20-40^{\circ} \mathrm{C}$ for the annealed samples at $-76.62(\mathbf{m}),-76.71($ ( $),-76.95$ (目) and -77.20 (国) ppm.

As shown in Figure 8.9, the trends are obvious for all three components of the sample PBFP and NC 5\%: where the $\mathrm{T}_{1}$ increases with increasing temperature with similar sensitivity, and those of the NC 5\% are consistently longer than of those in PBFP. This suggests that the domain sizes corresponding to the NC $5 \%$ and are consistently smaller than those of PBFP as they are more mobile. Hence, the presence of filler suppresses crystallite growth in the non-annealed samples. Similarly, for the annealed samples, in Figure 8.10, all four components of the annealed NC 5\% sample have longer $T_{1}$ values than in annealed PBFP. Furthermore, the absolute values of the $T_{1}$ in the annealed samples are shorter than their non-annealed counterparts. This implies that there is a significant increase in crystallite growth upon annealing; however, the presence of nano-filler suppresses it.

The comparison of ${ }^{19} \mathrm{~F} \mathrm{~T}_{1}$ values by component at higher temperature is shown in Figure 8.11 for all four samples. The NC $5 \%$ sample always has the longest $\mathrm{T}_{1}$ values for 
all components. Those of the PBFP have the second longest $T_{1}$, which is followed by the annealed NC 5\% and annealed PBFP having the smallest values. Again, samples with nano- $\mathrm{TiO}_{2}$ filling always have a larger $\mathrm{T}_{1}$ value than non-filled samples and the annealed samples always has a smaller $\mathrm{T}_{1}$ value than the corresponding non-annealed samples. Additionally, the $T_{1}$ values increase with increasing temperature for all components implies that the polymer sidechain undergoes a fast motion with relative to its larmor frequency. The ${ }^{19} \mathrm{~F} \mathrm{~T}_{1}$ results for all components increase after nano-filling for both PBFP and annealed $\mathrm{PBFP}$, suggesting that the presence of nano- $\mathrm{TiO}_{2}$ increases mobility and therefore suppresses crystallite growth.

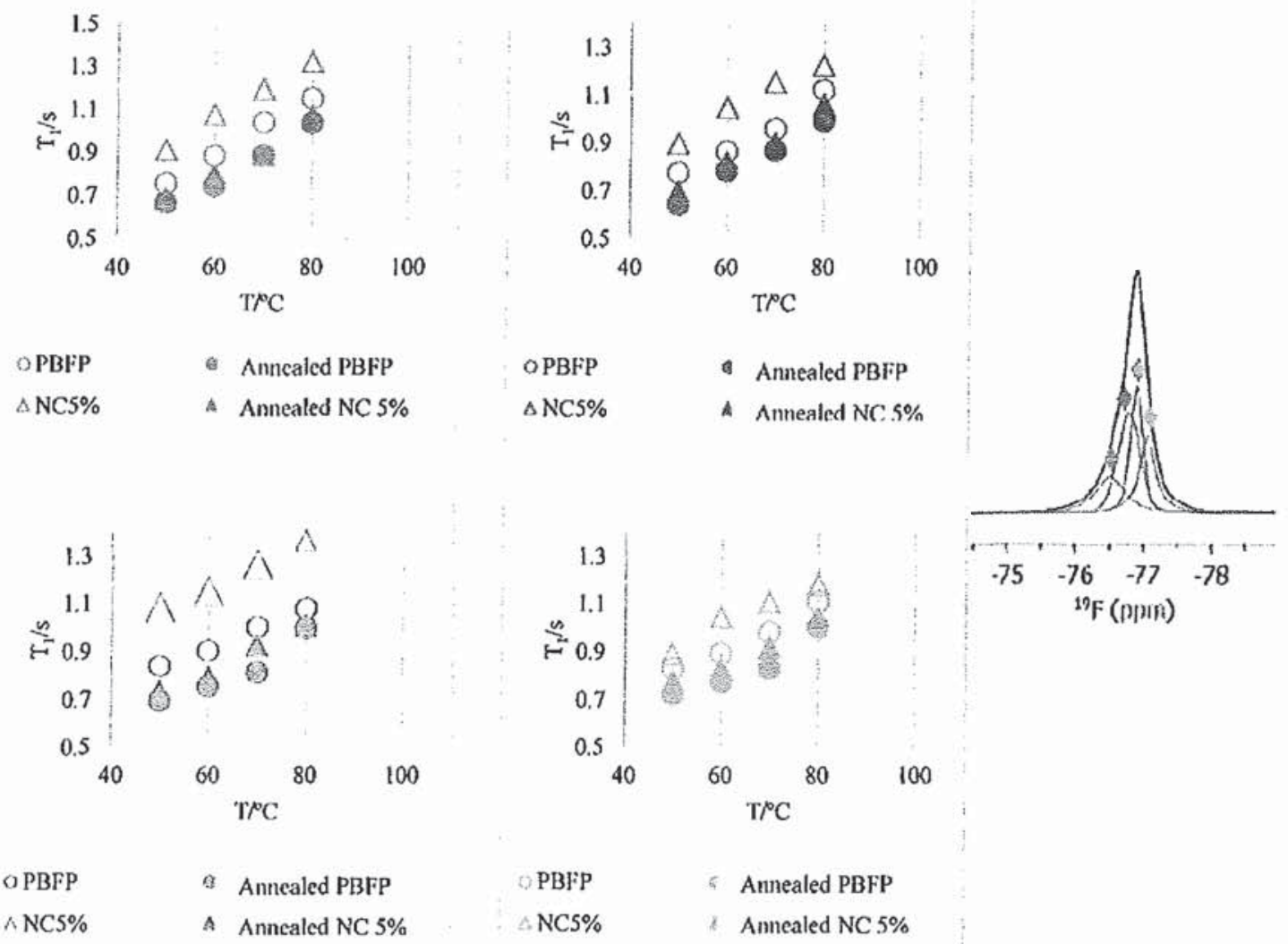

Figure 8.11: ${ }^{19} \mathrm{~F}$ spin-lattice relaxation time $T_{1}$ for the three components obtained by deconvolution of the ${ }^{19} \mathrm{~F}$ solid-state NMR spectra of PBFP, annealed PBFP, PBFP with $5 \%$ of nano- $\mathrm{TiO}_{2}$, and annealed sample of $\mathrm{PBFP}$ with $5 \%$ of nano- $\mathrm{TiO}_{2}$ over the temperature range of 50 to $80^{\circ} \mathrm{C}$ 


\subsection{2 ${ }^{19} \mathrm{~F}$ solid-state NMR $\mathrm{T}_{2}$ relaxation time of samples with different preparation methods}

The low temperature ${ }^{19} \mathrm{~F} \mathrm{~T}_{2}$ relaxation results of the non-annealed samples provide further support for the three-componcnt model: two of them are narrow originating from mobile domains, and one is broad arising from a rigid domain. For the PBFP and NC 5\%, at low temperature, the broad component at $-76.73 \mathrm{ppm}$ has distinctly small $\mathrm{T}_{2}$ values, relatively small $\mathrm{E}_{\mathrm{a}}$ values, and broad line-width at $538 \mathrm{~Hz}$. In contrast, the remaining two components appearing at -76.64 and $-76.93 \mathrm{ppm}$, have long $\mathrm{T}_{2}$ and slightly smaller $\mathrm{E}_{\mathrm{a}}$. This is somewhat at odds with the previously proposed model using the $\mathrm{T}_{1}$ data, where the broad component was seen to be made up of two contributions. $\mathrm{T}_{2}$ is not sensitive to the subtle differences in motion that led to both components to be observed in the $\mathrm{T}_{1}$ data; hence one cannot expect biexponential behaviour in this case. Thus, it consisting of two components can neither be supported nor refuted by these results.

The model for the annealed samples is somewhat more complex, compared to the non-annealed counterparts, as they have a broad component of much larger linewidth, and there is an additional narrow component, thus these spectra are difficult to reconcile with the four line model proposed for the $\mathrm{T}_{1}$ results. The broad component at $-76.62 \mathrm{ppm}$, with line width $1647 \mathrm{~Hz}$, has the smallest $T_{2}$ values for both annealed PBFP and annealed NC $5 \%$ over the temperature range. Thus it definitely originates from the rigid environments. One could consider that it actually is composed of two components of similar width near $800 \mathrm{~Hz}$; however no bi-exponential behaviour is seen, making it difficult to be confident in such a conclusion. The remaining three components appearing at $-76.71,-76.95$ and $77.20 \mathrm{ppm}$ have similar longer $\mathrm{T}_{2}$ than the broad component, and thus come from more mobile environments, which are further supported by their much reduced line widths. As 
the component at $-77.20 \mathrm{ppm}$ makes such a small contribution and its inconsistent relaxation behaviour with the other two components, one cannot rule out that it arises from an artefact in the fitting procedure. In other words no significant error is introduced by assuming there are only 2 narrow components.

At high temperature, a consistent four-component model is used for all samples, which is consistent with the two mobile and two less mobile components proposed from the $T_{1}$ results. The components at -76.42 and $-76.76 \mathrm{ppm}$ have similar $E_{\mathrm{a}}$ values and relative smaller $T_{2}$, suggesting that they arise from the same rigid environment. This is also supported by their broader line width. The other two components at -76.92 and $-77.10 \mathrm{ppm}$ have relative longer $T_{2}$ and smaller $E_{a}$, and thus originate from the mobile phases at low temperature. The two broader lines at high temperature are assumed to come from two broad components at low temperature. 


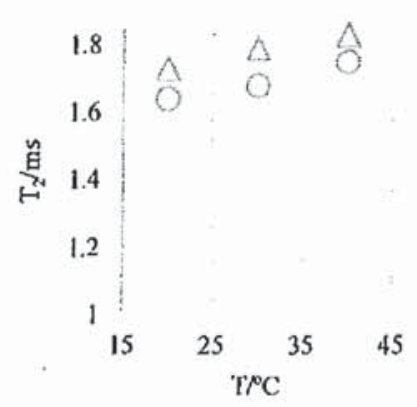

O PBFP $\triangle \mathrm{NCS} \%$

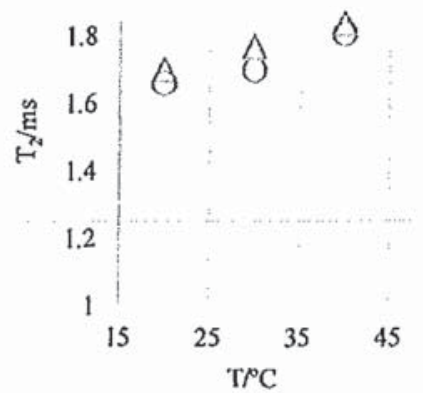

O PBFP $\triangle \mathrm{NC} 5 \%$

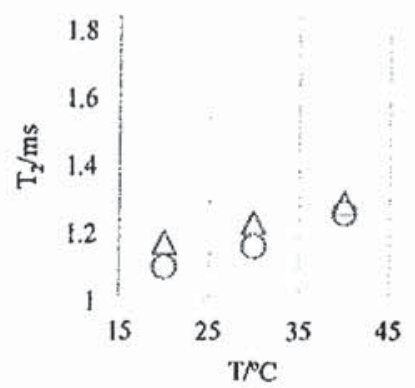

OPBFP $\triangle N C 5 \%$

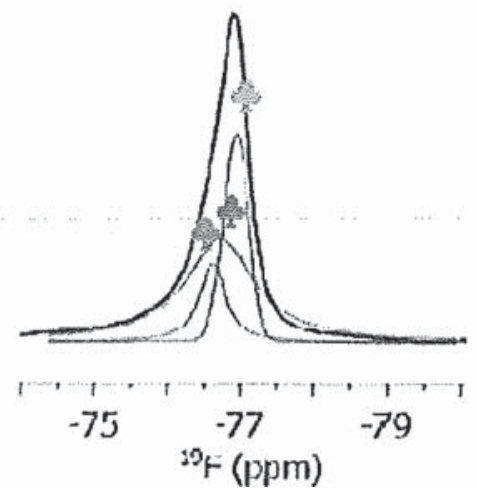

Figure 8.12: ${ }^{19} \mathrm{~F}$ transverse relaxation time $T_{2}$ for the three components obtained by deconvolution of the ${ }^{19} \mathrm{~F}$ solid-state NMR spectra of PBFP, PBFP with $5 \%$ of nano$\mathrm{TiO}_{2}$ over the temperature range of 20 to $40^{\circ} \mathrm{C}$

The comparison of the $T_{2}$ values for each component between the four samples is presented in Figure 8.12-14 for the temperature ranges from 20 to $40{ }^{\circ} \mathrm{C}$ and 50 to $80^{\circ} \mathrm{C}$. Figures 8.12m8.13 show that the NC 5\% samples have a longer $T_{2}$ than PBFP and the annealed NC $5 \%$ persistently have a longer $\mathrm{T}_{2}$ values than annealed PBFP. In Figure 8.14 shows that the NC $5 \%$ sample for 4 components always has the longest $T_{2}$ values, while those of the PBFP next longest, followed by the annealed NC 5\% and the annealed PBFP. Samples with nano- $\mathrm{TiO}_{2}$ filling always have a larger $\mathrm{T}_{2}$ value than non-filled samples and the annealed samples always has a smaller $T_{2}$ value than the corresponding non-annealed samples. 

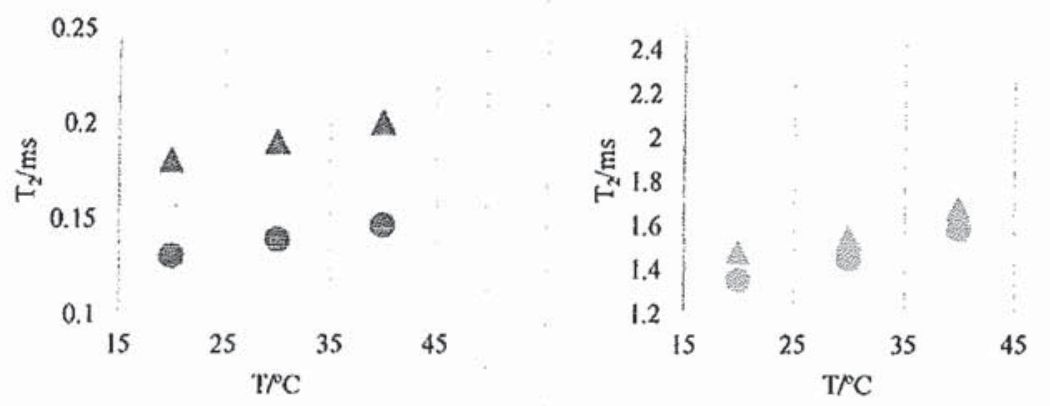

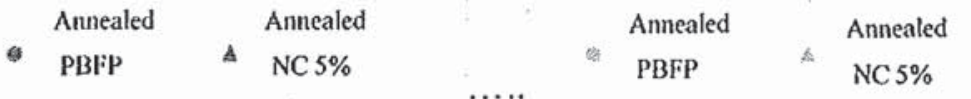
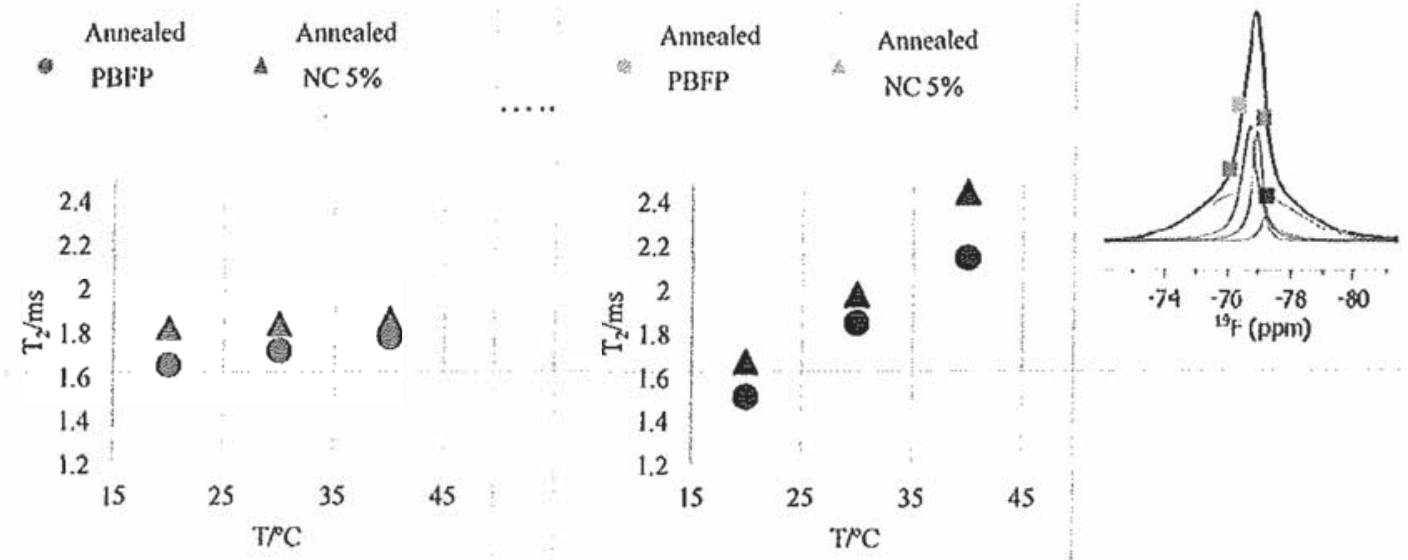

- Annealed

Annealed

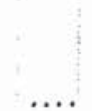

Annealed

- PBFP

Annealed
NC $5 \%$

Figure 8.13: ${ }^{19} \mathrm{~F}$ transverse relaxation time $T_{2}$ for the three components obtained by deconvolution of the ${ }^{19} \mathrm{~F}$ solid-state NMR spectra of annealed PBFP, annealed PBFP with $5 \%$ of nano- $\mathrm{TiO}_{2}$ over the temperature range of 20 to $40^{\circ} \mathrm{C}$

For high temperatures the differences in $T_{2}$ in all four components between the annealed samples are difficult to distinguish. In contrast the differences in $T_{2}$ between PBFP and annealed PBFP are more obvious for the components at -76.92 and $-77.10 \mathrm{ppm}$. Furthermore the differences in $\mathrm{T}_{2}$ between PBFP and NC 5\% are also seen at $-76.76 \mathrm{ppm}$. In general, the differences in $\mathrm{T}_{2}$ values are most pronounced at higher temperatures, and can be seen between non-annealed and annealed samples which are more pronounced than

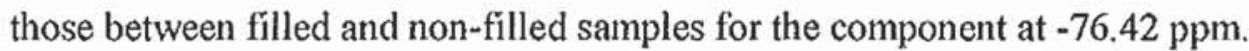



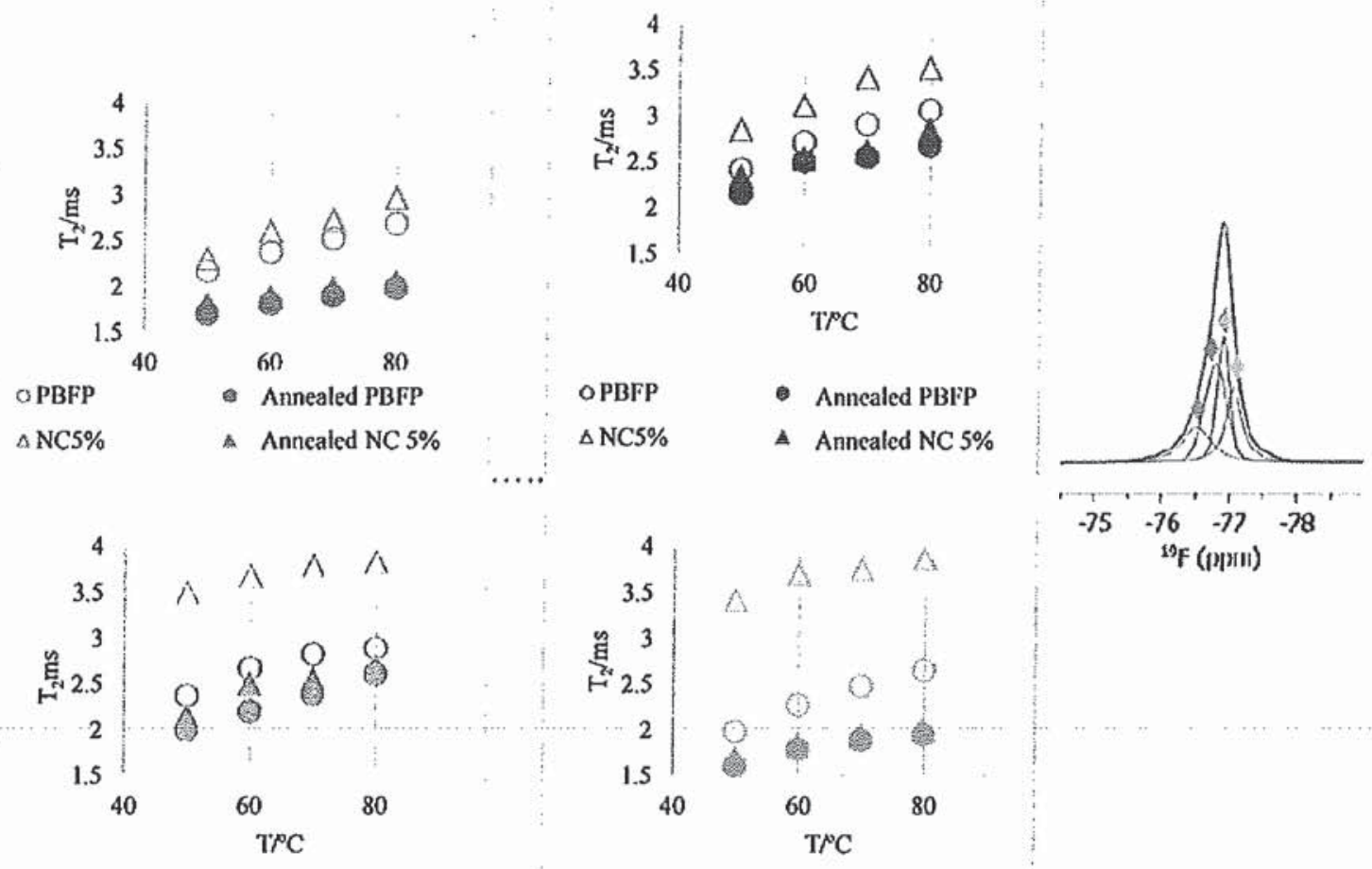

$\begin{array}{llllll}\text { OPBFP } & \rightarrow \text { Annealed PBFP } & \text { PBFP } & \text { Annealed PBFP } \\ \wedge \text { NC5\% } & \text { A Annealed NC 5\% } & \text { A NC5\% } & \text { A } & \text { Annealed NC 5\% }\end{array}$

Figure 8.14: $\quad{ }^{19} \mathrm{~F}$ transverse relaxation time $\mathrm{T}_{2}$ for the four components obtained by deconvolution of the ${ }^{19} \mathrm{~F}$ solid-state NMR spectra of PBPF, annealed PBFP, PBFP with $5 \%$ nano$\mathrm{TiO}_{2}$, and annealed PBFP with $5 \%$ of nano- $\mathrm{TiO}_{2}$ over the temperature range of 50 to $80^{\circ} \mathrm{C}$

The behaviour of $T_{2}$ with temperature in the high temperature range for -76.42 and $-76.76 \mathrm{ppm}$ provide support for the previous conclusion that they originate from the broad component at $-4.55 \mathrm{ppm}$ at lower temperature. The narrower distribution in $\mathrm{T}_{2}$ between samples suggests smaller absolute changes in mobility. This implies the crystallite s sizes are larger, as larger crystallites will be slower to increase their mobility.

Similar to the ${ }^{31} \mathrm{P}$ relaxation analysis, the ${ }^{19} \mathrm{FT}_{1} / \mathrm{T}_{2}$ for all components as a function of $1000 / \mathrm{T}(\mathrm{K})$ from $20-80^{\circ} \mathrm{C}$ are presented in Table $8.51-8.52$, to gain more insight into the domain size and mobility represented by each components, and their variation with 
preparation methodologies. The correlation time of ${ }^{19} \mathrm{~F}$ is a direct indication of the mobility of the sidechain. Large $T_{1} / T_{2}$ values indicate rigidity of environments, whereas small values imply mobile environments. Since the sidechain undergoes faster motion than the backbone, having much smaller correlation times, the ${ }^{19} \mathrm{~F} \mathrm{~T}_{1} / \mathrm{T}_{2}$ values are significantly smaller when compared to the corresponding ${ }^{31} \mathrm{P}$ values.

For the non-annealed samples, each component has two $T_{1} / T_{2}$ values at low temperature - one large and one small. This supports the previous assertion that a broad component lies underneath all three narrow components. Thus the non-annealed samples are composed of two broad and two narrow components. For the annealed samples, the component at $-76.62 \mathrm{ppm}$ has remarkably large $T_{1} / T_{2}$ values and long correlation times over $20-40{ }^{\circ} \mathrm{C}$, for two samples, which supports the suggestion that this component represents large rigid domain exhibiting a tightly packed structure. 
Table 8.51:

${ }^{19} \mathrm{~F} \mathrm{~T}_{1} / \mathrm{T}_{2}$ ratios of PBFP, annealed PBFP, NC $5 \%$ and annealed NC $5 \%$ with the change of temperature over $20-40^{\circ} \mathrm{C}$ obtained with an MAS rate of $10 \mathrm{kHz}$

\begin{tabular}{|c|c|c|c|c|c|c|c|}
\hline \multirow{5}{*}{ PBFP } & \multirow{3}{*}{$\begin{array}{c}\mathrm{CS} / \mathrm{ppm} \\
\text { Width/Hz } \\
20\end{array}$} & \multicolumn{2}{|c|}{$\begin{array}{c}-76.64 \\
(\%)\end{array}$} & \multicolumn{2}{|c|}{$\begin{array}{c}-76.73 \\
(\&)\end{array}$} & \multicolumn{2}{|c|}{$\begin{array}{c}-76.93 \\
(6)\end{array}$} \\
\hline & & \multicolumn{2}{|c|}{171} & \multicolumn{2}{|c|}{538} & \multicolumn{2}{|c|}{191} \\
\hline & & $500(55)$ & $244(38)$ & 573 & 400 & 325 & 247 \\
\hline & 30 & $503(55)$ & $273(41)$ & 586 & 397 & 393 & 226 \\
\hline & 40 & $497(55)$ & $263(40)$ & 571 & 429 & 387 & 249 \\
\hline \multirow{3}{*}{ NC 5\% } & 20 & $358(46)$ & $202(35)$ & 487 & 410 & 382 & 288 \\
\hline & 30 & $408(50)$ & $229(37)$ & 520 & 463 & 373 & 328 \\
\hline & 40 & $421(50)$ & $333(45)$ & 543 & 434 & 402 & 342 \\
\hline
\end{tabular}

Note: $T_{1}=$ spin-lattice relaxation time; $T_{2}=$ spin-spin relaxation time; $C S=$ chemical shift in ppm; Width = linewidth at lalf height in $\mathrm{Hz}$; $\mathrm{NC} 5 \%$ poly[bis(trifluoroethoxy)phosphazene/ $\mathrm{TiO}_{2}-5 \%$; correlation times in nanosecond are shown in the brackets for the contributions at $-76.64 \mathrm{ppm}$

\begin{tabular}{|c|c|c|c|c|c|}
\hline & CS/ppm & $\begin{array}{c}-76.62 \\
\text { (घ) }\end{array}$ & $\begin{array}{c}-76.71 \\
(*)\end{array}$ & $\begin{array}{c}-76.95 \\
\text { (日) }\end{array}$ & $\begin{array}{c}-77.20 \\
\text { (घ) }\end{array}$ \\
\hline \multirow{4}{*}{$\begin{array}{c}\text { annealed } \\
\text { PBFP }\end{array}$} & Width/Hz & 1647 & 266 & 166 & 127 \\
\hline & 20 & $3077(100)$ & $304(43)$ & 273 & 325 \\
\hline & 30 & $3381(100)$ & $310(43)$ & 291 & 292 \\
\hline & 40 & $3562(100)$ & $329(45)$ & 298 & 284 \\
\hline \multirow{3}{*}{$\begin{array}{l}\text { Annealed } \\
\text { NC 5\% }\end{array}$} & 20 & $2611(100)$ & $331(45)$ & 286 & 347 \\
\hline & 30 & $2684(100)$ & $338(45)$ & 310 & 313 \\
\hline & 40 & $2950(100)$ & $339(45)$ & 317 & 273 \\
\hline
\end{tabular}

Note: $T_{1}=$ spin-lattice relaxation time; $T_{2}=$ spin-spin relaxation time; $C S=$ chemical shift in ppm; Width = linewidth at half height in $\mathrm{Hz}$; annealed PBFP $=$ annealed poly[bis(trifluoroethoxy)phosphazene at $220^{\circ} \mathrm{C}$ for 2 hours; annealed NC $5 \%=$ annealed $\mathrm{PBFP} / \mathrm{TiO}_{2}-5 \%$ at $220^{\circ} \mathrm{C}$ for 2 hours; correlation times in nanosecond are shown in the brackets for the contributions at -76.62 and $-76.71 \mathrm{ppm}$

At higher temperature, the ${ }^{19} \mathrm{FT}_{1} / \mathrm{T}_{2}$ values for all four components fall into the same range from 300-500, the similar corresponding correlation times around $50 \mathrm{~ns}$, indicating that they have similar mobility and domain size. This is due to the polymer starting to undergo transformation from the 3D-crystal phases to the 2-D liquid crystal phase at its $\mathrm{T}(1)$ temperature around $60^{\circ} \mathrm{C}$. During this transition the polymer backbone motion increases dramatically, resulting in much reduced linewidths, while the sidechains reorganize. Since ${ }^{19} \mathrm{~F}$ is primarily sensitive to sidechain motion, which is already in the fast motion regime, the differences between environments are subtle at these elevated temperatures, making it difficult to differentiate between components. 
Table 8.52:

${ }^{19} \mathrm{~F} \mathrm{~T}_{1} / \mathrm{T}_{2}$ ratios of PBFP, annealed PBFP, NC $5 \%$ and annealed NC $5 \%$ with the

\begin{tabular}{|c|c|c|c|c|c|}
\hline \multirow[t]{2}{*}{ Samples } & $\mathrm{CS} / \mathrm{ppm}$ & $\begin{array}{c}-70.42 \\
(\diamond)\end{array}$ & $\begin{array}{c}-70.76 \\
(\phi)\end{array}$ & $\begin{array}{c}-76.92 \\
(\diamond)\end{array}$ & $\begin{array}{c}-77.10 \\
(\hat{0})\end{array}$ \\
\hline & Width/Hz & 239 & 181 & 103 & 125 \\
\hline \multirow{5}{*}{ PBFP } & 50 & $350(46)$ & 319 & 355 & $419(50)$ \\
\hline & 60 & $373(47)$ & 319 & 338 & $393(49)$ \\
\hline & 70 & $414(50)$ & 329 & 354 & $396(49)$ \\
\hline & 80 & $428(51)$ & 366 & 374 & $419(50)$ \\
\hline & 50 & $392(49)$ & 298 & 348 & $448(52)$ \\
\hline \multirow{4}{*}{$\begin{array}{l}\text { Annealed } \\
\text { PBFP }\end{array}$} & 60 & $410(50)$ & 313 & 343 & $433(51)$ \\
\hline & 70 & $469(53)$ & 340 & 341 & $439(51)$ \\
\hline & 80 & $522(56)$ & 370 & 383 & $515(56)$ \\
\hline & 50 & $396(49)$ & 314 & 309 & $260(40)$ \\
\hline \multirow{4}{*}{ NC 5\% } & 60 & $414(50)$ & 337 & 313 & $283(41)$ \\
\hline & 70 & $439(51)$ & 338 & 333 & 294 (42) \\
\hline & 80 & $447(52)$ & 348 & 356 & $305(43)$ \\
\hline & 50 & $382(48)$ & 298 & 341 & $466(53)$ \\
\hline \multirow{3}{*}{$\begin{array}{c}\text { annealed } \\
\text { NC 5\% }\end{array}$} & 60 & $423(50)$ & 321 & 316 & $451(52)$ \\
\hline & 70 & $452(52)$ & 342 & 363 & $474(53)$ \\
\hline & 80 & $522(56)$ & 372 & 376 & $519(55)$ \\
\hline \multicolumn{6}{|c|}{$\begin{array}{l}\text { Note: } \mathrm{T}_{1}=\text { spin-lattice relaxation time; } \mathrm{T}_{2}=\text { spin-spin relaxation time; four sample }=\text { solvent cast } \\
\mathrm{PBFP} \text {, annealed solvent cast } \mathrm{PBFP}, \mathrm{PBFP} / \mathrm{TiO}_{2}-5 \% \text { and annealed } \mathrm{PBFP} / \mathrm{TiO} \mathrm{O}_{2}-5 \% ; \mathrm{CS}=\text { chemical } \\
\text { shift in ppm; Width = linewvidth at half height in } \mathrm{Hz} \text {; correlation times in nanosecond are shown in } \\
\text { the brackets for the contributions at }-76.42 \text { and }-77.10 \mathrm{ppm}\end{array}$} \\
\hline
\end{tabular}
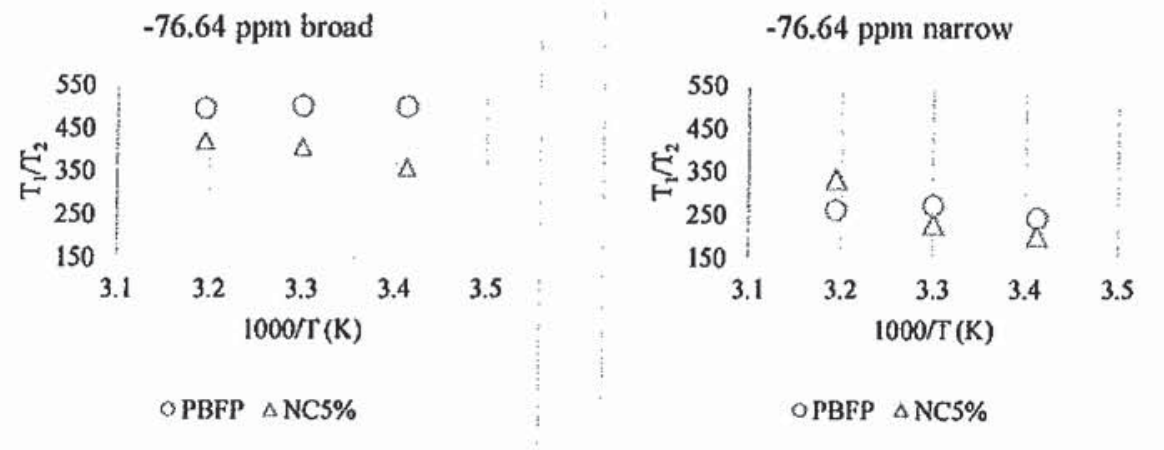

Figure 8.15: Two examples of ${ }^{19} \mathrm{~F} \mathrm{~T}_{1} / \mathrm{T}_{2}$ ratio with respect to the changes of temperature $(1000 / \mathrm{T})$ for one broad contribution (Left) and one narrow contribution (Right) over 20 to 40 ${ }^{\circ} \mathrm{C}$ for sample PBFP and NC $5 \%$ obtained by deconvolution of the ${ }^{31} \mathrm{P}$ solid-state NMR spectra at an MAS rate of $10 \mathrm{kHz}$

In Figure 8.15, the broad and the narrow contributions at $-76.64 \mathrm{ppm}$ are shown to illustrate the $T_{1} / T_{2}$ trends from $20-40{ }^{\circ} \mathrm{C}$. The $T_{1} / T_{2}$ values increase with increasing temperature, which is essentially the same for all the other components, indicating that the sidechain mobility decreases slightly with temperature. Decreasing mobility of the side 
chain with increasing temperature implies that the sidechains are reorganizing as the phase change initiates to the mesophase. In both cases the $T_{1} / T_{2}$ values and correlation times of NC $5 \%$ is smaller than pure PBFP, and suggest that nano- $\mathrm{TiO}_{2}$ increases sidechain motion. Furthermore nano- $\mathrm{TiO}_{2}$ seems to enhance the rate at which the sidechain motion increases with temperature, which means that the rate of change to the meso-phase is impeded by the nano-filler.

In Figure 8.16 , the trends in $\mathrm{T}_{1} / \mathrm{T}_{2}$ from $20-40{ }^{\circ} \mathrm{C}$ for the broad and narrow components at -76.62 and $-76.1 \mathrm{ppm}$ in the annealed samples are shown. The $\mathrm{T}_{1} / \mathrm{T}_{2}$ ratios increase with increasing temperature, indicating that sidechain motion decreases similarly to the non-annealed samples. The $T_{1} / T_{2}$ for the nano-filled material is much smaller for both contributions, again suggesting that nano- $\mathrm{TiO}_{2}$ increases sidechain motion. In addition, the broad components are most affected by nano-filling as indicated by the large difference in $T_{1} / T_{2}$ between the samples. Also the sensitivity to temperature is much greater for the broad component meaning that the nano-filler slows the phase change more strongly for the less mobile environments.
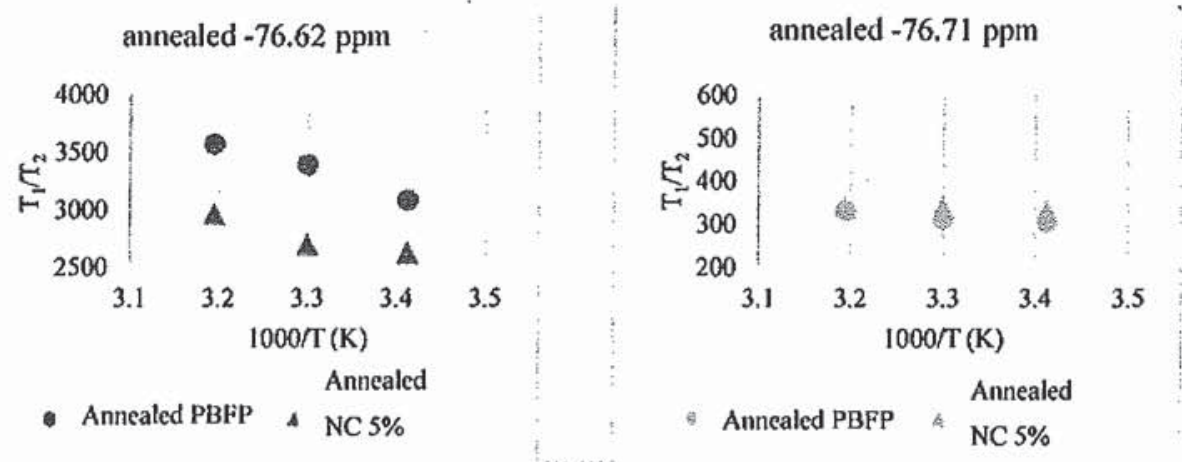

Figure 8.16: Two examples of ${ }^{19} \mathrm{~F} T_{1} / T_{2}$ ratio with respect to the changes of temperature $(1000 / T)$ for one broad contribution (Left), and one narrow contribution (Right) over 20 to 40 ${ }^{\circ} \mathrm{C}$ for sample annealed PBFP and annealed $\mathrm{NC} 5 \%-\mathrm{TiO}_{2} 5 \%$ obtained by deconvolution of the ${ }^{31} \mathrm{P}$ solid-state NMR spectra at an MAS rate of $10 \mathrm{kHz}$ 

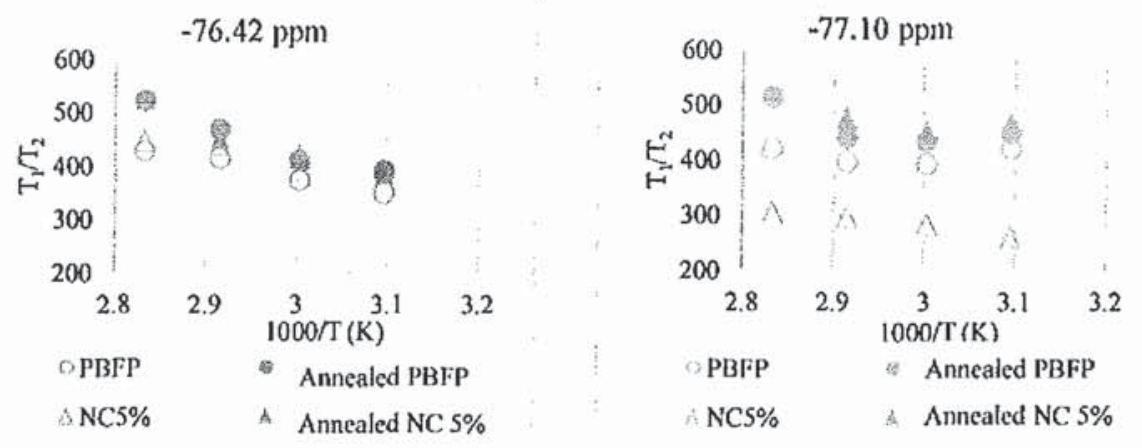

Figure 8.17: Two examples of ${ }^{19} \mathrm{~F} \mathrm{~T}_{1} / \mathrm{T}_{2}$ ratio with respect to the changes of temperature $(1000 / \mathrm{T})$ for one broad contribution (Left) and one narrow contribution (Right) over 50 to 80 ${ }^{\circ} \mathrm{C}$ of PBFP, annealed PBFP, PBFP with $5 \%$ of nano- $\mathrm{TiO}_{2}$, and annealed sample of PBFP with $5 \%$ of nano- $\mathrm{TiO}_{2}$ obtained by deconvolution of the ${ }^{31} \mathrm{P}$ solid-state NMR spectra at an MAS rate of $10 \mathrm{kHz}$

The $T_{1} / T_{2}$ ratios for the broad and the narrow components at -76.42 and $-77.10 \mathrm{ppm}$ are plotted in Figure 8.17 for all four samples at high temperature. The $T_{1} / T_{2}$ ratios consistently commence at their smallest values increasing with temperature, hence their correlation times increase with temperature. These decreasing sidechain motion with temperature correlated with rearrangements in the sidechain upon transformation to mesophase, continuing the trend seen in the low temperature range. The spreads between the $T_{1} / T_{2}$ ratios are much larger for the component at $-77.10 \mathrm{ppm}$ between samples, especially for NC $5 \%$ sample, which also exhibits more pronounced temperature sensitivity. This means that this component represents an environment that experience large increase in motion upon the addition of the nano-filler, which are less pronounced with annealing. 


\subsection{3 ${ }^{19} \mathrm{~F}$ solid-state $\mathrm{NMR} \mathrm{T}_{1 p}$ relaxation time of samples with different preparation methods}

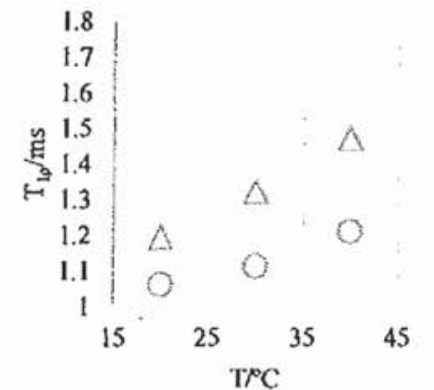

OPBFP $\triangle \mathrm{NC5} \%$

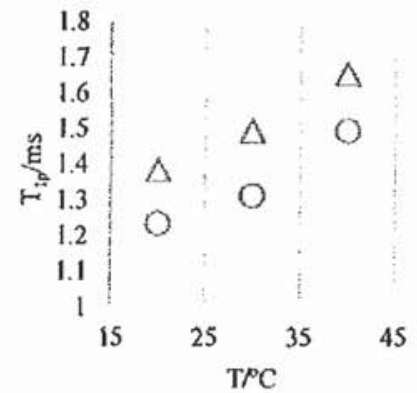

o PBPF $\triangle \mathrm{NC} 5 \%$

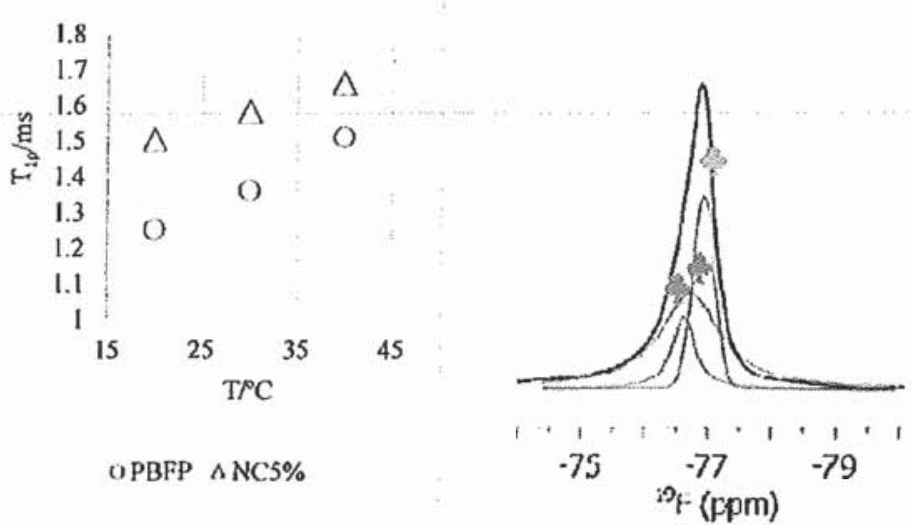

Figure 8.18: $\quad{ }^{19} \mathrm{~F}$ spin-lattice relaxation time $\mathrm{T}_{1_{\mathrm{p}}}$ in the rotating frame for the three components obtained by deconvolution of the ${ }^{31} \mathrm{P}$ solid-state NMR spectra of PBFP, and PBFP with $5 \%$ of nano. $\mathrm{TiO}_{2}$ over the temperature range of 20 to $40^{\circ} \mathrm{C}$

Each component of ${ }^{19} \mathrm{~F} \mathrm{~T}_{1 \rho}$ spectrum of $\mathrm{PBPF}$ and $\mathrm{PBFP} / \mathrm{TiO}_{2}-5 \%$ with different preparations over the temperature range of $20-40^{\circ} \mathrm{C}$ are compared in Figure 8.18. All three components increase in $\mathrm{T}_{\mathrm{Ip}}$ with temperature and the NC 5\% sample always have longer $\mathrm{T}_{1 p}$ values than in PBFP. There seems to be little difference in temperature sensitivity in

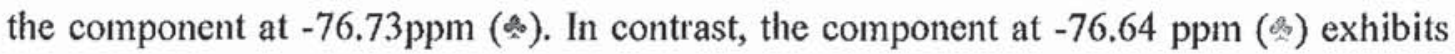
higher temperature sensitivity in $5 \% \mathrm{NC}$; while that at $-76.93 \mathrm{ppm}(\dot{*})$ has higher sensitivity in the neat polymer. This implies that the presence of filler destabilizes the crystallites in the $5 \%$ nano- $\mathrm{TiO}_{2}$ PBFP $\mathrm{NC}$ with respect to neat polymer for the more rigid domain, suggesting the crystallite sizes in this phase are smaller in the filled polymer. 


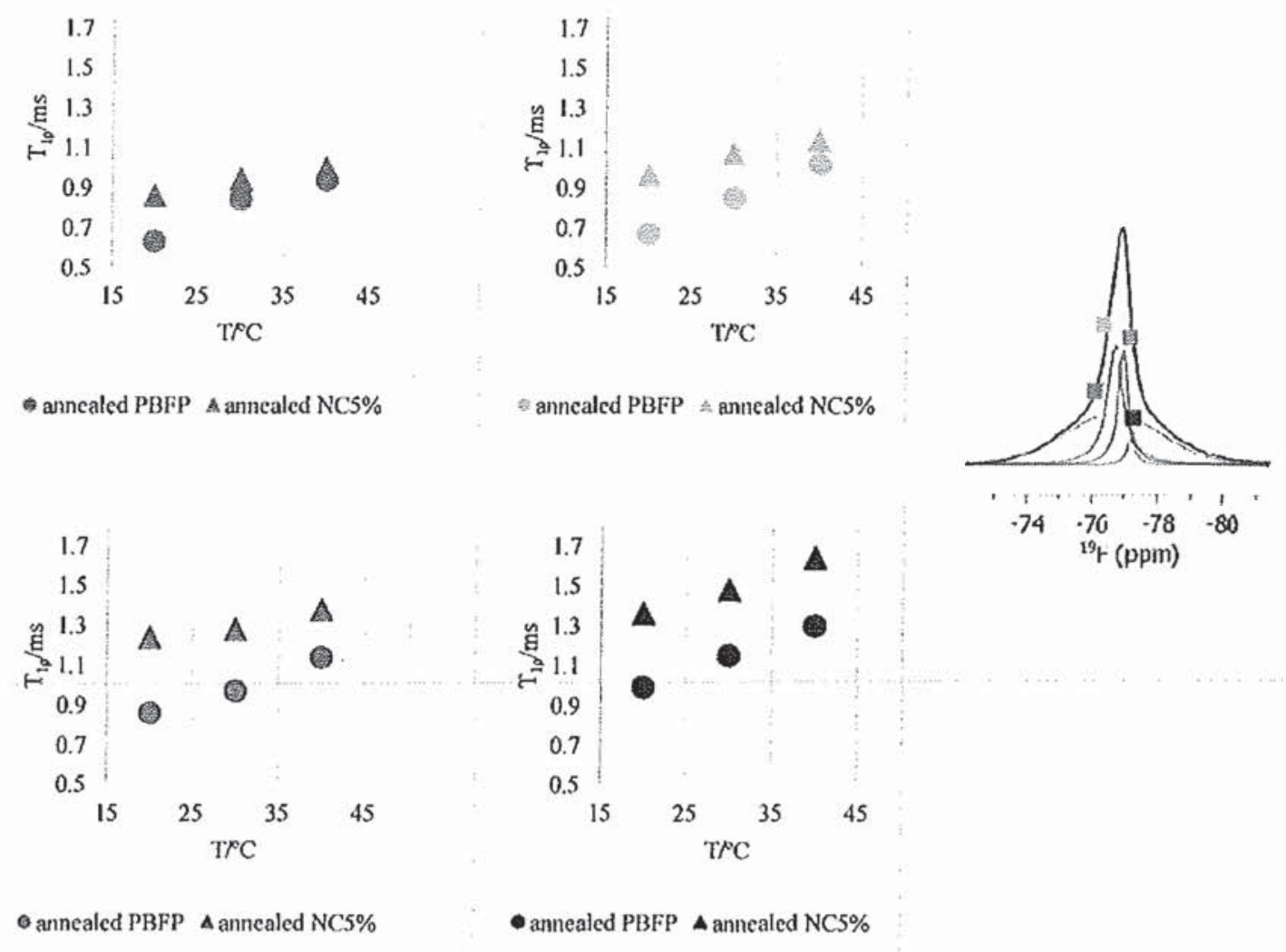

Figure 8.19: $\quad{ }^{19} \mathrm{~F}$ spin-lattice relaxation time $T_{1 p}$ in the rotating frame for the three components obtained by deconvolution of the ${ }^{31} \mathrm{P}$ solid-state NMR spectra of annealed PBFP, and annealed PBFP with $5 \%$ of nano- $\mathrm{TiO}_{2}$ over the temperature range of 20 to 40 ${ }^{\circ} \mathrm{C}$

The ${ }^{19} \mathrm{~F} \mathrm{~T}_{\mathrm{ip}}$ spectra of the annealed samples from $20-40^{\circ} \mathrm{C}$ at for the components at $76.62(\mathrm{a}),-76.71(\mathrm{~s}),-76.95(\mathrm{a})$ and $-77.20 \mathrm{ppm}(\mathbf{m})$ are presented in Figure 8.19. Fast motion is indicated by increasing $\mathrm{T}_{\mathrm{l} \rho}$ with temperature for all four components. Moreover, in the annealed $\mathrm{NC} 5 \%$ sample all four components have longer $\mathrm{T}_{\mathrm{lp}}$ values compared to the annealed PBFP. Furthermore the temperature sensitivity of $T_{1 p}$ of the filled sample is consistently weaker for all four components, suggesting the crystallite sizes are smaller in all environments. The difference is most striking for the broad component at $-76.62 \mathrm{ppm}$ (i) which have the shortest $T_{1 \rho}$ values, suggesting that the presence of the filler in the rigid domain decreases the domain sizes. 


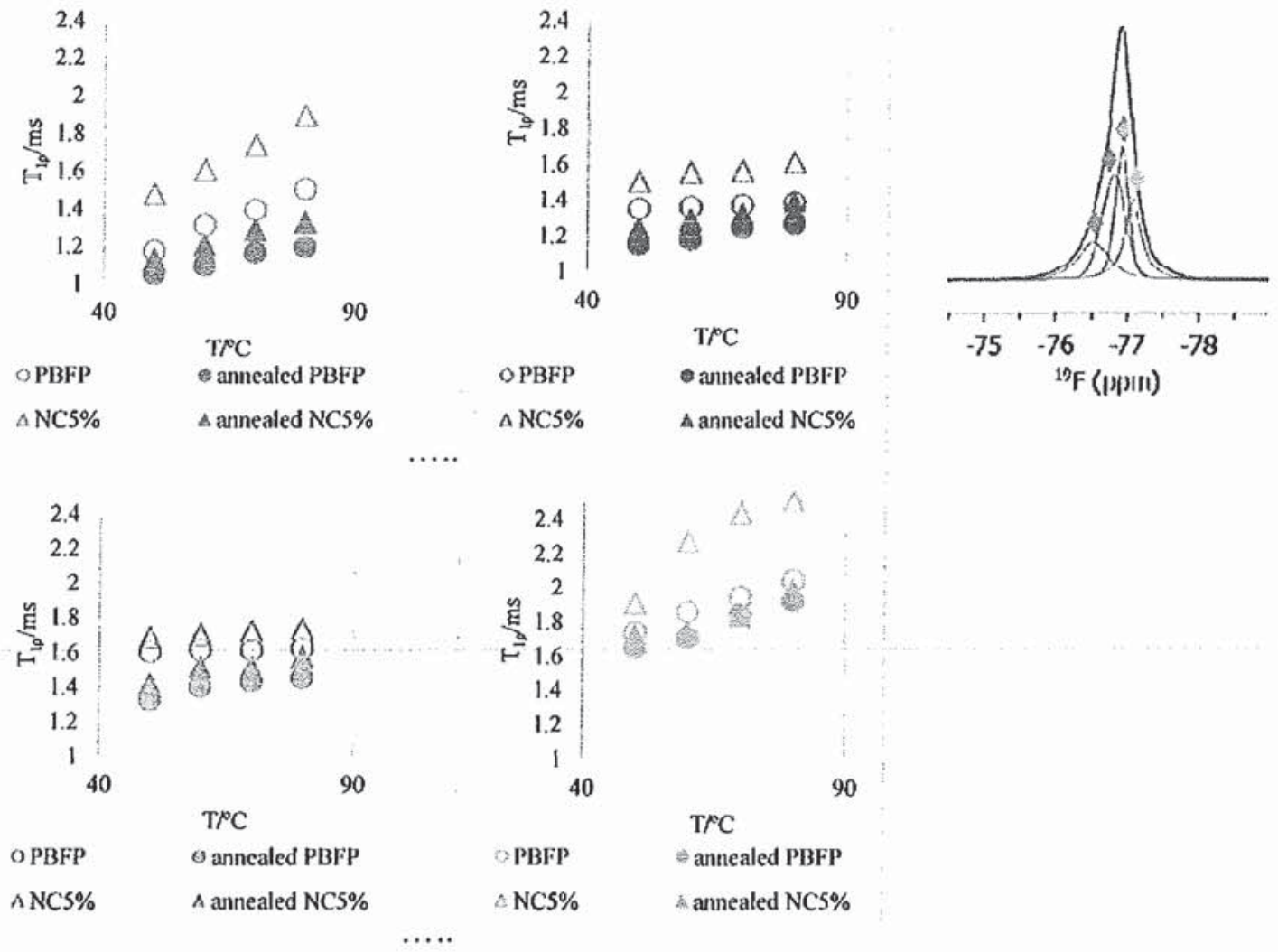

Figure 8.20: $\quad{ }^{19} \mathrm{~F}$ spin-lattice relaxation time $T_{10}$ in the rotating frame for the three components obtained by deconvolution of the ${ }^{31} \mathrm{P}$ solid-state NMR spectra of PBFP, annealed PBFP, PBFP with $5 \%$ nano- $\mathrm{TiO}_{2}$ and PBFP with $5 \%$ of nano- $\mathrm{TiO}_{2}$ over the temperature range of 50 to $80^{\circ} \mathrm{C}$

The $\mathrm{T}_{1 \mathrm{p}}$ values of ${ }^{19} \mathrm{~F}$ over the temperature range $50-80^{\circ} \mathrm{C}$ for the components at -

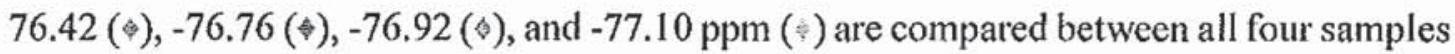
in Figure 8.20. The filled samples always have a larger $T_{I_{p}}$ than the non-filled samples, and also, the annealed samples consistently have smaller $T_{i p}$ values compared with the nonannealed samples. The components at $-76.42(\dot{ })$ and $-76.76(\diamond) \mathrm{ppm}$ have the largest variation between samples, especially between the annealed and non-annealed samples, whereas that $-76.92(\hat{\beta})$ exhibits the least variation. The broad component at $-76.42 \mathrm{ppm}$ $(\phi)$ in the non-annealed samples has the highest temperature sensitivity and exhibits the largest change upon filling. This provides support that nano-filling decreases crystallite 
size in the less mobile environments. Furthermore, this supports the previous conclusion that $-76.42(\hat{s})$ and $-76.76(\hat{s})$ ppm originate from the broad component at low temperature, while $-76.92($ ) $)$ corresponds to a highly mobile environment. 


\section{Reference}

1 Mcdonald, P. J.; Lane, D. M., Polymer Surfaces and Interfaces III 1997: 237-267.

2 Nicotetra, I.; Khalfan, A.; Goenaga, G.; Zhang, T., Ionics 2007, 14: 243-253.

3 Baglio, V.; Arico, A. S.; Antonucci, V., Journal of Power Sources 2006, 163: 52-55

4 Davies, G. L.; Corr, S. A.; Meledandri, C. J., ChemphysChem 2011, 12: 772-776.

5 Heisaburo, S., Biopolymers 1980, 19: 509-522. 


\section{CHAPTER 9}

\section{NMR CROSS POLARIZATION RESULTS $\left({ }^{19} \mathrm{~F} \rightarrow{ }^{31} \mathrm{P} \mathrm{CP}\right.$ and $\left.{ }^{1} \mathrm{H} \rightarrow{ }^{31} \mathrm{P} \mathrm{CP}\right)$ FOR PBFP AND ITS NANOCOMPOSITES}

\section{$9.1{ }^{19} \mathrm{~F} \rightarrow{ }^{31} \mathrm{PCP}$ NMR Spectroscopy for PBFP/Annealed PBFP and NC 5\%/Annealed NC 5\%}

The ${ }^{31} \mathrm{P}$ NMR relaxation times in chapter 8 showed that some components in the ${ }^{31} \mathrm{P}$ NMR spectrum exhibit bi-exponential behaviour with two distinct relaxation times indicating that the deconvolution model is not complete, even though it does not seem to be apparent in the spectra. In addition, when all the samples were heated from $20-80^{\circ} \mathrm{C}$, the spectra showed a sudden change near $50^{\circ} \mathrm{C}$, where the broad component at $-4.55 \mathrm{ppm}$ disappears and at least two new much narrower components appear. Both of these phenomena indicate that there must be more than four components in the ${ }^{31} \mathrm{P}$ NMR spectrum, and that the additional contribution must arise from the crystalline domain. Otherwise they should have been observed at lower temperature. To verify this hypothesis, a systematic study of the ${ }^{19} \mathrm{~F} \rightarrow{ }^{31} \mathrm{P}$ and ${ }^{1} \mathrm{H} \rightarrow{ }^{31} \mathrm{P}$ cross polarization dynamics was undertake ${ }^{2}$, which emphasises the rigid component signals over the mobile signals, and are furthermore independent of ${ }^{31} \mathrm{P} \mathrm{T}_{1}$. In this manner, any signals with very long ${ }^{31} \mathrm{P} \mathrm{T}_{1}$ values that may have been missed owing to saturation in the DP ${ }^{31} \mathrm{P}$ NMR spectra can be observed. The long $\mathrm{T}_{1}$ of ${ }^{31} \mathrm{P}$ and the low signal to noise ratio in the ${ }^{31} \mathrm{P}$ to ${ }^{1} \mathrm{H}$ and ${ }^{31} \mathrm{P}$ to ${ }^{19} \mathrm{~F}$ NMR CP experiment, due to unfavourable $\mathrm{CP}$ rates and short $\mathrm{T}_{1 \rho}$ times, made it impractical to obtain the complementary data sets for the $\mathrm{CP}$ dynamics ${ }^{3}$. Despite this limitation it is still possible to extract all the $\mathrm{CP}$ parameters from the ${ }^{1} \mathrm{H}$ to ${ }^{31} \mathrm{P}$ and ${ }^{19} \mathrm{~F}$ to ${ }^{31} \mathrm{P}$ results ${ }^{4}$. 


\subsection{1 ${ }^{19} \mathrm{~F} \rightarrow{ }^{31} \mathrm{P}$ CP NMR spectroscopy of PBFP}
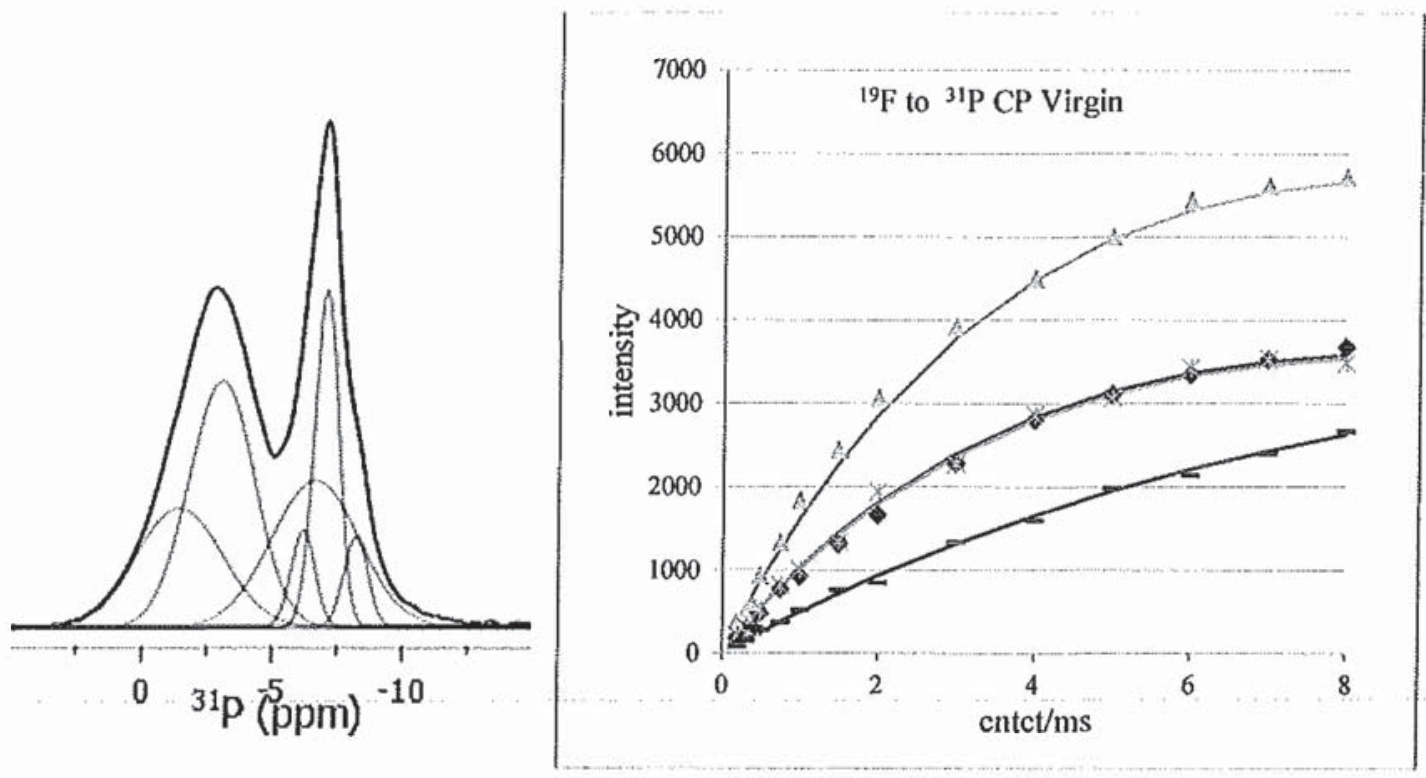

Figure 9.1: $\quad{ }^{19} \mathrm{~F}$ to ${ }^{31} \mathrm{P} \mathrm{CP}$ for PBFP deconvolution model (a, left); ${ }^{19} \mathrm{~F}$ to ${ }^{31} \mathrm{P}$ CP curve for PBFP (b, right) obtained with an MAS at $10 \mathrm{kHz}$ at $20^{\circ} \mathrm{C}$

Figure 9.1(a) shows the ${ }^{31} \mathrm{P}$ NMR spectra of PBFP obtained at a spinning rate of 10 $\mathrm{kHz}$ without proton decoupling using ${ }^{19} \mathrm{~F}$ to ${ }^{31} \mathrm{P} \mathrm{CP}$ pulse sequence. The spectra were fitted to a six-component model that includes three broad and three sharp components. The three narrow components at $-4.93,-8.38$ and $-9.48 \mathrm{ppm}$ have spectral width of 343,219 and 219 $\mathrm{Hz}$, which together have percentage area over $20 \%$, as presented in Figure 9.1 (a). These presumably correspond to the two narrow components in the original model given in Figure 7.10, and Table 7.4. Moreover, the ${ }^{31} \mathrm{p} \mathrm{T}_{1 \mathrm{p}}$ for the three components at $-2.32,-4.04$ and $7.96 \mathrm{ppm}$ are all $25 \mathrm{~ms}$, the corresponding ${ }^{19} \mathrm{~F} \mathrm{~T}_{1 \rho}$ values are $5 \mathrm{~ms}$ consistent with a rigid environment. In addition, the sum of the narrow components have much longer ${ }^{31} \mathrm{PT}_{1 \rho}$ and ${ }^{19} \mathrm{FT}_{1 \rho}$ values at 55 and $10 \mathrm{~ms}$, respectively, which is consistent with higher mobility. The $T_{1 p}$ values of both nuclei are longer than those shown in chapter 8 , as in this case they occur under mutual decoupling conditions, hence the heteronuclear coupling scales differently, reducing its contribution to the relaxation rate. 
Table 9.1: $\quad{ }^{19} \mathrm{~F}$ to ${ }^{31} \mathrm{P} \mathrm{CP}$ curve parameters of PBFP at $20^{\circ} \mathrm{C}$ obtained with an MAS rate of 10

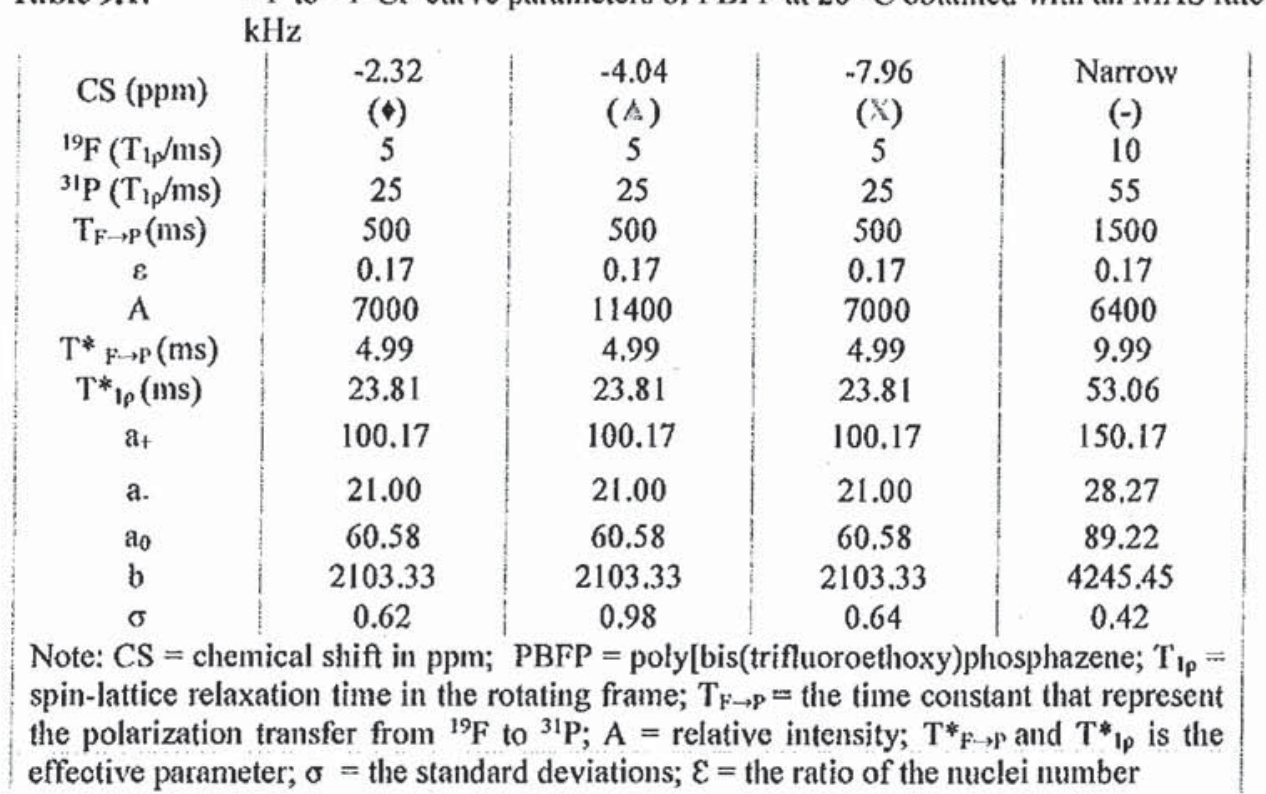

The CP curve (shown in figure 9.1(b)) for ${ }^{19} \mathrm{~F}$ to ${ }^{31} \mathrm{P}$ for sample PBFP is fitted to the function:

$$
\begin{gathered}
\mathrm{M}_{x}(t)=A\left\{-\exp \left(-\frac{t_{c p}}{T_{F p}^{*}}\right)+\exp \left(-\frac{t_{c p}}{T_{1 \rho}^{*}}\right)\right. \\
a_{+}=a_{0}+\sqrt{a_{0}^{2}-b} \\
a_{-}=a_{0}-\sqrt{a_{0}^{2}-b}
\end{gathered}
$$

where $T_{F P}^{*}, T_{1 \rho}^{*}$ are effective parameters adapted to the CP taking place between two abundant spins ${ }^{4}$, such as ${ }^{19} \mathrm{~F}$ and ${ }^{31} \mathrm{P}$, for which the expressions $T_{F p}^{* *}, T_{1 \rho}^{*}$ were introduced in chapter $1^{5}$. The expression above (Eqn. 9.1) characterizes the signal intensity of ${ }^{31} \mathrm{P}$ as a function of contact time, $t_{c p}$, where $A$ is the maximum signal intensity and $\varepsilon$ is the ratio of the number of ${ }^{31} \mathrm{P}$ to ${ }^{19} \mathrm{~F}$ spins, determined by the stoichiometry of PBFP as $1 / 6$. The parameters determined from the fitting of the experimental CP curves to Equation 9.1 are summarized in Table 9.1. The four solid lines represent the predicted CP curves for three 
broad and one narrow (sum of all narrow signals) components, where the corresponding symbols represent the experimental data. The quality of the fit between the predicted CP curve and the experimental data are indicated by the relative standard deviation ( $\sigma$ ) with respect to A, shown in Table 9.1.

The time constant $\mathrm{T}_{\mathrm{F}-\mathrm{P}}$ represents the polarization transfer from ${ }^{19} \mathrm{~F}$ to ${ }^{31} \mathrm{P}$ is determined by the analysis using the effective CP parameter. The $\mathrm{T}_{\mathrm{F}-\mathrm{p}}$ for the three broad components is $500 \mathrm{~ms}$, which implies an average dipolar coupling on the order of $2 \mathrm{~Hz}$, indicating an average static distance of $28.4 \AA$; however the true distance is much shorter as the dipolar coupling is scaled by rapid $\mathrm{C}-\mathrm{C}$ bond rotation of the $-\mathrm{CF}_{3}$ group. The same TF-P value for the three broad components implies that the sidechain conformation and motion are essentially the same for all three crystalline components. The Tr.p for the narrow component is much longer resulting from much enhanced sidechain motion in highly mobile environment as compared to that in the crystalline phases.

The relative intensity (A) of the signal at $-4.04 \mathrm{ppm}$ is 11400 , which is the largest, indicating it is the predominating component. The narrow components have an intensity totalling 6400 , indicating the relatively large contribution of the amorphous domain in the non-annealed neat polymer in excess of $16 \%$. The two equal intensities $(A)$ at -2.32 and $7.96 \mathrm{ppm}$ indicate the presence of either two essentially equally populated crystalline forms or two inequivalent ${ }^{31} \mathrm{P}$ environments in one crystalline form, in which case they would be represent the predominant contribution. Furthermore, the similar line width for the components at -2.32 and $-7.96 \mathrm{ppm}$ implies that they have similar dipolar coupling environments, meaning that the number of neighbouring ${ }^{31} \mathrm{P}$ nuclei and their average internuclear distances are the same. Also, the broad contribution between -2.32 and -4.04 
ppm has two components. This is in consistent with bi-exponential $\mathrm{T}_{1}$ behaviour seen previously.

\subsection{2 ${ }^{19} \mathrm{~F} \rightarrow{ }^{31} \mathrm{P}$ CP NMR spectroscopy of annealed PBFP}

Figure 9.2(a) shows the ${ }^{31}$ P NMR spectra of annealed PBFP obtained at a spin rate of $10 \mathrm{kHz}$ using ${ }^{19} \mathrm{~F}$ to ${ }^{31} \mathrm{P} \mathrm{CP}$ pulse sequence. The spectra were fitted into a five-component model that includes four broad and one sharp components. Two of the three narrow components previously seen in Figure 9.1 are lost, leaving only the most intense signal with a total percentage area less than $5 \%$, which is consistent with annealing decreasing its amorphous domain proportion. Additionally, one new broad component that appears at $5.57 \mathrm{ppm}$ is observed. Moreover, the ${ }^{31} \mathrm{PT}_{1 \rho}$ values for the four broad components at -2.32 , $-4.04,-7.96$ and $-5.57 \mathrm{ppm}$ are all $25 \mathrm{~ms}$, the corresponding ${ }^{19} \mathrm{~F} \mathrm{~T}_{\mathrm{I}_{\mathrm{p}}}$ values are $5 \mathrm{~ms}$, this is in consistent with the assumption that those four components represent the rigid domain. The narrow component has a much longer ${ }^{31} \mathrm{P}_{1 \rho}$ and ${ }^{19} \mathrm{~F} \mathrm{~T}_{1 \rho}$ value at $50 \mathrm{~ms}$ and $20 \mathrm{~ms}$, respectively. This is consistent with the higher mobility in the amorphous domain.
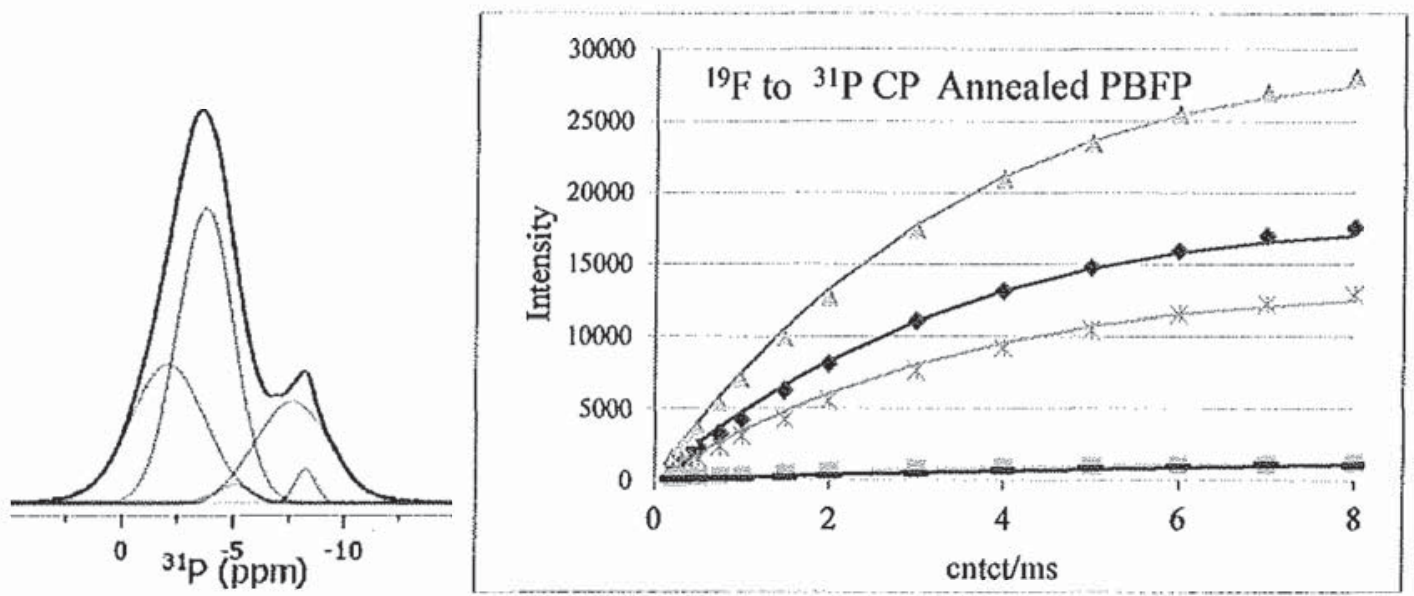

Figure 9.2: $\quad{ }^{19} \mathrm{~F}$ to ${ }^{31} \mathrm{P} \mathrm{CP}$ of annealed PBFP deconvolution model (a, left); ${ }^{19} \mathrm{~F}$ to ${ }^{31} \mathrm{P} \mathrm{CP}$ curve for annealed PBFP (b, right) obtained with an MAS at $10 \mathrm{kHz}$ at $20^{\circ} \mathrm{C}$ 
The CP curve (shown in Figure $9.2(\mathrm{~b})$ ) for both ${ }^{19} \mathrm{~F}$ to ${ }^{31} \mathrm{P}$ for annealed PBFP is fitted to Equation (9.1). The parameters determined from the fitting of the experimental CP curves to Equation (9.1) are summarized in Table 9.2.

Table 9.2: $\quad{ }^{19} \mathrm{~F}$ to ${ }^{31} \mathrm{P} \mathrm{CP}$ curve parameters of annealed PBFP at $20^{\circ} \mathrm{C}$ obtained with an MAS

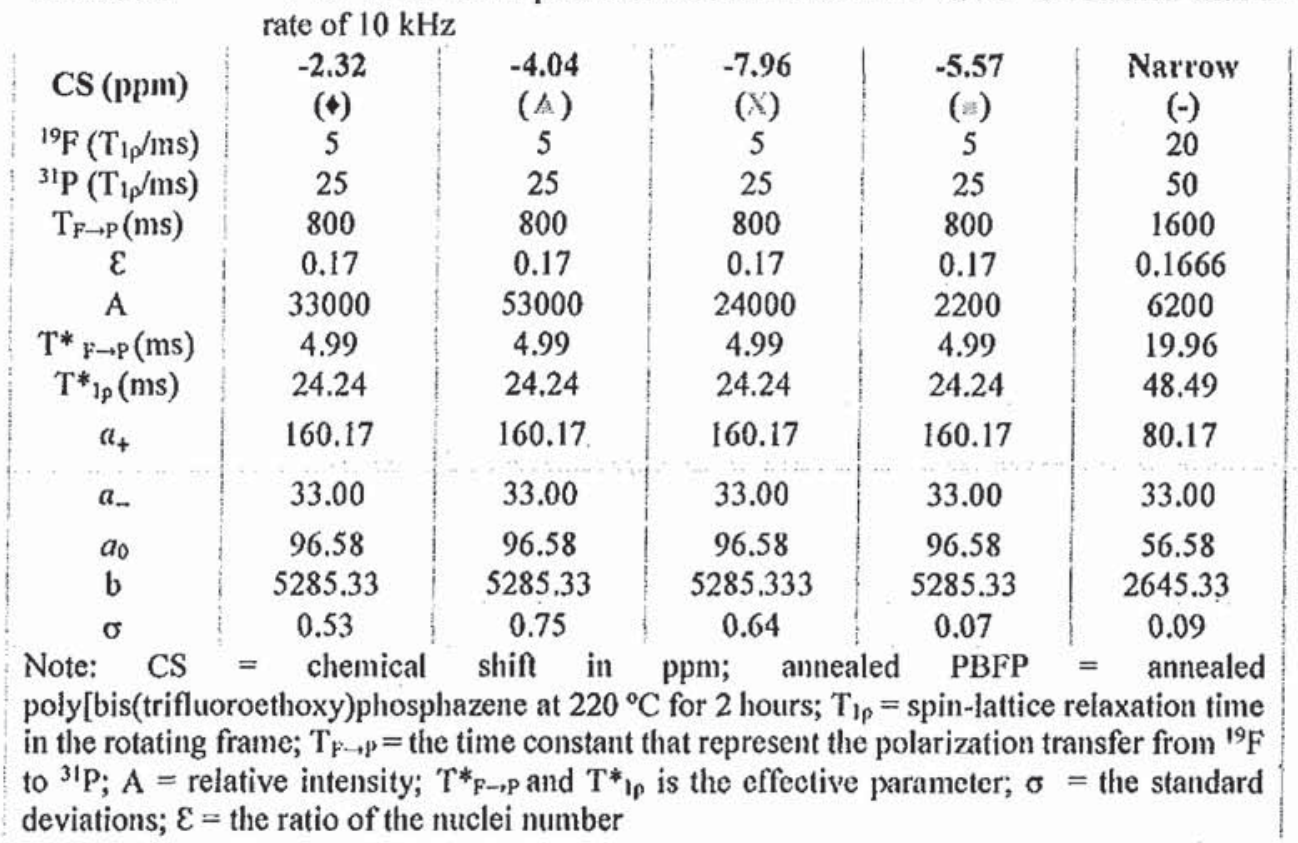

The $\mathrm{T}_{\mathrm{F} \rightarrow \mathrm{P}}$ for the four broad components are all $800 \mathrm{~ms}$, indicating an average dipolar coupling on the order of $1.25 \mathrm{~Hz}$, and an average static distance of $33.2 \AA$, implying that the side chain conformation and motion is the same for the rigid crystalline components. The weaker dipolar coupling between the ${ }^{19} \mathrm{~F}$ and ${ }^{31} \mathrm{P}$ results in a slightly enhance sidechain motion in this phase. Moreover, the largest relative intensity (A) at -4.04 ppm with a value at 53000 indicates that it is still the predominating component. The relative intensities at $2.32,-7.96$, and $-5.57 \mathrm{ppm}$ have changed with respect to the non-annealed polymer, implying that distribution the crystalline phases have changed upon annealing. 


\subsection{3 ${ }^{19} \mathrm{~F} \rightarrow{ }^{31} \mathrm{P}$ CP NMR spectroscopy of NC $5 \%$}

Figure 9.3 (a) shows the ${ }^{31}$ PNMR spectra of NC $5 \%$ obtained at a spin rate of $10 \mathrm{kHz}$ without proton decoupling using ${ }^{19} \mathrm{~F}$ to ${ }^{31} \mathrm{P} \mathrm{CP}$ pulse sequence. The spectra were fitted into a six-component model that includes four broad components and two narrow components. The model is similar as for sample annealed PBFP, where a small broad component appears at $-5.41 \mathrm{ppm}$, and the narrow component in table 9.3 represents the sum of the two sharp signals at -8.47 and $-9.65 \mathrm{ppm}$. Moreover, the ${ }^{31} \mathrm{P} \mathrm{T}_{\mathrm{l}_{\mathrm{p}}}$ for the components at $-2.32,-4.04,-$ $5.41,-7.96$ and $-5.41 \mathrm{ppm}$ are all $35 \mathrm{~ms}$, the corresponding ${ }^{19} \mathrm{~F}_{1_{\mathrm{p}}}$ at $5 \mathrm{~ms}$ are congruent with a rigid environment. The narrow component (sum of the two sharp components) has much longer ${ }^{31} \mathrm{P}_{1 p}$ and ${ }^{19} \mathrm{~F} \mathrm{~T}_{1 p}$ values at 50 and $10 \mathrm{~ms}$, respectively, as listed in Table 9.3.
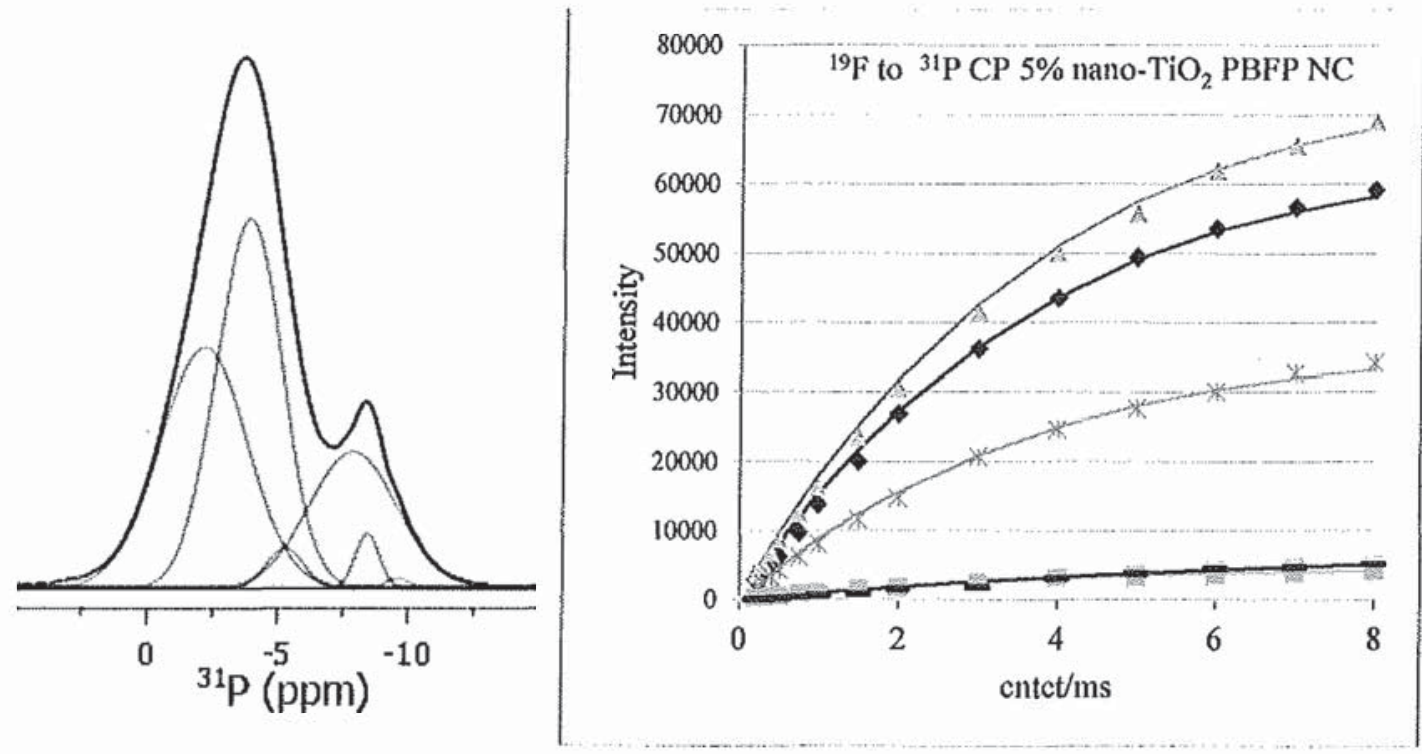

Figure 9.3: $\quad{ }^{19} \mathrm{~F}$ to ${ }^{31} \mathrm{P} \mathrm{CP}$ for $\mathrm{PBFP} / \mathrm{TiO}_{2} 5 \%$ deconvolution model (a, left); ${ }^{19} \mathrm{~F}$ to ${ }^{31} \mathrm{P} \mathrm{CP}$ curve for $\mathrm{PBFP} / \mathrm{TiO}_{2} 5 \%$ (b, right) obtained with an $\mathrm{MAS}$ at $10 \mathrm{kHz}$ at $20^{\circ} \mathrm{C}$

The CP curve (shown in Figure $\left(9.3\right.$ (b)) for ${ }^{19} \mathrm{~F}$ to ${ }^{31} \mathrm{P}$ for sample NC $5 \%$ is fitted to Equation (9.1). The parameters determined from the fitting of the experimental CP curves to Equation (9.1) are summarized in Table 9.3. 
Table 9.3: $\quad{ }^{19} \mathrm{~F}$ to ${ }^{31} \mathrm{P} \mathrm{CP}$ curve parameters of $\mathrm{PBFP} / \mathrm{TiO}_{2} 5 \%$ at $20^{\circ} \mathrm{C}$ obtained with an MAS rate of $10 \mathrm{kHz}$

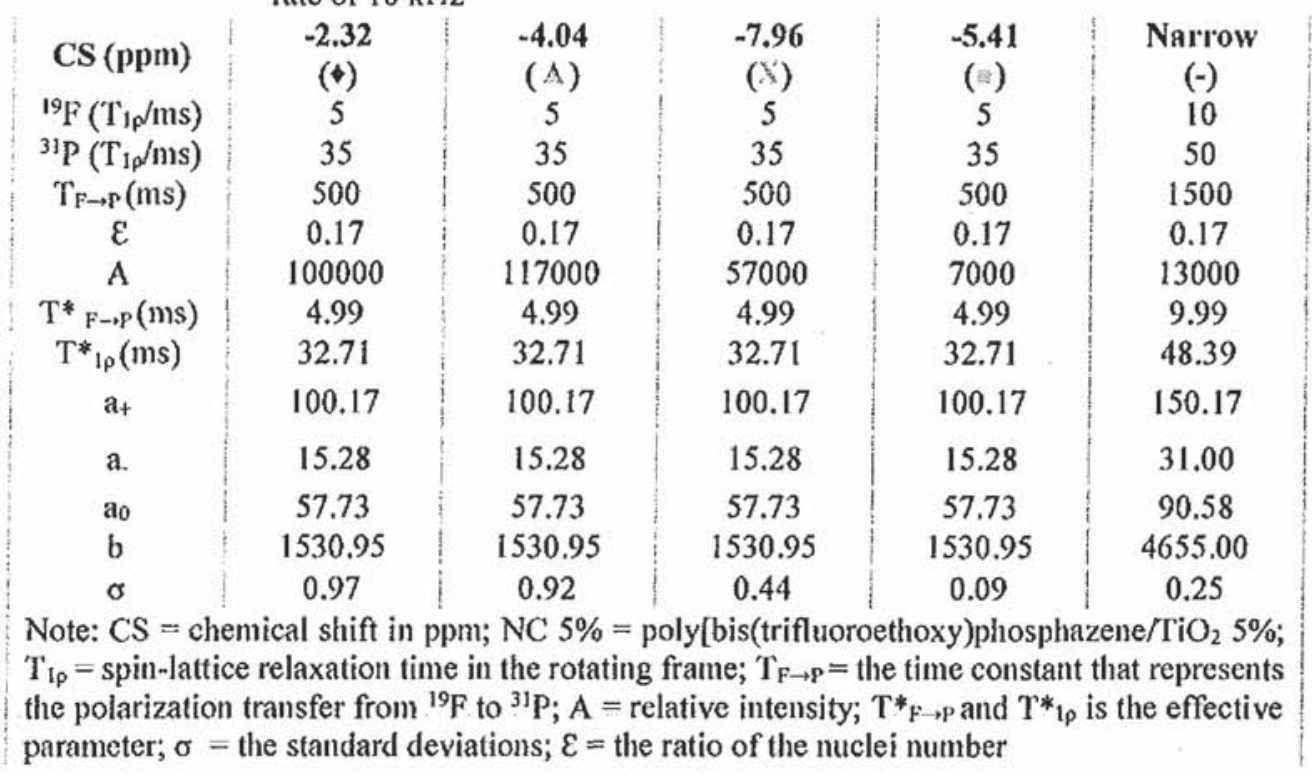

In Table 9.3, the $\mathrm{T}_{\mathrm{p} \rightarrow \mathrm{p}}$ for the four broad components are all $500 \mathrm{~ms}$, indicating an average dipolar coupling on the order of $2 \mathrm{~Hz}$, and an average static distance of $28.4 \AA$, implying that the side chain conformation and mobility are essentially the same for all the crystalline phases. The coupling between the ${ }^{19} \mathrm{~F}$ and ${ }^{31} \mathrm{P}$ is unchanged upon nano-filling, which means that the presence of the $\mathrm{TiO}_{2}$ crystallites does not significantly influence the sidechain motion and $-\mathrm{CF}_{3}$ rotation in the crystalline phases. In addition, the largest relative intensity (A) at $-4.04 \mathrm{ppm}$ with a value at 117000 indicates that it is still the predominating component. The relative intensity (A) at $-2.32,-7.96$, and $-5.41 \mathrm{ppm}$ are changed with respect to the neat polymer, implying that the distribution of the crystalline phases is influenced by nano-filling. The relative intensity of the narrow components indicate there is about $4 \%$ amorphous content, which suggests that the filler destabilizes the amorphous phase. 


\subsection{4 ${ }^{19} \mathrm{~F} \rightarrow{ }^{31} \mathrm{P}$ CP NMR spectroscopy of annealed NC 5\%}

Figure 9.4 (a) shows the ${ }^{31}$ P NMR spectra of ammealed NC $5 \%$ obtained at a spin rate of $10 \mathrm{kHz}$ without proton decoupling using ${ }^{19} \mathrm{~F}$ to ${ }^{31} \mathrm{P} \mathrm{CP}$ pulse sequence. The spectra were fitted into a six-component model including four broad and two sharp components, similar to the model for NC 5\%. Moreover, the ${ }^{31} \mathrm{P}$ and ${ }^{19} \mathrm{~F} \mathrm{~T}_{1 \rho}$ values for these broad components are 20 and $5 \mathrm{~ms}$, respectively, which is consistent with a rigid environment. The sum of the narrow components has the ${ }^{31} \mathrm{PT}_{1 \rho}$ value at $50 \mathrm{~ms}$ and a much longer ${ }^{19} \mathrm{~F} \mathrm{~T}_{1 \rho}$ at $20 \mathrm{~ms}$, which would be expected for the highly mobile amorphous phase.

The CP curves in Figure 9.4 (b) for ${ }^{19} \mathrm{~F}$ to ${ }^{31} \mathrm{P}$ for sample NC $5 \%$ is fitted to Equation (9.1). The parameters are determined from the fitting of the experimental CP curves to Equation 9.1 are summarized in Table 9.4.
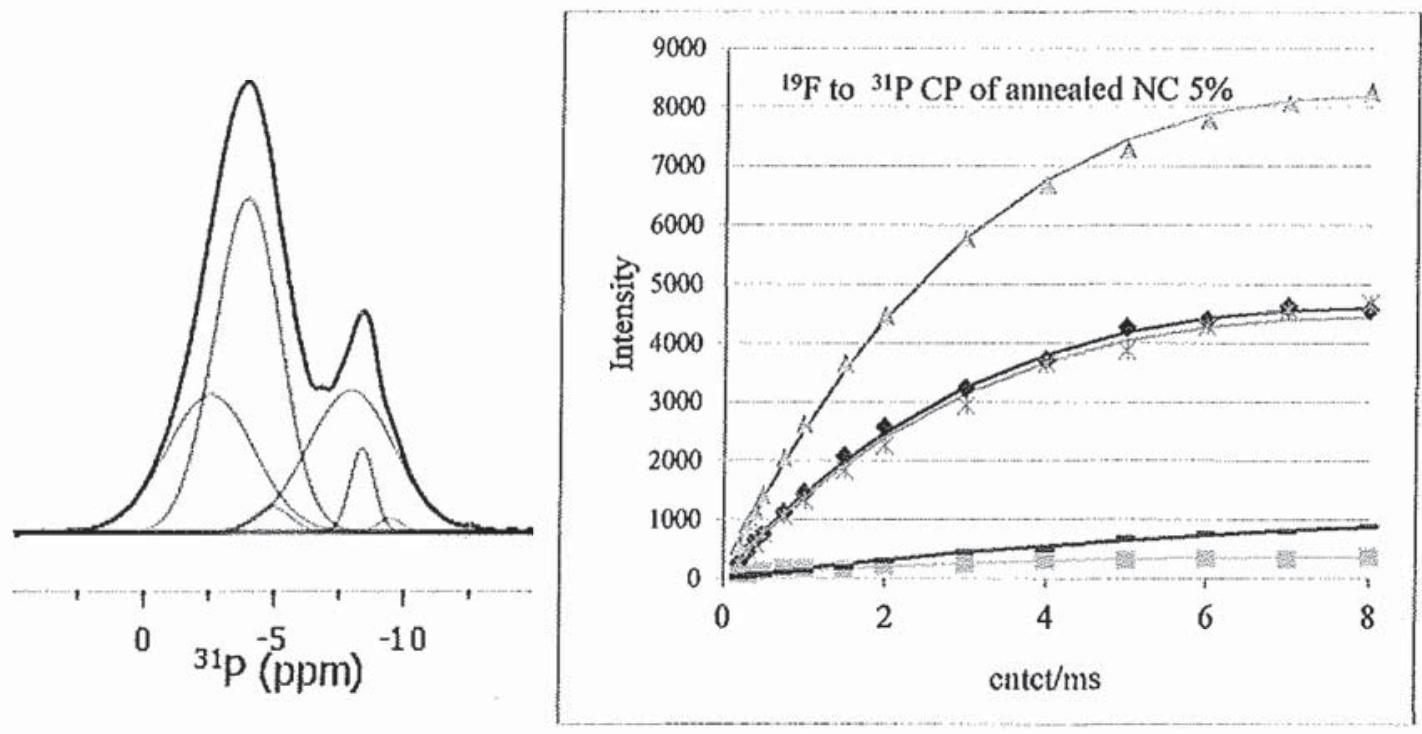

Figure 9.4: $\quad{ }^{19} \mathrm{~F}$ to ${ }^{31} \mathrm{P} C \mathrm{CP}$ for $\mathrm{PBFP}$ deconvolution model (a, left); ${ }^{19} \mathrm{~F}$ to ${ }^{31} \mathrm{P} \mathrm{CP}$ curve $(\mathrm{b}$, right) of annealed $\mathrm{PBFP} / \mathrm{TiO}_{2} 5 \%$ obtained with an MAS at $10 \mathrm{kHz}$ at $20^{\circ} \mathrm{C}$

The $\mathrm{T}_{\text {F-P }}$ value for the four broad components is $800 \mathrm{~ms}$, which implies an average dipolar coupling on the order of $1.25 \mathrm{~Hz}$, indicating an average static distance of $33.2 \AA$. The same $T_{\text {F-p }}$ value for the four broad components implies that the sidechain conformation 
and mobility is essentially the same for the crystalline components. The reduced dipole coupling is the same as in the annealed neat polymer, suggesting that nano-filling does not affect the sidechain mobility obviously nor $-\mathrm{CF}_{3}$ rotation to a significant extent in the crystalline phases of the annealed material. Additionally, the relative intensity (A) at -3.91 ppm is 18000 , which is the largest one, indicating it is the predominating component. Again the amorphous content is much reduced over the neat polymer at about $10 \%$, which is consistent with the annealing process; however, it does suggest that the nano-filler does alter the change in crystalline phase distribution upon annealing.

Table 9.4: $\quad{ }^{19} \mathrm{~F}$ to ${ }^{31} \mathrm{P} \mathrm{CP}$ curve parameters of annealed $\mathrm{PBFP}_{\mathrm{TiO}} 5 \%$ obtained with an $\mathrm{MAS}$

$\mathrm{CS}(\mathrm{ppm})$
${ }^{19} \mathrm{~F}\left(\mathrm{~T}_{1 \mathrm{p}} / \mathrm{ms}\right)$
${ }^{31} \mathrm{P}\left(\mathrm{T}_{1 \rho} / \mathrm{ms}\right)$
$\mathrm{T}_{\mathrm{F} \rightarrow \mathrm{p}}(\mathrm{ms})$
$\mathcal{E}(\mathrm{ms})$
$\mathrm{A}$
$\mathrm{T}^{*}{ }_{\mathrm{p} \rightarrow \mathrm{p}}(\mathrm{ms})$
$\mathrm{T}^{*}{ }_{1 p}(\mathrm{~ms})$
$\mathrm{a}_{+}$
a.
$\mathrm{a}_{0}$
b
$\sigma$
Note: CS
rate of $10 \mathrm{kHz}$ at $20^{\circ} \mathrm{C}$

poly[bis(trifluoroethoxy)phosphazene $/ \mathrm{TiO}_{2} 5 \% ; \mathrm{T}_{1 \rho}=$ spin-lattice relaxation time in the rotating frame; $\mathrm{T}_{\mathrm{F} \rightarrow \mathrm{P}}=$ the time constant that represents the polarization transfer from ${ }^{19} \mathrm{~F}$ to ${ }^{31} \mathrm{P} ; \mathrm{A}=$ relative intensity; $T *_{F \rightarrow P}$ and $T *_{1 \rho}$ is the effective parameter; $\sigma=$ the standard deviations; $\mathcal{E}=$ the ratio of the nuclei number 


\section{$9.2{ }^{1} \mathrm{H} \rightarrow{ }^{31} \mathrm{PCP}$ NMR Spectroscopy of PBFP/Annealed PBFP and NC 5\%/ Annealed NC 5\%}

\subsection{1 ${ }^{1} \mathrm{H} \rightarrow{ }^{31}$ P CP NMR spectroscopy of PBFP}

Figure 9.5 (a) shows that the ${ }^{31}$ P NMR spectra of PBFP were obtained at a spin rate of $10 \mathrm{kHz}$ without fluorine decoupling using ${ }^{1} \mathrm{H}$ to ${ }^{31} \mathrm{PCP}$ pulse sequence. The spectra were fitted into a six component model that includes three broad and three sharp components, which is the same as the model used for ${ }^{1} \mathrm{H}$ to ${ }^{31} \mathrm{P}$ CP for PBFP in Figure 9.1 (a).
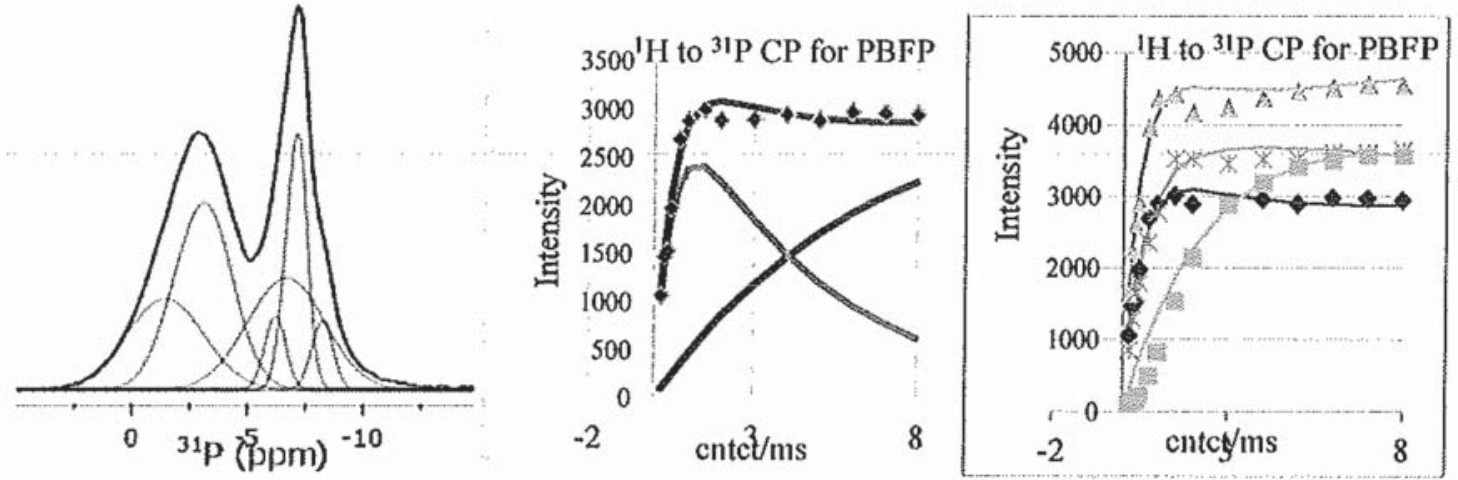

Figure 9.5:

${ }^{1} \mathrm{H}$ to ${ }^{31} \mathrm{P}$ CP for sample PBFP deconvolution model (left, a); ${ }^{1} \mathrm{H}$ to ${ }^{31} \mathrm{P}$ for one component, including the sum of two composition (blue); the faster CP curve (green); and slow CP curve (red)(middle, b); ' $\mathrm{H}$ to ${ }^{31} \mathrm{P} \mathrm{CP}$ curve for sample PBFP (right, c) ) obtained with an MAS at $10 \mathrm{kHz}$ at $20^{\circ} \mathrm{C}$

The $\mathrm{CP}$ curve in Figure 9.5 (c) for ${ }^{1} \mathrm{H}$ to ${ }^{31} \mathrm{P}$ for sample PBFP is fitted to the function:

$$
M_{x}(t)=A\left\{-\exp \left(-\frac{t_{c p}}{T_{H p}^{*}}\right)+\exp \left(-\frac{t_{c p}}{T_{i p}^{*}}\right)\right\}
$$

Similar to Equation (9.1), $T_{H P}^{*}, T_{1 \rho}^{*}$ are effective parameters adapted to the CP taking place between ${ }^{1} \mathrm{H}$ and ${ }^{31} \mathrm{P}$. The corresponding parameters determined from the fitting of the experimental CP curves to Equation 9.4 are summarized in Table 9.5. The results show that there are two CP curves for each component in Figure 9.5 (a). More specifically, for one CP curve series, the component at $-1.39,-3.02$, and $-6.66 \mathrm{ppm}$ have ${ }^{31} \mathrm{P} \mathrm{T}_{1 \mathrm{p}}$ at $0.65 \mathrm{~ms}$ and ${ }^{1} \mathrm{H} \mathrm{T}_{1 \rho}$ at 7-9 ms. In the other $\mathrm{CP}$ curve series, the $T_{1 \rho}$ values for ${ }^{31} \mathrm{P} \mathrm{T}_{1_{\mathrm{p}}}$ and ${ }^{1} \mathrm{H} \mathrm{T}_{1_{\rho}}$ are 10 
and $200 \mathrm{~ms}$, for the three broad components, and 3.4 and $30 \mathrm{~ms}$, for the one narrow component.

Table 9.5: $\quad{ }^{1} \mathrm{H}$ to ${ }^{31} \mathrm{P}$ CP curve parameters for sample PBFP of part 1 (left) and part 2 (right) and

\begin{tabular}{|c|c|c|c|c|c|c|c|c|}
\hline & \multicolumn{8}{|c|}{ the standard deviation obtained with an MAS at $10 \mathrm{kHz}$ at $20^{\circ} \mathrm{C}$} \\
\hline $\operatorname{CS}(\mathrm{ppm})$ & $\begin{array}{c}-1.39 \\
(\$)\end{array}$ & $\begin{array}{l}-3.02 \\
(A)\end{array}$ & $\begin{array}{l}-6.66 \\
(X)\end{array}$ & $\mathrm{CS}(\mathrm{ppm})$ & $\begin{array}{c}-1.39 \\
(\bullet)\end{array}$ & $\begin{array}{c}-3.02 \\
(A)\end{array}$ & $\begin{array}{l}-6.66 \\
(X)\end{array}$ & $\begin{array}{l}\text { Narrow } \\
\text { (㵷) }\end{array}$ \\
\hline${ }^{1} \mathrm{H}\left(\mathrm{T}_{1, \rho} / \mathrm{ms}\right)$ & 7 & 7 & 9 & ${ }^{\prime} \mathrm{H}\left(\mathrm{T}_{\mathrm{t} \rho} / \mathrm{ms}\right)$ & 200 & 200 & 200 & 30 \\
\hline${ }^{31} \mathrm{P}\left(\mathrm{T}_{1 \mathrm{p}} / \mathrm{ms}\right)$ & 0.65 & 0.65 & 0.65 & ${ }^{31} \mathrm{P}\left(\mathrm{T}_{1 \mathrm{p}} / \mathrm{ms}\right)$ & 10 & 10 & 10 & 3.4 \\
\hline $\mathrm{T}_{\mathrm{H} \rightarrow \mathrm{P}}(\mathrm{ms})$ & 2.5 & 1.5 & 11 & $\mathrm{~T}_{\mathrm{H} \rightarrow \mathrm{P}}(\mathrm{ms})$ & 30 & 20 & 20 & 800 \\
\hline$\varepsilon$ & 0.25 & 0.25 & 0.25 & $\varepsilon$ & 0.25 & 0.25 & 0.25 & 0.25 \\
\hline A & 3600 & 5000 & 3600 & A & 3900 & 6500 & 3800 & 5350 \\
\hline $\mathrm{T}^{*} \mathrm{H} \rightarrow \mathrm{P}(\mathrm{ms})$ & 0.51 & 0.44 & 0.61 & $\mathrm{~T}^{*} \mathrm{H} \rightarrow \mathrm{P}(\mathrm{ms})$ & 7.37 & 6.47 & 6.47 & 3.386 \\
\hline $\mathrm{T}^{*}{ }_{1 \rho}(\mathrm{ms})$ & 4.55 & 3.96 & 7.55 & $\mathrm{~T}^{*}{ }_{\mathrm{i} \rho}(\mathrm{ms})$ & 90.40 & 77.2800 & 77.28 & 29.72 \\
\hline$a_{+}$ & 4.90 & 3.39 & 17.94 & $a_{+}$ & 4.07 & 3.09 & 3.09 & 236.30 \\
\hline a. & 0.55 & 0.38 & 1.46 & a. & 0.33 & 0.26 & 0.26 & 26.92 \\
\hline$a_{0}$ & 2.73 & 1.89 & 9.70 & $a_{0}$ & 2.2 & 1.675 & 1.675 & 131.61 \\
\hline b & 2.69 & 1.29 & 26.14 & b & 1.35 & 0.8 & 0.8 & 6360 \\
\hline
\end{tabular}

CS (ppm)

$\sigma$

$$
\begin{array}{r}
-1.39 \\
0.29
\end{array}
$$

-3.02
0.42

$-6.66$

Narrow

0.11

Note: $\mathrm{CS}=$ chemical shift in ppm; PBFP = poly[bis(trifluoroethoxy)phosphazene; $\mathrm{T}_{\mathrm{lp}}=$ spinlattice relaxation time in the rotating frame; $T_{F \rightarrow P}=$ the time constant that represents the polarization transfer from ${ }^{19} \mathrm{~F}$ to ${ }^{31} \mathrm{P} ; \mathrm{A}=$ relative intensity; $\mathrm{T}^{*} \mathrm{~F} \rightarrow \mathrm{P}$ and $\mathrm{T}^{*}{ }_{1 \mathrm{p}}$ is the effective parameter; $\sigma=$ the standard deviations; $\mathcal{E}$ is the ratio of the nuclei number

Two different $T_{1 \rho}$ values for ${ }^{1} \mathrm{H}$ and ${ }^{31} \mathrm{P}$ were used to calculate the $T_{H P}^{*}, T_{1 \rho}^{*}$, separately, as listed in table 9.5. One of the ${ }^{1} \mathrm{H}$ to ${ }^{31} \mathrm{P}$ CP curve is similar to the ${ }^{19} \mathrm{~F}$ to ${ }^{31} \mathrm{P}$ that the $\mathrm{CP}$ curve builds up slower, while the remaining four compositions build up comparably faster. In Figure 9.5 (middle), the component at $-1.39 \mathrm{ppm}$ is chosen to show that the CP dynamics is composed of two contributions (this is also observed in remaining broad components). The total CP curves for three of the broad and one narrow (the sum of three sharp signals) components are shown in Figure 9.5(c).

The $\mathrm{T}_{\mathrm{H} \cdot \mathrm{p}}$ for each contribution to the broad components are very different, implying that there is a large disparity in their average dipolar couplings. This means that there are two distinct sidechain conformations of different mobility in each of these crystalline environments, which is consistent with the bi-exponential $T_{1}$ behaviour previously 
observed. Multimode CP dynamics was not seen in the ${ }^{19} \mathrm{~F}$ to ${ }^{31} \mathrm{P} \mathrm{CP}$ as it was obscured by the predominant scaling caused by rapid $-\mathrm{CF}_{3}$ rotation. The extremely small $\mathrm{T}_{\mathrm{H}-\mathrm{P}}$ values corresponds to averaged dipolar couplings of 400,666 and $90 \mathrm{~Hz}$, with static internuclear distances of $4.96,4.18,8.14 \AA$, respectively, suggesting very little sidechain motion. The longer more uniform $\mathrm{T}_{\mathrm{H}-\mathrm{P}}$ values between 20 to $30 \mathrm{~ms}$ in the second contribution to the $\mathrm{CP}$ dynamics, with average dipolar couplings of 33.3 to $50 \mathrm{~Hz}$, have corresponding static distances of 9.01 to $11.35 \AA$. This indicates a small but significant scaling effect due to increased sidechain motion. The relative intensity (A) at $-3.02 \mathrm{ppm}$ is the largest one amongst the broad components for both of $\mathrm{CP}$ curves parameters, as shown in Table 9.5, indicating it is the predominating component. The narrow components have $\mathrm{T}_{\mathrm{H}-\mathrm{P}}$ value of $800 \mathrm{~ms}$, with average dipolar coupling of $1.25 \mathrm{~Hz}$, which corresponds to a static distance of $33.9 \AA$, indicates very rapid sidechain motion as would be expected in the amorphous phase. The relative intensity of the narrow components indicate that there is approximately $16 \%$ amorphous content.

\subsection{2 ${ }^{1} \mathrm{H} \rightarrow{ }^{31} \mathrm{P} \mathrm{CP}$ NMR spectroscopy for samples annealed PBFP}

Figure 9.6 (a) shows the ${ }^{31} \mathrm{P}$ NMR spectra of annealed PBFP using ${ }^{1} \mathrm{H}$ to ${ }^{31} \mathrm{PCP}$ pulse sequence obtained at a spin rate of $10 \mathrm{kHz}$ without fluorine decoupling. The spectra were fitted into a four-component model, all of which are broad (no narrow signal are observed), and a new broad component at $-4.00 \mathrm{ppm}$ has appeared upon annealing. For the rapid $\mathrm{CP}$ curve, ${ }^{31} \mathrm{P}_{\mathrm{t}_{\mathrm{p}}}$ for -0.90 and $-2.46 \mathrm{ppm}$ are $3.1 \mathrm{~ms}$, for -6.33 and $-4.00 \mathrm{ppm}$ are $2.1 \mathrm{~ms}$, and the corresponding ${ }^{1} H T_{1 \rho}$ are $2 \mathrm{~ms}$, which is consistence with a rigid environment. While 
the slower CP curve for those components have ${ }^{31} \mathrm{P} \mathrm{T}_{1 \rho}$ at $10 \mathrm{~ms}$ and ${ }^{1} \mathrm{H} \mathrm{T}_{1 \rho}$ at $200 \mathrm{~ms}$, indicating enhanced mobility.
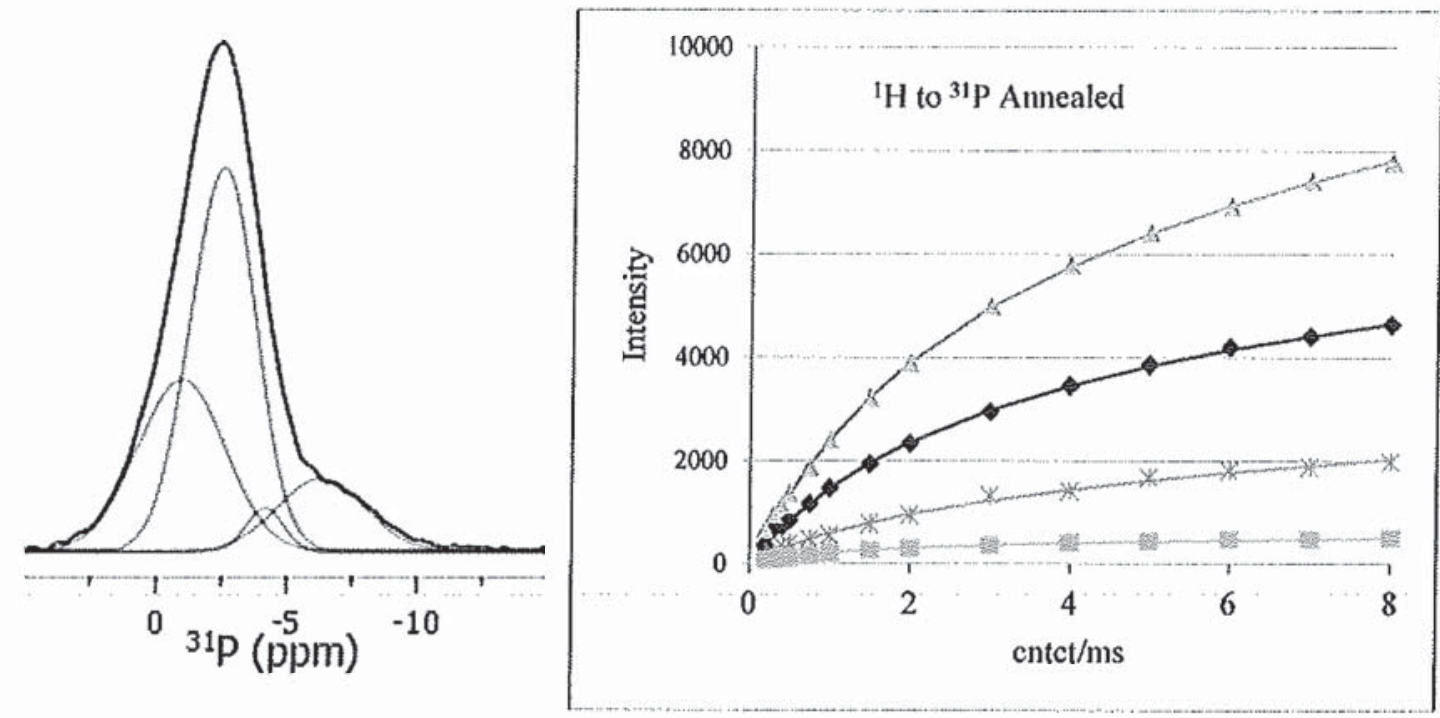

Figure 9.6: $\quad{ }^{1} \mathrm{H}$ to ${ }^{31} \mathrm{P} \mathrm{CP}$ for sample annealed PBFP deconvolution model (left); ${ }^{1} \mathrm{H}$ to ${ }^{31} \mathrm{P} \mathrm{CP}$ curve for sample annealed PBFP (right) obtained with an MAS at $10 \mathrm{kHz}$ at $20^{\circ} \mathrm{C}$

The multi-mode ${ }^{1} \mathrm{H}$ to ${ }^{31} \mathrm{P} \mathrm{CP}$ curves for each component in Figure 9.6 (b) for annealed PBFP are fitted to Equation (9.4). The corresponding fitting parameters are summarized in Table 9.6. Note that the contribution of the rapid CP dynamics is smaller compared to the slower dynamics. In the non-annealed sample these contribution were approximately equal. Annealing has changed this ratio to approximately 2:1.

The $\mathrm{T}_{\mathrm{H} \cdot \mathrm{P}}$ values for the broad components in the fast CP curves are uniformly $9 \mathrm{~ms}$, while in the slow CP curve, the $\mathrm{T}_{\mathrm{H} \cdot \mathrm{P}}$ values are $30-35 \mathrm{~ms}$. The average dipolar couplings are thus 110 and $28-33 \mathrm{~Hz}$, which have corresponding average static distances of 7.62 and 11.38-12.02 $\AA$, respectively. This means that there are two distinct sidechain conformations of different mobility in each of these crystalline environments. The mobility in all the crystalline environments is higher than seen in the neat polymer, suggesting that the annealing favour crystal phases with enhanced sidechain motion. 
Table 9.6: $\quad$ ' $\mathrm{H}$ to ${ }^{31} \mathrm{P}$ CP curve parameters for sample annealed PBFP of part 1 (left); part 2 (right) and the deviation error (bottom) obtained with an MAS at $10 \mathrm{kHz}$ at $20^{\circ} \mathrm{C}$

\begin{tabular}{|c|c|c|c|c|c|c|c|c|c|}
\hline \multirow[b]{2}{*}{$\mathrm{CS}(\mathrm{ppm})$} & & & & & & & & & \\
\hline & $\begin{array}{c}-0.90 \\
(\diamond)\end{array}$ & $\begin{array}{c}-2.46 \\
(\mathrm{~A})\end{array}$ & $\begin{array}{c}-6.33 \\
(X)\end{array}$ & $\begin{array}{c}-4.00 \\
(\mathrm{a})\end{array}$ & CS (ppm) & $\begin{array}{c}-0.90 \\
(\downarrow)\end{array}$ & $\begin{array}{l}-2.46 \\
(\triangle)\end{array}$ & $\begin{array}{c}-6.33 \\
(X)\end{array}$ & $\begin{array}{c}-4.00 \\
(\otimes)\end{array}$ \\
\hline${ }^{1} \mathrm{H}\left(\mathrm{T}_{\mathrm{lp}} / \mathrm{ms}\right)$ & 3.1 & 3.1 & 2.1 & 2.1 & ${ }^{1} \mathrm{H}\left(\mathrm{T}_{1} \mathrm{p} / \mathrm{ms}\right)$ & 200 & 200 & 200 & 200 \\
\hline${ }^{11} \mathrm{P}\left(\mathrm{T}_{1 \rho} / \mathrm{ms}\right)$ & 2 & 2 & 2 & 2 & ${ }^{31} \mathrm{P}\left(\mathrm{T}_{\mathrm{lp}} / \mathrm{ms}\right)$ & 10 & 10 & 10 & 10 \\
\hline $\mathrm{T}_{\mathrm{H}-\mathrm{P}}(\mathrm{ms})$ & 9 & 9 & 9 & 9 & $\mathrm{~T}_{\mathrm{H} \rightarrow \mathrm{P}}(\mathrm{ms})$ & 30 & 30 & 30 & 33 \\
\hline$\varepsilon$ & 0.25 & 0.25 & 0.25 & 0.25 & $\varepsilon$ & 0.25 & 0.25 & 0.25 & 0.25 \\
\hline A & 3100 & 5000 & 2200 & 1300 & A & 7750 & 13000 & 3500 & 850 \\
\hline $\mathrm{T}^{*} \mathrm{H} \rightarrow \mathrm{P}(\mathrm{ms})$ & 1.61 & 1.61 & 1.58 & 1.58 & $\mathrm{~T}^{*}{ }_{\mathrm{H} \rightarrow \mathrm{P}}(\mathrm{ms})$ & 7.37 & 7.37 & 7.67 & 7.56 \\
\hline $\mathrm{T}^{*}{ }_{1 \rho}(\mathrm{ms})$ & 2.95 & 2.95 & 2.08 & 2.08 & $T *{ }_{1 \rho}(\mathrm{ms})$ & 90.40 & 90.40 & 96.01 & 93.83 \\
\hline a+ & 5.60 & 5.60 & 5.71 & 5.71 & a+ & 4.07 & 4.07 & 4.56 & 4.36 \\
\hline a. & 3.05 & 3.05 & 4.32 & 4.32 & a. & 0.33 & 0.33 & 0.36 & 0.35 \\
\hline$a_{0}$ & 4.33 & 4.33 & 5.02 & 5.02 & $a_{0}$ & 2.2 & 2.2 & 2.46 & 2.36 \\
\hline b & 17.09 & 17.09 & 24.70 & 24.70 & b & 1.35 & 1.35 & 1.66 & 1.53 \\
\hline
\end{tabular}

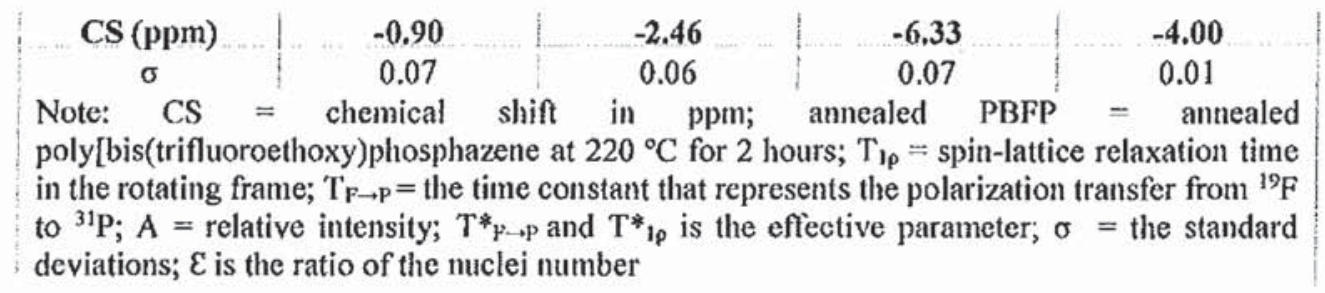

The relative intensity (A) at $-2.46 \mathrm{ppm}$ is the largest one amongst all the four broad components for both of $\mathrm{CP}$ curves parameter, indicating it is the predominating component. Moreover, the broad contribution between -0.90 and $-2.46 \mathrm{ppm}$ has to have components which is in consistent with its $\mathrm{T}_{1}$ bi-exponential relaxation previously observed.

\subsection{3 ${ }^{1} \mathrm{H} \rightarrow{ }^{31}$ P CP NMR spectroscopy of NC 5\%}

Figure 9.7 (a) shows the ${ }^{31} \mathrm{P}$ NMR spectra of NC $5 \%$ using ${ }^{1} \mathrm{H}$ to ${ }^{31} \mathrm{P}$ CP pulse sequence were obtained at a spin rate of $10 \mathrm{kHz}$ without fluorine decoupling. The spectra were fitted into a five-component model that include four broad components at $-0.90,-2.46$, -6.65 and $-3.67 \mathrm{ppm}$ and two sharp components at -6.85 and $-7.63 \mathrm{ppm}$. For the fast $\mathrm{CP}$ curve, the ${ }^{31} \mathrm{P}_{1_{1}}$ for these component at $-0.90,-2.46,-6.65$ and $-3.67 \mathrm{ppm}$ are $0.65 \mathrm{~ms}$ and 
the corresponding ' $\mathrm{H} \mathrm{T}_{\mathrm{l} \rho}$ are $7 \mathrm{~ms}$, which is consistent with a rigid phase. For the slower CP curves series, the ${ }^{31} \mathrm{P}_{1} \mathrm{~T}_{1 \rho}$ and ${ }^{1} \mathrm{H}_{1} \mathrm{~T}_{1 \rho}$ are 10 and $200 \mathrm{~ms}$, respectively, indicating higher mobility.
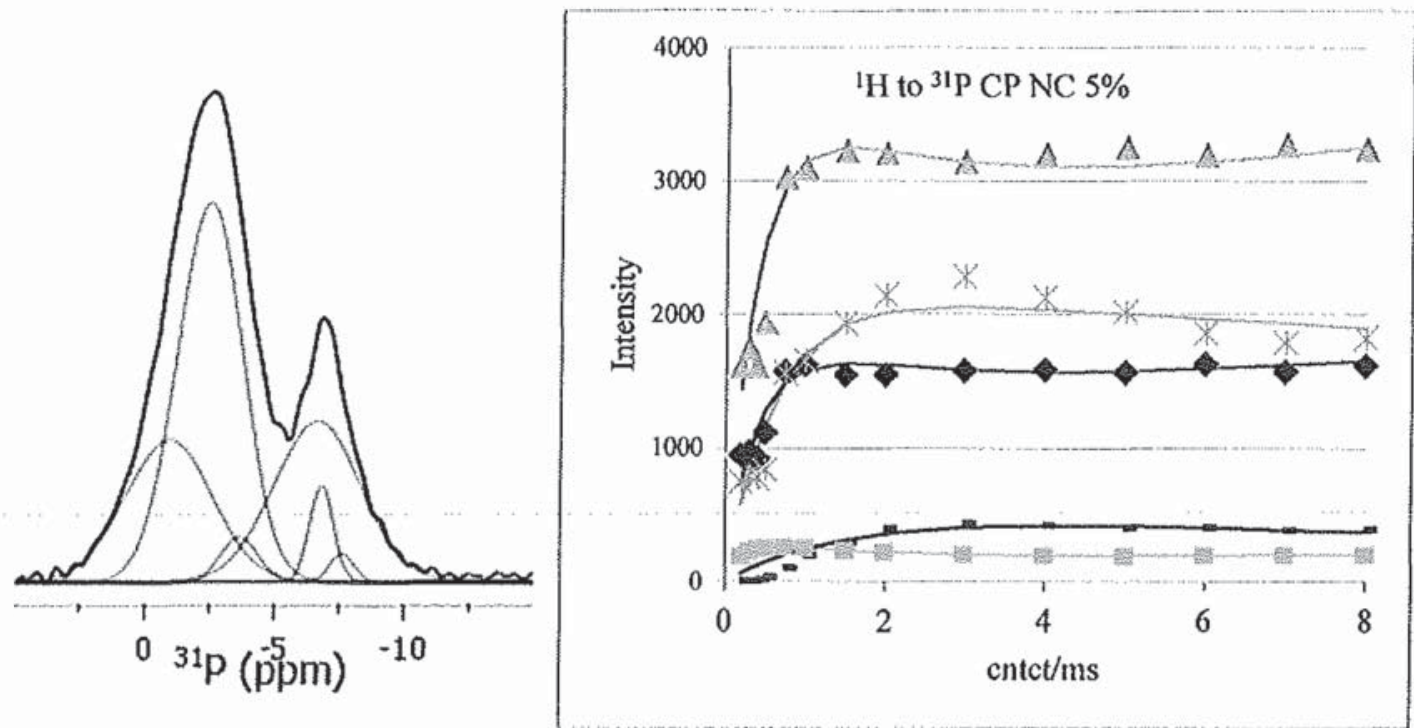

Figure 9.7: $\quad{ }^{1} \mathrm{H}$ to ${ }^{31} \mathrm{P} \mathrm{CP}$ for sample $\mathrm{NC} 5 \%-\mathrm{TiO}_{2}$ deconvolution model (left); ${ }^{1} \mathrm{H}$ to ${ }^{31} \mathrm{P} \mathrm{CP}$ curve for sample NC $5 \%$ (right) obtained with an MAS at $10 \mathrm{kHz}$ at $20^{\circ} \mathrm{C}$

The multimode ${ }^{1} \mathrm{H}$ to ${ }^{31} \mathrm{P} \mathrm{CP}$ curves for each component in Figure 9.7 (b) for $5 \% \mathrm{NC}$ PBFP are fitted in Equation (9.4). Table 9.7 shows the corresponding fitting parameters. Note that the contribution of the rapid CP dynamics is slightly smaller compared to the slower dynamics. Hence nano-filling seems to alter the distribution of the crystal phases somewhat favouring the phase with more mobile sidechains. 
Table 9.7: $\quad{ }^{1} \mathrm{H}$ to ${ }^{31} \mathrm{P}$ CP curve parameters for sample NC $5 \%$ of part 1 (left) and part 2 (right) obtained with an MAS at $10 \mathrm{kHz}$ at $20^{\circ} \mathrm{C}$

\begin{tabular}{|c|c|c|c|c|c|c|c|c|c|c|}
\hline CS (ppm) & $\begin{array}{c}-0.90 \\
(\diamond)\end{array}$ & $\begin{array}{c}-2.46 \\
(A)\end{array}$ & $\begin{array}{c}-6.65 \\
(X)\end{array}$ & $\begin{array}{c}-3.67 \\
(y)\end{array}$ & CS (ppm) & $\begin{array}{c}-0.90 \\
(4)\end{array}$ & $\begin{array}{c}-2.46 \\
\text { (A) }\end{array}$ & $\begin{array}{c}-6.65 \\
(X)\end{array}$ & $\begin{array}{c}-3.67 \\
(\text { ) }\end{array}$ & $\begin{array}{c}\text { Narrow } \\
(-)\end{array}$ \\
\hline${ }^{1} \mathrm{H}\left(\mathrm{T}_{1} / \mathrm{ms}\right)$ & 7 & 7 & 7 & 7 & ${ }^{1} \mathrm{H}\left(\mathrm{T}_{\mathrm{l} p} / \mathrm{ms}\right)$ & 200 & 200 & 200 & 200 & 30 \\
\hline${ }^{31} \mathrm{P}\left(\mathrm{T}_{\mathrm{ip}} / \mathrm{ms}\right)$ & 0.65 & 0.65 & 0.65 & 0.65 & ${ }^{31} \mathrm{P}\left(\mathrm{T}_{\mathrm{lp}} / \mathrm{ms}\right)$ & 10 & 10 & 10 & 10 & 3.3 \\
\hline $\mathrm{T}_{\mathrm{H} \rightarrow \mathrm{P}}(\mathrm{ms})$ & 1.1 & 1.1 & 11 & 0.3 & $\mathrm{~T}_{\mathrm{H} \rightarrow \mathrm{P}}(\mathrm{ms})$ & 30 & 30 & 30 & 20 & 800 \\
\hline E & 0.25 & 0.25 & 0.25 & 0.25 & $\varepsilon$ & 0.25 & 0.25 & 0.25 & 0.25 & 0.25 \\
\hline A & 1900 & 3800 & 2100 & 300 & A & 2500 & 4900 & 2500 & 300 & 600 \\
\hline $\mathrm{T}^{*}{ }_{\mathrm{H} \rightarrow \mathrm{P}}(\mathrm{ms})$ & 0.39 & 0.39 & 0.61 & 0.18 & $\mathrm{~T}_{\mathrm{H} \rightarrow \mathrm{P}}(\mathrm{ms})$ & 7.37 & 7.37 & 7.37 & 6.47 & 3.29 \\
\hline $\mathrm{T}^{*}{ }_{\mathrm{t} \rho}(\mathrm{ms})$ & 3.64 & 3.64 & 6.09 & 2.77 & $\mathrm{~T}^{*}{ }_{1 p}(\mathrm{~ms})$ & 90.40 & 90.40 & 90.40 & 77.28 & 29.72 \\
\hline$a_{+}$ & 2.80 & 2.80 & 17.94 & 1.65 & $a_{+}$ & 4.07 & 4.07 & 4.07 & 3.09 & 243.42 \\
\hline a. & 0.30 & 0.30 & 7.39 & 0.11 & a. & 0.33 & 0.33 & 0.33 & 0.26 & 26.91 \\
\hline$a_{0}$ & 1.55 & 1.55 & 1.81 & 0.88 & $a_{0}$ & 2.2 & 2.2 & 2.2 & 1.675 & 135.17 \\
\hline B & 0.85 & 0.85 & 32.39 & 0.18 & b & 1.35 & 1.35 & 1.35 & 0.8 & 6551.9 \\
\hline
\end{tabular}

\begin{tabular}{|c|c|c|c|c|c|}
\hline CS (ppm) & -0.9 & -2.46 & -6.65 & -3.67 & Narrow \\
\hline$\sigma$ & 0.32 & 0.78 & 0.52 & 0.09 & 0.22 \\
\hline
\end{tabular}

The $\mathrm{T}_{\mathrm{H}-\mathrm{P}}$ values for the broad components in the fast $\mathrm{CP}$ curve are uniformly $1.1 \mathrm{~ms}$ for 0.9 and-2.46 ppm, while that of $-6.65 \mathrm{ppm}$ is on an order of magnitude smaller at 11 ms. For the slow CP curve TH.P value is uniformly $30 \mathrm{~ms}$. In the former the average dipolar couplings are thus 900 and $90 \mathrm{~Hz}$, respectively, giving static internuclear distances of 5.11 and $11.01 \AA$, respectively. In the latter, the average dipolar coupling is $33 \mathrm{~Hz}$, with average internuclear distance of $15.38 \AA$. This means that there are two distinct sidechain conformations of different mobility in each of these crystalline environments; however the rigid environment at $-6.65 \mathrm{ppm}$ has more mobility than those at -0.9 and $-2.46 \mathrm{ppm}$. The difference in mobility is less pronounced than in the non-annealed samples, which is consistent with less obvious bi-exponential $T_{1}$ behaviour. The narrow signal has $T_{H \cdot P}$ value of $800 \mathrm{~ms}$, with an average dipole coupling of $1.25 \mathrm{~Hz}$, which indicate an average distance of $45.80 \AA$, thereby implying enhanced sidechain mobility in the amorphous phase. . 
The relative intensity $(\mathrm{A})$ at $-\mathbf{2 . 4 6} \mathrm{ppm}$ is the largest one amongst all the four broad components for both of $\mathrm{CP}$ curves parameter, as shown in Table 9.7, indicating it is the predominating component. The relative intensity of the narrow signals makes up $3 \%$ of the total, implying that nano-filling does reduce the amorphous component, thus destabilizing the amorphous phase.

\subsection{4 ${ }^{1} \mathrm{H} \rightarrow{ }^{31} \mathrm{P}$ CP NMR spectroscopy for annealed NC 5\%}
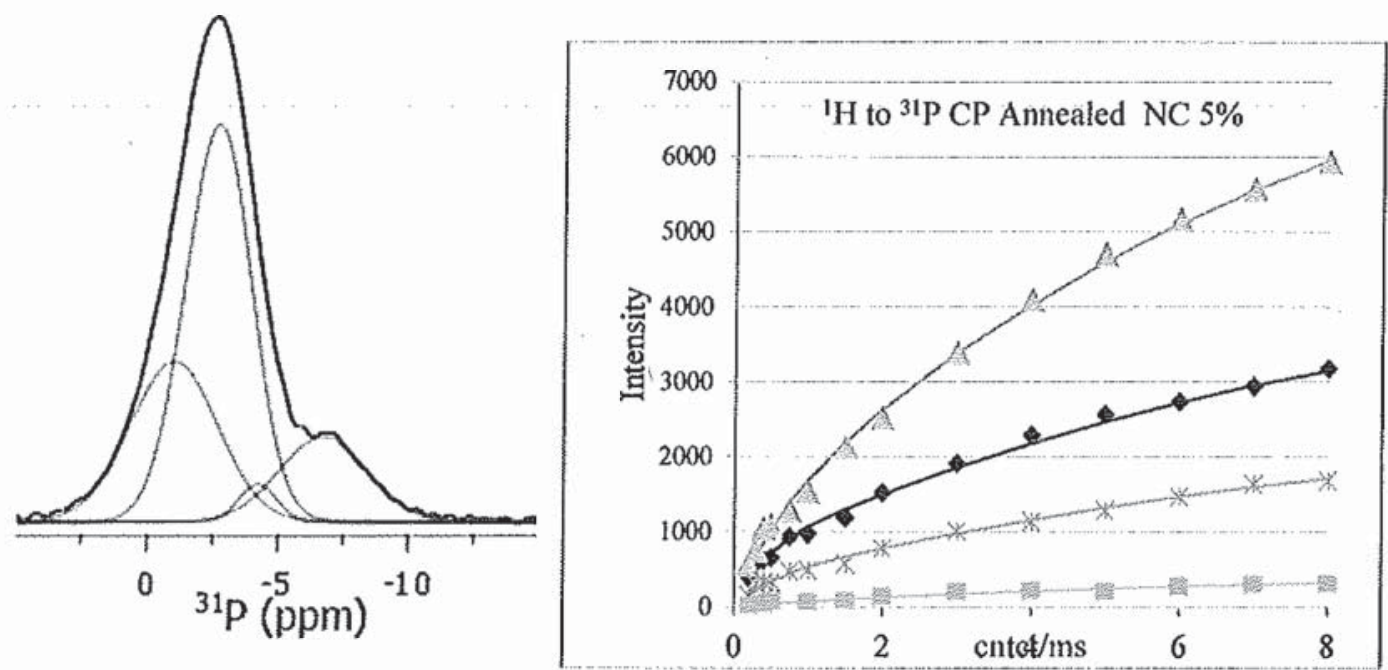

Figure 9.8: $\quad{ }^{1} \mathrm{H}$ to ${ }^{31} \mathrm{P} \mathrm{CP}$ for sample annealed NC 5\% deconvolution model (left); ${ }^{1} \mathrm{H}$ to ${ }^{31} \mathrm{P} \mathrm{CP}$ curve for sample annealed NC $5 \%$ (right) obtained with an MAS at $10 \mathrm{kHz}$ at $20^{\circ} \mathrm{C}$

The ${ }^{31} \mathrm{P}$ NMR spectra of annealed NC $5 \%$ using ${ }^{1} \mathrm{H}$ to ${ }^{31} \mathrm{P}$ CP pulse sequence were obtained at a spin rate of $10 \mathrm{kHz}$ without fluorine decoupling, as shown in Figure 9.8 (a). The spectra were fitted into a four-component model where all components are broad, which is similar to the model for sample annealed PBFP. For the fast $\mathrm{CP}$ curve, the ${ }^{31} \mathrm{P} \mathrm{T}_{1 \mathrm{p}}$ for components at $-1.08,-2.80$, and $-6.66 \mathrm{ppm}$ are $1.00 \mathrm{~ms}$ and the corresponding ${ }^{1} \mathrm{H} \mathrm{T}_{1 \mathrm{p}}$ are $3.10 \mathrm{~ms}$ and $2.10 \mathrm{~ms}$, which are consistent with a rigid environment. For the slower CP 
curve series, the ${ }^{31} \mathrm{P} \mathrm{T}_{1 p}$ and ${ }^{1} \mathrm{H} \mathrm{T}_{1 p}$ for those components are $10.00 \mathrm{~ms}$ and $200.00 \mathrm{~ms}$, separately, which is consistent with high mobility.

The CP curves in Figure 9.8 (b) for ${ }^{1} \mathrm{H}$ to ${ }^{31} \mathrm{P}$ for sample annealed $\mathrm{NC} 5 \%$ is fitted to Equation (9.4). The corresponding parameters determined from the fitting of the experiment curves to Equation (9.4) are summarized in Table 9.8.

Table 9.8: $\quad{ }^{1} \mathrm{H}$ to ${ }^{31} \mathrm{P}$ CP curve parameters for sample annealed NC $5 \%$ of part one (left) and

\begin{tabular}{|c|c|c|c|c|c|c|c|c|}
\hline CS (ppm) & $\begin{array}{c}-1.08 \\
(\downarrow)\end{array}$ & $\begin{array}{c}-2.80 \\
\text { (A) }\end{array}$ & $\begin{array}{c}-6.66 \\
(X)\end{array}$ & CS (ppm) & $\begin{array}{c}-1.08 \\
(4)\end{array}$ & $\begin{array}{c}-2.80 \\
(\mathrm{~A})\end{array}$ & $\begin{array}{c}-6.66 \\
(\mathrm{X})\end{array}$ & $\begin{array}{c}-4.20 \\
(1)\end{array}$ \\
\hline${ }^{1} \mathrm{H}\left(\mathrm{T}_{1 \mathrm{p}} / \mathrm{ms}\right)$ & 3.1 & 3.1 & 2.1 & ${ }^{1} \mathrm{H}\left(\mathrm{T}_{1 \rho} / \mathrm{ms}\right)$ & 200 & 200 & 200 & 200 \\
\hline${ }^{31} \mathrm{P}\left(\mathrm{T}_{1 \rho} / \mathrm{ms}\right)$ & 1 & 1 & 1 & ${ }^{31} \mathrm{P}\left(\mathrm{T}_{1 / \mathrm{p}} / \mathrm{ms}\right)$ & 10 & 10 & 10 & 10 \\
\hline $\mathrm{T}_{\mathrm{H} \rightarrow \mathrm{P}}(\mathrm{ms})$ & 1 & 1.9 & 1.9 & $\mathrm{~T}_{\mathrm{H} \rightarrow \mathrm{P}}(\mathrm{ms})$ & 30 & 35 & 35 & 33 \\
\hline $\mathrm{E}$ & 0.25 & 0.25 & 0.25 & $\mathrm{E}$ & 0.25 & 0.25 & 0.25 & 0.25 \\
\hline A & 800 & 1100 & 500 & A & 5400 & 10400 & 3000 & 570 \\
\hline$T^{*}{ }_{H \rightarrow p}(\mathrm{~ms})$ & 0.46 & 0.63 & 0.63 & $T^{*}{ }_{n \rightarrow P}(\mathrm{~ms})$ & 7.37 & 7.67 & 7.67 & 7.56 \\
\hline$T^{*}{ }_{1 p}(\mathrm{~ms})$ & 2.41 & 2.54 & 1.86 & $\mathrm{~T}^{*}{ }_{1 \rho}(\mathrm{ms})$ & 90.40 & 96.01 & 96.01 & 93.83 \\
\hline$a_{+}$ & 2.16 & 3.02 & 3.03 & $a_{+}$ & 4.07 & 4.56 & 4.56 & 4.36 \\
\hline a. & 0.41 & 0.75 & 1.02 & a. & 0.33 & 0.36 & 0.36 & 0.35 \\
\hline$a_{0}$ & 1.29 & 1.88 & 2.03 & $\mathrm{a}_{0}$ & 2.2 & 2.46 & 2.46 & 2.36 \\
\hline$b$ & 0.90 & 2.25 & 3.10 & b & 1.35 & 1.66 & 1.66 & 1.53 \\
\hline
\end{tabular}

\begin{tabular}{c|c|c|c|c}
$\mathrm{CS}(\mathrm{ppm})$ & -1.08 & -2.80 & -6.66 & -4.20 \\
$\sigma$ & $(\downarrow)$ & $(\mathrm{A})$ & $(\mathrm{X})$ & $(\approx)$ \\
& 0.16 & 0.22 & 0.10 & 0.07
\end{tabular}

Note: $\mathrm{CS}=$ chemical slift in ppm; $\mathrm{T}_{1 p}=$ spin-lattice relaxation time in the rotating frame annealed $\mathrm{NC} 5 \%=$ annealed poly[bis(trifluoroethoxy)phosphazene/ $/ \mathrm{TiO}_{2} 5 \%$ at $220^{\circ} \mathrm{C}$ for 2 hours; $T_{\mathrm{F} \rightarrow \mathrm{P}}=$ the time constant that represents the polarization transfer from ${ }^{19} \mathrm{~F}$ to ${ }^{31} \mathrm{P} ; \mathrm{A}=$ relative intensity; $\mathrm{T}_{\mathrm{F} \rightarrow \mathrm{p}}$ and $\mathrm{T}^{*}{ }_{1 \rho}$ is the effective parameter; $\sigma=$ the standard deviations; $\mathcal{E}$ is the ratio of the nuclei number

The $\mathrm{T}_{\mathrm{H}-\mathrm{P}}$ for the fast $\mathrm{CP}$ curve the broad components are 1 to $1.9 \mathrm{~ms}$, which are very close, implying that they may have similar dipolar coupling from 55 to $100 \mathrm{~Hz}$, with corresponding internuclear distances of $10.63-12.97 \AA$. This indicates that the difference in sidechain mobility is subtle amongst rigid portion of the crystalline components, which is faster than in the annealed neat polymer. This implies that the filler does effect the sidechain mobility in the rigid contribution of the broad components. The $\mathrm{T}_{\mathrm{H}-\mathrm{P}}$ values for the slow CP curve of the broad components are 30 to $35 \mathrm{~ms}$, implying that they may have 
dipolar coupling from 28 to $33 \mathrm{~Hz}$, with corresponding internuclear distances of 16.21$15.38 \AA$, which is essentially the same as seen for the annealed neat polymer. In addition, the relative intensity (A) at $-2.80 \mathrm{ppm}$ is the largest one amongst the broad components for both of $\mathrm{CP}$ curve parameters, indicating it is the predominating component. 


\section{Reference}

Kolodziejski, W.; Klinowski, J., Chemical reviews 2002, 102: 613-628

2 Taylorm, R. E.; Chin, N.; Dybowski, C., Journal of Molecular Structure 2007, 830: 147-155.

3 Aluas, M.; Tripon, C.; Filip, X.; FIlip, C., Journal of Optoelectronics and Advanced Materials 2007, 9; 664-667.

4 Shinji, A.; Robin, K. H,; Reinsberg, A., Journal of Magnetic Resonance 1999, 141: 91-103.

5 Naito, A.; McDowell, C. A., Journal of Chemical Physics 1986, 84: 4181-4186. 


\section{CHAPTER 10}

\subsection{Conclusion}

\section{CONCLUSION}

The ${ }^{31} \mathrm{P}$ and ${ }^{19} \mathrm{~F}$ solid-state NMR spectra reveal information about the polymer morphologies in both the polymer backbone and sidechain. The ${ }^{19} \mathrm{~F}$ and ${ }^{31} \mathrm{P}$ NMR spectra were deconvolved into several different components. The models were adjusted and validated to be consistent with all spectra for four samples over their corresponding temperature ranges. The relaxation data were used to give insight about how each component's morphology changes with variable temperature and with different sample treatments. The relaxation constants, $T_{1}, T_{2}$ and $T_{1 \rho}$, included, were used to assign the components as to their domain size and mobility. In addition, the cross polarization data were used to give more insights about the structural and morphological changes of the rigid components. At this stage, it becomes essential and necessary to assign the polymer components in the NMR spectra to different polymer phases, and thereby draw conclusions on the polymer morphological behaviors upon annealing and nano-filling.

Based on the discussion on chapter 7,8 , and 9 , the ${ }^{19} \mathrm{~F}$ and ${ }^{31} \mathrm{P}$ deconvolution models were refined and readjusted to make them the most reasonable fit for all data sets. The ${ }^{31} \mathrm{P}$ NMR spectra were fitted to two different models for the two different temperature ranges. At low temperature over $20-40^{\circ} \mathrm{C}$, totally three broad contributions are established based on the $\mathrm{T}_{1}, \mathrm{~T}_{2}$, and $\mathrm{T}_{1 \rho}$ data, together with the $\mathrm{CP}$ data, including ${ }^{19} \mathrm{~F}$ to ${ }^{31} \mathrm{P}$ and ${ }^{1} \mathrm{H}$ to ${ }^{31} \mathrm{P}$ cross polarization. Two narrow components are also revealed at this stage. At higher temperature, the three broad components are all become narrower, while they remain broader than the two narrow components. For ${ }^{19} \mathrm{~F}$ NMR spectra, at low temperature from 
20 to $40^{\circ} \mathrm{C}$, for both annealed and non-annealed samples, there are two broad and two narrow components observed.

The current understanding of the morphology of PBFP was described in chapter 2; however the most important aspects are briefly recapped and some additional isights are offered. Upon solvent casting, PBFP tends to form spherulites that are composed of either a 3D chain folded $\alpha$-orthorhombic crystal form, with unit cell parameters as: $a=10.14 \AA$, $\mathrm{b}=9.35 \AA$, and $\mathrm{c}=4.86 \AA$ or a $3 \mathrm{D}$ chain-folded $\beta$-monoclinic form $(\mathrm{a}=10.03 \AA, \mathrm{b}=9.37$ $\AA, c=4.86 \AA$ and $\gamma=0.91^{\circ}$ ), or a mixture of those two forms. After heating through its $T(1)$, the $\alpha$ form is transformed to a 2D pseudohexagonal meso-phase Upon further heating above $\mathrm{T}_{\mathrm{m}}$, the spherulitic structure melts and becomes isotropic. After the temperature is reduced below $T_{m}$, the isotropic melt returns to the $2 \mathrm{D}$ pseudohexagonal mesophase $(\delta)$. Further cooling below $\mathrm{T}(1)$, leads to the 3D $\gamma$-orthorhombic structure (as presented in Figure 10.1). The $\gamma$ cell has unit corresponding to four monomer units and its unit cell parameters are: $\mathrm{a}=20.60 \AA, \mathrm{b}=9.40 \AA$ and $\mathrm{c}=4.86 \AA$, the $\alpha$-orthorhombic cell is about one half the size of the $\gamma$-cell with two monomer units, and the hexagonal $\beta$ form is quite similar to $\alpha$-cell. Therefore, annealing treatment increases the proportion of the $\gamma$-cell, and correspondingly decrease the percentage of the $\alpha$-cell, while the proportion of the $\beta$-cell remain relatively stable. 

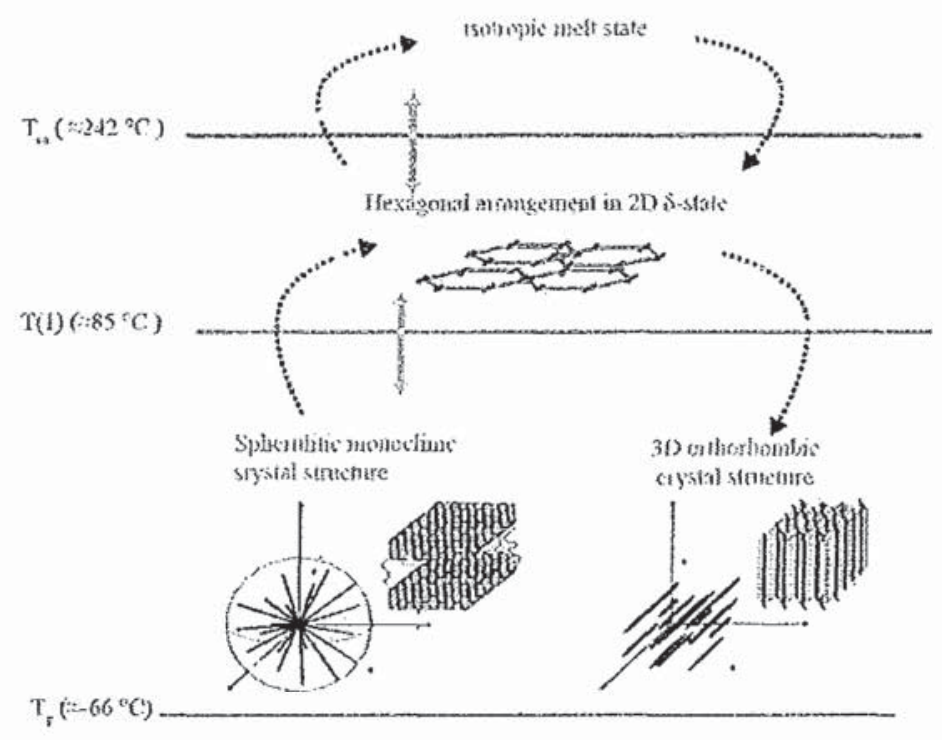

Figure 10.1 The transformation of PBFP over temperature

The different components in the ${ }^{31} \mathrm{P}$ NMR spectra should correspond to the different phase environments within the polymer. Based on the current morphological picture, the components of the established models for ${ }^{19} \mathrm{~F}$ and ${ }^{31} \mathrm{P}$ are assigned to the polymer phases as follows:

1) The $\alpha$ and $\gamma$ forms have the same cis-trans backbone configurations. To be more precise, the $\alpha$ crystalline form is more rigidly packed by having the smallest interchain spacing because of its chain folded formation, which results in a spherulitic morphology with largest domain size. Thus the ${ }^{31} \mathrm{P}$ NMR signal arising this phase should have the largest linewidth, longest $T_{1}$, shortest $T_{2}$, largest $T_{1} / T_{2}$, and longest correlation time values amongst all the phases. Furthermore, as it must be in the slow motion regime, its activation energy calculated from $T_{1}$ and $T_{1 p}$ should have negative values. In contrast, the $\gamma$ form has slightly larger interchain spacing and slightly smaller domain size because of its chain extended backbone configuration, suggesting it should have slight narrower linewidth than the $\alpha$ form and have the second largest $T_{1}$, the second smallest $T_{2}$, the second largest $T_{1} / T_{2}$ 
and second longest correlation time values. Its activation energy based on $T_{1}$ and $T_{1 p}$ should also be negative. In addition, the signals arising from $\alpha$ and $\gamma$ form should have the very similar chemical shifts as they have essentially the same backbone configuration. Moreover, because the $\alpha$ crystalline is more rigidly packed, the side chain motion of the $\alpha$ form is more restricted than the $\gamma$ form, suggesting that the ${ }^{19} \mathrm{~F}$ NMR signal from the $\alpha$ form is much broader.

2) The $\beta$ phase is more mobile due to its shorter chain length, thus is must have faster motion than observed in both $\alpha$ and $\gamma$ forms, resulting in much narrower line-widths. The $\beta$ components have slightly different backbone configuration than both $\alpha$ and $\gamma$ forms due to its greater configurational flexibility, where significant contributions from the cis-cis and trans-trans configurations are to be expected. This affects its chemical shifts, resembling values nearer to those in the more mobile amorphous and meso-phases. Thus, it is mostly likely that the $T_{l \rho}$ behavior in of $\beta$ phase should be further into the fast motion regime. Moreover, the sidechain of $\beta$ form is less restricted due to the large interchain spacing, thus its ${ }^{19} \mathrm{~F}$ NMR signal should be much narrower compared to those from $\alpha$ and $\gamma$ phase domains.

3) The hexagonal meso-phase ( $\delta$ ) has unit cell parameters as: $a=11.90 \AA, b=11.90$ $\AA, \mathrm{c}=4.86 \AA$. Its NMR signal should be observed when the temperature is raised to its $\mathrm{T}(1)$ transition temperature, i.e. around $60^{\circ} \mathrm{C}$. Thus when the temperature is approaching it $\mathrm{T}(1)$, the $\alpha$ phase proportion should decrease and extra component(s) should appear in the spectra for all four samples. Only this pseudohexagonal modification exists between its $\mathrm{T}(1)$ and $\mathrm{T}_{\mathrm{m}}$. Therefore, the signal from the $\delta$ phase should only be observed in the high temperature range, not at annealed PBFP or annealed NC 5\% after cooling down to room 
temperature. This hexagonal phase is liquid crystalline, and thus is much more dynamic than the $\alpha, \gamma$, and $\beta$ phases in both the polymer backbone and sidechain motion. The ${ }^{31} \mathrm{P}$ NMR signal originated from it should have a much narrower linewidth, shorter $\mathrm{T}_{1}$, longer $T_{2}$, longer $T_{1 \rho}$ and smaller $T_{1} / T_{2}$ ratio compared to other crystalline forms that are observed at the low temperature range ${ }^{2}$.

4) The amorphous phase has randomly coiled chains without consistent structural configuration, thus is the most loosely packed with large interchain spacing. Therefore it should have the faster backbone and sidechain motion than the crystalline phase. The ${ }^{31} \mathrm{P}$ NMR signal arising from it should have the narrowest linewidth, the smallest $T_{1}$ values, the largest $T_{2}$ and the smallest $T_{1} / T_{2}$ ratios.

For cast PBFP, all three crystalline phases should be observed, with the $\alpha$ crystalline phase dominating along with the amorphous phase. For annealed PBFP, all three crystalline phases should also be observed, with an increased proportion of the $\gamma$ form, along with decreased $\alpha$ and amorphous proportion compared to cast PBFP. The linewidth of the sum of the $\alpha$ and $\gamma$ crystalline components at -4.55 ppm should become narrower, because the chain folded $\alpha$ form crystalline is transformed to the $\gamma$ chain extended form upon annealing, leading the $\gamma$ form, which would have narrower linewidth, which is expected to dominate at this stage. While the $\beta$ form should remain relatively stable for both its linewidth and proportion as it does not transform to another crystalline phase or amorphous phase upon annealing.

Upon heating, both the crystalline and amorphous domain have increased backbone and sidechain motion, thus result in narrower spectral line-width. The crystalline phases still remain broader than those of the amorphous phases. The morphological and dynamic 
properties of the polymers also change when the temperatures approach their $T(1)$ values. To be more specific, the $\alpha$ phase melts to a pseudohexagonal mesophase, which would be more dynamic than other crystalline phase, thus it would have the narrowest line-width among all crystalline phases. The $\beta$ form becomes more mobile, resulting in its average chemical shifts and relaxation times becoming indistinguishable from the amorphous phases. The $\gamma$ form would also experience a faster motion than at low temperature. Therefore, in total two different models should be used to deconvolved the spectra, one for temperature range below its $\mathrm{T}(1)$, another for temperature above $\mathrm{T}(1)$ and below $\mathrm{T}_{\mathrm{m}}$.

At low temperature, based on the analysis of ${ }^{31} \mathrm{P}$ NMR data, the ${ }^{31} \mathrm{P}$ NMR spectra have two broad contributions around $-4.55 \mathrm{ppm}$, as proved by it $\mathrm{T}_{1}$ bi-exponential behavior in for all four samples. One of those broad contributions has a relatively long $T_{1}$ and the other one has a relatively short $T_{1}$, indicating that those two contributions originate from different morphological domains with different motions. The other three narrow components also have bi-exponential behaviors where one contribution has a $\mathrm{T}_{1}$ on the scale larger than $10 \mathrm{~s}$, which is similar to the time scale for component at $-4.55 \mathrm{ppm}$, while another contribution has a much shorter $T_{1}$ which is less than $5 \mathrm{~s}$. This suggests that there is one broad component sits underneath of those three narrow components, which is difficult to observe directly.

At high temperature, the relative broad contribution at $-4.55 \mathrm{ppm}$ is shifted to -7.37 $\mathrm{ppm}$, and the second broad contribution at $-4.55 \mathrm{ppm}$ is shifted to $-8.13 \mathrm{ppm}$, which is further supported by their similar $E_{a}$ values calculated from the $T_{1}$ and $T_{2}$ values. The broad component at $-9.79 \mathrm{ppm}$ is assigned to the broad component that was underneath of the 
three narrow components at the low temperature range, as approved by it broad line width and it similar $\mathrm{E}_{\mathrm{a}}$ values (based on the $\mathrm{T}_{1 \rho}$ data) to the component at $-7.37 \mathrm{ppm}$

Thus, at low temperature, three broad and three narrow contributions are established. The broadest contribution at $-4.55 \mathrm{ppm}$ is assigned to the $\alpha$ form crystalline, there are several phenomena that support this assignment. Firstly, it has the largest $\mathrm{T}_{1}$ value which could be more than $20 \mathrm{~s}$, indicating it undergoes the slowest motion. Also, its $T_{1} / T_{2}$ value are on the order of $10^{5}$, resulting in the longest correlation time among all contributions, suggesting that it originates from the most rigid, tightly packed crystalline phase with the large domain size. In addition, its $T_{1}$ values decrease with increasing temperature, indicating that it falls in the slow motion regime. Furthermore, the $\mathrm{T}_{1 \rho}$ values indicated its backbone local motion is slow with respect to the time scale of $13 \mu$ s. The second broad contribution at $-4.55 \mathrm{ppm}$ is assigned to the $\gamma$ form cell, as evidenced by its similar chemical shift to the $\alpha$ form crystalline, increased proportion upon annealing, its large $T_{1}$, and large $\mathrm{T}_{1} / \mathrm{T}_{2}$ ratio, which is only smaller than the $\alpha$ form. Also, the negative activation from $T_{1 p}$ value indicates that its backbone local motion is slow with respect to the time scale of the spin-locking field. The third broad component sits underneath of the narrow components is assigned to one broad component to arising from the $\beta$ from. As suggested by the large and similar $\mathrm{T}_{1}$ values for the three narrow components from their bi-exponential behavior; its chemical shift differs from those from the $\alpha$ and $\beta$ phases; and their $T_{1 \rho}$ values indicating local motion in of the backbone are slow with respect to the time scale of $13 \mu \mathrm{s}$.

At high temperature, the motion of both backbone and sidechain is enhanced, resulting a narrower linewidth, shorter $T_{1}$, longer $T_{2}$, smaller $T_{1} / T_{2}$ ratio, and the backbone local motion is faster with respect to $13 \mu$ s compared to their behaviors at the low 
temperature range. The component at $-7.37 \mathrm{ppm}$ originates from the $\alpha$ phase, which still has the broadest line-width at $220 \mathrm{~Hz}$, largest $T_{1}$ values, and large $T_{1} / T_{2}$ ratios. The signal appearing at $-9.79 \mathrm{ppm}$ is assigned to the $\beta$ form, based on the second largest $T_{1}$ and $T_{1} / T_{2}$ ratio. The component at -8.13 originates from the $\gamma$ phase, which has a much narrower line width than the $\alpha$ and $\beta$ phases because of its liquid crystal form, while still broader than the mobile phases. The chemical shifts of all three crystalline phases are shifted to lower chemical shifts which is mainly because the backbone configuration would undergo random rotations as the temperature increases, and that motion would result in significant proportions of cis-cis, trans-trans backbone configuration, leading to decreased chemical shifts.

The same assignment of polymer phases for the corresponding to components holds for the NC $5 \%$ and annealed NC $5 \%$ samples. The addition of nano- $\mathrm{TiO}_{2}$ suppresses the growth of the crystallites as indicated by decreased $T_{1}$ values, increased $T_{2}$ values, decreased $T_{1} / T_{2}$ ratios and thus decreased correlation times compared to those of their pure counterparts for the ${ }^{31} \mathrm{P}$ NMR spectra.

The CP data in chapter 9 provide more detailed information about the backbone in the rigid domains, as it emphasises the signal from the rigid over the mobile components, and is furthermore independent from accurately determined ${ }^{31} \mathrm{P}$ relaxation delay, where any broad contribution supressed due to saturation are now observed.

The ${ }^{19} \mathrm{~F}$ to ${ }^{31} \mathrm{P}$ CP shows that there are three broad components for PBFP, two of them deconvolved from the large broad component and appear at -2.32 and $-4.04 \mathrm{ppm}$. They can be assigned to the $\alpha$ and $\gamma$ crystalline form, respectively, because of their similar chemical shifts and the enhanced proportion for the component at -4.04 after annealing. The other 
component at $-7.96 \mathrm{ppm}$ is assigned to originate from the $\beta$ form. This assignment is consistent with that based on the relaxation analysis. For annealed PBFP, a new small component shows up at $-5.57 \mathrm{ppm}$ after annealing, and can be assigned to the $\gamma$ crystalline form. As proved by the increase of its total percentage, and the decreased proportion of the $\alpha$ phase, along with the relative stable proportion for the $\beta$ phase. For the sample of NC $5 \%$, four broad components are observed. The component at $-2.32 \mathrm{ppm}$ arises from the $\alpha$ crystalline phase, the component at -4.04 and -5.41 ppm come from the $\gamma$ crystalline domain, while the components at $-7.96 \mathrm{ppm}$ corresponds to the $\beta$ crystalline formation. In annealed NC $5 \%$, the sum of the proportion of components at -3.91 and $-4.96 \mathrm{ppm}$ increases after annealing. This supports the suggested assignment that they are the $\gamma$ form. Moreover, for non-annealed samples, the averaged dipolar coupling is on the order of $2 \mathrm{~Hz}$ which is larger than that of their annealed samples at $1.25 \mathrm{~Hz}$. This means that the motion of the sidechain and backbone is enhanced upon annealing, resulting in a larger interchain spacing. This indicates the suggestion that upon annealing the $\gamma$ phase proportion increases.

From the ${ }^{1} \mathrm{H} \rightarrow{ }^{31} \mathrm{P} \mathrm{CP}$ analysis, multimode $\mathrm{CP}$ dynamic behavior was observed for all four samples. The same phenomenon was not seen in the ${ }^{19} \mathrm{~F} \rightarrow{ }^{31} \mathrm{P} \mathrm{CP}$ as it was obscured by the rapid $-\mathrm{CF}_{3}$ rotation. The large difference between $\mathrm{T}_{\mathrm{H}-\mathrm{P}}$ for two crystalline environments indicates that there are two distinct sidechain conformations with different mobilities in each of these two crystalline domains. This multimode CP behavior is observed at -1.39 to $-3.02 \mathrm{ppm}$ proving that the signals form the $\alpha$ and $\gamma$ phases have the same backbone configuration and same chemical environments. The multimode for component at $-6.66 \mathrm{ppm}$ is also observed is because the $\beta$ phase has different backbone configuration which have different $\mathrm{CP}$ rate. 
For ${ }^{19} \mathrm{~F}$ at low temperature, the NMR spectra of PBFP and NC 5\% are deconvolved using three-component model. Each component shows a bi-exponential decay in the $\mathrm{T}_{1}$ data. All three have a one long $T_{1}$ on the order of 0.54 to $0.85 \mathrm{~s}$, and one short $\mathrm{T}_{1}$ in common on the order of 0.40 to $0.44 \mathrm{~s}$. In addition, all three components have common activation energies ranging from -5 to $-10 \mathrm{~kJ} \cdot \mathrm{mol}^{-1}$. This indicates that a broad component lies underneath all three contributions that was not previously observed. Therefore, the signal contains two broad components and two narrow components for non-annealed samples at low temperature. For annealed samples, the broad component at $-76.62 \mathrm{ppm}$ has a linewidth of $1647 \mathrm{~Hz}$, which is twice as broad as the component at $-76.73 \mathrm{ppm}$ with $538 \mathrm{~Hz}$ linewidth, suggesting that this broad contribution is composed of two similar small broad contributions. Therefore, the spectrum is composed of two broad components and three narrow components for annealed samples at low temperature.

At high temperature, the components at -76.42 and $-76.76 \mathrm{ppm}$ have smaller $\mathrm{T}_{1}$ than the other two components and similar $E_{a}$ values (based on the $T_{1}$ data) are both likely assigned to originate from the rigid component. Those two components should originate from two broad components at low temperature, which proves the assumption that there are two broad components for ${ }^{19} \mathrm{~F}$ NMR spectra at low temperature for all samples.

In addition to the phase assignments according to their morphological changes, the effect of nano- $\mathrm{TiO}_{2}$ filling on the morphology changes of PBFP and its nanocomposite is also studied. The nano- $\mathrm{TiO}_{2}$ filling decreases the total proportion of the $\alpha$ and $\beta$ crystalline phase for both annealed and non-annealed PBFP, as observed in Figure 7.4 and Figure 7.5. In addition, the presence of nano- $\mathrm{TiO}_{2}$ would inhibit the sidechain motion slightly as suggested by increased $T_{1} / T_{2}$ ratios and correlation times with temperature. However, it 
suppresses the growth of the crystallites as indicated by the increased $T_{1}$, increased $T_{2}$ and increased $\mathrm{T}_{1 \rho}$ for ${ }^{19} \mathrm{~F}$ signal, whose motion is on the fast motion regime compared to neat PBFP.

Moreover, in chapter 9 , based on the ${ }^{19} \mathrm{~F}$ to ${ }^{31} \mathrm{P} \mathrm{CP}$, the same $\mathrm{T}_{\mathrm{F} \rightarrow \mathrm{P}}$ between nanofilled samples and their pure counterparts indicates that $\mathrm{TiO}_{2}$ appears to have no significant influence on the sidechain motion and $-\mathrm{CF}_{3}$ rotation in all crystalline phases. The ${ }^{1} \mathrm{H}$ to ${ }^{31} \mathrm{P}$ $\mathrm{CP}$ indicated that nano-filling enhances the formation of the crystalline phase but suppresses the size of the crystallites. As indicated with the $\mathrm{NC} 5 \%$ sample, nano- $\mathrm{TiO}_{2}$ alters the proportion of the $\alpha$ and $\gamma$ crystal phases by favouring the phase with more mobile sidechains - the $\gamma$ phase. Because the ratio between the crystalline phase with more rapid CP rate (the $\alpha$ phase) to that of the crystalline phase with slower CP rate (the $\gamma$ phase) in NC 5\% is smaller than that in PBFP. This is further supported by the decreased amorphous component (3\%) in sample NC 5\% compared to that in sample PBFP (15\%). 


\section{Reference}

1 Nakamura, H.; Masuko, T.; Kojima, M.; Magill, J. H., Macromolecular Chemistry and Physics 1999, 2519-1519

2 Taylor, S. A.; White, J. L.; Elbaum, N. C., Macromolecules 1992, 25: 3369-3376. 